
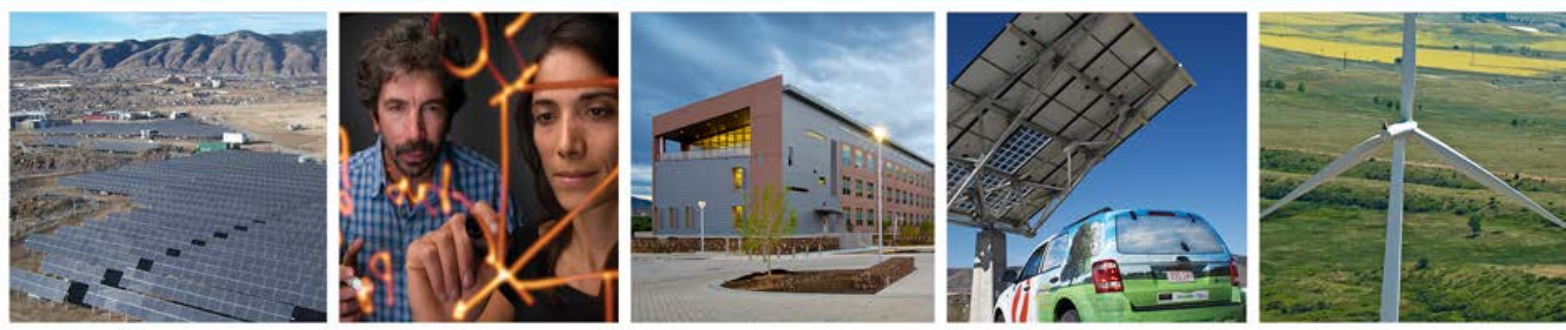

\title{
Best Practices Handbook for the Collection and Use of Solar Resource Data for Solar Energy Applications
}

M. Sengupta, ${ }^{1}$ A. Habte, ${ }^{1}$ S. Kurtz, ${ }^{1}$ A. Dobos, ${ }^{1}$ S. Wilbert, ${ }^{2}$ E. Lorenz, ${ }^{3}$ T. Stoffel, ${ }^{4}$ D. Renné,${ }^{5}$ C. Gueymard, ${ }^{6}$ D. Myers, ${ }^{7}$ S. Wilcox, ${ }^{4}$ P. Blanc, ${ }^{8}$ and R. Perez ${ }^{9}$

${ }^{1}$ National Renewable Energy Laboratory

${ }^{2}$ German Aerospace Center

${ }^{3}$ University of Oldenburg

${ }^{4}$ Solar Resource Solutions, LLC

${ }^{5}$ International Energy Agency Solar Heating and Cooling Programme

${ }^{6}$ Solar Consulting Services

${ }^{7}$ National Renewable Energy Laboratory-Retired

${ }^{8}$ Mines ParisTech

${ }^{9}$ State University of New York at Albany

This update was prepared in collaboration with International Energy Agency Solar Heating and Cooling Programme Task 36 and Task 46

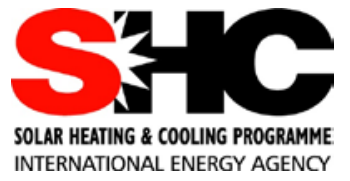

NREL is a national laboratory of the U.S. Department of Energy Office of Energy Efficiency \& Renewable Energy Operated by the Alliance for Sustainable Energy, LLC

This report is available at no cost from the National Renewable Energy Laboratory (NREL) at www.nrel.gov/publications.

Technical Report

NREL/TP-5D00-63112

February 2015

Contract No. DE-AC36-08GO28308 


\title{
Best Practices Handbook for the Collection and Use of Solar Resource Data for Solar Energy Applications
}

\author{
M. Sengupta,${ }^{1}$ A. Habte,${ }^{1}$ S. Kurtz, ${ }^{1}$ A. Dobos, ${ }^{1}$ S. Wilbert, ${ }^{2}$ \\ E. Lorenz, ${ }^{3}$ T. Stoffel, ${ }^{4}$ D. Renné,${ }^{5}$ C. Gueymard, ${ }^{6}$ D. Myers, ${ }^{7}$ \\ S. Wilcox, ${ }^{4}$ P. Blanc, ${ }^{8}$ and R. Perez ${ }^{9}$ \\ ${ }^{1}$ National Renewable Energy Laboratory \\ ${ }^{2}$ German Aerospace Center \\ ${ }^{3}$ University of Oldenburg \\ ${ }^{4}$ Solar Resource Solutions, LLC \\ ${ }^{5}$ International Energy Agency Solar Heating and Cooling Programme \\ ${ }^{6}$ Solar Consulting Services \\ ${ }^{7}$ National Renewable Energy Laboratory—Retired \\ ${ }^{8}$ Mines ParisTech \\ ${ }^{9}$ State University of New York at Albany \\ This update was prepared in collaboration with International \\ Energy Agency Solar Heating and Cooling Programme Task 36 \\ and Task 46
}

Prepared under Task No. SS13.3532

National Renewable Energy Laboratory 15013 Denver West Parkway Golden, CO 80401

303-275-3000 • www.nrel.gov
NREL is a national laboratory of the U.S. Department of Energy Office of Energy Efficiency \& Renewable Energy Operated by the Alliance for Sustainable Energy, LLC

This report is available at no cost from the National Renewable Energy Laboratory (NREL) at www.nrel.gov/publications.

\section{Technical Report}

NREL/TP-5D00-63112

February 2015

Contract No. DE-AC36-08GO28308 


\title{
NOTICE
}

This report was prepared as an account of work sponsored by an agency of the United States government. Neither the United States government nor any agency thereof, nor any of their employees, makes any warranty, express or implied, or assumes any legal liability or responsibility for the accuracy, completeness, or usefulness of any information, apparatus, product, or process disclosed, or represents that its use would not infringe privately owned rights. Reference herein to any specific commercial product, process, or service by trade name, trademark, manufacturer, or otherwise does not necessarily constitute or imply its endorsement, recommendation, or favoring by the United States government or any agency thereof. The views and opinions of authors expressed herein do not necessarily state or reflect those of the United States government or any agency thereof.

This report is available at no cost from the National Renewable Energy Laboratory (NREL) at www.nrel.gov/publications.

Available electronically at http://www.osti.gov/scitech

Available for a processing fee to U.S. Department of Energy and its contractors, in paper, from:

\author{
U.S. Department of Energy \\ Office of Scientific and Technical Information \\ P.O. Box 62 \\ Oak Ridge, TN 37831-0062 \\ phone: 865.576 .8401 \\ fax: 865.576 .5728 \\ email: mailto:reports@adonis.osti.gov
}

Available for sale to the public, in paper, from:

U.S. Department of Commerce

National Technical Information Service

5285 Port Royal Road

Springfield, VA 22161

phone: 800.553 .6847

fax: 703.605.6900

email: orders@ntis.fedworld.gov

online ordering: http://www.ntis.gov/help/ordermethods.aspx

Cover Photos: (left to right) photo by Pat Corkery, NREL 16416, photo from SunEdison, NREL 17423, photo by Pat Corkery, NREL 16560, photo by Dennis Schroeder, NREL 17613, photo by Dean Armstrong, NREL 17436, photo by Pat Corkery, NREL 17721. 


\section{Foreword}

The first version of this handbook was developed in response to a growing need by the solar energy industry for a single document addressing the key aspects of solar resource characterization. The solar energy industry has developed rapidly throughout the last few years, and there have been significant enhancements in the body of knowledge in the areas of solar resource assessment and forecasting. Thus, this second version of the handbook was developed from the need to update and enhance the initial version and present the state of the art in a condensed form for all of its users.

Although the first version of this handbook was developed by only researchers from the National Renewable Energy Laboratory, this version has additional contributions from an international group of experts primarily from the knowledge that has been gained through participation in the International Energy Agency's Solar Heating and Cooling Programme Task 36 and Task 46.

As in the first version, this material was assembled by scientists and engineers who have many decades of combined experience in atmospheric science, radiometry, meteorological data processing, and renewable energy technology development.

Readers are encouraged to provide feedback to the authors for future revisions and an expansion of the handbook's scope and content. 


\section{Preface}

As the world looks for low-carbon sources of energy, solar power stands out as the single most abundant energy resource on Earth. Harnessing this energy is the challenge for this century. Photovoltaics, solar heating and cooling, and concentrating solar power (CSP) are primary forms of energy applications using sunlight. These solar energy systems use different technologies, collect different fractions of the solar resource, and have different siting requirements and production capabilities. Reliable information about the solar resource is required for every solar energy application. This holds true for small installations on a rooftop as well as for large solar power plants. However, solar resource information is of particular interest for large installations, because they require a substantial investment, sometimes exceeding $\$ 1$ billion in construction costs. Before such a project is undertaken, the best possible information about the quality and reliability of the fuel source must be made available. That is, project developers need to have reliable data about the solar resource available at specific locations, including historic trends with seasonal, daily, hourly, and (preferably) subhourly variability to predict the daily and annual performance of a proposed power plant. Without this data, an accurate financial analysis is not possible.

In September 2008, the U.S. Department of Energy (DOE) hosted a meeting of prominent CSP developers and stakeholders. One purpose was to identify areas in which the DOE's CSP program should focus its efforts to help the industry develop and deploy projects. At the top of the priority list was the need to provide high-quality solar resource data and recommend to industry the best way to use these data for site selection and estimating power plant performance. The direct result was the National Renewable Energy Laboratory's (NREL's) first edition of Concentrating Solar Power: Best Practices Handbook for the Collection and Use of Solar Resource Data. The content was based on the experiences of scientists and engineers from industry, academia, and DOE for identifying the sources, quality, and methods for applying solar and meteorological data to CSP projects.

The International Energy Agency's (IEA's) Solar Heating and Cooling Programme (SHC) Task 36 on solar resource knowledge management and Task 46 on solar resource assessment and forecasting brought together the world's foremost experts in solar energy meteorology. This group of experts felt the need to create a collective document to disseminate the knowledge that was being developed through these tasks. It was decided that combining the efforts of the experts involved in the IEA task to build on the information in NREL's first version of this handbook would provide the best use of resources and deliver a handbook of outstanding quality to users. It was also decided that additional solar technologies, such as photovoltaics, would be incorporated along with additional aspects of energy meteorology that have become extremely important, such as solar forecasting.

This expanded version of the handbook presents detailed information about solar resource data and the resulting data products needed for each stage of a solar energy project, from initial site selection to systems operations. It also contains a summary of solar forecasting and its development throughout the last few years. This handbook is not meant to be read from cover to cover, but to be used as a reference during each project stage. The figure below lists these stages and shows which chapters contain information about the corresponding available data and resulting products. 


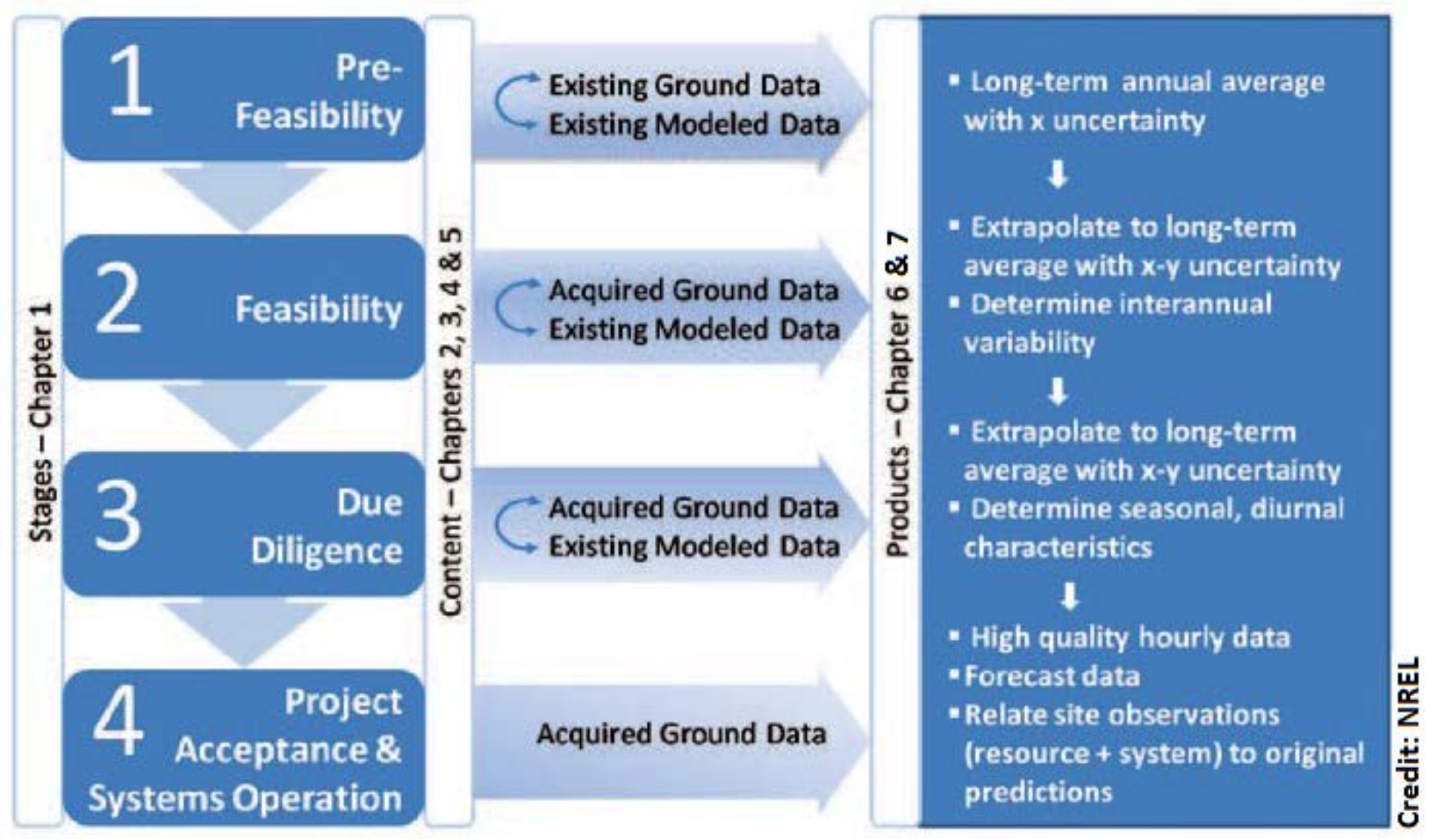

DOE's Solar Energy Technology Office, project developers, engineering procurement construction firms, utility companies, system operators, energy suppliers, financial investors, and others involved in solar energy systems planning and development will find this handbook to be a valuable resource for the collection and interpretation of solar resource data.

Further, this report serves as the final deliverable for the IEA SHC Task 36 on solar resource knowledge management, which ended in June 2011, and as an interim deliverable for the ongoing Task 46 on solar resource assessment and forecasting. As stated above, this report contains research findings from a number of experts from around the world who participated in Task 36 and are currently participating in Task 46. Future updates to this report are expected in June 2016 as the final deliverable to Task 46. 


\section{Acknowledgments}

This updated and expanded handbook is the collective effort of members of the Power Systems Engineering Center at the National Renewable Energy Laboratory (NREL), including Manajit Sengupta and Aron Habte; Craig Turchi and Mark Mehos from NREL's Buildings and Thermal Systems Center; Sarah Kurtz from NREL's Materials Applications and Performance Center; Aron Dobos from NREL's Strategic Energy Analysis Center; Stefan Wilbert from the German Aerospace Center, DLR; Tom Stoffel; Dave Renné, operating agent for the International Energy Agency's (IEA's) Solar Heating and Cooling Programme (SHC) Task 36 and Task 46; Daryl Myers; Steve Wilcox; Elke Lorenz from the University of Oldenburg, Germany; Steffen Stökler and Marion Schroedter-Homscheidt from the German Aerospace Center, DLR; Phillipe Blanc from Mines ParisTech in France; Richard Perez from State University of New York at Albany; and all of the participants of the IEA SHC Task 36 and Task 46. The critical reviews by our solar colleagues from industry, academia, and other federal agencies were invaluable to producing what we hope will be a useful reference for the solar power community. The coauthors are extremely grateful for the masterful editorial work by Katie Wensuc. The U.S Department of Energy's Solar Energy Technologies Program supported this work under DOE prime contract number DE-AC36-9-GO10337. The work performed by Dr. Elke Lorenz on the chapter "Forecasting Solar Radiation" was funded by the Federal Republic of Germany. Funding authority: Federal Ministry of Economics and Technology (BMWi). 


\section{List of Acronyms}

\begin{tabular}{|c|c|}
\hline $\mathrm{AC}$ & alternating current \\
\hline$\alpha_{\text {disk }}$ & solar disk angle (half angle) \\
\hline$\alpha_{\lim }$ & limit angle of pyrheliometer field of view \\
\hline $\mathrm{AM}$ & air mass \\
\hline AOD & aerosol optical depth \\
\hline$\alpha_{\text {slope }}$ & slope angle of pyheliometer field of view \\
\hline $\mathrm{AU}$ & astronomical unit \\
\hline ASTM & American Society for Testing and Materials \\
\hline AVHRR & Advanced Very High Resolution Radiometer \\
\hline BIPM & $\begin{array}{l}\text { Bureau International des Poids et Mesures } \\
\text { (International Bureau of Weights and Measures) }\end{array}$ \\
\hline BSRN & Baseline Surface Radiation Network \\
\hline $\mathrm{CPV}$ & concentrating photovoltaics \\
\hline $\operatorname{CSNI}\left(\alpha_{1}, \alpha_{2}\right)$ & $\begin{array}{l}\text { circumsolar normal irradiance emanating from the } \\
\text { region with distances between } \alpha_{1} \text { and } \alpha_{2} \text { from the } \\
\text { center of the solar disk }\end{array}$ \\
\hline CSR & circumsolar ratio \\
\hline CSP & concentrating solar power \\
\hline CST & concentrating solar technologies \\
\hline $\mathrm{COV}$ & coefficient of variation \\
\hline $\mathrm{DC}$ & direct current \\
\hline DEM & digital elevation model \\
\hline DHI & diffuse horizontal irradiance \\
\hline DISC & Direct Insolation Simulation Code \\
\hline DLR & $\begin{array}{l}\text { Deutsches Zentrum für Luft- und Raumfahrt } \\
\text { (German Aerospace Center) }\end{array}$ \\
\hline DNI & direct normal irradiance \\
\hline DOE & U.S. Department of Energy \\
\hline ESRA & European Solar Radiation Atlas \\
\hline ETR & extraterrestrial radiation \\
\hline FOV & field of view \\
\hline GFS & Global Forecast System \\
\hline GHI & global horizontal irradiance \\
\hline GOES & Geostationary Operational Environmental Satellite \\
\hline GUM & $\begin{array}{l}\text { Guide to the Expression of Uncertainty in } \\
\text { Measurements }\end{array}$ \\
\hline GTI & global titled irradiance \\
\hline IEA & International Energy Agency \\
\hline IFS & Integration Forecast System \\
\hline ISCCP & International Satellite Cloud Climatology Project \\
\hline ISIS & Integrated Surface Irradiance Study \\
\hline ISO & International Standards Organization \\
\hline $\mathrm{Kd}$ & $\begin{array}{l}\text { Diffuse horizontal radiation clearness index or } \\
\text { transmittance of the atmosphere }\end{array}$ \\
\hline
\end{tabular}




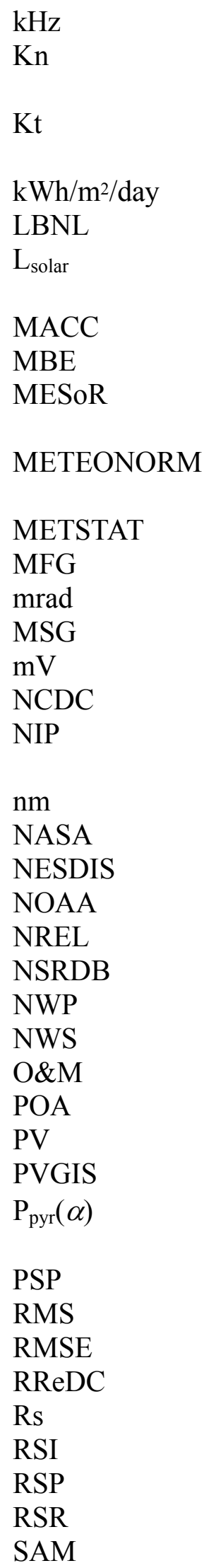

kiloHertz

Direct normal clearness index or transmittance of the atmosphere

Global horizontal radiation clearness index or transmittance of the atmosphere

kilowatt hours per square meter per day

Lawrence Berkeley National Laboratory

solar radiance profile for the solar disk and the circumsolar region

Monitoring Atmospheric Composition and Climate mean bias error

Management and Exploitation of Solar Resource Knowledge commercial data product of Meteotest, Bern, Switzerland meteorological-statistical solar radiation model Meteosat First Generation milliradian Meteosat Second Generation millivolt National Climatic Data Center Eppley Laboratory, Inc. Model Normal Incidence Pyrheliometer nanometer National Aeronautics and Space Administration NOAA's Satellite and Information Service National Oceanic and Atmospheric Administration National Renewable Energy Laboratory National Solar Radiation Database numerical weather prediction National Weather Service operations and maintenance plane of array photovoltaics photovoltaic geographical information system penumbra function evaluated at an angular distance $\alpha$ from the center of the sun precision spectral pyranometer root mean square root mean square error Renewable Resource Data Center responsivity rotating shadowband irradiometer rotating shadowband pyranometer rotating shadowband radiometer System Advisor Model 
SASRAB

SHC

SI

Si

SODA

SOLEMI

SOLMET

sr

SRB

SSE

SUNY

SURFRAD

SZA

TOA

TSI

TMM

TMY

$\mathrm{V}_{\text {unshade }}$

$\mathrm{V}_{\text {shade }}$

UV

$\mathrm{V}$

$\mathrm{W} / \mathrm{m}^{2}$

WMO

WRDC

WRC

WRF

WRR

$\mu \mathrm{V}$
Satellite Algorithm for Shortwave Radiation Budget

Solar Heating and Cooling Program

Système International

Silicon

Solar Radiation Data

Solar Energy Mining

Solar and Meteorological hourly dataset

steradian

Surface Radiation Budget

surface meteorology and solar energy

State University of New York

Surface Radiation Network

solar zenith angle

top of atmosphere

total solar irradiance (or solar constant)

typical meteorological month

typical meteorological year

voltage measured with unshaded pyranometer

voltage measured with shaded pyranometer

ultraviolet

volts

watts per square meter

World Meteorological Organization

World Radiation Data Center

World Radiation Center

Weather Research and Forecating

World Radiometric Reference

microvolt 


\section{Table of Contents}

1 Why Solar Resource Data Are Important to Solar Power.......................................................... 1

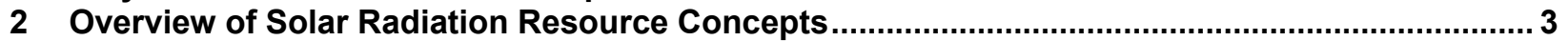

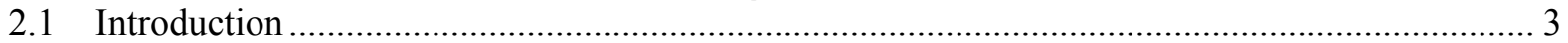

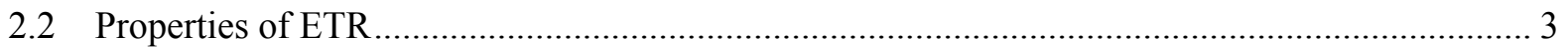

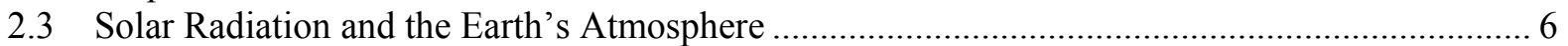

2.3.1 Relative Motions of the Earth and Sun ............................................................. 8

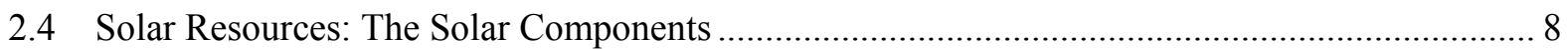

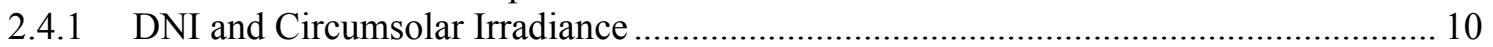

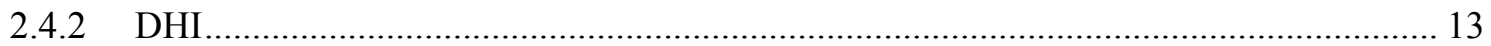

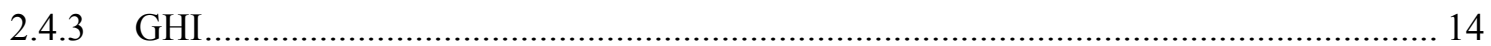

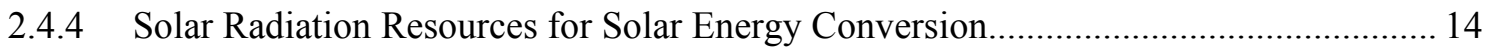

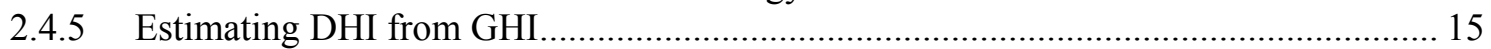

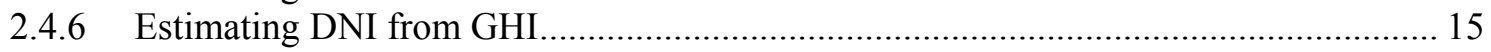

2.4.7 Estimating Solar Resources on a Tilted Surface ..................................................... 16

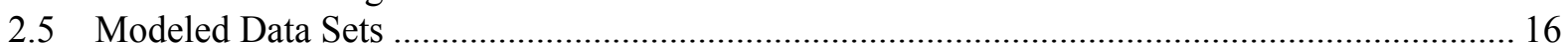

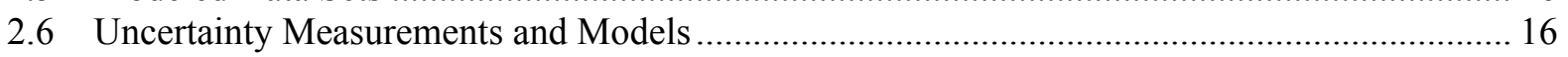

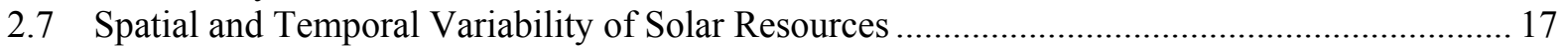

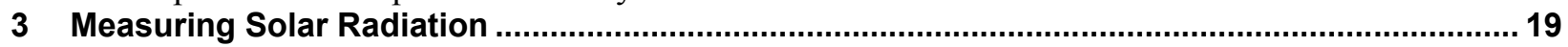

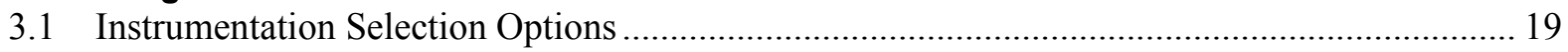

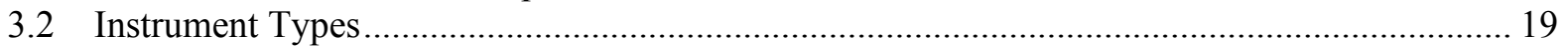

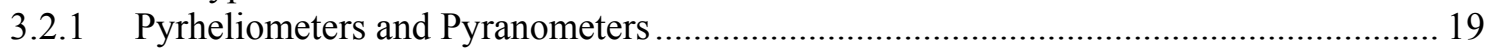

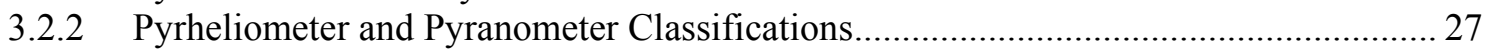

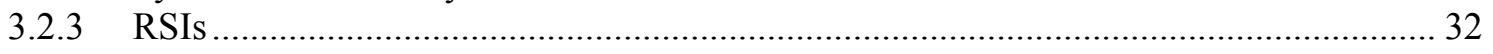

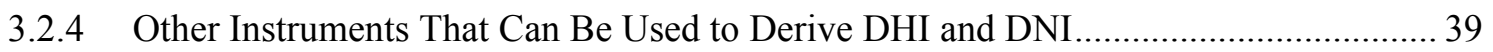

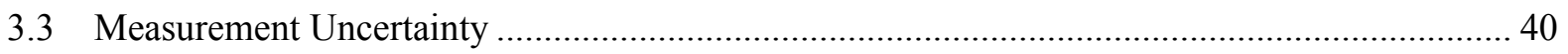

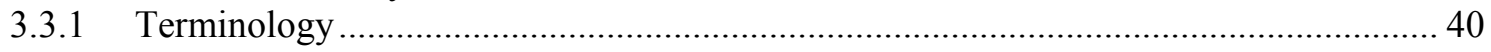

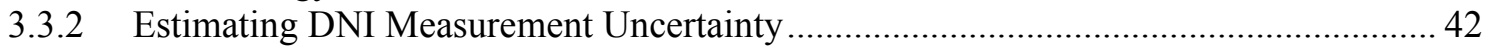

3.3.3 Estimating the Uncertainty of Pyrheliometer Calibrations ......................................... 42

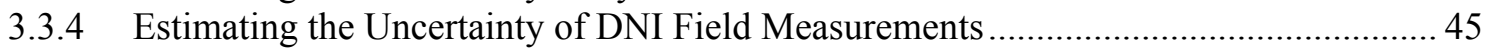

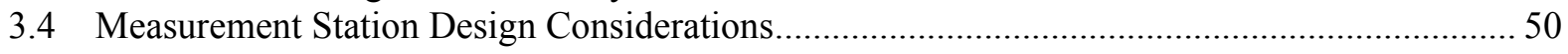

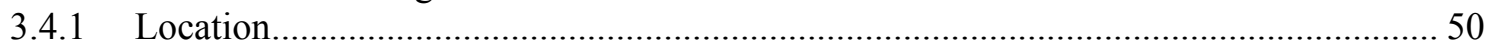

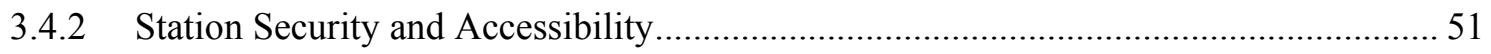

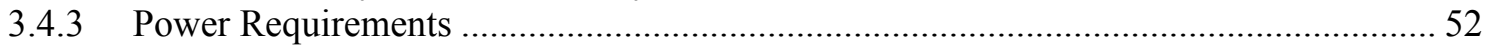

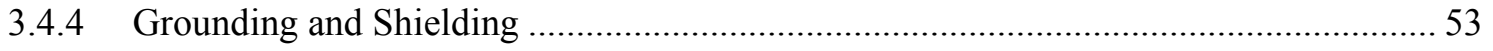

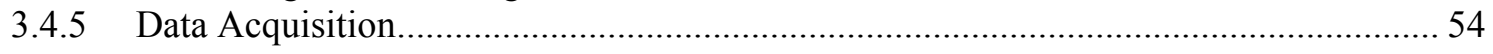

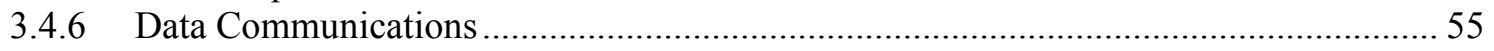

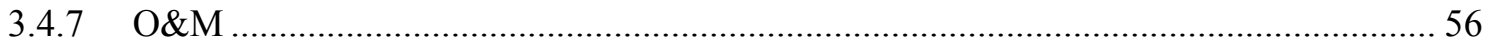

3.5 Data Quality Control, Data Correction, Data Quality Assessment, and Metadata..................... 58

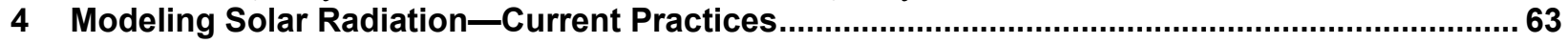

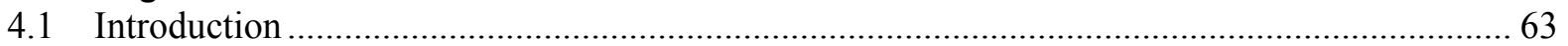

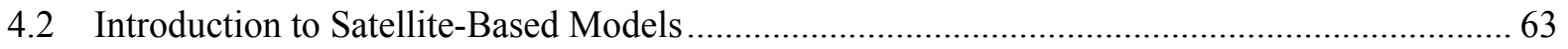

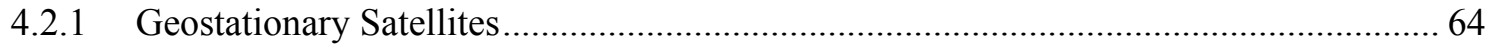

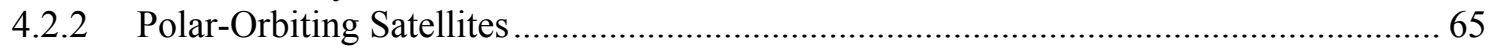

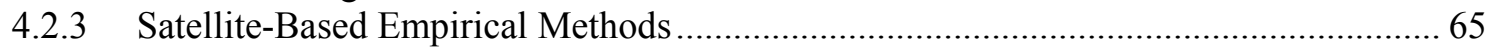

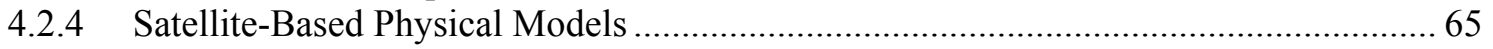

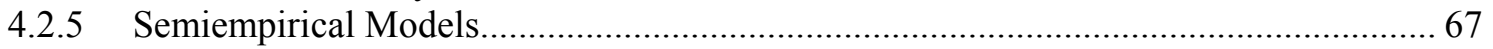

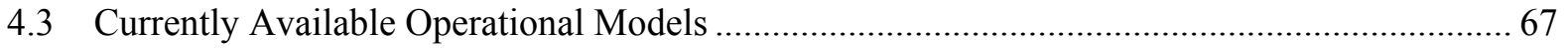

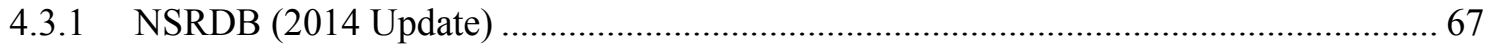

4.3.2 NASA/Global Energy and Water Cycle Experiment Surface Radiation Budget........... 68 


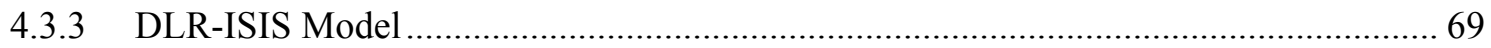

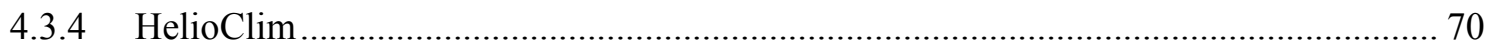

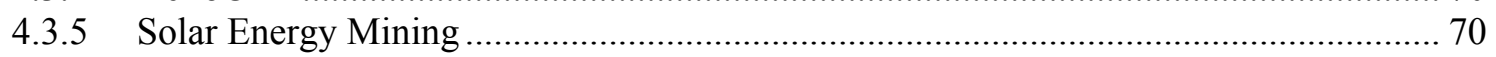

4.3.6 MACC-RAD Services................................................................................... 70

4.3.7 Perez/Clean Power Research.............................................................................. 71

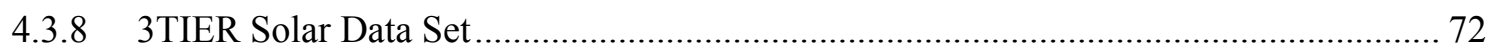

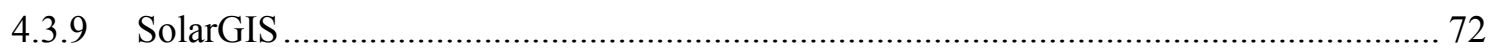

4.3.10 NOAA Global Surface Insolation Project ............................................................... 73

4.3.11 EnMetSol Model ............................................................................................. 73

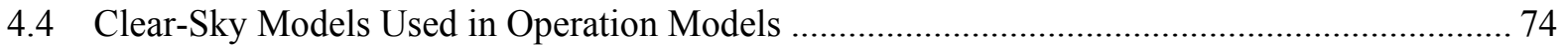

4.4.1 Bird Clear-Sky Model ................................................................................... 74

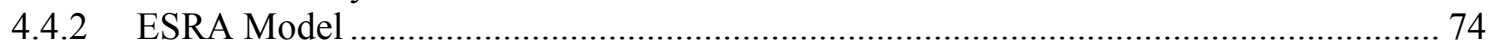

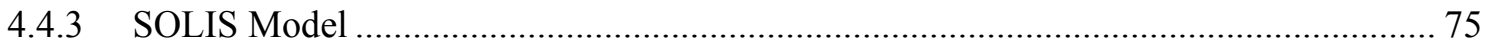

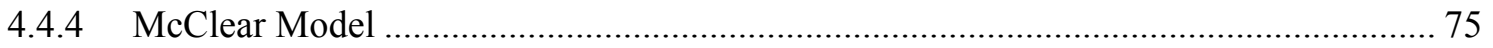

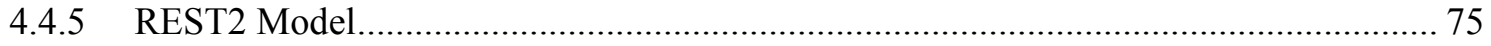

4.5 Model Uncertainty and Validation .............................................................................................. 76

$5 \quad$ Historical Solar Resource Data ...................................................................................... 82

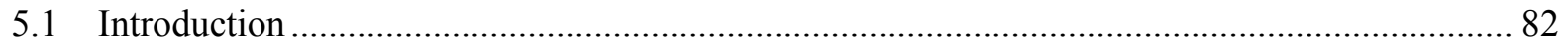

5.2 Solar Resource Data Characteristics .................................................................................. 82

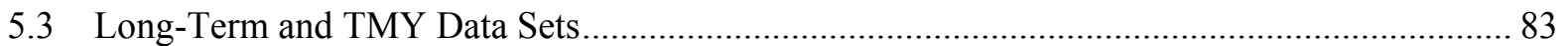

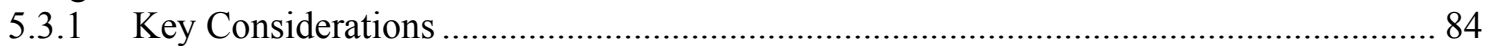

$5.4 \quad$ Solar Resource Data ………………………………………………………………….... 85

5.4.1 National Center for Environmental Protection/National Center for Atmospheric

Research Global Reanalysis Products ........................................................................ 85

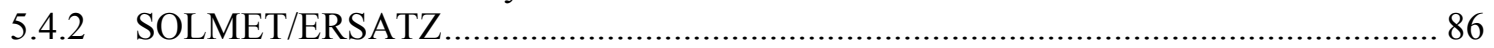

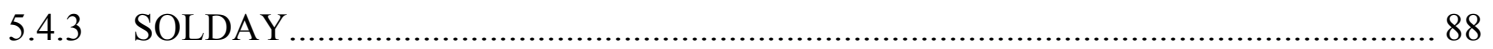

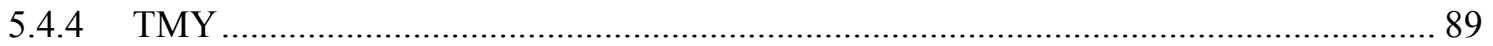

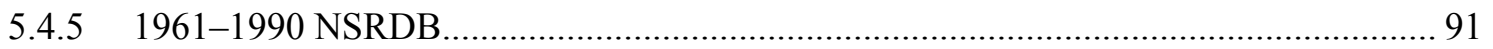

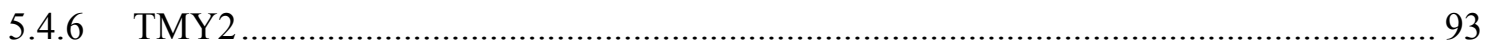

5.4.7 World Meteorological Organization WRDC ……………………………………..... 95

5.4.8 Western Energy Supply and Transmission Associates Solar Monitoring Network ....... 96

5.4.9 Pacific Northwest Solar Radiation Data Network ........................................................ 97

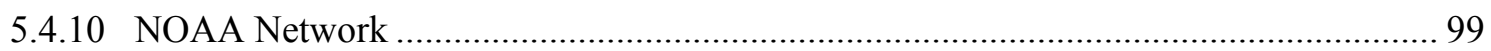

5.4.11 Solar Energy and Meteorological Research Training Sites.......................................... 100

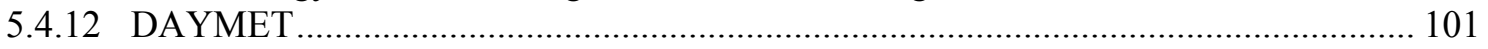

5.4.13 Solar Radiation Research Laboratory.................................................................. 101

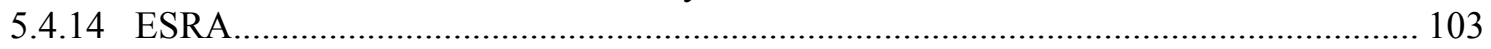

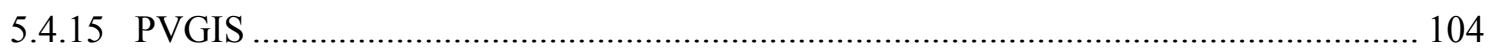

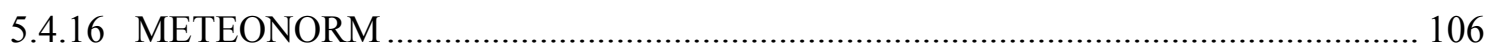

5.4.17 NASA Surface Meteorology and Solar Energy …………………………………... 107

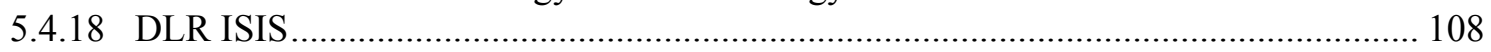

5.4.19 Historically Black Colleges and Universities Solar Measurement Network................ 109

5.4.20 Solar and Wind Energy Resource Assessment........................................................... 111

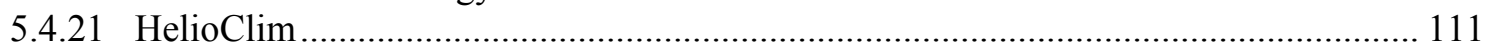

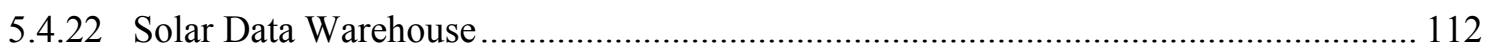

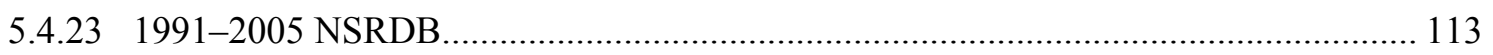

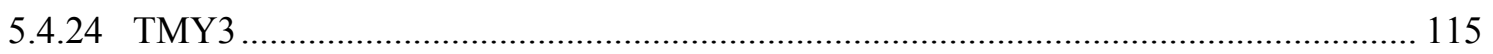

5.4.25 Management and Exploitation of Solar Resource Knowledge..................................... 119

5.4.26 International Daylight Measurement Program ……………………………………... 120

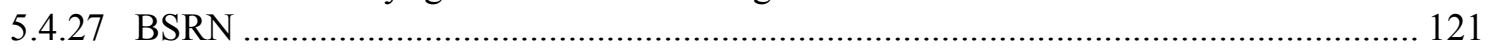

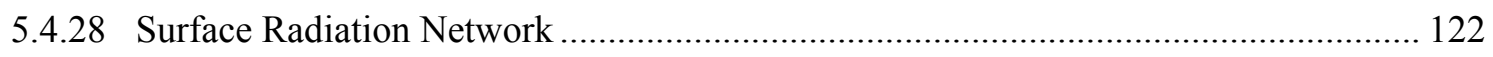




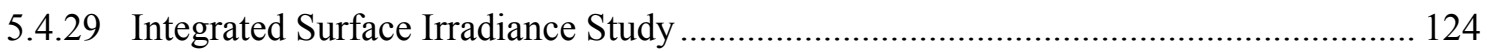

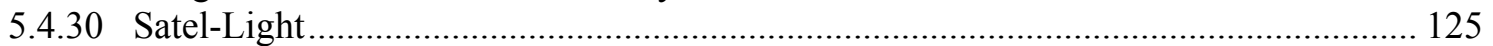

5.4.31 Atmospheric Radiation Measurement Program ...................................................... 126

5.4.32 3TIER Solar Time Series .............................................................................. 128

5.4.33 Clean Power Research—SolarAnywere............................................................... 129

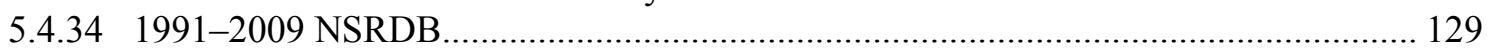

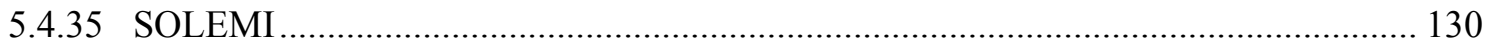

5.4.36 GeoModel Solar-SolarGIS ..................................................................... 130

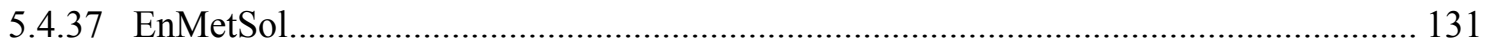

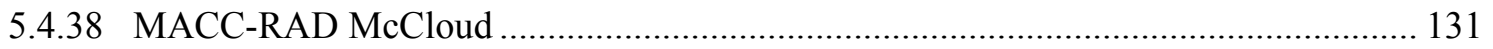

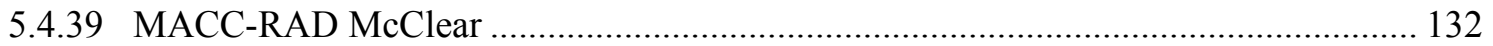

6 Applying Solar Resource Data to Solar Energy Projects ....................................................... 134

6.1 Data Applications for Site Screening and Prefeasibility Assessment ....................................... 136

6.1.1 Example for Review of Data Sources: DNI in the United States............................... 136

6.1.2 The Site Screening Process ……………………………………………………... 137

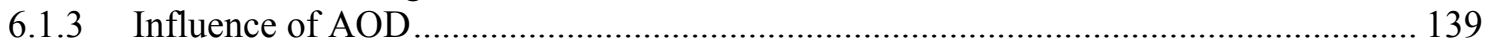

6.1.4 Comparison of Satellite-Derived Irradiation Resource Data Using Geographic Information System Tools ...................................................................................... 140

6.2 Data Applications for Feasibility, Engineering, and Financial Assessments ............................ 142

6.2.1 Extrapolating Short-Term Measured Data Sets........................................................... 143

6.2.2 Interannual Variability and Exceedance Probabilities ................................................. 146

6.2.3 Examples of Mean Irradiance Estimation and Hourly Data Selection Using NSRDB/SUNY, TMY3, and Measured Data............................................................... 148

6.3 Variability of the Solar Resource in the United States............................................................ 152

6.4 Applying Solar Resource Data to Planning Solar Energy Projects......................................... 157

6.4.1 Approaches to Estimating Yield of Non-Concentrating PV Projects .......................... 157

6.4.2 CST Plant Yield Calculation ................................................................................. 160

6.4.3 Flat-Plate Collector Yield Calculation ...................................................................... 162

6.4.4 Measurement of Solar Resource Data for Power Plant Characterization...................... 162

6.4.5 Evaluation of a Performance Guarantee..................................................................... 162

6.4.6 Monitoring Power Plant Performance ........................................................................ 163

6.5 Summary of Application of Solar Resource Data ............................................................... 163

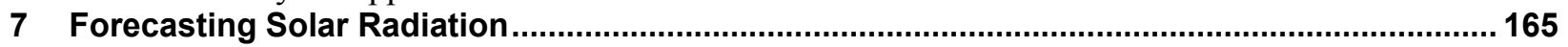

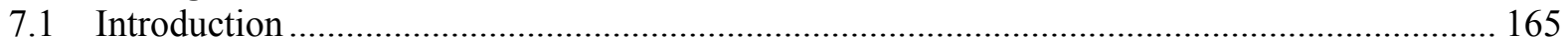

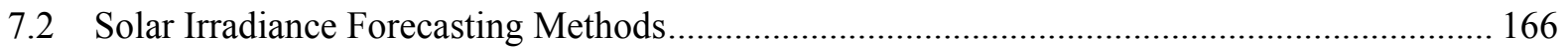

7.2.1 Irradiance Forecasting With Cloud Motion Vectors .................................................... 168

7.2.2 Satellite-Based Forecasts........................................................................................ 173

7.2.3 Irradiance Forecasting with NWPs..................................................................... 175

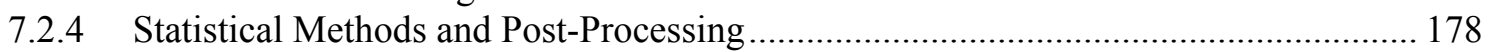

7.3 Evaluation of Irradiance Forecasts .................................................................................. 182

7.3.1 Measurement and Forecast Data ………………………………………………..... 183

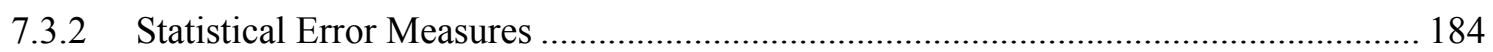

7.3.3 Evaluation in Dependence of Solar Elevation and Analysis of the Clear-Sky Index... 187

7.3.4 Persistence and Skill.................................................................................................... 190

7.3.5 Probability Density Function of the Clear-Sky Index ................................................. 191

7.3.6 Evaluation in Dependence on Cloud Variability and Spatial and Temporal Averaging192

7.3.7 Evaluation and Bias Correction in Dependence on Sun Elevation and Clear-Sky Index194

7.3.8 Comparison of Different Post-Processing Approaches to Site-Specific and Regional Forecasting ......................................................................................................... 196

8 Future Work

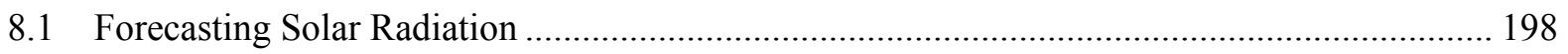




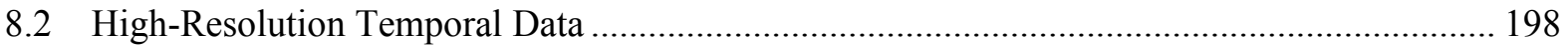

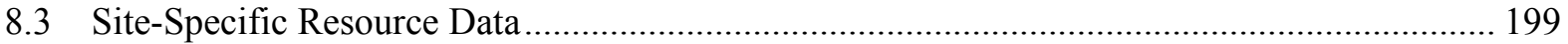

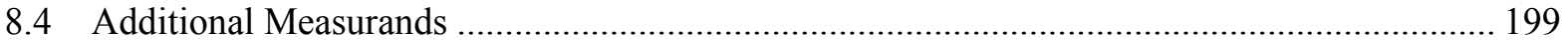

8.5 Effects of Climate Change on Solar Resource Assessments ................................................ 199

8.6 Need for Cross-Disciplinary Analysis Projects ..................................................................... 199

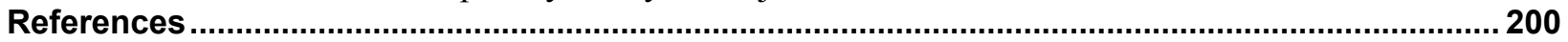

Chapter 2: Overview of Solar Radiation Resource Concepts....................................................... 200

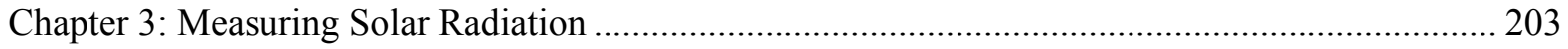

Chapter 4: Modeling Solar Radiation-Current Practices ............................................................. 208

Chapter 5: Historical Solar Resource Data.................................................................................. 216

Chapter 6: Applying Solar Resource Data to Concentrating Solar Power Projects ......................... 219

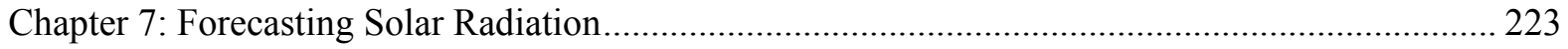

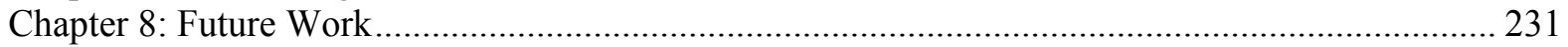

Appendix A: Radiometer Manufacturers and Distributors .......................................................... 232

Appendix B: International Energy Agency Solar Heating and Cooling Programme........................ 235 


\section{List of Figures}

Figure 2-1. The atmosphere affects the amount and distribution of solar radiation reaching the ground. Image from NREL

Figure 2-2. Three solar cycles show the variations of TSI in composite measurements from satellite-based radiometers (color coded) and model results produced by the World Radiation Center (WRC). Image used by permission of the Physical Meteorological Observatory in Davos, Switzerland

Figure 2-3. Schematic of the Earth's orbit. Image from Wikipedia .................................................... 6

Figure 2-4. Scattering of the direct-beam photons from the sun by the atmosphere produces diffuse radiation that varies with AM (Marion, Riordan, and Renné 1992). Image from NREL ........ 7

Figure 2-5. Apparent sun path variations during a year for Denver, Colorado. Image from the Universitity of Oregon Solar Radiation Monitoring Laboratory............................................................... 9

Figure 2-6. Solar radiation components resulting from interactions with the atmosphere. Image by Al

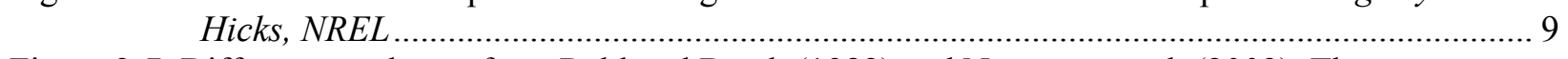

\section{9}

Figure 2-7. Different sunshapes from Rabl and Bendt (1982) and Neumann et al. (2002). The average solar disk angle and the recommended FOV of a pyrheliometer (WMO 2008) are shown as vertical lines. Image from Stefan Wilbert, DLR

Figure 2-8. Example of direct-beam monthly average daily total $\left(\mathrm{kWh} / \mathrm{m}^{2} /\right.$ day $)$ interannual variability from 1961 through 2005 for Daggett, California. Data from Wilcox et al. (2007). Image from

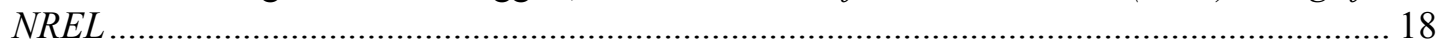

Figure 3-1. Thermopile assembly used in an Eppley Laboratory, Inc., model precision spectral pyranometer (PSP).

Figure 3-2. (Left) Typical photodiode detector and (right) spectral response of LI-COR pyranometers LI200SA. Photographs used by permission of LI-COR Biosciences, Inc. .....

Figure 3-3. Schematic of an Eppley Laboratory, Inc., model normal incidence pyrheliometer (NIP) (Bahm and Nakos 1979). Image from the former U.S. Energy Research and Development

Administration, now DOE ............................................................................................... 22

Figure 3-4. Pyrheliometers mounted on an automatic solar tracker. Photo from NREL......................... 22

Figure 3-5. Schematics of pyrheliometer alignment diopter configuration (Micek 1981). Image used by permission from Leonard Micek ................................................................................... 23

Figure 3-6. Multiple electrically self-calibrating absolute cavity radiometers mounted on solar trackers with control and data acquisition electronics. Photo from NREL ..................................... 24

Figure 3-7. The World Standard Group of six absolute cavity radiometers is used to define the WRR or DNI measurement standard. Photo from NREL …........................................................ 24

Figure 3-8. Schematic of the Eppley Laboratory, Inc., model AHF absolute cavity pyrheliometer. Image

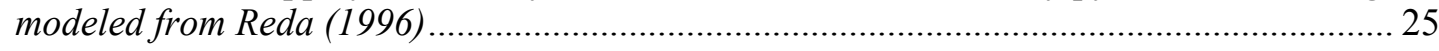

Figure 3-9. Schematic of the Eppley Laboratory, Inc., model PSP. Image from NREL …...................... 26 Figure 3-10. Kipp \& Zonen model CM22 pyranometers installed in CV2 ventilators. Photo from NREL 26

Figure 3-11. LI-COR model LI-200SA pyranometer with photodiode detector and acrylic diffuser fore optic. Photo from LI-COR

Figure 3-12. Four commercially available RSIs: (clockwise from upper left) An Irradiance, Inc., model RSR2; a Reichert GmbH RSP 4G (previously used by SM-AG); a Yankee Environmental Systems, Inc., model SDR-1; and a CSP-Services GmbH Twin-RSI. Photos by (clockwise from top) Irradiance, Inc.; Reichert GmbH RSP 4G; NREL; and CSP-Services ................. 33

Figure 3-13. Burst (sweep) with sensor signal and the derived GHI, shoulder values, and the DHI. Image

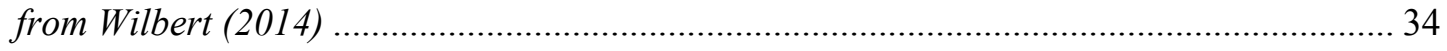

Figure 3-14. RSI calibration station at Plataforma Solar de Almería. Photo by Stefan Wilbert, DLR........ 38

Figure 3-15. Maximum, mean, and minimum RMS deviations of irradiance values with a time resolution of 10 minutes from 39 RSIs compared to thermopile sensors. The error bars show the maximum and the minimum deviation of the 39 data sets. Data sets for uncorrected raw 
values as well as for four different corrections were analyzed. Image from Geuder et al.

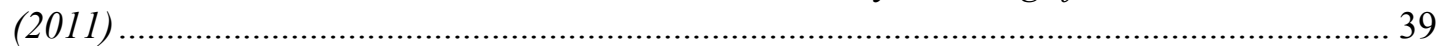

Figure 3-16. Delta-T Devices, Ltd., SPN1 ........................................................................................ 40

Figure 3-17. Calibration histories for two pyrheliometer control instruments spanning 12 years. Image

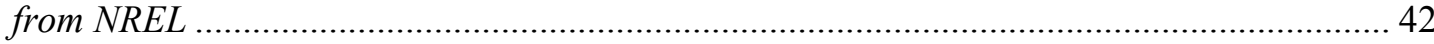

Figure 3-18. Pyrheliometer calibration results summarizing (left) Rs compared to SZA and (right) compard to local standard time. Image from NREL

Figure 3-19. Pyranometer calibration results summarizing Rs compared to (left) SZA and (right) local standard time. Image from Daryl Myers, NREL.

Figure3-20. Calibration traceability and accumulation of measurement uncertainty for pyrheliometers and pyranometers (coverage factor $\mathrm{k}=2$ ). Image from NREL ................................................ 49

Figure 3-21. Example of SERI quality-control data quality-assurance reporting. Image from NREL........ 60

Figure 3-22. Information flow of a quality-assurance cycle. Image from NREL .................................... 61

Figure 4-1. The location of the current geostationary satellites that provide coverage around the globe.

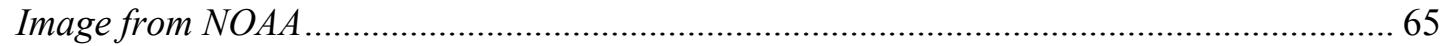

Figure 5-1. SOLDAY and SOLMET measurement stations (26 each). Image from NREL ..................... 88

Figure 5-2. Original 239 stations in the 1961-1990 NSRDB released in 1992 (NSRDB 1992 and 1995) and the 1,454 stations in the 1991-2005 NSRDB released in 2007. Image from NREL ....... 92

Figure 5-3. WRDC measurement stations. Image from NREL ......................................................... 96

Figure 5-4. Western Energy Supply and Transmission Associates Solar Monitoring Network of 52 measurement stations (1976-1980). Image from NREL

Figure 5-5. Pacific Northwest Solar Radiation Data Network operated by the University of Oregon. Image from NREL

Figure 5-6. NOAA Solar Monitoring Network of 39 stations (1977-1980). Image from NREL ............ 100 Figure 5-7. The Solar Energy and Meteorological Research Training Sites program provided the first 1minute measurements of multiple solar resource parameters for the United States. Image from NREL

Figure 5-8. NREL's main campus and the Solar Radiation Research Laboratory on South Table Mountain. Photo from NREL

Figure 5-9. Historically Black Colleges and Universities Solar Monitoring Network (1985-1996). Image from NREL

Figure 5-10. Example data quality summary for one of the 1,454 stations in the 1991-2010 NSRDB update. Image from Steve Wilcox, NREL

Figure 5-11. Annual mean daily total DNI distribution based on NSRDB/SUNY model results for 19982005 and the corresponding differences between the model and TMY3. (Red circles indicate DNI values from TMY3 < NSRDB/SUNY and blue circles indicate TMY3 >

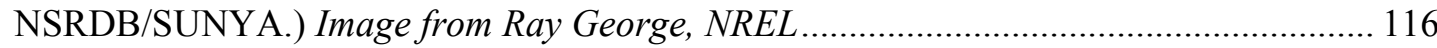

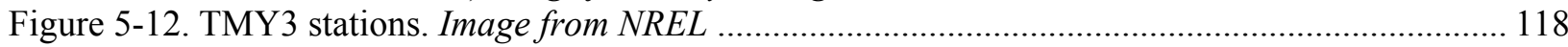

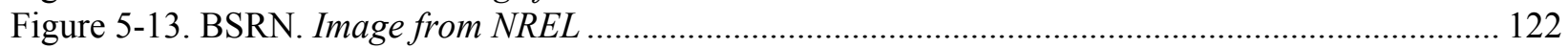

Figure 5-14. The SURFRAD network is operated by the Global Monitoring Division, Earth Systems Research Laboratory, NOAA. Image from NREL ............................................................ 123

Figure 5-15. DOE has operated the 23 Atmospheric Radiation Measurement stations in the southern Great Plains since 1997. Image from DOE.

Figure 6-1. The four stages of a solar power plant project

Figure 6-2. Geographic information system analysis for available site selection using DNI resource, land use, and 3\% terrain slope. Image from NREL

Figure 6-3. Geographic information sytsem analysis for available site selection using DNI resource, land use, and $1 \%$ terrain slope. Image from NREL 138

Figure 6-4. Yearly sum of DNI as calculated from five modeled data sets: METEONORM, PVGIS, NASA SSE, Satel-Light, and SOLEMI-(left) average of five databases $\left(\mathrm{kWh} / \mathrm{m}^{2}\right)$ and (right) relative standard deviation (\%). Graphics modified from Beyer et al. (2009) 140 
Figure 6-5. Resulting uncertainty when combining a base data set of $2 \%, 4 \%, 6 \%$, or $8 \%$ overall uncertainty with an additional data set of varying quality. Figure from Meyer et al. (2008)144

Figure 6-6. Upper left: Cumulative frequency distribution for training time of overlapping ground data and satellite time series. The arrow illustrates the difference between the two curves. Upper right: Corrected satellite cumulative frequency distribution for test period. Lower left: Mapping of original satellite irradiance values to original cumulative frequency distribution (arrow and bottom right image). The original cumulative frequency distribution is first mapped to produce a corrected cumulative frequency distribution, then the corrected cumulative frequency distribution is adjusted to satellite values. Green: ground data from training set; magenta: satellite data from training set; purple: satellite data from test set; yellow: corrected satellite data. Figure from Schumann et al. (2009)

Figure 6-7. Number of years to stabilize DNI and GHI in (clockwise from upper left) Burns, Oregon; Eugene, Oregon; Hermiston, Oregon; and Golden, Colorado. Image modified from Gueymard and Wilcox 2009.

Figure 6-8. NSRDB/SUNY 10-km grid cells near Harper Lake, California. The upper values shown in text boxes are averaged from (uncorrected) hourly files. The lower values are averaged DNI from corrected maps. The values in red show uncorrected time series mean values, which are substantially lower than the corrected map values. Image from NREL. 149

Figure 6-9. Monthly mean DNI for Harper Lake (Cell C2) and Daggett TMY3. Minimum and maximum values for cell $\mathrm{C} 2$ are also shown for each month. Image from NREL.

Figure 6-10. Desert Rock annual average GHI and DNI from satellite and measurements. Mean bias error is defined as (satellite - measured)/measured by 100\%. Image from NREL ........................ 152

Figure 6-11. Interannual DNI variability (COV as percent) for 1998-2005. Image from NREL ............. 153 Figure 6-12. A 3-by-3 grid layout with anchor cell in the center and 8 surrounding neighbor cells. Image from NREL 154

Figure 6-13. DNI spatial coefficient of variability for a (top) 3-by-3 cell matrix and (bottom) 5-by-5 cell matrix for the average DNI from 1998-2005. Image from NREL

Figure 6-14. The distribution of the pixels in each spatial variability analysis. The black center pixels were compared to each of the gray pixels. Images from NREL

Figure 6-15. Map showing spatial variability among neighboring pixels. Images from NREL ............... 156 Figure 6-16. Monthly standard deviation distributions in $\mathrm{kWh} / \mathrm{m}^{2} /$ day for the NSRDB gridded DNI and GHI data sets. Illustrations from NREL

Figure 7-1. Illustration of different forecasting methods for different spatial and temporal scales: TS = time-series modeling; CM-SI = cloud motion forecast based on sky-imagers; CM-sat = cloud motion forecast based on satellite images; and NWP . 168

Figure 7-2. Cloud information from sky imagers: (upper left) original images; (middle left) pixel intensities; (middle right) red-blue ratio, corrected with a clear-sky library; (upper right) cloud decision map; and (bottom) shadow map with irradiance measurements. Sky image and irradiance measurements taken in Jülich, Germany, on April 9, 2013 at 12:59_00UTC in the framework of the HOPE campaign (Macke et al. 2014). Images from the University of Oldenburg

Figure 7-3. Example of an optical-flow field. Sky image taken in Jülich, Germany, on April 19, 2013 at 15:30 UTC in the framework of the HOPE campaign (Macke and HOPE-Team forthcoming 2014], Madhavan, Kalisch, and Macke 2014). Image from the University of Oldenburg ... 172

Figure 7-4. Example sky imager-based 5-minute-ahead irradiance forecasts. Location: Universtity of California at San Diego, November 14, 2012. Image from University of California at San Diego Center for Energy Research .

Figure 7-5. Schematic overview for detection of cloud motion in satellite images. Images reproduced from Kuehnert et al. 2013 ........................................................................................... 175

Figure 7-6. Overview of the application of statistical methods and post-processing ............................. 179

Figure 7-7. Evaluation sites in Germany 183 
Figure 7-8. Scatterplots of predicted and measured GHI for (top) high-resolution SKA and (bottom) IFS forecasts. The original forecasts are shown in red, the forecast processed with the linear regression of GHI is shown in blue, and the regression equation is visualized in green. The data are from Dresden, Germany, March 1, 2013-February 28, $2014 .$.

Figure 7-9. (Solid lines with dots) RMSE and (dashed lines) bias of (red) $\mathrm{SKA}_{\mathrm{av}}$ and (blue) IFS GHI forecasts through the time of the day. The data are from 18 German Meteorological Service sites, March 1, 2013-February 28, 2014.

Figure 7-10. (Solid lines with dots) RMSE and (dashed lines) bias of (red) SKA av and (blue) IFS kt* forecasts through the (right) time of the day and (left) cosine of the SZA $\cos \Theta_{Z}$. The data are from 18 German Meteorological Service sites, March 1, 2013-February 28, 2014.

Figure 7-11. Scatterplot of predicted over measured kt* for (top) high-resolution SKA and (bottom) IFS forecasts. The original forecasts are shown in red, the forecast processed with the linear regression of $\mathrm{kt}^{*}$ is shown in blue, and the regression equation is visualized in green. The data are from Dresden, Germany, March 1, 2013-February 28, 2014; $\cos \left(\Theta_{Z}\right)>0.1$ for $\mathrm{kt} *$ evaluation, $\cos \left(\Theta_{\mathrm{Z}}\right)>0.0$ for GHI RMSE and bias.

Figure 7-12. Probability density function of the clear-sky index derived from (gray) measurements, (red) high-resolution SKA forecasts, and (blue) IFS forecasts. The data are from 18 German Meteorological Service sites, March 1, 2013-February 28, 2014; $\cos \left(\Theta_{Z}\right)>0.1$.

Figure 7-13. Example days comparing measurements to SKA forecasts with different spatial and temporal averaging - (red) SKA: nearest grid point with hourly resolution; (light blue) SKA $\mathrm{av}_{\mathrm{av}}$ : 5-hour moving average of clear-sky index of the average throughout 20-by-20 grid points. (Left) Clear-sky; data from Lindenberg, Germany, June 19, 2013; and (right) variable cloud conditions; data from Lindenberg, Germany, March 23, 2013.

Figure 7-14. RMSE in dependence of the standard deviation of $\mathrm{kt}^{*}{ }_{\text {meas }}$ (throughout 5 hours) for (left) SKA forecasts with the application of linear regression ([red] SKA: nearest grid point, [orange] LR: linear regression for GHI, and [yellow] LR-kt* for linear regression for kt*) and (right) with different spatial and temporal averaging ([red] SKA: nearest grid point, [dark blue] $\mathrm{SKA}_{20 \times 20}$ averaged throughout 20-by-20 grid points, [light blue] $\mathrm{SKA}_{\mathrm{av}}$ 5-hour gliding mean of clear-sky index of the average throughout 20-by-20 grid points, and [green] SKA $\mathrm{av}_{\mathrm{av}, \mathrm{LR} . \mathrm{kt} * \text { : }}$ linear regression of $\mathrm{kt}^{*}$ applied to $\mathrm{SKA}_{\mathrm{AV}}$ ). The data is from 18 German Meteorological Service sites, April 3, 2013-February 28, 2014; training set: last 30 days, all sites

Figure 7-15. (Left) Bias of IFS and (right) averaged $\mathrm{SKA}_{\mathrm{av}}$ forecasts in dependence of the cosine of the SZA and the predicted clear-sky index kt*. The data are from 18 German Meteorological Service sites, March 3, 2013-February 28, 2014.

Figure 7-16. (Left) RMSE of IFS and (right) averaged SKA forecasts in dependence of the cosine of the SZA and the predicted clear-sky index kt*. The data are from 18 German Meteorological Service sites, March 3, 2013-February 28, 2014.

Figure 7-17. RMSE of IFS and SKA GHI forecasts with different post-processing approaches for (left) single site forecasts and (right) regional forecasts, derived as mean value of all sites. The data are from 18 German Meteorological Service sites, April 3, 2013-February 28, 2014; training set: last 30 days, all sites...... 


\section{List of Tables}

Table 2-1. Radiometric Terminology and Units........................................................................... 4

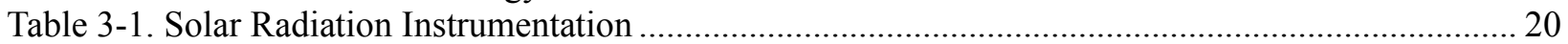

Table 3-2. WMO Characteristics of Operational Pyrheliometers for Measuring DNI ${ }^{\mathrm{a}}$........................... 28

Table 3-3. WMO Characteristics of Operational Pyranometers for Measuring GHI or DHI .................... 29

Table 3-4. ISO 9060 Specifications Summary for Pyrheliometers Used To Measure DNI ....................... 30

Table 3-5. ISO 9060 Specifications Summary for Pyranometers Used To Measure GHI and DHI .............. 31

Table 3-6. Estimated Pyrheliometer Calibration Uncertainties in Rsi ................................................. 44

Table 3-7. Example of Estimated Direct-Normal Subhourly Measurement Uncertainties (\%) ................. 45

Table 4-1. Regression Analysis of NASA SSE Compared to BSRN Bias and RMS Error for Monthly

Averaged Values from July 1983 through June 2006 ............................................................ 69

Table 4-2. HelioClim Compared to Ground Bias and RMS Error for Monthly Averaged Values from 1994

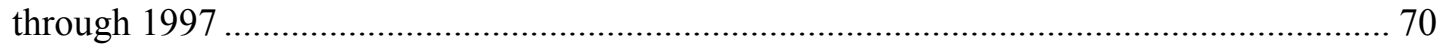

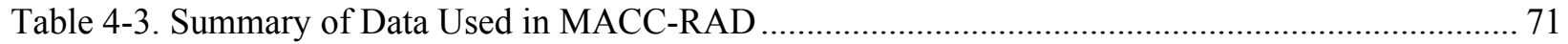

Table 4-4. Summary of Applications and Validation Results of Satellite Models- Empirical/Statistical

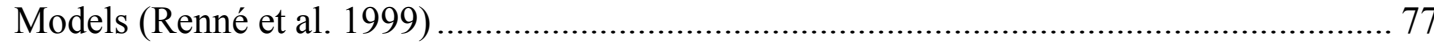

Table 4-5. Summary of Applications and Validation Results of Satellite Models-Empirical/Physical

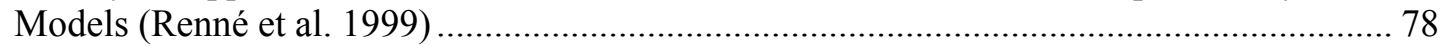

Table 4-6. Summary of Applications and Validation Results of Satellite Models - Broadband Theoretical

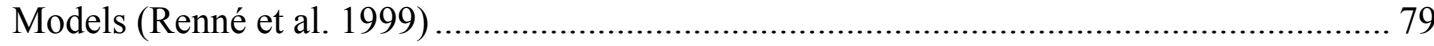

Table 4-7. Summary of Applications and Validation Results of Satellite Models- Spectral Theoretical

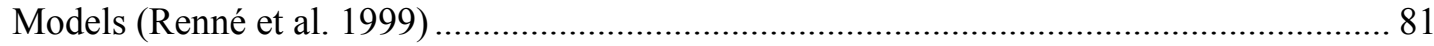

Table 5-1. Weighting Factors Applied to Cumulative Distributions ...................................................... 89

Table 5-2. Comparisons of TMY2 Data to 30 Years of NSRDB Data ................................................... 94

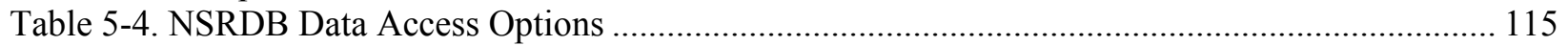

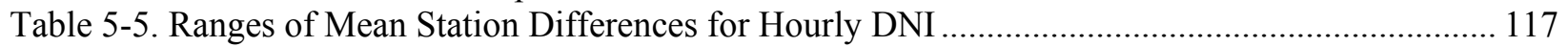

Table 5-6. Bias Differences (Test Data Minus Original 1961-1990 TMY) ........................................... 117

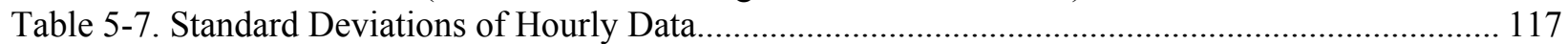

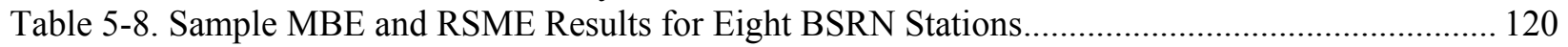

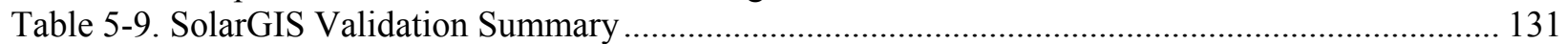

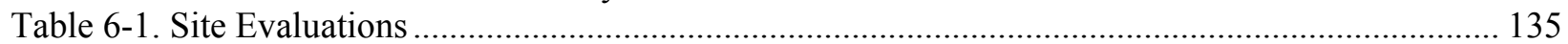

Table 6-2. Data Sources for DNI Estimation.................................................................................... 136

Table 6-3. Annual Mean Values of Global and Direct Radiation for Measured and Modeled Data at

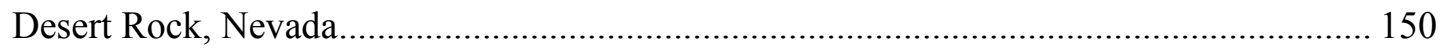




\section{Why Solar Resource Data Are Important to Solar Power}

Sunlight is the fuel for all solar energy generation technologies. Like any generation source, knowledge of the quality and future reliability of the fuel is essential to accurate analyses of system performance and the financial viability of a project. With solar energy systems, the variability of the supply of sunlight probably represents the single greatest uncertainty in a solar power plant's predicted performance. Solar resource data and modeling factor into three elements of a solar project's life:

- Site selection

- Predicted annual power plant output

- Temporal performance and operating strategy.

The first two items are interrelated. Site selection includes numerous factors, but a top priority is a good solar resource. For site selection, data from individual years and a representative annual solar resource are required to make comparisons to alternative sites and estimate power plant output. Because site selection is always based on historical solar resource data, and because changes in weather patterns occur from year to year, more years of data are better for determining a representative annual data set. Deriving a typical meteorological year (TMY) is described in Chapter 5. TMY data are used to compare the relative solar resource at alternative sites and to estimate the probable annual performance of a proposed solar power plant. Data from individual years are required to assess the annual variability that can be expected for a proposed location.

In the absence of long-term ground data, development of TMY data for large regions requires the use of models that rely mostly on satellite imagery. In regional terms, identifying prime solar resource areas is fairly simple. The southwestern United States, for example, has broad areas of excellent solar resource. However, narrowing down the data to a specific few square kilometers of land requires considering local impacts; although satellite data are very useful in mapping large regions, individual sites should be vetted by using ground-monitoring stations. Local measurements can be compared to same-day satellite data to test for bias in the satellite model results. Any correction in the satellite model can then be applied to the historical data sets. Correcting any bias in the satellite data will allow the modeler to more accurately apply multiple years of satellite data to generate an improved TMY data set for a site.

After a plant is built, resource data are immediately required to complete acceptance testing. The owner and financiers will insist on verifying that the power plant output meets its design specifications for a specific solar input. Often the acceptance tests will be for a short duration, perhaps a few days, but the owners will want to extrapolate the results to estimate annual performance. Annual performance estimates can be improved by comparing locally measured ground data to the satellite-derived data for the same time interval.

Accurate resource data will remain essential to a power plant's efficient operation throughout its service life. Comparison of plant output as a function of solar radiation resource is one global 
indicator of power plant performance. A drop in overall efficiency implies a degradation of one or more power plant components and indicates that maintenance is required.

Last, the realm of resource forecasting is becoming more important for plant dispatch as higher penetrations of solar power are planned for the electric grid. An accurate forecast can increase power plant profits by optimizing energy dispatch into the time periods of greatest value. Although not explicitly covered in this handbook, forecasting requires the same principles described here for historical resource assessment: proper use of satellite- and ground-based data sources and models. 


\section{Overview of Solar Radiation Resource Concepts}

\subsection{Introduction}

Describing the relevant concepts and applying a consistent terminology are important to the usefulness of any handbook. This chapter uses a standard palette of terms to provide an overview of the key characteristics of solar radiation, the fuel source for solar technologies.

Beginning with the sun as the source, we present an overview of the effects of the Earth's orbit and atmosphere on the types and amounts of solar radiation available for energy conversion. An introduction to the concepts of measuring and modeling solar radiation is intended to prepare the reader for the more in-depth treatment in Chapter 3 and Chapter 4. The overview concludes with an important discussion of the estimated uncertainties associated with solar resource data based on measurements and modeling methods used to produce the data.

\subsection{Properties of ETR}

Any object with a temperature above absolute zero emits radiation. With an effective temperature of approximately $6,000 \mathrm{~K}$, the sun emits radiation over a wide range of wavelengths, the solar spectral power distribution, or solar spectrum, commonly labeled from high-energy shorter wavelengths to lower energy longer wavelengths as gamma ray, x-ray, ultraviolet, visible, infrared, and radio waves. These are called spectral regions (Figure 2-1). Most (97\%) solar radiation is in the wavelength range of $290 \mathrm{~nm}$ to $3,000 \mathrm{~nm}$. Future references to broadband solar radiation refer to this spectral range.

Various different extraterrestrial spectral power distributions were derived based on ground measurements, extraterrestrial measurements, and physical models. Some of these spectra deviate strongly from currently accepted standard extraterrestrial spectra as presented in the American Society for Testing and Materials (ASTM) Standard E490 (2006).

Figure 2.1 shows the terrestrial and extraterrestrial spectrum of direct normal irradiance (DNI). Standardized terrestrial spectra for DNI and global hemispherical irradiance on a 37-degree south-facing tilted irradiance are presented in ASTM G173-03 (2006). 


\section{Spectral Solar Irradiance}

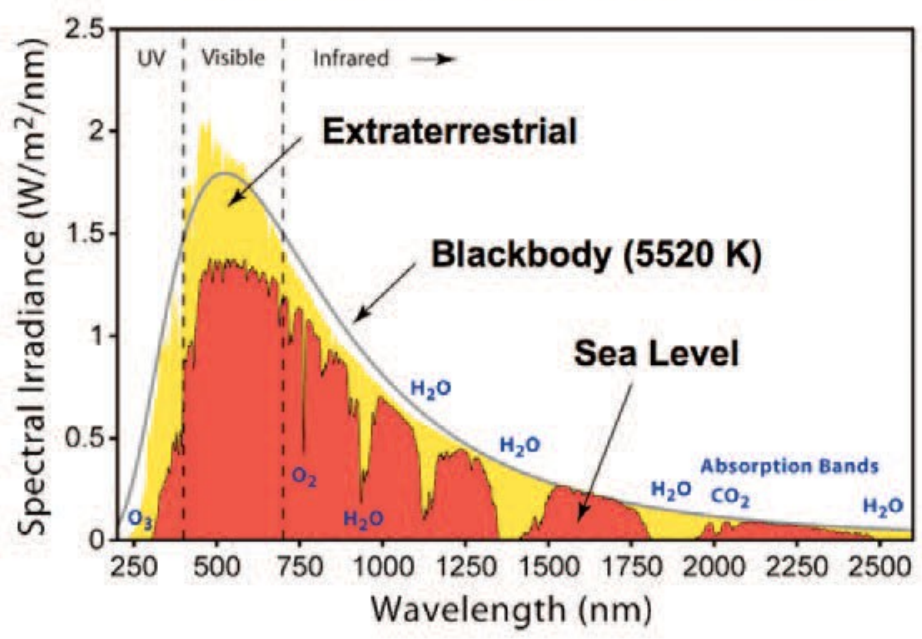

Figure 2-1. The atmosphere affects the amount and distribution of solar radiation reaching the ground. Image from NREL

Before continuing our discussion of solar radiation, it is important to understand a few basic radiometric terms. Radiant energy, flux, power, and other concepts used in this handbook are summarized in Table 2-1.

Table 2-1. Radiometric Terminology and Units

\begin{tabular}{|l|l|l|l|l|}
\hline \multicolumn{1}{|c|}{ Quantity } & Symbol & \multicolumn{1}{|c|}{ SI Unit } & Abbreviation & \multicolumn{1}{|c|}{ Description } \\
\hline $\begin{array}{l}\text { Radiant } \\
\text { energy }\end{array}$ & Q & joule & $\mathrm{J}$ & Energy \\
\hline $\begin{array}{l}\text { Radiant } \\
\text { flux }\end{array}$ & $\Phi$ & watt & $\mathrm{W}$ & $\begin{array}{l}\text { Radiant energy per unit of } \\
\text { time }\end{array}$ \\
\hline $\begin{array}{l}\text { Radiant } \\
\text { intensity }\end{array}$ & $\mathrm{I}$ & watt per steradian & $\mathrm{W} / \mathrm{sr}$ & $\begin{array}{l}\text { Power per unit of solar } \\
\text { angle }\end{array}$ \\
\hline $\begin{array}{l}\text { Radiant } \\
\text { emittance }\end{array}$ & $\mathrm{M}$ & $\begin{array}{l}\text { watt per square } \\
\text { meter }\end{array}$ & $\mathrm{W} / \mathrm{m}^{2}$ & $\begin{array}{l}\text { Power emitted from a } \\
\text { surface }\end{array}$ \\
\hline Radiance & $\mathrm{L}$ & $\begin{array}{l}\text { watt per steradian } \\
\text { per square meter }\end{array}$ & $\mathrm{W} / \mathrm{sr} / \mathrm{m}^{2}$ & $\begin{array}{l}\text { Power per unit solid angle } \\
\text { per unit of projected source }\end{array}$ \\
\hline Irradiance & $\mathrm{E}, \mathrm{I}$ & $\begin{array}{l}\text { watt per square } \\
\text { meter }\end{array}$ & $\mathrm{W} / \mathrm{m}^{2}$ & Power incident on a surface \\
\hline $\begin{array}{l}\text { Spectral } \\
\text { irradiance }\end{array}$ & $\mathrm{E}_{\lambda}$ & $\begin{array}{l}\text { watt per square } \\
\text { meter per nanometer }\end{array}$ & $\mathrm{W} / \mathrm{m}^{2} / \mathrm{nm}$ & $\begin{array}{l}\text { Power incident on a surface } \\
\text { per wavelength }\end{array}$ \\
\hline
\end{tabular}

The total radiant power from the sun is remarkably constant. In fact, the solar output (radiant emittance) has commonly been called the solar constant, but the currently accepted term is total solar irradiance (TSI), to account for the actual variability with time. There are cycles in the number of sunspots (cooler, dark areas on the sun) and general solar activity of approximately 11 years. Figure 2-2 shows a composite of space-based measurements of the TSI, normalized to 1 
astronomical unit (AU), the average Earth-sun distance, since 1975, encompassing the last three 11-year sunspot cycles (De Toma et al. 2004).

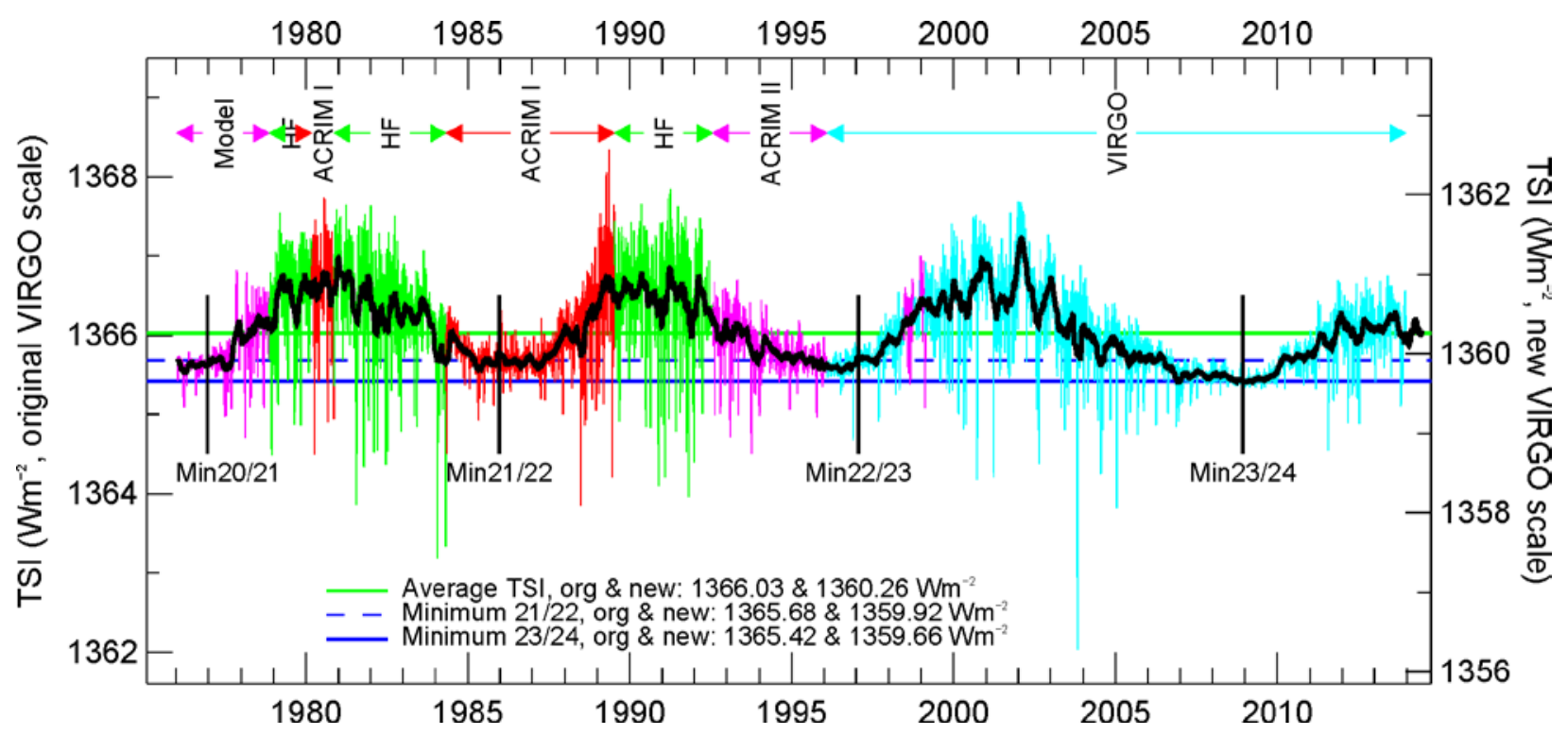

Figure 2-2. Three solar cycles show the variations of TSI in composite measurements from satellite-based radiometers (color coded) and model results produced by the World Radiation Center (WRC)..$^{1}$ Image used by permission of the Physical Meteorological Observatory in Davos, Switzerland

The measured variation in TSI resulting from the sunspot cycle is $\pm 0.2 \%$, only twice the precision (repeatability, not total absolute accuracy, which is approximately $\pm 0.5 \%$ ) of the most accurate radiometers measuring the irradiance in space. There is, however, some large variability in a few spectral regions, especially the ultraviolet (wavelengths less than $400 \mathrm{~nm}$ ), caused by solar activity.

The amount of radiation exchanged between two objects is affected by their separation distance. The Earth's elliptical orbit (eccentricity 0.0167) brings us closest to the sun in January and farthest from the sun in July. This annual variation results in variation of the Earth's solar irradiance of $\pm 3 \%$. The average Earth-sun distance is $149,598,106 \mathrm{~km}(92,955,953 \mathrm{miles})$, or 1 AU. Figure 2-3 shows the Earth's orbit in relation to the northern hemisphere's seasons, caused by the average 23.5-degree tilt of the Earth's rotational axis with respect to the plane of the orbit. The solar irradiance available at the top of atmosphere (TOA) is called the extraterrestrial radiation (ETR). ETR (see Equation 2-1) is the power per unit area, or flux density in watts per square meter $\left(\mathrm{W} / \mathrm{m}^{2}\right)$, radiated from the sun and available at the TOA. ETR varies with the Earthsun distance (r) and annual mean distance (r0):

$\operatorname{ETR} \operatorname{TSI}\left(\mathrm{r}_{0} / \mathrm{r}\right)^{2}$

\footnotetext{
${ }^{1}$ See http://www.pmodwrc.ch/pmod.php?topic=tsi/composite/SolarConstant.
} 


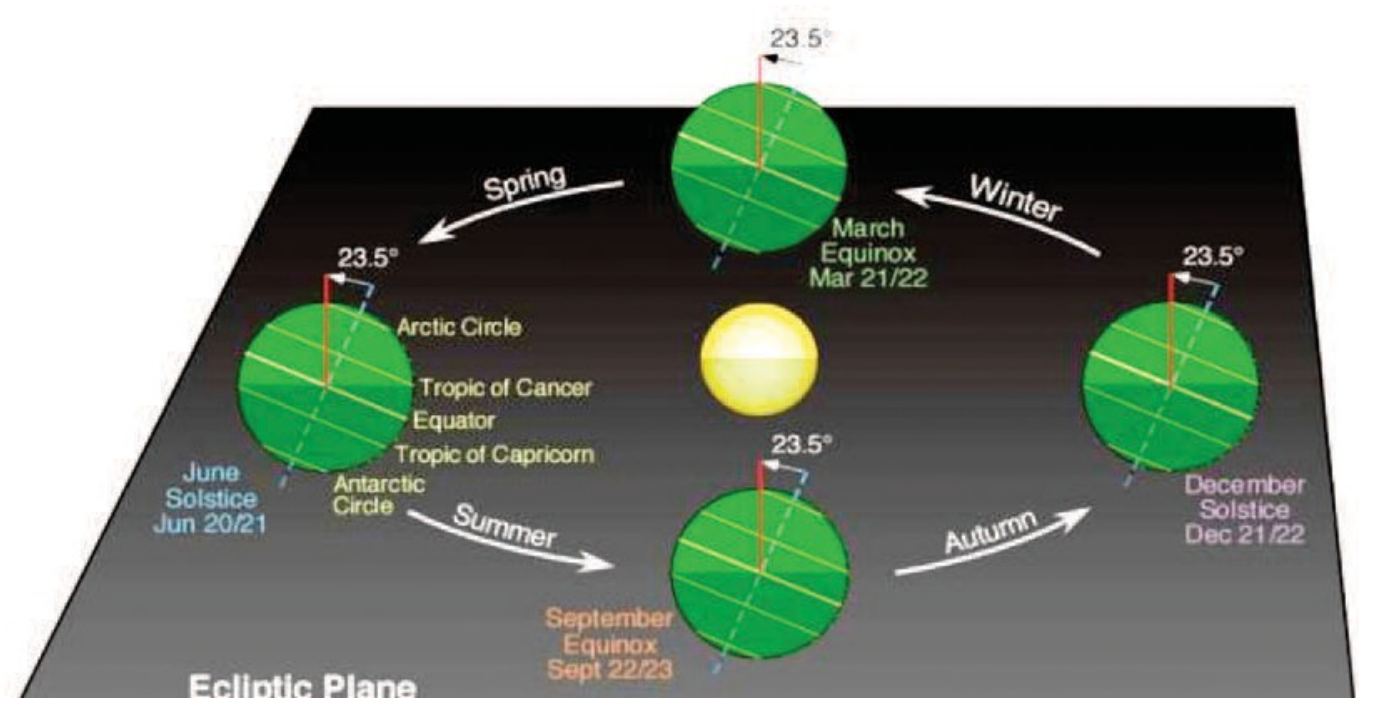

Figure 2-3. Schematic of the Earth's orbit. Image from Wikipedia

As measured by multiple satellites (with individual corrections and adjustments applied) throughout the past 30 years, the TSI is $1,366.1 \pm 7 \mathrm{~W} / \mathrm{m}^{2}$ at 1 AU. According to astronomical computations, such as those made by NREL's solar position software, the variation in the Earthsun distance causes the ETR to vary from approximately $1,415 \mathrm{~W} / \mathrm{m}^{2}$ around January 3 to approximately $1,321 \mathrm{~W} / \mathrm{m}^{2}$ around July 4 .

From the top of the atmosphere, the sun appears as a very bright disk with an approximate angular diameter of 0.5 degrees (the actual diameter varies by a small amount as the Earth-sun distance varies) surrounded by a completely black sky (apart from the light coming from stars and planets). This angle can be determined from the distance between the Earth and the sun and the sun's visible diameter. A point at the top of the Earth's atmosphere intercepts a cone of light from the hemisphere of the sun facing the Earth with a total angle of 0.5 degrees at the apex and a divergence angle from the center of the disk of 0.266 degree (half the apex angle, yearly average). Because the divergence angle is very small, the rays of light from the sun are nearly parallel; these are called the solar beam. In the following, we will discuss the interaction of the solar beam with the terrestrial atmosphere.

\subsection{Solar Radiation and the Earth's Atmosphere}

The Earth's atmosphere is a continuously variable filter for the solar ETR as it reaches the surface. Figure 2-4 illustrates the "typical" absorption of solar radiation by ozone, oxygen, water vapor, and carbon dioxide. The amount of atmosphere the solar photons must traverse, also called the atmospheric path length or air mass (AM), depends on the relative position of the observer with respect to the sun's position in the sky (Figure 2-4). By convention, air mass one (AM1) is defined as the amount of atmospheric path length observed when the sun is directly overhead from a location at sea level. AM is geometrically related to the solar zenith angle (SZA) as AM = secant of SZA, or $1 / \operatorname{Cos}(\mathrm{SZA})$. Because SZA is the complement of the solar elevation angle, AM is also equal to $1 / \mathrm{Sin}$ (solar elevation angle). Air mass two (AM2) occurs when the SZA is 60 degrees, and it has twice the path length of AM1. Weather systems, specifically clouds and storm systems, are the major elements that modify the ETR on its way to 
the surface or to a solar collector. The cloudless atmosphere also contains gaseous molecules, dust, aerosols, particulates, etc., which reduce the ETR as it moves through the atmosphere. This reduction is caused by absorption (capturing the radiation) and scattering (essentially a complex sort of reflection).

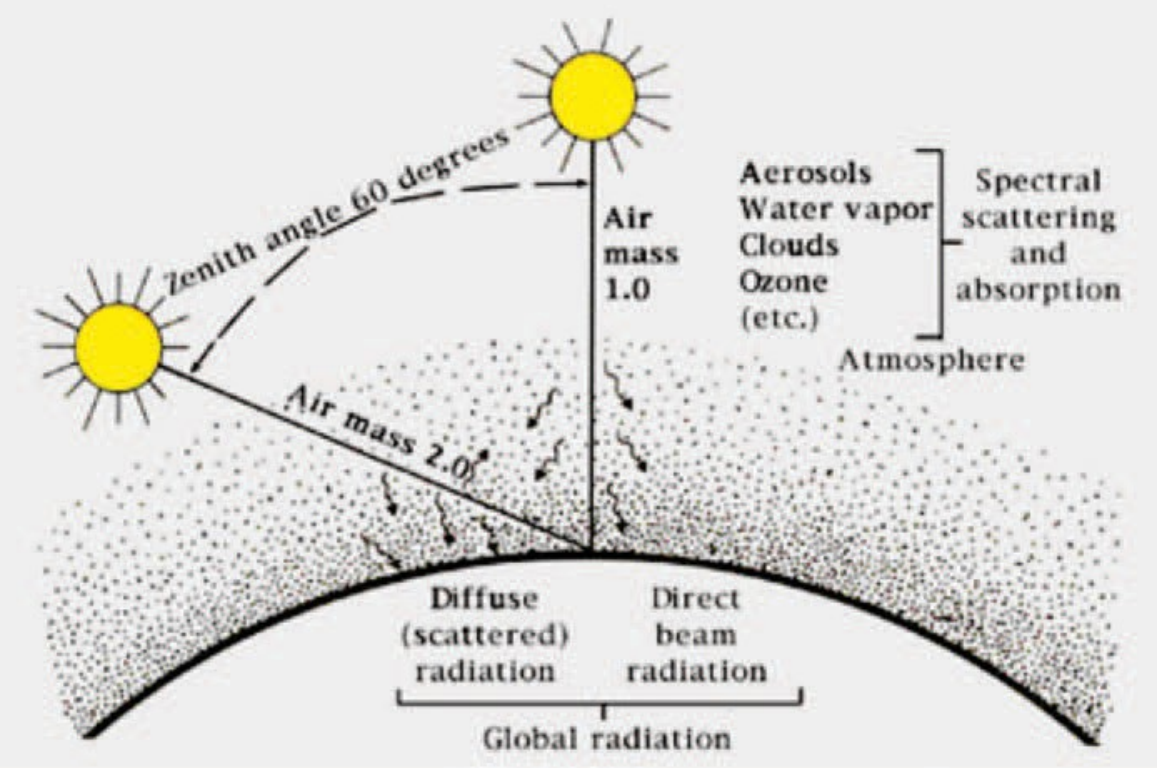

Figure 2-4. Scattering of the direct-beam photons from the sun by the atmosphere produces diffuse radiation that varies with AM (Marion, Riordan, and Renné 1992). Image from NREL

Absorption converts part of the incoming solar radiation to heat and raises the temperature of the absorber. The longer the path length through the atmosphere, the more radiation is absorbed and scattered. Scattering redistributes the radiation in the hemisphere of the sky dome above the observer, including reflecting part of the radiation back into space. The probability of scattering - and hence of geometric and spatial redistribution of the solar radiation-increases as the path (AM) from the TOA to the ground increases.

Part of the radiation that reaches the Earth's surface will be reflected back into the atmosphere. The actual geometry and flux density of the reflected and scattered radiation depend on the reflectivity and physical properties of the ground and constituents in the atmosphere, especially clouds.

Based on these interactions among the radiation and the atmosphere, the terrestrial solar radiation is divided into two components: direct beam radiation refers to solar photons that reach the surface without being scattered or absorbed; diffuse radiation refers to such photons that reach the observer after one or more interactions with the atmosphere. These definitions and their usage for solar energy will be discussed in detail in the following section on DNI.

Research into the properties of atmospheric constituents, ways to estimate them, and their influence on the magnitude of solar radiation in the atmosphere at various levels and at the ground continues and is of great importance to those who measure and model solar radiation fluxes (see Chapters 3 and 4). 


\subsubsection{Relative Motions of the Earth and Sun}

The amount of solar radiation available at the TOA is a function of the TSI and the Earth-sun distance at the time of interest. The slightly elliptical orbit of the Earth around the sun was briefly described above and shown in Figure 2-3. The Earth rotates around an axis through the geographical north and south poles, inclined at an average angle of approximately 23.5 degrees to the plane of the Earth's orbit. The resulting yearly variation in the solar input results in the climate and weather at each location. The axial tilt of the Earth's rotation also results in daily variations in the solar geometry throughout the course of a year.

In the northern hemisphere, at latitudes above the Tropic of Cancer $\left(23.5^{\circ} \mathrm{N}\right)$ near midday, the sun is low on the horizon during the winter and high in the sky during the summer. Summer days are longer as the sun rises north of east and sets north of west. Winter days are shorter as the sun rises south of east and sets south of west. Similar transitions take place in the southern hemisphere. All these changes result in changing geometry of the solar position in the sky with respect to a specific location. (See Figure 2-5 generated for Denver, Colorado, by a program available from the University of Oregon ${ }^{2}$.) These variations are significant and are accounted for in analyzing and modeling solar radiation components using solar position calculations such as NREL's Solar Position Algorithm. ${ }^{3}$

\subsection{Solar Resources: The Solar Components}

Radiation can be transmitted, absorbed, or scattered by an intervening medium in varying amounts depending on the wavelength (see Figure 2-1). Complex interactions of the Earth's atmosphere with solar radiation result in three fundamental broadband components of interest to solar energy conversion technologies:

- DNI-Solar (beam) radiation available (of particular interest to concentrating solar power, or CSP, and concentrating photovoltaic, or CPV, technology)

- Diffuse horizontal irradiance (DHI) - Scattered solar radiation from the sky dome (not including DNI)

- Global horizontal irradiance (GHI) - Geometric sum of the DNI and DHI (total hemispheric irradiance).

These basic solar components are reacted to the SZA by the expression

$$
\mathrm{GHI}=\mathrm{DNI} \times \mathrm{Cos}(\mathrm{SZA})+\mathrm{DHI}
$$

These components are shown in Figure 2-6.

\footnotetext{
${ }^{2}$ See http://solardat.uoregon.edu/SunChartProgram.html.

${ }^{3}$ See http://www.nrel.gov/midc/spa/.
} 


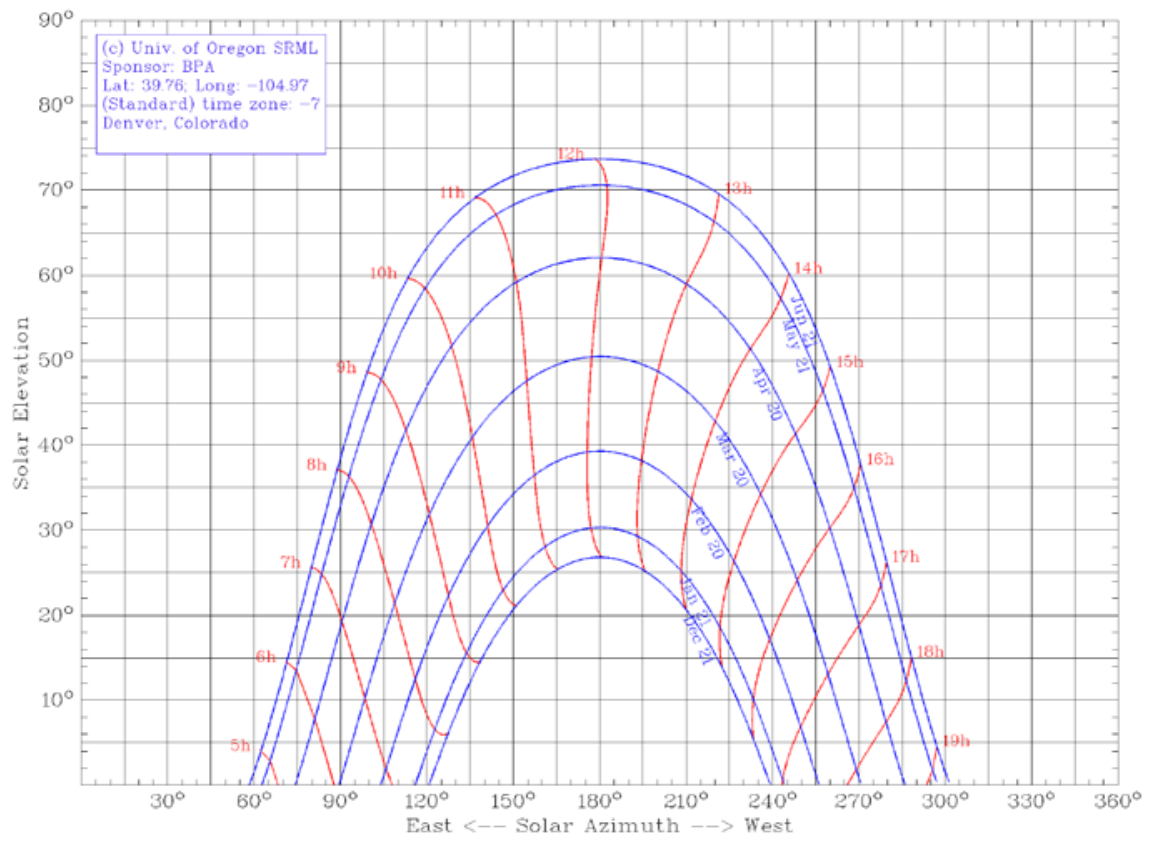

Figure 2-5. Apparent sun path variations during a year for Denver, Colorado. Image from the Universitity of Oregon Solar Radiation Monitoring Laboratory

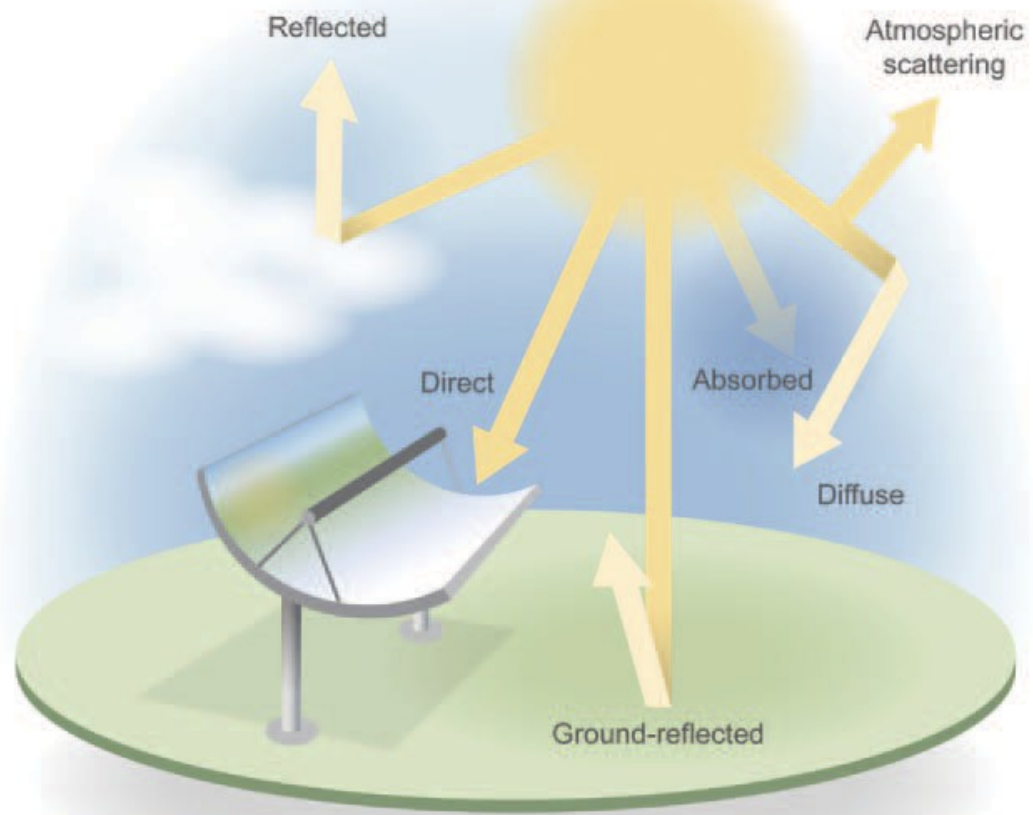

Figure 2-6. Solar radiation components resulting from interactions with the atmosphere. Image by Al Hicks, NREL 


\subsubsection{DNI and Circumsolar Irradiance}

The definition of DNI is the irradiance on a surface perpendicular to the vector from the observer to the center of the sun caused by radiation that did not interact with the atmosphere (WMO 2008). This strict definition is useful for atmospheric physics and radiative transfer models, but it results in a complication for ground observations: It is not possible to measure whether or not a photon was scattered if it reaches the observer from the direction in which the solar disk is seen. Therefore, DNI is interpreted differently in the world of solar energy. Direct solar radiation is understood as the "radiation received from a small solid angle centered on the sun's disk" (ISO 1990). The size of this "small solid angle" for DNI measurements is recommended to be $5 \cdot 10^{-3} \mathrm{sr}$ (corresponding to and approximate 2.5-degree half angle) (WMO 2008). This recommendation is approximately 10 times larger than the radius of the solar disk itself (yearly average 0.266 degree). This is because instruments for DNI measurements (pyrheliometers) have to track or follow the sun throughout its path of motion in the sky, and small tracking errors have to be expected. The large field of view (FOV) of pyrheliometers reduces the effect of such tracking errors.

To understand the definition of DNI and how it is measurement by pyrheliometers in more detail, the role of circumsolar radiation has to be discussed. Because of forward scattering of direct sunlight in the atmosphere, the circumsolar region closely surrounding the solar disk (solar aureole) looks very bright. The radiation coming from this region is called circumsolar radiation. For the typical FOV of modern pyrheliometers (2.5 degrees), circumsolar radiation contributes a variable amount, depending on atmospheric conditions, to the DNI measurement. This contribution can be quantified if the radiance distribution within the solar disk angle and the circumsolar region and the so-called penumbra function of the pyrheliometer is known. Both of these bits of information will be explained in the following. Such an explanation is of particular interest for concentrating solar technologies (CSP or CPV), because the contribution of circumsolar radiation to the yield of most concentrating power plants is less than the contribution from the DNI measurement. This effect has to be considered in the performance analysis of concentrating collectors to avoid overestimating the intercepted irradiance.

The first bit of information that is required to determine the effect of circumsolar radiation on the pyrheliometer is the solar and circumsolar radiance distribution. This distribution usually shows good radial symmetry around the center of the sun. Thus, it can be accurately described as a function of the angular distance from the center of the sun. This solar radiance profile, normalized to unity in the center of the sun, is called sunshape. The sunshape not varies with time and sky conditions, and the average sunshape determined for a specific site can also be very different from that of another location.

The solar radiance profile has been of interest for scientists of various specializations already for some centuries. In the middle of the 18th century, Bouguer carried out measurements of the disk radiance profile and found that the radiance decreases with increasing angular distance from the center of the sun (Mueller 1897). Between 1920 and 1955, the Smithsonian Institution measured the circumsolar irradiance coming from an annular region concentrically positioned around the sun under the lead of Dr. C.G. Abbot (Watt 1980).

Measurements of the solar radiance profile including the solar disk and the circumsolar region have been carried out by Lawrence Berkeley National Laboratory (LBNL) (Grether, Nelson, and 
Wahlig 1975). The measurements from LBNL are of special importance for solar energy, because nearly 180,000 measurements were collected from 11 different sites in the United States between 1976 and 1981 and were later digitally published in the LBNL reduced data base (Noring, Grether, and Hunt 1991). The instrument had a small circular aperture and measured the radiance coming from nearly point-like regions around and inside the solar disk. Other groups used analog photographic techniques to determine the solar radiance profile (for example, Deepak and Adams [1983] and Sandia National Laboratories [see Watt \{1980\}]). In the 1990s, charge-coupled device cameras were used by the Paul Scherrer Institute and the German Aerospace Center (DLR) (Schubnell 1992), an approach that was followed later by DLR until the end of the last century (Neumann et al. 1998). Recently, a method based on two commercial instruments (Visidyne's sun and aureole measurement system, Sytem Advisor Model (SAM), and a CIMEL sun photometer) was presented (Wilbert, Pitz-Paal, and Jaus 2013). Other instruments that measure the circumsolar irradiance are documented in Wilbert, Pitz-Paal, and Jaus (2012); Wilbert, Pitz-Paal, and Jaus (2013); Kalapatapu et al. (2012); and Wilbert (2014).

Figure 2-7 shows sunshapes derived from LBNL and the first DLR sunshape measurement system. Averages throughout several measurements are shown. A proposed "standard solar scan" was determined by Rabl and Bendt (1982) as an average from LBNL measurements. The term "standard" should not be misunderstood. Here it refers to an average of many sunshapes that deviate strongly from the so-called "standard solar scan." "DLR mean" shows an average sunshape derived from DLR's measurements as presented under this name in (Neumann et al. 2002). The other sunshapes are averages of sunshapes within different intervals of CSRs (circumsolar ratio) from (Neumann et al. 2002). They are named corresponding to the CSR interval that was used for the averaging. The CSR can be used to characterize the sunshape to some extent (Buie and Monger 2001). It is defined as

$$
\operatorname{CSR}\left(\alpha_{\mathrm{disk}}, \alpha_{\mathrm{lim}}\right)=\operatorname{CSNI}\left(\alpha_{\mathrm{disk}}, \alpha_{\mathrm{lim}}\right) /\left(\operatorname{CSNI}\left(\alpha_{\mathrm{disk}}, \alpha_{\text {lim }}\right)+\operatorname{DNI}\left(\alpha_{\mathrm{disk}}\right)\right)
$$

Here, $\operatorname{CSNI}\left(\alpha_{\text {disk }}, \alpha_{\text {lim }}\right)$ is the circumsolar normal irradiance observed in the circumsolar region between the angular distances $\alpha_{\text {disk }}$ and $\alpha_{\text {lim }}$ from the center of the sun. $\alpha_{\text {disk }}$ is the solar disk angle (half angle), and $D N I\left(\alpha_{\text {disk }}\right)$ is the disk irradiance (the normal irradiance caused by the radiation observed within the angular distance $\alpha_{d i s k}$ around the sun's center, independent of whether or not the photons were scattered).

The extent of the circumsolar region cannot be defined in a universally valid way. This is because different pyrheliometers and different concentrating collectors use radiation up to individual angular distances $\alpha_{\text {lim }}$ from the center of the sun. Hence, $\alpha_{\text {lim }}$ has to be selected depending on the investigated technology. For example, $\alpha_{\lim }=3.2^{\circ}$ is used for the CSR in the LBNL reduced database (Noring et al. 1991), and $\geq 4^{\circ}$ would be necessary to allow the complete description of a pyrheliometer measurement following World Meteorological Organization (WMO) recommendations (see next paragraph on penumbra functions).

For the physically exact interpretation of the CSR, $\alpha_{\text {disk }}$ is calculated as a function of time using the visible disk radius and the time dependent distance between the sun and the Earth (Wilbert, Pitz-Paal, and Jaus 2013). Depending on the authors and the measurement systems, other angles are used for $\alpha_{\text {disk. }}$ In Neumann et al. (2002), $\alpha_{\text {disk }}=4.65$ milliradians (mrad) is used accepting the error caused by neglecting the yearly variation of the solar disk angle due to the Earth's elliptic 
path around the sun. For the LBNL data, a slightly higher angle than the average solar disk angle is used for all measurements (Watt 1980) to avoid instrumental errors that caused an overestimation of the radiance close to the solar disk angle.

For CPV applications also, the spectral variation of the CSR has to be considered. Spectral CSR values for different wavelengths deviate strongly from each other and also from the corresponding broadband CSRs (Evans et al. 1980). Average ratios of broadband CSR to spectral CSR between 0.7 and 1.4 have been found for the visible and near-infrared spectrum with the LBNL instrument (for measurements around noon). The spectral dependence of these average ratios found for low CSR levels is opposite to that for high CSRs. Also, the scatter of these ratios for each of the wavelengths investigated by LBNL was quite high (Evans et al. 1980). Similar ratios were found in an analysis based on sunshapes predicted by the three-dimensional Monte Carlo radiative transfer model MYSTIC (Mayer 2009) that is part of the libRadtran package (Mayer and Kylling 2005) and SMARTS2 (Gueymard 2001) in Wilbert, Pitz-Paal, and Jaus (2013). Further, the broadband CSR and especially the spectral CSR depend on the AM.

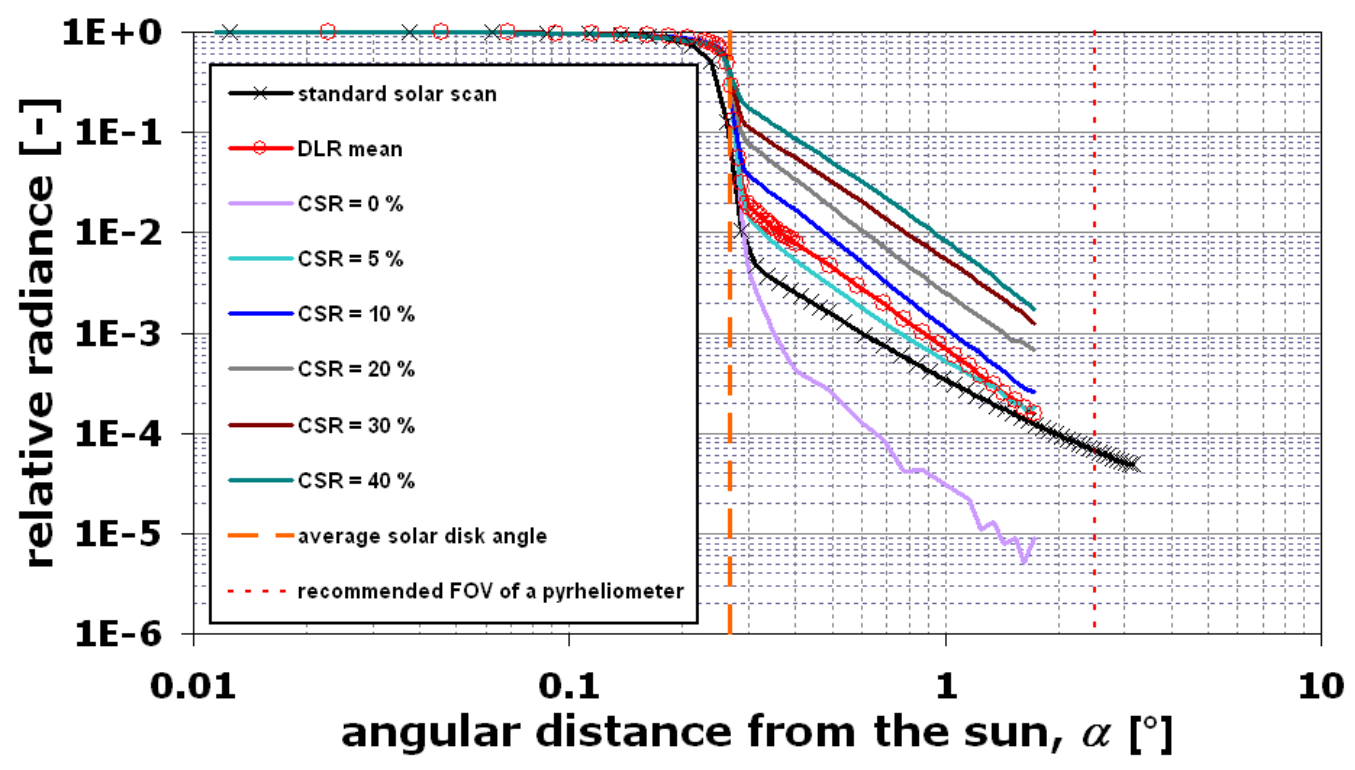

Figure 2-7. Different sunshapes from Rabl and Bendt (1982) and Neumann et al. (2002). The average solar disk angle and the recommended FOV of a pyrheliometer (WMO 2008) are shown as vertical lines. Image from Stefan Wilbert, DLR

The other bit of information to determine the effect of the circumsolar radiation on the DNI measurement is the penumbra function of the pyrheliometer. For pyrheliometers, the geometrical penumbra function evaluated at an angular distance a $\alpha$ from the center of the sun $P_{p y r}(\alpha)$ is defined as the fraction of parallel rays incident on the pyrheliometers aperture at $\alpha$ that reaches the sensor element. It can be calculated using the distance between the aperture window and the sensor element and their respective sizes (Pastiels 1959). Penumbra functions are defined equivalently to angular acceptance functions for CSP or CPV plants, for example, as used in Rabl and Bendt (1982). 
All radiation is detected for angular distances between 0 degrees and the slope angle $\alpha_{\text {slope }}$. For angular distances greater than the limit angle $\alpha_{\text {lim }}$, no radiation is detected by the instrument. The opening half angle (also FOV) is defined as the angle between the optical axis of the instrument and the vector from the center of the sensor element to the border of the instrument's entrance aperture. The opening angle is the average of the slope and the limit angle. WMO recommends that pyrheliometers should have an FOV of 2.5 degrees, a slope angle of 1 degree (WMO 2008), and it follows that the limit angle should be 4 degrees (all angles given as half angles).

Taking into account effects such as the spatial inhomogeneity of the sensor in addition to the geometry, the effective angular acceptance function is obtained (Major 1994).

The radiation accepted by a pyrheliometer $\mathrm{DNI}_{\text {exp }}$ for a known radially symmetric solar radiance profile $L_{\text {solar }}$ can be calculated as:

$$
\mathrm{DNI}_{\text {exp }}=2 \pi \cdot \int_{0}^{\alpha_{\text {lim }}} L_{\text {solar }}(\alpha) P_{\text {pyr }}(\alpha) \sin (\alpha) \cos (\alpha) d \alpha
$$

In this handbook, we understand DNI as $\mathrm{DNI}_{\text {exp }}$ following the typical usage in solar energy. Circumsolar radiation data are available from the LBNL Reduced Data Base. ${ }^{4}$ A detailed discussion of circumsolar radiation can be found in Blanc et al. 2014).

With the resurgence of interest in concentrating solar technology, there is a renewed interest and research in the amount of circumsolar radiation, or sunshapes, as affected by the variable properties of the atmosphere. This is especially relevant for power plant projects in regions in which no sunshape measurements have been performed so far.

\subsubsection{DHI}

A cloudless atmosphere absorbs and scatters radiation as the DNI penetrates to the ground. Parts of the DNI radiation are absorbed (removed) and reflected (scattered) in many other directions away from the path of this beam radiation. It is the scattered radiation we see as the sky radiation in the hemisphere above the ground. Lord Rayleigh (1871), Mie (1908), and Young (1981) developed theories for the mechanism of scattering in the atmosphere. These theories explain why the sky radiation appears blue (short wavelength, or blue light, is scattered more efficiently by atmospheric gases) and the solar disk tends to appear yellow and red at sunrise and sunset (the blue wavelengths are scattered a great deal out of our line of sight, but the longer red wavelengths from the solar disk come through unscattered). The sky radiation in the hemisphere above the local horizontal is called the DHI. A more technical and practical definition of DHI is that it represents all radiation from the sky dome except the DNI (considered to be the quasiparallel ray radiation from the solar disk; see also the detailed discussion in the previous paragraph). Corresponding to the definition of the experimental DNI (Equation 2-3), penumbra functions can be defined for instruments that measure DHI (Major 1992).

\footnotetext{
${ }^{4}$ See http://rredc.nrel.gov/solar/old_data/circumsolar/.
} 
DHI includes radiation reflected or scattered by clouds (if present) and ground-reflected radiation is re-reflected downward by the atmosphere or clouds. Sky-reflected radiation is difficult to model, because the photon interactions with the atmosphere are complex, clouds have varying compositions, and the ground has complex optical properties.

\subsubsection{GHI}

The total hemispherical solar radiation on a horizontal surface is the sum of the flux density resulting in the DNI at the given SZA, and the additional DHI:

$$
\mathrm{GHI}=\mathrm{DNI} \cdot \mathrm{Cos}(\mathrm{SZA})+\mathrm{DHI}
$$

SZA is the solar zenith angle computed from the date and time of measurement at a specific location.

This fundamental equation is the basis of most solar radiation measurement system designs, data quality assessments, and atmospheric radiative transfer models addressing the needs for solar resource data.

\subsubsection{Solar Radiation Resources for Solar Energy Conversion}

Determining the solar radiation components - GHI, DNI, or DHI, or some combination - that are applicable to a conversion system is the first step in evaluating design criteria and performance. Systems with concentrating optics rely on DNI availability. Low concentration systems may be able to use DHI radiation by light-trapping techniques. Flat-plate collectors, fixed or variable in their mounting, can use all radiation components. GHI is most often the only available measured, or modeled, solar radiation data. In this case, conversion models are used to derive estimates of the appropriate quantities (Perez et al. 1987). The solar radiation scientific research community, peer-reviewed publications, and published reports are presently used to evaluate, validate, and assess the quality of these conversion algorithms. Models for estimating solar radiation are constantly appearing and being evaluated (Badescu 2008). A few classic examples are discussed in the next sections.

Model inputs are typically limited to site location, hour of interest, and GHI for the hour, although some advanced models include more specialized inputs. We describe a quasi-physical model, the Direct Insolation Simulation Code (DISC) of Maxwell (1987), which is based on the following premises:

- A BIRD cleary-sky model is used to calculate clear-sky limits for the direct normal atmospheric transmittance.

- An exponential function of AM, similar in form to physical equations used to calculate energy transmission or propagation losses, is used to calculate deviations from clear-sky transmittance values, based on atmospheric composition.

- The equations for computing Kn and other direct normal coefficients are continuously variable relative to $\mathrm{Kt}$ and $\mathrm{AM}$ and reproduce real-world variations in the relationship between DNI and GHI throughout monthly intervals. 
The model is not a rigorous physical algorithm, because the coefficients for computing clear-sky transmittance values were derived from empirical regression analyses of measured DNI and GHI data from Atlanta, Georgia. Hourly average and thermopile radiometer data were used to derive the model. Applicability to higher time resolution (subhourly) data and solid-state (photodiode) radiometers that are subject to spectral effects, which do not sense the entire solar spectrum, is an open research question (Maxwell 1987).

\subsubsection{Estimating DHI from GHI}

During clear and partly cloudy conditions, diffuse irradiance on a horizontal surface, DHI, is often a relatively small part $(<30 \%)$ of the GHI. During overcast conditions, the GHI and DHI should be identical. When DHI measurements are not available, estimates of the diffuse may be needed in conjunction with GHI data to estimate DNI (as in the DISC model). DHI is also useful for daylighting applications. Many models based on empirical correlations between GHI and DHI data have been developed. Liu and Jordan (1960) developed a model for estimating monthly average hourly diffuse. Erbs, Klein, and Duffie (1982), Orgill and Hollands (1977), Iqbal (1983), Spencer (1982), and many others have developed algorithms for estimating hourly DHI. These algorithms generally use empirical correlations between the global clearness index, $\mathrm{Kt}$, and either the diffuse fraction, $\mathrm{K}$ ( = DHI/GHI), or the diffuse clearness index, Kd. Many hourly diffusefraction based models exist in the literature (Gueymard and Ruiz-Arias 2014)

- $\mathrm{Kt}=$ Clearness index or global horizontal transmittance of the atmosphere

$$
=\quad \mathrm{GHI} /\left[\mathrm{TSI} \cdot\left(\mathrm{r}_{0} / \mathrm{r}\right)^{2} \cdot \operatorname{Cos}(\mathrm{SZA})\right]
$$

- $\mathrm{Kd}=$ Diffuse transmittance of the atmosphere

$$
=\quad \mathrm{DHI} /\left[\mathrm{TSI} \cdot\left(\mathrm{r}_{0} / \mathrm{r}\right)^{2} \cdot \operatorname{Cos}(\mathrm{SZA})\right]
$$

\subsubsection{Estimating DNI from GHI}

DNI can be evaluated indirectly from the models of the previous section, once the global and diffuse components are known, or directly through other separation models that are not based on the diffuse fraction approach. Among the many models for estimating DNI from GHI, two of them are described below for reference purposes: the Maxwell DISC model and the Perez variation on this approach, DIRINT (Perez et al. 1990). This type of model is based on empirical relations between clearness indices Kt and Kn (Liu and Jordan 1960):

- $\mathrm{Kt}=$ Clearness index or global horizontal transmittance of the atmosphere

$$
=\quad \mathrm{GHI} / \mathrm{TSI} \cdot\left(\mathrm{r}_{0} / \mathrm{r}\right)^{2} \cdot \operatorname{Cos}(\mathrm{SZA})
$$

- $\mathrm{Kn}=$ Direct normal transmittance of the atmosphere

$$
=\quad \text { DNI/TSI } \cdot\left(\mathrm{r}_{0} / \mathrm{r}\right)^{2}
$$

where

- $\quad \mathrm{TSI}=$ Total solar irradiance (mean TSI, $\sim 1366.7 \mathrm{Wm}^{-2} \pm 7 \mathrm{Wm}^{-2}$ )

- $\mathrm{r}_{0}=$ mean Earth-sun distance $(149,598 \mathrm{~km})$ 
- $\mathrm{r}=$ Earth-sun distance at the time of interest

- $\mathrm{SZA}=$ Solar zenith angle at the time of interest.

\subsubsection{Estimating Solar Resources on a Tilted Surface}

Solar conversion systems as flat-plate collectors or non-concentrating photovoltaics (PV) are tilted toward the sun. Estimating or modeling the irradiance incident upon these systems is essential to the performance and yield evaluation of these systems. This irradiance is called plane-of-array (POA) irradiance or global titled irradiance (GTI). The GTI can be measured directly by pyranometers that are tilted as the collector plane. Some radiative transfer models also allow the direct modeling of the irradiance in this plane; however, GHI, DHI, and DNI data are more common. Hence, there are several transposition models that convert GHI or a set of GHI and DNI information for one timestamp to GTI (Reindl 1990, Liu 1979, Hay 1979, Klucher 1979, Gueymard 2008, Gueymard 1988, Temps 1977, Willmott 1982).

\subsection{Modeled Data Sets}

As mentioned above, long-term measured data sets are rare, have variable periods of record, and are not always easily accessed. Measurement networks or stations providing high-accuracy, upto-the-minute measured data are rare, although a wide variety of agricultural research station solar radiation data of highly variable quality is available. These types of data require careful evaluation and comparison to other sources of data, such as estimated or modeled data, to establish appropriateness of use. There are many sets of modeled solar radiation data, typically GHI, sometimes with DNI, and DHI. A few examples are the National Solar Radiation Database (NSRDB), the Swiss Meteotest METEONORM data set, the European Solar Radiation Atlas (ESRA), the National Aeronautics and Space Administration (NASA) surface meteorology and solar energy (SSE), and the European Commission's solar data (SODA) data sets. (See Chapter 5 for more details about these and other sources of data.)

Another popular modeled data set is the TMY for a specific location. TMY data sets were originally designed for simplified building heating and cooling load calculations. A TMY is meant to typify the meteorological conditions at a site of interest and is based on several years of meteorological data. Different algorithms to create TMYs and different data sets called TMYs exist. Further, similar algorithms and corresponding data sets exist that are called RMYs (representative meteorological years), RSYs (representative solar years), or TRYs (test reference years). Individual detailed descriptions of the properties of specific data sets will be discussed in Chapter 4 and Chapter 5.

These data sets are used primarily to estimate the relative performance of different conversion system designs with respect to a standard data set, and they are not appropriate for optimizing performance. Many software applications use TMY data to predict the typical performance of a solar conversion system, but this is not the purpose of the original TMY development.

\subsection{Uncertainty Measurements and Models}

Measurements of solar radiation are among the most uncertain in any measurement discipline. Empirical models developed from measured solar radiation data, and the validation of any model with measured data, always includes the measurement uncertainties in addition to the inherent model accuracy. 
Solar radiation models based on physics or correlations with meteorological variables are impossible to validate to an overall accuracy better than the uncertainty of the measured data. Measurement uncertainty analysis has been formalized by several organizations, including the International Bureau of Weights and Measurements (French acronym BIPM), and published by the International Standards Organization (ISO) as the Guide to the Expression of Uncertainty in Measurements, which is also known under the acronym GUM (BIPM et al. 1995).

The uncertainty of resource data will be addressed in all of the following chapters, because it is essential for the interpretation of the data independent of the method used for its determination.

\subsection{Spatial and Temporal Variability of Solar Resources}

Earlier we described the variation of the ETR as a function of the 11-year sunspot cycle (less than $\pm 0.2 \%)$, and the annual variation of the Earth-sun distance ( $\pm 3 \%)$. These are minor variations compared to the influences of the atmosphere, weather, climate, and geography on the variation of solar resources at the Earth's surface. Variations in solar radiation from month to month, especially in the latitudes outside the tropics, follow an annual pattern, generally during the summer, with lower values during the winter. The year-to-year variation in these patterns is called the interannual variability. The coefficient of variation $(\mathrm{COV}=$ ratio of the standard deviation to the mean of a set of given averages) can be used to quantify this variability. Studies of GHI and DNI distributions in the United States show that the range of GHI interannual variability is typically $8 \%$ to $10 \%$. This is generally approximately half, or less, of the variability of DNI, which can be $15 \%$ or more at the $68 \%$ confidence interval (Wilcox and Gueymard 2010).

However, studies show that the COV for annual averages of DNI can approach 10\%, depending on climate stability. For example, in the NSRDB 2005 update, Daily Statistics Files indicate that the COV for Daggett, California, is $6.2 \%$. Interannual COV for annual average GHI is typically $5 \%$. The COV is based on a single standard deviation and is typically approximately one-third the range of data in a sample.

In the continental United States, differences among radiation resources in the same months in different years are generally larger during the winter and smaller during the summer. Variations in weather and natural events such as forest fires, volcanic eruptions, dust clouds from drought regions, and agricultural activity all can contribute to interannual variations. Figure 2-12 compares the mean and maximum and minimum monthly average daily total DHI from the 1961-1990 NSRDB (modeled from meteorological data) to eight individual years of estimates based on satellite data (1998-2005) for Daggett, California. Variations much greater or somewhat smaller than this are shown for locations with more or less variable weather patterns. 


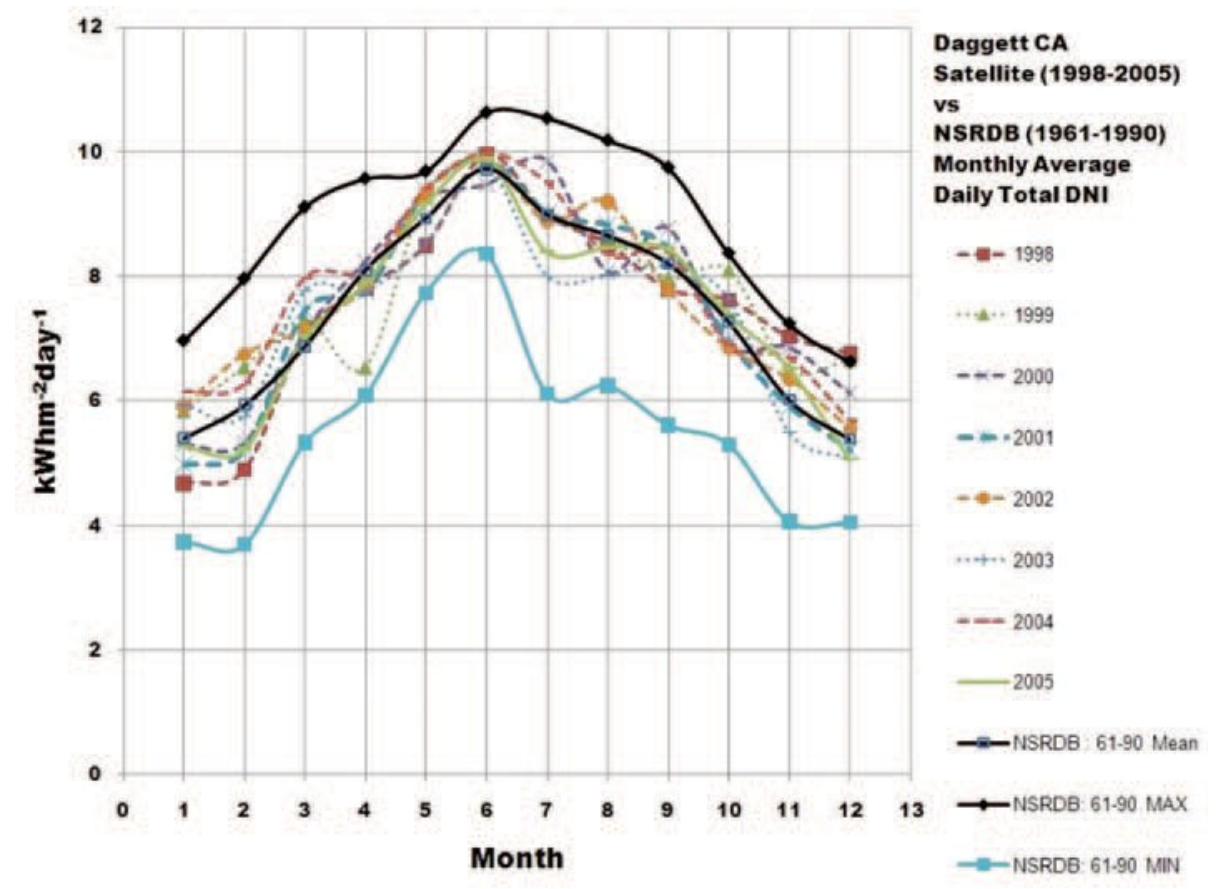

Figure 2-8. Example of direct-beam monthly average daily total $\left(\mathrm{kWh} / \mathrm{m}^{2} / \mathrm{day}\right)$ interannual variability from 1961 through 2005 for Daggett, California. Data from Wilcox et al. (2007). Image from NREL

Spatial variations in solar resources often come into question, especially if nearby or neighboring measured data are available for a site without measured data. Mountainous terrain or highly variable urban, agricultural, or other microclimate influences may contribute to high spatial variability of the solar resource. Analysis of measured and modeled data correlations to distances between stations has generally shown that correlations decrease with increasing station spacing and higher time resolution (e.g., 15-minute versus hourly) data integration periods. One study of 17 sites in Wisconsin showed that correlations for hourly data fell from 0.995 to 0.97 as spacing increased from $5 \mathrm{~km}$ to $60 \mathrm{~km}$. For 15 -minute data, the correlations fael from 0.98 to less than 0.75 at distances more than $100 \mathrm{~km}$ (see Chapter 6).

Prevailing winds and cloud motion patterns can also affect both spatial and temporal variability throughout distances from a few to hundreds of kilometers. A study of a dense solar measurement network in Oklahoma showed that correlations between stations degraded from $95 \%$ or better for nearby stations to less than $45 \%$ for stations greater than $300 \mathrm{~km}$ away, depending on the geographical relationship (east, west, northwest, etc.) between the stations. Barnett et al. (1998) provided a correlogram for these analyses. Attempts to interpolate between stations to estimate solar resources should be used with caution. Attention to the data sample period, geography, terrain, weather patterns, and spacing is important and requires careful analyses.

More details on this can be found in Chapter 4, Chapter 5, and Chapter 6 . 


\section{Measuring Solar Radiation}

Accurate measurements of the incoming irradiance are essential to solar power plant project design and implementation. Because irradiance data are relatively complex, and therefore expensive compared to other meteorological measurements, they are available for only a limited number of locations. This holds true especially for DNI. Increasingly, developers are in need of irradiance data for site resource analysis, system design, and plant operation. Irradiance measurements are also used to develop and test models for estimating irradiance and other solar irradiance components based on available surface meteorological observations or satellite remote sensing techniques. Irradiance measurements will also play an important role in developing solar resource forecasting techniques.

This chapter focuses on the instrument selection, installation, design, and operations and maintenance $(\mathrm{O} \& M)$ of measurement systems suitable for collecting irradiance resource measurements.

\subsection{Instrumentation Selection Options}

Before considering instrumentation options and the associated costs, the user must first evaluate the data accuracy or uncertainty levels that will satisfy the ultimate analyses based on the radiometric measurements. This ensures that the best value can be achieved after the available various measurement and instrumentation options are considered. (See the appendix for a list of radiometer manufacturers and distributors.)

By first establishing the project needs for radiometric data accuracy, the user can base instrument selection and the levels of effort necessary to operate and maintain the measurement system on an overall cost-performance determination. Specifically, the most accurate instrumentation (ISO 9060 first class; WMO "high quality" or ISO 9060 secondary standard) should not be purchased if the project resources cannot support the maintenance required to ensure measurement quality consistent with the radiometer design specifications and manufacturer recommendations. In such a case, alternative instrumentation with lower maintenance requirements, such as pyranometers with diffuser disks or rotating shadowband irradiometers (RSIs), should be preferred.

Redundant instrumentation is another important consideration to ensure confidence in data quality. Multiple radiometers within the project location and/or providing for the measurement of all three solar irradiance components (GHI, DHI, and DNI), regardless of the primary measurement need, can greatly enhance opportunities for post-measurement data quality assessment.

\subsection{Instrument Types}

Instruments designed to measure any form of radiation are called radiometers. In this section, we summarize the types of radiometers most commonly used to measure solar radiation resources for applications to solar energy technology needs.

\subsubsection{Pyrheliometers and Pyranometers}

Pyrheliometers and pyranometers are two types of radiometers used to measure solar irradiance. Their ability to receive solar radiation from two distinct portions of the sky distinguishes their designs. As described in Chapter 2, pyrheliometers are used to measure DNI, and pyranometers 
are used to measure GHI, DHI, or POA irradiances. Table 3-1 summarizes some key attributes of these two radiometers.

Table 3-1. Solar Radiation Instrumentation

\begin{tabular}{|l|l|l|l|}
\hline Radiometer Type & Measurement & FOV (Full Angle) & \multicolumn{1}{|c|}{ Installation } \\
\hline Pyrheliometer & DNI & $\begin{array}{l}5 \text { degrees to } \\
6.0 \text { degrees }\end{array}$ & $\begin{array}{l}\text { Mounted on automatic solar tracker } \\
\text { for alignment with the solar disk }\end{array}$ \\
\hline Pyranometer & DHI & $2 \pi$ steradians & $\begin{array}{l}\text { Mounted on stable horizontal } \\
\text { surface free from local obstructions }\end{array}$ \\
\hline Pyranometer & POA & $2 \pi$ steradians & $\begin{array}{l}\text { Mounted on automatic solar tracker } \\
\text { fitted with shading mechanism or on } \\
\text { a manually adjusted shadowband } \\
\text { platform to block DNI from detector } \\
\text { surface }\end{array}$ \\
\hline
\end{tabular}

${ }^{a}$ Optionally thermopile pyranometers are installed with powered ventilator and heating to reduce contamination of optical surfaces and thermal errors.

Pyrheliometers and pyranometers commonly use either a thermoelectric or photoelectric detector to convert solar flux $\left(\mathrm{W} / \mathrm{m}^{2}\right)$ into a proportional electrical signal (microvolts $\left.(\mu \mathrm{V}) \mathrm{dc}\right)$.

Thermoelectric detectors have an optically black coating that allows for a broad and uniform spectral response to all solar radiation wavelengths between approximately $300 \mathrm{~nm}$ and 3,000 nm (Figure 3-1). Because of the relatively large thermal mass of this detector design, the timeresponse characteristics are typically 1 second to 5 seconds. ${ }^{5}$ That is, the output signal lags the changes in solar flux. Common photoelectric detectors, however, generally respond to only the visible and near-infrared spectral regions from approximately $400 \mathrm{~nm}$ to $1,100 \mathrm{~nm}$ (Figure 3-2). These detectors have very fast time-response characteristics - on the order of microseconds. For either detector, as installed in commercially available instruments, the electrical signal generated by exposure to solar irradiance levels of approximately $1,000 \mathrm{~W} / \mathrm{m}^{2}$ is on the order of 10 millivolts $(\mathrm{mV}) \mathrm{DC}$ (assuming no amplification of the output signal and an appropriate shunt resistor for photodiode sensors). This rather low-level signal requires proper electrical grounding and shielding considerations during installation.

\footnotetext{
${ }^{5}$ Physically, the constant represents the time it takes the system's step response to reach $(1-1 / \mathrm{e})$ or approximately $36.8 \%$ of the total stimulus change.
} 


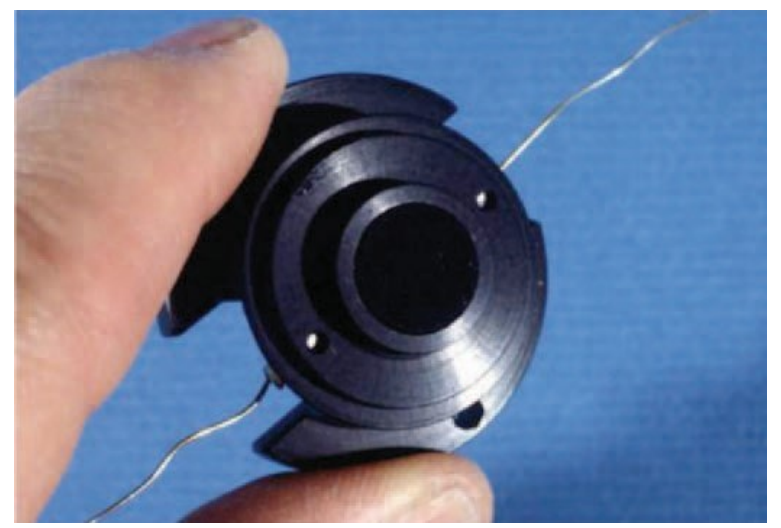

Figure 3-1. Thermopile assembly used in an Eppley Laboratory, Inc., model precision spectral pyranometer (PSP)

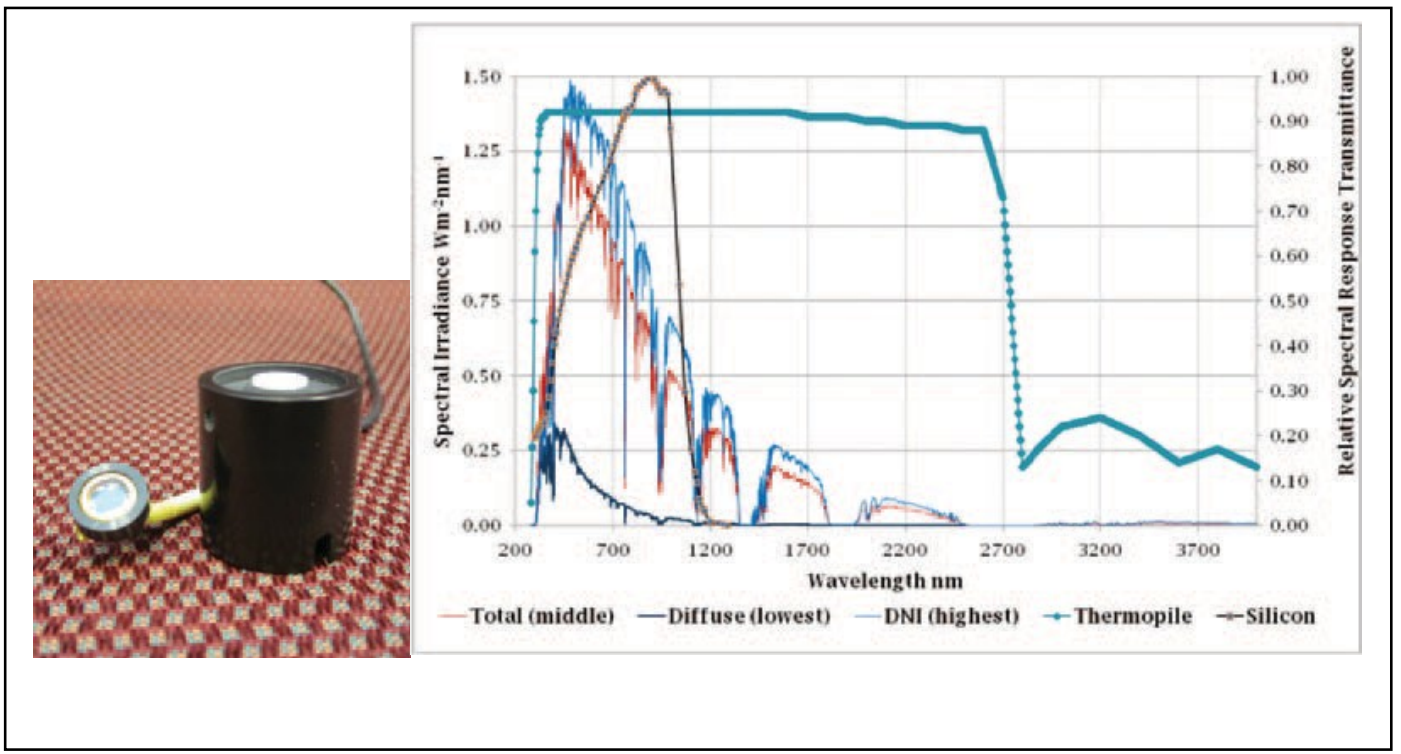

Figure 3-2. (Left) Typical photodiode detector and (right) spectral response of LI-COR pyranometers LI200SA. Photographs used by permission of LI-COR Biosciences, Inc.

All modern pyrheliometers have a 5-degree FOV, following WMO (2008) recommendations. However, the FOV of older instruments may be larger, such as 5.7 degrees to 10 degrees full angle." Pyrheliometers are mounted in automatic solar trackers to maintain the instrument's alignment with the solar disk and fully illuminate the detector from sunrise to sunset (Figure 3-3 and Figure 3-4). Alignment of the pyrheliometer with the solar disk is determined by a simple diopter, or a sighting device in which a small spot of light (the solar image) falls on a mark in the center of a target located near the rear of the instrument (Figure 3-5). By convention and to allow for small variations in tracker alignment, view-limiting apertures inside a pyrheliometer allow for the detection of radiation in a narrow annulus of sky around the sun (WMO 2008). This circumsolar radiation component is because of the forward scattering of radiation near the solar disk caused by cloud particles, atmospheric aerosols, and other constituents that can scatter solar radiation. Depending on the FOV - or, to be more precise, the sensor's penumbra function (see 
Chapter 2 section on "DNI and Circumsolar Irradiance") — and tracker alignment, pyrheliometer measurements include varying amounts of circumsolar irradiance contributions to the DNI.

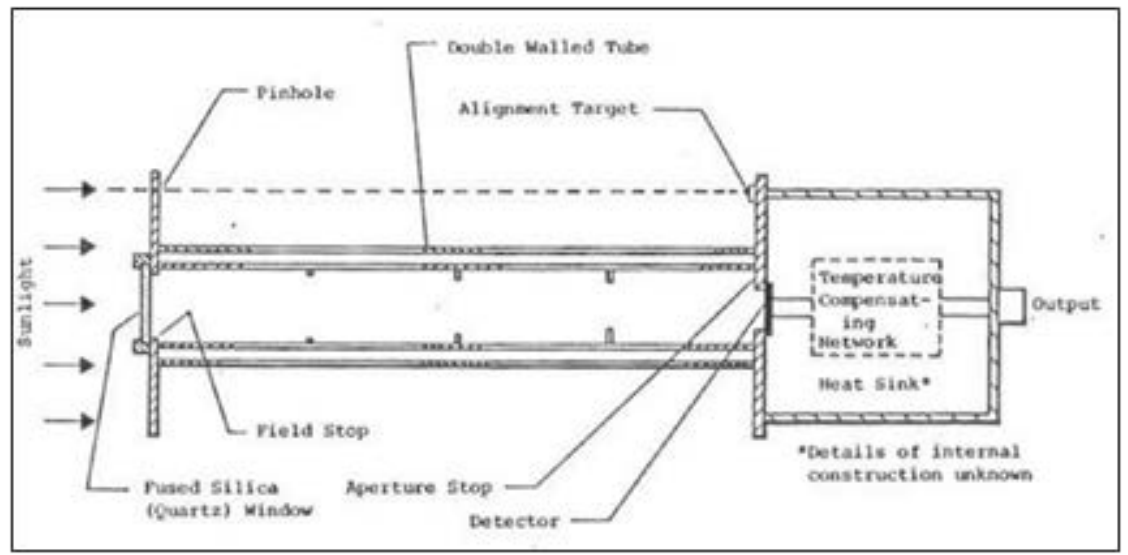

Figure 3-3. Schematic of an Eppley Laboratory, Inc., model normal incidence pyrheliometer (NIP) (Bahm and Nakos 1979). Image from the former U.S. Energy Research and Development Administration, now DOE

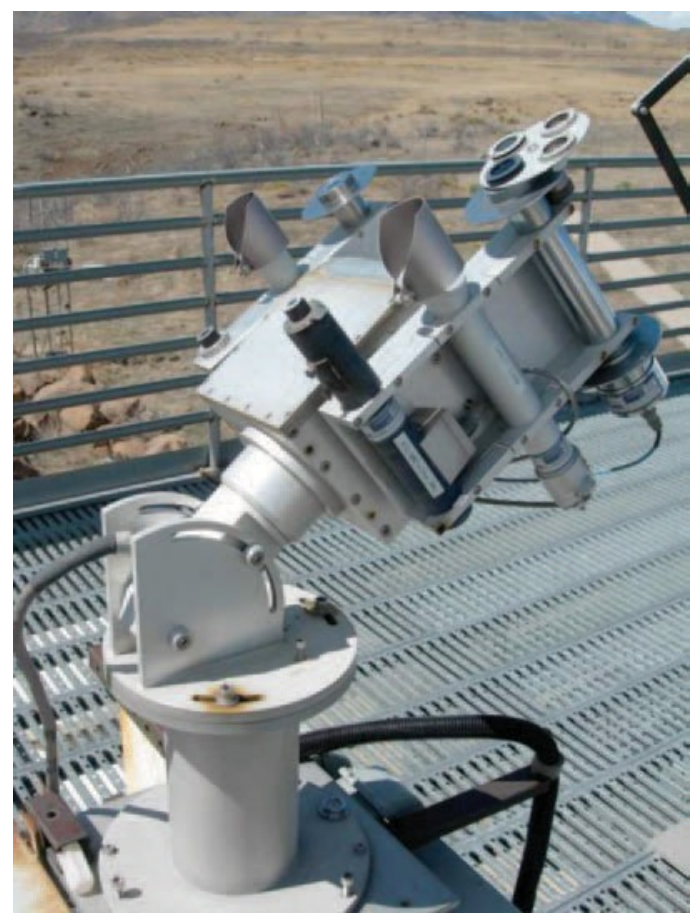

Figure 3-4. Pyrheliometers mounted on an automatic solar tracker. Photo from NREL 


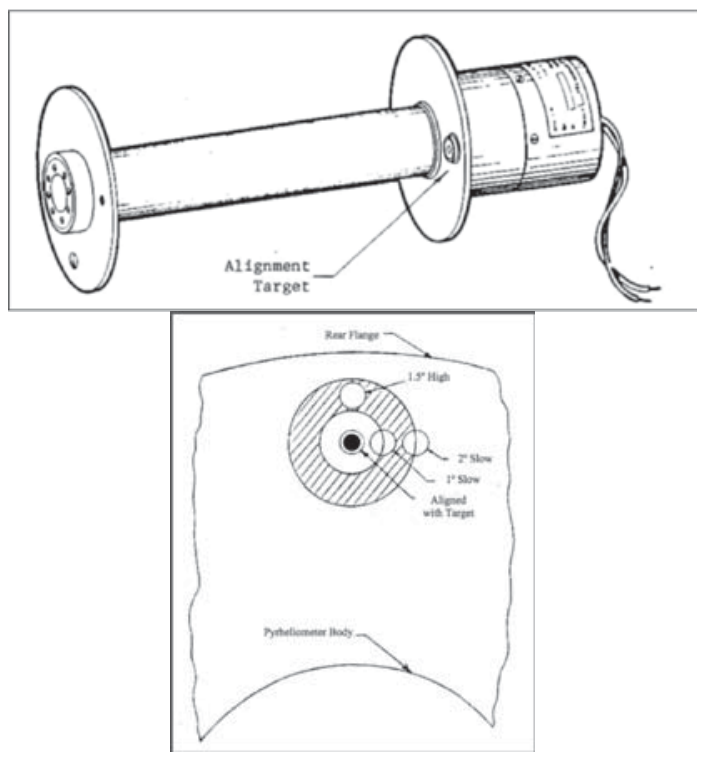

Figure 3-5. Schematics of pyrheliometer alignment diopter configuration (Micek 1981). Image used by permission from Leonard Micek

The most accurate measurements of DNI are accomplished using an electrically self-calibrating absolute cavity radiometer (see Figure 3-6). This type of pyrheliometer is the basis for the World Radiometric Reference (WRR), the internationally recognized detector-based measurement standard for DNI. The WMO World Standard Group of absolute cavity radiometers is shown in Figure 3-7 (Fröhlich 1991). By design, absolute cavity radiometers have no window and are therefore generally limited to fully attended operation during clear-sky conditions to protect the integrity of the receiver cavity (Figure 3-8). Removable windows and temperature-controlled allweather designs are available for automated continuous operation of these pyrheliometers; however, the installation of the protective window nullifies the "absolute" nature of the DNI measurement. The window introduces additional measurement uncertainties associated with the optical transmittance properties of the window (made from either quartz or calcium fluoride) and the changes to the internal heat exchange due to the now sealed system. 


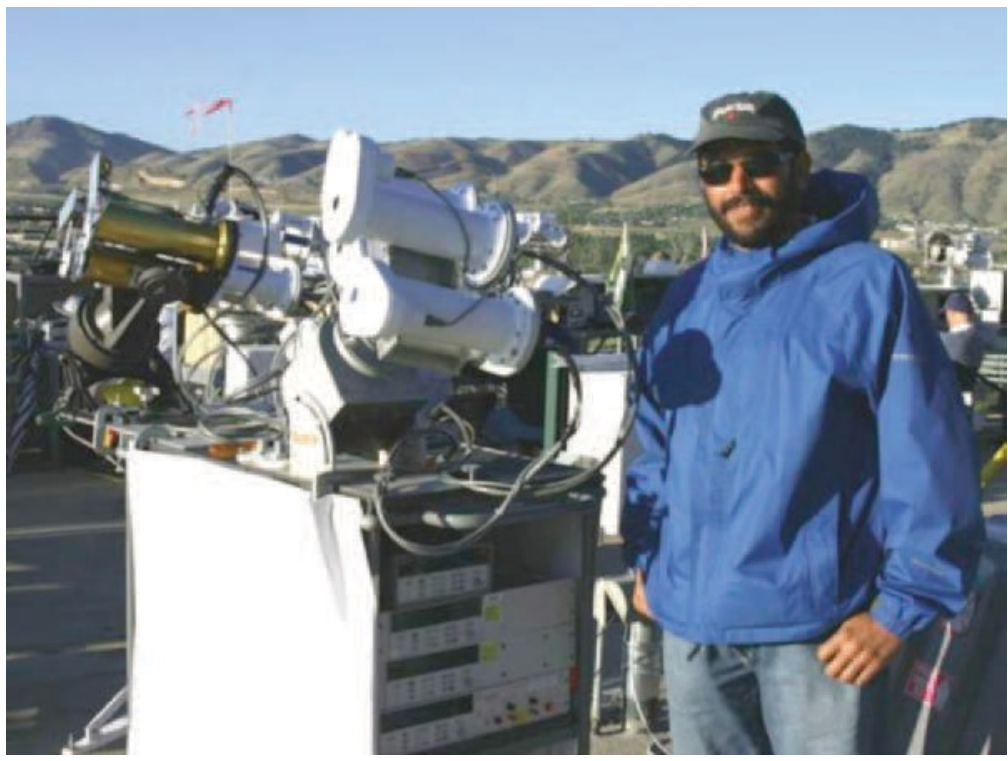

Figure 3-6. Multiple electrically self-calibrating absolute cavity radiometers mounted on solar trackers with control and data acquisition electronics. Photo from NREL

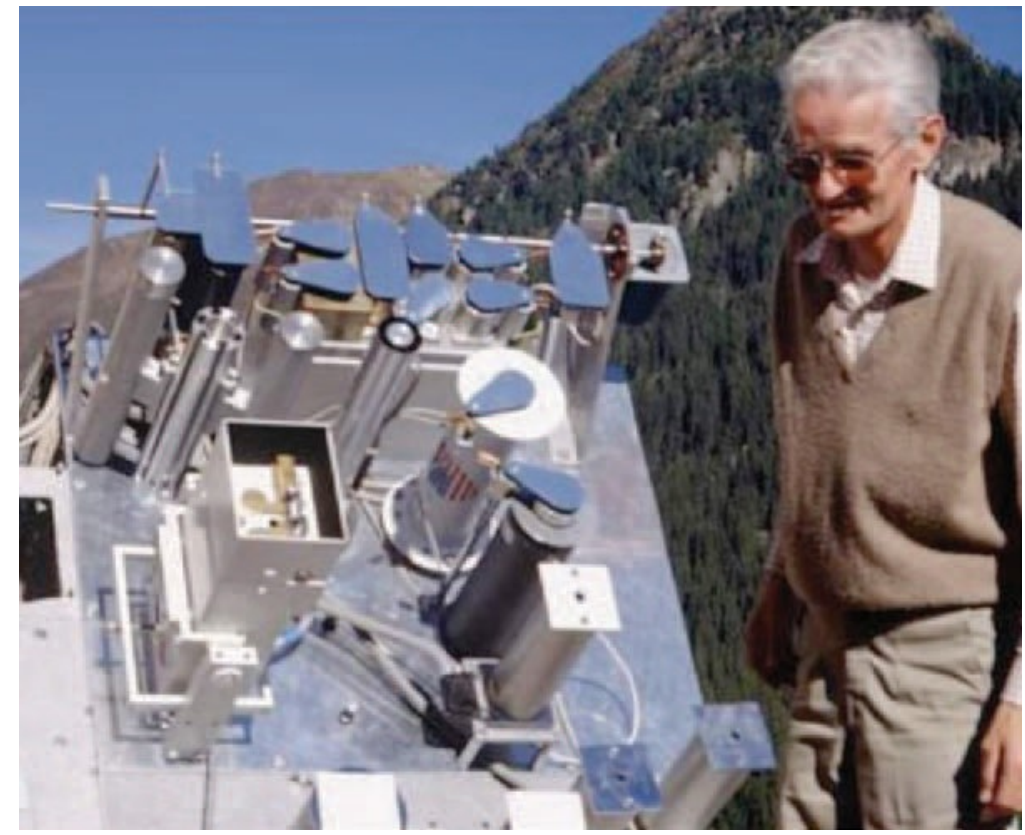

Figure 3-7. The World Standard Group of six absolute cavity radiometers is used to define the WRR or DNI measurement standard. Photo from NREL 


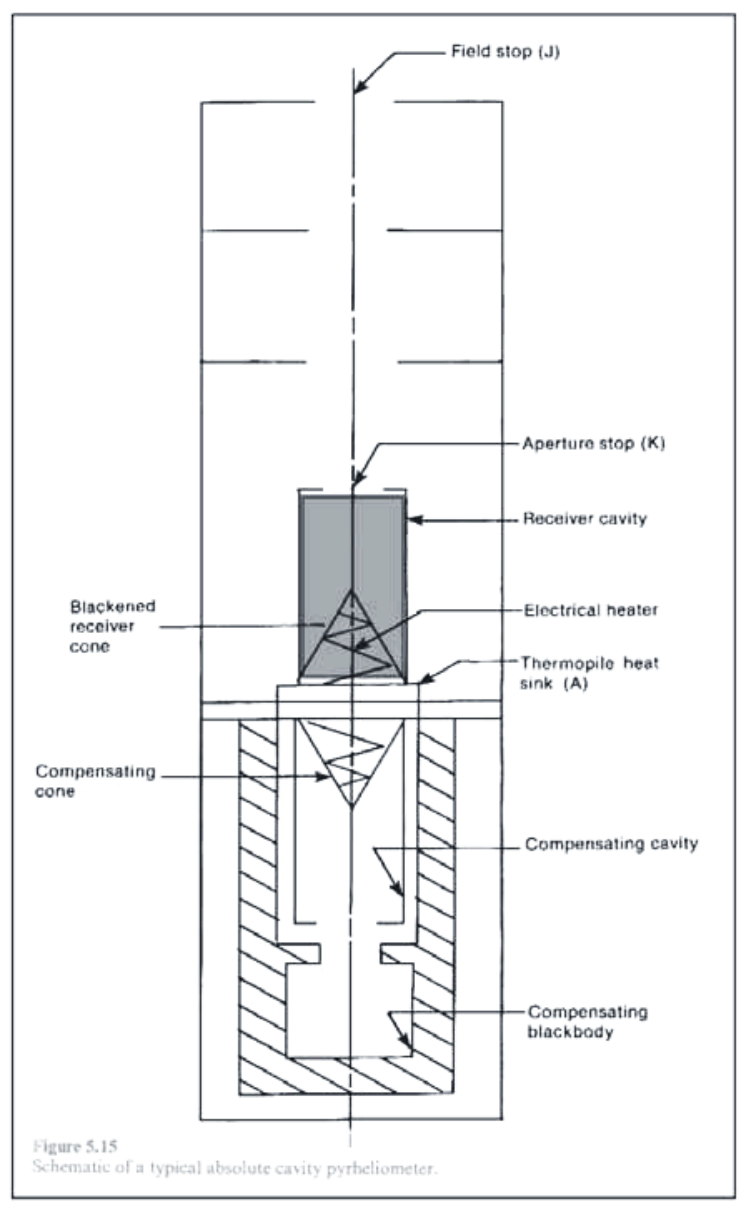

Figure 3-8. Schematic of the Eppley Laboratory, Inc., model AHF absolute cavity pyrheliometer. Image modeled from Reda (1996)

A pyranometer has a thermoelectric or photoelectric detector with a hemispherical or "fish-eye" FOV (360 degrees or $2 \pi$ steradians) (see Figure 3-9). This type of radiometer can be mounted on a horizontal platform to measure GHI. In this orientation, the pyranometer has a complete view of the sky dome. Ideally, the mounting location for this instrument is free of natural or artificial obstructions on the horizon. Alternatively, the pyranometer can be mounted tilted to measure tilted irradiance, vertical irradiance, or reflected irradiance.

The pyranometer detector is mounted under a protective precision-ground quartz (or other material) dome or a diffuser. Both designs protect the detector from the weather and provide optical properties consistent with receiving hemispheric solar radiation. Pyranometers can be fitted with ventilators that constantly blow air - sometimes heated - from under the instrument and over the dome (Figure 3-10). The ventilation reduces the potential for contaminating the pyranometer optics caused by dust, dew, frost, snow, ice, insects, or other material. Ventilators and heating also affect the thermal offset characteristics of pyranometers with single-black detectors (Vignola, Long, and Reda 2009). The ventilation devices can, particularly when heated, require a significant amount of electrical power $(5 \mathrm{~W}-20 \mathrm{~W})$, adding to the required capacity for on-site power generation in remote areas. 
Consistent with their low-cost design based on a photodiode detector, these pyranometer designs employ a diffuser above the detector (Figure 3-11). Acrylic diffusers can be more dust tolerant than optical glass domes (Maxwell et al. 1999).

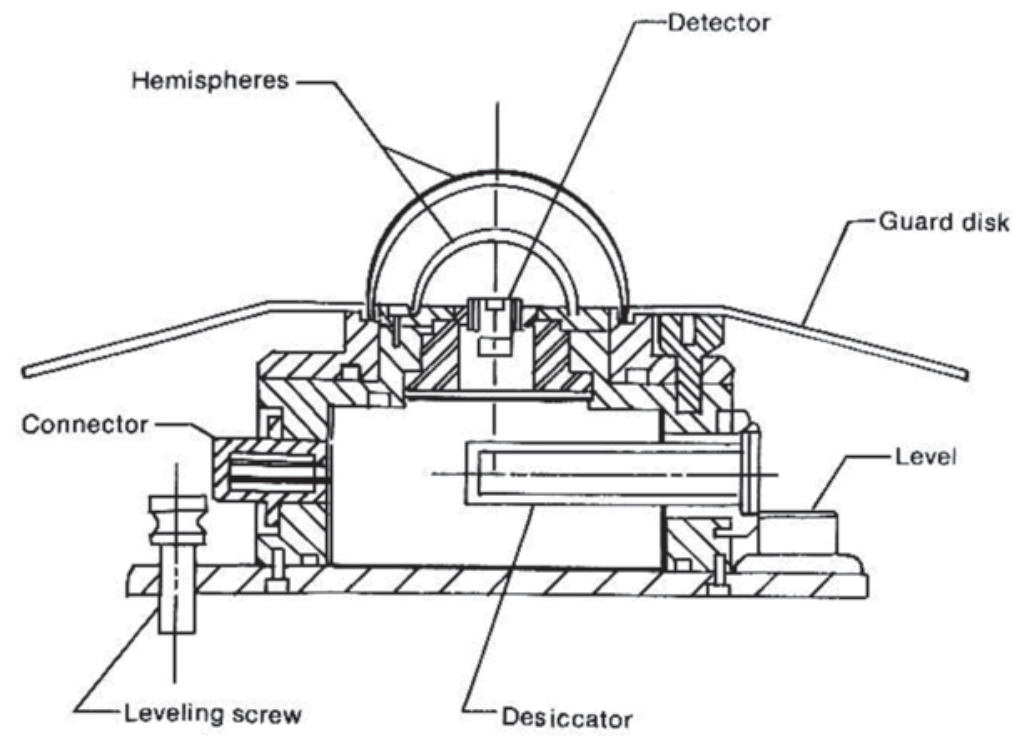

Figure 3-9. Schematic of the Eppley Laboratory, Inc., model PSP. Image from NREL

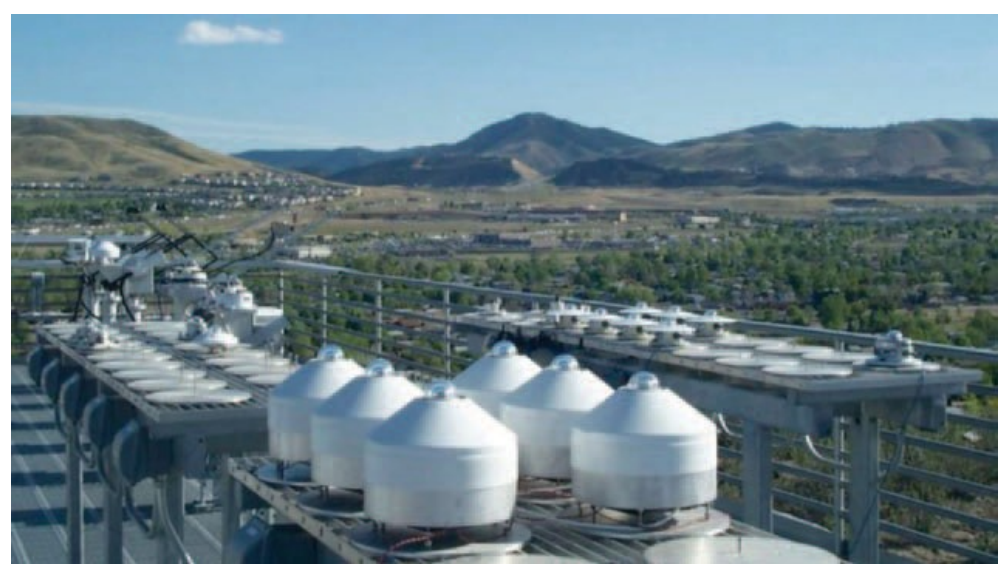

Figure 3-10. Kipp \& Zonen model CM22 pyranometers installed in CV2 ventilators. Photo from NREL 


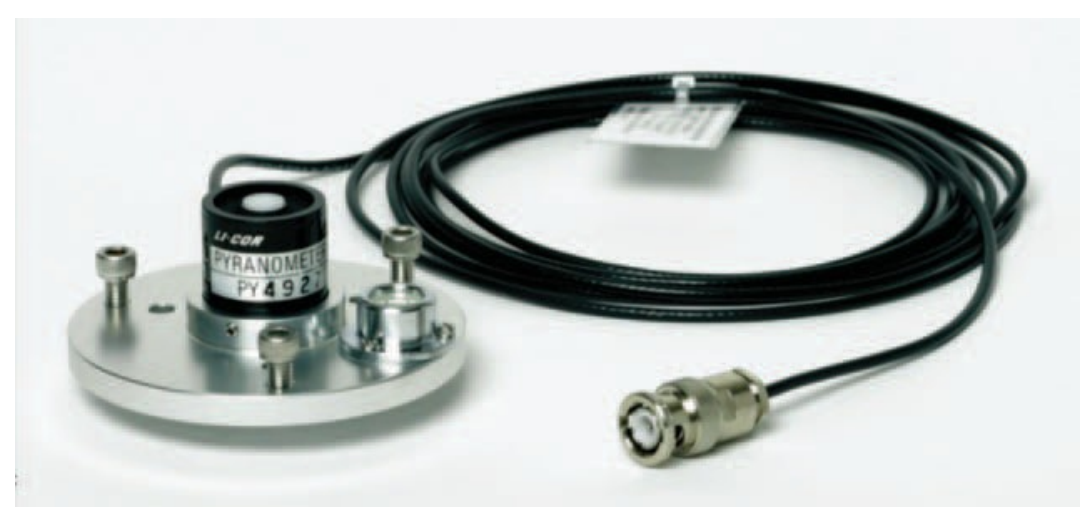

Figure 3-11. LI-COR model LI-200SA pyranometer with photodiode detector and acrylic diffuser fore optic. Photo from LI-COR

\subsubsection{Pyrheliometer and Pyranometer Classifications}

The ISO and the WMO have established classifications and specifications for the measurement of solar irradiance (ISO 1990, WMO 2008). We encourage the reader to review these documents in more detail as part of project planning for solar resource measurements before acquiring pyrheliometers or pyranometers.

Estimated measurement uncertainty is the basis for these pyrheliometer and pyranometer classifications. The WMO (2008) recognizes the difficulties associated with measuring solar irradiance:

It may be said generally that good quality measurements are difficult to achieve in practice, and for routine operations they can be achieved only with modern equipment and redundant measurements. Some systems still in use fall short of best practice, the lesser performance having been acceptable for many applications. However, data of the highest quality are increasingly in demand.

The WMO characteristics of operational pyrheliometers and pyranometers are presented in Table 3-2 and Table 3-3. The ISO specification lists for these radiometers are presented in Table 3-4 and Table 3-5. Our purpose for providing these classifications is to address questions about differences in data quality and to give the reader a better understanding of the data quality afforded by particular instrument classes. In the tables, Rs denotes responsivity, or signal per watt per square meter of flux, for example, millivolts $/\left(\mathrm{W} / \mathrm{m}^{2}\right)$. The reciprocal of Rs is the calibration factor used to convert signal to flux. 
Table 3-2. WMO Characteristics of Operational Pyrheliometers for Measuring $\mathrm{DNI}^{\mathrm{a}}$

\begin{tabular}{|c|c|c|c|}
\hline \multicolumn{2}{|c|}{ Characteristic } & High Quality $^{\mathrm{a}}$ & Good Quality $^{a}$ \\
\hline \multicolumn{2}{|c|}{ Response time (95\% response) } & $<15 s$ & $<30 s$ \\
\hline \multicolumn{2}{|c|}{$\begin{array}{l}\text { Zero offset-response to } 5-\mathrm{K} / \mathrm{h} \text { change in ambient } \\
\text { temperature }\end{array}$} & $2 \mathrm{~W} / \mathrm{m}^{2}$ & $4 \mathrm{~W} / \mathrm{m}^{2}$ \\
\hline \multicolumn{2}{|c|}{ Resolution-smallest detectable change in W/m² } & 0.51 & 1 \\
\hline \multicolumn{2}{|c|}{ Stability_change per year, percentage of full scale } & 0.1 & 0.5 \\
\hline \multicolumn{2}{|c|}{$\begin{array}{l}\text { Temperature response-percentage maximum error } \\
\text { caused by any change of ambient temperature within an } \\
\text { interval of } 50 \mathrm{~K}\end{array}$} & 1 & 2 \\
\hline \multicolumn{2}{|c|}{$\begin{array}{l}\text { Nonlinearity-percentage deviation from the responsivity } \\
\text { at } 500 \mathrm{~W} / \mathrm{m}^{2} \text { caused by any change of irradiance within } \\
\text { the range from } 100 \mathrm{~W} / \mathrm{m}^{2} \text { to } 1,100 \mathrm{~W} / \mathrm{m}^{2}\end{array}$} & 0.2 & 0.5 \\
\hline \multicolumn{2}{|c|}{$\begin{array}{l}\text { Spectral sensitivity-percentage deviation of the product } \\
\text { of spectral absorptance and spectral transmittance from } \\
\text { the corresponding mean within the range from } 300 \mathrm{~nm} \\
\text { to } 3,000 \mathrm{~nm}\end{array}$} & 0.5 & 1.0 \\
\hline \multicolumn{2}{|c|}{$\begin{array}{l}\text { Tilt response-percentage deviation from the respon- } \\
\text { sivity at } 0 \text { degrees tilt (horizontal) caused by a change in } \\
\text { tilt from } 0 \text { degrees to } 90 \text { degrees at } 1,000 \mathrm{~W} / \mathrm{m}^{2}\end{array}$} & 0.2 & 0.5 \\
\hline \multicolumn{4}{|c|}{ Achievable uncertainty ( $95 \%$ confidence level): } \\
\hline 1 -min totals & $\begin{array}{l}\text { Percent } \\
\mathrm{kJ} / \mathrm{m}^{2} \\
\mathrm{Wh} / \mathrm{m}^{2}\end{array}$ & $\begin{array}{l}0.9 \\
0.56 \\
0.16\end{array}$ & $\begin{array}{l}1.8 \\
1 \\
0.28\end{array}$ \\
\hline $1-\mathrm{h}$ totals & $\begin{array}{l}\text { Percent } \\
\mathrm{kJ} / \mathrm{m}^{2} \\
\mathrm{Wh} / \mathrm{m}^{2}\end{array}$ & $\begin{array}{l}0.7 \\
21 \\
5.83\end{array}$ & $\begin{array}{l}1.5 \\
54 \\
15.0\end{array}$ \\
\hline Daily totals & $\begin{array}{l}\text { Percent } \\
\mathrm{kJ} / \mathrm{m}^{2} \\
\mathrm{Wh} / \mathrm{m}^{2}\end{array}$ & $\begin{array}{l}0.5 \\
200\end{array}$ & $\begin{array}{l}1 \\
400\end{array}$ \\
\hline
\end{tabular}

${ }^{a}$ High quality means "near state of the art"; good quality refers to instruments for network operation. 
Table 3-3. WMO Characteristics of Operational Pyranometers for Measuring GHI or DHI

\begin{tabular}{|c|c|c|c|}
\hline Characteristic & $\begin{array}{l}\text { High } \\
\text { Quality }\end{array}$ & $\begin{array}{l}\text { Good } \\
\text { Quality }\end{array}$ & $\begin{array}{c}\text { Moderate } \\
\text { Quality }\end{array}$ \\
\hline Response time- $95 \%$ response & $<15 \mathrm{~s}$ & $<30 \mathrm{~s}$ & $<60 \mathrm{~s}$ \\
\hline $\begin{array}{l}\text { Zero offset-Response to } 200 \mathrm{~W} / \mathrm{m} 2 \text { net thermal } \\
\text { radiation (ventilated) Response to } 5-\mathrm{K} / \mathrm{h} \text { change in } \\
\text { ambient temperature }\end{array}$ & $\begin{array}{l}7 \mathrm{~W} / \mathrm{m}^{2} \\
2 \mathrm{~W} / \mathrm{m}^{2}\end{array}$ & $\begin{array}{l}7 \mathrm{~W} / \mathrm{m}^{2} \\
2 \mathrm{~W} / \mathrm{m}^{2}\end{array}$ & $\begin{array}{l}7 \mathrm{~W} / \mathrm{m}^{2} \\
2 \mathrm{~W} / \mathrm{m}^{2}\end{array}$ \\
\hline Resolution—smallest detectable change & $1 \mathrm{~W} / \mathrm{m}^{2}$ & $5 \mathrm{~W} / \mathrm{m}^{2}$ & $10 \mathrm{~W} / \mathrm{m}^{2}$ \\
\hline Stability-change per year, percentage of full scale & 0.8 & 1.5 & 3.0 \\
\hline $\begin{array}{l}\text { Directional response for beam radiation-the range of } \\
\text { errors caused by assuming that the normal incidence } \\
\text { Rs is valid for all directions when measuring, from any } \\
\text { direction, a beam radiation that has a normal incidence } \\
\text { irradiance of } 1,000 \mathrm{~W} / \mathrm{m}^{2}\end{array}$ & $10 \mathrm{~W} / \mathrm{m}^{2}$ & $20 \mathrm{~W} / \mathrm{m}^{2}$ & $30 \mathrm{~W} / \mathrm{m}^{2}$ \\
\hline $\begin{array}{l}\text { Temperature response-percentage maximum error } \\
\text { caused by any change of ambient temperature within } \\
\text { an interval of } 50 \mathrm{~K}\end{array}$ & 2 & 4 & 8 \\
\hline $\begin{array}{l}\text { Nonlinearity-percentage deviation from the Rs at } 500 \\
\text { W/m }{ }^{2} \text { caused by any change of irradiance within the } \\
\text { range from } 100 \mathrm{~W} / \mathrm{m}^{2} \text { to } 1,000 \mathrm{~W} / \mathrm{m}^{2}\end{array}$ & 0.5 & 1 & 3 \\
\hline $\begin{array}{l}\text { Spectral sensitivity-percentage deviation of the } \\
\text { product of spectral absorptance and spectral } \\
\text { transmittance from the corresponding mean within the } \\
\text { range from } 300 \mathrm{~nm} \text { to } 3,000 \mathrm{~nm}\end{array}$ & 2 & 5 & 10 \\
\hline $\begin{array}{l}\text { Tilt response-percentage deviation from the Rs at } \\
0 \text { degree tilt (horizontal) caused by a change in tilt from } \\
0 \text { degree to } 90 \text { degrees at } 1,000 \mathrm{~W} / \mathrm{m} 2\end{array}$ & 0.5 & 2 & 5 \\
\hline $\begin{array}{l}\text { Achievable uncertainty-95\% confidence level } \\
\text { Hourly totals } \\
\text { Daily totals }\end{array}$ & $\begin{array}{l}3 \% \\
2 \%\end{array}$ & $\begin{array}{l}8 \% \\
5 \%\end{array}$ & $\begin{array}{l}20 \% \\
10 \%\end{array}$ \\
\hline
\end{tabular}

Even among the instrument classifications and specifications, there can be some measurement uncertainty variations. The user should research various instrument models to gain familiarity with the design and measurement characteristics in view of a particular application (Myers and Wilcox 2009; Wilcox and Myers 2008; Habte, Wilcox, and Stoffel 2014). 
Table 3-4. ISO 9060 Specifications Summary for Pyrheliometers Used To Measure DNI

\begin{tabular}{|c|c|c|c|}
\hline \multicolumn{4}{|c|}{ Pyrheliometer Specification List } \\
\hline \multirow[b]{2}{*}{ Specification } & \multicolumn{3}{|c|}{ Class of Pyrheliometer } \\
\hline & $\begin{array}{c}\text { Secondary } \\
\text { Standard Class }\end{array}$ & First Class & Second \\
\hline Response time-95\% response & $<15 \mathrm{~s}$ & $<20 s$ & $<30 s$ \\
\hline $\begin{array}{l}\text { Zero offset } \\
\quad \text { Response to } 5-\mathrm{K} \mathrm{h}-1 \text { change in } \\
\text { ambient temperature }\end{array}$ & $\pm 1 \mathrm{Wm}^{-2}$ & $\pm 3 \mathrm{Wm}^{-2}$ & $\pm 6 \mathrm{Wm}^{-2}$ \\
\hline $\begin{array}{l}\text { Resolution-smallest detectable } \\
\text { change in } \mathrm{Wm}^{-2}\end{array}$ & $\pm 0.5 \mathrm{Wm}^{-2}$ & $\pm 1 \mathrm{Wm}^{-2}$ & $\pm 5 \mathrm{Wm}^{-2}$ \\
\hline $\begin{array}{l}\text { Stability-percentage of full scale, } \\
\text { change/year }\end{array}$ & $\pm 0.5 \%$ & $\pm 1 \%$ & $\pm 2 \%$ \\
\hline $\begin{array}{l}\text { Nonlinearity-percentage deviation } \\
\text { from the responsivity at } 500 \mathrm{~W} / \mathrm{m}^{2} \\
\text { because of change in irradiance } \\
\text { between } 100 \mathrm{Wm}-2 \text { and } 1,000 \mathrm{Wm}^{-2}\end{array}$ & $\pm 0.2 \%$ & $\pm 0.5 \%$ & $\pm 2 \%$ \\
\hline $\begin{array}{l}\text { Spectral selectivity-percentage } \\
\text { deviation of the product of the spectral } \\
\text { absorptance and the spectral } \\
\text { transmittance from the corresponding } \\
\text { mean between } 0.35 \mu \mathrm{m} \text { and } 1.5 \mu \mathrm{m}\end{array}$ & $\pm 0.5 \%$ & $\pm 1 \%$ & $\pm 5 \%$ \\
\hline $\begin{array}{l}\text { Temperature response-total } \\
\text { percentage deviation because of } \\
\text { change in ambient temperature within } \\
\text { an interval of } 50 \mathrm{~K}\end{array}$ & $\pm 1 \%$ & $\pm 2 \%$ & $\pm 10 \%$ \\
\hline $\begin{array}{l}\text { Tilt response-percentage deviation } \\
\text { from the responsitivity at } 0 \text { degrees tilt } \\
\text { (horizontal) because of change in tilt } \\
\text { from } 0 \text { degrees to } 90 \text { degrees at } 1,000 \\
W / \mathrm{m}^{-2} \text { irradiance }\end{array}$ & $\pm 2 \%$ & $\pm 0.5 \%$ & $\pm 2 \%$ \\
\hline $\begin{array}{l}\text { Traceability-maintained by periodic } \\
\text { comparison }\end{array}$ & $\begin{array}{l}\text { With a primary } \\
\text { standard } \\
\text { pyrheliometer }\end{array}$ & $\begin{array}{c}\text { With a } \\
\text { secondary } \\
\text { standard } \\
\text { pyrheliometer }\end{array}$ & $\begin{array}{l}\text { With a first- } \\
\text { class } \\
\text { pyrheliometer } \\
\text { or better }\end{array}$ \\
\hline
\end{tabular}


Table 3-5. ISO 9060 Specifications Summary for Pyranometers Used To Measure GHI and DHI

\begin{tabular}{|c|c|c|c|}
\hline \multicolumn{4}{|c|}{ Pyrheliometer Specification List } \\
\hline \multirow[b]{2}{*}{ Specification } & \multicolumn{3}{|c|}{ Class of Pyrheliometer ${ }^{a}$} \\
\hline & $\begin{array}{c}\text { Secondary } \\
\text { Standard Class }\end{array}$ & First Class & Second \\
\hline Response time-95\% response & $<15 \mathrm{~s}$ & $<30 \mathrm{~s}$ & $<60 \mathrm{~s}$ \\
\hline $\begin{array}{l}\text { Zero offset } \\
\quad \text { Response to } 200 \mathrm{Wm}^{-2} \text { net thermal } \\
\text { radiation (ventilated) }\end{array}$ & $\pm 7 \mathrm{Wm}^{-2}$ & $\pm 15 \mathrm{Wm}^{-2}$ & $\pm 30 \mathrm{Wm}^{-2}$ \\
\hline $\begin{array}{l}\text { Response to } 5-\mathrm{Kh}^{-1} \text { change in } \\
\text { ambient temperature }\end{array}$ & $\pm 2 \mathrm{Wm}^{-2}$ & $\pm 4 \mathrm{Wm}^{-2}$ & $\pm 8 \mathrm{Wm}^{-2}$ \\
\hline $\begin{array}{l}\text { Resolution-smallest detectable } \\
\text { change }\end{array}$ & $\pm 0.5 \%$ & $\pm 1 \%$ & $\pm 3 \%$ \\
\hline $\begin{array}{l}\text { Stability-percentage change in } \\
\text { responsivity per year }\end{array}$ & & & \\
\hline $\begin{array}{l}\text { Nonlinearity-percentage deviation } \\
\text { from the responsivity at } 500 \mathrm{~W} / \mathrm{m}^{2} \\
\text { because of change in irradiance } \\
\text { between } 100 \mathrm{Wm}-2 \text { and } 1,000 \mathrm{Wm}^{-2}\end{array}$ & $\pm 10 \mathrm{Wm}^{-2}$ & $\pm 20 \mathrm{Wm}^{-2}$ & $\pm 30 \mathrm{Wm}^{-2}$ \\
\hline $\begin{array}{l}\text { Directional response for beam radiation } \\
\text { (the range of errors caused by assuming } \\
\text { that the normal incidence responsivity is } \\
\text { valid for all directions when measuring, } \\
\text { from any direction, a beam radiation that } \\
\text { has a normal incidence irradiance of } \\
1,000 \mathrm{Wm}^{-2}\end{array}$ & $\pm 3 \%$ & $\pm 5 \%$ & $\pm 10 \%$ \\
\hline $\begin{array}{l}\text { Spectral selectivity-percentage deviation } \\
\text { of the product of the spectral absorptance } \\
\text { and the spectral transmittance from the } \\
\text { corresponding mean between } 0.35 \mu \mathrm{m} \\
\text { and } 1.5 \mu \mathrm{m}\end{array}$ & $2 \%$ & $4 \%$ & $8 \%$ \\
\hline $\begin{array}{l}\text { Temperature response-total percentage } \\
\text { deviation because of change in ambient } \\
\text { temperature within an interval of } 50 \mathrm{~K}\end{array}$ & $\pm 0.5 \%$ & $\pm 2 \%$ & $\pm 5 \%$ \\
\hline $\begin{array}{l}\text { Tilt response-percentage deviation } \\
\text { from the responsitivity at } 0 \text { degrees tilt } \\
\text { (horizontal) because of change in tilt } \\
\text { from } 0 \text { degrees to } 90 \text { degrees at } 1,000 \\
W / \mathrm{m}^{-2} \text { irradiance }\end{array}$ & & & \\
\hline
\end{tabular}

${ }^{a}$ The highest category for pyranometers is the secondary standard, because the most accurate determination of GHI has been suggested to be the sum of the DNI as measured by an absolute cavity radiometer and the DHI as measured by a secondary standard pyranometer shaded from the DNI by a disk. 


\subsubsection{Correction Functions for Systematic Errors of ISO First Class and Secondary Standard Instruments}

Some of the errors of pyrheliometers and pyranometers described in the previous section are systematical and can be reduced. An example of a systematic reduction is the correction of the solar zenith/cosine response. For example, instead of using only one calibration constant for a pyrheliometer, NREL determines calibration constants for different intervals of SZA. Similar correction curves for pyranometers are also measured and provided by some manufacturers.

Also, especially if the sensor temperature of pyranometers and pyrheliometers is measured using a temperature-dependent resistor close to the thermopile, a temperature correction can be applied too. Correction coefficients are supplied by the manufacturer.

Measurements from only black pyranometers can be corrected for the thermal offset using additional measurements from pyrgeometers. Alternatively, a less accurate correction can be made based on estimations of the thermal offset from the often negative measurements collected during the night (Dutton et al. 2000).

\subsubsection{RSIs}

RSIs use a pyranometer that is periodically shaded by a motorized shadowband that moves across the detector's FOV (Figure 3-12). The principle of operation of RSIs is to measure GHI when unshaded and DHI when shaded. Then DNI is calculated using the following equation relating GHI, DHI, and DNI:

$$
\mathrm{DNI}=(\mathrm{GHI}-\mathrm{DHI}) \operatorname{Cos}(\mathrm{SZA})
$$

RSIs are often called RSRs (rotating shadowband radiometers) or RSPs (rotating shadowband pyranometer), depending on the instrument manufacturer. The notation RSI refers to all such instruments measuring irradiance by use of a rotating shadowband. There are two types of RSIs: RSIs with continuous rotation and RSIs with discontinuous rotation. 

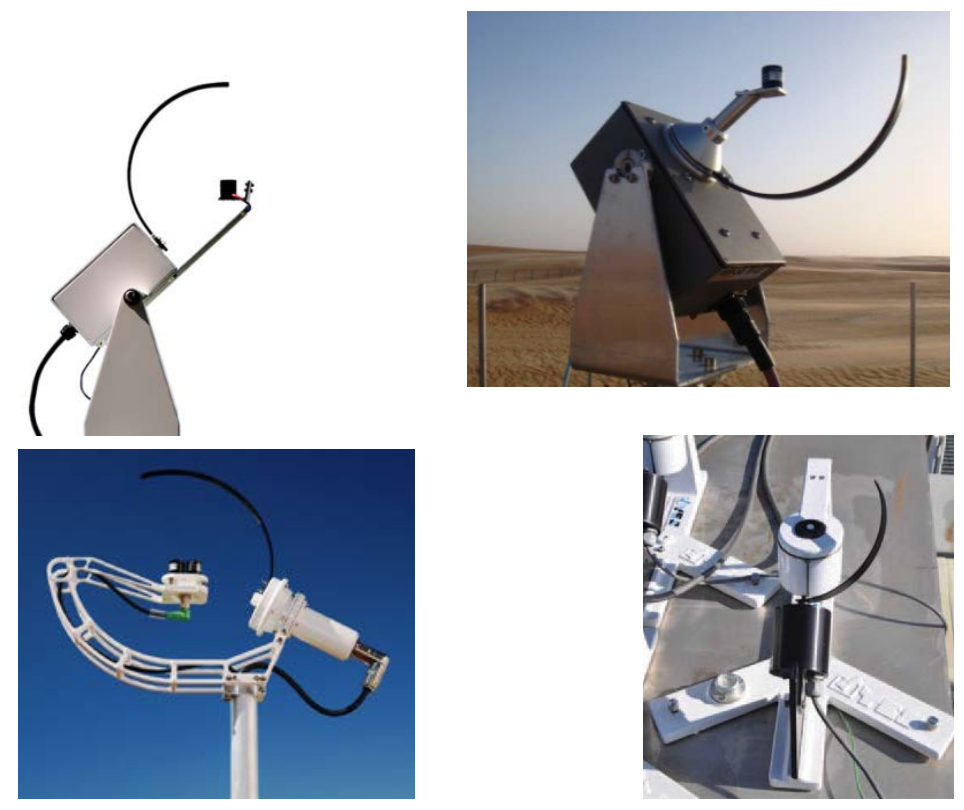

Figure 3-12. Four commercially available RSIs: (clockwise from upper left) An Irradiance, Inc., model RSR2; a Reichert GmbH RSP 4G (previously used by SM-AG); a Yankee Environmental Systems, Inc., model SDR-1; and a CSP-Services GmbH Twin-RSI. Photos by (clockwise from top) Irradiance, Inc.; Reichert GmbH RSP 4G; NREL; and CSP-Services

The operational principal of RSIs with continuous rotation is shown in Figure 3-13. At the beginning of the rotation, the shadowband is below the pyranometer, in its rest position. The rotation is performed with constant angular velocity and takes approximately 1 second. During the rotation, the irradiance is measured with a high and constant sampling rate (approximately 1 $\mathrm{kHz}$ ). This measurement is called burst or sweep. At the beginning of the rotation, the pyranometer measures GHI. In the moment when the center of the shadow falls on the center of the sensor, it approximately detects DHI; however, the shadowband covers some portion of the sky so that the minimum of the burst is less than the DHI. Thus, so-called shoulder values are determined by curve analysis algorithms.

Such algorithms are usually implemented in the data logger program and use the maximum of the absolute value of the burst's slope to find the position of the "shoulder values." The difference between the GHI and the average of the shoulder values is added to the minimum of the curve to obtain the DHI. Subsequently, DNI is calculated by the data logger using GHI, DHI, and the actual sun height angle calculated by the known time and coordinates of the location, as stated above. All of the RSIs shown in Figure 3-12 except for the SDR-1 work with a continuous rotation. 


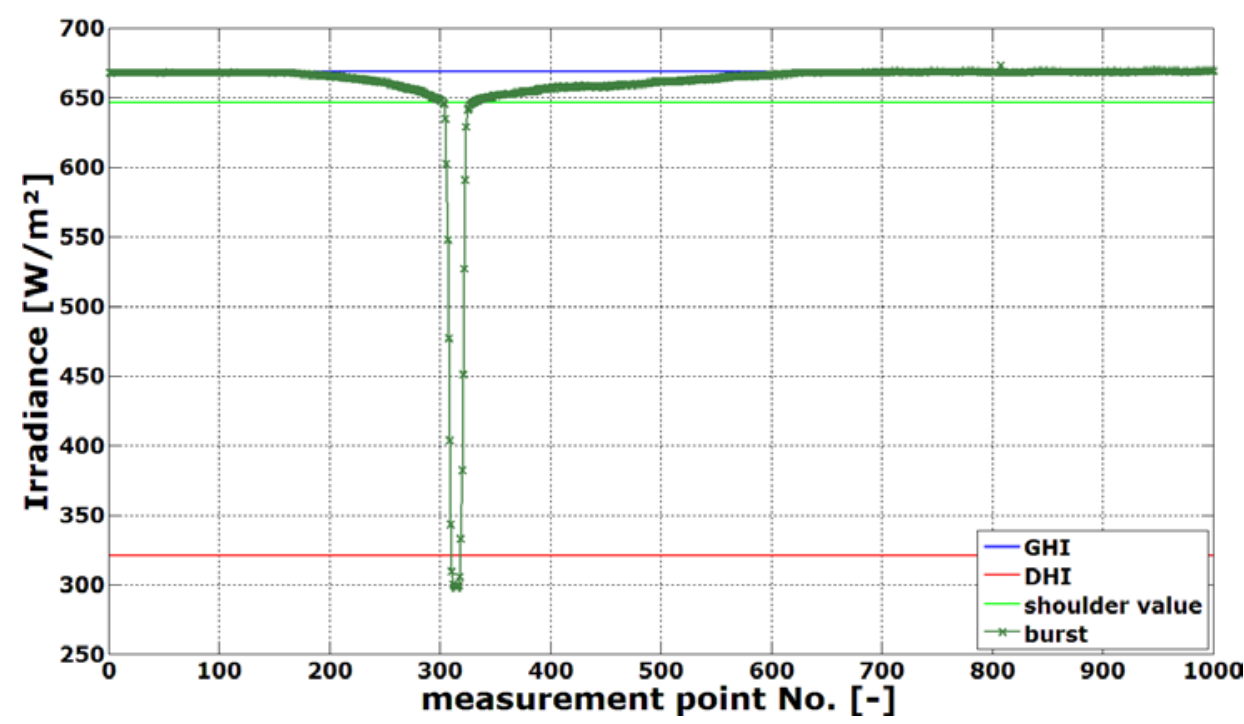

Figure 3-13. Burst (sweep) with sensor signal and the derived GHI, shoulder values, and the DHI. Image from Wilbert (2014)

RSIs with discontinuous rotation do not measure the complete burst, but only four points of it. First, the GHI is measured while the shadowband is in the rest position. Then the shadowband rotates from the rest position toward the position at which it nearly shades the pyranometer, stops, and a measurement is taken (e.g., during 1 second for the SDR-1 in Figure 3-12). Then it continues the rotation toward the position at which the shadow lies centered on the pyranometer, and another measurement is taken. The last point is measured in a position at which the shadow just passed the pyranometer. The measurement with the completely shaded sensor is used equivalently to the minimum of the burst, as shown in Figure 3-13. The two measurements for which the shadow is close to the sensor are used equivalently to the shoulder values. The shoulder values are used to correct for the portion of the sky blocked by the shading band.

The two types of RSIs have advantages and disadvantages. An RSI with continuous rotation of the shadowband needs a pyranometer with a fast response time $(<1$ second, e.g., approximately $10 \mu \mathrm{s}$ ); thus, thermal sensors as described in ISO 9060 cannot be used. Instead, semiconductor sensors are used - for example, the Silicon (Si)-pyranometer LI-200SA shown in Figure 3-11. Because of the nonhomogeneous spectral response of such Si pyranometers (see Figure 3-2), the measurement accuracy of secondary standard thermal pyranometers cannot be reached and correction functions for this systematic error have to be applied to reach the required accuracy for the resource assessment. These functions will be discussed below.

RSIs with discontinuous rotation can use sufficiently long measurement times for each of the four points to allow the application of thermal pyranometers (e.g., the Yankee TSR-1 thermopile shadowband radiometer); thus, the spectral error of a Si pyranometer detector can be avoided. However, the discontinuous rotation is connected to other disadvantages compared to the continuous rotation. Although RSIs with continuous rotation are not affected by small azimuth alignment errors ( \pm approximately 5 degrees), the azimuth alignment of RSIs with discontinuous rotation is crucial for their accuracy. Also, the accuracy of the sensor's coordinates and the time is more important for the discontinuous rotation. If the shadowband stops in the wrong position, 
the DHI measurement fails. Further, the duration of the measurement with a discontinuous rotation increases the measurement uncertainty. This is especially relevant if thermal sensors are used and if the sky conditions are not stable (e.g., cloud passages). If the GHI and the sky radiance distribution change during the measurement of the four points, the data used to determinme the DHI will deviate noticeably from the true value. This complication is not very relevant for the continuously rotating RSIs, because the rotation takes approximately only 1 second.

Both RSIs use radiometers with diffusers that have varying spectral transmittance over different spectral regions. The calibration of the radiometers themselves are usually performed either throughout a wide range of clear to cloudy conditions (and averaged) or selected clear conditions. In both cases, not all the relative variations throughout all spectral regions can be accounted for in the radiometer calibrations. This leads to additional uncertainties related to the spectral transmittance of the diffusers and varying spectral conditions in the field.

DHI is typically determined one or four times a minute, but GHI measurements can be sampled in a higher frequency without the rotation of the shadowband-for example, every second. The variation of the GHI also contains some information about the change in DNI. Different algorithms are used to determine the average of the DHI and DNI between two DHI measurements using the more frequent GHI measurement.

The initially lower accuracy of RSIs compared to ISO 9060 first class pyrheliometers and secondary standard pyranometers is often compensated by some advantages of RSIs. Because of their low soiling susceptibility (Pape et al. 2009, Geuder and Quaschning 2006, Maxwell et al. 1999), low power demand, and comparatively lower cost (instrumentation and O\&M), RSIs show significant advantages compared to thermal sensors when operated under the measurement conditions of remote weather stations.

Without the application of corrections of the systematic deviations and a matched calibration method, RSIs yield only an uncertainty of $5 \%$ to $10 \%$ and more. This accuracy is notably improved with proper calibration of the sensors and the correction functions, which are described in the following. Most instrument providers also offer post-processing software or services that include these correction functions. Users should inquire of the manufacturer about whether such post-processing is part of the instrument package and is readily available.

Because of the stated disadvantages of RSIs with discontinuous rotation and the higher relevance of RSIs with continuous rotation for solar energy applications, we focus on RSIs with $\mathrm{Si}$ pyranometers in the following. More information about RSIs with discontinuous rotation can be found in Harrison, Michalsky, and Berndt (1994).

\subsubsection{Correction Functions and Calibration Procedures for RSIs}

The main systematic errors of RSIs with Si sensors are caused by the spectral response of the $\mathrm{Si}$ pyranometers, their cosine response, and the temperature dependence.

Several research groups have developed correction functions that reduce the systematic errors of RSIs. In all cases, the Si pyranometer of the RSI is a LICOR LI-200SA. Whereas temperature 
correction is similar in all versions (King and Myers 1997; Geuder, Pulvermüller, and Vorbrügg 2008), the methods for the spectral and cosine corrections vary among the publications.

Alados, Batlles, and Olmo (1995) use tabular factors for different sky clearness and skylight brightness parameters and a functional correction depending on the incidence angle. King and Myers (1997) propose functional corrections in dependence on AM and the angle of incidence derived primarily for GHI. This approach was further developed by Augustyn et al. (2002) and Vignola (2006), including also diffuse and subsequently direct beam irradiance. The combination of the GHI correction presented in Augustyn et al. (2002) combined with the diffuse correction from Vignola (2006) provides a complete set of corrections for LICOR LI-200SA-based RSIs. Independently, a method for DNI, GHI, and DHI correction was developed by the DLR using functional corrections, including a particular spectral parameter composed from GHI, DHI, and DNI (Geuder, Pulvermüller, and Vorbrügg 2008). Additional corrections in dependence on AM and incidence angle were used. Another set of correction functions was developed by CSPServices and is presented in Geuder et al. (2011).

In addition to the corrections above, special calibration techniques are required for RSIs. At the time of this writing, RSIs with continuous rotation are equipped with LI-COR LI-200SA silicon pyranometers. They usually come precalibrated by the manufacturer LI-COR for global irradiance by outdoor comparisons with an Eppley pyranometer (PSP) with an accuracy of $<5 \%$ (LI-COR Biosciences 2005). Thus, an additional calibration (e.g., on-site or with respect to DHI, DNI, or GHI independently) of the RSIs can noticeably improve the accuracy.

Because of the rather narrow and inhomogeneous spectral response of the photodiodes and the combined measurement of DHI and GHI, only some aspects of the existing ISO standards for pyrheliometer and pyranometer calibration can be transferred to RSI calibration. The calibration methods described in ISO 9846 (ISO 1993) and ISO 9847 (ISO 1992) for pyranometers and in ISO 9059 (ISO 1990) for pyrheliometers are based on simultaneous solar irradiance measurements with test and reference instruments recorded with selected instrumentation. For calibrations using a reference pyrheliometer (ISO 1990, ISO 1993), measurements are taken under specified meteorological conditions. Following the recommendations of the standards, measurements should be taken near solar noon and when DNI is greater than $700 \mathrm{~W} / \mathrm{m}^{2}$. The angular distance of clouds from the sun has to be greater than 15 degrees for pyrheliometer calibration and greater than 45 degrees for pyranometers. Also, cloud cover should be less than $1 / 8$, the cloud movement has to be considered for the calibration, and Linke turbidities should be less than 6. The Linke turbidity is a measure of the attenuation of the cloudless atmosphere. The Linke turbidity coefficient represents the number of clean and dry atmospheres that result in the same attenuation as the real cloudless atmosphere. One method to derive the Linke turbitiy is presented in Ineichen and Perez (2002). For pyranometer calibrations using a reference pyranometer (ISO 1992), the sky conditions are less defined. The calibration interval for the latter case is adjusted depending on the sky conditions.

Calibrating RSI instruments involves calibrating for DNI, DHI and GHI, each of which possess distinctly different spectral power distributions. Because of the spectral response of the silicon detectors and/or diffusers, it is problematic to calibrate based on only a few series of measurements and under the special conditions defined in ISO 9847 and ISO 9059. This is possible only for thermal sensors because of their homogenous spectral response covering at 
least $300 \mathrm{~nm}$ to $3 \mu \mathrm{m}$ (> 99\% of the ASTM G173 airmass 1.5-DNI spectrum). A similar calibration for RSIs would need the additional assumption that all RSIs from one manufacturer have exactly the same known spectral and cosine response. Preferably, a wide variety of meteorological conditions have to be included in the calibration period and then the conditions should be characterized wisely during the calibration process. The accuracy of the calibration generally improves when the conditions during the calibration represent the conditions at the site where the RSI later is operated. In addition to the cloud cover, the influences of aerosols, water vapor, and site altitude on the solar spectrum have to be considered (Myers 2011). Calibrations with artificial radiation sources that lack the spectral power distributions of natural solar radiation components usually lack the variety of natural irradiation conditions; therefore, field calibrations under natural irradiation conditions are preferred.

For example, RSI calibrations are performed at NREL in Golden, Colorado, or by DLR on the Plataforma Solar de Almería in Spain. There, RSIs are operated parallel to ISO 9060 first class pyrheliometers and secondary standard pyranometers under real-sky conditions (see Figure 314). The duration of this calibration is from several hours until more than one year; thus, it provides a data base for the analysis of systematic signal deviations and measurement accuracy. An analysis of the dependence of the calibration constants on the duration of the calibration period for one RSI is presented in Geuder, Affolter, and Kraas (2012)). Data quality is analyzed and compared to the reference irradiances. RSI calibrations are performed according to the different methods. All published calibration techniques are based on the comparison of corrected RSI signals (using the existing correction functions described above) to irradiance measurements with thermal sensors.

Depending on the calibration method, one, two, or even three calibration constants are defined. The motivation for determining one calibration constant is that only one pyranometer is used and that the calibration based on GHI is less time consuming than a calibration for GHI, DHI, and DNI. Becaues of the pyranometer's spectral response, sensitivity for DHI, GHI, and DNI is not the same; hence, the application of two or three calibration constants is physically reasonable even though only one sensor is used. Examples for the drift of GHI calibration constants from Geuder, Pulvermüller, and Vorbrügg (2008) were investigated for nine sensors in Geuder et al. (2010). For recalibration periods between 2 years and $33 / 4$ years, changes of this calibration constant were below $1 \%$ in most cases. Recalibration is recommended at least every two years. An overview of RSI calibration methods is presented in Geuder et al. (2011), and more details can be found in Geuder, Pulvermüller, and Vorbrügg (2008), Kern (2010), and Geuder et al. (2010). A case study for the reached accuracy for different combinations of correction functions and calibration methods is summarized in the next paragraph. 


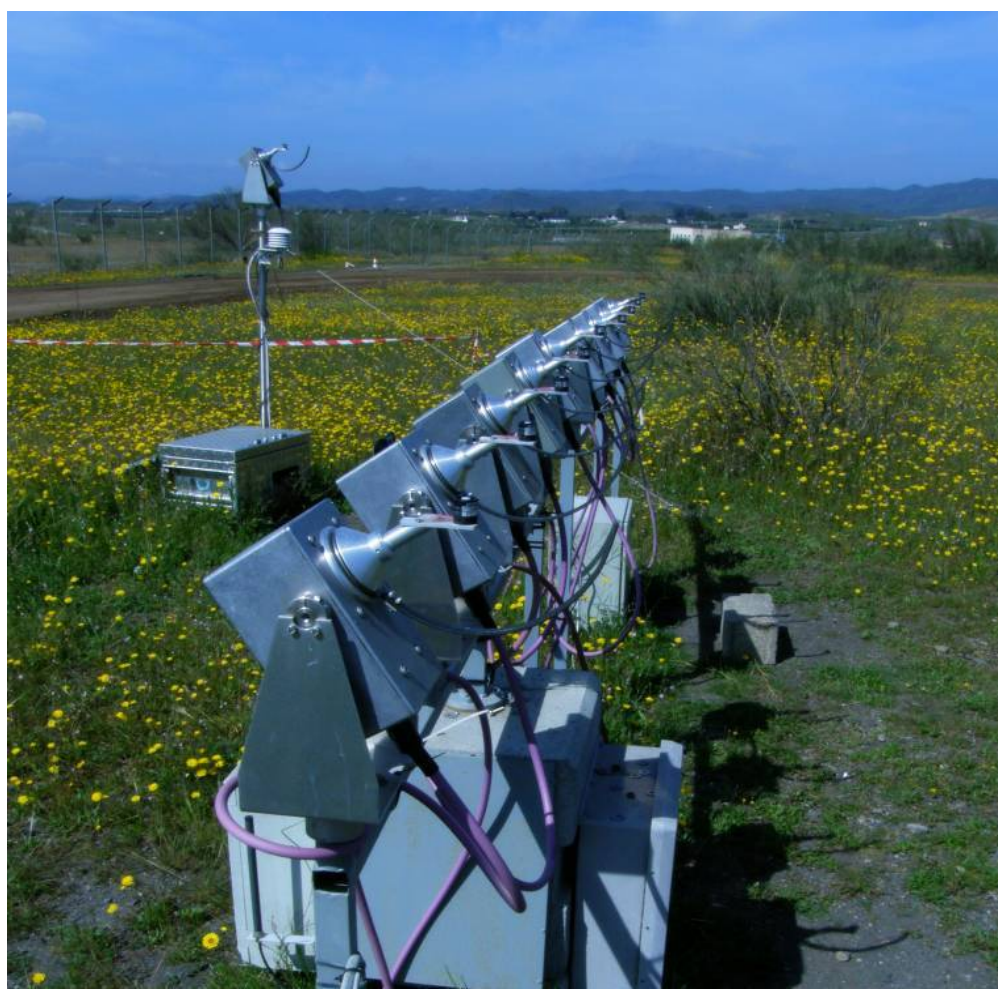

Figure 3-14. RSI calibration station at Plataforma Solar de Almería. Photo by Stefan Wilbert, DLR

\subsubsection{Accuracy of RS/s}

The accuracy of RSIs was investigated empirically in various case studies described below. In addition, the uncertainty of Si-based RSI sensors is also presented in this chapter in the section on "Estimating the Uncertainty of DNI Field Measurements."

As stated above, the accuracy of RSIs strongly depends on the correction functions used and the calibration. A comparative evaluation of different existing correction and calibration procedures for 39 RSIs is presented in Geuder et al. (2011). The largest part of the data set used in this case study has been collected at Plataforma Solar de Almería, but data from four additional sites in different climate zones and at different altitudes was also used. Geuder et al. (2010) present a case study with a focus on the site dependence of RSI performance. Including the analysis of uncorrected raw data (using LI-COR calibrations), five cases were compared: (1) the raw uncorrected data; (2) an algorithm from Geuder et al. (2011) called CSPS2011; (3) an algorithm from Geuder, Pulvermüller, and Vorbrügg (2008) with the notation DLR2008; (4) the GHI correction algorithm from Augustyn et al. (2002) combined with the diffuse correction from Vignola (2006) with one single calibration constant for GHI, DHI, and DNI, denoted US-1; and, finally, (5) the same as (4) but with three separate calibration constants for GHI, DNI, and DHI, called US-3.

The root mean square (RMS) deviations for the five analyzed cases is plotted in Figure 3-15. It was concluded that corrections US-3, DLR2008, and CSPS2011 yield noticeably better accuracies compared to raw data and US-1. The analysis also investigated the accuracy for the annual sums of GHI, DNI, and DHI. It was shown that the average of the absolute annual 
deviations for the 39 RSIs for DNI was less than 1\% for CSPS2011, DLR2008, and US-3. For the post-processing techniques CSPS2011 and DLR2008, this average was even below $0.5 \%$.

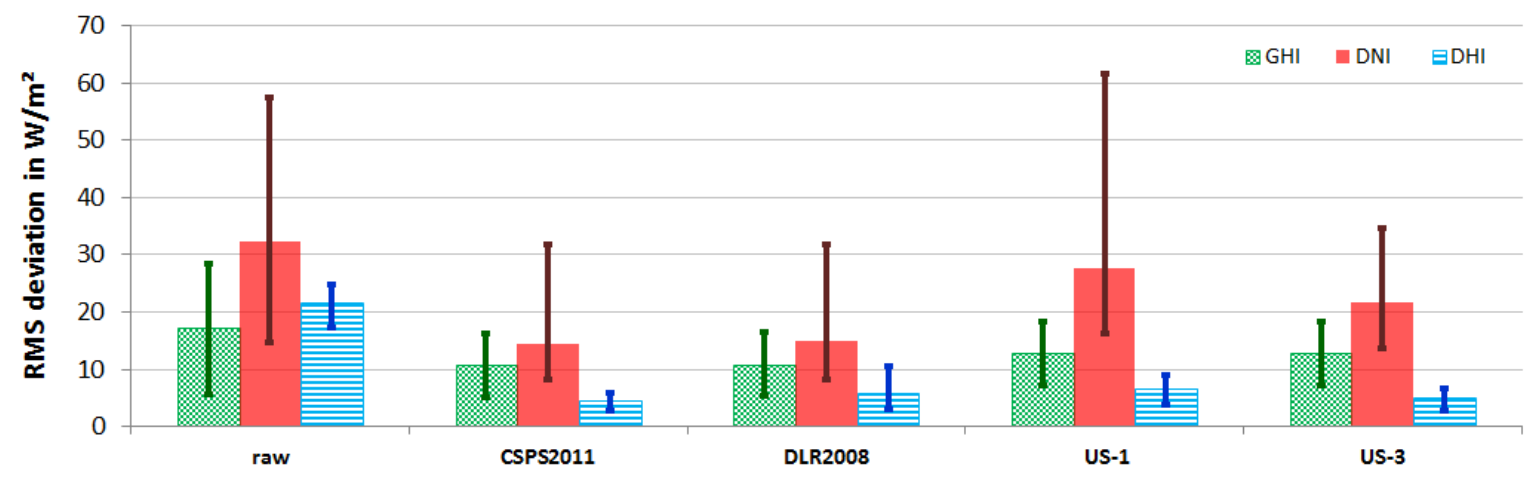

Figure 3-15. Maximum, mean, and minimum RMS deviations of irradiance values with a time resolution of 10 minutes from 39 RSIs compared to thermopile sensors. The error bars show the maximum and the minimum deviation of the 39 data sets. Data sets for uncorrected raw values as well as for four different corrections were analyzed. Image from Geuder et al. (2011)

An empirical estimation of the difference of subhourly DNI measurements after the application of correction functions and an RSI specific calibration is approximately 3\%. This fits with the results presented in Augustyn et al. (2002)) and Geuder, Pulvermüller, and Vorbrügg (2008)), and also to the uncertainty analysis presented below in the section on "Estimating the Uncertainty of DNI Field Measurements.” In other measurement campaigns, a comparable accuracy of RSI measurements was stated for annual scale as reached with properly maintained high-precision instruments such as pyrheliometers (Geuder et al. 2010). However, remaining aspects concerning the spectral corrections are stated in Geuder et al. (2010) and Myers (2011), so further improvements of the corrections and calibration techniques are being investigated.

\subsubsection{Other Instruments That Can Be Used to Derive DHI and DNI}

In addition to the already described instruments, other instruments might be used to derive DHI or DNI. For example, the Scanning Pyrheliometer/Pyranometer (SCAPP) (Bergholter and Dehne 1994) or the sunshine duration sensor Soni e3 (Lindner 1984) can also be used to derive DNI; however, these two sensors reach only lower accuracies compared to tracked pyrheliometers, thermal pyranometers with shading balls or disks, and RSIs, as documented in Geuder et al. (2006). It should be mentioned here that older analog sunshine recorders, such as the CampbellStokes sunshine recorder, do not deliver DNI and GHI. Sunshine duration might be used only in models to derive irradiation data if rough estimates are required (which is not the case for solar power plant projects). The Campbell-Stokes sunshine recorder focuses the direct beam by a simple spherical lens (glass ball) to create burn marks during clear periods (when DNI exceeds $120 \mathrm{Wm}^{-2}$ ). Campbell-Stokes sunshine recorders have been used for more than a century to measure solar radiation around the world (Iqbal 1983; Vignola, Michalsky, and Stoffel 2012), but such analog sunshine recorders are rather of historic importance.

An option to measure DHI and GHI with one instrument is the SPN1. The SPN1 is shown in Figure 3-16. Using the solar elevation angle and the GHI and DHI measurement, the DNI can be calculated. 


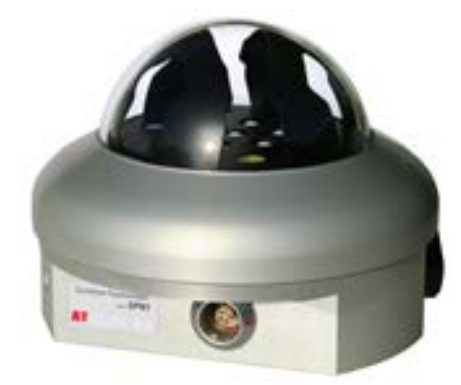

Figure 3-16. Delta-T Devices, Ltd., SPN1

The instrument consists of an array of seven thermopile radiation detectors that are distributed under a glass dome in a hexagonal pattern. The detectors are positioned under diffuser disks and a special shadow mask. The shape of the mask is selected such that for any position of the sun in the sky there will always be one or more detectors that are fully shaded from the sun and exposed to approximately half of the diffuse radiation (for completely overcast skies). Also, one or more detectors are exposed to the full solar beam for all positions. The minimum and the maximum readings of the seven detectors are used to derive GHI and DHI.

With this principle of operation, GHI, DHI, and DNI can be derived without any moving parts. Further, the SPN1's low power demand increases its suitability for operation in remote sites compared to DNI or DHI measurements involving solar trackers. First results about the instrument's accuracy indicate that the SPN1's DNI has higher errors compared to the DNI measured with RSIs (Vuilleumier et al. 2013). Further results about the SPN1 at six different locations worldwide can be found in a discussion paper by Badosa et al. (2014). The sensitivity of the SPN1 to sensor soiling is currently being investigated as well as the possibility of improving its accuracy with more sophisticated corrections.

\subsection{Measurement Uncertainty}

Every measurement only approximates the quantity being measured, and it is incomplete without a quantitative statement of uncertainty. Each element of a measurement system contributes to the final uncertainty of the data. Accurate measurements of solar irradiance depend on the radiometer design, hardware installation scheme, data acquisition method, measurement system O\&M, calibration method and frequency, and possible real-time or a posteriori corrections to the data. A successful measurement uncertainty analysis produces no properly measured data that exceed the expected range of uncertainty.

This overview of measurement uncertainty is based on Myers et al. (2002), Reda et al. (2011), Stoffel et al. (2000), and Wilcox and Myers (2008).

\subsubsection{Terminology}

Historically, uncertainty analysis treated sources of uncertainty in terms of random and bias error types. Random sources were related to the standard deviation or variance of measured data sets. Biases were estimates of deviations from a "true value" primarily based on engineering judgments of the measurement system performance. Total uncertainty (UT) was computed as the square root of the sum of the squares for these two error types: 


$$
\mathrm{UT}=\left[\Sigma(\text { Bias })^{2}+\Sigma(2 \cdot \text { Random })^{2}\right]^{1 / 2}
$$

where the factor of 2 in the random term was necessary to "inflate" the random component to provide an approximate $95 \%$ confidence interval for the computed value of UT, assuming the data were normally distributed (i.e., random).

The GUM is currently the accepted guide for measurement uncertainty (ISO 2008). GUM defines Type A uncertainty values as derived from statistical methods and Type B sources as evaluated by other means, such as scientific judgment, experience, specifications, comparisons, and calibration data. GUM defines the concept of a standard uncertainty (Ustd) for each uncertainty type, which is an estimate of an equivalent standard deviation (of a specified distribution) of the source of uncertainty. The combined uncertainty $\left(\mathrm{U}_{\mathrm{C}}\right)$ is computed from the Type A and Type B standard uncertainties summed under quadrature. The GUM replaces the historical factor of 2 with a coverage factor, $\mathrm{k}$ (which depends on the known or assumed statistical distribution of uncertainties) and computes the expanded uncertainty $\left(\mathrm{U}_{\mathrm{E}}\right)$ as:

$$
\mathrm{U}_{\mathrm{E}}=\mathrm{k} \cdot \mathrm{U}_{\mathrm{C}}=\mathrm{k} \cdot\left[\Sigma(\text { Type } \mathrm{B})^{2}+\Sigma(\text { Type } \mathrm{A})^{2}\right]^{1 / 2}
$$

For small samples $(\mathrm{n}<20)$ from a normal distribution, $\mathrm{k}$ may be selected from Student's $\mathrm{t}$ distribution. The coverage factor $(\mathrm{k})$ is usually in the range of 2 to 3 for confidence intervals of $95 \%$ and $99 \%$, respectively (Taylor and Kuyatt 1987 ). For a 95\% confidence interval, $U_{\mathrm{E}}$ is twice the value of $U_{C}$.

When a result, $R$, is functionally dependent on several variables, $x_{i}$, where $i=1$ to $n$, the propagation of error is used:

$$
\mathrm{U}_{\mathrm{R}}=\left[\sum_{\mathrm{i}}\left(\partial_{\mathrm{Xi}} \mathrm{R} \cdot \mathrm{e}_{\mathrm{i}}\right)^{2}\right]^{1 / 2}
$$

where

- $\mathrm{U}_{\mathrm{R}}=$ uncertainty in the resultant

- $\mathrm{e}_{\mathrm{i}}=$ estimated uncertainty in variable $\mathrm{X}_{\mathrm{i}}$

- $\mathrm{X}_{\mathrm{i}}=$ independent variable

- $\partial_{\mathrm{Xi}} \mathrm{R}=$ the partial derivative of the response $\mathrm{R}$ with respect to $\mathrm{X}_{\mathrm{i}}$ (sensitivity function for variable $\mathrm{X}_{\mathrm{i}}$ ).

The GUM procedure can be summarized in four steps (Reda 2011):

1. Determine the process measurement equation.

2. List or estimate the standard uncertainty for each variable in the measurement equation and for each component (curve-fitting uncertainty, environmental conditions uncertainty, etc.) that might introduce uncertainty to the measurement process.

3. Calculate the combined standard uncertainty using the root-sum-of-squares method of all standard uncertainties in Step 2 and the sensitivity coefficients obtained through partial derivative of the variables in the measurement equation. 
4. Calculate the expanded uncertainty by multiplying the combined standard uncertainty by the coverage factor, typically by applying Student's t-analysis to determine the appropriate coverage factor (typically 2 for $95 \%$ and 3 for $98 \%$ confidence, respectively, for large data sets).

\subsubsection{Estimating DNI Measurement Uncertainty}

Two measurement processes are applicable to DNI measurement uncertainty analysis:

- Pyrheliometer calibration

- Field measurements.

Uncertainty in measurements begins with the uncertainty in calibration references, calibration processes, and sensor design characteristics. For example, for thermopile sensors, a calibration constant is required to convert the output voltage to the required irradiance (Reda et al. 2008). These calibration constants are not temporally stable, as shown for the time series plot of calibration responsivities of two pyrheliometers in Figure3-17. The resulting uncertainty in calibration factors must then be combined with the influence of additional sources of uncertainty in the field measurement instrumentation, installation methods, data acquisition, and O\&M processes (Reda 2011).

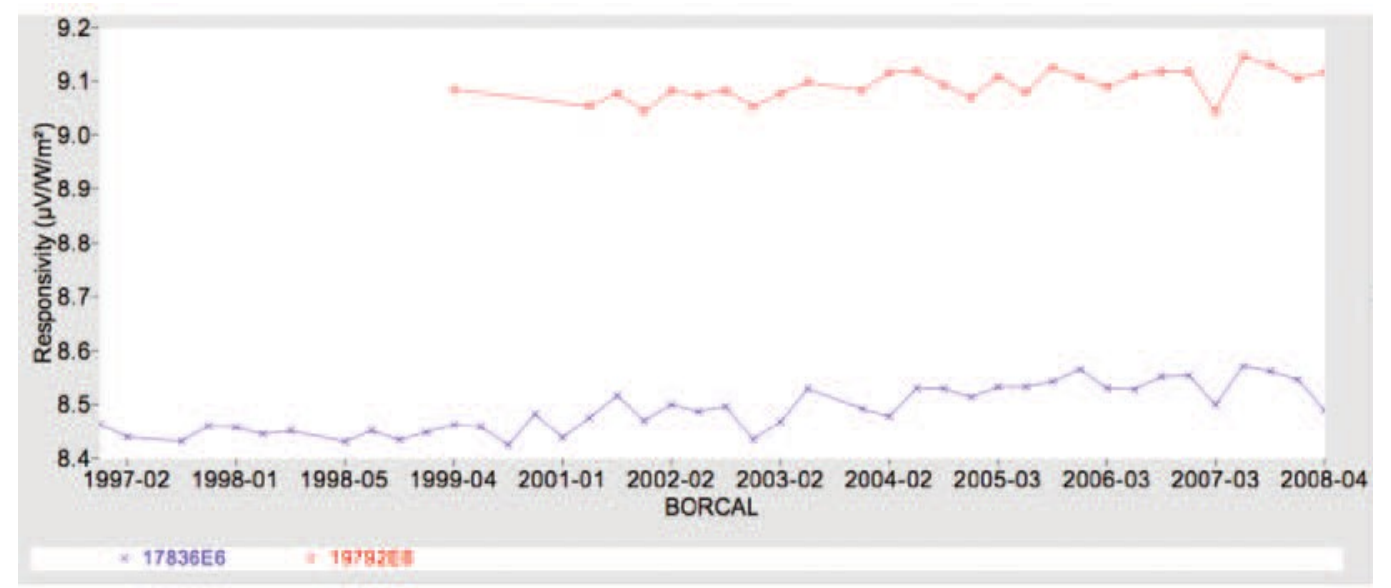

Figure 3-17. Calibration histories for two pyrheliometer control instruments spanning 12 years. Image from NREL

\subsubsection{Estimating the Uncertainty of Pyrheliometer Calibrations}

The internationally accepted Système Internationale (SI) traceable reference for the measurement of terrestrial solar radiation is the WRR. This internationally recognized measurement reference is a detector-based standard maintained by a group of electrically self-calibrating absolute cavity pyrheliometers at the WRC maintained by the Physical Meteorological Observatory in Davos, Switzerland. The present accepted inherent uncertainty in the WRR is $\pm 0.30 \%$ (Finsterle 2011). Reference cavity pyrheliometers used as national and institutional standards are calibrated by comparison to the World Standard Group of absolute cavity pyrheliometers (Figure 3-7) maintainted by the WMO- Physical Meteorological Observatory, which embody the WRR at international pyrheliometer comparisons conducted by the WRC once every five years. 
Transfer of calibrations from the WRR to national standards results in an expanded uncertainty ${ }^{6}$ for these measurement standards of $\pm 0.45 \%$ (Reda, Dooraghi, and Habte 2013). The annual transfer of calibrations from national reference absolute cavity radiometers to pyrheliometers for field measurements results in absolute uncertainty (in the calibration factors) of $\pm 1.0 \%$, primarily because of the environmental influences on the performance of field pyrheliometers. The calibration stability of commercially available pyrheliometers is generally less than a $1 \%$ change in responsivity (Rs) per year (see Figure 3-16). Results of a field pyrheliometer calibration during clear-sky comparisons using an absolute cavity are shown in Figure3-17. When finally deployed in the field, factors such as accuracy of solar tracking, data logger accuracy, cleanliness of the windows, and frequency of recalibration may contribute more sources of uncertainty resulting in typical uncertainties of $\pm 2.0 \%$ to $\pm 2.5 \%$ (or greater) in DNI measurements from a very carefully conducted, high-quality measurement system (Reda 2011).

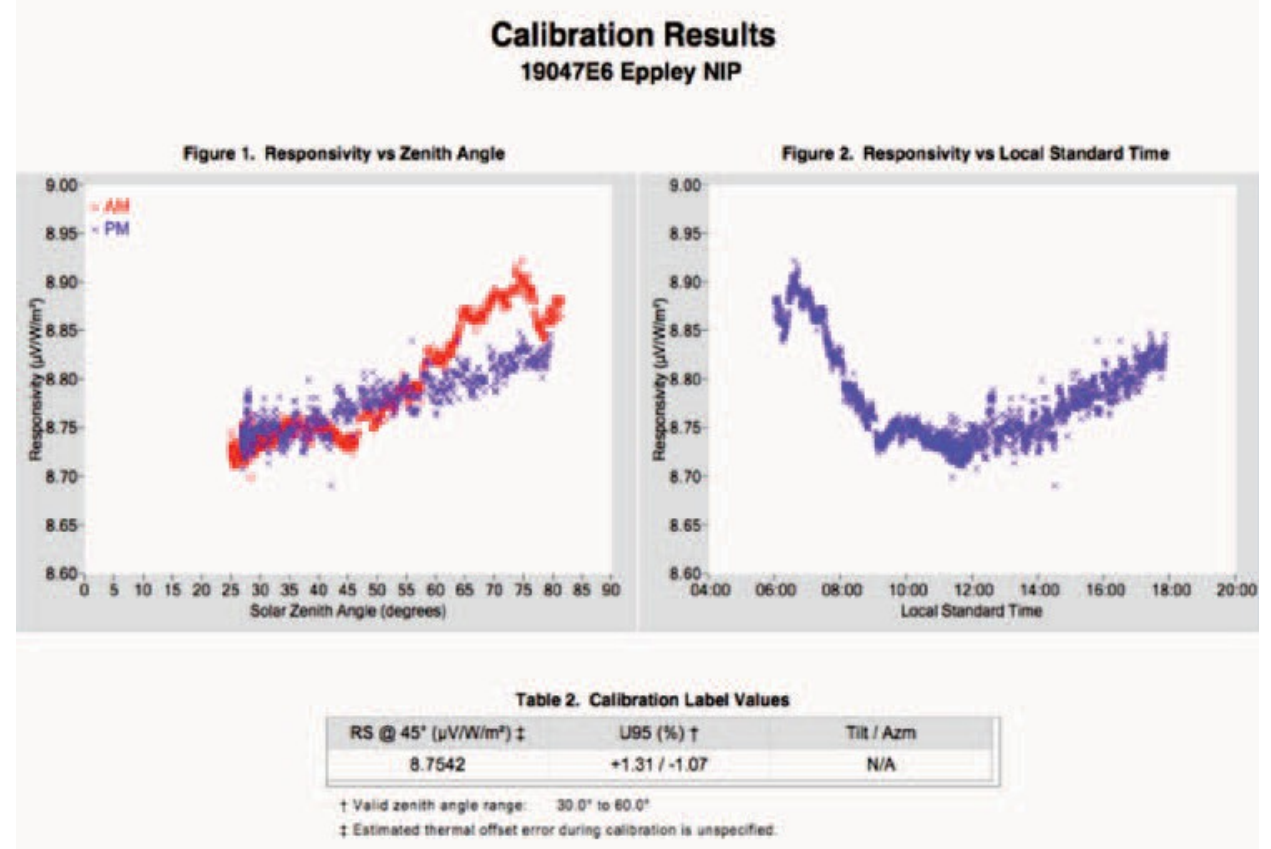

Figure 3-18. Pyrheliometer calibration results summarizing (left) Rs compared to SZA and (right) compard to local standard time. Image from NREL

The pyrheliometer responsivity (Rsi) is computed as the microvolts $(\mu \mathrm{V})$ per $\mathrm{W} / \mathrm{m}^{2}$ at each measurement comparison (i) typically made at 1 -second to 60 -second intervals with a reference or transfer standard radiometer (typically an electrically self-calibrating absolute cavity radiometer traceable to the WRR) and the output signal from the pyrheliometer under calibration:

$$
\mathrm{Rsi}=\mathrm{Vi} / \mathrm{REFi}
$$

where

\footnotetext{
${ }^{6}$ See measurement uncertainty terminology.
} 
- $\mathrm{Vi}=$ pyrheliometer output voltage $(\mu \mathrm{V})$

- $\mathrm{REFi}=$ reference DNI $\left(\mathrm{W} / \mathrm{m}^{2}\right)$.

Applying the GUM procedure to the pyrheliometer calibration, Table 3-6 summarizes the uncertainties for a $95 \%$ confidence interval for the individual pyrheliometer responsivity results.

Table 3-6. Estimated Pyrheliometer Calibration Uncertainties in Rsi

\begin{tabular}{|l|l|l|l|}
\hline Type A Error Source & Ustd (\%) & Type B Error Source & Ustd (\%) \\
\hline WRR transfer & 0.200 & WRR uncertainty (UE, k =2) & 0.3 \\
\hline $\begin{array}{l}\text { Absolute cavity responses to } \\
\text { environmental conditions }\end{array}$ & 0.013 & $\begin{array}{l}\text { Absolute cavity bias } \\
\text { responses to environmental } \\
\text { conditions }\end{array}$ & 0.013 \\
\hline Data logger precision & 0.0025 & Data logger bias $(9 \mu \mathrm{V} / 10 \mathrm{mV})$ & 0.09 \\
\hline $\begin{array}{l}\text { Pyrheliometer detector } \\
\text { temperature response }\end{array}$ & 0.25 & $\begin{array}{l}\text { Pyrheliometer detector } \\
\text { temperature response }\end{array}$ & 0.25 \\
\hline $\begin{array}{l}\text { Pyrheliometer detector linearity } \\
\text { Solar tracker alignment }\end{array}$ & 0.100 & $\begin{array}{l}\text { Event-to-event temperature } \\
\text { bias (10C) }\end{array}$ & 0.125 \\
\hline $\begin{array}{l}\text { variations } \\
\text { transmeliometer window spectral }\end{array}$ & 0.500 & $\begin{array}{l}\text { Solar tracker alignment bias } \\
\text { spectral transmittance }\end{array}$ & 0.125 \\
\hline $\begin{array}{l}\text { Electromagnetic interference and } \\
\text { electromagnetic field }\end{array}$ & 0.005 & $\begin{array}{l}\text { Electromagnetic interference } \\
\text { and electromagnetic field }\end{array}$ & 0.005 \\
\hline Total Type A & 0.125 & Total Type B & 0.665 \\
\hline
\end{tabular}

${ }^{\text {a }}$ Summed under quadrature

The combined uncertainty (UC) can be determined from the above standard uncertainties for Type A and Type B errors:

$$
\mathrm{UC}=\left[(0.615)^{2}+(0.665)^{2}\right]^{1 / 2}=0.906 \%
$$

The expanded uncertainty (UE) with a $95 \%$ confidence interval can therefore be computed based on the effective degrees of freedom (greater than 100 for pyrheliometer calibrations that can be based on more than 1,000 measurements throughout the course of a day) and a coverage factor, $\mathrm{k}$, of 2.0:

$$
\mathrm{UE}=2 \cdot \mathrm{UC}=1.8 \%
$$

Therefore, the expanded uncertainty of the calibration for each Rsi is $\pm 1.8 \%$.

With this in mind, the reader can review the radiometer calibration certificate issued for each instrument and contact the manufacturer for additional information about the calibration process. 
NREL assigns a single value for Rsi, Rs, corresponding to SZA $=45$ degrees and values of Rs for each 2-degree interval in the range of SZA encountered during the outdoor calibrations. ${ }^{7}$

\subsubsection{Estimating the Uncertainty of DNI Field Measurements}

Accounting for this calibration uncertainty and other sources of measurement errors (condition of radiometer optics and the relevant issues similar to those considered for the calibration measurement uncertainty estimates), the expanded measurement uncertainty for subhourly DNI measurements is $\pm 2.5 \%$ for a well-maintained measurement station equipped with a thermopilebased pyrheliometer and $\pm 5 \%$ for a photodiode-based RSR (Wilcox and Myers 2008). Table 3-7 identifies the uncertainty sources used for the overall uncertainty analysis of DNI measurements from two types of radiometers.

Table 3-7. Example of Estimated Direct-Normal Subhourly Measurement Uncertainties (\%)

\begin{tabular}{|c|c|c|c|c|c|}
\hline Type A Error Source & $\begin{array}{l}\text { Ustd (\%) } \\
\text { TP }^{\mathrm{a}}\end{array}$ & $\begin{array}{c}\text { Ustd (\%) } \\
\mathrm{Si}^{\mathrm{b}}\end{array}$ & Type B Error Source & $\begin{array}{l}\text { Ustd (\%) } \\
\text { TP }^{\mathrm{a}}\end{array}$ & $\begin{array}{c}\text { Ustd (\%) } \\
\mathrm{Si}^{\mathrm{b}}\end{array}$ \\
\hline $\begin{array}{l}\text { Fossilized }{ }^{\mathrm{C}} \text { calibration } \\
\text { error }\end{array}$ & 0.615 & 0.615 & $\begin{array}{l}\text { Fossilized calibration } \\
\text { error }\end{array}$ & 0.665 & 0.665 \\
\hline $\begin{array}{l}\text { Data logger precision } \\
( \pm 50 \mu \mathrm{V} / 10 \mathrm{mV})^{\mathrm{d}}\end{array}$ & 0.5 & 0.5 & $\begin{array}{l}\text { Data logger bias } \\
(1.7 \mu \mathrm{V} / 10 \mathrm{mV})^{\mathrm{d}}\end{array}$ & 0.02 & 0.02 \\
\hline $\begin{array}{l}\text { Si detector cosine } \\
\text { response }\end{array}$ & 0 & 0.5 & $\begin{array}{l}\text { Si detector cosine } \\
\text { response }\end{array}$ & 0 & 1.5 \\
\hline $\begin{array}{l}\text { Pyrheliometer detector } \\
\text { temperature response } \\
\left(\mathrm{D} 20^{\circ} \mathrm{C}\right)\end{array}$ & 0.25 & 0.05 & $\begin{array}{l}\text { Detector temperature } \\
\text { response }\end{array}$ & 0.25 & 0.05 \\
\hline $\begin{array}{l}\text { Pyrheliometer detector } \\
\text { linearity }\end{array}$ & 0.100 & 0.10 & $\begin{array}{l}\text { Day-to-day tempera- } \\
\text { ture bias }\left(10^{\circ} \mathrm{C}\right)\end{array}$ & 0.125 & 0.10 \\
\hline $\begin{array}{l}\text { Solar alignment varia- } \\
\text { tions (tracker or shade } \\
\text { band) and pyranometer } \\
\text { level for } \mathrm{Si}\end{array}$ & 0.2 & 0.10 & $\begin{array}{l}\text { Solar alignment varia- } \\
\text { tions (tracker or shade } \\
\text { band) and pyranometer } \\
\text { level for } \mathrm{Si}\end{array}$ & 0.200 & 0.20 \\
\hline $\begin{array}{l}\text { Pyrheliometer window } \\
\text { spectral transmittance }\end{array}$ & 0.1 & 1.0 & $\begin{array}{l}\text { Pyrheliometer window } \\
\text { spectral transmittance }\end{array}$ & 0.5 & 1.0 \\
\hline $\begin{array}{l}\text { Optical cleanliness } \\
\text { (blockage) }\end{array}$ & 0.2 & 0.1 & $\begin{array}{l}\text { Optical cleanliness } \\
\text { (blockage) }\end{array}$ & 0.25 & 0.1 \\
\hline $\begin{array}{l}\text { Electromagnetic } \\
\text { interference and } \\
\text { electromagnetic field }\end{array}$ & 0.005 & 0.005 & $\begin{array}{l}\text { Electromagnetic } \\
\text { interference and } \\
\text { electromagnetic field }\end{array}$ & 0.005 & 0.005 \\
\hline TOTAL Type $A^{e}$ & 0.889 & 1.382 & 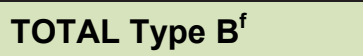 & 0.934 & 1.938 \\
\hline
\end{tabular}

${ }^{\mathrm{a}}$ Thermopile detector used for a pyrheliometer

${ }^{\mathrm{b}}$ Silicon diode pyranometer detector used for an RSR

${ }^{\mathrm{c}}$ Fossilized indicates that the calibration uncertainty is always carried forward into the field.

${ }^{\mathrm{d}}$ Typical manufacturer specified accuracy: $\pm 0.05 \%$ of full-scale range (typically $50 \mathrm{mV}$ ) $-25^{\circ} \mathrm{C}$ to $50^{\circ} \mathrm{C}$; assume $10-\mathrm{mV}$ signal, so \pm 50 microvolts $(\mu \mathrm{V})(0.5 \%)$ with $1.67 \mu \mathrm{V}$ resolution $(0.02 \%)$

${ }^{\mathrm{e}, \mathrm{f}}$ Summed under quadrature

\footnotetext{
${ }^{7}$ See www.nrel.gov/solar_radiation for additional information.
} 
The combined uncertainty, UC, can be determined from the above standard uncertainties for Type A and Type B errors for each detector type:

$$
\begin{aligned}
& \mathrm{U}_{\mathrm{CTP}}=\left[(0.889)^{2}+(0.934)^{2}\right]^{1 / 2}=1.29 \% \\
& \mathrm{U}_{\mathrm{CSi}}=\left[(1.382)^{2}+(1.938)^{2}\right]^{1 / 2}=2.38 \%
\end{aligned}
$$

The UE with a $95 \%$ confidence interval can therefore be computed based on the effective degrees of freedom (greater than 100 for pyrheliometer measurements that can be based on several thousand measurements throughout the course of a day) and a coverage factor, $\mathrm{k}$, of 2.0:

$$
\begin{aligned}
& \mathrm{U}_{\mathrm{Etp}}=2 \cdot \mathrm{U}_{\mathrm{CTP}}=2.58 \% \\
& \mathrm{U}_{\mathrm{ESi}}=2 \cdot \mathrm{U}_{\mathrm{CSi}}=4.76 \%
\end{aligned}
$$

The expanded uncertainty estimate of DNI from a thermopile pyrheliometer or silicon photodiode-based RSR is $\pm 2.6 \%$ and $\pm 4.76 \%$, respectively. Measured data should be examined carefully and periodically checked against field reference radiometers to identify conditions that exceed these limits, in which case problems with the radiometers, data acquisition systems, or other supporting equipment could be affecting the measurements. A year-long intercomparison of several models of commercially available and reference absolute cavity pyrheliometers conducted by the WMO in conjunction with laboratories at NREL and the National Oceanic and Atmospheric Administration (NOAA), located in Boulder, Colorado, produced results consistent with the estimations developed here (Michalsky et al. 2011).

\subsubsection{Uncertainty in Pyranometer Calibrations and Global Horizontal Irradiation Measurements}

The WRR is also the reference for the calibration of pyranometers used to measure GHI and DHI. Physically, it is assumed that the hemispherical detectors in a pyranometer respond only to the vertical component of the DNIVertical based on the SZA at the time of measurement:

$$
\text { DNIVertical }=\text { DNI } \cdot \operatorname{Cos}(\mathrm{SZA})
$$

The pyranometer detector is assumed to have no response to the horizontal component of DNIHorizontal:

$$
\text { DNIHorizontal }=\text { DNI } \cdot \operatorname{Sin}(\mathrm{SZA})
$$

Using the relationship described in Section 2.4 for GHI, DNI, DHI, and SZA:

$$
\mathrm{GHI}=\mathrm{DNI} \cdot \operatorname{Cos}(\mathrm{SZA})+\mathrm{DHI}
$$

we can compute the DNI as:

$$
\mathrm{DNI}=(\mathrm{GHI}-\mathrm{DHI}) / \mathrm{Cos}(\mathrm{SZA})
$$


The GHI and DHI are measured by unshaded and shaded pyranometers, respectively (see Chapter 3). Thus, given a reference DNI and DHI, we can use the above relationship to calibrate a single pyranometer.

By alternately shading and unshading the detector surface of a pyranometer on a clear day, the difference in output signal between shaded $\left(\mathrm{V}_{\text {shade }}\right)$ and unshaded $\left(\mathrm{V}_{\text {unshade }}\right)$ conditions can be compared to the reference DNI measurement to compute the Rs of the pyranometer under test:

$$
\operatorname{Rs}\left(\mathrm{V} / \mathrm{W} / \mathrm{m}^{2}\right)=\left[\left(\mathrm{V}_{\text {unshade }}-\mathrm{V}_{\text {shade }}\right) / \operatorname{Cos}(\mathrm{SZA})\right] / \mathrm{DNI}
$$

This is called the shade/unshade calibration technique, and it is described in more detail by Reda et al. (2003).

Alternatively, the radiometer can be calibrated by using a reference pyrheliometer to measure DNI and a continuously shaded pyranometer (calibrated using the above shade/unshade technique) to compute a reference GHI. The Rs of pyranometer(s) under calibration can be computed from their unshaded signal $\left(\mathrm{V}_{\text {unshade }}\right)$ :

$$
\text { Rs }\left(\mathrm{V} / \mathrm{W} / \mathrm{m}^{2}\right)=\mathrm{V}_{\text {unshade }} /(\mathrm{DNI} \cdot \cos (\mathrm{SZA})+\mathrm{DHI})
$$

Computing the Rs in this way is called the component summation calibration technique.

The shade/unshade and component summation techniques, when conducted throughout a range of SZA, demonstrate pyranometers - which by design have differing non-Lambertian, or nonideal response - as a function of SZA (or incidence angle) of the DNI. The differences in Rs as a function of SZA are like fingerprints or signatures for each individual (not only type) of pyranometer detector. Figure 3-18 shows that variations of pyranometer Rs can be symmetrical with respect to solar noon, or highly skewed, depending on the mechanical alignment of the pyranometer detector, detector surface structure, and detector absorber material properties. 


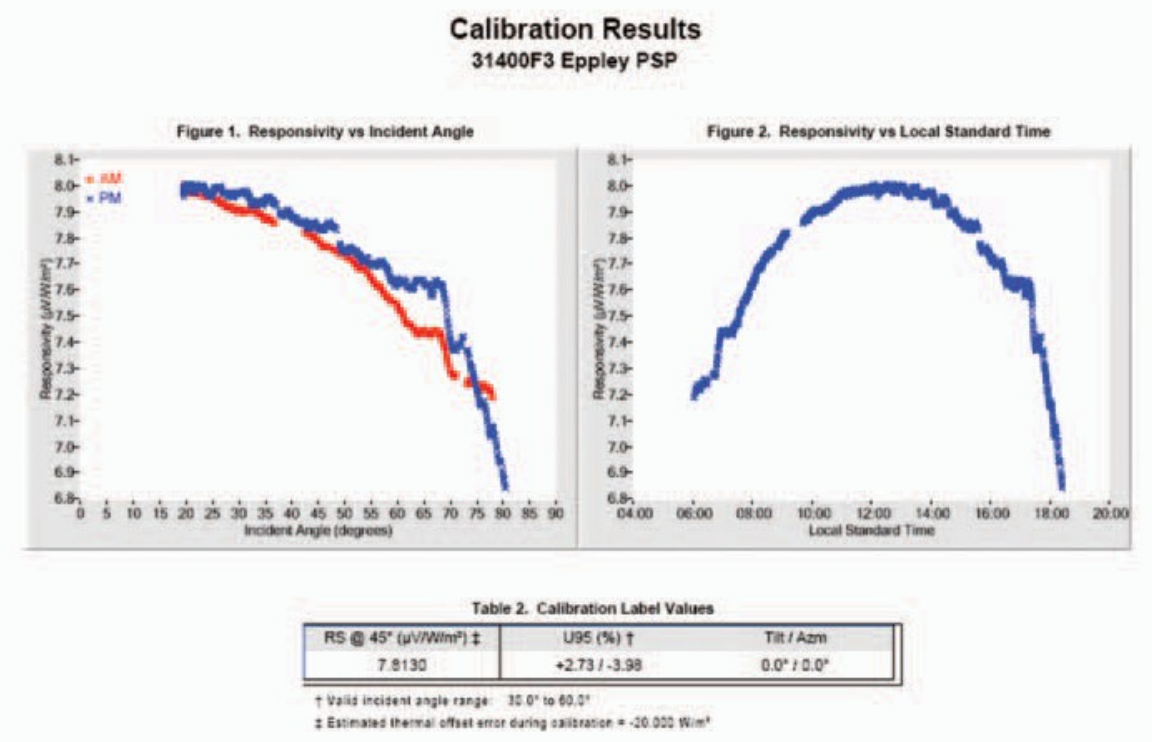

Figure 3-19. Pyranometer calibration results summarizing Rs compared to (left) SZA and (right) local standard time. Image from Daryl Myers, NREL

Typical calibration uncertainty for any sensor with respect to a WRR reference cavity radiometer is approximately $0.5 \%$ at any one very narrow range ( \pm 2 degrees to \pm 5 degrees) of zenith angle (Reda et al. 2008). Over a wide range of zenith angles ( 0 degrees to 85 degrees), the Rs can vary by 10 to 20 times that over a narrow range, or from $\pm 3 \%$ to $\pm 10 \%$ or even more. These effects then need to be combined with the field measurement influences, the same as with the DNI measurement uncertainty estimate (e.g., including pyranometer installation, data logger accuracy, cleanliness, spectral dependency, and temperature sensitivity).

These larger high-zenith-angle-related uncertainties occur throughout parts of the day (morning and afternoon) when the available solar resource is much smaller than typical midday resources, when the zenith angles are smaller. Because the maximum elevation (minimum zenith) angles vary throughout the seasons, the uncertainty in hemispherical radiation data will vary as well.

Even in the good measurement regime of midday, hemispherical field measurement uncertainty is typically two to three times that of direct-beam measurements, or $\pm 4 \%$ to $\pm 5 \%$, throughout a year, primarily because of these seasonal uncertainty variations. Better instrumentation design and careful applications of correction factors as a function of zenith angles are ways to improve (reduce) the uncertainty in GHI measurements. The alternative is to use high-quality DNI and DHI measurements using a tracking shading disk/ball to compute GHI. The measurement uncertainties for GHI then approach that of the DNI $( \pm 2 \%)$ for clear-sky measurements.

Figure 3-19 shows the calibration traceability for pyrheliometers used to measure DNI and pyranometers used to measure GHI or DHI and indicates how measurement uncertainties accumulate from calibration to field deployment. Broad arrow boxes show accumulated uncertainty at each phase of the process. The resulting field deployment uncertainties for pyrheliometers used for measuring DNI is $\pm 2.0 \%$. Measurement uncertainties for pyranometers 
used to measure $\mathrm{GHI}$ in the field range from $\pm 3.0 \%$ for SZA between 30 degrees and 60 degrees and up to $\pm 7 \%$ to $\pm 10 \%$ for SZA greater than 60 degrees.

The calibration and assessment of calibration and field uncertainties for pyrheliometers and pyranometers is described in detail in national and international standards (ASTM G167-05, ASTME 816-05, ASTM E824-05, ASTM G183-05, ISO 9059, ISO 90846, ISO 9847).

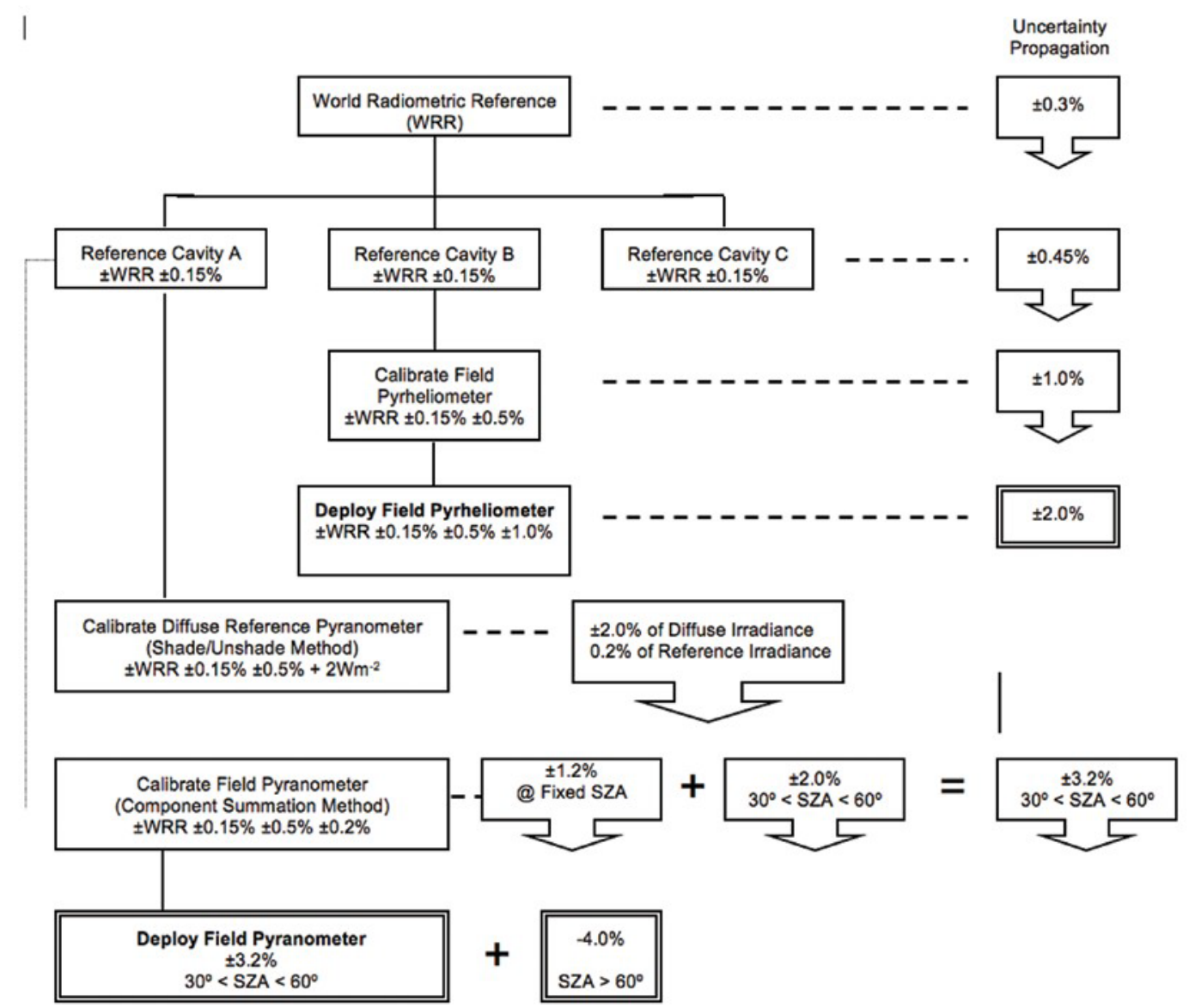

Figure3-20. Calibration traceability and accumulation of measurement uncertainty for pyrheliometers and pyranometers (coverage factor $\mathrm{k}=2$ ). Image from NREL

\subsubsection{Model Estimate Uncertainties}

Empirical models derived from measured data correlations with independent parameters inherently carry measurement uncertainty embedded in the ultimate model accuracy. Models based on $2 \%, 5 \%$, or $10 \%$ accurate measurements can be no more accurate than the data used to generate the model. Typically, scatter about model regression lines increases the random component of uncertainty further. Models based on first principles of physics and radiation transfer cannot be validated or verified to a level of accuracy greater than that of the measurements. Beware of claims of high accuracy in models or measurements without a thoroughly documented uncertainty analysis (Gueymard and Myers 2009). 


\subsection{Measurement Station Design Considerations}

To collect useful DNI resource data, the successful design and implementation of a solar resource measurement station or network of stations requires careful consideration of the elements summarized in this subsection.

\subsubsection{Location}

The primary purpose of setting up a solar resource measurement station is to collect data that allow an analyst to accurately characterize the solar irradiance and relevant meteorological parameters at a particular location. Ideally, the instruments would be collocated with the targeted analysis area, but in some cases separation distances may be tolerated depending on the complexities of local climate and terrain variations. Lower variability in terrain and climate generally translates to lower variability in the solar resource over larger spatial scales. These effects should be well understood before determining the final location of a measurement station. The proximity to the target area must also be weighed against operational factors, such as availability of power, communications, and access for maintenance, as discussed below. Considerations should also include the possible effects of local sources of pollution or dust - for example, traffic on a nearby dirt road that could degrade the measurements.

When measurement stations are constructed in metropolitan, industrial areas or even electrical substations, consideration should be given to possible sources of radio frequency signals that could impart unwanted noise in sensors or cables. For example, the same high building that would provide an attractive unobstructed site for solar measurements may also be the ideal location for radio or television broadcast towers or some other communication apparatus. Such sites should also be investigated for harmful effects of electromagnetic radiation on the health of station maintenance workers.

Instrument selection is a fundamental consideration, because measurements with greater accuracy will better reflect the actual resource; however, instrument placement is also an important consideration. If nearby objects - such as trees or buildings - shade the instruments for some period of time during the day, the resulting measurement will not truly represent the available solar resource. Distant objects - especially mountains - may be legitimate obstructions, as the shadows they cast are likely to produce an influence beyond the area local to the instruments. Conversely, nearby objects can potentially reflect solar radiation onto the instruments, likewise resulting in measurements that do not represent the local natural environment. Such cases could include a nearby wall, window, or other highly reflective object. The best practice is to locate instruments away from any objects that are in view of the instrument detector. The recommendations from WMO (2008) for radiation and all other measurands apply.

The easiest way to determine the quality of solar access is to scan the horizon for a full 360 degrees of azimuth and note the elevation of any objects protruding into the sky above the local horizon. Look for buildings, trees, antennae, power poles, and even power lines. Most locations will have some obstructions, but whether they will be significant in the context of the necessary measurements must be determined. Generally, pyranometers are very insensitive to sky blockage within approximately 5 degrees elevation above the horizon. Pyrheliometers, however, are more sensitive, because objects can completely block the DNI, depending on the daily path of the sun 
throughout the year. The amount of blockage time each day will be related the object's width and height above the horizon. The number of blockage days each year will depend on where along the horizon the object lies. To be a concern, the object must be in the area of the sun near sunrise or sunset, the time and azimuth of which vary throughout the year. For most of the horizon, objects blocking the sky will not be a factor, because the sun rises in a limited range in the east and sets likewise in the west during sunset (e.g., at $40^{\circ} \mathrm{N}$ latitude, sunrise near the summer solstice occurs at approximately $60^{\circ}$ from true north). However, the farther north in latitude the site is located, the greater the range of these sunrise and sunset areas of interest. A solar horizon map, or even a sketch of obstructions by elevation and azimuth, will help determine the areas where horizon objects will affect the measurement (see Figure 2-5).

Considerations for locating a station should also include environmental concerns, such as wildlife habitat, migratory paths, drainage, and antiquities or archeological areas.

\subsubsection{Station Security and Accessibility}

Tens of thousands or even hundreds of thousands of dollars can comprise measurement stations. Although this equipment is typically not the target of thieves seeking property for resale, it is still subject to theft and should be protected. Vandalism may be even more likely than theft. Unlike thieves, vandals typically care less about what they are vandalizing and more about their ability to destroy property with high value to its owner. The less visible and accessible the station is to the public, the less likely it will be the target of theft or vandalism. For example, instruments mounted on a rooftop are less likely to attract unwanted attention than those unprotected beside a highway. Lack of visibility is the best defense against vandalism, including damage from bullets or rocks.

Security fences should be used if people or animals are likely to intrude. Fencing should be at least $6 \mathrm{ft}$ tall, preferably with barbed wire, and fitted with locking gates in high-profile areas where intrusion attempts are likely. Less elaborate fences may suffice in areas that are generally secure and where only the curious need be discouraged from meddling with the equipment. In remote venues with few human hazards, cattle fence paneling (approximately $4 \mathrm{ft} \mathrm{tall}$ ) may be advisable if large animals roam the area. The fencing should be sturdy enough to withstand the weight of a large animal that may rub against the compound or otherwise be pushed or fall against the fence. It may not be possible to keep smaller animals out of the station compound, and precautions should be taken to ensure that the equipment, cabling, supports, etc., can withstand encounters with these animals. Coyotes, rodents, rabbits, birds, and other wildlife may be able to move through the wires or jump over or burrow under fences. In particular, signal cabling between modules or sensors at or near ground level is prone to gnawing by rodents and should be run through a protective conduit or buried. Any buried cable should either be specified for use underground or run through conduit approved for underground use. Underground utilities and other objects should be investigated before postholes are dug or anchors sunk.

If fences are used, they must be considered as a potential obstacle that can shade the instruments or reflect radiation to the instruments. The radiometers should be positioned above the fence line (including barbed wire), if only by a few millimeters, to prevent any shading of the sensor. This assumes that the pyranometer is mounted in a horizontal position and that the pyrheliometer is installed in a solar tracker. POA pyranometers should have an unobstructed view of the ground and sky in front of them. In any case, the recommendations from WMO (2008) concerning 
obstacles have to be followed. If nearby towers are unavoidable, the station should be positioned between the tower and the equator (e.g., to the south of the tower in the northern hemisphere) to minimize shading. The radiometers should be positioned as far as possible from the tower- at least several meters - so the tower blocks as little of the sky as possible (radiometer signal cables should be shorter than $50 \mathrm{~m}$ to avoid losses caused by line resistance). The tower should also be painted a neutral gray to minimize strong reflections that could contaminate the solar measurement. These guidelines assume that the tower is part of the measurement station proper and that the site operator has control of the placement or modification of the tower. Absent that control, the radiometers should be moved as far as possible from the tower.

Access to the equipment must also be part of a station construction plan. Because routine maintenance is a primary factor affecting data quality, provisions must be made for reasonable and easy access to the instruments. Factors here could include ease of access to cross-locked property, well-maintained all-weather roads, and roof access that might be controlled by other departments. Safety must also be a consideration. Locations that present hazardous conditionssuch as rooftops without railings or that require access using unanchored ladders - must be avoided.

\subsubsection{Power Requirements}

Ongoing measurements require a reliable source of electrical power to minimize system downtime from power outages. In some areas, power from the utility grid is reliable, and downtime is measured in minutes per year. In other areas, multiple daily power interruptions are routine. Depending on the tolerance of the required analysis to missing data, precautions should be taken to ensure that gaps in the data stream from power outages do not seriously affect the results. The most common and cost-effective bridge for power outages is an uninterruptible power supply. An uninterruptible power supply can also filter out unwanted or harmful line voltage fluctuations that can occur for a variety of reasons. It has internal storage batteries that are used as a source of power in the event of an alternating current (AC) power interruption. When the AC power is interrupted, internal circuitry makes an almost seamless switch from gridconnected $\mathrm{AC}$ power to $\mathrm{AC}$ provided through an inverter connected to the battery bank. When power is restored, the uninterruptible power supply recharges the internal battery from the AC line power. Power loss is detected quickly, as is switching to battery, and it is measured in milliseconds or partial line cycles. Some equipment may be particularly susceptible to even millisecond power interruptions during switching and should be identified through trial and error to avert unexpected downtime despite use of the uninterruptible power supply.

The uninterruptible power supply is sized according to:

- Operating capacity — amount of power in watts; it can continuously supply either on or off grid-connected AC power

- Longevity of battery power - how long the battery can last under anticipated maximum load.

Users should estimate the longest possible power outage and size the uninterruptible power supply for the maximum load of attached devices and the maximum period of battery capacity. Batteries should be tested regularly to ensure that the device can still operate per design specifications. Internal battery test functions sometimes report errors only when batteries are 
near complete failure and not when performance has degraded. A timed full-power-off test should be conducted periodically to ensure that the uninterruptible power supply will provide backup power for the time needed to prevent measurement system failure.

In remote locations where utility power is not available, local power generation should be devised. Options for on-site electrical power generation include PV or small wind turbine systems (or both) and gasoline- or diesel-fueled generators with battery storage. The renewable energy systems should be sized to provide enough energy for the maximum continuous load and power through several days of cloudy weather when solar generation would be minimal. This would include sites prone to persistent ground fog. The sizing is a function of the extremes of the solar climate and should consider the longest gap during reduced generation, the shortest recharge period available after discharge, and the generation capacity and storage necessary to provide uninterrupted power for the target location. Some oversizing is necessary to accommodate degradation of PV panels and battery storage, and consideration should be given to ambient temperature, which affects the ability of a battery to deliver energy. Sizing calculators are available to help with this effort. ${ }^{8}$

Equipment should be specified and tested for self-power-on capability in the event of a power outage. This ensures that when power is restored, the equipment will automatically resume measurements and logging without operator intervention. This is an important consideration for remote locations where considerable downtime might occur before personnel could be dispatched to restart a system.

\subsubsection{Grounding and Shielding}

Station equipment should be protected against lightning strikes and shielded from radio frequency interference that could damage equipment or reduce the validity of the measurements. Several books are available that describe techniques for grounding and shielding low-voltage signal cables (see, for example, Morrison [1998]). The reader is urged to consult available references or seek expert technical advice during the design of a solar resource measurement system.

In general, the following steps should be taken when designing and constructing a measurement station:

1. Use a single-point ground (e.g., a copper rod driven several feet into the ground) for all signal ground connections to prevent ground loops that can introduce noise or biases in the measurements.

2. Use twisted pair, shielded cables for low-voltage measurements connected as doubleended measurements at the data logger. Double-ended measurements require separate logger channels for + and - signal input conductors. These inputs do not share a common signal ground and therefore significantly reduce the possibilities for electrical noise introduced in the signal cable.

\footnotetext{
${ }^{8}$ See http://pvwatts.nrel.gov/.
} 
3. Physically isolate low-voltage sensor cables from nearby sources of electrical noise, such as power cables. (Do not run signal cables in the same bundle or conduit as AC power cables.) If a power cable must be near a signal cable, always position the two at right angles to each other. This limited contact will minimize the possibility of induced voltages in the signal cable. Also, although this case is not recommended, the data logger settings should be selected to avoid signal noise (integration time of the voltage measurement adjusted to AC frequency).

4. Metal structures such as masts and tripods should be connected to the ground to provide an easy path to the ground in the event of a lightning strike. This will help protect sensitive instruments. Electronic equipment often has a special ground lug and associated internal protection to help protect against stray voltages from lighting strikes. These should be connected with a heavy gauge wire to ground (12 American wire gauge or larger). Metal oxide varistors, avalanche diodes, or gas tubes can be used to protect signal cables from electrical surges such as lightning. These devices must be replaced periodically to maintain effectiveness. The replacement frequency is a function of the accumulated energy dissipated by the unit.

\subsubsection{Data Acquisition}

Data logging equipment should have performance specifications that do not degrade the potential measurement of the radiometer signals (e.g., analog-to-digital conversion of low-level direct current voltages, temperature response coefficients, and environmental limits of operation).

Most radiometers output a voltage, current, or resistance that is measured by a voltmeter, ammeter, or ohmmeter. The measured value is subsequently converted to engineering units through a multiplier and/or an offset determined by calibration to a recognized measurement standard. Data loggers should be chosen so that the measurement signal is consistent with the uncertainty of the sensor - for example, a much smaller uncertainty, perhaps 3 to 10 times smaller than the estimated measurement uncertainty associated with the radiometer. This is the accuracy ratio between the data logger and the radiometer. For example, typical specifications for a good data logger measuring a $10-\mathrm{mV}$ output from the radiometer accurate to $1 \%$, or $0.1 \mathrm{mV}$ $(100 \mu \mathrm{V})$, are on the order of total uncertainty (accuracy) of better than (less than) $0.1 \%$ of reading (or full scale) for the parameter in question, which would be $0.010 \mathrm{mV}$, or $10 \mu \mathrm{V}$. The logger should also have a range that can measure the voltage or resistance at near full scale to best capture the resolution of the data. For example, a sensor with a full-scale output of $10 \mathrm{mV}$ should be connected to a logger with a range that is at least but not below $10 \mathrm{mV}$. A logger with a $1-\mathrm{V}$ range may be able to measure $10 \mathrm{mV}$, but not with the desired precision. Most modern data loggers have several range selections, allowing the user to optimize the match for each instrument. Because of the nature of solar radiation, radiometers (e.g., pyranometers used for GHI measurements) can sometimes produce $200 \%$ or more of clear-sky readings under certain passing cloud conditions, and the logger range should be set to prevent over-ranging during unusual sky conditions.

Some radiometers use amplifiers to raise the instrument output to a higher range to better satisfy signal range matching requirements; however, such amplifiers require power and will add some uncertainty to the data with nonlinearity, noise, temperature dependence, or instability. Highquality amplifiers may minimize these effects and allow a reasonable trade-off between logger 
cost and data accuracy. Calibrations must be made of these radiometer systems by including the pyranometer or pyrheliometer and its uniquely associated amplifier.

The logging equipment should also have environmental specifications that are compatible with the environment where the equipment will be used. Loggers used inside an environmentally controlled building could have less stringent environmental performance specifications than one mounted outside in a desert or arctic environment. Equipment enclosures can create an internal environment several degrees above ambient air temperature because of solar heating (absorption by the enclosure materials), heat generated by electronic devices mounted inside, and lack of ventilation to help purge heat. Vent plugs are available to provide ventilation openings and prevent insects and water from entering the enclosure (e.g., Gore Tex vent plugs).

The sampling frequency and time statistics of the solar resource data should be determined from the desired data analysis requirements. For example, monthly means, daily totals, hourly, minute, or sub-1-minute data records can be useful. Data loggers can generally be configured to produce output of instantaneous or integrated values at any reasonable time period consistent with the radiometer time-response characteristics. The design should consider the current requirements and, if convenient and practical, future needs for additional analyses. A high-temporal-resolution data-logging scheme can be down sampled or integrated to longer time periods than the other way around. For example, transforming hourly data to 1-minute data with any certainty and accuracy is impossible if a specific data time series must be reproduced. Data logging equipment, data transfer mechanisms, and data storage can generally handle 1-minute data resolution, and this time realm should be considered the fundamental resolution in the data logger. Because most applications address the solar energy available over time, integrated data of sub-minute samples within the data logger (e.g., 1-second signal sampling) is a common method of data output regardless of the final data resolution required by the analysis. The output of instantaneous samples is much less likely to represent the available energy and should be avoided when configuring a data logger. If the size of a measured data set is a defining issue (e.g., limited data communications throughput), the user can determine the lowest temporal resolution necessary for the application and optimize the data collection accordingly.

\subsubsection{Data Communications}

Provisions should be made for transferring data from the data logger to a data processing facility. This is the basis for adequately frequent data control. Historically, data have been captured, transferred, and processed in various ways. The manual transfer of data recorded on strip charts physically carried or shipped from the observing station to a data center has been replaced by advances in electronics and telecommunications that allow remote data collection from nearly any location.

A telephone modem link that uses conventional dial-up phone lines to connect stations to data centers can now be replaced with cellular telephone technology, obviating the need for a physical connection between logger and phone line. The cell phone network is configured to provide virtual Internet links between a measurement station and the data center. Satellite up- and downlinks are also available for data transfers in areas that are not served by either wire- or cellbased phone service. Within the area of an observing station, short-distance wireless communications such as Wi-Fi connectivity may be useful to minimize the need for long cables between radiometers and data loggers. 
To prevent data loss in case of connection problems, the memory of the data logger should be selected appropriately. Memory extensions are available for many data loggers. Especially for high-temporal-resolution or many measurement channels, a direct connection between the logger and a PC with Internet connection should be considered, too.

\subsubsection{O\&M}

Proper O\&M practices are essential for acquiring accurate solar resource measurements. As addressed in this subsection, several elements in a chain form a quality system. Collectively, these elements produce accurate and reliable solar resource data: station location, measurement system design, equipment installation, data acquisition, and O\&M practices. Proper O\&M requires long-term consistency, attention to detail, and a thorough appreciation for the importance of preventative and corrective maintenance of sensitive equipment.

Calibrations are performed with clean instrument optics and a carefully aligned detector. To properly apply the calibration factor, the instrument should be kept in the same condition during field measurements. To maintain the calibration relationship between irradiance and radiometer output, proper cleaning and other routine maintenance is necessary. The maintenance process includes:

- Checking the alignment of the detector. Pyrheliometers must be accurately aligned with the solar disk for accurate DNI measurements. Pyranometer detectors must be horizontal for GHI and DHI measurements and accurately aligned with a flat-plate collector for POA measurements. The radiometer orientation should be checked periodically using the features described in Chapter 3. (In some cases, a carefully leveled pyranometer may produce GHI readings that are not symmetrical around solar noon under clear skies. If this cannot be attributed to any change in atmospheric composition (aerosols or water vapor), or optical asymmetries can be verified under strict laboratory conditions, the optical axis of the detector is probably not exactly vertical. This is a manufacturing defect.

- Cleaning the instrument optics. To properly measure the solar intensity, no contaminant should block or reduce the amount of sunshine falling on the detector. The outdoor environment provides many sources of such contamination, such as dust, precipitation, dew, plant matter, insects, and bird droppings. The sensors should be cleaned regularly to minimize the effect of contaminants on the measurements. Depending on the local conditions, this can require daily maintenance of unventilated or otherwise protected radiometers.

- Documenting the condition of the radiometer. For analysts to understand limitations of the data, conditions that affect the measurement must be documented. This includes substandard measurement conditions, but it is just as important to document proper operations to add credibility to the data set. Observations and notes provide a critical record of conditions that positively and negatively affect data quality.

- Documenting the environment. As a consistency check, note the sky and weather conditions at the time of maintenance when interpreting data from the radiometer, including measurements with unusual values.

- Documenting the infrastructure. The measurement station as a whole should be examined for general robustness. Any defects should be noted and corrected. 
Maintenance frequency depends on prevailing conditions that soil the instruments. This includes dust, rain, snow, birds, and insects. It is also depends on instrument type. Radiometer designs based on optical diffusers (such as LI-COR LI-200) are less susceptible to dust contamination than are instruments with clear optics (Myers et al. 2002). This may be caused by, in part, the area subject to soiling (e.g., a larger dome versus a smaller diffuser). Also, fine dust on the surface of a diffuser can become an integral part of the diffuser, and it may lessen the impact of the dust on the diffuser transmittance compared to that on a precision-ground optical dome. Soiling of the windowed or domed radiometers can quickly affect the measurement and increase by many-fold the measurement uncertainty. This is especially relevant for pyrheliometers (Geuder 2006). As described earlier, a pyranometer in a ventilator can reduce this risk of contamination; thus, the frequency and cost of maintenance should be considerations in instrument specification.

If a remote site will be difficult to maintain for extended periods, a higher class windowed instrument might not be optimal, despite its potential for better measurements. The cost of maintenance for a remote site may dominate the estimated cost of setting up and operating a station. This aspect should be anticipated when planning a measurement campaign.

A conservative maintenance schedule will support the credibility of the measurement data set and provide the analyst a base of justification when assigning confidence intervals for the data.

Daily inspection should be scheduled for instruments with clear optics, and twice monthly inspections should be scheduled for diffuser instruments. More frequent spot inspections should be conducted after significant weather events (e.g., dust storms, heavy rainfall, rainfall during periods with high optical depth, and storms). Radiometer optics may not necessarily soil within a 24-hour period, but the effects of soiling can best be mitigated with frequent inspection.

Radiometers should be carefully cleaned at each inspection, even if soiling appears minimal. Cleaning is generally a very short procedure, and it removes the possibility of differing interpretations of the need to clean among different technicians. With such a procedure in place, the analyst can claim with confidence that the instruments were kept clean according to the documented schedule.

Maintenance at remote measurement sites away from institutional or corporate employment centers will require finding a qualified person nearby who can perform the necessary maintenance duties. The qualifications for maintenance are generally nontechnical, but they require someone with the interest and disposition to reliably complete the tasks. As a rule, compensating these people for time and vehicle mileage - rather than seeking volunteersbecomes a worthwhile investment in the long run, because it sets up a firm contractual commitment to perform all necessary maintenance duties. Absent that formal relationship, it can become difficult to assert the need for reliable and regular attention.

All O\&M should be carefully documented with log sheets or preferably with electronic databases that contain enough information to reveal problems and solutions or that assert that the instruments were in good form when inspected. The exact times of the maintenance events should be noted, not estimations. For example, a button connected to the data logger that is pressed at the beginning and at the end of an inspection is recommended. The O\&M information 
enables an analyst to identify potentially bad data, and it provides important documentation to determine and defend the overall quality of the measurements.

\subsection{Data Quality Control, Data Correction, Data Quality Assessment, and Metadata}

The data quality is generally established when the measurement is taken. Little can be done after the fact to improve fundamental quality. For example, a poorly maintained station with dirty optics or misaligned instruments will produce data with presumed (or even apparent) errors, and the magnitude of those errors is not likely to be discernable until days or weeks later. There is no way to systematically reduce the uncertainty of such a measurement, and one can only guess at which corrections to make. In this context, data quality control involves a well-defined supervisory process by which station operators are confident that, when a measurement is taken with unattended instruments, the instruments are in a state that produces data of known quality. This process largely encompasses the calibration, inspection, and maintenance procedures discussed earlier, along with log sheets and other items that document the condition of the station. It also includes a critical inspection or assessment of the data to help detect problems not evident from physical inspection of the instruments.

Data quality assessment is a method by which data quality can be judged based on criteria for a particular application. Several particular errors of meteorological data can be detected by automatic screening algorithms. Corresponding tests are documented in a number of publications, including Long and Dutton (2002); Maxwell, Wilcox, and Rymes (1993); Wilcox and Cormack (2011); Journée and Bertrand (2011); and Espinar et al. (2011). Data can be compared to certain physical limits that have been determined to be reasonable, with redundant or complementary measurements, or with physical or empirical models, all of which will provide some degree of independent measure for a quality judgment; however, dependent on how strict the screening parameters and their corresponding values are chosen, too many or too few events may be detected. Moreover, the values of some parameters are site dependent according to corresponding weather conditions; therefore, the results of the automatic screening always demand a manually check of an expert to ensure their validity. Also, further influences on the data potentially known by the supervisor have to be included as comments or flags.

The interpretation and application of solar resource measurements depend greatly on the efforts to record and include metadata relevant to the observations. This includes not only site location; local horizon survey; data acquisition system(s); input signal channel assignments; radiometer types, models, serial numbers, calibration histories, and installation schemes; but also information on eventual post processing of the data and maintenance records. An example of online metadata is available from NREL's Solar Radiation Research Laboratory. ${ }^{9}$ Such metadata should be included with the archiving of the measured solar resource data. For example, influences that have to be documented may included damaged or misaligned sensors, maintenance works on the instruments, detection of soiled sensors and subsequent sensor cleaning, obstructed sensors, and temporarily erroneous calibration constants in the program code. These events are frequently not detected automatically or sometimes not even detectable

\footnotetext{
${ }^{9}$ See www.nrel.gov/midc/srrl bms.
} 
by automatic quality-control screening tools. Hence, manual checks are required, as discussed in Geuder et al. (2014). However, automatic tests such as those presented in the following contain important and valuable information and should be performed.

One common method for evaluating DNI, GHI, and DHI quality is a three-component coupling test. As described in Chapter 2, the measurements of DNI and DHI can be combined mathematically to derive a global measurement, as described in Equation 2-4. When all three components are measured, measurement redundancy is apparent, because any one component can be derived from the other two; thus, in the context of quality assurance, expected values for each component can be calculated from the other two. This method helps quantify the relative error among the three components, although it does not necessarily determine strictly which measurement - or measurements - are in error. However, operational knowledge of the instruments and trackers can provide valuable insight into likely errors. For example, a misaligned tracker would cause either a low DNI or high diffuse measurement (low DNI from a poorly aligned pyrheliometer or high diffuse from a poorly aligned shading disk). With this knowledge, and an observation of trends in the magnitude of flagging, a data quality expert can quickly spot common operational errors. The measurement of the three redundant componentsrather than only a single measurement or two components of specific interest - is a significant and important tool for data quality analysis, and it should be strongly considered when specifying instrumentation for a station.

For example, the SERI QC software (Maxwell et al. 1993) produces flags that can be plotted (see Figure 3-14). The process generates a data quality value, or flag, for each data point based upon a normalization process involving the dimensionless parameters-clearness or cloudiness index, $(\mathrm{Kt})$, effective diffuse horizontal transmittance $(\mathrm{Kd})$, and direct beam transmittance $(\mathrm{Kn})$ derived from the corresponding ETR. The plot on the left indicates more severe flags from among the three components, plotted here by day of month (y axis) and hour of day (x axis), the least error in the dark blue, and the greatest error in red. Further, the other three plots correspond respectively to normalized GHI, DNI, and DHI measurements, providing the analyst with additional information to pinpoint the measurement causing the error. 


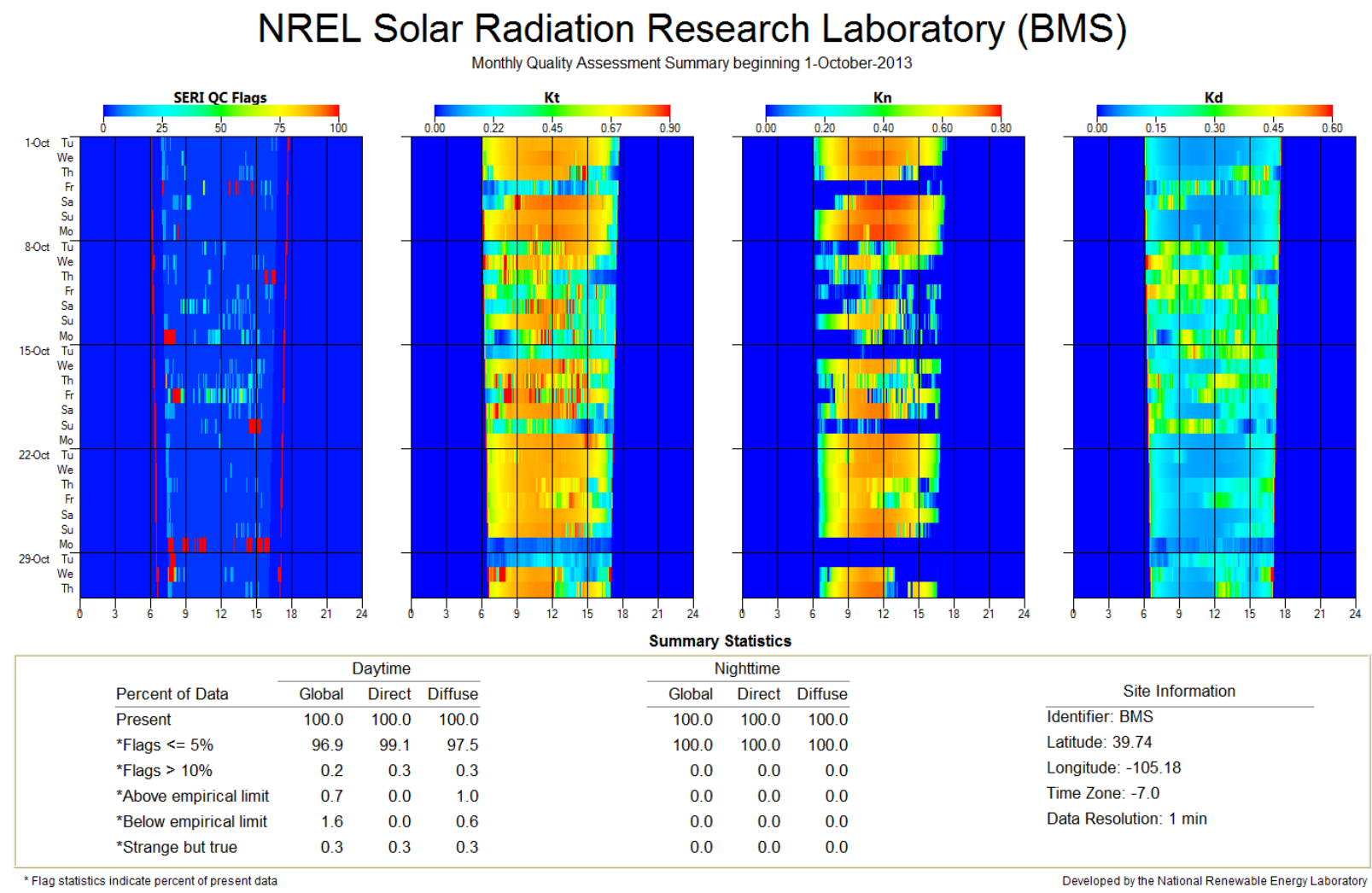

Figure 3-21. Example of SERI quality-control data quality-assurance reporting. Image from NREL

In the case shown in Figure 3-21, the three-component data (GHI, DNI, and DHI) were submitted to the SERI QC software, which performs the three-component coupling test in the realm of the clearness index, or $\mathrm{Kt}, \mathrm{Kn}$, and $\mathrm{Kd}$ (see Chapter 2). This $\mathrm{K}$-space value normalizes the solar irradiance values to remove the effect of the SZA. Thus, in K-space

$$
\mathrm{Kt}=\mathrm{Kn}+\mathrm{Kd}
$$

Or, rearranged, the deviation from this equation of component coupling can be quantified as the residual from

$$
\mathrm{E}=\mathrm{Kt}-\mathrm{Kn}-\mathrm{Kd}
$$

Perfect component coupling would result in $\varepsilon=0$, and any nonzero value indicates some disagreement among the instruments; however, this method does not reveal which component or components are in error, only that there is some disagreement. Further, instrument errors in opposing directions could result in a false zero value.

Despite these ambiguities, a knowledgeable analyst can confidently detect measurement errors in most typical measurement scenarios. In the case of Figure 3-21, for each minute data record containing the three components, the residual was plotted as a quality flag, with red color flags (left column) indicating a greater deviation or apparent error. The actual $\mathrm{Kt}$, $\mathrm{Kn}$, and $\mathrm{Kd}$ values are also plotted in the next three columns; the red roughly correlates to higher irradiance, and the 
dark blue represents the least clear. Data from an RSI consist of GHI, DNI, and DHI and are derived from a single pyranometer. To some extent, this limitation can be mitigated by including a secondary unshaded (GHI) pyranometer on the RSI to provide some redundant measurements. This enhancement is a relatively low-cost method of adding confidence in the measurements and can be included in a two- or three-component quality-assessment test.

The three-component methods described here are generally more reliable than a simple clear-sky data analysis in which some conclusions are drawn based on modeled or other expected values of the clear-sky data. Significant day-to-day variations in cloudless, clear-sky data can occur because of variations in atmospheric constituents, such as aerosols or water vapor. Thus, such variation can make it difficult to draw conclusions about possible instrument error without specific information regarding other critical atmospheric components.

A successful quality-control process requires elements of quality assessment and feedback. Figure 3-22 depicts a quality-assurance cycle that couples data acquisition with quality assessment and feedback.

\section{The Quality Assurance Cycle}

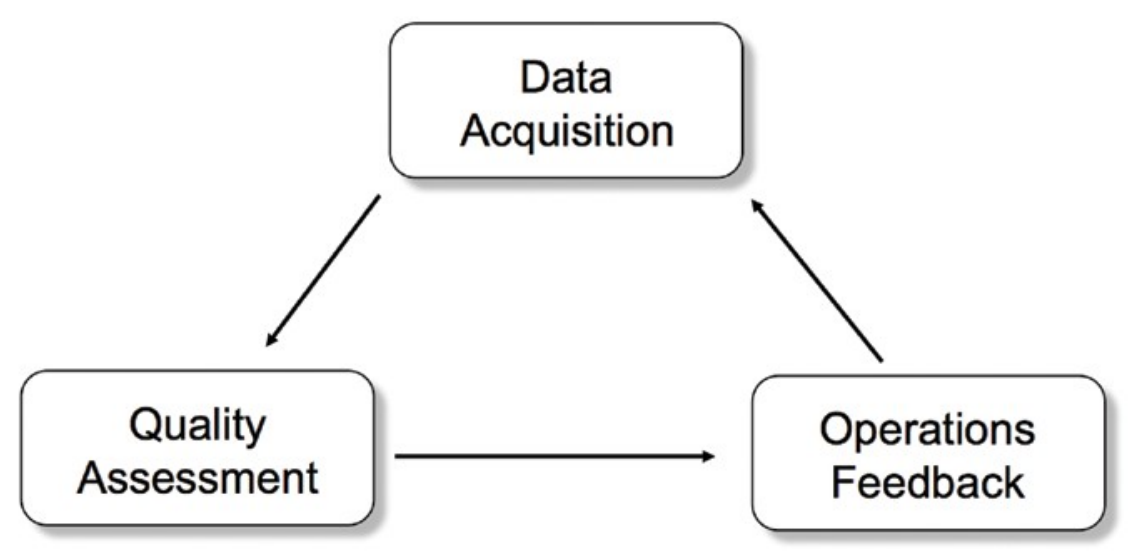

Figure 3-22. Information flow of a quality-assurance cycle. Image from NREL

As shown in Figure 3-22, the information flows from data acquisition to quality assessment, where some criteria are used to establish data quality. The results of the quality assessment are analyzed and formed into feedback that goes back to the data acquisition module. The activities in the boxes can take several forms. For example, quality assessment could be the daily site inspection, and the analysis and feedback could be a simple procedure that corrects equipment malfunctions. Or the quality assessment could be a weekly summary of data flags, and the analysis provides a determination of specific instrument error that is transmitted back to maintenance personnel with instructions to correct deficiencies or further troubleshoot problems.

The faster the cycle runs, the sooner errors will be detected, and the fewer bad data will be collected during failure modes. Conversely, if the site is inspected infrequently, the chances 
increase that a large portion of the data set would be contaminated with substandard equipment. More than one quality-assurance cycle can — and likely will — run at any time, each with a different period and emphasis, as noted above: daily inspection, weekly quality reports, monthly summaries, etc.

One practical aspect of this cycle is the importance of positive feedback - a regular report back to site personnel of error conditions and of high-quality operations or data sets exceeding quality thresholds. This positively reinforces a job well done and keeps site operators cognizant that data are being used and that their efforts are an integral part of an ongoing process.

The quality-assurance cycle is a deliberate part of the quality-control process, and it should be well defined and funded to maintain a consistency of data quality over time. After the quality of the data is determined, corresponding conclusions have to be made for the further use of the data. In every case, the quality-assurance has to be included in the data set as metadata. In some cases, the quality of the data can even be improved based on the quality assurance. For example, data gaps from one sensor can be filled with the redundant data. Gap filling is a complex topic that is not described in detail here. To calculate daily, monthly, or yearly sums, gap filling will nearly always be necessary, and it is recommended that the reader considers various publications concerning the topic for this type of correction.

Another systematic error that might be corrected concerns the calibration. If the recalibration of a sensor shows a noticeable change relative to the calibration constant that was used sligtly before the recalibration, the data might be reprocessed with a corrected calibration constant. For sun photometers, this kind of post processing is known from the AERONET Level 2 data (Holben 1998). A noticed change of the calibration constant is assumed to be linear in time, and the data between the two calibrations is then reprocessed with a time series of linearly changing calibration constants.

Also, the systematic effects of soiling on the resource assessment can be reduced by some extent. This requires that an eventual change of the DNI caused by the sensor cleaning is documented. One example for the correction of DNI data can be found in Geuder (2006); however, such a correction can result only in accurate data if the effect of the soiling is small at all times $(<1 \%)$. The availability of rough correction methods does not affect the requirement frequently clean the instruments. 


\section{Modeling Solar Radiation-Current Practices}

\subsection{Introduction}

High-quality solar resource assessment accelerates technology deployment by making a positive impact on decision making and reducing uncertainty in investment decisions. GHI and/or DNI are the quantities of interest for resource assessment and characterization at a particular location. Surface-based measurements of DNI and GHI can be made only on a relatively sparse network, given the costs of O\&M. Nevertheless, observations from ground networks have been used in conjunction with models to create maps of surface solar radiation. Another option is to use information from geostationary satellites to estimate GHI and DNI at the surface (Perez and Ineichen 2002; Pinker and Laszlo 1992). Because geostationary satellite coverage is available at regular intervals on a fixed-grid surface, radiation can be available for the entire globe (at least between approximately -60 degrees and +60 degrees latitudes) at temporal and spatial resolutions representative of a particular satellite.

Solar radiation models that use only ground-measured input parameters were used in the past when satellite or weather-model-derived data was not available. Examples of such models are briefly mentioned in this paragraph for historic reasons. One popular historic model type is based on data from the Campbell Stokes sunshine duration recorder (see Section 3.2.4). The monthly mean GHI is derived using a regression fit to the number of clear hours measured by the sunshine recorder's burn marks. Then regression coefficients are calculated using GHI measurements. There are digital sunshine recorders available that can be used to provide input to these models. The exact method to calculate GHI using sunshine recorder information is empirical and therefore specific to each geographical area and not standardized. Moreover, the meteorological services of some countries, such as the United States and Canada, have stopped measuring sunshine duration because of the limited quality and significance of this measurement, which is not standardized and varies from country to country. In the absence of surface radiation measurements, estimates of surface radiation can also be made using meteorological ground measurements such as cloud cover, temperature, visibility, and water vapor in a radiative transfer model (Marion and Wilcox 1994). Methods have been developed over the years and have been used to create earlier versions of the NSRDB, for instance (George et al. 2007). The METSTAT model uses information about cloud cover, water vapor, ozone, and aerosol optical depth (AOD) to develop empirical correlations to compute atmospheric transmittance extinction during both clear- and cloudy-sky conditions.

This chapter contains an introduction to satellite-based models, information about currently operational models that provide surface radiation data for current or recent periods, a summary of radiative transfer models used in the operational models, and a discussion of uncertainty in solar-based resource assessment.

\subsection{Introduction to Satellite-Based Models}

The goal of satellite-based irradiance models is to use observed information about TOA radiances and albedos to calculate GHI and DNI. During the last decades, satellite-based retrievals of GHI have been used - for example, for climate studies (Justus, Paris, and Tarpley 1986). These methods can be primarily divided empirical and physical methods (Pinker, Frouin, and Li 1995; Schmetz 1989; Myers 2013). The empirical methods are based on developing 
relationships between satellite- and ground-based observations; the physical methods estimate surface radiation directly from satellite information using retrieval schemes to determine the atmospheric properties important to radiative transfer. Empirical methods generally produce only GHI and require additional models to calculate DNI from GHI.

\subsubsection{Geostationary Satellites}

Geostationary satellites near the equator provide continuous global coverage. (Measurements are usable up to 66 degrees north and south latitudes because of the Earth's curvature; see Figure 41). As an example of satellite coverage, the Geostationary Operational Environmental Satellite (GOES) series covers North and South America every 3 hours and the Northern Hemisphere, including the United States, every 30 minutes. Two GOES satellites (GOES-East, or GOES-12; and GOES-West, or GOES-11) operate concurrently and provide 30-minute coverage for the entire United States. The imager instrument on the current GOES satellites measures at 5 wavelength bands, or spectral regions.

The visible channel $(0.64 \mu \mathrm{m})$ has a nominal $1-\mathrm{km}$ resolution; the infrared channels $(3.9 \mu \mathrm{m}, 6.5$ $\mu \mathrm{m}, 10.7 \mu \mathrm{m}$, and $12 \mu \mathrm{m}$ ) have 4-km resolution. The next (future) series of GOES satellites that are expected in 2015 will have a new instrument called the Advanced Baseline Imager, with 5minute coverage at $1-\mathrm{km}$ resolution for 16 channels ( 6 in the visible and near-infrared).

The European Organization for the Exploitation of Meteorological Satellites Union owns the METEOSAT series of satellites that covers Europe and Africa as well as the Indian Ocean. The visible and infrared imager on the METEOSAT first-generation satellites (up to METEOSAT 7) had 3 channels in the visible, water vapor, and infrared. The visible channel produced 8-km nadir resolution; the infrared channel's nadir resolution was $5 \mathrm{~km}$. Repetition frequency is imagery every 30 minutes. The Spin Enhance Visible and Infrared Imager on the METEOSAT Second Generation (MSG) satellites (METEOSAT 8 onward) provide satellite imagery every 15 minutes at a nominal 3-km resolution for 11 channels (Schmetz et al. 2002). The 12th channel, a highresolution visible channel, has a nadir resolution of $1 \mathrm{~km}$.

The Japanese Multifunctional Transport Satellite covers East Asia and the Western Pacific at a 4$\mathrm{km}$ spatial and 30-minute temporal resolution taking measurement in 5 channels. It replaced the GMS series of satellites, which has been in operation since 1977. 


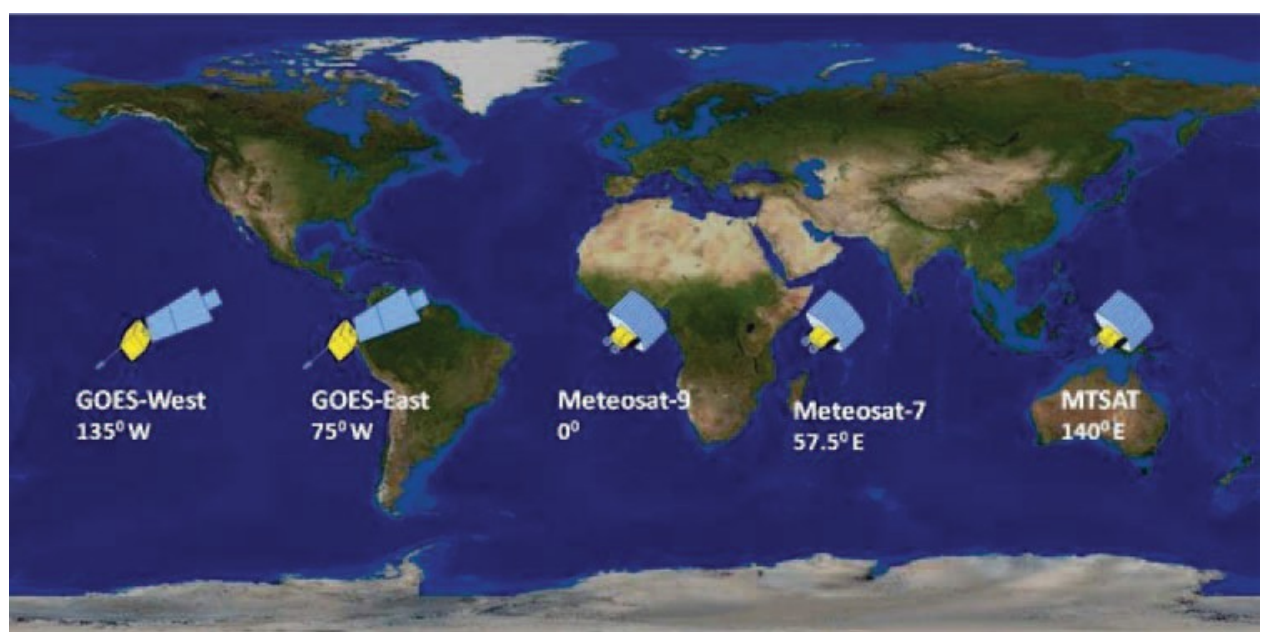

Figure 4-1. The location of the current geostationary satellites that provide coverage around the globe. Image from NOAA

\subsubsection{Polar-Orbiting Satellites}

Polar-orbiting satellites are also used to continuously sense the Earth and retrieve cloud properties and solar radiation at the surface. An example of one such instruments is the Advanced Very High Resolution Radiometer on the NOAA series of polar-orbiting platforms. Another recent example is the Moderate Resolution Imaging Spectroradiometer instrument on NASA's Aqua and Terra satellites. Although polar orbiters provide global coverage, their temporal coverage is limited because of their orbit, in which they essentially cover a particular location only once a day at the lower latitudes.

\subsubsection{Satellite-Based Empirical Methods}

Satellite-based empirical methods consider a pseudo-linear correlation between the atmospheric transmittance and the measurement sensed by the satellite. These methods (correlations) have been used to create regression relationships between what is simultaneously observed by a satellite and ground-based instruments (Cano et al. 1986; Hay, Hanson, and Hanson 1978; Justus, Paris, and Tarpley 1986; Tarpley 1979). Hay, Hanson, and Hanson (1978) created a regression model that relates atmospheric transmittance to the ratio of incoming to outgoing radiation at TOA. The transmittance is then used to compute GHI.

In this method, the coefficients of the regression model change significantly based on location and need to be trained with surface observations (Nunez 1990) to produce accurate results. The Tarpley method also used the well-known relation between surface radiation, the TOA radiation (both upwelling and downwelling), and atmospheric transmittance to create three separate regression equations. The regression equations were classified based on sky conditions labeled as clear, partly cloudy, and cloudy and used accordingly.

\subsubsection{Satellite-Based Physical Models}

Physical models generally use radiative transfer theory to directly estimate surface radiation based on first principles. These can be classified as either broadband or spectral, depending on whether the radiative transfer calculations involve a single broadband calculation or multiple calculations in different wavelength bands. 
The National Renewable Energy Laboratory (NREL), University of Wisconsin, and NOAA collaborated to produce the integration of the Satellite Algorithm for Shortwave Radiation Budget (SASRAB) products into NREL's 4-km by 4-km gridded NSRDB (2014 update). Gridded NSRDB uses a two-stage scheme to first retrieve cloud properties and then use those properties in the SASRAB model to calculate surface radiation (Pinker and Laszlo 1992). The goal of the project is to create a half-hourly, 4-km gridded DNI, GHI, and DHI product of known quality and accuracy for use in the NSRDB.

The broadband method of Gautier, Diak, and Masse (1980) used thresholds depending on multiple days of satellite pixel measurements to determine clear and cloudy skies. Separate clearsky and cloudy-sky models were then used to compute surface DNI and GHI. The clear-sky model initially included water vapor and Rayleigh scattering but progressively added ozone (Diak and Gautier 1983) and aerosols (Gautier and Frouin 1984). Assuming that attenuation caused by the atmosphere does not vary from clear to cloudy conditions, Dedieu, Deschamps, and Kerr (1987) created a method that combines the impact of clouds and the atmosphere. This method again uses a time series of images to determine clear-sky for computing surface albedo. Darnell et al. (1988) created a parameterized model to calculate surface radiation using a product of the TOA insolation, atmospheric transmittance, and cloud transmittance. Developed with data from polar-orbiting satellites, this model used collocated surface and satellite measurements to create relationships between cloud transmittance and planetary albedo.

Möser and Raschke (1983) created a model based on the premise that GHI is related to fractional cloud cover and used it with METEOSAT data to estimate solar radiation over Europe (Möser and Raschke 1984). The fractional sky cover was determined to be a function of satellite measurements in the visible channel. This method uses radiative transfer modeling (Kerschegens, Pilz, and Raschke 1978) to determine the clear- and overcast-sky boundaries. Stuhlmann, Rieland, amd Raschke (1990) have since enhanced the model to include elevation dependence and additional constituents as well as multiple reflections in the all-sky model.

An important spectral model developed by Pinker and Ewing (1985) divided the solar spectrum into 12 intervals and applied the Delta-Eddington radiative transfer (Joseph, Wiscombe, and Weinman 1976) to a three-layer atmosphere. The primary input to the model is cloud optical depth that can be provided from various sources. This model was enhanced by Pinker and Laszlo (1992) and used in conjunction with cloud information from the International Satellite Cloud Climatology Project (ISCCP) (Schiffer and Rossow 1983). Another physical method involves the use of satellite information from multiple channels to derive cloud properties (Stowe, Davis, and McClain 1999) and then computes DNI and GHI using the cloud properties in a radiative transfer model. This method, called CLOUDS, was originally developed for the polar-orbiting satellite data. It uses the advanced, very high-resolution radiometer (AVHRR) instrument on NOAA satellites (Stowe, Davis, and McClain 1999) and has been modified and enhanced to obtain cloud properties from the GOES satellites (Heidinger 2003; Pavlonis, Heidinger, and Uttal 2005). The cloud information is then input to the Pinker and Laszlo (1992) model to produce surface radiation.

Physical models are computationally more intensive than empirical and semiempirical (see next section) models. An advantage of physical models is that they can use additional channels from 
new satellites (such as MSG) to improve cloud property retrieval and, hence, surface radiation modeling.

\subsubsection{Semiempirical Models}

Semiempirical models are so classified because of their hybrid approach to retrieving surface radiation from satellite observations in which normalized satellite-observed reflectance is related to GHI at the surface.

Cloud-cover indices that use visible satellite imagery are first created with budget equations between TOA and surface radiation. Those indices are then used to modify clear-sky GHI and estimate GHI at the ground consistent with the cloud scene. DNI can then be derived from GHI and the clear-sky DNI.

The Cano model (Cano et al. 1986) was modified by Diabate et al. (1988) and Moussu et al. (1989), who used METEOSAT data to develop the HELIOSAT model to create solar resource. This data is available commercially from Mines ParisTech (see Chapter 5). Models such as those developed by Perez et al. (2002) also evolved from Cano et al. (1986) and are currently being used to estimate GHI and DNI. For the United States, data sets created using the Perez et al. (2002) model for the period between 1998 and 2009 are available for free from NREL.

\subsection{Currently Available Operational Models}

The following sections present examples of currently available operational models. Only a selection of models is found. Further public, scientific, and commercial operational models exist and might be of interest for the resource analysis.

\subsubsection{NSRDB (2014 Update)}

To fulfill the needs of solar energy technologies, NREL in collaboration with the University of Wisconsin and NOAA produced a physics-based satellite-derived solar radiation data set as part of the NSRDB. The satellite-based data are available every 30 minutes for 4-km-resolution pixels. The data fields include a solar radiation and meteorological data set. The current release of the data set encompasses the years from 2005 to 2012 .

The model uses a two-stage scheme that retrieves cloud properties and uses those properties in the SASRAB model to calculate surface radiation (Pinker and Laszlo 1992). The product initially generates the cloud properties using the AVHRR Pathfinder Atmospheres-Extended (PATMOS$\mathrm{x}$ ) algorithms (Heidinger et al. 2014) while calculating the GHI and DHI using SASRAB; however, the existing SASRAB model for solar resource assessment has been shown to underestimate DNI and GHI during clear-sky conditions and especially during low SZA conditions. This is because the SASRAB algorithm requires a background reflectance field to calculate solar insolation for the current image. This is generated by recording the second-darkest value for each image pixel from the previous 28 days. This is a visible channel measurement that has been shown to be adversely affected by the Earth's surface reflectivity. Thus, desert environments, snow, or any high-albedo conditions that existed at the time of the background calibration force the model to assume it was actually caused by high atmospheric aerosols. This means that the SASRAB clear-sky shortwave radiation results will often be underestimated compared to the actual surface radiation. At the time of this algorithm's development, in the 
early 1980s, aerosol content over land via satellite imagery did not exist; however, with satellite aerosol data now available, NREL used this opportunity to correct the current and historical SASRAB data (Sengupta et al. 2014a and Sengupta et al. 2014b). The time-series irradiance data for each pixel were quality-checked to ensure that they were within acceptable physical limits, gaps were filled, and the UTC time stamp was shifted to local standard time. Finally, the GOES East and West data sets were blended to create a contiguous national data set of irradiance data for the period from 2005 to 2012 .

\subsubsection{NASA/Global Energy and Water Cycle Experiment Surface Radiation Budget}

To serve the needs of the World Climate Research Programme, Whitlock et al. (1995) developed a global Surface Radiation Budget (SRB) data set using cloud information from the ISCCP C1 data set at a $250-\mathrm{km}$ by $250-\mathrm{km}$ (approximately 2.5 degrees by 2.5 degrees) resolution every 3 hours (Schiffer and Rossow 1983; Zhang et al. 2004). Information from the ISCCP-C1 data set is used as an input into the Pinker and Laszlo (1992) model and the Darnell et al. (1988) model.

The currently available version is the NASA/ Global Energy and Water Cycle Experiment SRB Release-3.0 data sets that contain global 3-hourly, daily, monthly/3-hourly, and monthly averages of surface longwave and shortwave radiative parameters on a 1-degree by 1-degree grid. Primary inputs to the models include:

- Visible and infrared radiances and cloud and surface properties inferred from ISCCP pixel-level (DX) data

- Temperature and moisture profiles from the GEOS-4 reanalysis product obtained from the NASA Global Modeling and Assimilation Office

- Column ozone amounts constituted from a total ozone mapping spectrometer and TIROS Operational Vertical Sounder archives and the Stratospheric Monitoring-group's Ozone Blended Analysis, an assimilation product from NOAA's Climate Prediction Center.

The SRB data set is available from multiple sources. The Surface meteorology and Solar Energy (SSE) Web site provide SRB data in a version that is more applicable to renewable energy. ${ }^{10}$ SRB data sets are also available from the Clouds and the Earth's Radiant Energy System project. ${ }^{11}$ Additionally, the Fast Longwave and Shortwave Radiative Fluxes (FLASHFlux) project makes real-time SRB data. ${ }^{12}$ Both projects use global observations from Clouds and the Earth's Radiant Energy System and moderate-resolution imaging spectroradiometer instruments. Table 4-1 shows the estimated bias and RMS error between measured WMO Baseline Surface Radiation Network (BSRN) monthly averages of the three solar radiation components. The NASA SSE accuracy and methodology are documented on the SSE Web site.

\footnotetext{
${ }^{10}$ See http://eosweb.larc.nasa.gov/sse/.

${ }^{11} \mathrm{See}$ https://eosweb.larc.nasa.gov/project/ceres/ceres table.

${ }^{12}$ See https://eosweb.larc.nasa.gov/project/ceres/flashflux-12 table.
} 
Table 4-1. Regression Analysis of NASA SSE Compared to BSRN Bias and RMS Error for Monthly Averaged Values from July 1983 through June $2006^{13}$

\begin{tabular}{|l|l|r|r|}
\multicolumn{1}{|c|}{ Parameter } & \multicolumn{1}{|c|}{ Region } & Bias (\%) & RMS (\%) \\
\hline GHI & Global & -.01 & 10.25 \\
& $60^{\circ}$ poleward & -1.18 & 34.37 \\
& $60^{\circ}$ equatorward & 0.29 & 8.71 \\
\hline $\mathrm{DHI}$ & Global & 7.49 & 29.34 \\
& $60^{\circ}$ poleward & 11.29 & 54.14 \\
& $60^{\circ}$ equatorward & 6.86 & 22.78 \\
\hline $\mathrm{DNI}$ & Global & -4.06 & 22.73 \\
& $60^{\circ}$ poleward & -15.66 & 33.12 \\
& $60^{\circ}$ equatorward & 2.40 & 20.93 \\
\hline
\end{tabular}

\subsubsection{DLR-ISIS Model}

Similar to the NASA SSE data sets discussed in Section 4.3.2, the DLR-ISIS data $\operatorname{set}^{14}$ is a 21 year DNI and GHI data set ( $280 \mathrm{~km}$ by $280 \mathrm{~km}$ every 3 hours) based on the ISCCP cloud product covering the period from July 1983 through December 2004. The cloud products are used in a 2stream radiative transfer model (Kylling, Stamnes, and Tsay 1995) to compute DNI and GHI. The correlated-k method from Kato et al. (1999) is used to compute atmospheric absorption in the solar spectrum. Scattering and absorption in water clouds are analyzed using the parameterization of $\mathrm{Hu}$ and Stamnes (1993); ice cloud properties are obtained from Yang et al. (2000) and Key et al. (2002). Fixed effective radii of $10 \mu \mathrm{m}$ and $30 \mu \mathrm{m}$ are used for water and ice clouds, respectively. The radiative transfer algorithm and parameterizations are included in the radiative transfer library libRadtran (Mayer and Kylling 2005). ${ }^{15}$

The complete method for creating the DLR-ISIS data set using the ISCCP cloud products and the libRadtran library is outlined in Lohmann et al. (2006). The cloud data used for the derivation of the DLR-ISIS data set are taken from the ISCCP FD (global radiative flux data product) input data set (Zhang et al. 2004), which is based on ISCCP D1 cloud data. (See the ISCCP homepage for more information about cloud data sets. ${ }^{16}$ ) It provides 3 -hour cloud observations on a $280-\mathrm{km}$ by $280-\mathrm{km}$ equal area grid. The whole data set consists of 6,596 grid boxes on 72 latitude steps of 2.5 degrees. This grid is maintained for the DLR-ISIS data set.

ISCCP differentiates among 15 cloud types. The classification includes three intervals of optical thickness in three cloud levels: low, middle, and high clouds. Low and middle cloud types are further divided into water and ice clouds; high clouds are always ice clouds.

For DLR-ISIS, optical thickness, cloud top pressure, and cloud phase given in the ISCCP data set are processed to generate clouds for the radiative transfer calculations. One radiative transfer

\footnotetext{
${ }^{13}$ From https://eosweb.larc.nasa.gov/cgi-bin/sse/sse.cgi? + s06\%23s 06.

${ }^{14} \mathrm{See}$ http://www.pa.op.dlr.de/ISIS/.

${ }^{15}$ See www.libradtran.org/doku.php.

${ }^{16}$ See http://isccp.giss.nasa.gov.
} 
calculation is carried out for each occurring cloud type assuming 100\% cloud coverage, plus one calculation for clear sky. For the final result, irradiances are weighted with the cloud amount for each cloud type and for clear sky, respectively.

\subsubsection{HelioClim}

The HELIOSAT method based on Cano et al. (1986) is used to produce the HelioClim database (Rigollier et al. 2004) that uses METEOSTAT data. ${ }^{17}$ HelioClim covers Europe, Africa, the Mediterranean Basin, the Atlantic Ocean, and part of the Indian Ocean. Mines ParisTech Armines produced data that can be accessed through the SODA Service. ${ }^{18}$ Mines ParisTech produced the method HELIOSAT-2 in November 2002, partly with the support of the European Commission; HELIOSAT-4 is being developed by Mines ParisTech and the DLR. Table 4-2 shows representative differences through comparisons of HelioClim modeled data and ground measurements in Europe and Africa between 1994 and 1997 (Lefèvre, Wald, and Diabate 2007).

Table 4-2. HelioClim Compared to Ground Bias and RMS Error for Monthly Averaged Values from 1994 through 1997

\begin{tabular}{|l|l|c|c|}
\multicolumn{1}{c|}{ Parameter } & \multicolumn{1}{|c|}{ Region } & Bias (\%) & RMS (\%) \\
\hline \multirow{3}{*}{ GHI } & Europe & $-9 \%$ to $-1 \%$ & $25 \%$ \\
& Africa & $-3 \%$ to $+4 \%$ & $18 \%$ \\
\hline
\end{tabular}

\subsubsection{Solar Energy Mining}

Solar Energy Mining (SOLEMI) is a service from DLR that provides irradiance data commercially and for scientific purposes. The data are based on global atmospheric data sets (aerosol, water vapor, ozone) from different earth observation sources and climate models as well as cloud data from Meteosat. GHI and DNI data sets are available every hour at a $2.5-\mathrm{km}$ resolution and cover Europe and Africa (1991-2012) and Asia (1999-2012). SOLEMI combines the established HELIOSAT method described by Cano et al. (1986) with schemes from Schillings et al. (2003a) and Rigollier, Lefèvre, and Wald (2004).

\subsubsection{MACC-RAD Services}

Within the radiation subproject (MACC-RAD), existing historical and daily updated databases HelioClim-3 and SOLEMI for monitoring incoming surface solar irradiance were further developed. The new service is called HelioClim-4, and it is jointly provided by DLR and Mines ParisTech Armines. Data are made available both via the Copernicus portal ${ }^{19}$ and the SODA service. ${ }^{20}$ The European Earth observation programme Copernicus aims to provide environmental information to support policy makers, public authorities, and both public and commercial users. Data are provided under the Copernicus data policy, which includes free availability for any use, including commercial use. The preoperational atmosphere service of Copernicus is currently provided through the FP7 projects MACC and MACC-II (Monitoring Atmospheric Composition and Climate). MACC combines state-of-the-art atmospheric modeling

\footnotetext{
${ }^{17}$ See http://www.soda-is.com/eng/helioclim/heliosat.html.

${ }^{18}$ See http://www.soda-is.com/eng/index.html.

${ }^{19}$ See http://www.gmes-atmosphere.eu.

${ }^{20}$ See http://www.soda-pro.com.
} 
on aerosols with Earth observation data to provide information services covering European air quality, global atmospheric composition, climate, and ulttraviolet and solar energy.

The user's guide (Espinar et al. 2014) describes the data, methods, and operations used to deliver time series of solar radiation available at ground surface. The new HELIOSAT-4 method is based on the decoupling solution proposed by Oumbe et al. (2014). In the case of an infinite plane-parallel single- and double-layered cloud, the solar irradiance at ground level computed by a radiative transfer model can be approximated by the product of the irradiance during a clear atmosphere, McClear model (Lefèvre et al. 2013; see section 4.6.4) and a modification factor because of cloud properties and ground albedo only (McCloud) (Espinar et al. 2014).

Table 4-3. Summary of Data Used in MACC-RAD

\begin{tabular}{|l|l|l|l|}
\hline \multicolumn{1}{|c|}{ Variable } & \multicolumn{1}{|c|}{ Data Sources } & Temporal Resolution & Spatial Resolution \\
\hline Aerosols properties and type & MACC & $3 \mathrm{~h}$ & 1.125 degrees \\
\hline Cloud properties and type & APOLLO (DLR) & $15 \mathrm{~min}$ & $3 \mathrm{~km}$ to $10 \mathrm{~km}$ \\
\hline Total column content in ozone & MACC & $3 \mathrm{~h}$ & 1.125 degrees \\
\hline Total column content in water vapor & MACC & $3 \mathrm{~h}$ & 1.125 degrees \\
\hline Ground albedo & Mines ParisTech & $\begin{array}{l}\text { Climatology of } \\
\text { monthly values }\end{array}$ & $6 \mathrm{~km}$ \\
\hline
\end{tabular}

Within McCloud, the clear-sky index, $K c$, is computed by the means of a lookup table approach with SZA, cloud optical depth, and ground albedo as input parameters. Four types of clouds are discriminated: water or mixed-phase clouds at low, medium, and high altitudes and optically thin ice clouds. Cloud information from the Meteosat Second Generation (MSG) satellites are provided by the APOLLO (AVHRR Processing scheme Over clouds, Land and Ocean; Kriebel, Saunders, and Gesell 1989; Kriebel et al. 2003) algorithm. APOLLO provides quantities related to cloud for each pixel (3 km at nadir) and every 15 minutes. Among these quantities are a mask (cloud-free/cloudy) and the type of the cloud (low, medium, high, thin). Cloud coverage-i.e., the fraction of a pixel covered by a cloud, expressed in percent - is derived for each type of cloud separately. Table 4-3 shows an overview of the data used in MACC-RAD.

\subsubsection{Perez/Clean Power Research}

The Perez et al. (2002) method (herein referred to as the Perez State University of New York [Perez SUNY] model) for computing GHI and DNI is based on the concept that atmospheric transmittance is directly proportional to the TOA planetary albedo (Schmetz 1989). This method is being applied to the GOES satellites and is currently available from Clean Power Research. ${ }^{21}$ The concept of using satellite-based measurements of radiance assumes that the visible imagery demonstrates cloud cover for high levels of brightness and lower levels for more clear-sky conditions (e.g., dark ground cover). The method is outlined below, and readers are referred to Perez et al. (2002) for additional details. The method:

\footnotetext{
${ }^{21}$ See www.cleanpower.com.
} 
- Normalizes the satellite measurement with the SZA to remove effects of solar geometry

- Creates a dynamic range of satellite measurements using time-series information for each pixel

- Calculates a "cloud index" for every pixel in an image by scaling with the dynamic range for the corresponding pixel that was created in the previous step

- Uses the SOLIS model (Mueller et al. 2004) to create a GHI map for clear-sky (GHIclr)

- Calculates GHI by scaling GHIclr with the cloud index

- Calculates clear-sky DNI (DNIclr) and DNI from GHIclr and GHI, respectively, using the DIRINT model (Perez et al. 1992)

- Calculates clear-sky DNI from the Bird (see Section 4.6.1) model (DNIclr, Bird) using water vapor, ozone, and AODs as inputs (DNIclr, Bird is estimated to be more accurate than DNIclr.)

- Scales DNIclr, Bird with the ratio of DNI and DNIclr from Step 6 to calculate the DNI.

The above steps are used to calculate DNI and GHI from satellite visible imagery. Some additional corrections and ancillary data are used to make the product more accurate. These include:

- Using snow cover information from the National Snow and Ice Data Center to reset the lower bound of the dynamic range

- Using surface elevation from the U.S. Geological Survey's digital elevation models (DEMs) to adjust for atmospheric optical depth based on elevation

- Adjusting the lower bound of the dynamic range for high AM effects

- Adjusting for specular reflection caused by the angle between the sun and the satellite

- Adjusting the cloud index to a clearness index using a nonlinear conversion process and applying the clearness index to GHI calculations.

\subsubsection{TIER Solar Data Set}

3TIER developed a global solar radiation data set for both GHI and DNI. It follows the method of Perez et al. (2002) using independently developed algorithms. This 12-plus-years data set is available for global locations at a $3-\mathrm{km}$ resolution from $1997 .{ }^{22}$

\subsubsection{SolarGIS}

A new model for the high-performance calculation of global and direct irradiances has been implemented for the region covered by the Meteosat, GOES, and MTSAT satellites covering land between latitudes $60^{\circ} \mathrm{N}$ and $50^{\circ} \mathrm{S}$. The model philosophy is based on the principles of the HELIOSAT-2 calculation scheme (Hammer et al. 2003) and the model by Perez et al. (2002), and it is implemented to operationally process satellite data at a full spatial and temporal resolution.

\footnotetext{
${ }^{22}$ See http://www.3tier.com/static/ttcms/us/documents/education/3TIER-Solar-Satellite-Data-Introduction.pdf.
} 
The model was developed by GeoModel Solar (Cebecauer, Suri, and Perez 2010). The enhancements include:

- Multispectral satellite information to improve classification of snow/land/cloud signals

- A new algorithm to find lower bound values preserving diurnal variability

- Implementation of backscatter correction

- Variable upper bound for dynamic range and cloud index calculations

- Simplified SOLIS clear-sky model

- Downscaling with a high-resolution DEM to include local variability of solar irradiance.

In particular, the following algorithms are implemented:

- Satellite model: modified version of HELIOSAT by Perez et al. (2002), adapted for multispectral MSG data, with improvements of snow classification and cloud index determination

- DHI: Perez model, Perez et al. (1987)

- DNI: DirIndex, Perez et al. (1992 2002)

- Snow detection: Dürr and Zelenka (2009)

- Clear-sky model: broadband simplified Solis (Ineichen 2008)

- Terrain disaggregation: Ruiz-Arias et al. (2010).

\subsubsection{NOAA Global Surface Insolation Project}

NOAA is currently running a physical model that produces GHI at an approximate 4-km resolution for the northern hemisphere. ${ }^{23}$ Its output is called the GOES Surface Insolation Product. The GOES Surface Isolation Product algorithm follows a two-step process and

1. Uses multichannel GOES satellite information and ancillary data sets, including snow cover, surface albedo, and digital elevation to retrieve cloud properties (Heidinger 2003)

2. Uses the cloud properties from Step 1 to produce GHI (Pinker and Laszlo 1992; Laszlo et al. 2008).

Although the GOES Surface Isolation Product was primarily developed to estimate sea surface temperature for coral bleaching and numerical weather prediction (NWP) applications, it can be tailored to CSP needs, because DNI is currently produced, but not saved, in the official product (Laszlo, personal communication).

\subsubsection{EnMetSol Model}

The EnMetSol method is a technique for determining the global radiation at ground by using data from a geostationary satellite (Beyer et al. 1996; Hammer et al. 2003). It is

\footnotetext{
${ }^{23}$ See http://www.ospo.noaa.gov/Products/land/gsip/index_v3.html.
} 
used in combination with a clear-sky model to evaluate the three irradiance parameters: global horizontal, direct irradiance, and diffuse irradiance. The key parameter of the method is the cloud index $n$, which is estimated from the satellite measurements and related to the transmissivity of the atmosphere. The method is used for Meteosat firstand second-generation and GOES data.

The method

- Is performed on historical data, near real time data, and used for short term forecasting (intraday)

- Normalizes the satellite measurement with the SZA to remove the effects of solar geometry

- Calculates a "cloud index" for every pixel in an image by applying the HELIOSAT method

- Uses the clear-sky model of Dumortier (Fontoynont et al. 1998, Dumortier 1998) with the Remund (2009) METEONORM HR high-resolution database for the turbidity input. This model is applied when calculating near-real-time and forecasts of global horizontal and tilted irradiance as input for PV power predictions. The all-sky DHI is calculated with a diffuse fraction model (Lorenz 2007).

- Uses the SOLIS model (Mueller et al. 2004) in combination with monthly averages of AOD (Kinne et al. 2005) and water vapour content (Kalnay 1996) as input parameters to calculate DNI or spectrally resolved solar irradiance. The DNI for all sky conditions is calculated with a beam fraction model (Hammer et al. 2009).

\subsection{Clear-Sky Models Used in Operation Models}

\subsubsection{Bird Clear-Sky Model}

The Bird clear-sky model (Bird and Hulstrom 1981) is a broadband algorithm that produces estimates of clear-sky direct beam, hemispherical diffuse, and total hemispherical solar radiation on a horizontal surface. The model is based on parameterization built using radiative transfer computations and is composed of simple algebraic expressions. Model results are expected to agree within $\pm 10 \%$ with detailed high-resolution spectral and broadband physics based radiative transfer models. The model can be used at 1-min or better resolutions, and it can duly accept inputs at that frequency if available. However, in the absence of high temporal resolution input parameters, climatological or annual average values could be used as inputs to the model. The Bird clear-sky model also forms the basis of the clear-sky part of METSTAT, with only minor modifications. The performance of these two models has been assessed rigorously and compared to other algorithms (Badescu et al. 2012; Gueymard 1993, 2003a, 2003b, and 2012; Gueymard and Myers 2008; Gueymard and Ruiz-Arias 2015).

\subsubsection{ESRA Model}

The ESRA model is another example of a clear-sky model. Used in the HELIOSAT-2 model that retrieves GHI from satellites, this model computes DNI, GHI, and DHI using Rayleigh optical depth, elevation, and the Linke turbidity factor as its inputs. The performance of the model has been evaluated at various locations (Badescu et al. 2012, Gueymard and Myers 2008, Gueymard 2012, and Gueymard and Ruiz-Arias 2015). 


\subsubsection{SOLIS Model}

The SOLIS model (Mueller et al. 2004) is a simple clear-sky model that can calculate DNI, GHI, and diffuse radiation based on an approximation to the Lambert-Beer relation for computing DNI:

$$
\mathrm{I}=\mathrm{I}_{0} \mathrm{e}^{\left(-\mathrm{M}^{*} \tau\right)}
$$

where

- $\tau$ is the atmospheric optical depth at a specific (monochromatic) wavelength

- $\mathrm{M}$ is the optical AM

- $\mathrm{I}_{0}$ is the TOA direct radiation

- I is the DNI at the surface for a monochromatic wavelength.

This equation is modified to account for slant paths and adapted for GHI and diffuse. The modified Lambert-Beer relation (Mueller et al. 2004) is

$$
I(S Z A)=I_{0} e^{(-\tau 0 / \operatorname{Cos} a(S Z A)}
$$

where

- I(SZA) is the irradiance associated with the empirical factor, $a$, used to compute the DNI, DHI, or GHI ( $\mathrm{a}=1$ for DNI)

- $\tau 0$ is the vertical broadband optical depth of the atmosphere

- $\quad$ SZA is the solar zenith angle.

The Beer-Lambert equation is a simple relationship, because it accounts for monochromatic DNI and is impacted only by atmospheric attenuation. On the other hand, DHI and GHI are broadband values that contain energy that is scattered by the atmosphere. The empirical factor is used as an adjustment factor to compute GHI and DHI, as explained in Mueller et al. (2004).

\subsubsection{McClear Model}

The fast clear-sky model called McClear implements a fully physical modeling replacing empirical relations or simpler models used before. It exploits the recent results on aerosol properties and total column content in water vapor and ozone produced by the MACC project. McClear irradiances were compared to 1-minute measurements made under clear-sky conditions at several BSRN stations representative of various climates. For global, respectively direct irradiance, the correlation coefficients range from 0.95 to 0.99 , resp. 0.86 to 0.99 . The bias ranges from 14 to $25 \mathrm{~W} / \mathrm{m}^{2}$, resp. 49 to $+33 \mathrm{~W} / \mathrm{m}^{2}$. The root mean square error (RMSE) ranges from $20 \mathrm{~W} / \mathrm{m}^{2}\left(3 \%\right.$ of the mean observed irradiance) to $36 \mathrm{~W} / \mathrm{m}^{2}(5 \%)$, resp. $33 \mathrm{~W} / \mathrm{m}^{2}(5 \%)$ to 64 $\mathrm{W} / \mathrm{m}^{2}(10 \%)$.

\subsubsection{REST2 Model}

The high-performance REST2 model is based on transmittance parameterizations over two distinct spectral bands separated at $0.7 \mu \mathrm{m}$. The model's development and its benchmarking are 
described by Gueymard (2008). REST2 has been thoroughly validated and compared to other irradiance models under varied atmospheric conditions, including extremely high aerosol loads (Gueymard 2012, Gueymard 2014, Gueymard and Myers 2008, Gueymard and Ruiz-Arias 2015, and Sengupta and Gotseff 2013).

The model is now used in many solar-related applications, including the benchmarking of the radiative output of the Weather Research and Forecasting (WRF) model (Ruiz-Arias et al. 2012), operational derivation of surface irradiance components using MODIS satellite observations (Chen, Zhuang, and He 2014), improvement in GHI to DNI separation modeling (Vindel, Polo, and Antonanzas-Torres 2013), and development of future climate scenarios (Fatichi, Ivanov, and Caporali et al. 2011). REST2 is also being used at NREL and is integrated into its suite of algorithms that will produce future versions of the NSRDB.

\subsection{Model Uncertainty and Validation}

It is important to understand the accuracy of satellite measurements compared to surface data. A satellite pixel provides an estimate of surface radiation based on cloud and aerosol information spread throughout a certain area; the surface observations are based on an instrument viewing the sky from a point. If the satellite pixel size is small enough, parallax errors enter into the comparison. Terrain effects may also influence a comparison in which cloudiness may vary within a short distance. According to Perez et al. (1987), satellite-based retrievals of DNI are accurate to $10 \%$ to $12 \%$. According to Renné et al. (1999) and Zelenka et al. (1999), the targetspecific comparison to ground-based observations will have an RMSE of at least 20\%; the timespecific pixelwide accuracy is $10 \%$ to $12 \%$ on an hourly basis.

The various empirical and theoretical methods discussed above have been tested for accuracy. Although there is no standardized method for accuracy assessment, the authors have mostly reported root mean square deviation and mean bias error (MBE) (absolute or relative). As an example, the physical model of Darnell et al. (1988) was used to compute surface radiation using cloud information from the ISCCP-C1 data. The results were then compared to surface observations collected by the World Radiation Data Center (WRDC) by Darnell and Staylor (1992). The root mean square deviation from this comparison was found to be approximately 16 $\mathrm{W} / \mathrm{m}^{2}$, and the mean bias was approximately $4 \mathrm{~W} / \mathrm{m}^{2}$. (See Tables 4-4 through 4-7.) It should also be noted that interpretation of reported errors is dependent on the spatial and temporal resolution of the data being compared, and that the relative errors in DNI are always larger than in GHI-opposite to what occurs with high-quality measurements. 
Table 4-4. Summary of Applications and Validation Results of Satellite ModelsEmpirical/Statistical Models (Renné et al. 1999)

\begin{tabular}{|c|c|c|c|c|c|}
\hline References & Objective & $\begin{array}{l}\text { Satellite Data/ } \\
\text { Study Period }\end{array}$ & $\begin{array}{l}\text { Location/ } \\
\text { Resolution }\end{array}$ & Methodology & Accuracy \\
\hline Nullet 1987 & $\begin{array}{l}\text { GHI over the } \\
\text { tropical } \\
\text { Pacific }\end{array}$ & $\begin{array}{l}\text { ESSA 1,3,5,7; } \\
\text { ITOS I, NOAA } \\
\text { 1,2; Feb. 1965- } \\
\text { Jan. } 1973\end{array}$ & $\begin{array}{l}\text { Tropical } \\
\text { Pacific, } \\
\text { monthly, } 2.5 \\
\text { km by } 2.5 \mathrm{~km}\end{array}$ & $\begin{array}{l}\text { Cloud cover by } \\
\text { Sadler, Oda, and } \\
\text { Kilonsky 1976; } 2 \\
\text { irradiance models }\end{array}$ & $\begin{array}{l}\text { Three islands } \\
\text { (annual) - } \\
0.5 \% \text { to } \\
+4.4 \%\end{array}$ \\
\hline $\begin{array}{l}\text { Shaltout and } \\
\text { Hassen } \\
1990\end{array}$ & $\begin{array}{l}\text { Seasonal } \\
\text { maps of } \\
\text { daily } \mathrm{GHI} \\
\text { and } \mathrm{DHI}\end{array}$ & $\begin{array}{l}\text { METEOSAT } \\
1100 \text { LST } \\
\text { cloud-cover } \\
\text { images }\end{array}$ & $\begin{array}{l}\text { Egypt, } 1 \\
\text { observation/d, } \\
2.5 \mathrm{~km} \text { by } 2.5 \\
\mathrm{~km} \text { (visible) } \\
\text { and } 5 \mathrm{~km} \text { by } \\
5 \mathrm{~km}\end{array}$ & $\begin{array}{l}\text { Linear regression } \\
\text { with } 24 \text { ground } \\
\text { stations }\end{array}$ & $\begin{array}{l}\mathrm{GHI} \pm 7.0 \% \\
\mathrm{DHI} \pm 12.5 \%\end{array}$ \\
\hline $\begin{array}{l}\text { Delorme, } \\
\text { Gallo, and } \\
\text { Oliveiri } 1992\end{array}$ & $\begin{array}{l}\text { Real-time } \\
\text { daily images }\end{array}$ & $\begin{array}{l}\text { METEOSTAT, } \\
\text { visible, Mar. } \\
\text { 15-Jun. 30, } \\
1990\end{array}$ & $\begin{array}{l}\text { Southern } \\
\text { France, } \\
\text { daily, } 367 \mathrm{~km} \\
\text { by } 725 \mathrm{~km}\end{array}$ & $\begin{array}{l}\text { "Gistel" model } \\
\text { applied to WEFAX } \\
\text { images }\end{array}$ & $\begin{array}{l}\text { Generally } \\
\text { high } \\
\text { inaccuracies }\end{array}$ \\
\hline $\begin{array}{l}\text { Ben Djemaa } \\
\text { and } \\
\text { Delorme } \\
1992 \\
\end{array}$ & $\begin{array}{l}\text { Comparison } \\
\text { to } 7 \text { ground } \\
\text { stations }\end{array}$ & $\begin{array}{l}\text { METEOSAT } \\
\text { B2, Oct. 1985- } \\
\text { Sept. } 1986\end{array}$ & $\begin{array}{l}\text { Tunisia, daily, } \\
30 \mathrm{~km} \text { by } \\
30 \mathrm{~km}\end{array}$ & $\begin{array}{l}\text { "Gistel" model } \\
\text { applied to B2 data } \\
\text { for daily values }\end{array}$ & $\begin{array}{l}0 \%-10 \% \\
(51 \% \text { of data) } \\
-10 \% \text { to } 0 \% \\
(38 \% \text { of data) }\end{array}$ \\
\hline
\end{tabular}


Table 4-5. Summary of Applications and Validation Results of Satellite ModelsEmpirical/Physical Models (Renné et al. 1999)

\begin{tabular}{|c|c|c|c|c|c|}
\hline References & Objective & $\begin{array}{l}\text { Satellite Data/ } \\
\text { Study Period }\end{array}$ & $\begin{array}{l}\text { Location/ } \\
\text { Resolution }\end{array}$ & Methodology & Accuracy \\
\hline $\begin{array}{l}\text { Nunez } \\
1990\end{array}$ & $\begin{array}{l}\text { Solar energy } \\
\text { for Australian } \\
\text { cities }\end{array}$ & $\begin{array}{l}\text { GMS,1986- } \\
1988\end{array}$ & $\begin{array}{l}8 \text { Australian } \\
\text { cities, Daily, } \\
219 \text { km by } \\
177 \text { km }\end{array}$ & $\begin{array}{l}\text { Simple } \\
\text { physical } \\
\text { model by } \\
\text { author }\end{array}$ & $\begin{array}{l}<10 \%(6 \\
\text { cities })>10 \% \\
(2 \text { cities })\end{array}$ \\
\hline $\begin{array}{l}\text { Tarpley } \\
1979\end{array}$ & $\begin{array}{l}\text { GHI from } \\
\text { GOES }\end{array}$ & Summer 1997 & $\begin{array}{l}\text { USA Great } \\
\text { Plains, daily } \\
\text { totals from } \\
\text { hourly images, } \\
50 \mathrm{~km} \text { by } \\
50 \mathrm{~km}\end{array}$ & $\begin{array}{l}\text { Empirical } \\
\text { relation to } \\
\text { ground } \\
\text { stations } \\
\text { coupled with } \\
\text { physical } \\
\text { models }\end{array}$ & $\begin{array}{l}\text { RMSE < } \\
10 \% \text { (daily) } \\
\text { RMSE <20\% } \\
(1 \text { image/d) }\end{array}$ \\
\hline $\begin{array}{l}\text { Klink and } \\
\text { Dollhopf } \\
1986\end{array}$ & $\begin{array}{l}\text { Resource } \\
\text { assessment } \\
\text { for Ohio }\end{array}$ & GOES, 1982 & $\begin{array}{l}8 \text { stations in } \\
\text { Ohio, } 50 \mathrm{~km} \text { by } \\
50 \mathrm{~km}\end{array}$ & Tarpley 1979 & \begin{tabular}{|l|}
$10 \%-12 \%$ \\
RMSE \\
(snow-free) \\
$-3.5 \%$ MBE \\
\end{tabular} \\
\hline $\begin{array}{l}\text { Czeplak, } \\
\text { Noia, and } \\
\text { Ratto } 1991\end{array}$ & $\begin{array}{l}\text { Comparisons } \\
\text { of Tarpley } \\
\text { method }\end{array}$ & $\begin{array}{l}\text { METEOSAT } \\
\text { visible, Nov. } \\
1986\end{array}$ & $\begin{array}{l}\text { Western } \\
\text { Germany, } 8 \mathrm{~km} \\
\text { by } 8 \mathrm{~km}\end{array}$ & Tarpley 1979 & \begin{tabular}{|l}
$21 \%$ RMSE \\
(daily) $11 \%$ \\
RMSE \\
(monthly)
\end{tabular} \\
\hline $\begin{array}{l}\text { Frulla et al. } \\
1988\end{array}$ & $\begin{array}{l}\text { Solar } \\
\text { radiation over } \\
\text { Argentina }\end{array}$ & $\begin{array}{l}\text { GOES-E, } \\
1982-1983\end{array}$ & $\begin{array}{l}\text { Northern } \\
\text { Argentina, } \\
\text { daily, } 1 \mathrm{~km} \text { by } \\
1 \mathrm{~km}\end{array}$ & Tarpley 1979 & $\begin{array}{l}\text { RMSE 10\%- } \\
15 \% \text { (daily) } \\
\text { RMSE 25\% } \\
\text { (hourly) }\end{array}$ \\
\hline $\begin{array}{l}\text { Diabate } \\
\text { et al. } 1989\end{array}$ & $\begin{array}{l}\text { Establish a } \\
\text { HELIOSAT } \\
\text { station }\end{array}$ & $\begin{array}{l}\text { METEOSAT, } \\
\text { 1983-1985 }\end{array}$ & $\begin{array}{l}\text { European and } \\
\text { eastern } \\
\text { Mediterranean, } \\
\text { hourly }\end{array}$ & $\begin{array}{l}\text { HELIOSAT } \\
\text { (Cano et al. } \\
\text { 1986; } \\
\text { Moussu } \\
\text { et al. 1989) }\end{array}$ & $\begin{array}{l}\text { RMSE } \\
0.06 \mathrm{kWh} / \mathrm{m}^{2}\end{array}$ \\
\hline
\end{tabular}


Table 4-6. Summary of Applications and Validation Results of Satellite ModelsBroadband Theoretical Models (Renné et al. 1999)

\begin{tabular}{|c|c|c|c|c|c|}
\hline References & Objective & $\begin{array}{c}\text { Satellite } \\
\text { Data/Study } \\
\text { Period }\end{array}$ & $\begin{array}{l}\text { Location/ } \\
\text { Resolution }\end{array}$ & Methodology & Accuracy \\
\hline $\begin{array}{l}\text { Frouin } \\
\text { et al. } 1988\end{array}$ & $\begin{array}{l}\text { Compare } \\
\text { Gautier's } \\
\text { method to } 5 \\
\text { empirical } \\
\text { models }\end{array}$ & $\begin{array}{l}\text { GMS, } \\
1986-1988\end{array}$ & $\begin{array}{l}8 \text { Australian } \\
\text { cities/daily, } \\
219 \text { km by } \\
177 \text { km }\end{array}$ & $\begin{array}{l}\text { Gautier, } \\
\text { Diak, and } \\
\text { Masse 1980, } \\
\text { with } \\
\text { refinements }\end{array}$ & $\begin{array}{l}\text { RMSE } 12.0 \\
W^{2} \text { (daily) } \\
\text { MBE }-4.9 \\
W^{2}\end{array}$ \\
\hline $\begin{array}{l}\text { Gautier } \\
1988\end{array}$ & $\begin{array}{l}\text { GHI over } \\
\text { oceanic } \\
\text { regions }\end{array}$ & $\begin{array}{l}\text { Summer } \\
1997\end{array}$ & $\begin{array}{l}\text { USA Great } \\
\text { Plains, daily } \\
\text { totals from } \\
\text { hourly } \\
\text { images, } 50 \\
\text { km by } 50 \mathrm{~km} \\
\end{array}$ & $\begin{array}{l}\text { Gautier, } \\
\text { Diak, and } \\
\text { Masse 1980, } \\
\text { with } \\
\text { refinements }\end{array}$ & $\begin{array}{l}\text { RMSE } 12 \\
\mathrm{Wm}^{2} \text { or } 5 \% \\
\text { (daily) } \mathrm{MBE} \\
6 \mathrm{Wm}^{2}\end{array}$ \\
\hline $\begin{array}{l}\text { Darnell } \\
\text { et al. } 1988\end{array}$ & $\begin{array}{l}\text { GHI estimates } \\
\text { using sun- } \\
\text { synchronous } \\
\text { satellites }\end{array}$ & $\begin{array}{l}\text { GOES } \\
1982\end{array}$ & $\begin{array}{l}8 \text { stations in } \\
\text { Ohio, } 50 \mathrm{~km} \\
\text { by } 50 \mathrm{~km}\end{array}$ & $\begin{array}{l}\text { GHI } \\
\text { technique } \\
\text { from sun- } \\
\text { synchronous } \\
\text { satellites }\end{array}$ & $\begin{array}{l}\text { RMSE } \\
19.2 \% \\
\text { (daily) } \\
2.7 \% \\
\text { (monthly) }\end{array}$ \\
\hline $\begin{array}{l}\text { Dedieu, } \\
\text { Descahmps, } \\
\text { and Kerr } \\
1987\end{array}$ & $\begin{array}{l}\text { Calculate GHI } \\
\text { and albedo } \\
\text { from } \\
\text { METEOSAT }\end{array}$ & $\begin{array}{l}\text { METEOSAT } \\
\text { visible, Nov. } \\
1986\end{array}$ & $\begin{array}{l}\text { Western } \\
\text { Germany, } 8 \\
\text { km by } 8 \mathrm{~km}\end{array}$ & $\begin{array}{l}\text { Physical } \\
\text { relationship } \\
\text { between } \\
\text { computed } \\
\text { TOA and } \\
\text { satellite } \\
\text { values }\end{array}$ & $\begin{array}{l}\text { RMSE } \\
19.5 \% \\
\text { (hourly, } \\
\text { noon) } \\
\text { RMSE 6.7\% } \\
\text { (monthly) }\end{array}$ \\
\hline
\end{tabular}

The Kolmogorov-Smirnov test (Massey Jr. 1951) is a rigorous method that is currently gaining acceptance for benchmarking satellite-retrieved GHI and DNI with ground-based observations. This test has the advantage of being nonparametric and is therefore not distribution dependent. It compares the distributions of GHI and DNI obtained from the two sources.

A detailed analysis of uncertainty in models in general is beyond the scope of this handbook; however, it is important to indicate possible sources of these uncertainties. A detailed discussion about the relative importance of these uncertainties is provided in Cebecauer et al. (2011). One important issue of DNI and GHI assessments is the AOD of the atmosphere. Depending on its composition, an aerosol can scatter and/or absorb the incident irradiance and thus affect the DNI and to a lesser extent the GHI. This interaction is called atmospheric extinction. The proportion of absorption and scattering is determined by the aerosol type. For example, mineral dust is a mostly scattering aerosol; black carbon is highly absorbing. To calculate DNI, we need only the aerosol extinction, but GHI calculations are more accurate if the scattering and absorption components are available. AODs vary over the wavelength range, and the use of a single broadband AOD results in additional uncertainties. Climatological AODs can be used for resource assessment but sometimes lead to large DNI errors. This happens in areas of biomass burning, urban air pollution, and dust storms when the use of climatology results smooth out episodic events and ultimately lead to an underestimation of DNI. 
It is difficult to discriminate between clouds and snow cover on the ground by using the satellite visible imagery. Because snow results in an elevated reflection of sunlight, the satellite image may be interpreted as being cloud covered. This results in an underestimation of GHI and DNI. The use of multiple satellite channels in the visible and infrared can solve this issue.

Specular reflection, especially from sandy desert surfaces during certain times of the day, may result in interpretation of the satellite image as cloudy and thus an underestimation of GHI and DNI. This issue can be resolved by theoretically estimating the probability of specular reflection and factoring that into the calculation of surface radiation. 
Table 4-7. Summary of Applications and Validation Results of Satellite ModelsSpectral Theoretical Models (Renné et al. 1999)

\begin{tabular}{|c|c|c|c|c|c|}
\hline References & Objective & $\begin{array}{l}\text { Satellite } \\
\text { Data/Study } \\
\text { Period }\end{array}$ & $\begin{array}{l}\text { Location/ } \\
\text { Resolution }\end{array}$ & Methodology & Accuracy \\
\hline $\begin{array}{l}\text { Möser and } \\
\text { Raschke } \\
1984\end{array}$ & $\begin{array}{l}\text { Solar radiation } \\
\text { over Europe }\end{array}$ & $\begin{array}{l}\text { METEOSAT- } \\
\text { I-II, Jun. } \\
1979 \text { and } \\
\text { Apr. } 1982\end{array}$ & $\begin{array}{l}\text { Europe, } \\
\text { daily ( } 3 \text { to } 6 \\
\text { images } / \mathrm{d} \text { ), } \\
25 \mathrm{~km} \text { by } \\
25 \mathrm{~km}\end{array}$ & $\begin{array}{l}\text { Normalized } \\
\text { reflected } \\
\text { radiance; } \\
\text { 2-stream } \\
\text { radiative } \\
\text { transfer } \\
\text { model }\end{array}$ & $\begin{array}{l}\text { RMSE 5\%- } \\
6 \% \text { (monthly) } \\
\text { RMSE 10\%- } \\
14 \% \text { (daily) } \\
\text { Daily RE } \\
<20 \% \text { (no } \\
\text { snow) > 20\% } \\
\text { (snow) }\end{array}$ \\
\hline $\begin{array}{l}\text { Stuhlmann } \\
\text { et al. } 1990\end{array}$ & $\begin{array}{l}\text { Improve IGMK } \\
\text { model of } \\
\text { Möser and } \\
\text { Raschke 1983 } \\
\text { (cloud } \\
\text { transmittance) }\end{array}$ & $\begin{array}{l}\text { METEOSAT } \\
\text { ISCCP B2 }\end{array}$ & $\begin{array}{l}\text { Europe, } \\
\text { Africa, } \\
\text { Western } \\
\text { South } \\
\text { Africa, } \\
30 \mathrm{~km}- \\
50 \mathrm{~km}\end{array}$ & $\begin{array}{l}\text { Explicitly } \\
\text { account for } \\
\text { multiple } \\
\text { reflections } \\
\text { among } \\
\text { surface and } \\
\text { atmospheric } \\
\text { layers; } \\
\text { improved } \\
\text { clear-sky } \\
\text { algorithm }\end{array}$ & $\begin{array}{l}\text { Monthly } \\
\text { means } \\
\text { generally } \\
\text { within } \pm 10 \% \\
\text { (better over } \\
\text { Europe) }\end{array}$ \\
\hline $\begin{array}{l}\text { Pereira et } \\
\text { al. } 1996\end{array}$ & Surface GHI & $\begin{array}{l}\text { METEOSAT- } \\
\text { II, 1985- } \\
1986\end{array}$ & $\begin{array}{l}\text { Brazil } \\
\text { monthly }\end{array}$ & $\begin{array}{l}\text { IGMK model } \\
\text { (Sthuhlman } \\
\text { et al. 1990) }\end{array}$ & $\begin{array}{l}\text { RMSE } 13 \% \\
\text { MBE } 7 \%\end{array}$ \\
\hline $\begin{array}{l}\text { Raschke et } \\
\text { al. } 1991\end{array}$ & $\begin{array}{l}\text { Solar radiation } \\
\text { atlas for Africa }\end{array}$ & $\begin{array}{l}\text { METEOSAT } \\
\text { ISSCP B2, } \\
\text { 1985-1986 }\end{array}$ & $\begin{array}{l}\text { Africa, } \\
30 \mathrm{~km}-50 \\
\mathrm{~km}(\mathrm{IGMK}), \\
2.5 \mathrm{~km} \\
\text { (HELIOSAT) } \\
\text { monthly } \\
\text { (derived } \\
\text { from 3-h } \\
\text { values) }\end{array}$ & $\begin{array}{l}\text { IGMK } \\
\text { (HELIOSAT } \\
\text { for selected } \\
\text { areas over } \\
\text { western } \\
\text { Africa) }\end{array}$ & $\begin{array}{l}\text { RMSE }-8 \% \text { to } \\
16 \% \text { (monthly) } \\
\text { MBE }-2 \% \text { to } \\
8 \% \text { (monthly) }\end{array}$ \\
\hline $\begin{array}{l}\text { Pinker and } \\
\text { Laszlo } \\
1992\end{array}$ & $\begin{array}{l}\text { Global SRB } \\
\text { estimates }\end{array}$ & $\begin{array}{l}\text { ISCCP C1 } \\
\text { (based on } \\
\text { ISSCP B3) } \\
\text { Jul. 1983 }\end{array}$ & $\begin{array}{l}\text { Global } 2.5 \\
\text { degrees } \\
\text { latitude by } \\
2.5 \text { degrees } \\
\text { longitude }\end{array}$ & $\begin{array}{l}\text { Pinker and } \\
\text { Ewing } 1985\end{array}$ & $\begin{array}{l}\text { High level of } \\
\text { consistency } \\
\text { on global } \\
\text { scale }\end{array}$ \\
\hline
\end{tabular}




\section{Historical Solar Resource Data}

\subsection{Introduction}

Understanding the long-term spatial and temporal variability of available solar resources is fundamental to any assessment of solar energy potential. Information derived from historical solar resource data can be used to make energy policy decisions, select optimum energy conversion technologies, design systems for specific locations, and operate and maintain installed solar energy conversion systems. Historical solar resource data can be the result of insitu measurement programs, satellite remote-sensing methods, or meteorological model outputs. As described in the previous chapters, each type of data has different information content and applicability.

This chapter summarizes historical solar resource data available for the United States and selected international locations. It is an inventory of representative sources of solar radiation data and provides a summary of important data characteristics associated with each data source (e.g., period of record, temporal and spatial resolutions, available data elements, and estimated uncertainties).

NREL and other agencies have made every effort to make data products that are as useful, robust, and as representative as possible; however, the responsibility for applying the data correctly resides with the user. A thorough understanding of the data sources, how they are created, and their limitations is vital to proper application of the resource data to analyses and subsequent decision-making. Discussion and examples of the use of several of these data sets for solar energy applications are presented here. Users are encouraged to read the pertinent sections of this chapter before applying solar resource and meteorological data.

Measured solar irradiance data can provide detailed temporal information for a specific site. Because solar radiation measurement stations are challenging to operate and the data collected are not used for routine weather forecasts, they are few in number and have limited data collection records. The largest national measurement network for obtaining hourly solar resource data in the United States was the 39-station NOAA network, which operated from 1977 through 1980 (See Section 5.4.10). Currently, measured solar irradiance in some form is available from more than 3,000 sites in the United States that are operated by various interests producing data with a wide range of data quality. (See Section 5.4.15 on the photovoltaic geographical information system, or PVGIS.)

Satellite-based observations and mesoscale meteorological models address the needs for understanding the spatial variability of solar radiation resources throughout a range of distances. Present state-of-the-art models provide estimates for GHI and DNI at spatial resolutions of 10 $\mathrm{km}$ or less for the United States. The rapidly growing needs for more accurate solar resource information throughout shorter temporal and smaller spatial scales require the user to fully appreciate the characteristics of all available data, especially those from historical sources.

\subsection{Solar Resource Data Characteristics}

Characterizing the available solar resources for solar energy applications is important for all aspects of realizing the full potential of this utility-scale energy source. Energy policy decisions, engineering designs, and system deployment considerations require an accurate understanding of 
the relevant historical solar resource data, the ability to assess the accuracy of current solar measurement and modeling techniques, and forecasts of the levels of solar irradiance for various temporal and spatial scales.

Solar resource data can be the result of in-situ measurement programs, remote sensing instruments, or meteorological modeling outputs. Each type of data product has different information content and applicability.

Measured solar irradiance data can provide information about the temporal variability at a specific site. Practical radiometer designs were developed in the early 1900s to determine the sun's energy output based on high-altitude measurements of DNI made with pyrheliometers (Hulstrom 1989). To address the needs of agriculture for monitoring such quantities as evapotranspiration, the U.S. Weather Bureau (now National Weather Service) deployed a national radiometer network in the1950s to collect GHI. Since then, radiometer design and data acquisition system performance advanced considerably.

The earliest records of solar flux measurements were based on thermopile-type pyranometer signals recorded and stored on analog strip charts to determine daily amounts of solar flux on a horizontal surface. Today, 1-minute (or shorter) digital recordings are available from fastresponse silicon photodiodes and improved thermopile- type pyranometers and pyrheliometers that are deployed in regional measurement networks to provide solar energy resource data for a variety of applications.

Historically, there have been four radiometer calibration reference scales: Ångström Scale ( $\AA$ S; created in 1905), Smithsonian Scale (SS; created in 1913), International Pyrheliometric Scale (IPS; created in 1956), and the WRR (1979). The relative differences among these scales can introduce a data bias on the order of $2 \%$. The user should be aware of this potential bias in data measured before 1979 .

- $\quad \mathrm{WRR}=1.026(\AA \AA \mathrm{S} 1905)$

- $\quad \mathrm{WRR}=0.977(\mathrm{SS} 1913)$

- $\quad$ WRR $=1.022$ (IPS 1956).

Modeled solar resource data derived from available surface meteorological observations and satellite measurements provide estimates of solar resource potential for locations lacking actual measurements. These modeling methods address the needs for improved spatial resolution of the resource data. The first national effort to model solar resources in the 1970s advanced our understanding of solar radiation distributions based on the then available historical measurements at 26 locations to an additional 222 meteorological observing stations with detailed records of hourly cloud amounts and other relevant data (see Section 5.4.2 on SOLMET/ERSATZ). Today, satellite-based observations of clouds are used to model subhourly surface solar fluxes with a 4-km spatial resolution (see Sengupta et al. [2014]).

\subsection{Long-Term and TMY Data Sets}

Understanding the time frame, or period of record, associated with solar resource and other meteorological data is important for conducting useful analyses. These weather-driven data have 
fluctuations that can range from seconds to years and longer. Long-term data can be representative of the climate if the period of record is at least 30 years. By convention, the meteorological community has deemed that according to the 1933 International Meteorological Conference in Warsaw, a 30-year interval is sufficient to reflect longer-term climatic trends and filter the short-term interannual fluctuations and anomalies. Climate "normals" are recomputed each decade to address temperature, pressure, precipitation, and other surface meteorological variables. Note that the term normal is not equivalent to "average" and has a specific meaning in the meteorological and climatological community. Namely, normal refers to the 30 -year average of an observed parameter that is updated every 10 years (Arguez and Vose 2011). Thus, the averaging period shifts every 10 years.

Often plant project developers require "typical" meteorological information for a potential plant site for prefeasibility studies. A TMY data set provides designers and other users with a small sized annual data set that holds 8,760 hourly meteorological values that typify conditions at a specific location throughout a longer period, such as the 30 -year climatic normal. Different types of TMYs exist. Twelve TMMs (typical meteorological months) selected on the basis of their similarity of individual cumulative frequency distributions for selected data elements comprise the TMY data set. The longer-term distributions are determined for that month using data from the full period of record. The TMMs are then concatenated, essentially without modification, to form a single year with a serially complete data record. The resulting TMY data set contains measured and modeled time-series solar radiation and surface meteorological data, although some hourly records may contain filled or interpolated data for periods when original observations are missing from the data archive.

TMY data sets are widely used by building designers and others for rough modeling of renewable energy conversion systems. Although not designed to provide meteorological extremes, TMY data have natural diurnal and seasonal variations and represent a year of typical climatic conditions for a location. A TMY data set should not be used to predict weather or solar resources for a particular period of time, nor is it an appropriate basis for evaluating real-time energy production or the detailed power plant design. Rather, a TMY data set represents conditions judged to be typical throughout a long period, such as 30 years. Because it represents typical rather than extreme conditions, it is not suited for designing systems and their components to meet the worst-case weather conditions that could occur at a location.

The next section describes the three versions of TMY data for the United States. In 1978, Sandia National Laboratories produced the first TMY for 248 locations using long-term weather and solar data from the 1952-1975 Solar and Meteorological (SOLMET) hourly data set and ERSATZ database (Hall et al. 1978). In 1994, NREL developed the TMY2 using data from the 1961-1990 NSRDB (Marion and Urban 1995). In 2007, NREL released a 15-year updated NSRDB for 1991-2005 (Wilcox 2007) that formed the basis of the TMY3 data set.

\subsubsection{Key Considerations}

Applying solar and meteorological data from different sources requires attention to these key considerations:

- Period of record. Influenced by many factors, solar resource data vary from year to year, seasonally, monthly, weekly, daily, and on timescales down to a few seconds. Thus, 
climate normals are based on 30 years of meteorological data. But the 30 -year averaging period is updated (shifted) every 10 years. The normal for one period will not likely be the same as a normal for previous or successive periods. Another popular approach is to determine a TMY data set from a statistical analysis of multiyear data to derive a single year of data that are representative of a longer-term record. Comparative analyses must account for any natural differences that result from the periods when the data were acquired.

- Temporal resolution. Solar resource data can range from annually averaged dailyintegrated power $\left(\mathrm{kWh} / \mathrm{m}^{2} /\right.$ day) typically used for mapping resource distributions to 1second samples of irradiance $\left(\mathrm{W} / \mathrm{m}^{2}\right)$ for operational time-series analyses. Other considerations depend on the data type.

- Spatial coverage. The area represented by the data can range from a single station to a sample geographic region to a global perspective.

- Spatial resolution. Ground-based measurements are site specific. Current satelliteremote sensing estimates can be representative of $10-\mathrm{km}$ by $10-\mathrm{km}$ or smaller areas.

- Data elements and sources of the data. The usefulness of solar resource data may depend on the available data elements (e.g., DNI) and whether the data were measured, modeled, or produced in combination.

- Data quality control and quality assessments. Descriptions of the measurement operations, model validation methods, and data adjustments or corrections are key metadata elements.

- Estimated uncertainties. Stated uncertainties should include a description of the methodology used to provide this information.

- Availability. Data are distributed in the public domain, for purchase, or license.

- Updates. The need to include the most recent data and other revisions can require regular database updates.

\subsection{Solar Resource Data}

An inventory of solar resource data sources is presented in chronological order.

The attributes of each data source are presented using the list of key considerations (see sidebar). DNI data are available from these sources or can be estimated by using available models and the data elements present in each data set.

\subsubsection{National Center for Environmental Protection/National Center for Atmospheric Research Global Reanalysis Products}

Products from National Center for Environmental Prediction/National Center for Atmospheric Research Reanalysis Project are archived in the data set called ds090.0. The resolution of the global Reanalysis model is $209 \mathrm{~km}$ with 28 vertical levels. Results are available at 6-hour intervals. Although the initial plan was to reanalyze the data for a 40-year period (1957-1996), production has gone back to 1948 and is going forward continuously. Plans call for rerunning the entire period as next generation models are ready (Kalnay et al. 1996; Kistler et al. 2001). 
There are more than 80 variables, including incoming solar radiation (GHI), temperature, relative humidity, and wind components, in several coordinate systems. They are organized as different subgroups in the archive. Some special periods are analyzed more than once to provide data for special research studies.

The Research Data Archive is maintained by the Computational and Information Systems Laboratory at the National Center for Atmospheric Research. The National Center for Atmospheric Research is sponsored by the National Science Foundation. The original data are available from the Research Data Archive in data set number ds090.0. ${ }^{24}$

- Period of record: $1948-2009$

- Temporal resolution: 6-hour $\left(\mathrm{W} / \mathrm{m}^{2}\right)$

- Spatial coverage: Global

- Spatial resolution: 2.5 degrees (nominal)

- Data elements and sources: $\mathrm{GHI}$ and more than 80 variables, including geopotential height, temperature, relative humidity, and $\mathrm{U}$ and $\mathrm{V}$ wind components, in several coordinate systems, such as a 17-pressure-level stack on 2.5- by 2.5-degree grids, 28 sigma-level stacks on 192 by 94 Gaussian grids, and 11 isentropic-level stacks on a 2.5by 2.5 -degree grid

- Data quality control and assessment: No information

- Estimated uncertainties: None stated

- Availability: University Center for Atmospheric Research, Computational and Information Systems Laboratory Research Data Archive: http://rda.ucar.edu/datasets/ds090.0/

- Updates: Monthly.

\subsubsection{SOLMET/ERSATZ}

In response to the energy crisis in the mid-1970s, NOAA and the Energy Research and Development Administration (later the U.S. Department of Energy; DOE) funded the "rehabilitation" of surface meteorological and solar measurement data to create the SOLMET hourly data set. SOLMET data were derived from the best available solar radiation measurements from 26 stations operated by the National Weather Service (NWS) (National Climatic Data Center [NCDC] 1978 and 1979a). Additional ERSATZ data, literally an "inferior substitute," were modeled from available hourly and 3-hour cloud and other surface meteorological observations to expand the data coverage by an additional 222 NWS stations. The SOLMET/ERSATZ database was created to address the needs of the solar energy research and development community. The database provided:

- A single source of merged suitable solar measurements and meteorological data

- Data consistent with SI units

\footnotetext{
${ }^{24}$ See http://rda.ucar.edu.
} 
- Time-series data so users can access the information in true solar and standard time

- Time-series data so users will be aware of the selected meteorological observation that is closest to the time of the solar observation (e.g., selected to be the observation nearest to the midpoint of the solar hour)

- Data recorded in local standard time for conversion to solar time

- A data format with additional solar radiation parameters (direct and tilted, normal incidence, diffuse, and net), as well as additional measurements (ultraviolet and other spectral regions) to be available from stations in the future

- Historical solar radiation data (including the ETR field) converted to the same international scale based on a solar constant value of $1,377 \mathrm{~W} / \mathrm{m}^{2}$

- Eliminated undesirable format features that were inherent in the past data sources, such as overpunches and blanks

- Missing observations and observations that were estimated via models (e.g., bright sunshine duration and cloud regression models)

- Solar GHI data as they were originally observed and that provided the user with data corrected for all known scale, instrument, and calibration problems in addition to a data set corrected via a model.

This database provides some of the earliest measurements of solar irradiance from a national network.

- Period of record: December 1951 through December 1976.

- Temporal resolution: Hourly (hour ending in local solar time)

- Spatial coverage: United States and territories (Figure 5-1)

- Spatial resolution: 26 measurement stations and 222 modeled stations

- Data elements and sources: ETR, GHI (observed-SOLMET or modeled-ERSATZ, engineering corrected, standard-year corrected), direct normal radiation (estimated from global), minutes of sunshine, clouds (ceiling height, total and opaque cloud fractions, and information for up to four cloud layers), and surface meteorological conditions (temperature, wind speed, pressure, snow cover, horizontal visibility, sky condition, and current weather)

- Estimated uncertainties: Based on comparisons to subsequent NOAA network measurements from 1977 to 1980 , the monthly mean daily total SOLMET GHI and DNI accuracies are $\pm 7.5 \%$ and $\pm 10 \%$, respectively. Similarly, the monthly mean daily total ERSATZ GHI and DNI accuracies are $\pm 10 \%$ and $\pm 20 \%$. The modeling method eliminated any evidence for long-term trends in atmospheric opacity resulting from volcanic eruption, urbanization, or other causes. The uncertainty of individual hourly values is higher than the monthly mean daily statistics.

- Availability: NCDC, NOAA, NOAA's Satellite and Information Service (NESDIS), U.S. Department of Commerce: http://www.ncdc.noaa.gov/ 
- Updates: Released in 1978, the SOLMET/ERSATZ database was replaced in 1992 by the 1961-1990 NSRDB.

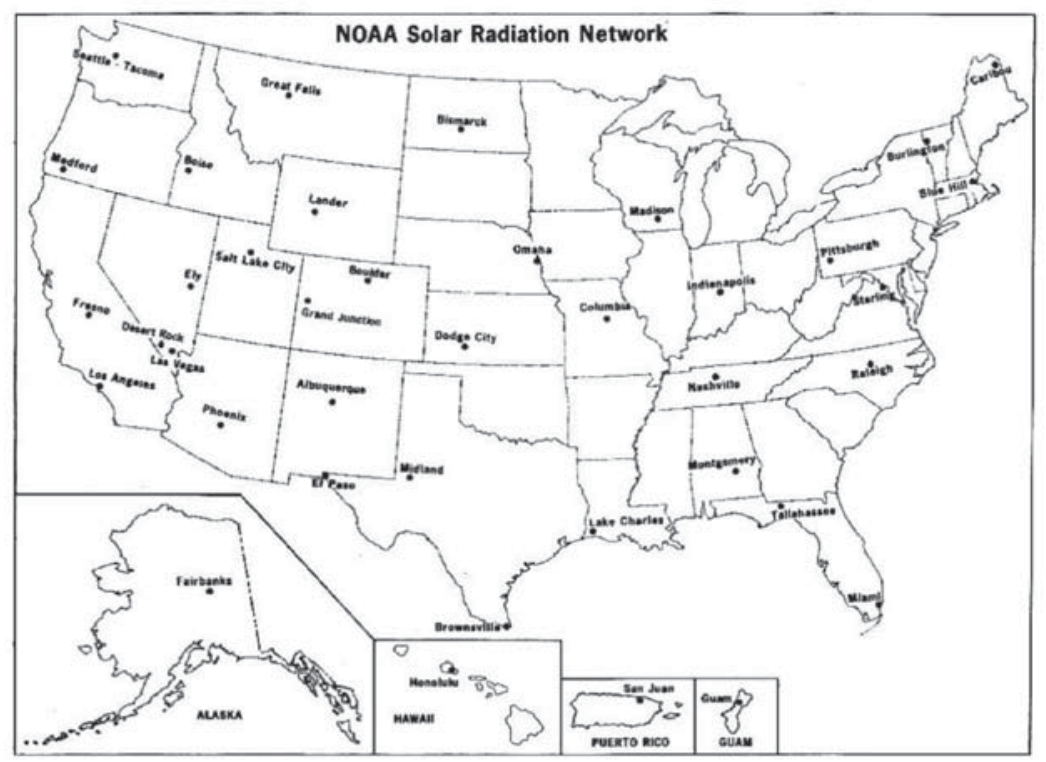

Figure 5-1. SOLDAY and SOLMET measurement stations (26 each). Image from NREL

\subsubsection{SOLDAY}

SOLDAY is the second of two data rehabilitation projects completed for NOAA and the Energy Research and Development Administration (now DOE) to produce a solar resource data set with merged suitable solar measurements and meteorological data consistent with SI (NCDC 1979b). The daily GHI data were reformatted by removing all known procedural and instrumental errors and including all available meteorological elements. Rehabilitated hourly solar measurement stations used in SOLMET were not selected for the SOLDAY format to eliminate data redundancy. Daily GHI data were based on recorded solar radiation on strip charts and daily amounts obtained for a part of the time by summing hand-computed hourly values. For the remainder of the time, daily sums were obtained from a mechanical integrator. This data set provides some of the earliest measurements of solar radiation and complements the geographic distribution of the SOLMET measurement stations.

- Period of record: January 1952 through December 1976

- Temporal resolution: Daily

- Spatial coverage: Continental United States (Figure 5-1)

- Spatial resolution: 26 measurement stations

- Data elements and sources: Computed times of daily sunrise and sunset, ETR (based on solar constant $=1,377 \mathrm{~W} / \mathrm{m}^{2}$ ), measured GHI from mechanical integrators and strip charts and daily amounts calculated by summing hand-computed hourly values, minutes, and percent of possible sunshine, temperature (maximum, minimum, mean), precipitation, snowfall, snow depth, weather codes, and sky cover from hourly observations. None of the 26 SOLDAY measurement stations are in the hourly SOLMET data set. 
- Data quality control and assessment: Individual station histories note pertinent information, making it possible to properly interpret the data. If more than 60 days elapsed between clear solar noon irradiance values, no sky cover/sunshine model was used to fill the irradiance data gaps.

- Estimated uncertainties: Based on the known measurement characteristics of the Eppley Laboratory, Inc., Model 50 and Model PSP pyranometers used to measure GHI at SOLDAY stations, the estimated daily total irradiances are expected to be within $\pm 10 \%$. Measured data from Model PSP radiometers were not corrected for thermal offsets that were discovered much later.

- Availability: NCDC, NOAA, NESDIS, U.S. Department of Commerce: http://www.ncdc.noaa.gov/

- Updates: Released in 1979, the SOLDAY database was replaced in 1992 by the 19611990 NSRDB.

\subsubsection{TMY}

A TMY data set provides a single year of hourly data for solar radiation and other meteorological elements that permit performance comparisons of system types and configurations for one or more locations. Different versions of TMYs exist. Here, the first TMY data set is described. A general description of TMYs can be found at the beginning of the chapter; the other versions of TMYs are described later in this chapter. It should be repeated that a TMY is not necessarily a good indicator of conditions throughout the next year, or even the next 5 years; rather, it represents conditions judged to be typical throughout a long period, such as 30 years. It should also be repeated that TMYs represent typical rather than extreme conditions and that TMYs are therefore not suited for designing systems and their components to meet the worst-case weather conditions that could occur at a location.

The first TMY data set is a subset of the hourly SOLMET measurement and ERSATZ model estimates for 248 locations in the United States and territorial possessions. The TMY data consists of typical months concatenated to form a complete year of 8,760 hourly records. The TMMs were selected in part by comparing weighted cumulative distribution functions of nine data elements, as shown in Table 5-1, to the long-term distributions. Examining the weighted sum of the 13 Finkelstein-Schafer statistics for each year and persistence characterized by frequency and run length above and below fixed long-term percentiles resulted in 5 "candidate years" for the month in question. The final selection of a TMM was somewhat subjective; years with small weighted sum statistics, small deviations, and "typical" run structures were chosen.

TMY data provide hourly GHI and DNI solar data and other surface meteorological elements.

Table 5-1. Weighting Factors Applied to Cumulative Distributions

\begin{tabular}{|l|c|c|c|c|c|c|c|c|c|c|}
\multirow{2}{*}{ Version } & \multicolumn{5}{|c|}{ Temperature } & \multicolumn{3}{c|}{ Wind Velocity } & \multicolumn{3}{c|}{ Solar Radiation } \\
\cline { 2 - 12 } & \multicolumn{4}{|c|}{ Dry Bulb } & \multicolumn{3}{c|}{ Dew Point } & & & \\
& Max. & Min. & Mean & Max. & Min. & Mean & Max. & Mean & GHI & DNI \\
\hline TMY & $1 / 24$ & $1 / 24$ & $2 / 24$ & $1 / 24$ & $1 / 24$ & $2 / 24$ & $2 / 24$ & $2 / 24$ & $12 / 24$ & NA \\
\hline TMY2-3 & $1 / 20$ & $1 / 20$ & $2 / 20$ & $1 / 20$ & $1 / 20$ & $2 / 20$ & $1 / 20$ & $1 / 20$ & $5 / 20$ & $5 / 20$ \\
\hline
\end{tabular}


- Period of record: One year representative of the SOLMET/ERSATZ data period 19521976

- Temporal resolution: Hourly

- Spatial coverage: United States and territories (Figure 5-1)

- Spatial resolution: 26 measurement stations and 222 modeled stations

- Data elements and sources: ETR, GHI (observed-SOLMET or modeled-ERSATZ, engineering corrected, standard-year corrected), direct normal radiation (estimated from global), minutes of sunshine, clouds (ceiling height, total and opaque cloud fractions, and information for up to four cloud layers), and surface meteorological conditions (temperature, wind speed, pressure, snow cover, horizontal visibility, sky condition, and current weather)

- Data quality control and assessment: Measured hourly GHI determined from strip chart recordings and labeled as "observed" data. Known instrument corrections for temperature response were applied to observed GHI and labeled as "engineering corrected" data. Measured data from single-black thermopile radiometers were not corrected for thermal offsets that were discovered much later. Clear-sky model estimates of pyranometer calibration changes were applied to observed GHI and labeled as "standard year irradiance corrected" data. The clear-sky model was also used to fill missing GHI observations. Only the standard year irradiance data field is serially complete. All SOLMET DNI data were computed based on a regression relationship between observed hourly global and DNI measurements taken at five measurement stations: Albuquerque, New Mexico; Livermore, California; Raleigh, North Carolina; Maynard, Massachusetts; and Fort Hood, Texas. All ERSATZ GHI and DNI data were estimated from clear-sky models and available cloud observations.

- Estimated uncertainties: The uncertainty of the data sets that were used for the creation of the TMY can be found in this chapter in the section about the SOLMET/ERSATZ data. The deviation between the TMY and the site-specific actual measurements are not discussed here. A comparison of the TMY to the TMY2 can be found in the following section on the TMY2 in this chapter.

- Availability: NCDC, NOAA, NESDIS, U.S. Department of Commerce: http://www.ncdc.noaa.gov/

- Updates: TMY was released in 1978. TMY Version 2 (TMY2) is based on the 19611990 NSRDB and was available in 1994. TMY Version 3 (TMY3) is based on input data for 1976-2005 from the 1961-1990 NSRDB, Version 1.1 and the 1991-2005 NSRDB update. TMY3 was available in 2008.

Further, NREL recently created a gridded TMY, TDY, and TGY data set for the continental United States. This gridded typical data set is derived from the $10-\mathrm{km}$ by $10-\mathrm{km}$ gridded NSRDB data (for years 1998-2009). The methodology used is similar to that of the TMY2 and TMY3 production; however, additional typical data sets such as TDY and TGY were developed, whereby the weighting focused on the irradiance components of the data rather than the meteorological data. The weighting factor for each solar component was 20/20 for TDY production and 20/20 during the TGY production. 


\subsubsection{1-1990 NSRDB}

NREL completed the 1961-1990 NSRDB in 1992 (NREL 1992). The database consists of serially complete hourly modeled (93\%) and measured (7\%) solar radiation data for 239 locations in the United States. Data records include associated meteorological measurements such as temperature, humidity, cloud cover, and visibility. Measured solar radiation data are included in the data sets when available for 52 NSRDB primary stations, but among those no station has more than a few years of measured data. All remaining GHI solar data were modeled using the METSTAT solar radiation model (Maxwell 1998). The METSTAT model was designed to accept hourly cloud information from the then readily available data from trained NWS observers. DNI measurements were available from primary stations; otherwise, these data were modeled from available meteorological data.

The NSRDB contains statistical summaries computed from the hourly data for the entire period of record for all stations. For the solar radiation data, these statistics include the average and standard deviations of the daily total solar energy (DNI, DHI, and GHI) for each station-yearmonth and each station-year. The 30-year averages and the standard deviations of monthly and annual means from 1961 through 1990 are also provided. For the meteorological elements, only monthly, annual, and 30-year averages were computed.

The hourly statistical products include monthly, annual, and 30-year averages and standard deviations for each hour of the day for GHI, DNI, and DHI. The averages can be used to prepare average diurnal profiles of hourly solar energy. The hourly values have also been binned in 24 $50-\mathrm{Wh} / \mathrm{m}^{2}$ bins from $0 \mathrm{Wh} / \mathrm{m}^{2}$ to $1,200 \mathrm{Wh} / \mathrm{m}^{2}$. The mean number of hourly values falling into each bin has been determined for each station-month for the 30-year period of record from 1961 through 1990. These statistics can be used to plot histograms and determine cumulative frequency distributions.

A solar radiation persistence product was created for each station-month by calculating the number of times the daily total solar radiation energy persisted above or below set thresholds for periods from 1 day to 15 days. These calculations were performed for the entire 30 -year period from 1961 to 1990.

- Period of record: $1961-1990$

- Temporal resolution: Hourly

- Spatial coverage: United States, Guam, and Puerto Rico (Figure 5-2)

- Spatial resolution: 239 stations (56 stations have some radiation measurements)

- Data elements and sources: Hourly GHI, DNI, DHI, ETR, direct normal ETR, total sky cover, opaque sky cover, ceiling height, dry-bulb temperature, dew point temperature, relative humidity, atmospheric pressure, horizontal visibility, wind speed, wind direction, present weather, AOD, total precipitable water, snow depth, and number of days since last snowfall. Approximately $93 \%$ of the irradiance data were modeled from cloud observations. Measured DNI are available from primary stations. 


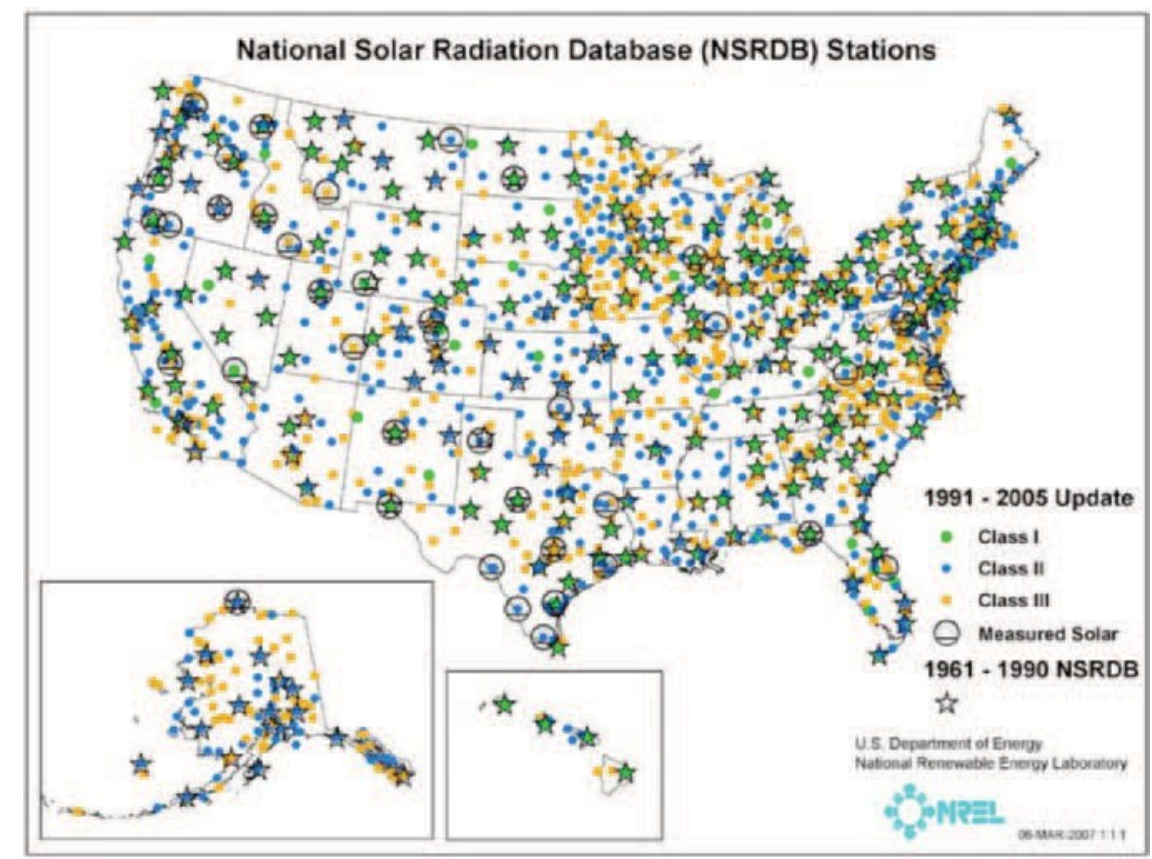

Figure 5-2. Original 239 stations in the 1961-1990 NSRDB released in 1992 (NSRDB 1992 and 1995) and the 1,454 stations in the 1991-2005 NSRDB released in 2007. Image from NREL

- Data quality control and assessment: An automated data processing method was developed to apply quality flags to each hourly solar radiation and meteorological element. These flags provide information about the source and uncertainty of a data element, allowing the user to evaluate its usefulness. Because of the difficulties frequently encountered when measuring solar radiation and the resultant unknown quality of some solar radiation data, a major effort was undertaken to develop procedures and software for performing post-measurement quality assessments of these data. Such assessments were needed to ensure that the data selected for model development and other applications were of the highest quality available. The assessments were also needed to calculate the uncertainty of measured solar radiation data. A quality-assessment software package (SERI QC) was developed to address these needs (Maxwell et al. 1993). SERI QC is based on the establishment of boundaries or limits within which acceptable data are expected to lie. This is similar to previous quality-assessment procedures that used extraterrestrial values for the upper limit and zero for the lower limit within which solar radiation data were expected. SERI QC increased the sophistication of this approach by establishing much more restrictive boundaries specific to each stationmonth. Measured data from single-black thermopile radiometers were not corrected for thermal offsets that were discovered much later.

- Estimated uncertainties: Statistics about the quality of the solar radiation data were determined by calculating the percentage of the hourly values to which each source and uncertainty flag was assigned. These percentages were calculated for each station-year and for the 30-year period of record and are available as a separate product.

- Availability: NCDC, NOAA, NESDIS, U.S. Department of Commerce Renewable Energy Data Sources. Solar data only and documentation are maintained by the NREL 
Renewable Resource Data Center (RReDC):

http://rredc.nrel.gov/solar/old data/nsrdb/1961-1990/

- Updates: Released in 1992; updated in 2007 (Wilcox 2007).

\subsubsection{TMY2}

TMY provides a single year of hourly data for solar radiation and other meteorological elements that permit performance comparisons of system types and configurations for one or more locations. A TMY is not necessarily a good indicator of conditions throughout the next year, or even the next 5 years; rather, it represents conditions judged to be typical throughout a long period of time, such as 30 years. Because they represent typical rather than extreme conditions, they are not suited for designing systems and their components to meet the worst-case conditions occurring at a location.

TMY2 was developed from the 1961-1990 NSRDB. Succeeding the older 1952-1975 SOLMET/ ERSATZ database, the NSRDB accounted for any 1975-1990 climate changes and provided more accurate values of solar radiation for several reasons, including because it provided:

- A better model for estimating values (More than $90 \%$ of the solar radiation data in both databases are modeled.)

- More measured data, some of which are DNI

- Improved instrument calibration methods

- Rigorous procedures for assessing data quality.

A comparison of the older and newer databases provided an incentive for developing the TMY2s. On an annual basis, $40 \%$ of the NSRDB and SOLMET/ERSATZ stations were in disagreement for values of GHI by more than 5\%; some stations showed disagreement of up to $18 \%$ (Marion and Myers 1992). For DNI, $60 \%$ of the NSRDB and SOLMET/ERSATZ stations were in disagreement by more than $5 \%$; some showed disagreement of up to $33 \%$. Disagreement between the two databases is even greater when compared on a monthly basis.

An analysis of cloud cover data indicated little or no change for the two periods; consequently, most of the disagreement for NSRDB and SOLMET/ERSATZ data is attributed to differences in reconstructing the instrument calibrations and differences in the solar radiation models (Marion and Urban 1995). Because of differences in the databases from which they were derived, the old TMYs and the new TMY2s will differ. For some stations the differences may be minor, but for others will be significant.

For the TMY2 and the more recent TMY3 data (see Section 5.4.13 on the Solar Radiation Research Laboratory), the selection of the months in the typical year included a weighting index for DNI radiation (see Table 5-1). This improves the agreement between annual DNI for the TMY and the 30-year annual average by a factor of approximately 2 (based on 20 geographically representative NSRDB stations), as follows. When only GHI is used for the solar index, the TMY annual direct radiation values for the 20 stations were within $4 \%$ (95\% confidence level) of 
the 30 -year annual average. Using both GHI and DNI indices reduced the differences to $2 \%$, with no adverse effect on GHI comparisons.

Because they represent typical rather than extreme conditions, TMYs are not suited for designing systems and their components to meet the worst-case conditions at a location.

- Period of record: One year representative of the 1961-1990 NSRDB data period

- Temporal resolution: Hourly

- Spatial coverage: United States and territories (Figure 5-1)

- Spatial resolution: 239 stations representing the 1961-1990 NSRDB

- Data elements and sources: Hourly GHI, DNI, DHI, ETR, direct normal ETR, total sky cover, opaque sky cover, ceiling height, dry-bulb temperature, dew point temperature, relative humidity, atmospheric pressure, horizontal visibility, wind speed, wind direction, present weather, AOD, total precipitable water, snow depth, and number of days since last snowfall. Approximately $93 \%$ of the irradiance data were modeled from surface observations of clouds. Measured DNI is available from primary stations. The format of the TMY2 data files is different from the format used for the NSRDB and the original TMY data files.

- Data quality control and assessment: The data are serially complete; each hourly record in the file contains values for solar radiation, illuminance, and meteorological elements. A two-character source and uncertainty flag is attached to each data value to indicate whether the data value was measured, modeled, or missing, and to provide an estimate of the uncertainty of the data value. Measured data from single-black thermopile radiometers were not corrected for thermal offsets that were discovered much later.

- Estimated uncertainties: The TMY2 data were compared to 30-year NSRDB data sets to show differences in mean values between TMY2 data and long-term data for the same stations. Comparisons were made on a monthly and an annual basis for GHI, DNI, and south-facing latitude tilt radiation; and for heating and cooling degree-days. These comparisons give general insights into how well, with respect to long-term conditions, the TMY2s portray the mean solar resource and the dry-bulb temperature environment for simulations of solar energy conversion systems and building systems. On an annual basis, the TMY2s compare closely to the 30 -year data sets. The monthly comparisons are less favorable than the annual comparisons (Table 5-2).

Table 5-2. Comparisons of TMY2 Data to 30 Years of NSRDB Data

\begin{tabular}{|l|c|c|}
\multicolumn{1}{|c|}{ Data Element } & \multicolumn{2}{c|}{ Confidence Interval $\left(\mathrm{kWh} / \mathrm{m}^{2} / \mathrm{d}\right)$} \\
& Monthly & Annual \\
\hline $\mathrm{GHI}$ & \pm 0.20 & \pm 0.06 \\
\hline $\mathrm{DNI}$ & \pm 0.50 & \pm 0.16 \\
\hline $\begin{array}{l}\text { Global on tilted surface } \\
\text { (tilt angle = site latitude) }\end{array}$ & \pm 0.29 & \pm 0.09 \\
\hline
\end{tabular}


- Availability: NREL RReDC: http://rredc.nrel.gov/solar/old_data/nsrdb/1961-1990/tmy2/

- Updates: TMY2 was released in 1994. TMY3 is based on input data for 1976-2005 from the 1961-1990 NSRDB, Version 1.1, and the 1991-2005 NSRDB update. TMY3 was available in 2008 (see Section 5.4.24).

\subsubsection{World Meteorological Organization WRDC}

Established in 1962, the WRDC is one of the recognized world data centers sponsored by the WMO. Located at the Main Geophysical Observatory in St. Petersburg (formerly Leningrad), Russian Federation, the WRDC has collected, archived, and published solar radiation data from observing stations from around the world in accordance with Resolution 31 of WMO Executive Committee XVIII, which ensures the availability of these data for research by the international scientific community. Daily total GHI measurements comprise most of the data from the more than 1,000 sites that have contributed to the archive. Some diffuse, sunshine duration, and radiation balance observations are also submitted. Data are submitted primarily by national meteorological services from contributing countries. Some recent hourly measurements are present for a few measurement stations. Dense coverage is available for the western European continent; whereas the South American continent has large unrepresented areas.

- Period of record: 1964 -present

- Temporal resolution: Daily totals with some hourly measurements at a few sites

- Spatial coverage: Global (Figure 5-3)

- Spatial resolution: More than 1,000 measurement stations

- Data elements and sources: Primarily daily total GHI, radiation balance, and sunshine duration, but some DHI and DNI. Some hourly measurements are available from a few sites.

- Data quality control and assessment: In an effort to ameliorate the differing practices among submitting countries, the WRDC has a long-term practice of processing data arrays from many stations; however, the processing of data, and especially quality control, is carried out without knowledge of in-situ weather conditions.

- Estimated uncertainties: No information

- Availability: http://wrdc-mgo.nrel.gov and http://wrdc.mgo.rssi.ru. ${ }^{25}$

\footnotetext{
${ }^{25}$ For more detailed information, contact Voeikov Main Geophyical Observatory, World Radiation Data Centre, 7 , Karbyshev Str., 194021, St. Petersburg, Russian Federation. Telephone: 812-297-43-90; fax: 812-297-86-61. Please direct any comments or suggestions regarding the Web site to Dr. Anatoly V. Tsvetkov, Head of WRDC. Telephone: 812-295-04-45; email: wrdc@main.mgo.rssi.ru or tsvetkov@main.mgo.rssi.ru
} 


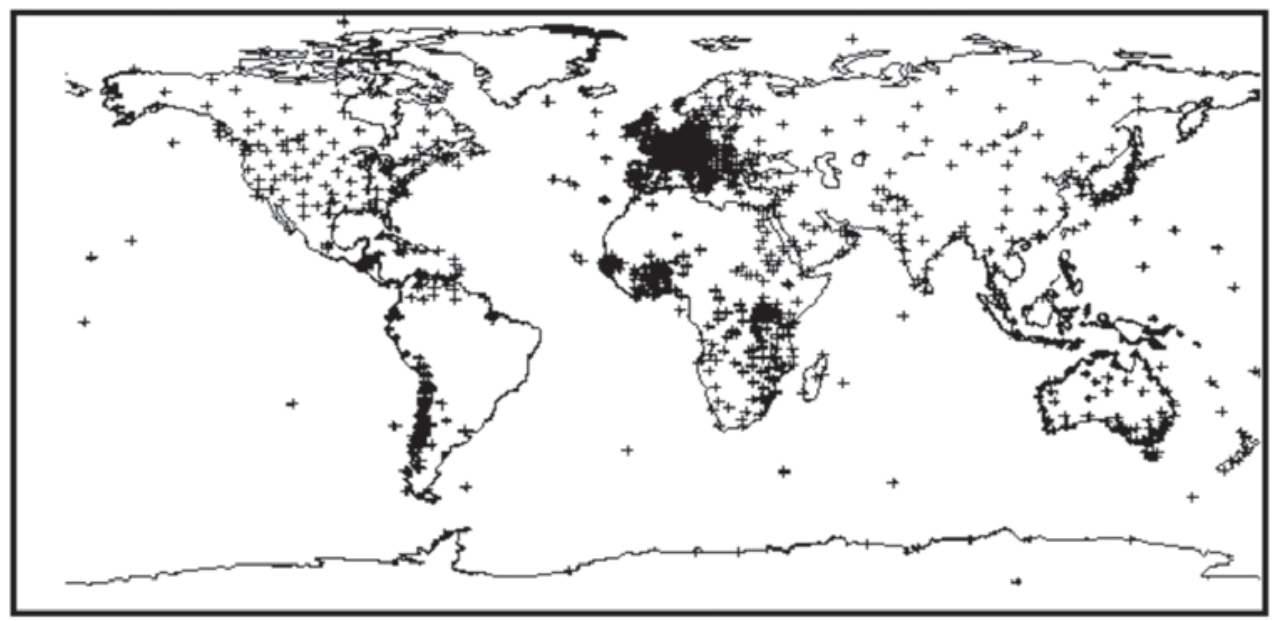

Figure 5-3. WRDC measurement stations. Image from NREL

\subsubsection{Western Energy Supply and Transmission Associates Solar Monitoring Network}

In the mid-1970s, Southern California Edison submitted a proposal to Western Energy Supply and Transmission Associates to expand the solar monitoring effort outside the Southern California Edison service territory in an effort to establish an accurate solar resource database. The resulting Western Energy Supply and Transmission Solar Monitoring Network eventually included 52 stations in 6 western states (Arizona, California, Colorado, Nevada, New Mexico, and Wyoming). The network operated for 5 years from 1976 to 1980 collecting 15-minute GHI, solar DNI, and dry-bulb temperatures. Not all stations were in operation all 5 years, nor did all collect all data parameters. Thirteen stations reported data in the first West Associates Network publication in 1976. All told, during the approximately $4 \frac{1}{2}$ years of network operation, 52 stations gathered data on GHI and ambient temperature. Twenty-six also reported DNI measurements.

- Period of record: $1976-1980$

- Temporal resolution: 15 -minute

- Spatial coverage: Arizona, California, Colorado, Nevada, New Mexico, and Wyoming

- Spatial resolution: 52 measurement stations (Figure 5-4). 


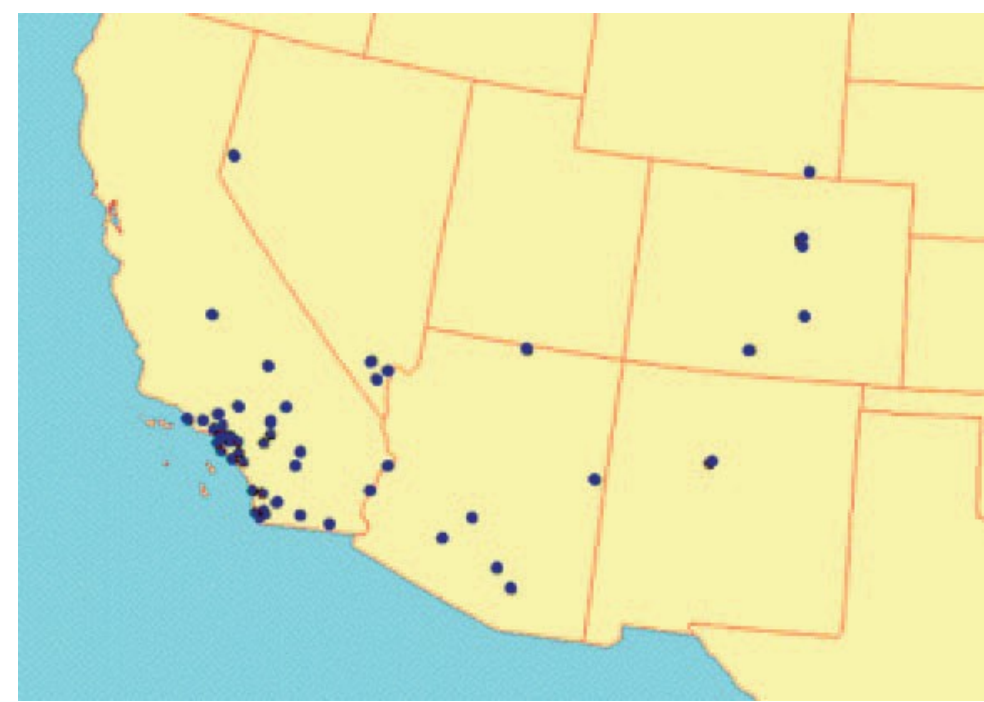

Figure 5-4. Western Energy Supply and Transmission Associates Solar Monitoring Network of 52 measurement stations (1976-1980). Image from NREL

- Data elements and sources: GHI, DNI, and dry-bulb temperature measured with pyranometers (Eppley Black and White, Eppley PSP, and the Spectrolab Spectrosun SR75) and pyrheliometers (Eppley NIP) in automatic solar trackers. DNI was measured at 26 of the 52 stations.

- Data quality control and assessment: Southern California Edison instituted a rigorous program of radiometer maintenance and calibration for the Western Energy Supply and Transmission Associates Solar Monitoring Network. Procedures included maintenance to be performed once per week at stations that monitored GHI and dry-bulb temperature. The pyranometer dome was cleaned and the electronics package checked for correct operation. At stations that also monitored DNI, additional procedures called for maintenance to be performed three times per week. During this maintenance, the pyrheliometer was cleaned and the semiautomatic solar tracker was adjusted for changes in declination and azimuth. All network radiometers were calibrated twice per year to the WRR. Measured data from single-black thermopile radiometers were not corrected for thermal offsets that were discovered much later.

- Estimated uncertainties: Accounting for the frequency of maintenance and radiometer calibrations, the daily total GHI and DNI are likely accurate to $\pm 5 \%$ and $\pm 8 \%$. (DNI uncertainty estimate accounts for semiautomatic operation of the solar tracker requiring manual adjustment for changing solar declination.)

- Availability: NREL RReDC: http://rredc.nrel.gov/solar/pubs/wa/wa index.html

- Updates: Released in 1981.

\subsubsection{Pacific Northwest Solar Radiation Data Network}

The University of Oregon's Pacific Northwest Solar Data Network has the longest continuous record of measured DNI in the United States. Beginning in 1977 with an 11-station network, the goal has remained to provide high-quality scientific data for solar energy resource evaluation and long-term climate studies. The work is made possible by the Bonneville Power Administration, 
Energy Trust of Oregon, Eugene Water and Electric Board, Emerald People's Utility District, NREL, Northwest Power and Conservation Council, and Oregon BEST. Information about the monitoring stations, solar data, software tools, and educational material is available from the University of Oregon's Solar Radiation Monitoring Laboratory. ${ }^{26}$

- Period of record: 1977 -present

- Temporal resolution: 5-minute

- Spatial coverage: Idaho, Montana, Oregon, Utah, Washington, and Wyoming

- Spatial resolution: 39 measurement stations (Figure 5-5)

- Data elements and sources: GHI, DNI, DHI, global irradiance on tilted surfaces (various), spectral irradiance (various), and surface meteorological data (temperature, relative humidity, dew-point temperature, barometric pressure, precipitation, cloud cover, snow depth, etc.)

- Data quality control and assessment: A two-digit data quality control flag is assigned to each data value to identify the type of data (observed, corrected, interpolated, computed, missing, or rejected). Radiometers are calibrated annually with periodic onsite checks with traveling references.

- Estimated uncertainties: Based on the instrument selections, installation, and O\&M practices, the estimated uncertainties for corrected daily total irradiances are DNI $\pm 2 \%$, $\mathrm{GHI} \pm 5 \%$, and $\mathrm{DHI} \pm 15 \%+5 \mathrm{~W} / \mathrm{m}^{2}$

- Availability: http://solardat.uoregon. edu/SolarData.html

- Updates: Continuous.

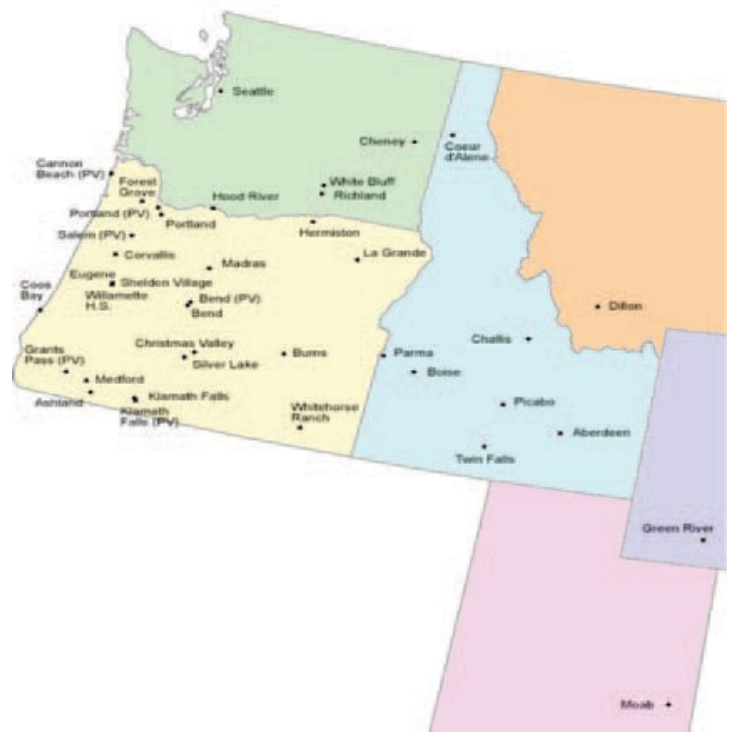

Figure 5-5. Pacific Northwest Solar Radiation Data Network operated by the University of Oregon. Image from NREL

${ }^{26}$ See http://solardat.uoregon.edu/index.html. 


\subsubsection{NOAA Network}

Coincident with the rehabilitation of historical data from NWS in the 1970s, DOE and NOAA cofunded the reconstruction of the NWS solar measurement network. The new network of 39 stations was instrumented with new Eppley Laboratory, Inc., model PSP pyranometers and model NIP pyrheliometers for measuring GHI and DNI. Seven stations had shaded PSP pyranometers for measuring DHI. New data acquisition systems were installed to digitally sample the radiometer signals at 1-minute intervals and provide strip chart records as a backup medium. Radiometers were calibrated annually at NOAA's solar research facility in Boulder, Colorado, using references traceable to the WRR. Network data were processed and disseminated on 9-track magnetic tape reels by NCDC. These data represent the most complete set of solar resource measurements from the largest federally operated measurement network ever fielded in the United States.

- Period of record: $1977-1980$

- Temporal resolution: Hourly

- Spatial coverage: United States and territories (Figure 5-6)

- Spatial resolution: 39 NWS measurement stations

- Data elements and sources: GHI, DNI, DHI (7 stations), air temperature, relative humidity, cloud amounts, barometric pressure, wind speed and direction at $10 \mathrm{~m}$, precipitation, snow cover, and weather codes measured according to standard NWS operating procedures. Radiation measurements are digitally recorded from 1-minute instantaneous samples with redundant strip chart recordings.

- Data quality control and assessment: Data processing performed at the NCDC using standard procedures that included visual inspection of strip chart records. Radiometers were calibrated annually in Boulder, Colorado, with reference radiometers traceable to the WRR. Monthly data reports and digital data files were produced by the NCDC. Measured data from single-black thermopile radiometers were not corrected for thermal offsets that were discovered much later.

- Estimated uncertainties: Based on the instrument selections, installation, and O\&M practices, the estimated uncertainties for corrected daily total irradiances are $\mathrm{DNI} \pm 2 \%$, $\mathrm{GHI} \pm 5 \%$, and $\mathrm{DHI} \pm 15 \%+5 \mathrm{~W} / \mathrm{m}^{2}$.

- Availability: NCDC; National Environmental, Satellite, Data, and Information Service; NOAA; U.S. Department of Commerce: http://www.ncdc.noaa.gov/

- Updates: Final release of TD-9736 occurred in 1983. 


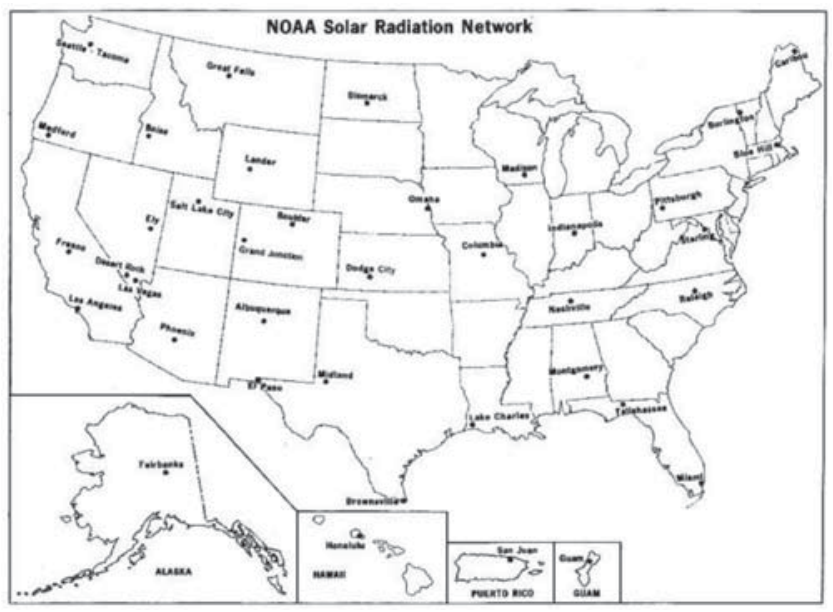

Figure 5-6. NOAA Solar Monitoring Network of 39 stations (1977-1980). Image from NREL

\subsubsection{Solar Energy and Meteorological Research Training Sites}

Recognizing the need to provide an educated workforce and advance the knowledge of solar radiation and meteorological measurements, DOE and the Solar Energy Research Institute (now NREL) solicited responses from U.S. universities and colleges to participate in what became the Solar Energy and Meteorological Research Training Sites program. Central to the multiyear effort was the requirement to maintain the data from 4 of the original 6 participants as part of its RReDC (data from Davis, California, and Honolulu, Hawaii, were never made available) and to produce a minimum of 12 months of solar resource data from precision instruments with measurements collected at 1-minute intervals.

- Period of record: $1979-1983$

- Temporal resolution: 1-minute

- Spatial coverage: Fairbanks, Alaska; Atlanta, Georgia; Albany, New York; San Antonio, Texas (see Figure 5-7)

- Spatial resolution: Four measurement stations

- Data elements and sources: GHI, DNI, and DHI; global irradiance on tilted surfaces (varies), infrared irradiances, ultraviolet and other spectral irradiance (varies), and surface meteorological conditions (temperature, relative humidity, pressure, visibility, wind speed and direction at $10 \mathrm{~m}$, precipitation, etc.).

- Data quality control and assessment: Research-quality data from proper instrument selection, installation, and maintenance. Data were used to develop automated qualityassessment methods. Measured data from single-black thermopile radiometers were not corrected for thermal offsets that were discovered much later.

- Estimated uncertainties: Based on radiometer types, installation, and O\&M, the data uncertainties for daily irradiances were GHI $\pm 7 \%$, DNI $\pm 3 \%$, and $\mathrm{DHI} \pm 15 \%+5 \mathrm{~W} / \mathrm{m}^{2}$.

- Availability: NREL's RReDC: http://rredc.nrel.gov/solar/old_data/semrts/

- Updates: Released in 1985. 


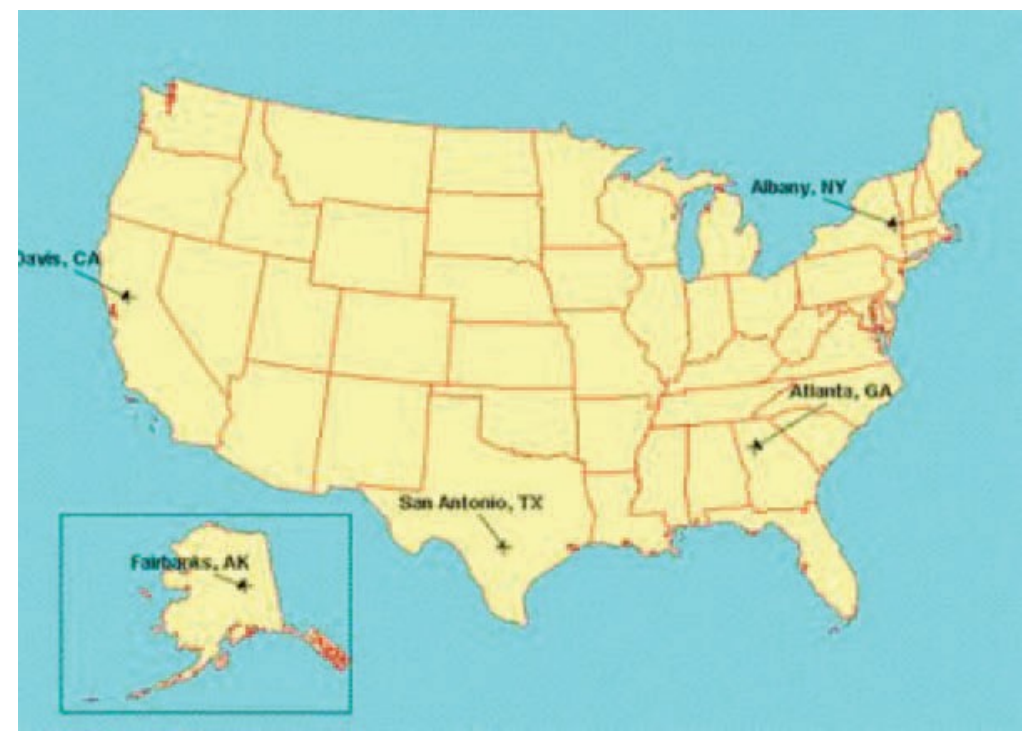

Figure 5-7. The Solar Energy and Meteorological Research Training Sites program provided the first 1-minute measurements of multiple solar resource parameters for the United States. Image from NREL

\subsubsection{DAYMET}

DAYMET generates daily surfaces of temperature, precipitation, humidity, and GHI throughout large regions of complex terrain. The model was developed at the University of Montana's Numerical Terradynamic Simulation Group to meet the needs for high-resolution, daily meteorological and climatological data necessary for plant growth model inputs (Thornton, Hasenauer, and White 2000; Thornton and Running 1999). A DEM and daily observations of minimum and maximum temperatures and precipitation from ground-based meteorological stations were used to produce an 18-year daily data set (1980-1997) as a continuous surface at a $1-\mathrm{km}$ resolution. A wide range of summary and point daily data throughout the conterminous United States is available.

- Period of record: $1980-1997$

- Temporal resolution: Daily

- Spatial coverage: Continental United States

- Spatial resolution: $1 \mathrm{~km}$

- Data elements and sources: GHI, air temperature (minimum and maximum), relative humidity, and precipitation

- Data quality control and assessment: No information

- Estimated uncertainties: No information

- Availability: http://daymet.ornl.gov/.

\subsubsection{Solar Radiation Research Laboratory}

The Solar Radiation Research Laboratory was established at the Solar Energy Research Institute (now NREL) in 1981 to provide continuous measurements of the solar resources, outdoor 
calibrations of pyranometers and pyrheliometers, and to characterize commercially available instrumentation. The Solar Radiation Research Laboratory is an outdoor laboratory located on South Table Mountain, a mesa providing excellent solar access throughout the year, overlooking Denver. Beginning with the basic measurements of DNI, GHI, and DHI at 5-minute intervals, the Solar Radiation Research Laboratory Baseline Measurement System now produces more than 130 data elements at 1-minute intervals that are available from the Measurement and Instrumentation Data Center Web site. ${ }^{27}$

- Period of record: $1981-$ present

- Temporal resolution: 5-minute (beginning July 15, 1981); 1-minute (beginning January 13, 1999)

- Spatial coverage: Golden, Colorado (Figure 5-8)

- Spatial resolution: Research measurement station.

Data elements and sources: GHI, DNI, DHI (from shadowband and tracking disk), global on tilted surfaces, reflected solar irradiance, ultraviolet, infrared (upwelling and downwelling), photometric and spectral radiometers, sky imagery, and surface meteorological conditions (temperature, relative humidity, barometric pressure, precipitation, snow cover, and wind speed and direction at multiple levels).

Data quality control and assessment: Daily instrument maintenance (M-F) with automated data quality control based on real-time examinations of redundant instrumentation and internal consistency checks using the SERI QC methodology (Maxwell et al. 1993). Operators are notified of equipment problems by automatic e-mail messages generated by the data acquisition and processing system. Radiometers are recalibrated at least annually with reference instruments traceable to the WRR. An instrument characterization study is available (Wilcox and Myers 2008, Gueymard and Myers 2009, and Habte et al. 2014). Beginning in 2000, measured data from single-black thermopile radiometers are corrected for thermal offsets that were discovered at that time.

- Estimated uncertainties: Based on the instrument selections, installation, and O\&M practices, the estimated uncertainties for corrected daily total irradiances are $\mathrm{DNI} \pm 2 \%$, $\mathrm{GHI} \pm 5 \%$, and $\mathrm{DHI} \pm 15 \%+5 \mathrm{~W} / \mathrm{m}^{2}$ (GHI data from thermopile-based detectors under clear-sky conditions can exhibit a bias of up to $-2.5 \%$ if not corrected for thermal offsets).

- Availability: NREL Measurement \& Instrumentation Data Center: http://www.nrel.gov/midc/srrl_bms/

- Updates: Data are updated at least hourly.

\footnotetext{
${ }^{27}$ See www.nrel.gov/midc/srrl_bms.
} 


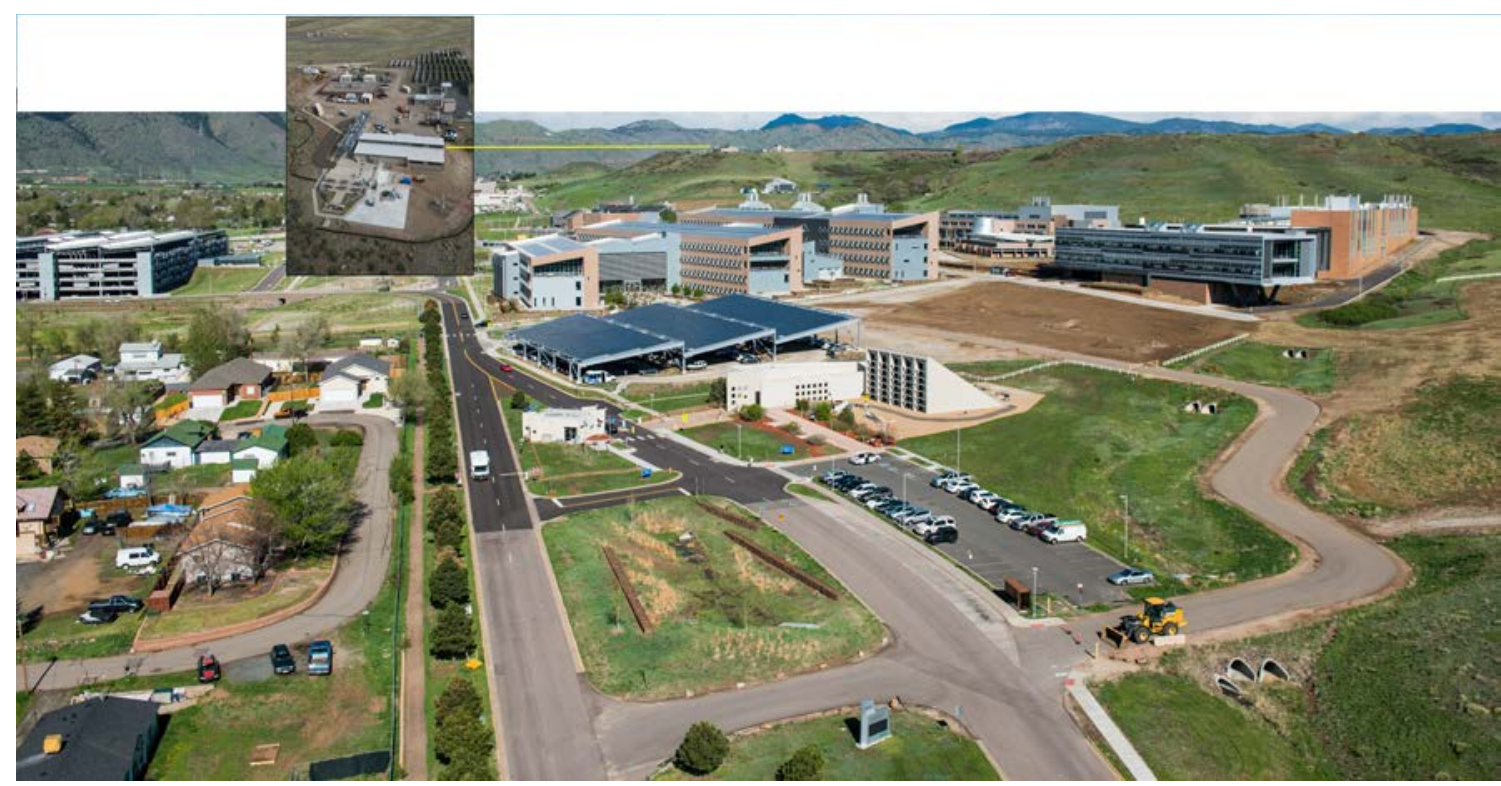

Figure 5-8. NREL's main campus and the Solar Radiation Research Laboratory on South Table Mountain. Photo from NREL

\subsubsection{ESRA}

This atlas is a software package offering solar resources for Europe in a broad sense, from Ural to Azores and from Northern Africa to the Polar Circle. It is a powerful tool for architects, engineers, meteorologists, agronomists, local authorities, tourism professionals, researchers, and students. It covers the period from 1981 through 1990. The volume containing the CD-ROM database offers spatial (approximately every $10 \mathrm{~km}$ ) and temporal knowledge for different time scales (from climatologically means for more than 700 stations to hourly values for 7 stations) on the solar resources: irradiation (global and its components), sunshine duration, as well as air temperatures, precipitation, water vapor pressure, and air pressure in a number of stations.

The software uses the database in either a map or a station mode at the user's choice. More than 50 maps provide information about global irradiation, direct and diffuse components, and the clearness index. After a station has been selected, the program looks for all the data available for this station. The software includes algorithms covering solar geometry, optical properties of the atmosphere, estimation of hourly slope irradiation under cloudless skies, estimation of solar irradiation values (from daily to hourly values, conversion from horizontal to titled surfaces), spectral irradiance, illuminance, and daily mean profiles of temperature and other statistical quantities (central moments, extremes, probability, cumulative probability, and utilization curves). Graphics can be displayed in two or three dimensions.

- Period of record: $1981-1990$

- Temporal resolution: Monthly and annual average daily totals $\left(\mathrm{kWh} / \mathrm{m}^{2} /\right.$ day $)$

- Spatial coverage: Europe

- Spatial resolution: $10 \mathrm{~km}$ 
- Data elements and sources: GHI, DNI, and DHI, sunshine duration, air temperatures, precipitation, water vapor pressure, and air pressure in a number of stations

- Data quality control and assessment: No information

- Estimated uncertainties: No information

- Availability: Les Presses Mines Paris Tech: http://www.minesparistech.fr/Ecole/Culture-scientifique/Presses-des-mines/\#54. See also http://www.sodais.com/eng/index.html

- Updates: No information.

\subsubsection{PVGIS}

PVGIS provides a map-based inventory of solar energy resource and assessment of the electricity generation from PV systems in Europe, Africa, and southwestern Asia. It is part of the SOLAREC action that contributes to the implementation of renewable energy in the European Union as a sustainable and long-term energy supply. As the basis for PVGIS, the Joint Research Center of the European Commission has developed a solar radiation database from climatologic data homogenized for Europe and available in the ESRA, using the r.sun model and the interpolation techniques s.vol.rst and s.surf.rst. These geographic resource analysis support system routines are described with references available from the Joint Research Center Web site. $^{28}$

The model algorithm estimates beam, diffuse, and reflected components of the clear-sky and real-sky global irradiance/irradiation on horizontal or inclined surfaces. The total daily irradiation $\left(\mathrm{Wh} / \mathrm{m}^{2}\right)$ is computed by the integration of the irradiance values $\left(\mathrm{W} / \mathrm{m}^{2}\right)$ calculated at regular time intervals throughout the day. For each time step during the day, the computation accounts for sky obstruction (shadowing) by local terrain features (hills or mountains) calculated from the DEM.

The database consists of raster maps representing 12 monthly averages and 1 annual average of daily sums of global irradiation for horizontal surfaces, as well as those inclined at angles of 15, 25, and 40 degrees. In addition to these data, raster maps of clear-sky irradiation, the Linke turbidity, ${ }^{29}$ and the ratio $\mathrm{DHI} / \mathrm{GHI}$ were computed.

- Period of record: $1981-1990$

- Temporal resolution: Annual average $\left(\mathrm{kWh} / \mathrm{m}^{2}\right)$

- Spatial coverage: Europe

- Spatial resolution: $1 \mathrm{~km}$ aggregated to 5 arc-minutes $(\sim 8 \mathrm{~km})$

- Data elements and sources: GHI, DNI, DHI, and POA irradiance based on these inputs for the European subcontinent:

\footnotetext{
${ }^{28}$ See http://re.jrc.ec.europa.eu/pvgis/solres/solresref.htm.

${ }^{29}$ The Linke turbidity factor is an approximation to model the atmospheric absorption and scattering of clear sky DNI solar radiation due to water vapor and aerosols.
} 
- Monthly averages of daily sums of global and diffuse irradiation measured or calculated for 566 ground meteorological stations distributed over the region. The averages represent the period from 1981 to 1990; the data were collected within the ESRA project.

- Linke turbidity derived from the global database (Remund et al. 2003), available also at the SODA.

- DEM with a grid resolution of $1 \mathrm{~km}$ by $1 \mathrm{~km}$; derived from the USGS SRTM data

- CORINE land cover with grid resolution of $100 \mathrm{~m}$ by $100 \mathrm{~m}$

- GLC2000 (Global Land Cover 2000) with a grid resolution of $1 \mathrm{~km}$ by $1 \mathrm{~km}$ GISCO database (copyright of the EuroGeographics Association for the administrative boundaries).

- VMAP0 (Vector Map) and ESRI data.

For the Mediterranean Basin, Africa, and southwestern Asia:

- HelioClim-1 database, consisting of daily sums of GHI calculated from Meteosat Prime images throughout the whole disc. The values represent the period from 1985 to 2004, and the original spatial resolution is a 15 -arc by 15 -arc min (approximately $30 \mathrm{~km}$ by $30 \mathrm{~km}$ on the equator); the data were processed by the HELIOSAT-2 method (Rigollier, Bauer, and Wald 2000)

- Linke turbidity derived from the global database (Remund et al. 2003), available also at the SODA

- DEM with original grid resolution of $1 \mathrm{~km}$ by $1 \mathrm{~km}$; derived from the USGS SRTM data

- Global Land Cover 2000 with original grid resolution of $1 \mathrm{~km}$ by $1 \mathrm{~km}$

○ VMAP0 data.

- Data quality control and assessment: A cross-validation was applied to estimate the predictive accuracy of the modeling approach that better explains the distribution of errors further from the locations with known measurements. The cross-validation error shows the maximum possible error that might occur at the given point if it were not taken into consideration in the interpolation. The average yearly MBE from cross-validation is smaller: $1 \mathrm{Wh} / \mathrm{m}^{2}(0.03 \%)$; but the range of monthly averages of MBE is higher: from -3 $\mathrm{Wh} / \mathrm{m}^{2}$ in January to $4 \mathrm{Wh} / \mathrm{m}^{2}$ in August. The cross-validation RMSE is higher, within the interval from 97 to $299 \mathrm{Wh} / \mathrm{m}^{2} /$ day (4.7\% to $\left.11.2 \%\right)$; and the yearly average is 146 $\mathrm{Wh} / \mathrm{m}^{2}(4.5 \%)$.

- Estimated uncertainties: The model accuracy of the PVGIS values in the database was evaluated against the input meteorological data used in the computation. Comparing the yearly averages of the daily GHI, the MBE is $8.9 \mathrm{Wh} / \mathrm{m}^{2}(0.3 \%)$ and the RMSE is 118 $\mathrm{Wh} / \mathrm{m}^{2}(3.7 \%)$. The average RMSE of the PVGIS data is almost the same as it is for ESRA, and the PVGIS approach shows better performance from October to April. Its advantage is linking the terrain features to changes in radiation fields and considering the shadowing effects. Comparisons of GHI data from 563 measurement stations with 
PVGIS (Version 2) and ESRA raster maps, respectively, indicate that the RMSE of the results from the original measurements of daily global irradiation occur within an interval of $68 \mathrm{Wh} / \mathrm{m}^{2}$ to $209 \mathrm{Wh} / \mathrm{m}^{2}$. In relative terms, it is within the interval of $3.2 \%$ to $7.8 \%$; the RMSE values peak in winter months. The comparison of the ESRA interpolation approach shows that although the overall accuracy is practically the same (the yearly average of the RSME for ESRA is $113 \mathrm{Wh} / \mathrm{m}^{2}$, i.e., 3.5\%), the PVGIS modeled values are slightly better during the period from October to April and poorer in summer months.

- Availability: Joint Resarch Center of the European Commission, Institute for Energy, Renewable Energy Unit: http://re.jrc.ec.europa.eu/pvgis/download/download.htm.

\subsubsection{METEONORM}

METEONORM 6.1 (Edition 2009) is a comprehensive meteorological reference incorporating a catalogue of meteorological data and calculation procedures for solar applications and system design at any desired location in the world. METEONORM addresses the needs of engineers, architects, teachers, planners, and anyone interested in solar energy and climatology by providing access to a unified set of data, models, and software tools.

\section{- Database Properties}

- Climatological data from more than 8,055 weather stations (1,422 recording GHI)

- Measured parameters: monthly means of global radiation, temperature, humidity, precipitation, days with precipitation, wind speed and direction, and sunshine duration

○ Time periods 1961-1990 and 1996-2005 for temperature, humidity, precipitation, and wind speed selectable

○ Updated global radiation database for 1981-2000

- Use of satellite data for areas with low density of weather stations

- Inclusion of climate change projections (Hadley CM3 model).

- Model Overview

- Interpolation models to calculate mean values for any site in the world

- 1-minute time resolution for radiation parameters

- Calculation of radiation for inclined surfaces with updated models

- Enhanced temperature and humidity generation for building simulation

- Software functions

- Import of user data (including current data by Internet)

- Effects of high horizon considered in radiation calculation (high horizon calculated automatically for all mountain regions)

- 28 output formats as well as user-definable output format

- 5 languages supported: English, French, German, Italian, and Spanish

- Manual in English, maps and illustrations included on CD-ROM. 
- Period of record: 1981-2000 (GHI database); current user data also accepted by the software

- Temporal resolution: 1-minute and hourly modeled data

- Spatial coverage: Global

- Spatial resolution: Data from 8,055 meteorological stations are interpolated to establish weather data at any specified point

- Data elements and sources: Measured: monthly means of GHI, temperature, humidity, precipitation, wind speed and direction, and bright sunshine duration. Modeled: 1-minute and hourly typical years radiation parameters (GHI, DNI, DHI, global on a tilted surface, downwelling infrared, luminance, and ultraviolet-A and -B), precipitation, and humidity parameters (dew point, relative humidity, mixing ratio, psychrometric temperature).

- Data quality control and assessment: With the Version 6.1 database, solar energy systems can be consistently simulated in all parts of the world. The interpolation errors are within the variations of climate from one year to the next. Extensive testing and validation of the radiation models are documented in the "Handbook Part II: Theory" available from $\mathrm{http}$ ://meteonorm.com/images/uploads/downloads/mn71 theory.pdf.

- Estimated uncertainties: Interpolation of $\mathrm{GHI}-\mathrm{MBE}=0 \mathrm{~W} / \mathrm{m}^{2}$; RSME $=15 \mathrm{~W} / \mathrm{m}^{2}$; for yearly mean GHI- $17 \mathrm{~W} / \mathrm{m}^{2}(10 \%)$

- Availability: METEOTEST GmgH, Bern, Germany: www.meteonorm.com

- Updates: Periodic.

\subsubsection{NASA Surface Meteorology and Solar Energy}

The Prediction of Worldwide Energy Resource project was initiated in 2003 to improve the subsequent releases of SSE and to create new data sets applicable to other industries from new satellite observations and the accompanying results from forecast modeling. The Prediction of Worldwide Energy Resource Web interface currently encompasses the SSE data set, tailored for the renewable energy industry, as well as parameters tailored for the sustainable buildings community and the bioenergy/agricultural industries. ${ }^{30}$ In general, the underlying data behind the parameters used by each of these industries are the same: solar radiation and meteorology, including surface and air temperatures, moisture, and winds.

The data are on a 1-degree longitude by 1-degree latitude equal-angle grid covering the entire globe (64,800 regions). The data are generated using the NASA GEOS Version 4 (GEOS 4) Multiyear Assimilation Time Series Data. The GEOS 4 data set has a spacing of 1.25 degrees of longitude by 1 degree of latitude. Bilinear interpolation is used to produce 1-degree by 1-degree regions.

The solar energy data are generated using the Pinker and Laszlo shortwave algorithm (Pinker and Laszlo 1992). Cloud data are taken from the ISCCP DX data set. These data are on an equal area grid with an effective $30-\mathrm{km}$ by $30-\mathrm{km}$ pixel size. The output data are generated on a nested grid

\footnotetext{
${ }^{30}$ See http://power.larc.nasa.gov.
} 
containing 44,016 regions. The nested grid has a resolution of 1 degree latitude globally and a longitudinal resolution ranging from 1 degree in the tropics and subtropics to 120 degrees at the poles. This in turn is regridded to a 1-degree equal-angle grid (360 longitudes by 180 latitudes). The regridding method is by replication, wherein any grid region that is larger than 1 degree by 1 degree is subdivided into 1-degree by 1-degree regions, each with the same value as the original.

SSE estimates were compared to ground site data on a global basis. Radiation parameters were compared to data from the BSRN (NASA 2008). Summary results are presented in Table 4-1.

See the NASA SSE Web site. ${ }^{31}$ The source data were downloaded from the SSE Web site at Data Retrieval: Meteorology and Solar Energy $>$ Global data sets as text files. The tabular data were then converted to the shapefile format.

- Period of record: July 1983-June 2005

- Temporal resolution: Monthly and annual average daily totals $\left(\mathrm{kWh} / \mathrm{m}^{2} /\right.$ day $)$

- Spatial coverage: Global

- Spatial resolution: 1 degree

- Data elements and sources: GHI, DNI, and DHI from a satellite remote sensing model. Also available: Estimates of clear-sky GHI, DNI, and DHI and tilted surface irradiance, temperature, pressure, humidity, precipitation, and wind speed

- Estimated uncertainties: Based on comparisons to surface measurements available from the BSRN, the 23-year monthly mean daily total irradiance uncertainties (Bias\%/RMSE\%) for mid-latitudes have been determined for GHI $(0.29 \% / 8.71 \%)$, DHI $(6.86 \% / 22.78 \%)$, and DNI $(2.40 \% / 20.93 \%)$

- Availability: NASA SSE Web site: http://eosweb.larc.nasa.gov/sse/

- Updates: Release 6.0 data set (January 2008).

\subsubsection{DLR ISIS}

The Deutsches Zentrum für Luft-und Raumfahrt (DLR) irradiance at the surface derived from ISCCP cloud data (DLR-ISIS) data set gives an overview of the available TSI worldwide based on radiative transfer model results using cloud properties and cloud amount data supplied from the ISCCP. ${ }^{32}$ The radiative transfer model also uses atmospheric aerosol optical thickness determined from the NASA-GISS data set (Lohmann et al. 2006).

With more than 21 years of model estimates, the data can be used to derive stable long-term averages, evaluate the variability of irradiance from year to year, and study the effects of extreme atmospheric conditions on the irradiance at the surface, for example, after a volcano eruption. The three-hour temporal resolution of ISIS enables the study of daily cycles; however, the spatial resolution of $280 \mathrm{~km}$ by $280 \mathrm{~km}$ is too coarse for site selection (see Section 4.3 .5 on SOLEMI).

\footnotetext{
${ }^{31} \mathrm{See}$ http://eosweb.larc.nasa.gov/sse/.

${ }^{32}$ See http://isccp.giss.nasa.gov.
} 
- Period of record: July 1983-December 2004

- Temporal resolution: 3-hour

- Spatial coverage: Global

- Spatial resolution: $280 \mathrm{~km}$ by $280 \mathrm{~km}$

- Data elements and sources: DNI and GHI from a radiative transfer model using cloud and aerosol inputs

- Data quality control and assessment: Comparison of monthly mean daily total DLRISIS DNI to data from 78 stations shows an average underestimation of $3 \%$ for monthly means. For DLR-ISIS GHI, validation with data from 89 stations indicates an overestimation of monthly means by $3 \%$.

- Estimated uncertainties: No information

- Availability: http://www.pa.op.dlr.de/ISIS/.

\subsubsection{Historically Black Colleges and Universities Solar Measurement Network}

The Historically Black Colleges and Universities Solar Radiation Monitoring Network operated from July 1985 through December 1996. Funded by DOE, the 6-station network provided 5minute averaged measurements of global and diffuse horizontal solar irradiance. The data were processed at NREL to improve the assessment of the solar radiation resources in the southeastern United States (Marion 1994). Three of the stations also measured the DNI with a pyrheliometer mounted in an automatic sun tracker. Historical Historically Black Colleges and Universities data available online include quality-assessed 5-minute data, monthly reports, and plots.

In January 1997, the HBCU sites became part of the CONFRRM solar monitoring network.

- Period of record: $1985-1996$

- Temporal resolution: 5-minute

- Spatial coverage: Southeastern United States: Daytona Beach, Florida; Savannah, Georgia; Itta Bena, Mississippi; Elizabeth City, North Carolina; Orangeburg, South Carolina; and Bluefield, West Virginia

- Spatial resolution: Six measurement stations (Figure 5-9). 


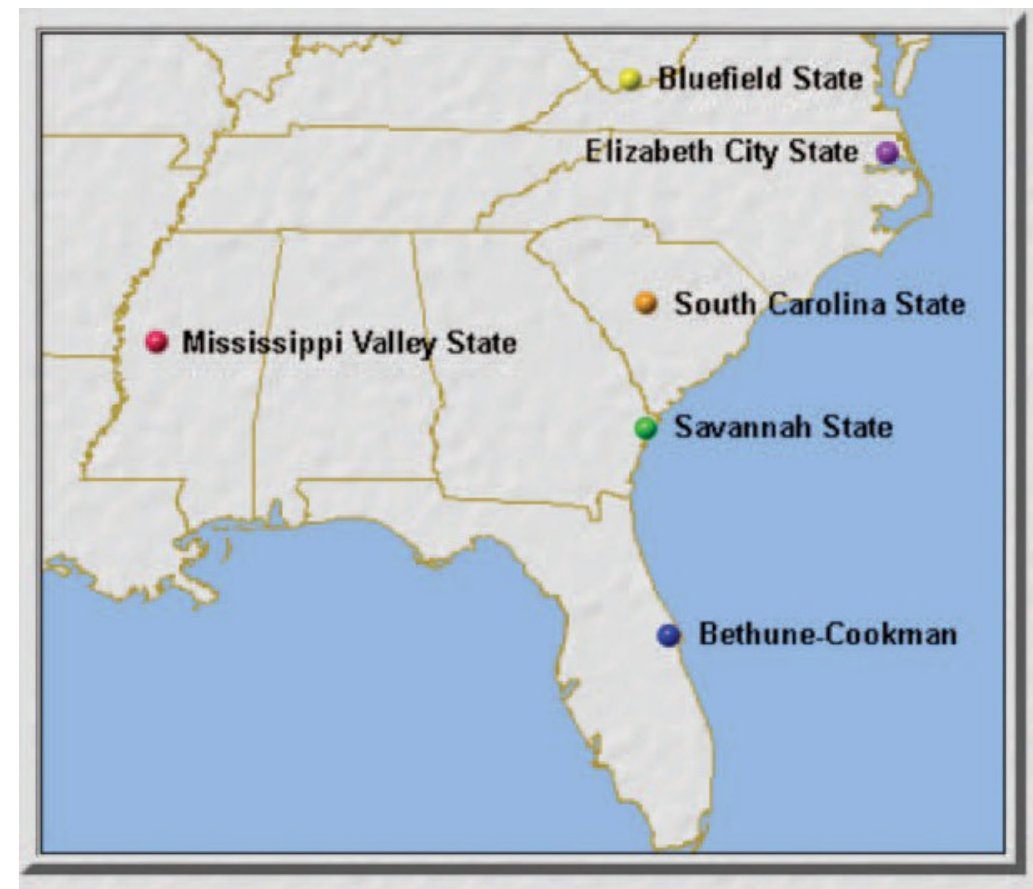

Figure 5-9. Historically Black Colleges and Universities Solar Monitoring Network (1985-1996). Image from NREL

Data elements and sources: GHI, DNI (at three stations), DHI (shadowband) from measurements by the Eppley Laboratory, Inc. Model PSP pyranometers and Model NIP pyrheliometers mounted in automatic solar trackers (LI-COR Model 2020). Radiometers were maintained daily and calibrated annually at NREL using the broadband outdoor radiometer calibration process (Myers et al. 2002) with reference standards traceable to the WRR.

- Data quality control and assessment: The station operators inspected the instrumentation daily to ensure that the radiometers were clean and properly aligned. Data were processed at NREL using SERI QC software to assign each data value a twodigit quality flag. Measured data from single-black thermopile radiometers were not corrected for thermal offsets that were discovered much later.

- Estimated uncertainties: Based on the instrument selections, installation, and O\&M practices, the estimated uncertainties for corrected daily total irradiances are measured $\mathrm{DNI} \pm 2 \%$, computed DNI from measured $\mathrm{GHI}$ and $\mathrm{DHI} \pm 8 \%, \mathrm{GHI} \pm 5 \%$, and $\mathrm{DHI} \pm$ $15 \%+5 \mathrm{~W} / \mathrm{m}^{2}$.

- Availability: NREL RReDC: http://rredc.nrel.gov/solar/old data/hbcu/ (includes qualityassessed monthly data files, monthly summary reports, and monthly irradiance plots)

- Updates: Final data released in 1997. Measurements from the Elizabeth City State University station continue to be available from the NREL Measurement \& Instrumentation Data Center: http://www.nrel.gov/midc/ecsu/. 


\subsubsection{Solar and Wind Energy Resource Assessment}

The Solar and Wind Energy Resource Assessment tool provides easy access to high-quality renewable energy resource information and data to users around the world. Its goal is to help facilitate renewable energy policy and investment by making high-quality information freely available to key user groups. Solar and Wind Energy Resource Assessment products include geographic information systems and time-series data, along with links to energy optimization tools needed to apply these data. To view additional information about the available resources or tools, select one of the links in the Resource Information or Analysis Tools section on the Web site. ${ }^{33}$ These products are being offered through a team of international experts and their incountry partners.

- Period of record: Moderate resolution: 1985-1991; high resolution: 1998-2002

- Temporal resolution: Monthly and annual average daily totals $\left(\mathrm{kWh} / \mathrm{m}^{2} /\right.$ day)

- Spatial coverage: Moderate resolution: South America, Central America, Africa, South and East Asia, Caribbean, Mexico, Middle East (Israel, Palestine/Jordan, Lebanon, Syria, Iraq, Yemen, Saudi Arabia [partial], and Kuwait). High resolution: Guatemala, Belize, El Salvador, Honduras, Nicaragua, partial Mexico (Oaxaca), Cuba, Afghanistan, Pakistan, partial Mexico (Chiapas, Vera Cruz, northern Mexico to 24 degrees latitude), Dominican Republic, Bhutan, India (NW), Ethiopia, Ghana, Ethiopia, Kenya, Sri Lanka, Nepal, Bangladesh, Western China, and United Arab Emirates

- Spatial resolution: Moderate resolution: 40-km; high resolution: $10 \mathrm{~km}$

- Data elements and sources: GHI, DNI (DHI), and POA from model estimates based on surface meteorological observations and/or satellite remote sensing input data

- Data quality control and assessment: No information

- Estimated uncertainties: No information

- Availability: Designed by the Solar and Wind Energy Resource Assessment program and maintained by UNEP/GRID-Sioux Falls:

○ http://maps.nrel.gov/swera

- Products for Brazil were developed by Brazil's National Institute of Space Research and Laboratory of Solar Energy/Federal University of Santa Catarina. More information about INPE is available at http://www.inpe.br/ingles/index.php. Products developed by DLR are available from http://www.dlr.de/tt/desktopdefault.aspx/tabid-2885/4422_read-6548/

- Updates: New data sets are made available on a continuing basis.

\subsubsection{HelioClim}

HelioClim is a family of databases comprising solar irradiance and irradiation values available at ground level. HelioClim data are modeled from Meteosat imagery covering Europe, Africa, the Mediterranean Basin, the Atlantic Ocean, and part of the Indian Ocean. Three databases on the

\footnotetext{
${ }^{33}$ See http://maps.nrel.gov/swera.
} 
HelioClim server are presently operated by the Mines ParisTech Armines Center for Energy and Processes. The Mines ParisTech Armines receives Meteosat data from Eumetsat and processes them in real time. It produces the databases HelioClim that can be accessed through the SODA Service.

- Period of record: 1985-present.

- Temporal resolution: 15 -minute

- Spatial coverage: Europe and Africa

- Spatial resolution: $5 \mathrm{~km}$

- Data elements and sources: Hourly and daily GHI from satellite remote sensing mode

- Data quality control and assessment: Web-based data quality programs compare the data to the extraterrestrial irradiation and data provided by a clear-sky model for the day or hour and generate a data quality report. The report explains anomalies in the HelioClim data

- Estimated uncertainties: No information

- Availability: Mines ParisTech Armines Center for Energy and Processes. www.helioclim.org/radiation/index.html. See also: www.soda-is.com/eng/index.html

- Updates: There are presently three databases: $\mathrm{HC}-1, \mathrm{HC}-2$, and $\mathrm{HC}-3$. Work continues on the most recent database, HC-3. An improved method HELIOSAT-4 to process Meteosat images is under preparation; it will create the database HC-4.

\subsubsection{Solar Data Warehouse}

The Solar Data Warehouse accesses climate data from more than 30 measurement networks across the United States and provides hourly and daily data from more than 3,000 stations. Measurements from these networks are converted to a uniform format and combined into a consistent data set.

- Period of record: Varies from 5 to 25 years ago to the present

- Temporal resolution: Hourly and daily

- Spatial coverage: Continental United States

- Spatial resolution: More than 3,000 measurement stations

- Data elements and sources: GHI

- Data quality control and assessment: Most of the radiometers are medium-quality pyranometers. Spatial and temporal comparisons of data among multiple nearby stations are used to identify anomalous data. Continual (weekly) adjustments to quality control routines because of addition, relocation, and discontinuation of measurement stations.

- Estimated uncertainties: Data from 13 NSRDB Class 1 measurement stations were compared to 16 Solar Data Warehouse stations separated by less than $40 \mathrm{~km}$ for the period from 2003 to 2005 . The average daily error was $9.85 \%$, and the RMSE was 19.0 $\mathrm{W} / \mathrm{m}^{2}$. 
- Availability: http://www.solardatawarehouse.com/.

\subsubsection{1-2005 NSRDB}

The 1991-2005 NSRDB update contains hourly solar radiation (including GHI, DNI, and GHI) and meteorological data for 1,454 stations. This update builds on the 1961-1990 NSRDB, which contains data for 239 stations (see Figure 5-3). The update includes the conventional time series for NSRDB ground stations as well as a 1/10-degree gridded data set from SUNY-Albany that contains hourly solar records for 8 years (1998-2005) for the United States (except Alaska above 60 degrees latitude) for approximately 100,000 pixel locations (at a nominal 10-km by 10-km pixel size). To increase data quantity, developers relaxed the standard of serial completeness mandated by the 1961-1990 NSRDB. In the update, the stations were classified by data quality. The 221 Class I stations have a complete hourly data set for the period from 1991 to 2005 period and were produced with the best available input data. The 637 Class II stations have a complete hourly data record, but they have a higher uncertainty because of lower quality input data (because of NWS automation of weather observations in the mid-1990s). The 596 Class III stations contain gaps in the data period but contain at least 3 years of data that may be useful for some applications.

A significant difference between the 1961-1990 and 1991-2005 NSRDBs involves data storage. In the original database, measured data were merged with modeled data such that a seamless data set of solar radiation values was produced-i.e., the model essentially filled gaps in the measured data. The updated database includes separate fields for both modeled and measured data, which allows users the flexibility to select modeled, or, if available, measured data for an application.

See the NSRDB user manual. ${ }^{34}$

- Period of record: $1991-2005$

- Temporal resolution: Hourly

- Spatial coverage: United States

- Spatial resolution: 1,454 locations and 10-km by 10-km grid (1998-2005) (Figure 5-3).

- Data elements and sources: Computed or modeled data: ETR on surfaces horizontal and normal to the sun, GHI, DNI, and DHI. Measured or observed data: total sky cover, opaque sky cover, dry-bulb temperature, dew point temperature, relative humidity, station pressure, wind speed and direction, horizontal visibility, ceiling height, precipitable water, AOD, surface albedo, and precipitation.

- Data quality control and assessment: Each data element has been assigned flags indicating the source and estimated uncertainty. Thirty-three measurement sites were used for the model evaluation based on their instrumentation, period of record, and proximity to NWS sites (Figure 5-10).

\footnotetext{
${ }^{34}$ See www.nrel.gov/docs/fy07osti/41364.pdf.
} 


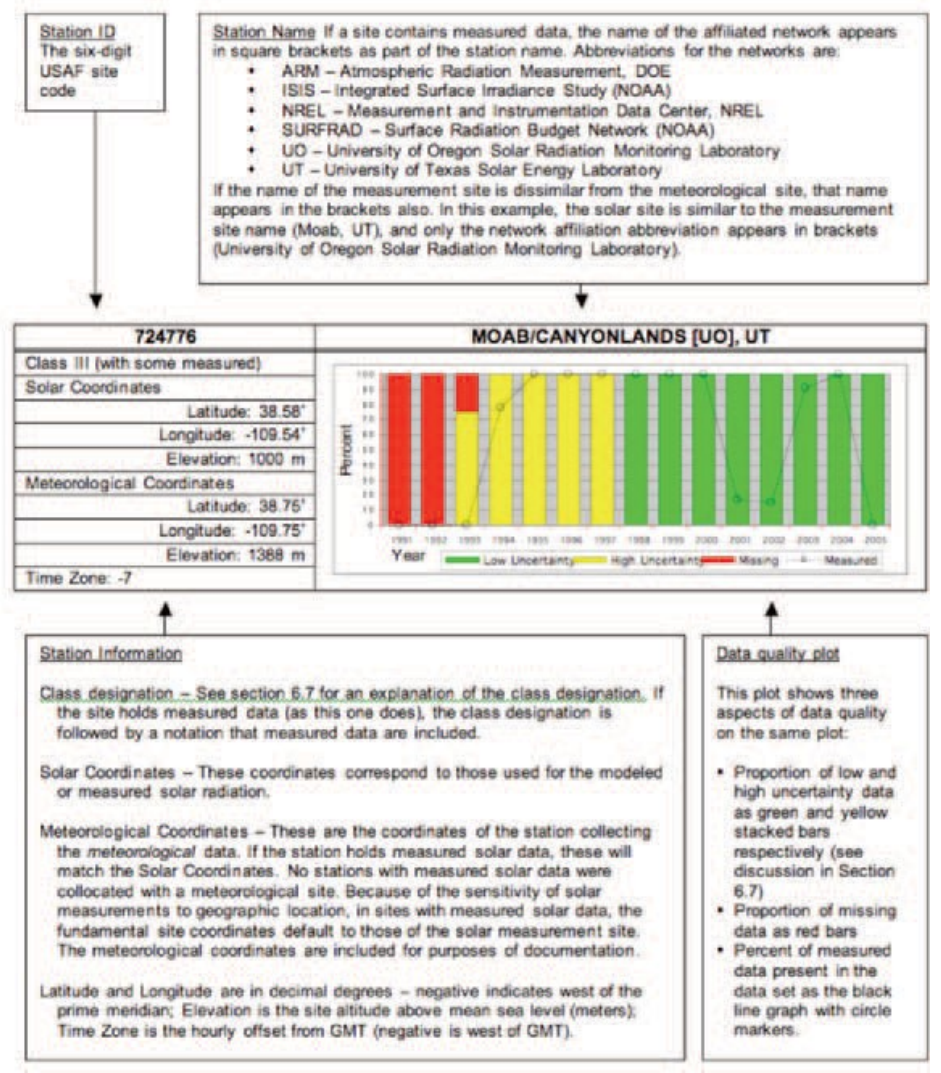

Figure 5-10. Example data quality summary for one of the 1,454 stations in the 1991-2010 NSRDB update. Image from Steve Wilcox, NREL

- Estimated uncertainties: Base uncertainty estimates were made for the two models used to generate the database. The base uncertainty of the surface model, METSTAT (Maxwell 1998), was determined from results that used high-quality model input data and compared the model output to measured data. Measured data from single-black thermopile radiometers were not corrected for thermal offsets that were discovered much later. This base uncertainty was then modified for the increased uncertainty of filled meteorological or the Automated Surface Observing System data when such input data were used. Similarly, the base uncertainty of the satellite remote sensing model (Perez et al. 2002) was determined in the model evaluation and then increased for periods of snow cover or high latitude - circumstances known to degrade model performance. Hourly uncertainties for modeled data range from $8 \%$ during optimal conditions to more than $25 \%$ for less-than-optimal input data. Additional information is available from Zelenka et al. (1999).

- Availability: Data are available from the NREL and NCDC, as shown in Table 5-4

- Updates: Released in 2007. 
Table 5-4. NSRDB Data Access Options

\begin{tabular}{|l|c|l|}
\multicolumn{1}{c|}{ Data Set } & Distributor & \multicolumn{1}{c|}{ URL } \\
\hline $\begin{array}{l}\text { NSRDB solar fields } \\
\text { (no meteorological data) }\end{array}$ & NCDC & ftp://ftp.ncdc.noaa.gov/pub/data/nsrdb-solar/ \\
\hline SUNY 10-km gridded data & NCDC & ftp://ftp.ncdc.noaa.gov/pub/data/nsrdb-solar/ \\
\hline $\begin{array}{l}\text { NSRDB statistical } \\
\text { summaries }\end{array}$ & NCDC & ftp://ftp.ncdc.noaa.gov/pub/data/nsrdb-solar/ \\
\hline
\end{tabular}

\subsubsection{TMY3}

The TMY3 data were produced using input data for 1976-2005 from the 1961-1990 NSRDB, Version 1.1 and the 1991-2005 NSRDB update. Because the 1961-1990 NSRDB has 239 sites and the 1991-2005 NSRDB update has more than 1,400 sites, production of the TMY3 data was designed to maximize both the number of stations and the number of years from which to characterize the typical conditions (Wilcox and Marion 2008). At sites where data are available for 30 years, the base time period for the TMY algorithm spans 1976-2005. For the remaining sites, the base time period spans 1991-2005.

Except for a few changes to the weighting criteria, which account for the relative importance of the solar radiation and meteorological elements, the TMY2 and TMY3 data sets were created using procedures similar to those developed by Sandia National Laboratories to create the original TMYs from the 1952-1975 SOLMET/ERSATZ data (Table 5-1). Minor changes to the algorithm were made between the TMY2 and TMY3 production runs. A small change to the persistence criteria better accommodates selecting a TMM for periods of records with fewer years. Also, computer code was removed that prioritized the selection of months with measured solar data because less than 1\% of the data records in the 1991-2005 NSRDB update contain measured data. The effects of these changes between the TMY2 and TMY3 algorithm were evaluated as part of the TMY3 production process. In the context of producing data sets with similar characteristics, these effects were small (Wilcox and Myers 2008). In practice, however, there are differences in the apparent solar resources among the data available as TMY2, TMY3, and the 8-year annual means of the NSRDB/SUNY model. Figure 5-11 illustrates the differences of annual mean daily total DNI for 8 years of NSRDB/ SUNY model estimates and the TMY3 data based on data from 1976 to 2005. 


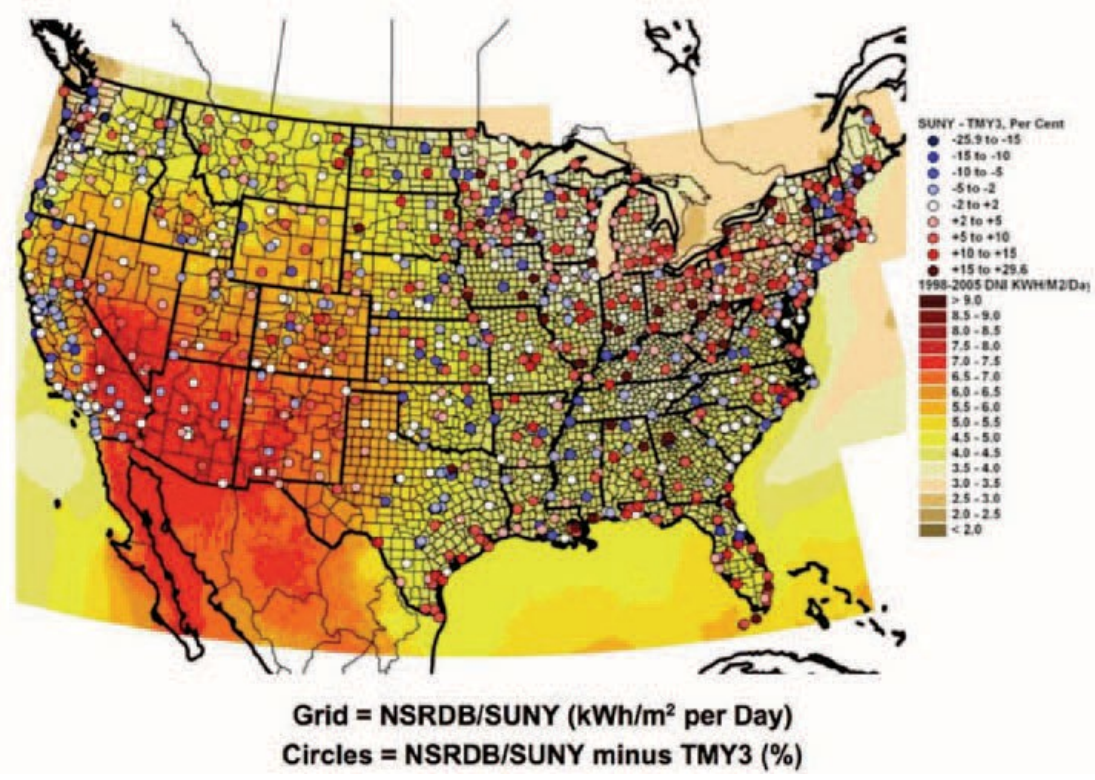

Figure 5-11. Annual mean daily total DNI distribution based on NSRDB/SUNY model results for 1998-2005 and the corresponding differences between the model and TMY3. (Red circles indicate DNI values from TMY3 < NSRDB/SUNY and blue circles indicate TMY3 > NSRDB/SUNYA.) Image from Ray George, NREL

Missing meteorological data have been filled to provide serially complete records as input for modeling the TMY3 solar radiation fields. Filled meteorological data fields (which are flagged in the data file) may also be useful for certain renewable energy applications; however, the filled data are not suitable for climatological studies.

To help guide the development and process validation for the TMY3, a 1961-1990 TMY was created with the updated software using data from the TD3282 NSRDB data set distributed by the NCDC. This data set was created solely for algorithm evaluation purposes, and no data have been released. Missing meteorological fields were filled according to methods used for the 19912005 NSRDB update. To evaluate the effects of drawing from differing periods of time for the input data set, we compared each of the following year-span subgroups using the original 19611990 TMY data set as a benchmark:

- 1961-1990 (30 years for evaluating software algorithm changes)

- 1976-2005 (for evaluating an updated TMY from a 30-year data set)

- 1991-2005 (for evaluating an updated TMY from a 15-year data set)

- 1998-2005 (for evaluating an updated TMY from an 8-year data set).

The TMY software was run on each data set to create TMYs for the 233 sites common to all subgroups (several sites among the 239 in the TMY2 data set did not have sufficient data for this analysis). We calculated a mean value for each parameter by site for each subgroup TMY. 
Although mean values of any data element are only a minor consideration in the TMY algorithm, they are one characteristic of climate and are a simple method of detecting large shifts or errors in the results. The ranges of the mean differences (the largest possible mean difference at any one site) in DNI for all stations, except Alaska and Hawaii, between the original 1961-1990 TMY2 and TMY3 data interval subgroups) are shown in Table 5-5.

Table 5-5. Ranges of Mean Station Differences for Hourly DNI

\begin{tabular}{|l|c|}
\multicolumn{1}{|c|}{ Data Interval } & Range of Station-Mean DNI Differences ${ }^{\mathrm{a}}\left(\mathrm{W} / \mathrm{m}^{2}\right)$ \\
\hline $1961-1990$ & \pm 15 \\
\hline $1975-2005$ & \pm 25 \\
\hline $1991-2005$ & \pm 40 \\
\hline $1998-2005$ & \pm 45 \\
\hline
\end{tabular}

a Differences computed as "new TMY3" minus original TMY2 hourly DNI values at each of the 233 stations. Larger mean differences in DNI, approaching $-100 \mathrm{~W} / \mathrm{m}^{2}$, were computed for stations in Alaska and Hawaii and require further study.

The mean biases and standard deviations for these comparison data sets are shown in Table 5-6 and Table 5-7. The statistics are found by determining the mean of sun-up data for the solar parameters and the mean of all data for meteorological parameters. Biases are determined as the test TMY data set minus the original 61-90 TMY. This information may give the user some indication of the increased uncertainty in the data (particularly noticeable in Table 5-7) with the smaller source data sets. The years corresponding to the eruptions of volcanoes El Chichón and Mount Pinatubo (1982-1984 and 1992-1994, respectively) are not represented among the selected years. The TMY algorithm explicitly excluded these years, because the effects of increased aerosols on solar radiation for those years are considered atypical.

Table 5-6. Bias Differences (Test Data Minus Original 1961-1990 TMY)

\begin{tabular}{|l|c|c|c|c|}
\multicolumn{1}{c|}{ Parameter } & $1961-1990$ & $1976-2005$ & $1991-2005$ & $1998-2005$ \\
\hline Direct normal W/m & -5.9 & -1.1 & -7.9 & -1.7 \\
\hline Global horizontal W/m ${ }^{2}$ & -4.0 & -5.7 & -15.2 & -11.7 \\
\hline Dry-bulb temperature ${ }^{\circ} \mathrm{C}$ & 0.07 & 0.39 & 0.77 & 0.94 \\
\hline Dew point temperature ${ }^{\circ} \mathrm{C}$ & 0.08 & 0.33 & 0.81 & 1.08 \\
\hline Wind speed $\mathrm{m} / \mathrm{s}$ & 0.02 & -0.1 & -0.3 & -0.4 \\
\hline
\end{tabular}

Table 5-7. Standard Deviations of Hourly Data

\begin{tabular}{|l|c|c|c|c|}
\multicolumn{1}{c|}{ Parameter } & $1961-1990$ & $1976-2005$ & $1991-2005$ & $1998-2005$ \\
\hline Direct normal W/m ${ }^{2}$ & 6.7 & 11.9 & 21.0 & 32.5 \\
\hline Global horizontal W/m ${ }^{2}$ & 2.8 & 5.3 & 10.0 & 15.1 \\
\hline Dry-bulb temperature ${ }^{\circ} \mathrm{C}$ & 0.22 & 0.37 & 0.49 & 0.77 \\
\hline Dew point temperature ${ }^{\circ} \mathrm{C}$ & 0.28 & 0.43 & 0.57 & 0.82 \\
\hline Wind speed $\mathrm{m} / \mathrm{s}$ & 0.12 & 0.20 & 0.30 & 0.34 \\
\hline
\end{tabular}


Data quality flags were assigned to each hourly data value to indicate the source and uncertainty, except for the computed values for extraterrestrial horizontal and extraterrestrial direct normal radiation. The source flag indicates whether the data were measured, modeled, or missing, and the uncertainty flag provides an estimate of the uncertainty of the data. Usually, the source and uncertainty flags are the same as those in the NSRDB, from which the TMY files were derived. In the case of the TMY3 data files, the uncertainties are expressed as plus-minus percent rather than the coded uncertainty used in the TMY2 files. Uncertainty values apply to the data with respect to actual values at the time stamp and not to how typical a particular hour is for a future month and day. The uncertainty values represent the plus or minus interval about the data value that contains the true value $95 \%$ of the time.

The uncertainty assigned to modeled solar radiation data includes primarily the model bias error and, to a lesser extent, the random error component, which could be several times larger for partly cloudy skies (Wilcox 2007). For partly cloudy skies, an hour can be composed of large or small amounts of sunshine, depending on whether the sun is mostly free of or occluded by the clouds. Consequently, modeled hourly values may depart significantly from true values for partly cloudy skies. The uncertainty assigned to modeled solar radiation data represents the average uncertainty for a large number of model estimates (such as for a month). When averaging large data sets, random errors tend to cancel, leaving only the bias error.

- Period of record: $1991-2005$

- Temporal resolution: Hourly

- Spatial coverage: United States and territories

- Spatial resolution: 1,020 locations (Figure 5-12)

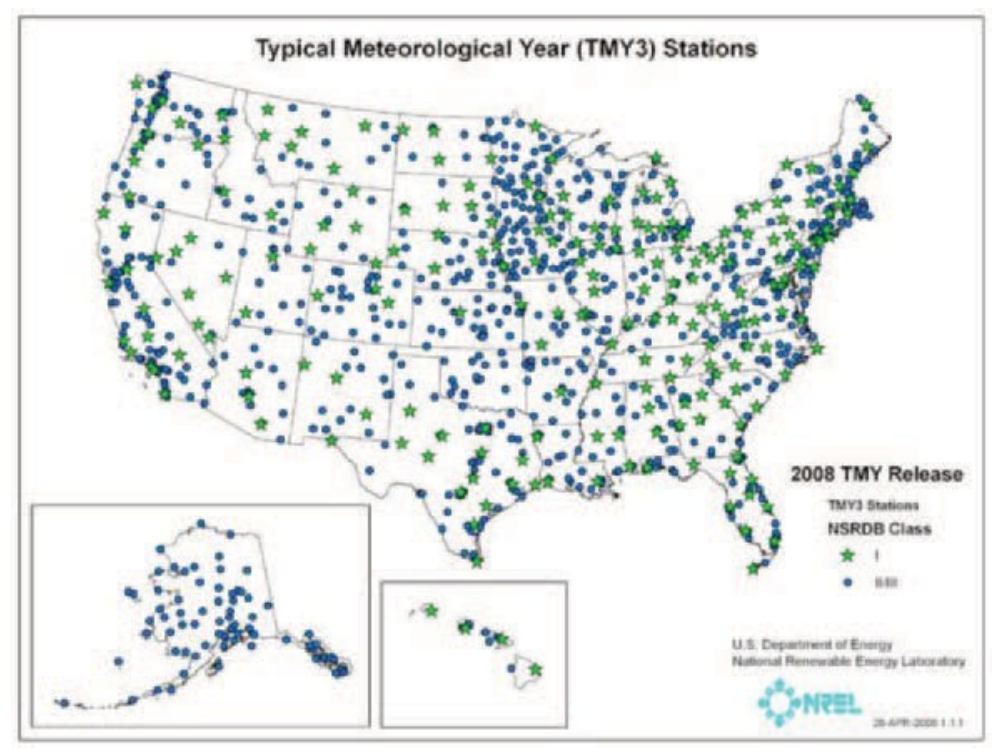

Figure 5-12. TMY3 stations. Image from NREL

- Data elements and sources: Computed or modeled data: ETR on surfaces horizontal and normal to the sun, GHI and illuminance, DNI and illuminance, DHI and illuminance, zenith luminance. Measured or observed data: total sky cover, opaque sky cover, dry- 
bulb temperature, dew-point temperature, relative humidity, station pressure, wind speed and direction, horizontal visibility, ceiling height, precipitable water, AOD, surface albedo, and precipitation

- Data quality control and assessment: Each data element has been assigned flags indicating the source and estimated uncertainty

- Estimated uncertainties: Base uncertainty estimates were made for the two models used to generate the database. The base uncertainty of the surface model was determined from results that used high-quality model input data and compared the model output to measured hourly data. This base uncertainty was then modified for the increased uncertainty of filled meteorological or the Automated Surface Observing System data when such hourly input data were used. Similarly, the base uncertainty of the satellite remote sensing model was determined in the model evaluation and then increased for periods of snow cover or high latitude - circumstances known to degrade model performance. Hourly uncertainties for modeled data range from $8 \%$ during optimal conditions to more than $25 \%$ for less-than-optimal input data.

- Availability: The NREL RReDC: http://RReDC.nrel.gov/solar/old_data/nsrdb/19912005/tmy3/.

\subsubsection{Management and Exploitation of Solar Resource Knowledge}

The Management and Exploitation of Solar Resource Knowledge (MESoR) project started in June 2007 for the purpose of removing the uncertainty and improving the management of solar energy resource knowledge. The results of past and present large-scale initiatives in Europe will be integrated, standardized, and disseminated uniformly to facilitate their effective exploitation by stakeholders. The project will contribute to preparation of the future roadmap for research and development and strengthening of the European position in the international field. The project includes activities in user guidance (benchmarking of models and data sets; handbook of best practices), unification of access to information (use of advanced information technologies; offering one-stop-access to several databases), connecting to other initiatives (INSPIRE of the European Union, Prediction of Worldwide Energy Resource of NASA, Solar Heating and Cooling and Photovoltaic Power Systems of the International Energy Agency (IEA), GMES/GEO) and to related scientific communities (energy, meteorology, geography, medicine, ecology), and information dissemination (stakeholders involvement, future research and development, communication). MESoR is supported as a coordination action by the European Commission.

- Period of record: 1991-2005: Europe and Africa; 1999-2006: Asia

- Temporal resolution: Hourly

- Spatial coverage: Europe, Western Asia, Africa, parts of Australia, South America

- Spatial resolution: $2.5 \mathrm{~km}$

- Data elements and sources: GHI, DNI, DHI from ground measurements and modeling results

- Data quality control and assessment: Benchmarking data include ground measurements available from BSRN, International Daylight Measurement Program, 
Global Atmospheric Watch, and others. Time-series data analyzed for MBE, RMSE, and Kolmogrov-Smirnov Test statistics

- Estimated uncertainties: Sample MBE and RSME results for eight BSRN stations are shown in Table 5-8

- Availability: DLR: www.mesor.org/.

Table 5-8. Sample MBE and RSME Results for Eight BSRN Stations

\begin{tabular}{|l|c|c|c|c|c|c|c|c|}
\hline \multirow{2}{*}{ Timescale } & $\begin{array}{c}\text { Mean } \\
\left(\mathrm{Wm}^{-2}\right)\end{array}$ & $\begin{array}{c}\text { MBE } \\
(\%)\end{array}$ & $\begin{array}{c}\text { RMSE } \\
(\%)\end{array}$ & $\mathrm{R}^{2}$ & $\begin{array}{c}\text { Mean } \\
\left(\mathrm{Wm}^{-2}\right)\end{array}$ & $\begin{array}{c}\text { MBE } \\
(\%)\end{array}$ & $\begin{array}{c}\text { RMSE } \\
(\%)\end{array}$ & $\mathrm{R}^{2}$ \\
\hline Hour & 87.3 & $.93 \%$ & 8.79 & .97 & 67.8 & 0.73 & 6.83 & .87 \\
\hline Day & NA & NA & 1.08 & .99 & NA & NA & 3.58 & .95 \\
\hline Month & NA & NA & .95 & .99 & NA & NA & .69 & .99 \\
\hline Year & NA & NA & .66 & .99 & NA & NA & .92 & .99 \\
\hline
\end{tabular}

\subsubsection{International Daylight Measurement Program}

The International Daylight Measurement Program was initiated in the framework of Technical Committee 3.07 of the International Commission on Illumination by Derrick Kendrick of the University of Adelaide, Australia. The year 1991 was designated the International Daylight Measurement Year on the occasion of the International Commission on Illumination quadrennial conference. Researchers from around the world took this opportunity to start measurement stations based on standard conventions developed by the program. In conjunction with the IEA Solar Heating and Cooling Program (SHC), the International Daylight Measurement Program measurements and modeling of spectral radiation continued through 1994.

- Period of record: $1991-1994$

- Spatial coverage: Australia, Canada, China, France, Germany, Greece, India, Indonesia, Israel, Japan, Korea, The Netherlands, New Zealand, Portugal, Russia, Singapore, Slovakia, Spain, Sweden, Switzerland, United Kingdom, and the United States

- Spatial resolution: 43 measurement stations

- Data elements and sources: GHI, DNI, DHI, zenith luminance, illuminance (including vertical surfaces), air temperature, relative humidity (or dew point), wind speed and direction, bright sunshine duration, sky imagers, and sky scanners

- Data quality control and assessment: International Daylight Measurement Program guidelines address the use of physical limits (acceptance thresholds) and comparisons of measurements to validated models that account for various sky conditions and solar position. The stand-alone program, AQCCIE, is also available.

- Estimated uncertainties: No information

- Availability: École Nationale des Travaux Publics: $\underline{\text { http://idmp.entpe.fr/. }}$ 


\subsubsection{BSRN}

In 1992, the World Climate Research Programme Radiative Fluxes Working Group initiated a new BSRN to support the research projects of the World Climate Research Programme and other scientific programs needing high-quality and continuous measurements of the irradiances at the Earth's surface. Some years later, the BSRN incorporated into the World Climate Research Programme Global Energy and Water Cycle Experiment Radiation Panel.

The objective of the BSRN is to provide observations of the best possible quality for shortwave and long-wave surface radiation fluxes by using a high sampling rate. These readings are taken from a small number of selected stations, in contrasting climatic zones, together with collocated surface and upper-air meteorological data and other supporting observations. The uniform and consistent measurements throughout the BSRN network are used to

- Monitor the background (least influenced by immediate human activities that are regionally concentrated) shortwave and long-wave radiative components and their changes by using the best methods currently available

- Provide data to validate and evaluate satellite-based estimates of the surface radiative fluxes

- Produce high-quality observational data for comparison to climate model calculations and to develop local and regionally representative radiation climatological analyses.

At present, approximately $60 \mathrm{BSRN}$ stations are in operation. These stations measure different sets of radiation values. Some carry out only basic measurements according to the BSRN Technical Plan (Hegner et al. 1998). Other stations carry out other measurements in addition to the basic measurements. Some stations also perform synoptic observations, upper air soundings, ozone measurements, and expanded measurements. More stations are being established.

The BSRN database is based on PANGAEA (named after the Pangaea theory). This Publishing Network for Geoscientific \& Environmental Data is an Open Access library aimed at archiving, publishing, and distributing georeferenced data from Earth system research. Data descriptions (metadata) of all data sets are visible and include the principal investigator's name and e-mail for contact. Online data access is offered to anybody who accepts the data release guidelines. ${ }^{35}$

In addition to the Web-based PANGAEA access, the original station-to-archive files (without derived quantities and quality flags) can be obtained via the FTP server. ${ }^{36}$

The BSRN data have become widely known for their research quality and are used for model development and validation.

- Period of record: $1992-$ present

- Temporal resolution: 1-minute

- Spatial coverage: Global

\footnotetext{
${ }^{35}$ See www.pangaea.de/PHP/BSRN Status.php to access the data and see a list of current stations.

${ }^{36}$ See ftp.bsrn.awi.de. Contact Gert.Koenig-Langlo@awi.de.
} 
- Spatial resolution: 40 measurement stations (Figure 5-13).

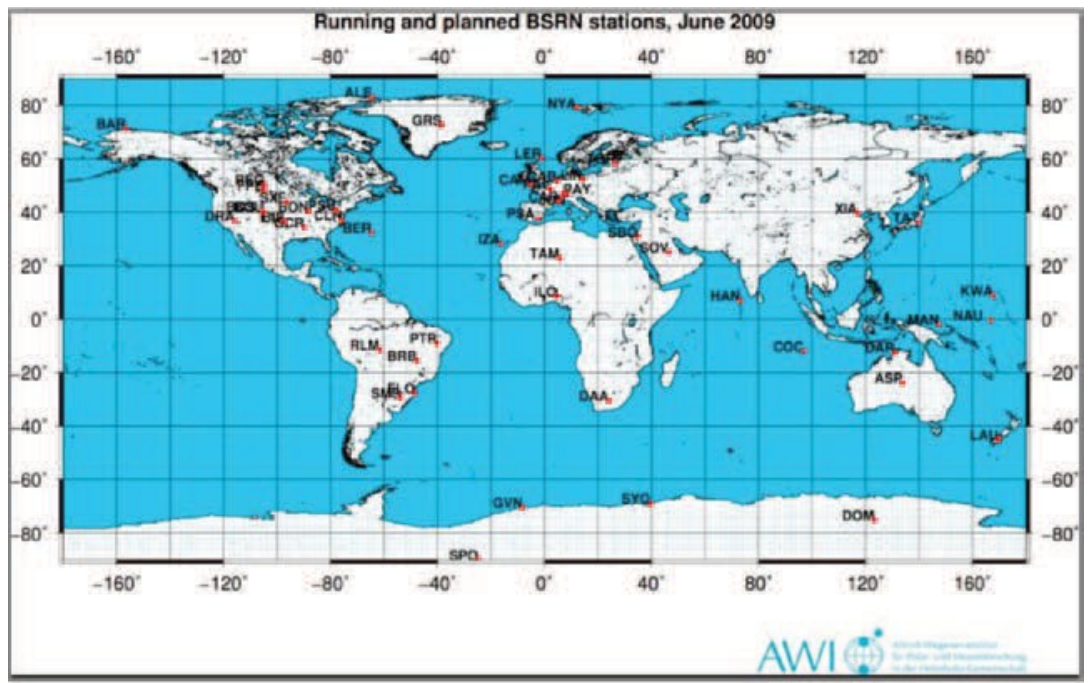

Figure 5-13. BSRN. Image from NREL

Data elements and sources: The number and type of measurements vary with station. Basic radiation measurements include GHI, DNI, DHI, downwelling infrared irradiance, upwelling infrared irradiance, and upwelling (reflected) shortwave irradiance. Measurements are from radiometers of various manufacturers. Synoptic meteorological observations, upper air measurements, and numerous expanded and supporting measurements are available.

Data quality control and assessment: Measurement station design and O\&M practices must conform to established BSRN requirements. The station scientist is responsible for measurements and data quality. For each month, the station scientist produces a station-toarchive file, according to Hegner et al. (1998).

- Estimated uncertainties: The World Climate Research Programme of the WMO established the standards of measurement for the BSRN. The stated accuracies are 15 $\mathrm{W} / \mathrm{m}^{2}$ for broadband solar measurements and $110 \mathrm{~W} / \mathrm{m}^{2}$ for thermal infrared measurements.

- Availability: The WRMC provides Web-based and FTP data access: www.bsrn.awi.de/en/home/

- Updates: The BSRN data archive is maintained by the WRMC and updated regularly: www.bsrn.awi.de/en/home/wrmc/.

\subsubsection{Surface Radiation Network}

The Surface Radiation (SURFRAD) Network was established in 1993 through the support of NOAA's Office of Global Programs to support climate research with accurate, continuous, longterm measurements of the SRB over the United States.

Currently, seven SURFRAD stations operate in climatologically diverse regions: Montana, Colorado, Illinois, Mississippi, Pennsylvania, Nevada, and South Dakota. This represents the first time that a monitoring network in the United States was designed to measure the complete 
SRB. The site selection process for SURFRAD was a collaborative effort among NOAA, NASA, and university scientists. Locations were chosen with the intent of best representing the diverse climates of the United States. Special consideration was given to places where the landform and vegetation are homogeneous throughout an extended region so that the point measurements would be qualitatively representative of a large area.

Each station is equipped to measure broadband solar and infrared irradiances, including DNI, to compute the net surface fluxes. Measurements of the spectral irradiance are collected to provide the relative amounts of ultraviolet and photosynthetically active radiation. Photometric measurements at selected solar irradiance wavelengths can be used to estimate the AOD (important for determining DNI and amounts of forward scattering - circumsolar irradiance), total column ozone, and precipitable water vapor. Surface meteorological measurements, including all-sky digital cameras for measuring cloud cover, complete the instrumentation.

Data are downloaded, quality controlled, and processed into daily files that are distributed in near real-time by anonymous FTP and the Internet. Observations from SURFRAD have been used to evaluate satellite-based estimates of surface radiation and to validate hydrologic, weather prediction, and climate models. Quality assurance is built into the design and operation of the network and ensure continuous high-quality product.

The station in Boulder, Colorado, is an operating SURFRAD station and serves as a calibration facility for network instruments as well as for spectroradiometers operated by several North American agencies that monitor ultraviolet radiation.

- Period of record: 1993 -present

- Temporal resolution: Data are reported as 3-minute averages of 1-second samples before January 1, 2009, and 1-minute averages on and after January 1, 2009.

- Spatial coverage: United States

- Spatial resolution: Seven stations: Montana, Colorado, Illinois, Mississippi, Pennsylvania, Nevada, and South Dakota (Figure 5-14)

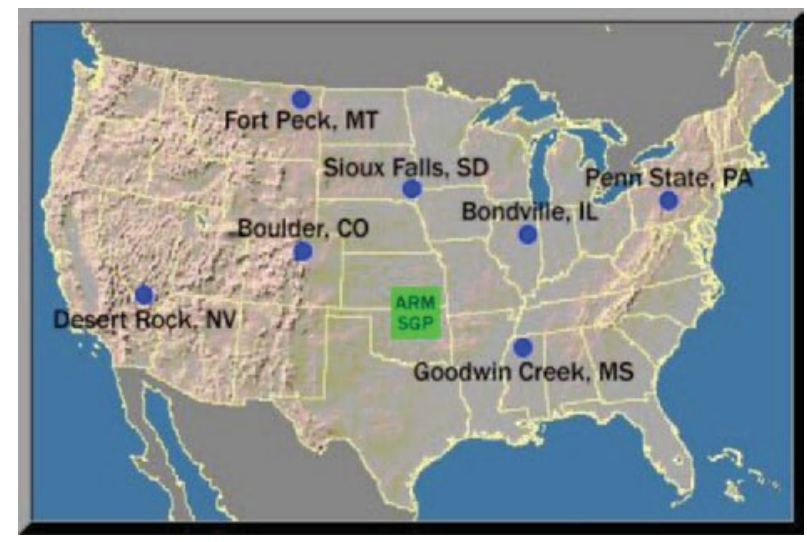

Figure 5-14. The SURFRAD network is operated by the Global Monitoring Division, Earth Systems Research Laboratory, NOAA. Image from NREL 
- Data elements and sources: GHI, DNI, DHI, downwelling infrared irradiance, upwelling infrared irradiance, and upwelling (reflected) shortwave irradiance. Photosynthetically active radiation, solar net radiation, infrared net radiation, air temperature, relative humidity, wind speed and direction (10 m AGL), and all-sky images

- Data quality control and assessment: The stations are regularly maintained and data are downloaded, quality controlled, and processed into daily files that are distributed in near real time by anonymous FTP and the World Wide Web. Radiometers are recalibrated annually, and field measurements are compared to standards as part of the instrument exchange procedure. Data elements are assigned an individual quality-assessment flag. The redundancy of three-component solar measurements (global, direct, and diffuse) provides a useful tool for quality control of the SURFRAD data by examining the internal consistency of these measurements at any time interval.

- Estimated uncertainties: Based on the instrument selections, installation, and O\&M practices, the estimated uncertainties for corrected daily total irradiances are measured $\mathrm{DNI} \pm 2 \%$, computed DNI from measured GHI and $\mathrm{DHI} \pm 8 \%$, computed GHI from measured DNI and $\mathrm{DHI} \pm 5 \%$, measured GHI corrected for unshaded pyranometer thermal offsets $\pm 5 \%$, and DHI $\pm 15 \%+5 \mathrm{~W} / \mathrm{m}^{2}$. SURFRAD has adopted the standards for measurement set by the BSRN as developed by the World Climate Research Programme of the WMO. The stated accuracies are $15 \mathrm{~W} / \mathrm{m}^{2}$ for broadband solar measurements and $110 \mathrm{~W} / \mathrm{m}^{2}$ for thermal infrared measurements. To achieve these ambitious goals, the broadband solar instruments are calibrated at NREL against standards traceable to the WRC in Davos, Switzerland.

- Availability: NOAA, Earth Systems Research Laboratory, Global Monitoring Division, in Boulder, Colorado: ftp://ftp.srrb.noaa.gov/pub/data/surfrad and www.srrb.noaa.gov. SURFRAD data are also submitted to the BSRN archives: www.bsrn.awi.de/

- Updates: Continuous data updates accommodate the latest measurements.

\subsubsection{Integrated Surface Irradiance Study}

The Integrated Surface Irradiance Study (ISIS) is a continuation of earlier NOAA surface-based solar monitoring programs. ISIS addresses questions of spatial distributions and time trends at sites selected to be regionally representative and long-term continuous records of observations. Data from 1995 to 2008 are archived at the NCDC from 10 stations: Albuquerque, New Mexico; Bismarck, North Dakota; Desert Rock, Nevada; Hanford, California; Madison, Wisconsin; Oak Ridge, Tennessee; Seattle, Washington; Salt Lake City, Utah; Sterling, Virginia; and Tallahassee, Florida. Data consist of 15-minute averaged measurements with standard deviations and minimum/maximum values based on 1-second samples of GHI using the Eppley Laboratory, Inc., Model PSP pyranometer; DNI using a Model NIP pyrheliometer; diffuse irradiance using models PSP or 8-48 pyranometers; ultraviolet-B (UVB) irradiance using a solar light ultraviolet biometer; GHI using a silicon cell pyranometer, plus its maximum, minimum, photosynthetically active radiation, and GHI using RSRs with photodiode detectors; and SZA.

The network ceased operation in January 2006 because of funding limitations.

- Period of record: $1995-2006$ 
- Temporal resolution: 15-minute

- Spatial coverage: Continental United States

- Spatial resolution: 9 stations (Figure 5-14)

- Data elements and sources: GHI, DNI, DHI, and Global UVB

- Data quality control and assessment: These data are provisional. The NOAA Solar Radiation Research has attempted to produce the best data set possible; however, the data quality is constrained by measurement accuracies of the instruments and the quality of the calibrations. Regardless, the Solar Radiation Research Branch attempts to ensure the best quality possible through quality assurance and quality control. The data were subjected to automatic procedures as the daily files were processed. Data were subjected only to this first-level check and a daily eye check before being released.

Quality assurance methods were in place to protect against premature equipment failure in the field and postdeployment data problems. For example, all instruments at each station were exchanged annually for newly calibrated instruments. Calibrations were performed by world-recognized organizations with pyranometers and pyrheliometers calibrated at NREL to the WRR. Calibration factors for the UVB instrument were transferred from three standards maintained by the Solar Radiation Research Branch's National UV Calibration Facility in Boulder. In general, all of the standards collected by the Solar Radiation Research Branch and NREL were traceable to the National Institute of Standards and Technology or its equivalent.

- Estimated uncertainties: Based on the instrument selections, installation, and O\&M practices, the estimated uncertainties for corrected daily total irradiances are measured $\mathrm{DNI} \pm 2 \%$, computed $\mathrm{DNI}$ from measured $\mathrm{GHI}$ and $\mathrm{DHI} \pm 8 \%$, GHI $\pm 5 \%$, and $\mathrm{DHI} \pm$ $15 \%+5 \mathrm{~W} / \mathrm{m}^{2}$

- Availability: NOAA, Earth Systems Research Laboratory, Global Monitoring Division, Boulder, Colorado: $\mathrm{ftp}$ ://ftp.srrb.noaa.gov/pub/data/isis/

- Updates: First released in 1995 and updated through 2005 with subsequent measurements.

\subsubsection{Satel-Light}

The European database of daylight and solar radiation is based on Meteosat images and a model that uses an estimation of cloud cover to produce a cloud index to produce GHI data. The DNI data are derived from GHI using the Page model (Page 1996). The Satel-Light server provides these data in map form for all of Europe.

- Period of record: $1996-2000$

- Temporal resolution: 30-minute

- Spatial coverage: Europe

- Spatial resolution: $\sim 5 \mathrm{~km}$

- Data elements and sources: DNI, GHI, DHI, POA, horizontal illuminance, tilted illuminance, and sky luminance distribution. 
- Data quality control and assessment: The satellite-based model results have been compared to measurements from 25 stations. End user products generated from the satellite estimates were also compared to those generated from ground measurements at five stations (Dumortier 1998; Olseth and Skartveit1998)

- Estimated uncertainties: Measurements of GHI from 25 sites in Europe were used to evaluate model performance for all sky conditions. The resulting annual mean bias deviation for GHI ranged from $-1 \%$ to $3 \%$ and a root mean square deviation ranged from $20 \%$ (south of Europe with a high frequency of sunny skies) to $40 \%$ (north of Europe with a high frequency of cloudy skies)

- Availability: www.satellight.com/indexgS.htm.

\subsubsection{Atmospheric Radiation Measurement Program}

The Atmospheric Radiation Measurement Climate Research Facility is a DOE national user facility for the study of global change by the national and international research community. Research at this facility includes the study of alterations in climate, land productivity, oceans or other water resources, atmospheric chemistry, and ecological systems that may alter the capacity of the Earth to sustain life. Measuring solar and infrared irradiances is an important source of data for this research. Continuous measurements of surface radiative flux are made in three geographic areas of the world. Beginning in 1997, Atmospheric Radiation Measurement began operating 23 solar infrared stations in the southern Great Plains located in parts of Kansas and Oklahoma. Ground Radiation (GNDRAD) and Sky Radiation (SKYRAD) stations are located at three sites in the tropical western Pacific and two sites in the North Slope of Alaska. Known for the research quality of these measurements, the data are used for a variety of atmospheric model validations.

Important ancillary data, such as AOD, precipitable water vapor, cloud cover and optical depth, surface albedo, spectral irradiance, and atmospheric profiles of temperature, pressure, and water vapor are also available from the Atmospheric Radiation Measurement facility

- Period of record: 1997-present

- Temporal resolution: 20-second instantaneous samples and 1-minute averages of 2second scans

- Spatial coverage: Southern Great Plains, North Slope of Alaska, and tropical western Pacific (Figure 5-15). 


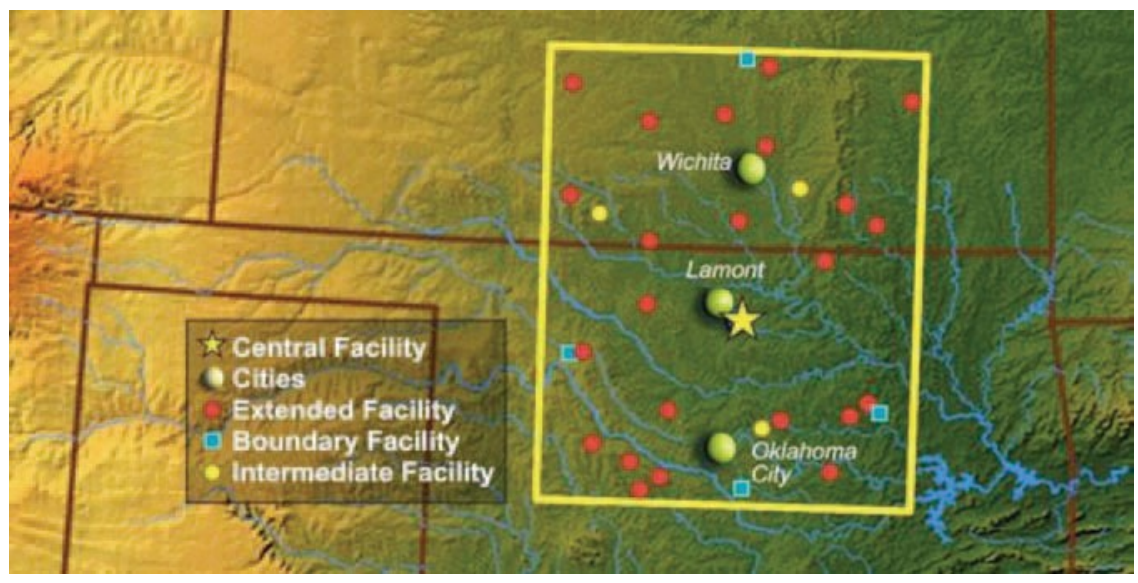

Figure 5-15. DOE has operated the 23 Atmospheric Radiation Measurement stations in the southern Great Plains since 1997. Image from DOE

- Spatial resolution: 23 stations (southern Great Plains), 2 stations (North Slope of Alaska), and 2 stations (tropical western Pacific)

- Data elements and sources: GHI, DNI, DHI, DIR, UIR, and upwelling (reflected) shortwave irradiance. Measurements from the Eppley Laboratory, Inc., Model PSP (GHI, DHI, and upwelling shortwave irradiance), Model 8-48 (DHI after 2000), Model NIP (DNI), and Model PIR (DIR and UIR)

- Data quality control and assessment: Measurement stations are inspected daily (North Slope of Alaska and tropical western Pacific stations) to biweekly (southern Great Plains, except for central facility stations that are maintained daily) for preventative and corrective maintenance. Data are processed using data quality-assessment methods based on SERI QC, checked visually as time-series plots, and compared to relevant ancillary measurements and model outputs (e.g., clear-sky solar irradiance model results). The pyranometer data are corrected for known thermal offsets. The Atmospheric Radiation Measurement Data Quality Office reports on the health and status of the data at hourly and daily intervals. Each measurement is assigned a data quality flag. Radiometers are calibrated annually at the Radiometer Calibration Facility near Lamont, Oklahoma, and control and reference radiometers are compared to standards maintained by NREL. All pyranometers and pyrheliometer calibrations are traceable to the WRR.

- Estimated uncertainties: Based on the instrument selections, installation, and O\&M practices, the estimated uncertainties for corrected daily total irradiances are measured $\mathrm{DNI} \pm 2 \%, \mathrm{GHI} \pm 5 \%$, and $\mathrm{DHI} \pm 15 \%+5 \mathrm{~W} / \mathrm{m}^{2}$

- Availability: DOE, Atmospheric Radiation Measurement Climate Research Facility: www.arm.gov. Data sets are labeled SIRS, SKYRAD, and GNDRAD. SIRS data are also submitted to the BSRN archives: www.bsrn.awi.de/

- Updates: Continuous data updates accommodate latest measurements, and value-added products are available. 


\subsubsection{TIER Solar Time Series}

The data set covering the Western Hemisphere is based on more than 12 years of half-hourly high-resolution (roughly 1-km) visible satellite imagery from GOES data (GOES East, GOES West, and GOES South) using the broadband visible wavelength channel. The satellite data set was collected from January 1997 to present and has been processed to create hourly values of GHI, DNI, and DHI at a horizontal resolution of roughly $3 \mathrm{~km}$.

3TIER processes the satellite images based on a combination of in-house research and algorithms published in peer-reviewed scientific literature. These algorithms contain parameters and coefficients that are based on empirical fits to observational data. To develop and validate their model, 3TIER used observations from the SURFRAD, BSRN, NSRDB, Bureau of Meteorology (Australia), National Institute of Water and Atmospheric Research (New Zealand), Indian Meteorology Department, Linke Turbidity Database from Mines ParisTech, and snow data from the 24-km data set developed by the National Snow and Ice Data Center.

The basic processing scheme follows the SUNY model (Perez et al. 2002) with a few key improvements made within the 3TIER algorithms. These include a higher spatial and temporal resolution, an in-house developed seasonal variability correction factor, an in-house developed empirical fitting of the data to ground station measurements, and the integration of instantaneous irradiance values to determine the hourly value. Each improvement results in a lower RMSE compared to the NREL/NSRDB Update/SUNY data set (1998-2005).

- Period of record: January 1997-Present

- Temporal resolution: $\sim 30$-minute instantaneous and 1-hour averages

- Spatial coverage: Western Hemisphere and much of Asia and Oceania

- Spatial resolution: 2 arc-min $(\sim 3 \mathrm{~km})$

- Data elements and sources: GHI, DNI, and DHI from model estimates based on satellite remote sensing input data

- Data quality control and assessment: The irradiance data are based on the model developed by Perez et al. (2002) with proprietary improvements for increased spatial and temporal resolution, seasonal variability correction factor, empirical fitting of the modeled data to ground station measurements, and integration of instantaneous irradiance values to determine the hourly value. Surface radiation measurements from ground stations operated for the BSRN, SURFRAD, and other regional networks as identified by the NSRDB were used to validate the 3TIER model

- Estimated uncertainties: Analyses of continental United States based on 36 observing stations for the years 1998 through 2005 indicate the following RSME and bias values in $\mathrm{W} / \mathrm{m}^{2}$ for each irradiance component: GHI [77/4], DNI [181/4] and DHI [63/4]

- Availability: 3-TIER: www.3tier.com/products/

- Updates: Continuous. 


\subsubsection{Clean Power Research-SolarAnywere}

SolarAnywhere is a Web-based service that provides hourly estimates of the solar irradiance based on satellite images and atmospheric data using algorithms developed and maintained by Dr. Richard Perez and the State University of New York at Albany (Perez et al. 2002).

- Period of record: 1998 -present

- Temporal resolution: Hourly

- Spatial coverage: Continental United States and Hawaii

- Spatial resolution: $10 \mathrm{~km}$.

- Data elements and sources: GHI, DNI, wind speed, and ambient air temperature

- Data quality control and assessment: The Perez/SUNY model was developed and has been validated using surface irradiance measurements from selected SURFRAD stations

- Estimated uncertainties: Based on comparisons to measured data from 10 stations in the United States (Perez et al. 2002), the annual average hourly RSME and MBE for GHI are $14.0 \%$ and $0.8 \%$, respectively, and for DNI, $29.8 \%$ and $0.9 \%$, respectively

- Availability: Clean Power Research: www.cleanpower.com/SolarAnywhere

- Updates: Model version control information available.

\subsubsection{1-2009 NSRDB}

The 1991-2009 NSRDB update contains hourly solar radiation (including GHI, DNI, and GHI) and meteorological data for 1,454 stations.

This update builds on the 1991-2005 NSRDB, which contains data for 1,454 stations (see Figure 5-3). The update includes the conventional time series for NSRDB ground stations as well as a 1/10-degree gridded data set from Clean Power Research (CPR) that contains hourly solar records for 12 years (1998-2009) for the United States (except Alaska above 60 degrees latitude) for approximately 100,000 pixel locations (at a nominal $10-\mathrm{km}$ by $10-\mathrm{km}$ pixel size). In the update, the stations were classified by data quality. The 242 Class I stations have a complete hourly data set for the 1991-2005 period and were produced with the best available input data. The 618 Class II stations have a complete hourly data record, but they have a higher uncertainty because of lower quality input data (due to NWS automation of weather observations in the mid1990s). The 594 Class III stations contain gaps in the data period but contain at least 3 years of data that may be useful for some applications.

As mentioned in the NSRDB user manual (Wilcox, 2012), a significant difference between the 1961-2005 and 1991-2009 NSRDBs is that the latter contains updated satellite data from Clean Power Research, which implemented several model refinements in SolarAnywhere since the SUNY data set that was used in the 1991-2005 update. Further, the latter has a different cloud cover input to the METSTAT model in which the ASOS cloud algorithm for the METSTAT model was changed after research showed that ASOS tends to overestimate cloud cover at cloudy sites. However, other than these changes, the 1991-2009 data set is similar to the 19912005 update. 
- Period of record: 1991-2009

- Temporal resolution: Hourly

- Spatial coverage: United States

- Spatial resolution: 1,454 locations and 10-km by 10-km grid (1998-2009) (Figure 5-2)

- Data elements and sources: Computed or modeled data: ETR on surfaces horizontal and normal to the sun, GHI, DNI, and DHI. Measured or observed data: total sky cover, opaque sky cover, dry-bulb temperature, dew point temperature, relative humidity, station pressure, wind speed and direction, horizontal visibility, ceiling height, precipitable water, AOD, surface albedo, and precipitation

- Data quality control and assessment: Each data element has been assigned flags indicating the source and estimated uncertainty. Thirty-three measurement sites were used for the model evaluation based on their instrumentation, period of record, and proximity to NWS sites (Figure 5-10)

- Availability: NSRDB user manual: http://www.nrel.gov/docs/fy12osti/54824.pdf and http://RReDC.nrel.gov/solar/old_data/nsrdb/1991-2010/.

\subsubsection{SOLEMI}

SOLEMI is a service set up by DLR providing high-quality irradiance data based on Meteosatdata with a nominal spatial resolution of $2.5 \mathrm{~km}$ and half-hourly temporal resolution. Solar radiation maps and hourly time series will be available for almost half the Earth's surface.

- Period of record: No information

- Temporal resolution: 30-minute

- Spatial coverage: Global

- Spatial resolution: $2.5 \mathrm{~km}$

- Data elements and sources: No information

- Data quality control and assessment: No information

- Estimated uncertainties: No information.

- Availability: DLR: www.solemi.com/home.html

- Updates: No information.

\subsubsection{GeoModel Solar-SolarGIS}

The SolarGIS database is derived from Meteosat, GMS, MTSAT, and GOES satellite data and ECMWF and NOAA atmospheric parameters using in-house algorithms and computing infrastructure.

- Period of record: 1994, 1999, 2007-present (depends on the region)

- Temporal resolution: 15- and 30-minute

- Spatial coverage: Land area, worldwide, between latitudes $60^{\circ} \mathrm{N}$ and $50^{\circ} \mathrm{S}$

- Spatial resolution: $\sim 3 \mathrm{~km}$ (at the equator) down scaled to $\sim 80 \mathrm{~m}$ using SRTM-3 DEM 
- Data elements and sources: DNI, GHI, DHI, GTI, and air temperature (2-m AGL) and others

- Data quality control and assessment: Model data compared to measurements from 50 stations in Europe and North Africa (Ineichen 2014)

- Estimated uncertainties: See Table 5-9 for summary statistics based on comparisons to measurement stations in Europe and North Africa

- Availability: http://solargis.info.

Table 5-9. SolarGIS Validation Summary

\begin{tabular}{|l|l|l|l|}
\hline Component & Number of Stations & MBE & RMSE \\
& 18 & $0 \%$ & $\begin{array}{l}17 \% \text { (hourly) } \\
8 \% \text { (daily) } \\
4 \% \text { (monthly) }\end{array}$ \\
\hline DNI & & & $\begin{array}{l}34 \% \text { (hourly) } \\
21 \% \text { (daily) } \\
10 \% \text { (monthly) }\end{array}$ \\
\hline
\end{tabular}

\subsubsection{EnMetSol}

The EnMetSol database is based on Meteosat images and a model based on a cloud index to derive irradiance data. Data can be requested at the University of Oldenburg.

- Period of record: 1995-present-1995-2004 is based on Meteosat First Generation(MFG); 2005-present is based on Meteosat Second Generation (MSG)

- Temporal resolution: 30-minute for MFG; 15-minute for MSG

- Spatial coverage: Continental Europe, Canary Islands, Turkey, and Israel

- Spatial resolution: $2.5 \mathrm{~km}$ for $\mathrm{MFG} ; 1 \mathrm{~km}$ for $\mathrm{MSG}$

- Data elements and sources: GHI, DHI and DNI

- Data quality control and assessment: The EnMetSOL model has been validated with 18 stations for the 2004-2011 period (Ineichen 2013). Moreover, a daily quality check is performed with data from more than 300 European meteorological stations

- Estimated uncertainties: Based on comparisons to measured data from 18 stations (Ineichen 2013), the annual average hourly RSME and MBE for GHI are $17.0 \%$ and $0.8 \%$, respectively, and for DNI, 37\% and $0 \%$, respectively. The deviation of site-specific longterm mean values of GHI range from $-6 \%$ to $+5 \%$ for non-mountainous sites (average 3\%) and for DNI from $-14 \%$ to $14 \%$ for all sites (average $9 \%$ )

- Availability: University of Oldenburg: www.energiemeteorologie.de.

\subsubsection{MACC-RAD McCloud}

The preoperational atmosphere service of Copernicus is currently provided through the FP7 projects MACC and MACC-II. MACC combines state-of-the-art atmospheric modeling on aerosols with Earth observation data to provide information services covering European air quality, global atmospheric composition, climate, and UV and solar energy. Within the radiation 
subproject (MACC-RAD), existing historical and daily updated databases HelioClim-3 and SOLEMI for monitoring incoming surface solar irradiance are further developed. The new service is called HelioCllim-4, and it is jointly provided by DLR and Mines ParisTech Armines. Data are made available both via the Copernicus portal and the SODA service.

- Period of record: 2004-present

- Temporal resolution: 15-minute

- Spatial coverage: Europe/Africa/Middle East/Atlantic Ocean.

- Spatial resolution: Spatial resolution is the original pixel of the Meteosat image. Approx. $3 \mathrm{~km}$ at satellite nadir and $5 \mathrm{~km}$ at mid-latitude. The nearest satellite pixel is chosen

- Data elements and sources: Global and direct irradiances

- Data quality control and assessment: Input quality control, regular benchmarking against ground stations, regular monitoring the consistency and detecting possible trends.

- Estimated uncertainties: Results for 15-minute means of irradiance compared to BSRN stations: bias for global irradiance between $4 \mathrm{~W} \mathrm{~m}^{-2}$ and $34 \mathrm{~W} \mathrm{~m}^{-2}$; RMSE is approximately $90 \mathrm{~W} \mathrm{~m}^{-2}$ for most stations; and its relative value ranges between $15 \%$ and $20 \%$ of the mean observed irradiance for stations in desert and Mediterranean climate and between $26 \%$ and $45 \%$ for rainy climate with mild winters. Bias for direct irradiance is between $-44 \mathrm{~W} \mathrm{~m}^{-2}$ and $+46 \mathrm{~W} \mathrm{~m}^{-2}$, and the RMSE ranges from $81 \mathrm{~W} \mathrm{~m}^{-2}(32 \%$ of the mean observed irradiance) to $189 \mathrm{~W} \mathrm{~m}^{-2}(64 \%)$

- Availability: Copernicus: http://www.gmes-atmosphere.eu; SODA: http://www.sodapro.com

- Updates: Continuous.

\subsubsection{MACC-RAD McClear}

The fast clear-sky model called McClear implements a fully physical modeling replacing empirical relations or simpler models used before. It exploits the recent results on aerosol properties and total column content in water vapor and ozone produced by the MACC project. Data are made available both via the Copernicus and the SODA service.

- Period of record: $2004-$ present

- Temporal resolution: 1-minute

- Spatial coverage: Global

- Spatial resolution: Interpolated to the point of interest

- Data elements and sources: Global and direct irradiances for clear-sky cases

- Data quality control and assessment: Input quality control, regular benchmarking against ground stations, regular monitoring of consistency and detecting possible trends

- Estimated uncertainties: For global irradiance, the correlation coefficient ranges between 0.95 and 0.99 ; for direct irradiance: 0.86 and 0.99 . The bias for global irradiance 
ranges from -14 and $25 \mathrm{~W} \mathrm{~m}^{-2}$ to -49 and $+33 \mathrm{~W} \mathrm{~m}^{-2}$ for direct irradiance. The RMSE ranges from $20 \mathrm{~W} \mathrm{~m}^{-2}$ (3\% of the mean observed global irradiance) and $36 \mathrm{~W} \mathrm{~m}^{-2}$ (5\% of the mean observed global irradiance) and $33 \mathrm{~W} \mathrm{~m}^{-2}(5 \%$ of the mean observed direct irradiance) and $64 \mathrm{~W} \mathrm{~m}^{-2}$ (10\% of the mean observed direct irradiance)

- Availability: Copernicus: http://www.gmes-atmosphere.eu; SODA: http://www.sodapro.com

- Updates: Continuous. 


\section{Applying Solar Resource Data to Solar Energy Projects}

This chapter provides a summary of the tools and techniques for evaluating specific solar energy sites based on all available information as well as guidance on steps to improve the on-site determination of the solar resource relevant to the type of solar technology that is being considered. The overall goal is to help the project developer and investor obtain the best estimates of the solar resource and weather information to address four stages of a solar energy project evaluation and operation (see Figure 6.1).

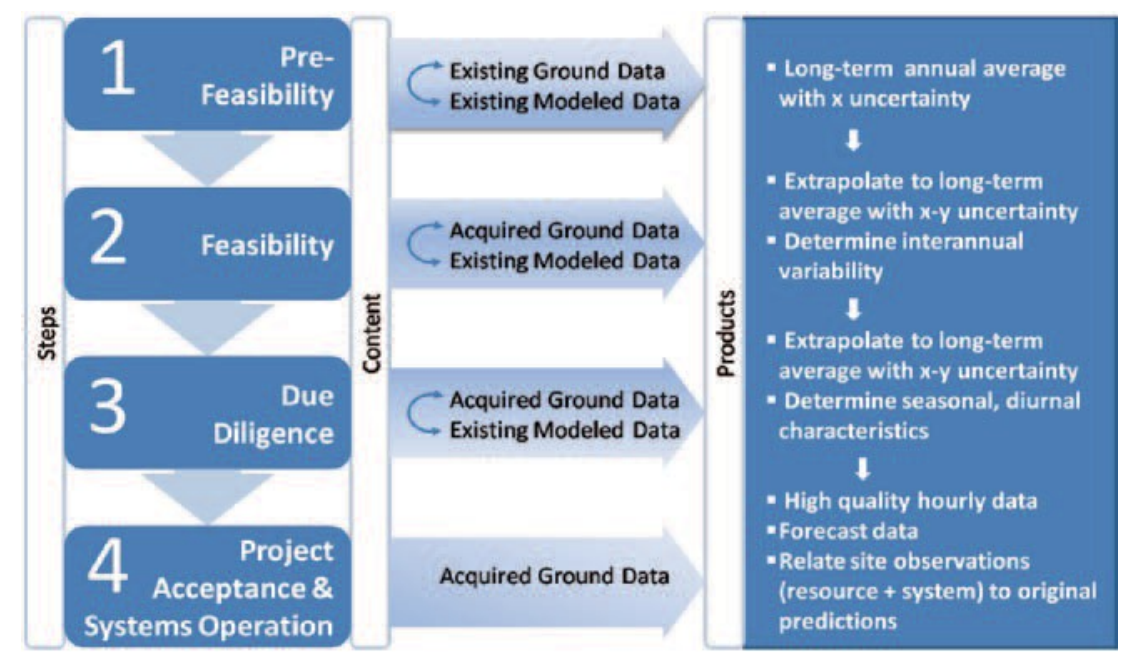

Figure 6-1. The four stages of a solar power plant project

Ideally, a potential solar power plant site will have several years of high-quality on-site data, using the measurement and metrology procedures described in Chapter 3, in formats directly relevant to the type of technology being considered; however, in the current solar energy market, such data are not usually available, and project developers must rely on a number of techniques to provide the most accurate determination of site resource characteristics based on any available information sources. In the United States, these data sources might include some limited on-site measurements of varying quality, access to nearby measurements that may or may not be precisely applicable to the site because of spatial and temporal variability, access to satellitederived irradiance estimates, or access to nearby modeled ground stations, such as those found in the NSRDB. In the latter case, both hourly statistics throughout the entire length of the NSRDB period and TMY data representing either 15 years or 30 years of solar resource data modeled from ground observations might be available. Most ground stations in the NSRDB provide modeled estimates of the solar resource based on cloud cover and other weather observations obtained at the station and not on actual solar measurements (see Chapter 5).

Assuming that no high-quality on-site data are available during the site screening and prefeasibility stages, annual energy estimates must be derived from historical data sets such as the NSRDB or satellite-derived data from commercial vendors. During feasibility assessments, including engineering analysis and due diligence, some periods of high-quality measurements are assumed to be available at the site; however, these relative short-term measurements must be 
extrapolated to long-term records that capture seasonal trends and the interannual variability of solar resources for the site. During the system acceptance and site operation stages, reliance should be on high-quality ground-based measurements, perhaps supplemented to some extent by ongoing satellite-derived measurements for the region.

The project developer should consult Table 6-1 when evaluating sites through the various stages of project development.

Table 6-1. Site Evaluations

\begin{tabular}{|c|c|c|}
\hline $\begin{array}{c}\text { Evaluation } \\
\text { Step }\end{array}$ & Question & Solutions and Insights \\
\hline \multirow[t]{2}{*}{$\begin{array}{l}\text { Site } \\
\text { selection }\end{array}$} & $\begin{array}{l}\text { What proposed site } \\
\text { location(s) need(s) to be } \\
\text { evaluated? }\end{array}$ & \\
\hline & $\begin{array}{l}\text { Has a single site been } \\
\text { chosen? }\end{array}$ & $\begin{array}{l}\text { If not, is the developer making a choice from among two or } \\
\text { more sites or "prospecting" from a wider area? If choosing } \\
\text { among multiple sites, the developer will benefit from using } \\
\text { maps and graphical techniques to evaluate both the } \\
\text { estimated resource and the uncertainty of those resource } \\
\text { estimates. See examples below. }\end{array}$ \\
\hline $\begin{array}{l}\text { Predicted } \\
\text { plant output } \\
\text { throughout } \\
\text { its project life }\end{array}$ & $\begin{array}{l}\text { How can short-term data } \\
\text { sets that provide pro- } \\
\text { jections throughout the next } \\
\text { few years be extended to } \\
\text { long-term ( } 30-y) \text { projections } \\
\text { so that projections of cash } \\
\text { flow throughout the life of } \\
\text { the project can be made? }\end{array}$ & $\begin{array}{l}\text { Different locations may have different interannual variability- } \\
\text { e.g., locations more subject to a monsoon effect will have } \\
\text { higher interannual variability in the summer months. Typically, } \\
\text { on-site data cover at most a few years, so we discuss } \\
\text { procedures for extrapolating these data sets to long-term } \\
\text { projections using longer-term (up to } 45-y \text { ) modeled irradiance } \\
\text { data as well as how to relate the nearest ground stations to } \\
\text { site-specific data. }\end{array}$ \\
\hline \multirow[t]{2}{*}{$\begin{array}{l}\text { Temporal } \\
\text { performance } \\
\text { and system } \\
\text { operating } \\
\text { strategies }\end{array}$} & $\begin{array}{l}\text { How important are } \\
\text { seasonal and diurnal } \\
\text { patterns of the solar } \\
\text { resource? }\end{array}$ & $\begin{array}{l}\text { Many solar energy projects will produce electricity for the } \\
\text { public utility grid. If time-of-day pricing has been implemented } \\
\text { for the consumer, an understanding of the diurnal patterns } \\
\text { and monthly mean values during those months when time-of- } \\
\text { day pricing is in place may be more important than the } \\
\text { estimate of the annual average. For example, if a CSP } \\
\text { project includes thermal storage, the need to analyze when } \\
\text { the system will build up storage compared to when the } \\
\text { system provides power to the grid during daylight hours also } \\
\text { emphasizes the importance of understanding the diurnal } \\
\text { patterns. Thermal storage greatly mitigates the effect of } \\
\text { system intermittency, but accurate or realistic daily, hourly, or } \\
\text { subhourly solar radiation data may still be needed. }\end{array}$ \\
\hline & $\begin{array}{l}\text { Are data needed that most } \\
\text { closely match actual } \\
\text { concurrent utility load data } \\
\text { to conduct grid-integration } \\
\text { studies and system } \\
\text { intermittency? }\end{array}$ & $\begin{array}{l}\text { In this case, daily, hourly, or even subhourly data may be } \\
\text { needed for a specific time period, which cannot be provided } \\
\text { by TMY data. }\end{array}$ \\
\hline
\end{tabular}




\begin{tabular}{|l|l|l|}
$\begin{array}{c}\text { Evaluation } \\
\text { Step }\end{array}$ & \multicolumn{1}{|c|}{ Question } & \multicolumn{1}{|c|}{ Solutions and Insights } \\
\hline & $\begin{array}{l}\text { What are the temporal and } \\
\text { spatial characteristics of the } \\
\text { data sources available to } \\
\text { the developer, and how do } \\
\text { these characteristics } \\
\text { influence the evaluation of } \\
\text { system performance? }\end{array}$ & $\begin{array}{l}\text { Example: Measured solar data apply to a specific location } \\
\text { and are usually recorded at short time intervals (6 min), then } \\
\text { averaged to the desired time interval (often hourly in the early } \\
\text { project phase) }\end{array}$ \\
& $\begin{array}{l}\text { Example: Surface modeled data (e.g., NSRDB/METSTAT) } \\
\text { are somewhat smoothed, because they are based on cloud } \\
\text { cover observations that can be seen from a point location, } \\
\text { typically a circle 40-km in radius, averaged over an } \\
\text { approximate 30-min period } \\
\text { Example: Satellite data usually represent snapshots in time } \\
\text { because of the scanning characteristics of the onboard } \\
\text { radiometers and are typically considered to range from nearly } \\
\text { instantaneous to approximate 5-min averages. For SUNY } \\
\text { satellite data used in the NSRDB, the individual pixel size is 1 } \\
\text { km, and the pixel is at the center of the 10-km grid cell. } \\
\text { Newer satellite-based methodologies now average the 1-km } \\
\text { pixel to 3-km or 5-km grid cells. }\end{array}$ \\
\hline
\end{tabular}

\subsection{Data Applications for Site Screening and Prefeasibility Assessment}

\subsubsection{Example for Review of Data Sources: DNI in the United States}

In the first step, the available data sources for the region of interest and the irradiance component of interest have to be reviewed. Several sources were presented in Chapter 4 and Chapter 5. The following example is for DNI and locations in the United States, mostly in the Southwest. The selected data sources in Table 6-2 are those most likely to be used by a project developer for a concentrating solar technologies (CST) plant in this region. Similar data sources may be available for other locations (see Chapter 3).

Table 6-2. Data Sources for DNI Estimation

\begin{tabular}{|l|l|l|l|}
\multicolumn{1}{|c|}{ Source } & $\begin{array}{c}\text { Period of } \\
\text { Record }\end{array}$ & \multicolumn{1}{|c|}{ Origin } & \multicolumn{1}{c|}{ Comments } \\
\hline NSRDB/SUNY & $\begin{array}{l}1998- \\
2009\end{array}$ & $\begin{array}{l}\text { CPR } \\
\text { SolarAnywhere } \\
\text { (See Chapter 4) } \\
\text { monthly and } \\
\text { annual mean } \\
\text { DNI values }\end{array}$ & $\begin{array}{l}\text { Monthly and annual mean values } \\
\text { available for uniform grid (CONUS } \\
\text { and HI) with 0.1-degree spacing. DNI } \\
\text { values for approximately 2,100 grid } \\
\text { cells in the Southwest have been } \\
\text { adjusted upward to correct for } \\
\text { satellite model underestimates of } \\
\text { DNI in areas of high surface albedo } \\
\text { (snow, sand, salt flats). }\end{array}$ \\
\hline $\begin{array}{l}\text { NSRDB/SUNY } \\
\text { GNidded hourly } \\
\text { DNI values }\end{array}$ & $1998-$ & $\begin{array}{l}\text { CPR- } \\
\text { SolarAnywhere } \\
\text { (See Chapter 4) }\end{array}$ & $\begin{array}{l}\text { Hourly time-series data available } \\
\text { from NREL's Solar Prospector and } \\
\text { NCDC Web sites in different formats. } \\
\text { The mean DNI values have not been } \\
\text { corrected for the surface albedo } \\
\text { issue. }\end{array}$ \\
\hline TMY2 & $1961-$ & $\begin{array}{l}\text { 1961-1990 } \\
\text { NSRDB (See } \\
\text { Chapter 4) }\end{array}$ & $\begin{array}{l}\text { The annual and monthly mean } \\
\text { DNI for the selected "typical" } \\
\text { months may not agree with the } \\
\text { 30-year monthly means for the } \\
\text { same station. }\end{array}$ \\
\hline
\end{tabular}




\begin{tabular}{|l|l|l|l|}
\multicolumn{1}{|c|}{ Source } & $\begin{array}{l}\text { Period of } \\
\text { Record }\end{array}$ & \multicolumn{1}{|c|}{ Origin } & \multicolumn{1}{|c|}{ Comments } \\
\hline TMY3 & $\begin{array}{l}1991- \\
2005\end{array}$ & $\begin{array}{l}\text { 1991-2005 } \\
\text { NSRDB (See } \\
\text { Chapter 4) }\end{array}$ & $\begin{array}{l}\text { Based on 24 years of data for sites } \\
\text { near the 1961-1990 NSRDB } \\
\text { locations and 12 years of data for } \\
\text { remaining sites. Years with large } \\
\text { amounts of stratospheric aerosol } \\
\text { loading caused by volcanic eruptions } \\
\text { are excluded from the selection. The } \\
\text { mean DNI values may NOT agree } \\
\text { with the long-term means for the } \\
\text { same location. }\end{array}$ \\
\hline $\begin{array}{l}\text { DNI } \\
\text { measurements }\end{array}$ & $\begin{array}{l}1977- \\
\text { present }\end{array}$ & $\begin{array}{l}\text { Various (See } \\
\text { Chapter 4) }\end{array}$ & $\begin{array}{l}\text { Measurement networks in } \\
\text { operation 1977-1980 (NOAA } \\
\text { network) and 1993-present } \\
\text { (SURFAD) }\end{array}$ \\
\hline $\begin{array}{l}\text { Surface } \\
\text { weather } \\
\text { observations }\end{array}$ & $\begin{array}{l}1961- \\
2010\end{array}$ & $\begin{array}{l}\text { NSRDB (See } \\
\text { Chapter 4) }\end{array}$ & $\begin{array}{l}\text { Observations for 15-year and 30- } \\
\text { year data sets available from } \\
\text { NSRDB (usually NWS stations } \\
\text { located at airports). Most reliable } \\
\text { source. }\end{array}$ \\
\hline $\begin{array}{l}\text { Modeled } \\
\text { weather data }\end{array}$ & $\begin{array}{l}1998- \\
2005\end{array}$ & $\begin{array}{l}\text { North } \\
\text { American } \\
\text { Regional } \\
\text { Reanalysis }\end{array}$ & $\begin{array}{l}\text { Data from model with 32-km spatial } \\
\text { resolution and 3-h time resolution. } \\
\text { Advisable for user to calculate } \\
\text { average temperature and dew point } \\
\text { for times of interest for comparison to } \\
\text { other (best available observations } \\
\text { from nearest site) }\end{array}$ \\
\hline
\end{tabular}

\subsubsection{The Site Screening Process}

In the early stages of project development, a prefeasibility assessment of possible sites is undertaken. A desired outcome at this stage is the estimated annual energy production that could be expected from the solar energy system in various proposed locations. Historical solar resource data sets are generally used in this stage, often in the form of maps, such as the NSRDB/SUNY gridded maps (Table 6-2). These data sets use a fairly consistent methodology to reliably identify the regions of highest solar potential. The maps should be used to make a preliminary assessment of solar resource, assuming a fairly large potential for error (approximamtely 15\%). Thus, if a desirable level of solar resource is $7.0 \mathrm{kWh} / \mathrm{m}^{2} /$ day, sites with mapped resource values down to approximately $6.0 \mathrm{kWh} / \mathrm{m}^{2} /$ day should be considered.

Examples of a "first order" prefeasibility assessment include the analysis of CST potential in the southwestern United States conducted by NREL's Concentrating Solar Power Program (Mehos and Perez 2005). ${ }^{37}$ Using geographic information system screening techniques, resource maps were developed that highlighted regions potentially suitable for project development after various land use constraints - such as protected land areas, sloping terrain, and distance from transmission-were taken into consideration (Figure 6-2 and Figure 6-3). The results of these studies show that even with these constraints vast areas in the southwestern United States are

\footnotetext{
${ }^{37}$ See www.nrel.gov/csp/maps.html.
} 
potentially suitable for CST development (Mehos and Perez 2005). Maps such as these have been valuable to project developers to highlight specific regions in which various levels of site prospecting and prefeasibility analysis can take place.

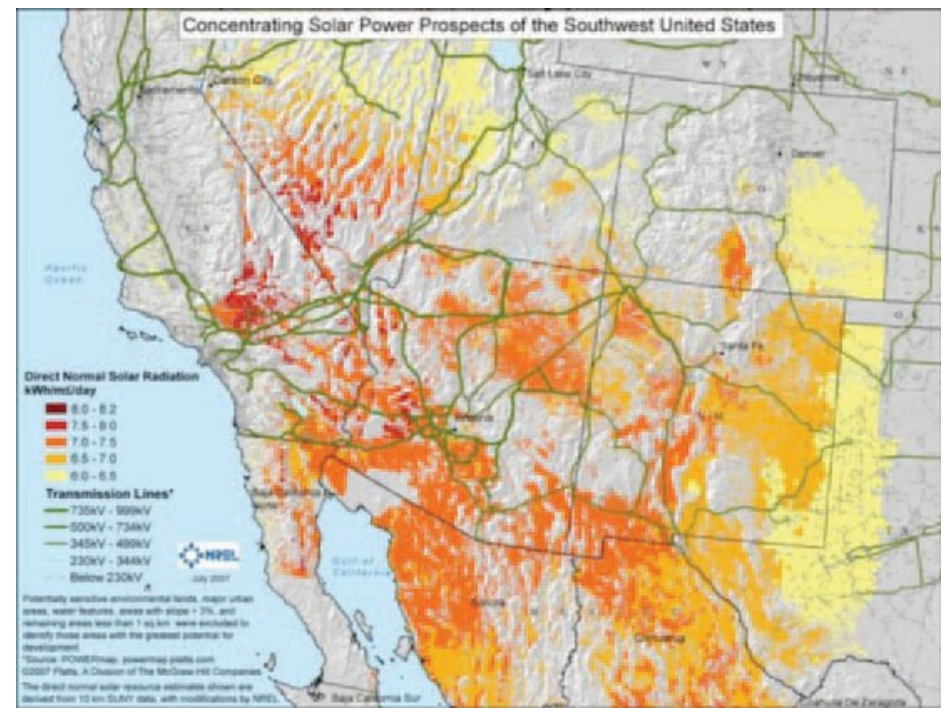

Figure 6-2. Geographic information system analysis for available site selection using DNI resource, land use, and $3 \%$ terrain slope. Image from NREL

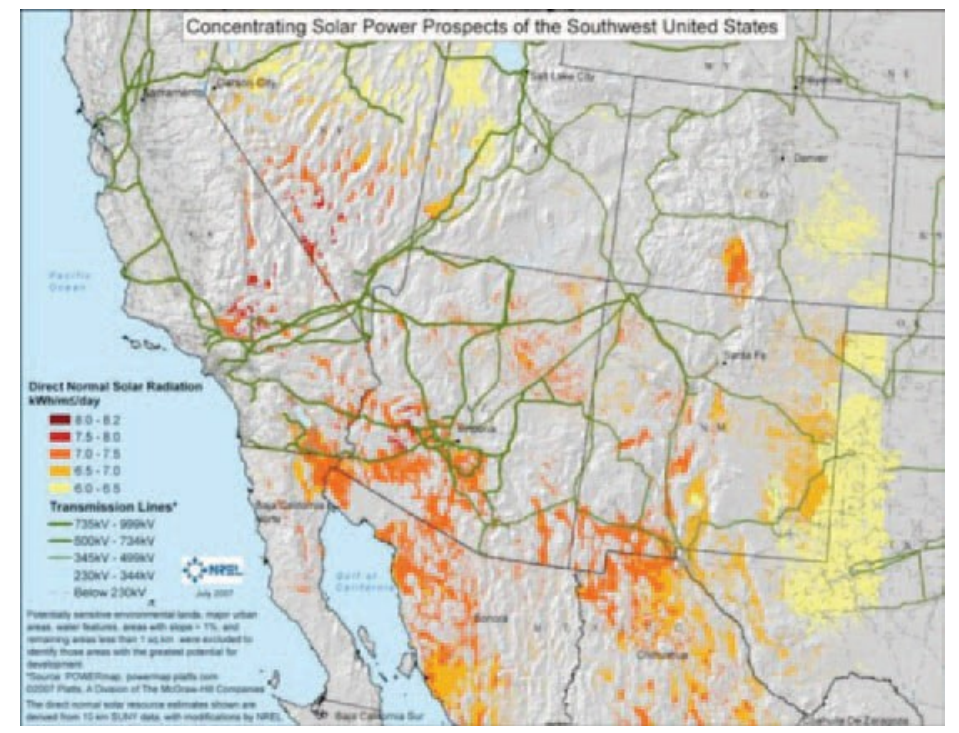

Figure 6-3. Geographic information sytsem analysis for available site selection using DNI resource, land use, and $1 \%$ terrain slope. Image from NREL

With the introduction of powerful, easy-to-use tools such as the Solar Advisor Model, ${ }^{38}$ greenius, ${ }^{39}$ and the NREL Solar Power Prospector Web site, ${ }^{40}$ many analysts now expect to use time-dependent modeling of their prospective solar systems as part of the preliminary analysis.

\footnotetext{
${ }^{38}$ See www.nrel.gov/analysis/analysis tools tech sol.html.

${ }^{39}$ See http://freegreenius.dlr.de/.

${ }^{40}$ See http://maps.nrel.gov/node/10/.
} 
Considerable care must be taken to choose the correct irradiance data sets for input to the model. Experts recommend multiple years of at least hourly input data, rather than data from only one year or even TMYs, to assess the effects of interannual variability of the solar resource on yearto-year system performance. Each hourly data set should be evaluated at least to determine whether the monthly mean values from hourly data match the best estimate of monthly mean values at the proposed site (Meyer et al. 2008).

\subsubsection{Influence of $A O D$}

For solar energy projects, a key step in site screening is to implement a concept we call clean air prospecting. This is of special importance for CST projects, because DNI is more strongly affected by AOD than GHI is. In deserts and other areas with high solar resource, most sites have low annual cloud cover. In these locations, the annual average irradiance is strongly influenced by the AOD. Knowing the AOD characteristics is vital to assessing the solar resource and the performance of solar installations.

AOD is a measure of haze and smoke effects in irradiance that are not caused by clouds. Sources of AOD include dust and particulates, air pollution, smoke from wildfires and agricultural burning, and sea salt (near coastlines). Solar facilities should, if possible, be sited at locations that are protected from sources of these aerosols. For rural areas with low AOD, the irradiance averages from the satellite-derived (gridded) data are more likely to be correct, if it can be confirmed that the area is indeed protected from sources of aerosols.

The analyst should ask the following questions about the site:

- What are sources of potential aerosols? These may include:

○ Dust storms

- Air pollution

○ Fires

○ Proximity to urban areas

○ Proximity to power plants, mines, etc.

- Does the area have good visibility most of the time? Are distant hills or features visible without the effects of haze?

- No visible haze would indicate that the AOD is indeed low, and, therefore, the irradiance is similar to the modeled map values.

- If the area is known to have some form of visible haze, aerosols may (or may not) be a problem at the site. Further research or measurements may be necessary.

Typically, areas on the fringes of cities are zones of higher uncertainty in AOD. Also, areas on the fringes of metropolitan areas may be good candidates for solar energy for economic and infrastructure reasons. New measured irradiance data may be necessary to resolve whether a site is sufficiently protected from sources of aerosols. 


\subsubsection{Comparison of Satellite-Derived Irradiation Resource Data Using Geographic Information System Tools}

A study conducted by the MESoR project in Europe (Beyer et al. 2009) provides insights into the spatial distribution of uncertainty of the estimates of irradiance by relative cross-comparison of five data sources: METEONORM, Satel-Light, NASA SSE, SOLEMI, and PVGIS.

The map-based comparison is performed as a type of relative benchmarking of solar databases. It does not point to the "best" database, but it gives an indication of the user's uncertainty at any location in the region by comparing data from different sources. Because the spatial products cover different periods of time, this comparison also introduces uncertainty resulting from the interannual variability of solar radiation. The maps of long-term averages of irradiance yearly sums are cross-compared. The map of standard deviation from the average indicates the combined effect of differences among the databases, and in this study it is used as an indicator of model uncertainty.

As shown on the maps of standard deviation (Figure 6-4), the solar industry in some regions in Europe might expect higher variability in the outputs from the analyzed databases. These variations are found primarily in complex climatic conditions, such as mountainous regions, and density and quality of input data. Significant differences are found in some regions with high DNI potential, such as the Balkan region, Greece, parts of the Iberian Peninsula, and Italy.
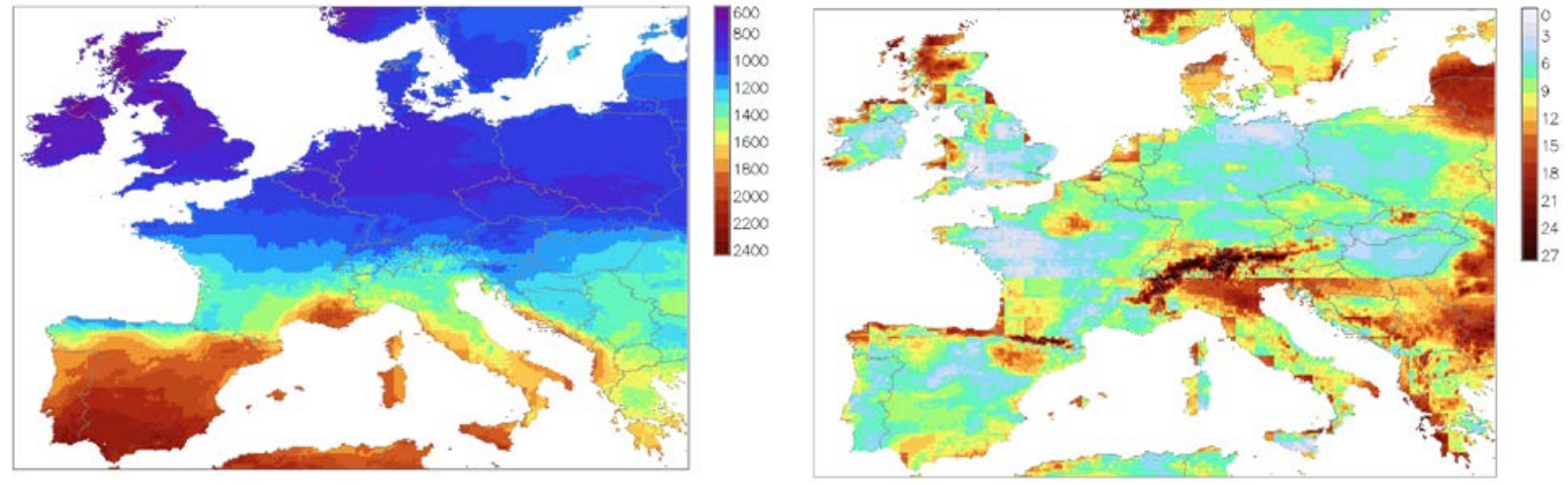

Figure 6-4. Yearly sum of DNI as calculated from five modeled data sets: METEONORM, PVGIS, NASA SSE, Satel-Light, and SOLEMI-(left) average of five databases $\left(\mathrm{kWh} / \mathrm{m}^{2}\right)$ and (right) relative standard deviation (\%). Graphics modified from Beyer et al. (2009)

The MESoR map comparison studies have provided the following results:

- There is an inherent difference between in-situ (ground) and satellite observations and in the methods of processing these data. Databases relying on the interpolation of ground observations (PVGIS Europe and partially METEONORM) are sensitive to the quality and completeness of ground measurements (especially those from earlier time periods) and density of the measurement network. PVGIS and METEONORM include long-term statistical averages, and some geographical regions may show higher uncertainty because of the lower concentration of measurement sites with varying data consistency. The satellite-derived databases (NASA SSE, SOLEMI, and Satel-Light) offer time series with 
high time resolutions (3-hour, hourly, and 30-minute data, respectively) and provide spatially continuous coverage, but the results may be affected by a higher uncertainty of the cloud cover assessment when the ground is covered by snow and ice and for low sun angles; however, these regions are typically not high-value sites for solar energy applications.

- Terrain effects (e.g., differences in AM, shadowing by surrounding terrain) play a role in solar radiation modeling in hilly and mountainous regions. The spatial resolution of the input data and the selected DEM have direct impacts on the accuracy of the estimates. A coarse-resolution DEM results in a smoother spatial pattern of solar irradiance, which also affects the regional mean of the irradiation; however, a high-resolution DEM is presently being used only in METEONORM and PVGIS. Databases with coarser spatial resolution (e.g., NASA SSE) provide global estimates; however, for studies at a local level they may show higher deviations, because they smooth out local climate and terrain features.

- Like cloudiness, AOD is highly variable throughout time and space. Its measurement requires sophisticated instrumentation and complex satellite models. The numerous AOD data sets available to the solar radiation modeling community come from various sources; however, except for a few - such as AERONET, ${ }^{41}$ the SKYNET branch measurements, ${ }^{42}$ and temporally highly resolved modeled data such as MATCH (M. SchroedterHomscheidt and A. Oumbe, 2013) - they represent only climate (averaged) values for a few years, which do not address high-frequency changes.

- DNI is sensitive to the determination of cloud index that attenuates the solar irradiance reaching the surface. With the older generation satellites (MFG), effects of snow, ice, and fog interfere with cloud detection. This often leads to underestimation of DNI, especially in mountainous regions. The current satellite instrument MSG Spin Enhance Visible and Infrared Imager, in orbit since 2004, provides a high-quality calibrated signal with stable and known properties over continents, and with high information potential of 11 multispectral channels. This shows promising improvements in cloud detection.

- DNI is more sensitive than GHI to atmospheric parameters. The quality and spatial detail of satellite-derived databases are determined by input data used in the models, primarily parameters describing the optical state of the atmosphere, such as Linke atmospheric turbidity, or the analytical data sets (ozone, water vapor, and aerosols). After cloudiness, the effect of aerosols represented by AOD is the most important variable affecting DNI (Gueymard and George 2005).

The studies conducted so far provide only a preliminary outline of the state of the art of current knowledge of irradiance. Such a data comparison as provided above does not fully address the needs of the solar energy industry, so further work is needed to improve our knowledge and decrease the uncertainties.

\footnotetext{
${ }^{41}$ See http://aeronet.gsfc.nasa.gov.

${ }^{42}$ See http://www.euroskyrad.net/sites.html.
} 


\subsection{Data Applications for Feasibility, Engineering, and Financial Assessments}

After one or more candidate sites have been selected for an engineering feasibility assessment, a common problem facing solar power plant project developers is how to produce data sets that allow for the most reliable calculation of annual or interannual system performance when only short-term ground measurements, along with other estimated data sources, are available. In the wind energy industry, solutions to this problem are known as measure-correlate-predict (Thøgersen et al. 2007). Measure-correlate-predict is based on various statistical procedures whereby short-term on-site measurements are related to nearby long-term measurements to obtain estimates of a site's long-term wind energy potential and interannual variability. The correlation is then used to predict resources for the new site.

The problem tends to be more complex for the wind industry than for the solar industry, because:

- Wind resources are generally much more variable spatially than solar resources

- Wind characteristics can vary significantly with height above the ground, which complicates the comparison of short-term to long-term measurements if the heights of the two measurement systems are different

- Wind resources must take speed and direction into account, which complicates the measure-correlate-predict statistical procedures

- With the exception of reanalysis data or surface wind observations from national weather service stations, reliable long-term data relevant to a proposed wind site, such as those developed from a satellite methodology for solar radiation resources, are generally lacking for wind resources.

For these reasons, it is not necessary to employ some of the more complex measure-correlatepredict methods available in the literature for wind energy assessments to solar power plant analyses. We suggest a simpler approach that should be reasonably viable. Readers interested in learning more about wind-energy-related measure-correlate-predict methods will find a good summary of various approaches in Thøgersen et al. (2007).

The degree of accuracy required for system performance and energy yield estimates depends on the stage of project development, as follows:

- Prefeasibility stage. Specific sites are evaluated to determine whether they may be suitable for development and thus require more comprehensive evaluation.

- Feasibility stage. Sites have been selected for actual project implementation, and system design and energy performance estimates become very important. At this stage, more comprehensive knowledge of the annual resource as well as seasonal and diurnal characteristics, with known accuracies, is required. After (or concurrent with) this detailed analysis, due diligence on the chosen project site is required, which involves accurate cash flow analysis throughout the life of the project. In this case, accurate longterm site performance estimates are required, and the variability of the system output from year to year (caused by interannual variability of the resource, again within wellestablished confidence limits) is required. 


\subsubsection{Extrapolating Short-Term Measured Data Sets}

The basic methodology for obtaining an estimate of the annual solar resource suitable for prefeasibility analysis that can be used to make energy yield estimates is to acquire available long-term site estimates, such as satellite-derived estimates or nearby modeled station values (such as those available through the NSRDB or TMYs). These data sets and their uncertainties have already been described in previous chapters and sections. When short-term on-site estimates from new solar radiation measurements are available, they can be used to reduce the uncertainty of the modeled estimates (Gueymard and Wilcox 2009). This process becomes critical in the project feasibility and due diligence stages of project development.

Three methods by which we can combine the short- and long-term data to obtain a more accurate estimate of the long-term solar resource (such as what may be needed for project feasibility studies) are discussed here.

The ratio method assumes that at least two independent data sets are available: an on-site measurement data set (presumed to be relatively short term), and a long-term climatological data set, such as a satellite-derived database or a nearby long-term measurement station or modeled data. Ideally, at least part of the two data sets should be concurrent. If there is no concurrency in the data, the ratio method can still be applied, but the uncertainty of the resulting long-term onsite data profile will likely be much higher than if concurrent data periods are available. This method is described in Gueymard and Wilcox (2009). Basically, the method involves calculating the ratios of a selected averaging period of the concurrent data sets, such as hourly or monthly averages, then applying these ratios to the balance of the long-term data set to produce a longterm estimate for the site.

There are several important considerations to applying this approach, especially if the long-term data set involves the use of satellite-derived data for the same location as the site data. Although the ratio method removes biases between the short-term and long-term data sets, the biases may, in fact, vary from year to year or from season to season. Variations in biases suggest that the cross-correlation between the two concurrent measurement sources is less than 1.0, and lower cross-correlation values indicate more uncertainty associated with extrapolating short-term data to long-term means (Gueymard and Wilcox 2009). Consider these possible scenarios:

- In an ideal scenario, there is low month-to-month variability in biases between the reference data and on-site measurements. Under these circumstances, a simple correction factor based on the ratio method should be acceptable for extrapolating the short-term data set.

- A second scenario is high random variability between the short-term on-site data and the long-term reference data source, meaning that an accurate extrapolation to a longer-term value at the site will have high uncertainty.

- A third scenario is when there are strong seasonal trends in the data, which may require additional years of on-site data to better confirm or define the trend. This scenario would ultimately lead to long-term extrapolations with low uncertainty.

A second method is to combine two different data sets by weighting each. They could be weighted equally, or, as suggested by Meyer et al. (2008), the weighting can be determined 
based on the inverse of the uncertainty of each data set. By assuming that the deviations from truth follow a normal distribution and are statistically independent, the Gaussian law for error propagation can be applied. Meyer et al. (2008) then provide curves showing how the additional data sets do not need to be of the same high quality as the base data set to add value to the combined data sets (Figure 6-5).

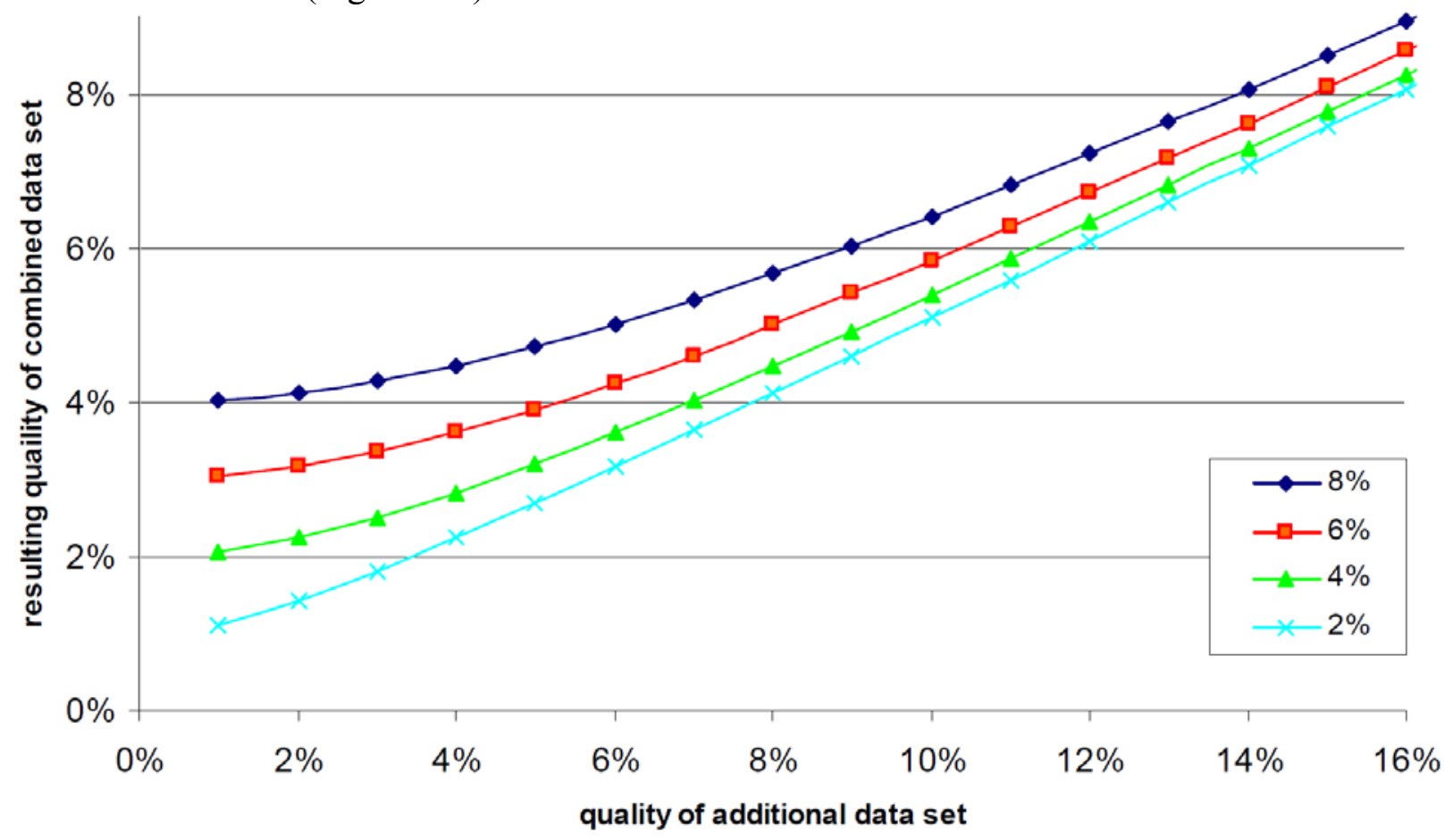

Figure 6-5. Resulting uncertainty when combining a base data set of $2 \%, 4 \%, 6 \%$, or $8 \%$ overall uncertainty with an additional data set of varying quality. Figure from Meyer et al. (2008)

Meyer et al. (2008) show that by using more than two data sets, the resulting quality of the combined data set can be even further improved. For example, when the base data set has an uncertainty of $4 \%$, the resulting data set can be improved by adding two data sets with a moderate $7 \%$ quality rather than $10 \%$; however, if the two additional data sets have an uncertainty of $10 \%$ or more, the base data set cannot be improved. Therefore, data sets with such high uncertainties should not be used. If the analyst uses this method, he or she should be prepared to demonstrate that the incorporated data sets are truly independent and there are no correlations (similar instrumentation and measurement protocols, common estimates for model parameters such as aerosols or clouds). This methodology of combining the uncertainties of various input data sets to provide the resulting uncertainty of the "best guess" DNI estimate for a site is elaborated in a more recent paper by Meyer et al. (2008).

Another approach for reducing the uncertainty of long-term satellite-derived data sets using highquality short-term ground data has been developed by Schumann et. al (2011). In this method, the frequency distribution of the ground-based data is used to improve the satellite-derived data. Their method has resulted in greatly reduced bias errors and improved Kolmogorov-Smirnov Integrals in the satellite data, especially for DNI estimates, even when as little as 3 months of ground data are available. In particular, their method shows that a full year of ground data are 
sufficient to obtain significant improvements in long-term satellite-derived DNI data sets, resulting in greatly improved bankable data for purposes of financing large-scale CSP plants. Although their method was less successful in improving GHI data, they point out that there is generally less of a need to correct GHI data.

Their method builds on results from earlier studies (Carow 2008, Beyer et. al 2010) in which the frequency distribution, rather than a simple ratio technique, is employed to improve the satellitederived data, noting that the frequency distribution of DNI has a strong influence on the power production of CSP plants, just as the wind speed frequency distribution has a strong influence on the power production of wind plants. The method of "training" the satellite data using overlapping time series from ground measurements is demonstrated in Figure 6-6. The differences in cumulative frequency distributions (training data) are applied to the test satellite data, producing a corrected data set (upper right image). The lower panels then show how the cumulative frequency distributions of the satellite data are "mapped" to a new frequency distribution based on the correction factors developed with the ground data.
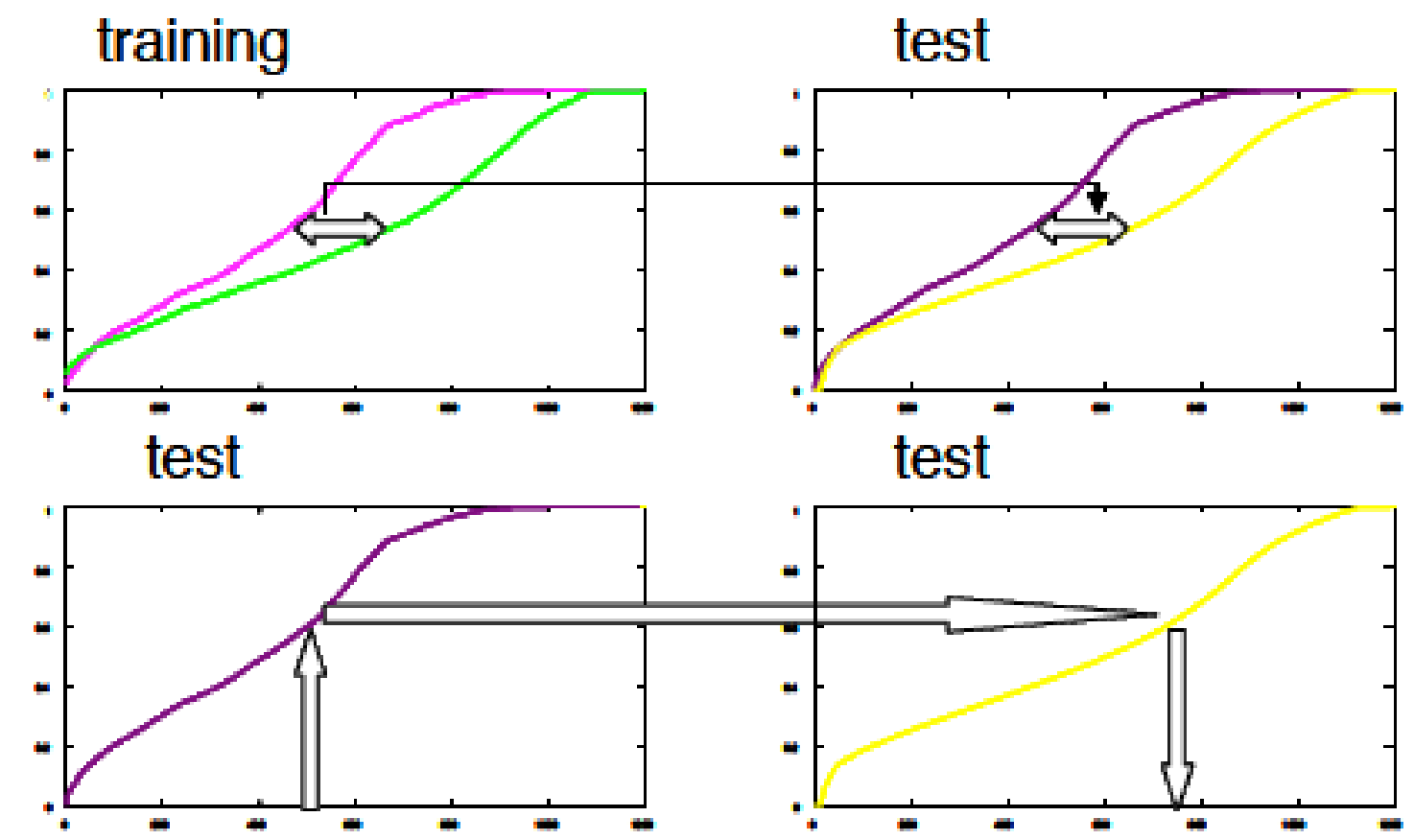

Figure 6-6. Upper left: Cumulative frequency distribution for training time of overlapping ground data and satellite time series. The arrow illustrates the difference between the two curves. Upper right: Corrected satellite cumulative frequency distribution for test period. Lower left: Mapping of original satellite irradiance values to original cumulative frequency distribution (arrow and bottom right image). The original cumulative frequency distribution is first mapped to produce a corrected cumulative frequency distribution, then the corrected cumulative frequency distribution is adjusted to satellite values. Green: ground data from training set; magenta: satellite data from training set; purple: satellite data from test set; yellow: corrected satellite data. Figure from Schumann et al. (2009)

Despite the improvements offered by the method of Schumann et al (2009), significant biases in the DNI estimates remain, as demonstrated by Killius and Schroedter-Homscheidt (2012). A 
method to reduce the bias errors to near zero, and especially to minimize the biases of the highest radiation values that are used for design purposes, has been presented by Mieslinger et al. (2014). The method was tested against two high-quality ground stations: Plataforma Solar de Almería (southern Spain) and Tamanrasset (Algeria); and four different satellite-derived methodologies: DLR, University of Oldenburg Department of Energy and Semiconductor Research, GeoModel Solar, and HelioClim-3. Unlike the previous methods, the method of Mieslinger et al. (2014) takes into consideration not only the systematic but also the random errors that are found when comparing satellite to ground data. Random errors are caused by parallax errors as well as by the difficulty in determining the vertical thickness of clouds from the satellite data used in the four methodologies applied here.

The basis of the adaption method is to apply a third order polynomial relationship rather than a simple regression model, to account for both the systematic and the random errors. The polynomial function also addresses results in significant improvements in the differences between measured and modeled data at high irradiance values. Using the criteria that an adapted data set should demonstrate a relative mean bias comparable to high-quality ground measurements $( \pm 2 \%)$, the polynomial adaption method was shown to exceed this limit, especially when the $\mathrm{HC} 3$ data are removed from the testing. It was also demonstrated that forcing the bias to zero considerably reduces the Kolmogorov-Smirnov integrals of the satellitederived values. Nevertheless, to validate this method further, it is clear that additional testing should be applied to more sites to investigate the performance of the method in different climatic zones and topographies.

Other methods have been proposed in the literature (Bender et al. 2011; Cebecauer and Suri 2010; Gueymard et al. 2012; Harmsen, Tosado-Cruz, and Mecikalski 2014; and Thuman, Schnitzer, and Johnson 2012), and they are typically used by commercial data providers. This is because the short-term measured data set must first be subjected to a stringent quality-control procedure and then to more or less complex statistical methods. There is currently no study that compares the performance of these methods, the details of which are often proprietary. Finally, an innovative optimal interpolation method has been proposed to perform the necessary corrections to long-term data time series at a regional scale rather than for one specific site at a time (Ruiz-Arias et al. 2015).

\subsubsection{Interannual Variability and Exceedance Probabilities}

Studies have also been undertaken to determine how long surface measurements at a proposed site should be taken before the true long-term mean is captured. This is important when no concurrent data sets are available and yet project finance decisions must still be made. Another way to look at the problem is to ask how representative a short-term (perhaps 1-y) measurement is to the "true" climatological (nominally 30-y) mean? In the wind industry, a rule of thumb is that it takes 10 years of on-site wind measurements to obtain a mean annual wind speed that is within $\pm 10 \%$ of the true long-term mean, which is generally required by financial institutions. But in a case with only 1 year or 2 years of on-site measurements, these data may be all that are available to a financial institution conducting due diligence on a project.

Gueymard and Wilcox (2009) begin to address this problem through an analysis of the 8-year SUNY data set used in the updated 1991-2005 NSRDB with coincident measured hourly data from 37 sites from various networks in the conterminous United States; however, only 4 stations 
had continuous measurements for 25 years or more, which are needed to determine the climatological average. These stations are Burns, Eugene, and Hermiston, Oregon, and NREL's Solar Radiation Research Laboratory station on top of South Table Mountain near Golden, Colorado.

Tomson, Russak, and Kallis (2008) show that the mean annual global irradiation in any year is virtually independent of the previous year, which means that 11 years of on-site measurements does not represent the long-term mean. Thus, Gueymard and Wilcox (2009) examined the longterm data from the 4 stations mentioned to address questions about how many years of measurements it takes to converge to the long-term mean and whether the variability in annual radiation changes significantly from one site to another.

For these stations, the results from Gueymard and Wilcox (2009) show that, first, there is much lower interannual variability in GHI than in DNI. GHI is almost always within $\pm 5 \%$ of the true long-term mean after only one year of measurements, regardless of which year these measurements are taken. The situation is quite different for DNI, however. After only one year of measurements, the study shows that the estimate of the average DNI is no better than $\pm 10 \%$ to $\pm 20 \%$ of the true long-term mean. Another way of stating this finding is that the COV for DNI is generally two to three times higher than the COV for GHI.

At 2 of the sites, upward of 10 years of measurements are required to be within $\pm 5 \%$ of the true long-term mean, which is consistent with the findings in the wind energy industry.

Financial institutions prefer to evaluate the risk of uncertainty with solar resource data in terms of exceedance probabilities (e.g., P50 or P90). P50 is the result of achieving an annual energy production based on the long-term median resource value. For this value, the probability of reaching a higher or lower energy value is 50:50. For example, TMYs represent the P50 value. For an exceedance probability of P90, the risk that an annual energy value is not reached is $10 \%$ ( $90 \%$ of all values in a distribution exceed the P90 value).

Figure 6-7 provides other interesting observations about multiple years of DNI measurements, particularly for Hermiston and Burns, which are in the arid eastern part of Oregon. Even in Golden, Colorado, a cloudier than average year will strongly influence negative anomalies, but these generally converge to zero more quickly than do the positive anomalies. Another factor, especially for clear sites, is that AOD becomes the primary influence on DNI variability; events such as volcanic eruptions or regional forest fires produce significant AOD anomalies that could be the main cause of the asymmetries shown in Figure 6-7.

These results indicate the importance of having a second, independent quality data set, such as a satellite-derived data set, available to reduce the uncertainty of the long-term average DNI estimates for a proposed CST site to provide reasonable due diligence of a plant's estimated performance throughout the life of the project. 

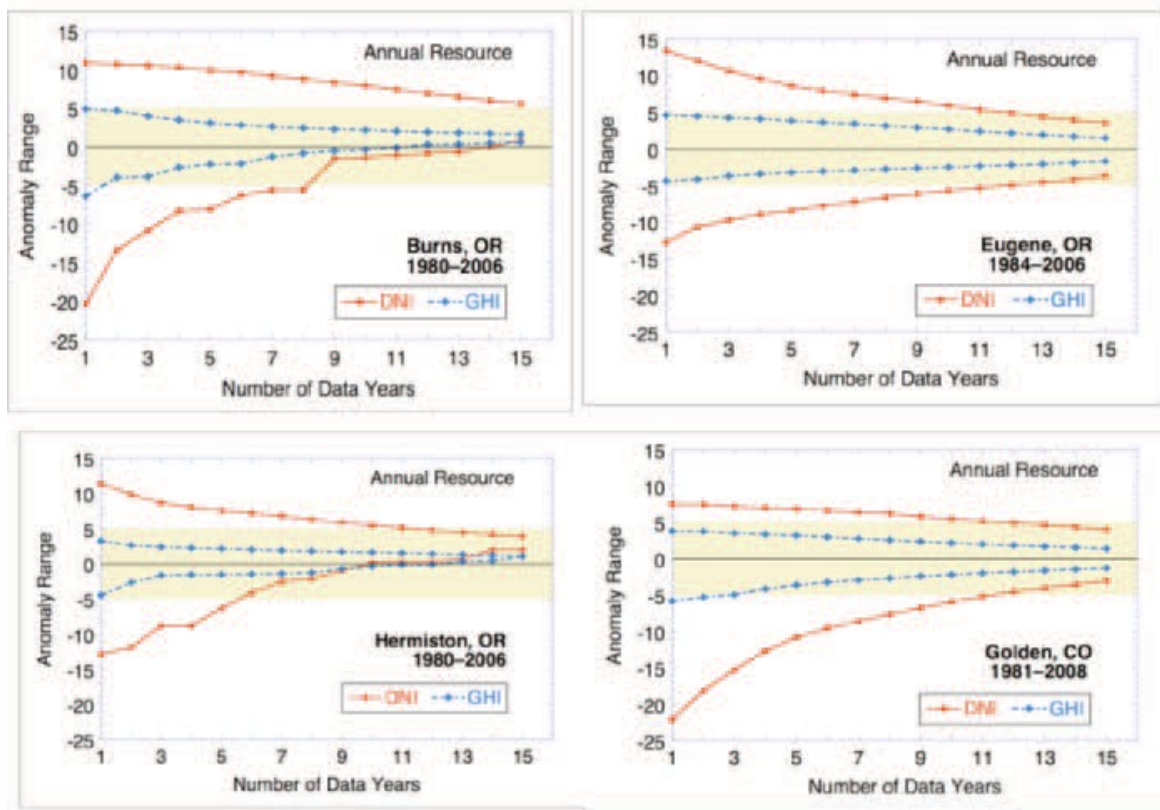

Figure 6-7. Number of years to stabilize DNI and GHI in (clockwise from upper left) Burns, Oregon; Eugene, Oregon; Hermiston, Oregon; and Golden, Colorado. Image modified from Gueymard and Wilcox 2009

\subsubsection{Examples of Mean Irradiance Estimation and Hourly Data Selection Using NSRDB/SUNY, TMY3, and Measured Data}

Example 1 is a proposed trough plant near Harper Lake, California. Harper Lake is actually a dry lake bed with very bright salt deposits on the surface. Our goals for exploring this example are to:

- Determine the best estimate for mean DNI by month and year for a chosen site

- Procure one or more years of time series DNI (and weather) data for use in timedependent modeling (CST plant models or electrical grid models).

To quickly assess the annual and monthly mean DNI, we use the Solar Prospector ${ }^{43}$ with satellite ground surface imagery (from Google Maps) as background. Figure 6-8 shows nine NSRDB/SUNY grid cells in the area near Harper Lake in the Mojave Desert. The values of average DNI can be obtained from Solar Prospector. The upper value is the mean DNI from the hourly data, which is not corrected for specular reflection. The lower is from the map, which has been corrected for this artifact.

Next, we look at the mean DNI values, by month, for the 8 years of data from the 1998-2005 NSRDB. We do this for the desired location and a few nearby locations. If the map value and the hourly averaged values are different by more than $0.2\left(\mathrm{kWh} / \mathrm{m}^{2} /\right.$ day $)$, the grid-cell map is corrected. In this example, Cells B1 and B2 were corrected. If the candidate site for a solar power plant is located in cell B2, the analyst could select hourly data from another cell that has

\footnotetext{
${ }^{43}$ See http://maps.nrel.gov/node/10/.
} 
not been corrected, such as A2 or $\mathrm{C} 2$. This procedure will ensure that the hourly simulations (e.g., Solar Advisor Model for CSP) ${ }^{44}$ produce results that are more consistent with the mean value at the proposed site.

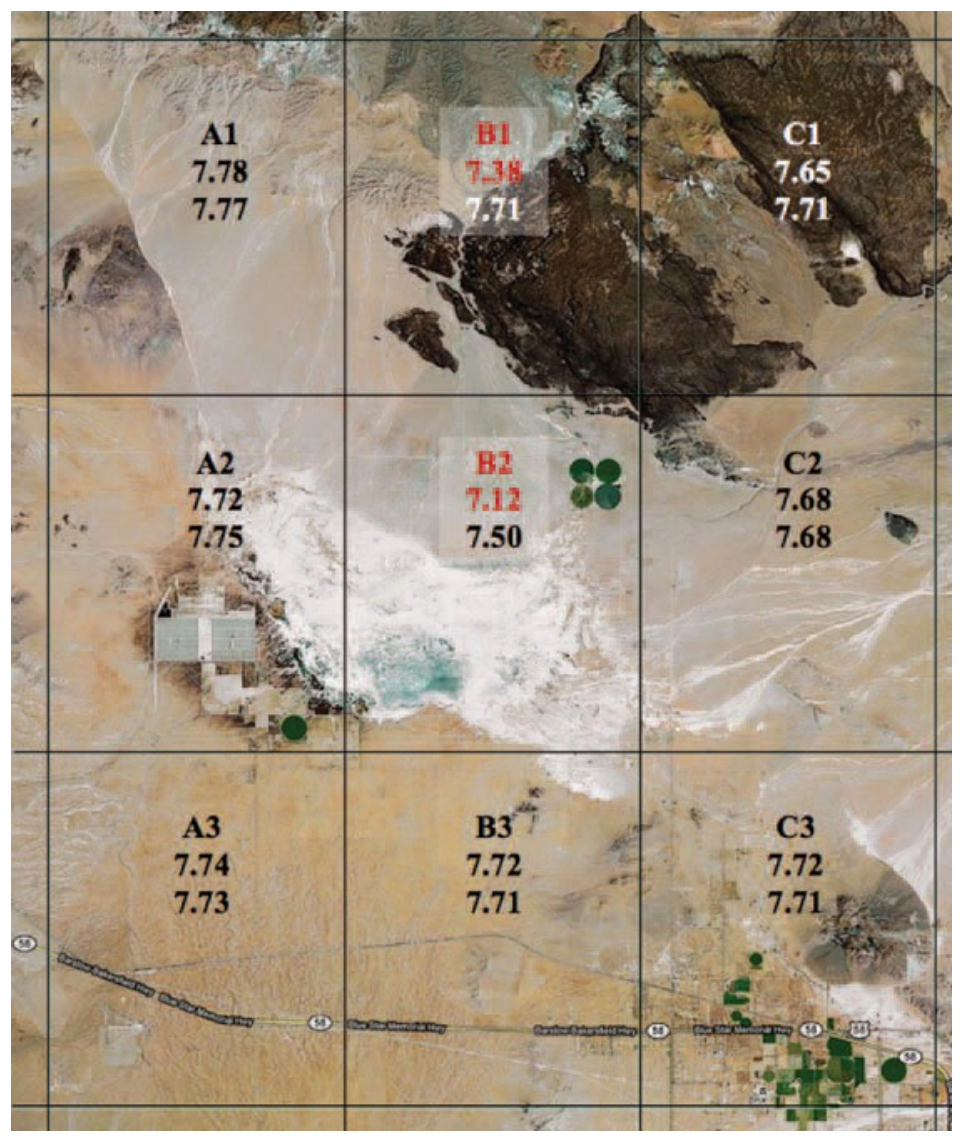

Figure 6-8. NSRDB/SUNY 10-km grid cells near Harper Lake, California. The upper values shown in text boxes are averaged from (uncorrected) hourly files. The lower values are averaged DNI from corrected maps. The values in red show uncorrected time series mean values, which are substantially lower than the corrected map values. Image from NREL

In general, cells in need of correction have bright or uneven areas, especially near the center. Adjacent cells with a darker, more uniform background will have more reliable hourly DNI data. The goal is to select the correct time series to match the estimate of the mean values. The Google Map shows that the time-series data from the selected cell (B2) should not be used, because the time series produces different (lower) means. The SUNY team developed corrections to this artifact; in the near future, corrected maps will be available that avoid this problem.

Although it is not recommended, the user could choose one of the TMY2 or TMY3 data sets to act as a surrogate for the 8 years of data. If a TMY2 or TMY3 data set is proposed as a surrogate for this site, the data set should be carefully evaluated for applicability of the mean values in space and time. Figure 6-9 shows the monthly DNI values for the C2 site and the nearby

\footnotetext{
${ }^{44}$ See www.nrel.gov/analysis/sam/.
} 
Daggett, California, TMY3, which is a higher quality, Class I, NSRDB site. In this case, the TMY3 may be a suitable surrogate for the site-specific SUNY data.

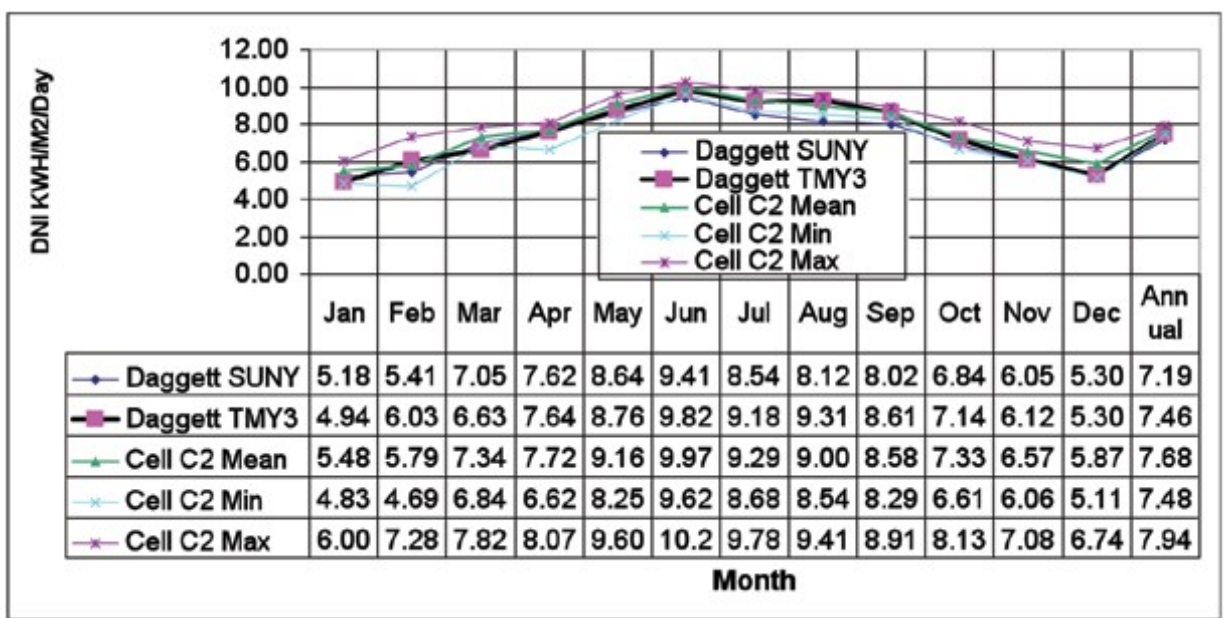

Figure 6-9. Monthly mean DNI for Harper Lake (Cell C2) and Daggett TMY3. Minimum and maximum values for cell $\mathrm{C} 2$ are also shown for each month. Image from NREL

Example 2 is a proposed plant site near Desert Rock, Nevada. We assume for this example that we have chosen the NSRDB/SUNY data for preliminary analysis, and we have obtained new measured data for the desired site. We show the effects on the annual DNI and GHI estimate of including measured and modeled data. Table 6-3 shows the results of using 8 years of modeled NSRDB data with 2 years of measured data (2004-2005), year 2004 of measured data only, and year 2005 of measured data only.

Table 6-3. Annual Mean Values of Global and Direct Radiation for Measured and Modeled Data at Desert Rock, Nevada

\begin{tabular}{|l|c|c|c|c|}
\multirow{2}{*}{\multicolumn{1}{c|}{ Measured Time Period }} & \multicolumn{4}{c|}{$\left(\mathrm{kWh} / \mathrm{m}^{2} /\right.$ day $)$} \\
\cline { 2 - 5 } & $2004-2005$ & 2004 Only & 2005 Only & $1998-2005$ \\
\hline Model global & 5.615 & 5.656 & 5.574 & 5.622 \\
\hline Model direct & 7.642 & 7.720 & 7.564 & 7.658 \\
\hline Measured global & 5.703 & 5.799 & 5.607 & \\
\hline Measured direct & 7.564 & 7.901 & 7.227 & \\
\hline MBE global & $-1.54 \%$ & $-2.46 \%$ & $-0.58 \%$ & \\
\hline MBE direct & $1.04 \%$ & $-2.28 \%$ & $4.67 \%$ & \\
\hline Adjusted direct 8-year mean & 7.579 & 7.833 & 7.300 & \\
\hline Meyer-corrected mean DNI & 7.582 & 7.859 & 7.305 & \\
\hline Meyer MBE direct & $0.8 \%$ & $-1.8 \%$ & $3.6 \%$ & \\
\hline Meyer-adjusted 8-year mean & 7.597 & 7.793 & 7.386 & \\
\hline
\end{tabular}


We adjust the average DNI from the 8-year period using the bias error from our observed data with the simple "ratio method" described above. The bias error using both years is a relatively low value of $1.04 \%$. The bias errors from individual years are higher and do not show a consistent pattern. The adjusted direct is the new estimate of the long-term mean DNI, and it is simply the 8-year mean DNI (7.658) times (1.0 - MBE). The method of Meyer et al. (2008) described previously can also be used advantageously here. If we assume the uncertainty is $3 \%$ for measured data and 10\% for SUNY data, we can calculate the corrected means for all the months we have both measured and modeled data. If we adopt this value as our best guess for the actual DNI for the years 2004 and 2005, then our new bias error is (SUNY-Meyer)/Meyer, and our bias errors are smaller. The Meyer estimate is calculated using the following equation:

$$
\mathrm{I}_{\mathrm{est}}=\left(\mathrm{I}_{\mathrm{me}} / \mathrm{U}_{\mathrm{me}}=\mathrm{I}_{\mathrm{mo}} / \mathrm{U}_{\mathrm{mo}}\right) /\left(1 / \mathrm{U}_{\mathrm{me}}+1 / \mathrm{U}_{\mathrm{mo}}\right)
$$

where

- $\quad$ the Meyer estimate $=\mathrm{I}_{\mathrm{est}}$

- $\mathrm{I}_{\mathrm{me}}=$ measured value

- $\mathrm{I}_{\mathrm{mo}}=$ modeled value

- $\mathrm{U}_{\mathrm{me}}=$ measurement uncertainty $(0.03)$

- $\mathrm{U}_{\mathrm{mo}}=$ modeled uncertainty (0.10).

Monthly mean values of GHI and DNI are shown for the Desert Rock site (see Figure 6-10). For many months, especially during 2005, the bias errors are very small for GHI and large for DNI. GHI and DNI bias errors are well correlated in 2004, but not in 2005. One interpretation is that the principal source of error during 2004 is the cloud estimation, and the principal source of error in 2005 is in the AOD.

A small error in global radiation along with a large overestimate of the DNI indicates that AOD at the site may have been much higher than the estimated AOD used in the satellite model. A diligent analyst might pursue an explanation for the higher than normal AOD and ask whether higher levels of AOD could be caused by dust storms, forest fires, or a general underestimation of the AOD. The average monthly values shown in Figure 6-10 would be helpful in pinpointing the cause of the problem. 


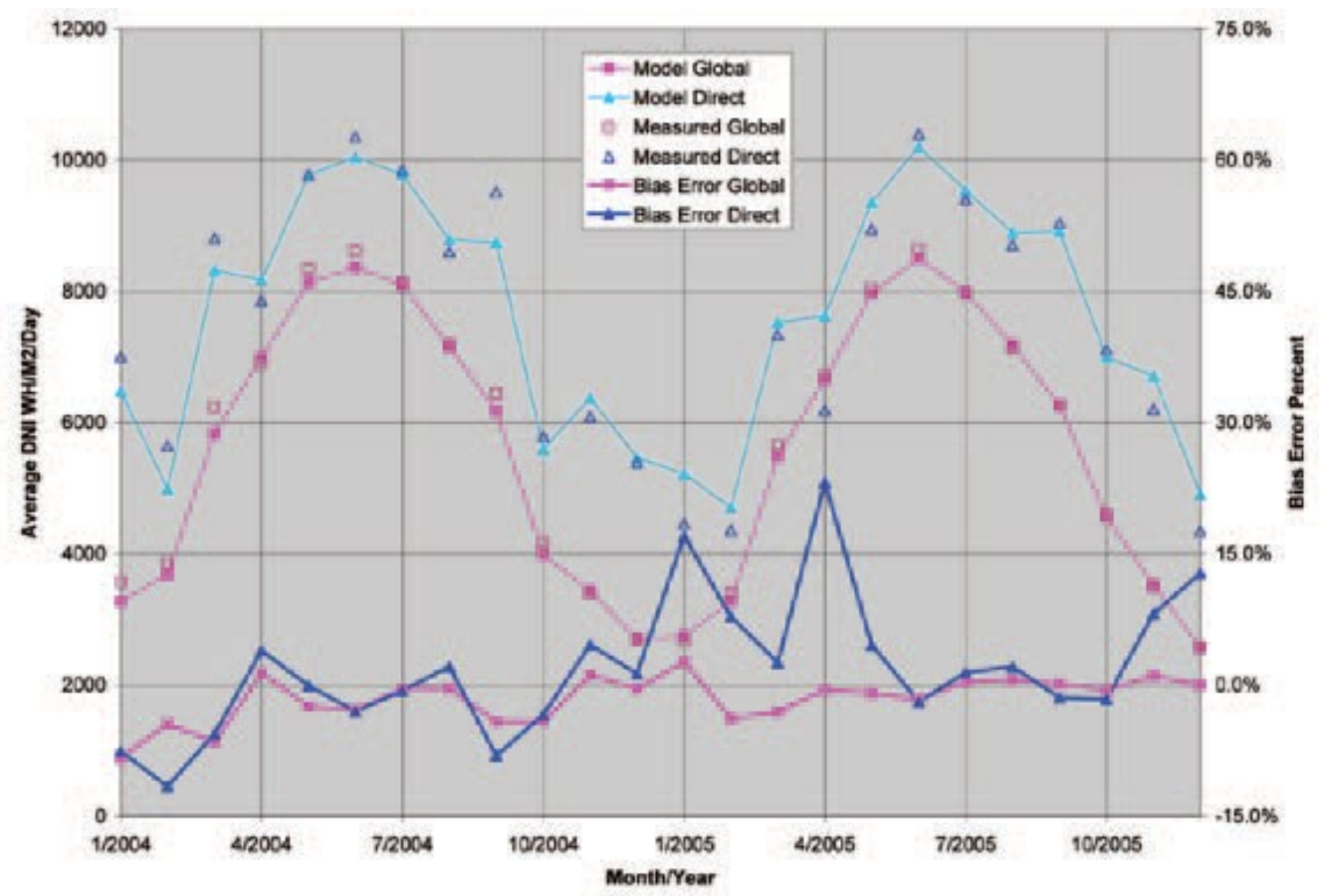

Figure 6-10. Desert Rock annual average GHI and DNI from satellite and measurements. Mean bias error is defined as (satellite - measured)/measured by $100 \%$. Image from NREL

The monthly values in Figure 6-10 show large shortfalls in the measured DNI in January and April 2005, indicating higher than normal AOD. After the likely cause has been determined, the analyst should then assess whether that phenomenon might be more prevalent in the future or if it is possibly a rare event.

The broadband AOD may be estimated from the new DNI measurements using a clear-sky model such as REST2 (Gueymard 2013), with supplemental data to estimate total column water vapor. These values can then be used to adjust the modeled DNI estimates; however, AOD is also highly variable from month to month and from year to year (and also on smaller timescales), so it would take several years of data to show conclusively that the mean AOD used in the satellite model needs to be adjusted at this site.

In this example (see Table 6-3), the new estimate for the 2-year data set DNI, $7.597 \mathrm{kWh} / \mathrm{m}^{2} / \mathrm{day}$, is less than $1 \%$ different from the 8 -year model estimate of 7.658 . With only 1 year of measurements, the errors are larger, up to $3.6 \%$.

\subsection{Variability of the Solar Resource in the United States}

The variability of the solar resource is an important consideration in the need to adequately characterize the variability with measurements and for predicting future solar power plant performance. This analysis disregards predictable variability, such as that caused by site latitude and time of day, and concentrates on less predictable behavior caused by climate. The solar variability is closely related to the variability of climate in time and space, because atmospheric forces and constituents have a strong impact on the amount of solar radiation absorbed, reflected, or otherwise prevented from reaching the Earth's surface. 
With knowledge of the likelihood of variability from year to year, users are provided some justification for selecting a particular period of time for measurements adequate to characterize the solar resource. Likewise, with knowledge of variability across distance, users can make some statement of the applicability of a measurement to a location some distance away. Knowledge of variability then becomes valuable when deciding how long to make measurements at a particular location and whether the character of the solar resource at that location can be extended to other nearby locations.

Gueymard and Wilcox (2011) have analyzed 8 years of data (1998-2005) from the NSRDB in the realms of temporal and spatial variability. The analysis summarized the values in each $10-\mathrm{km}$ by $10-\mathrm{km}$ cell in the SUNY satellite-derived data in the NSRDB and calculated monthly mean daily totals, annual mean daily totals, and mean daily total for the entire 8-year period. The values were analyzed by temporal and spatial variability.

Temporal variability. For each cell, the 8 annual values were used to calculate a COV. The 8year mean irradiance $<$ Ep $>$ and each annual value Ei were used to derive the standard deviation of the data set. Because there are no missing values, the standard deviation simplifies to

$$
\sigma_{\mathrm{t}}=\left[\left(<\mathrm{E}_{\mathrm{p}}>-\mathrm{E}_{\mathrm{i}}\right)^{2 / 8}\right]^{1 / 2}
$$

The temporal COV is

$$
\mathrm{C}_{\mathrm{t}}=\sigma_{\mathrm{t}} /<\mathrm{E}_{\mathrm{p}}>
$$

To understand the variability in a seasonal scope, the process was repeated on a monthly levelfor example, the 8 Januarys, Februarys, etc. The results, expressed in percent, represent the variability in the solar resource year by year at the cell's geographic location. The resulting COV for DNI for all cells plotted as a contour map of the United States is shown in Figure 6-11, which provides a quick visual measure of differences in interannual variability. The temporal COV for the 48 U.S. states ranges from a low of $0.49 \%$ in south-central Washington to a high of $15.8 \%$ in northwest Washington (which has an interesting contrast of climate within a single state).

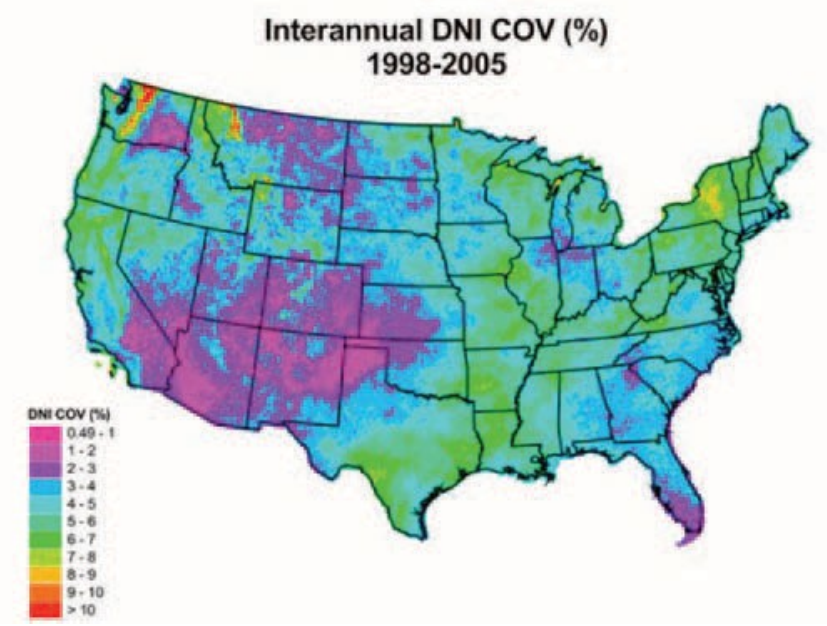

Figure 6-11. Interannual DNI variability (COV as percent) for 1998-2005. Image from NREL 
Spatial variability. The 8 -year daily total irradiation means for each $10-\mathrm{km}$ by $10-\mathrm{km}$ cell were compared to a matrix of surrounding cells to determine the variability of the solar resource within the matrix (see Figure 6-12).

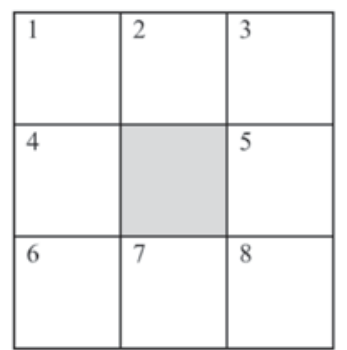

Figure 6-12. A 3-by-3 grid layout with anchor cell in the center and 8 surrounding neighbor cells. Image from NREL

Here, the standard deviations of the surrounding cells were calculated as

$$
\sigma_{\mathrm{S}}=\left[\Sigma\left(\mathrm{E}_{\mathrm{p}}-\mathrm{E}_{\mathrm{i}}\right)^{2 / \mathrm{n}}\right]^{1 / 2}
$$

The spatial COV is

$$
C_{t}=\frac{\sigma_{t}}{<E_{p}>}
$$

The same process was applied to the 8-year means on a monthly level-all Januarys, Februarys, etc.

Two matrix sizes were analyzed: a 3 by 3 (see Figure 6-12) and a 5 by 5 . These represent areas of approximately $30 \mathrm{~km}$ by $30 \mathrm{~km}$ and $50 \mathrm{~km}$ by $50 \mathrm{~km}$, respectively, and likewise roughly represent an area within $15 \mathrm{~km}$ and $25 \mathrm{~km}$ of a measurement site. The results for DNI, expressed in percent, are mapped in Figure 6-13, which provides a quick visual representation of how the solar resource varies throughout space. For DNI, the values range from $0.12 \%$ in central Missouri to approximately $11.5 \%$ along a corridor between Los Angeles and San Bernardino, California. Variability tends to be higher in coastal areas (particularly the California coast) and in mountainous areas. Greater variability occurs in the 5-by-5 matrix, which is to be expected because of the effects of terrain. Further, the general pattern of high and low variability remains the same between the two maps, indicating that in locations of significant variability, the magnitude is much a function of distance. 


\section{Spatial DNI COV (\%) of Annual Average 1998-2005}
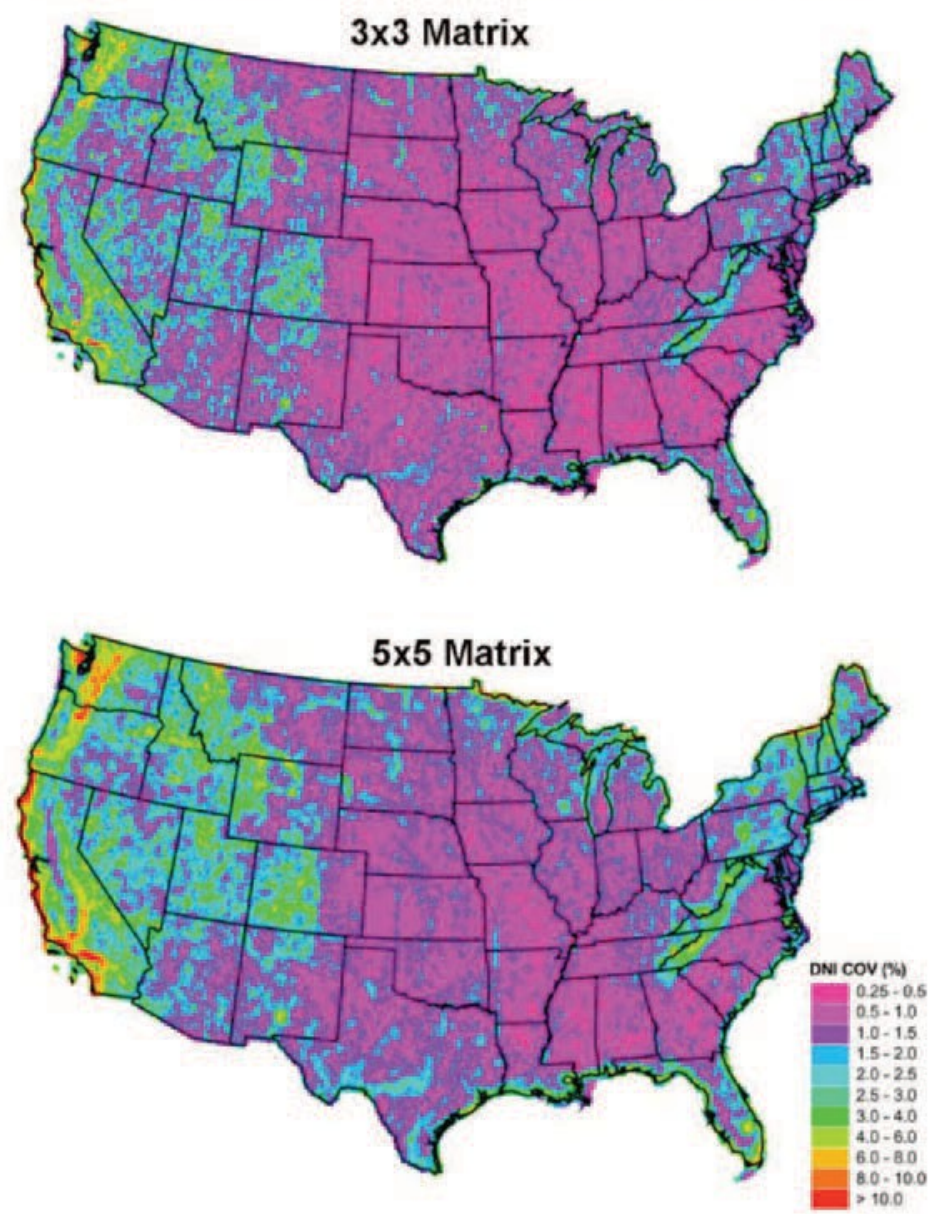

Figure 6-13. DNI spatial coefficient of variability for a (top) 3-by-3 cell matrix and (bottom) 5-by-5 cell matrix for the average DNI from 1998-2005. Image from NREL

The underlying data for these maps are available from NREL to provide users with the actual values for each $10-\mathrm{km}$ by $10-\mathrm{km}$ cell both in units of $\% \mathrm{COV}$ and $\mathrm{Wh} / \mathrm{m}^{2}$. Users should be cautioned that the 8-year period may not be long enough to produce definitive variability values, and the uncertainty of this analysis has not been defined. NREL plans to update this data set by drawing from a longer period of record; however, the results here are very likely accurate enough to reveal the relative variability of the solar resource throughout the United States.

Using these variability statistics, users can better understand the extent of measurements required to best characterize the solar resource for a particular application. In areas with low interannual variability, a shorter measurement period may suffice. In areas with low spatial variability, a measurement station could possibly represent the solar resource at nearby locations, negating the need for additional measurements. An analyst can use this information to better build confidence in a data set as being sufficient for an analysis and can use these data to understand the consistency of future solar power plant performance and how that relates to the economic viability of a particular location. 
Similar analysis was carried out for the gridded TMY data set (1998-2009) (Habte et al. 2014). The spatial variability analysis was implemented by comparing a center pixel to neighboring pixels (Figure 6-14). A COV value was generated for each configuration shown in Figure 6-15.

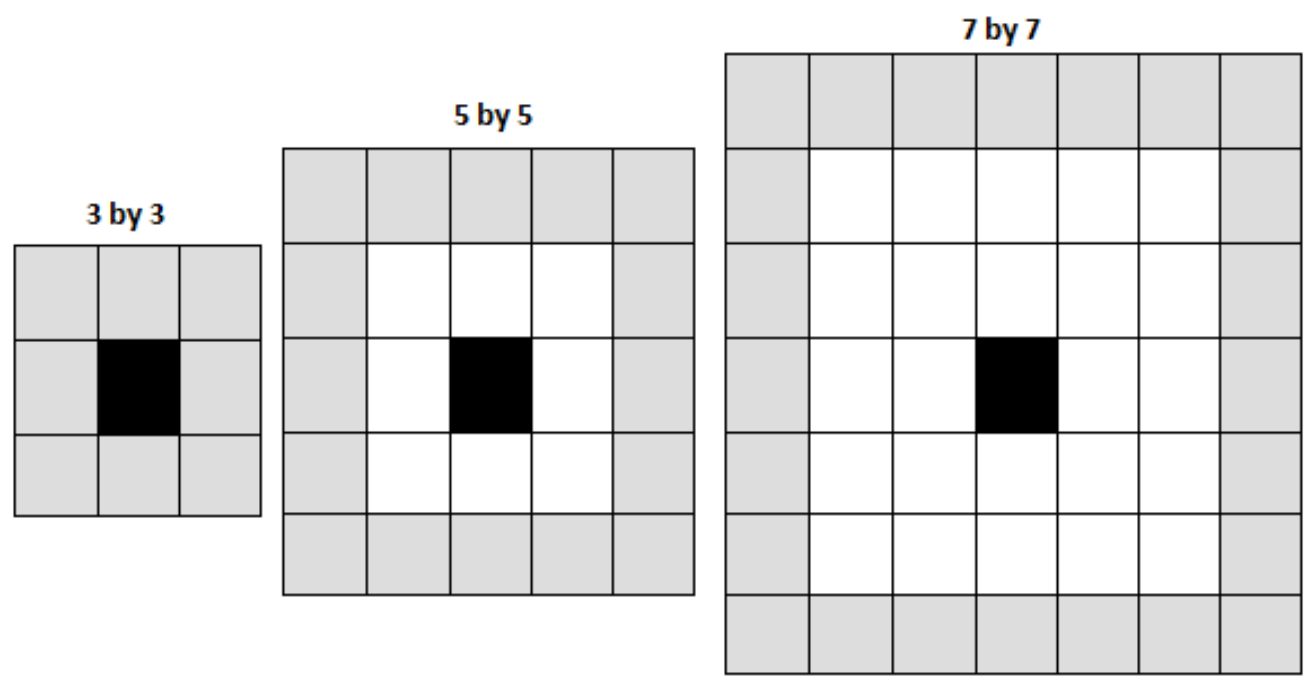

Figure 6-14. The distribution of the pixels in each spatial variability analysis. The black center pixels were compared to each of the gray pixels. Images from NREL
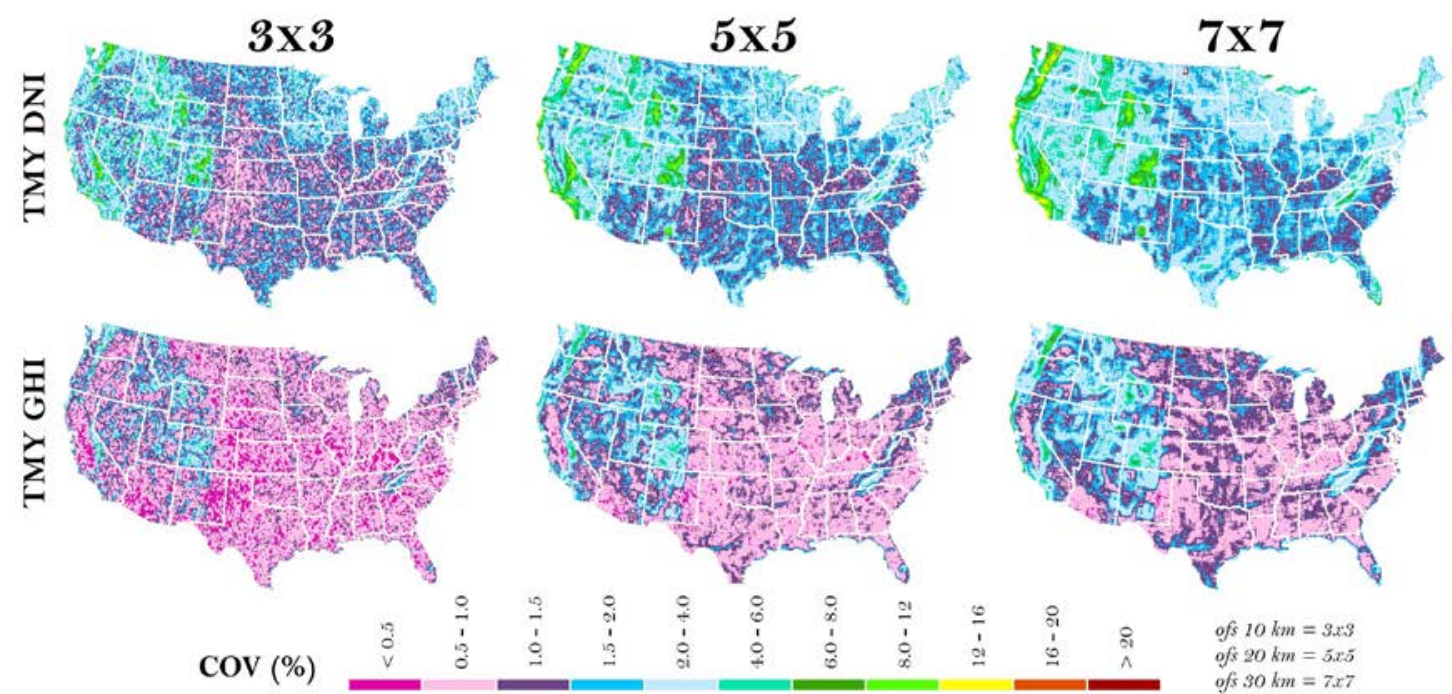

\section{Annual Spatial COV in 3x3, 5x5, and 7x7 Matrices}

Figure 6-15. Map showing spatial variability among neighboring pixels. Images from NREL

The higher variation in the DNI compared to the GHI was attributed to the opacity of sky and also aerosols (Gueymard and Wilcox 2011). The magnitude of variation (COV) in the DNI increased rapidly as the distance between the center pixel and the farthest pixel increased (Figure 6-15). The variation of DNI with adjacent pixels could provide system performance analysts with essential information about how the system energy output will be spatially variable in certain locations. 
The temporal variability was also analyzed using a standard deviation for the monthly gridded NSRDB (1998-2009) data. The purpose of this analysis was to understand the effectiveness of the TMY data in representing the long-term variations. Figure 6-16 shows the standard deviation distribution of the GHI and DNI data set for all pixels in the United States. The yellow box illustrates confidence interval coverage of $95 \%$, and the red line is the mean point of the $95 \%$ confidence interval data.
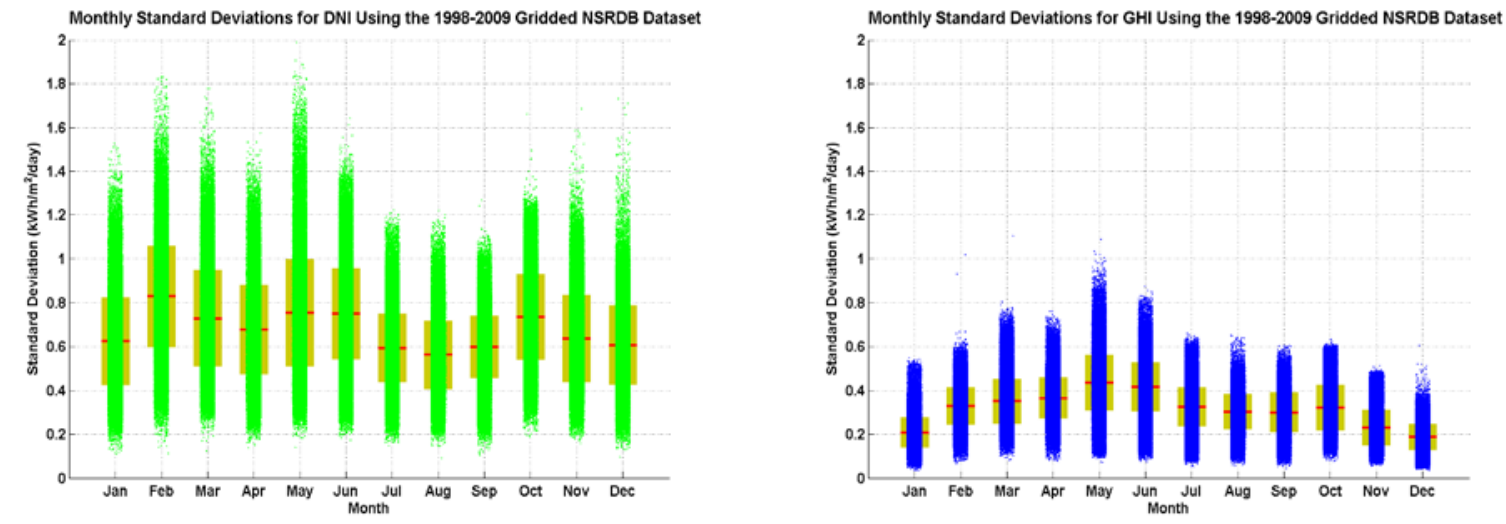

Figure 6-16. Monthly standard deviation distributions in $\mathrm{kWh} / \mathrm{m}^{2} /$ day for the NSRDB gridded DNI and GHI data sets. Illustrations from NREL

As illustrated above, the standard deviations of the irradiance for each month provide useful information about the temporal variability of a typical data set. The annual temporal variations included in the typical data set do not depict the monthly variations; therefore, the user of the TMY data set must assess the importance of shorter term (monthly) compared to long-term (annual) temporal variability when applying the data to a specific system performance project.

\subsection{Applying Solar Resource Data to Planning Solar Energy Projects}

This section first provides a summary of general approaches of using solar resource data (as described in Chapters 1-5) to estimate the yield from solar energy systems. First, PV systems are discussed, followed by concentrating systems and flat-plate collectors. The second part of the chapter discusses a variety of approaches for monitoring solar resource data at an existing power plant to better understand the plant's performance.

\subsubsection{Approaches to Estimating Yield of Non-Concentrating PV Projects}

The value of electricity generated by a PV plant depends on the amount of electricity generated and the grid's need for that electricity at the time it is generated. A quantitative understanding of the solar resource for the intended location and orientation of the PV array is essential to both of these. This section gives a high-level overview; more detailed descriptions of PV system modeling can be found in Ellis et al. (2011). 
We describe three general approaches to estimating PV system yield. These are presented in order of increasing accuracy.

\subsubsection{Performance Ratio Method}

The output of a PV plant can be characterized by the performance ratio metric, which describes the fraction of electricity generated by the plant relative to what the plant would generate if it always operated at its nameplate efficiency (International Electrotechnical Commission 1998). Typically, new PV plants operate with performance ratios of $0.8 \pm 0.1$ (van Sark et al. 2012). Thus, if the annual solar resource available for a given site and given array orientation (fixed or tracking) is determined, the annual output of the system can be estimated according to Eq. 6-6:

$$
\text { Annual output }(\mathrm{kWh} / \mathrm{y})=0.8 \mathrm{X} \text { solar resource }\left(\mathrm{kWh} / \mathrm{m}^{2} / \mathrm{y}\right) \mathrm{X} \text { PV plant size }(\mathrm{kW})
$$

Where the PV plant size is derived from the sum of the module nameplate ratings as characterized under standard test conditions $\left(1,000 \mathrm{~W} / \mathrm{m}^{2}\right)$. The uncertainty of this estimate must include the variability of the solar resource from year to year and the variability of the performance ratio (van Sark et al. 2012). Contributors to low performance ratios include

- Shading losses

- Soiling or snow-coverage losses

- High-temperature operation

- Undersized inverters so that the inverters "clip" the plant output part of the time

- Older plants that have experienced degradation

- Modules that have below nameplate performance (Today, many manufacturers bin modules so that the performance is equal to or greater than the nameplate value; whereas in past years manufacturers often placed modules in the bin with the larger nameplate value.)

Contributors to high performance ratios include:

- Operation in a cool climate

- Modules with low temperature coefficients (Typically, CdTe and high-efficiency silicon modules tend to have the lowest temperature coefficients.)

- Modules that generate power well above the nameplate rating.

Van Sark et al. (2002) found a few older systems with performance ratios less than $50 \%$, so Equation 1 must be used cautiously. The performance ratio method is particularly useful if one desires to compare performance of existing systems or when it is desired to quickly use solar resource data that is not available through performance models as presented in the next sections. Otherwise, using more sophisticated performance models is likely to be the better approach for estimating PV plant output.

\subsubsection{Simple PV Performance Models}

PVWatts is presented as an example for a simple performance model in the following. 
NREL's PVWatts calculator is a free online tool that estimates the electric energy production of a grid-connected roof- or ground-mounted PV system based on a few simple inputs. ${ }^{45}$ The user enters a street address or geographic coordinates of the system's location, specifies the system size and array orientation, and provides some information about the system's cost and electricity rates. PVWatts calculates estimated values for the system's annual, monthly, and hourly energy production and for the monetary value of the electricity produced.

By default, PVWatts uses the TMY2 data set for locations in the United States. Other solar resource data options are the TMY3 data set, the 10-km SolarAnywhere satellite-based data set, and a collection of TMY data for more than 300 international locations. Advanced users of PVWatts may change default assumptions for losses because of shading, soiling, and other factors, and PVWatts allows tracking systems to be modeled as well. In 2014, a major update to PVWatts's algorithms was deployed to make the results more accurate and give additional flexibility for advanced users who may want to select different module technology types or customize system sizing.

Full details about the underlying PVWatts algorithms can be found in Dobos (2014). PVWatts provides a very convenient and more accurate method than the performance ratio described in the previous section, so it is recommended any time that a quick estimate is needed.

\subsubsection{Detailed PV System Performance Models}

More accurate estimates of PV system performance can be obtained by setting up a detailed model of the PV plant that includes choosing specific modules and inverters, an array layout, detailed losses, and shading analysis. Examples for freely available programs that include such detailed performance models are $\mathrm{SAM}^{46}$ and greenius (Quaschning et al. 2001). ${ }^{47}$ Other commercial programs exist, such as PV*SOL and PVSYST. As an example, the software SAM will be presented in the following.

SAM is a desktop software developed by NREL that enables the calculation of both detailed system performance and economics for a PV plant (Blair et al. 2014).

SAM integrates several detailed models and databases of thousands of commercially available components that are used to accurately predict the performance of specific PV modules and inverters and their combinations in an array. SAM supports complex system designs that may have multiple subarrays and estimations of shading losses for systems arranged in regularly spaced rows as well because of their irregular obstructions and detailed accounting of other losses in the system. SAM's PV model calculates energy production in each hour of the year using the user's selection of any solar resource data file, including TMY2, TMY3, EPW, and SolarAnywhere satellite data. The accuracy of the estimation of annual yield from the PV plant is still dependent on the uncertainty and variability of the solar resource, but the more sophisticated component and system-level modeling can more accurately model the response of a PV plant to changing meteorological conditions. The accuracy of the model is often dependent on the user's

\footnotetext{
${ }^{45}$ See http://pvwatts.nrel.gov.

${ }^{46} \mathrm{See}$ http://sam.nrel.gov.

${ }^{47} \mathrm{See}$ http://freegreenius.dlr.de.
} 
ability to estimate losses from soiling and/or snow. SAM has been compared to measured power plant performance data for numerous systems to validate its estimations (Freeman et al. 2013) as well as to other free and commercial PV modeling tools (Freeman et al. 2014).

SAM combines its PV plant performance model with an economic analysis that handles complex utility tariff structures, incentives, plant installation and operating cost information, and financial structures to calculate a full multi-year cash flow and economic metrics, such as levelized cost of electricity and net present value. These outputs may facilitate decision making for people involved in the PV industry, such as project managers and engineers, policy analysts, technology developers, and energy systems researchers.

\subsubsection{CST Plant Yield Calculation}

CST plant yield models consist of an optical performance model and a model for the conversion of concentrated light to electricity, process heat, or chemical energy. In the case of CSP, the conversion from concentrated light to electricity involves a solar receiver model, the transfer of the heat to the turbine, and the storage and the operation of the turbine. For CPV, the module performance, the inverters, and, if present, also a battery system must be modeled.

Because the solar resource data are used as inputs to the optical performance model, we focus on the description of these models in the following. Some of the latter-mentioned optical performance models also include software modules for the conversion to electricity or also costs (e.g., SAM, greenius, HFLCAL).

Optical performance models can be separated into different categories: ray-tracing tools, analytical optical performance models, and models that determine the optical performance with lookup tables or parameterizations of the solar position relative to the collector. To better understand the use of the DNI data in power plant models, we briefly introduce different types of optical performance models and tools.

\subsubsection{Ray-Tracing Models}

The available solar radiation can be described as a multitude of solar rays transmitted from the sun to the concentrators and finally to the receiver. Ray-tracing tools such as STRAL (Belhomme et al. 2009), SolTRACE (Wendelin 2003), MIRVAL (Leary and Hankins 1979), or SPRAY (Buck 2010) calculate the path of the sun's rays from the sunshape to the receiver by application of physical laws. Monte Carlo techniques are often implemented to allow for tractable calculation times.

For the sake of illustration, one method for ray tracing that is available in SPRAY is explained in the following. The method selects one concentrator element after another and traces a given number of rays from the current element. After calculating the vector to the center of the sun, the appropriate sunshape is included. This is done by calculating an angular deviation of the ray vector from the center of the sun based on the probability density function corresponding to the user-defined sunshape. The specific ray under scrutiny is then related to a power calculated as the product of the incident DNI and the projected area of the current concentrator element divided by the number of rays per element. Then the path of the ray is followed until it reaches the receiver. This ray-tracing method can be based on actual measurements of the plant 
geometry. Because not only the intercepted power but also its distribution on the receiver is determined, ray-tracing tools are also used for the detailed design of the plant components.

\subsubsection{Analytical Optical Performance Models}

The Bendt-Rabl model (Bendt et al. 1979; Bendt and Rabl 1981) is another type of calculation method that uses an analytical approach. To accelerate calculations, analytical equations are derived and solved to describe the ray's path through the optical system. For example, the model suggested by Bendt and Rabl can be used for parabolic troughs and solar dishes. In a first step, an angular acceptance function is determined from the design geometry. The angular acceptance function is defined by the fraction of rays incident on the aperture at an angle that reaches the receiver. The second step of the Bendt-Rabl method is to determine an effective source that includes both the user-defined sunshape and the deviations from the design geometry. The optical errors of a CST collector are described as Gaussian-distributed independent uncertainties. Their combination is also a Gaussian distribution with a standard deviation, which is often called an optical error. The function that describes the optical errors is then combined with the sunshape using convolution. For line-focusing systems, such as parabolic troughs, a further integration step is required, because the effect of circumsolar radiation on the incident irradiance depends strongly on angle.

Finally, the intercepted radiation can be determined by integrating the product of the effective source and the acceptance function.

Bendt and Rabl (1981) also describe an alternative order of the calculation steps that combines the angular acceptance function and the optical errors to the so-called "smeared acceptance function," which is then combined with the sunshape.

Similar analytical methods are used in HELIOS (Vittitoe and Biggs 1981), DELSOL (Kistler 1986) and HFLCAL (Schwarzbözl 2009).

\subsubsection{Lookup Table-Based Optical Performance Models}

The fastest way to determine the optical performance of a CST collector uses only parameterizations or lookup tables that describe the change of the optical performance with solar position. The necessary parameters can be derived from experimental data, the aforementioned analytical performance models, or ray-tracing tools. Only one constant sunshape is typically described by these simple models, which constitutes a limitation.

Such lookup tables or parameterizations are used in SAM (Blair et al. 2014) and greenius (Quaschning et al. 2001; Dersch, Schwarzbözl, and Richert 2011).

\subsubsection{Required Meteorological Input for CST Models}

The DNI is the most important meteorological input parameter for CST models; however, further parameters must be provided for accurate yield analysis. High wind speed might force the plant operators to set the collectors to their stow position. Thermal losses are influenced by wind (convection) and ambient temperature. Humidity and pressure have an effect on the thermodynamic performance of CSP plants. A discussion of the influence of the different meteorological parameters can be found in Chhatbar and Meyer (2011). Recently, other parameters such as soiling and the extinction of radiation between the mirror and the receivers 
have gained interest. (For extinction, see Pitman and Vant-Hull [1982], Sengupta and Wagner [2011], Ballestrín and Marzo [2011], and Hanrieder et al. [2012]. For soiling, see Sarver, AlQaraghuli, and Kazmerski [2013] and Wolfertstetter et al. [2014]).

Directly connected to the DNI itself is its angular distribution, the sunshape. Depending on the used CST model, the sunshape has to be provided by the user, too. The first two types of models mentioned above - ray-tracing and analytical models - need the sunshape and DNI as input variables. The third model type (lookup tables) requires only DNI as input; whereas assumptions about the sunshape are included as fixed settings in the model. A discussion about the influence of the selection of the sunshape data can be found in Wilbert (2014).

\subsubsection{Flat-Plate Collector Yield Calculation}

Regarding PV, the relevant solar input for flat-plate collector yield calculation is the POA irradiance, though other meteorological parameters also influence the system output. Ambient temperature, wind, and soiling have to be mentioned. Flat-plate thermal collector models usually include a thermal receiver model, thermal losses of the piping, parasitic losses, and a storage model. One example for software for the yield analysis of flat-plate collectors is greenius (see Section 6.4.1). Although the POA irradiance is the physically relevant irradiance, the separate specification of DNI and DHI can be of interest. Individual incidence angle modifiers can be used to determine the efficiency of the DNI and DHI conversion, respectively, for a given solar position. For example, in greenius, GHI can be provided as an input parameter for the model. GHI is then converted to DNI and DHI in the greenius software.

\subsubsection{Measurement of Solar Resource Data for Power Plant Characterization}

The performance of a solar energy system is directly linked to the current meteorological conditions. For flat-plate thermal collectors and PV, the production is roughly proportional to the POA irradiance. For CST, DNI is the most important parameter; however, other meteorological influences also exist, as discussed above. The following are a few of the many reasons to measure meteorological conditions for comparison to solar system performance:

- Evaluate a performance guarantee (acceptance testing)

- Assess power plant performance for improved yield predictions for the installed and future plants

- Identify conditions of poor performance, including evidence of soiling, shading, hardware malfunction, or degradation, which might lead to warranty replacement, etc.

\subsubsection{Evaluation of a Performance Guarantee}

Different methods exist for evaluating the performance guarantee. In all cases, measurements of the solar resource are involved.

For CST, acceptance tests involve DNI. For flat-plate thermal collectors and PV, the yield prediction is generally based on GHI. So, it is also common for a performance guarantee to use GHI as the basis for determining whether a plant has performed as promised; however, some companies have noted that the performance characterization of a PV plant can be accomplished with a lower uncertainty if the irradiance is measured in the POA (removing the uncertainty of the transposition of the GHI irradiance to the irradiance in the POA) and if irradiance sensors are 
chosen to match the expected response of the PV modules (reducing angle-of-incidence and spectral effects). If a company chooses to specify the performance guarantee in terms of POA irradiance measurements, then the financial assumptions for the project need to consider that the plant efficiency characterized during the evaluation of the performance guarantee may differ from the efficiency that would be found from the model based on historical data for GHI. Also, the placement of all sensors must be confirmed to (1) be in the correct plane (which is easy to confirm when the sensor is in the horizontal plane, but not as easy for other orientations) and (2) experience the promised ground albedo nearby if the sensor is not in the horizontal plane (Kurtz et al. 2014).

Further meteorological parameters have to be considered too, as discussed above for the yield prediction. Depending on the size of the solar system, more than one measurement point must be considered if the evaluation also includes partly cloudy sky conditions. Acceptance tests for CSP systems are discussed in Kearney (2011), Kearney (2013), and Janotte (2012).

\subsubsection{Monitoring Power Plant Performance}

During power plant operation, knowledge of the current meteorological conditions and the status of the plant are of high importance. Also, the future development of the meteorological conditions is relevant. Therefore, the solar resource measurements and forecasts are included in many solar energy systems. For CSP installations such measurements and forecasts are fundamental. Although many PV plants can operate successfully without any intervention, measurements and forecasts are also advantageous for PV. There can be value to washing a PV array, and equipment malfunctions can be detected more quickly if the PV plant output is being continually compared to the expected output based on the meteorological conditions.

For CST, DNI measurements are involved along with the other parameters mentioned above. The closest correlation between PV plant performance and irradiance is obtained by monitoring the POA by using a reference cell or reference module that closely matches the PV module response. Although the PV community is not in agreement regarding use of this approach for performance guarantees, there is wide agreement that a matched reference cell in the POA is the best choice when the goal is to identify the need for power plant maintenance.

\subsection{Summary of Application of Solar Resource Data}

Depending on the technology of interest, DNI, GHI, and/or POA irradiance is required.

Different data sets are used for the different phases of a solar energy project. For small PV installations or domestic flat-plate receivers, a more simple procedure than the following presentation might be sufficient.

Before making site-specific measurements (prefeasibility, feasibility stages):

1. Use screening maps (e.g., NSRDB ) and other criteria (grid connection, etc.) or choose candidate sites.

2. Assess monthly/yearly mean irradiance values from a long-term data set (e.g., NSRDB 8year data set). Compare it to nearby sites that have more years of data. Create a set of best-guess target values for the monthly mean irradiance. 
3. Assess the uncertainty of irradiance means in the target values. The uncertainty is higher if the site:

A. Is close to strong gradients in average irradiance

B. Is close to strong gradients in AOD (especially if DNI data are required)

C. Is subject to possible enhanced aerosols (close to urban areas, mines, power plants, etc.)

D. Has bright surface albedo, or highly variable albedo.

4. Adjust the expected monthly mean irradiance values upward or downward, based on these parameters.

5. Choose hourly data sets to match expected mean value; at the same time, have diurnal and seasonal patterns close to those of the candidate site.

6. Assess the data to see how closely these mean values match the expected patterns (if using TMY data).

Site-specific ground measurements must be included in the analysis for bigger solar energy projects. If no high-quality data are available for at least 1 year, new measurements have to be collected following the methods described in this handbook.

After new ground measurements are available (according to the later stages in the project development-i.e., project qualification phase):

7. Realistically assess the quality of the new measured irradiance data.

8. Use the ratio method or Meyer method to compare measured and modeled data and create updated estimates of monthly mean irradiance.

9. Use a comparison of measured and modeled irradiance to assess the variability of aerosols.

10. Prepare the best possible data sets and multiple year as well as exceedance values, based on all available data, for final simulation runs.

11. Evaluate the interannual variability and check to determine whether global dimming or brightening is an issue in the region of interest. 


\section{Forecasting Solar Radiation}

\subsection{Introduction}

Solar resource forecasting becomes important in the operation phase of solar power plants (see Fig 6.1). Power generation from solar energy systems is highly variable. Especially in the case of PV systems, it cannot easily be matched to the electricity demand like power generated with conventional plants. In the case of CSP plants, grid effects are less problematic because of the thermal inertia of the heat transfer medium and other plant components together with the storage facilities; however, temporally and spatially varying irradiance has to be considered for the operation of CSP plants. Hence, the variability of the solar resource introduces new challenges to the operation of single solar power plants, and - with the expected integration of large shares of solar power-for the organization, structure, and management of all levels of electricity supply systems. In this context, reliable predictions of solar power production are getting more and more important as a basis for efficient management and operation strategies as well as for solar energy trading.

Already today, solar power prediction systems are an essential part of the grid and system control in countries with substantial solar power generation. For example, in Germany, which had an installed PV power capacity of $36 \mathrm{GW}_{\text {peak }}$ at the end of 2013 , more than $40 \%$ of the total load can be covered by PV power on sunny summer days at noon. According to the German Renewable Energy Sources act, transmission system operators are in charge of marketing and balancing the overall fluctuating PV power feed-in, making regional forecasts for the control areas necessary. Additionally, there is the option of direct marketing of PV power, based on forecasts for the respective PV plants' output. PV power is first offered on the day-ahead auction at the European Power Exchange, requiring day-ahead forecasts of PV power. Adaptations based on forecasts for several hours ahead can be made on the intraday market, when electricity may be traded until 45 minutes before delivery begins. Remaining deviations between scheduled and needed power are adjusted using balancing power. A similar procedure for the Californian electricity market is described in Kleissl (2013). High accuracy of PV power forecasts on different spatial and temporal scales is very important for cost-efficient grid integration, because large day-ahead forecast errors can cause either very high or negative prices on the intraday market; whereas intraday forecast errors determine the need for costly balancing power.

Several studies on the value of solar irradiance and power forecasting for solar energy applications and on basic requirements for these forecasts have been presented recently. Perez, Moore, and Stackhouse (2007) evaluate forecasts' end-use operational accuracy, focusing on their ability to accurately predict the effective capacity of grid-connected PV power plants. Kleissl (2013) describe stakeholder needs from the perspective of independent system operators as well as energy traders. The requirements for a nowcasting and short-term forecasting system to support microgrid or island systems with respect to stability and power quality are specified in Rikos et al. (2008). Wittmann et al. (2008) and Kraas et al. (2013) use case studies to show the economic benefit of using direct solar irradiance forecasts for optimized operation strategies of solar thermal power plants. Hirsch et al. (2014) are dedicated to using 6-hour forecasts (nowcasting) to operate CST plants.

Another application is the use of solar radiation forecasts for the energy management of a solarassisted district heating grid, as proposed in Steinmaurer (2008). An overview of different 
applications of solar power forecasting is given in Dumortier et al. (2009). Also, industry representatives and private sector consultants are indicating an increasing need for reliable shortterm and day-ahead forecasting as more PV and CSP installations are tied to electricity grids.

Following the new and rapidly evolving situation on the energy market with a strong need for accurate solar power predictions, increasing effort during the last years has been spent on developing irradiance and solar power prediction models. In particular, solar resource forecasting has been a major activity under the IEA SHC's Task 36, "Solar Resource Knowledge Management," and the follow-up Task 46, "Solar Resource Assessment and Forecasting." These tasks provide an excellent opportunity to bring researchers together from around the world to share their approaches and experiences in developing, implementing, and evaluating solar resource forecasts. So far, the joint work has focused on GHI and PV applications.

In this chapter, we give an overview of basic concepts of irradiance forecasting by referring to selected examples rather than by giving a review of the state of the art - which can be found in Inman, Pedro, and Coimbra (2013); Kleissl et al. (2013); and Lorenz and Heinemann (2012)and an extensive list of existing operational models as done for resource data sets in this handbook. The selection of the models presented here is motivated by the work completed in the IEA SHC's Task 36 and Task 46. The evaluation and comparison of different irradiance forecasting approaches is another focus of this chapter. The text focuses on GHI; DNI is discussed in less detail, because fewer results have been published compared to GHI. Nevertheless, DNI forecasting is of great importance for the solar industry, and first results and products exist. Research on direct irradiance forecasting is an ongoing task, with additional results expected in the near future.

\subsection{Solar Irradiance Forecasting Methods}

Depending on the application and the corresponding requirements with respect to forecast horizon and temporal and spatial resolutions, different data and forecast models are appropriate, as illustrated in Figure 7-1.

- Intra-hour forecasting with high spatial and temporal resolutions requires on-site observations of irradiance and/or cloud conditions.

- Statistical time-series models with local irradiance measurements as input are used to provide point forecasts up to a few hours ahead with a temporal resolution from minutes to 1 hour. They benefit from the high autocorrelation for short time lags in time series of solar irradiance; however, changes in cloud conditions, such as by approaching clouds, can hardly be predicted.

- Information about clouds and their motion in the surroundings of a given site can be obtained with ground-based sky imagers. Based on these observations, future cloud conditions and irradiance are extrapolated with a temporal resolution down to minutes or even below and a spatial resolution in the range of $10 \mathrm{~m}$ to $100 \mathrm{~m}$. Forecast horizons of sky-imager forecasts are typically up to 15 minutes to 30 minutes ahead.

- Forecast up to several hours ahead are operationally derived by analyzing and extrapolating clouds and cloud motion in satellite images that have a broad coverage (see 
also Section 4.4). The spatial resolutions are approximately $1 \mathrm{~km}$ to $5 \mathrm{~km}$ for the current generation of geostationary satellites, and images are generated in every 15 minutes to 30 minutes.

- NWP models are employed for forecast horizons from several hours to several days ahead. Predicting dynamic changes of the atmosphere, including formation or dissolution of clouds as well as advection, essentially relies on physical modeling. NWP models describe the physical and dynamical processes in the atmosphere by numerically solving the governing equations of the atmosphere on a grid starting from observed initial conditions. Global NWPs cover the Earth with spatial resolutions ranging from approximately 0.125 degrees to approximately 0.5 degrees and temporal resolutions of 1 hour to 3 hours. Local or regional models are employed for downscaling global model forecasts for specific regions to a finer grid of typically $3 \mathrm{~km}$ to $10 \mathrm{~km}$ with hourly resolutions.

The application of statistical models is beneficial for all horizons, ranging from very short-term forecasting with times-series models based on local measurements to forecasting for several days ahead by applying model output statistics to the output of NWP models for local refinement. Whichever model is used for forecasting, partly stochastic and systematic errors remain. These errors may be reduced with statistical models by learning from historic data sets of forecasted, measured, or satellite-derived irradiance. In particular, there is a high potential for improving forecast accuracy by combining different models with statistical learning approaches, which allow for an optimum assembling of different input data, depending on forecast horizon and weather situation.

Basic features and characteristics of the different approaches to predict surface solar irradiance are briefly introduced below. 


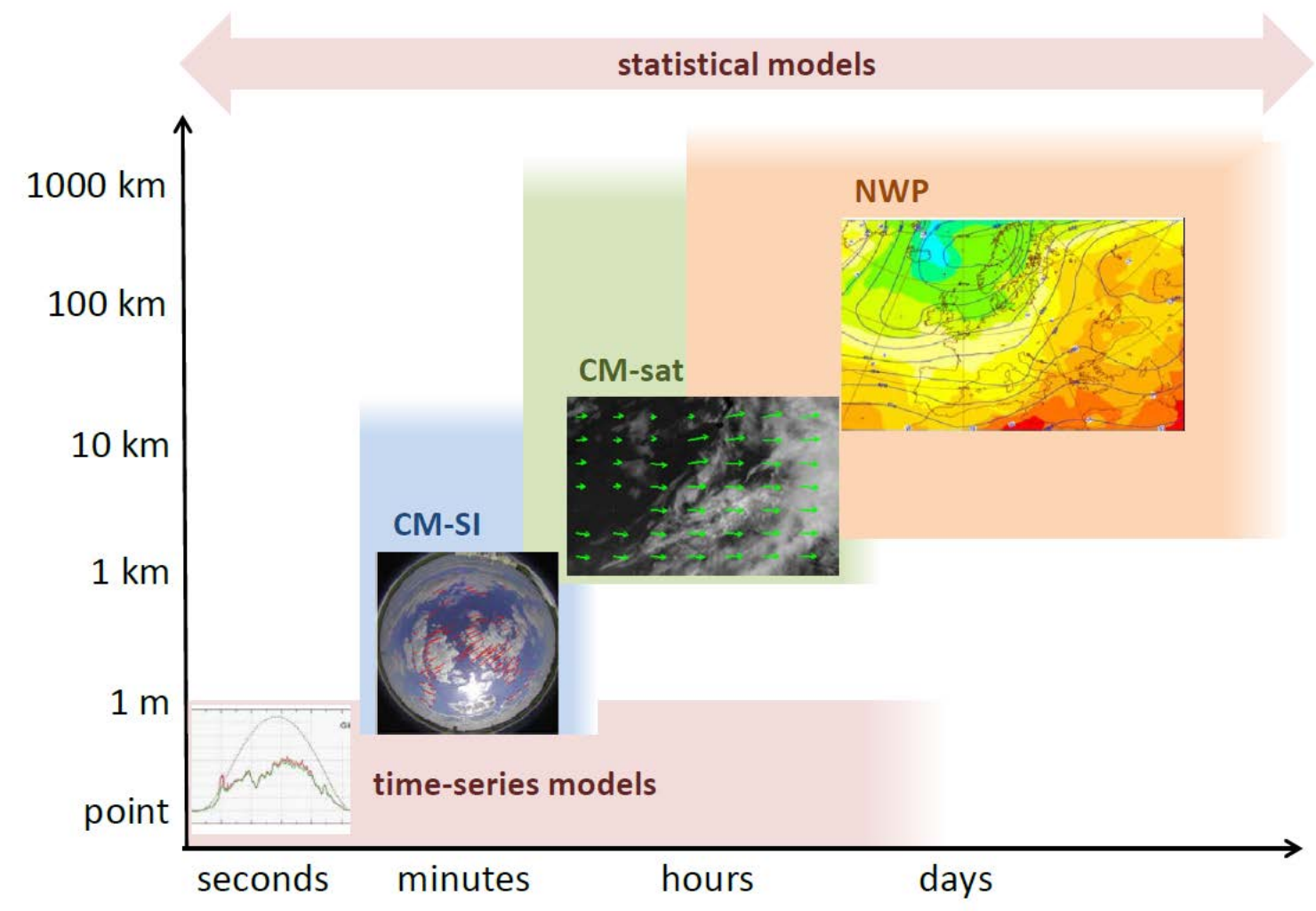

Figure 7-1. Illustration of different forecasting methods for different spatial and temporal scales: TS = time-series modeling; CM-SI = cloud motion forecast based on sky-imagers; CM-sat = cloud motion forecast based on satellite images; and NWP

\subsubsection{Irradiance Forecasting With Cloud Motion Vectors}

For short-term horizons, the temporal change in cloud patterns is strongly governed by horizontal advection, with the shape of clouds often remaining rather stable. Techniques detecting clouds and cloud motion in sufficient detail therefore provide valuable information for irradiance forecasting in the corresponding time scales. Evidently, the performance of this forecasting method is degraded when local cloud formation and dissipation processes, such as strong thermal convection, are dominant over cloud advection.

The following basic steps comprise forecasting based on cloud motion vectors:

- Images with cloud information ("cloud images") are derived from satellite or groundbased sky-imager measurements

- Assuming stable cloud structures and optical properties for the considered temporal and spatial scales, cloud motion vectors (CMV) are determined by identifying matching cloud structures in consecutive "cloud images"

- To predict future cloud conditions, the calculated motion vectors are applied to the latest available "cloud image"-i.e., cloud motion is extrapolated using the additional assumption of persistent cloud speeds and velocity

- Forecasts of site-specific solar irradiance are inferred from the predicted "cloud images." 


\subsubsection{Forecasting Using Ground-Based Sky Imagers}

Solar irradiance forecasts in the subhourly range with very high temporal and spatial resolutions can be derived from ground-based sky images. In particular, they have the potential for capturing sudden changes in irradiance, often referred to as ramps, on a temporal scale of minutes or even less (see Figure 7-4). Cloud fields may be resolved in high detail, allowing partial cloud cover on large PV installations to be modeled and forecasted (see Figure 7-2). Maximum possible forecast horizons strongly depend on cloud conditions-i.e., cloud height and velocity. They are limited by the time the monitored cloud scene has passed the location or area of interest, typically up to 15 minutes to 30 minutes ahead.

Short-term irradiance forecasting based on ground-based sky imagers is a rather new research field; a review of the state of the art is given in Urquhart et al. (2013). Currently, there is no defined standard for sky-imaging hardware, camera calibration, or image processing techniques. Systems in use range from commercially available low-cost web-cam-based sky cameras to highquality prototype systems developed at research institutes. These use digital cameras with charge-coupled device chips and large fish-eye lenses for photography and industry applications - for example, the sky imaging systems specifically designed for solar energy purposes at the University of California at San Diego (Urquhart et al. 2014). 

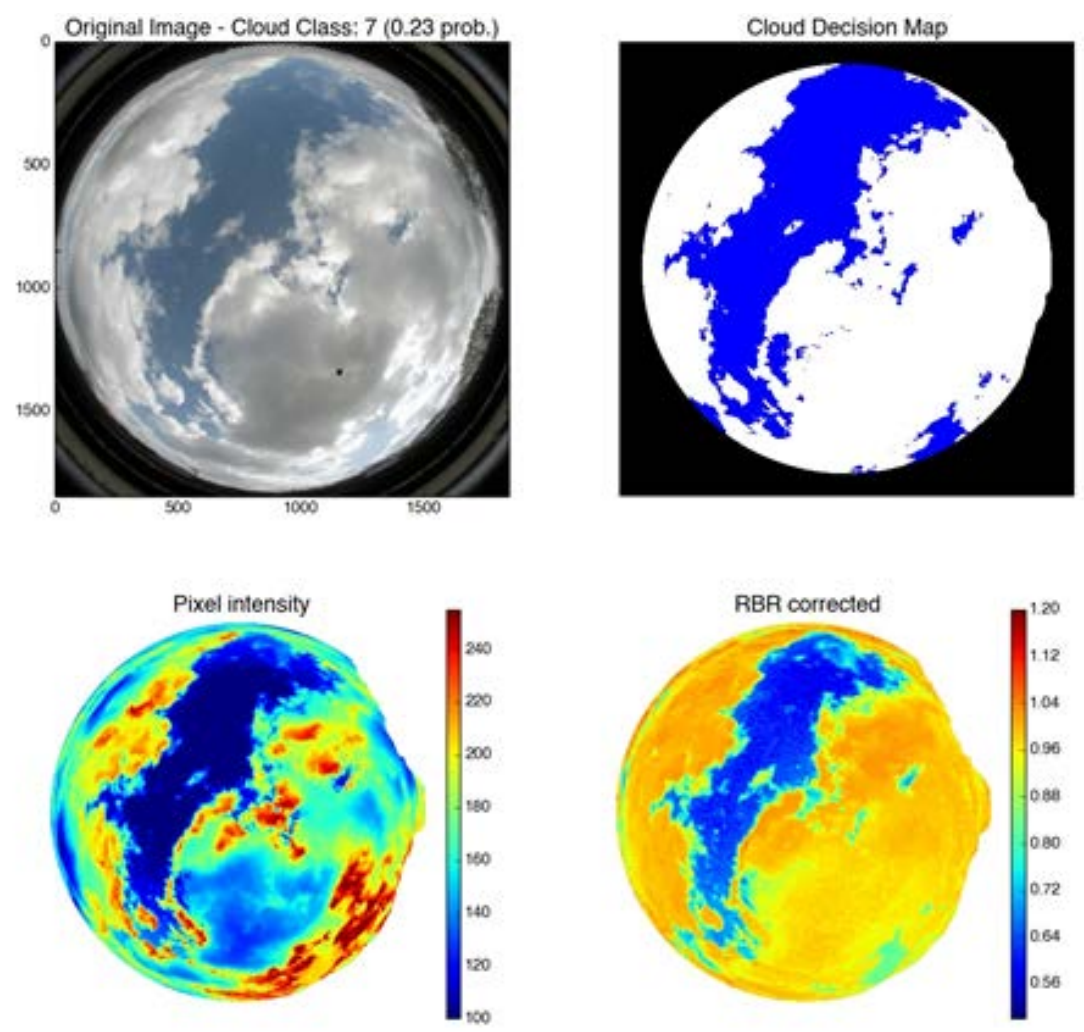

2013-04-19 12:59:00 UTC Juelich

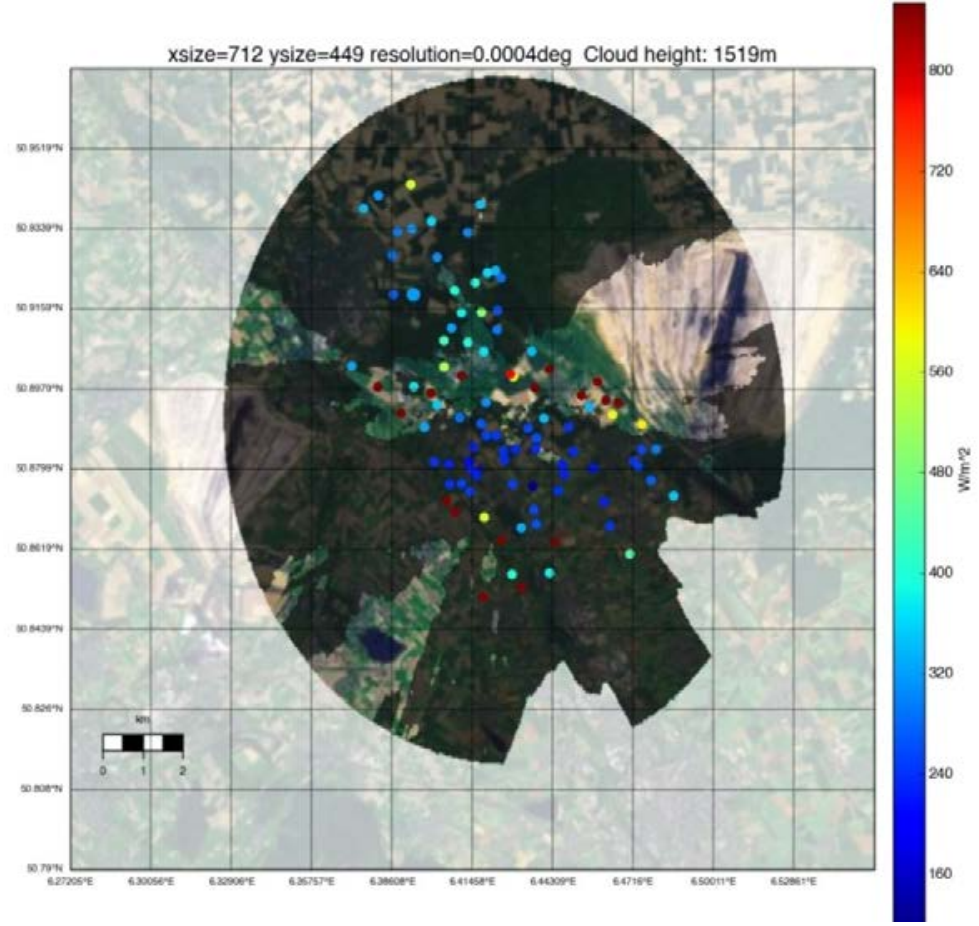

Figure 7-2. Cloud information from sky imagers: (upper left) original images; (middle left) pixel intensities; (middle right) red-blue ratio, corrected with a clear-sky library; (upper right) cloud decision map; and (bottom) shadow map with irradiance measurements. Sky image and irradiance measurements taken in Jülich, Germany, on April 9, 2013 at 12:59_00UTC in the framework of the HOPE campaign (Macke et al. 2014). Images from the University of Oldenburg 
Cloud detection from sky-imager pictures is performed by evaluating different image properties. The red-to-blue ratio (RBR, Figure 7-2, middle right) is a main indicator for clouds because of different spectral-scattering properties of clouds (high RBR) and clear sky (low RBR) (Shields, Johnson, and Koehler 1993; Long and DeLuisi 1998). Pixel intensities (Figure 7-2, middle left) are also related to cloud cover and may be exploited as an additional feature for cloud detection. Binary cloud decision maps (Figure 7-2, top right) can be derived on the basis of threshold procedures - for example, by evaluating the RBR in relation to a clear-sky library (Chow et al. 2011) to account for nonuniform clear-sky signal over sky hemisphere in dependence on the position of the sun. Cloud detection is particularly difficult in the circumsolar region because of saturated pixel information with high RBR values not only for cloudy but also for clear conditions. Gauchet et al. (2012) consider the circumsolar area and the solar disk separately with an image segmentation approach, distinguishing also clear skies, bright, and dark clouds. Ghonima et al. (2012) propose a method to differentiate between thin and thick clouds for various atmospheric conditions using a clear-sky library. Additional information on the cloud type in the monitored scene, which also gives an indication on cloud optical thickness and cloud height, can be obtained by a cloud classification algorithm (for example, Heinle, Macke, and Srivastav 2010).

Transformation of the derived cloud maps to real-world coordinates requires information about cloud height, which together with the position of the sun determines the position of cloud shadows at the surface; therefore, it is essential for modeling and forecasting irradiance fields with high spatial resolutions. Note, however, that a single-point forecast for the position of the sky imager does not require information about cloud height but can be derived by simply evaluating the movement of cloudy pixels toward the pixel at the position of the sun. Different options to determine cloud height include ground-based observations, satellite methods, and the evaluation of sky-imager data. Most accurate information on cloud-base height is obtained from ceilometers (lidars), typically employed at airport weather stations. Cloud-top height retrieval from satellite images (see Chapter 4) gives spatially continuous information but shows larger uncertainties. Different methods to determine cloud height using information from more than one sky imager are shortly introduced in Urquhart et al. (2013) and Prahl et al. (2014).

Detection of cloud motion is the next step to derive irradiance forecasts. Chow et al. (2011) identify cloud motion based on a normalized cross-correlation procedure-i.e., by maximizing the cross-correlation between shifted areas in two consecutive images. Alternatively, cloud movement may be analyzed by applying optical flow techniques (for example, as in Lucas and Kanade 1981 and Wood-Bradely, Zapata, and Pye 2012) to subsequent images (see Figure 7-3). The derived cloud motion vectors are then used to project the observed cloud scenes in the future.

Cloud shadows maps at the surface (see Figure 7-2, bottom) are produced by projecting the forecasted cloud scenes with their assigned height using information about the position of the sun. Finally, solar irradiance is estimated from these cloud shadow maps. Without information about cloud optical properties and other atmospheric parameters, this is not a trivial task. Local irradiance or PV power measurements can be used to estimate irradiance or PV power for cloudy and clear skies. Urquhart et al. (2013) analyze frequency distributions of PV power normalized to clear-sky conditions to determine a clear and a cloudy mode and to assign them to shaded and unshaded cells, respectively. Gauchet et al. 2012 propose the use of a regression model in 
combination with a clear-sky model to estimate the surface solar irradiance from segmented sky images with information about clear-sky, bright, and dark clouds; circumsolar area; and solar disk.

High-quality irradiance measurements are essential for further algorithm development. In particular, the analysis of irradiance fields with high spatial resolutions requires measurements from a dense network of observation sites, such as the high-quality data set collected during the HOPE campaign (Macke and HOPE-Team, 2014] and Madhavan, Kalisch, and Macke 2014; Figure7-2, bottom). Measurements with more than 90 photodiode pyranometers distributed throughout an area of $10 \mathrm{~km}$ by $10 \mathrm{~km}$ close to Jülich in Germany where taken from April to July 2013.

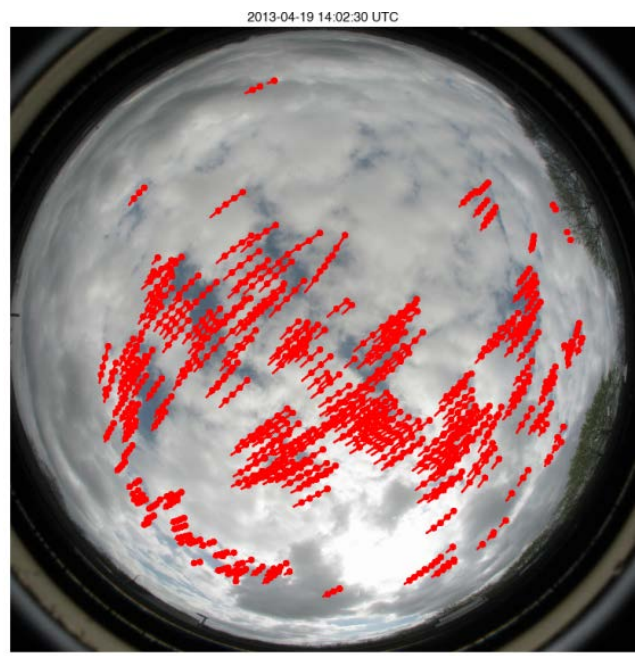

Figure 7-3. Example of an optical-flow field. Sky image taken in Jülich, Germany, on April 19, 2013 at 15:30 UTC in the framework of the HOPE campaign (Macke and HOPE-Team forthcoming 2014], Madhavan, Kalisch, and Macke 2014). Image from the University of Oldenburg 


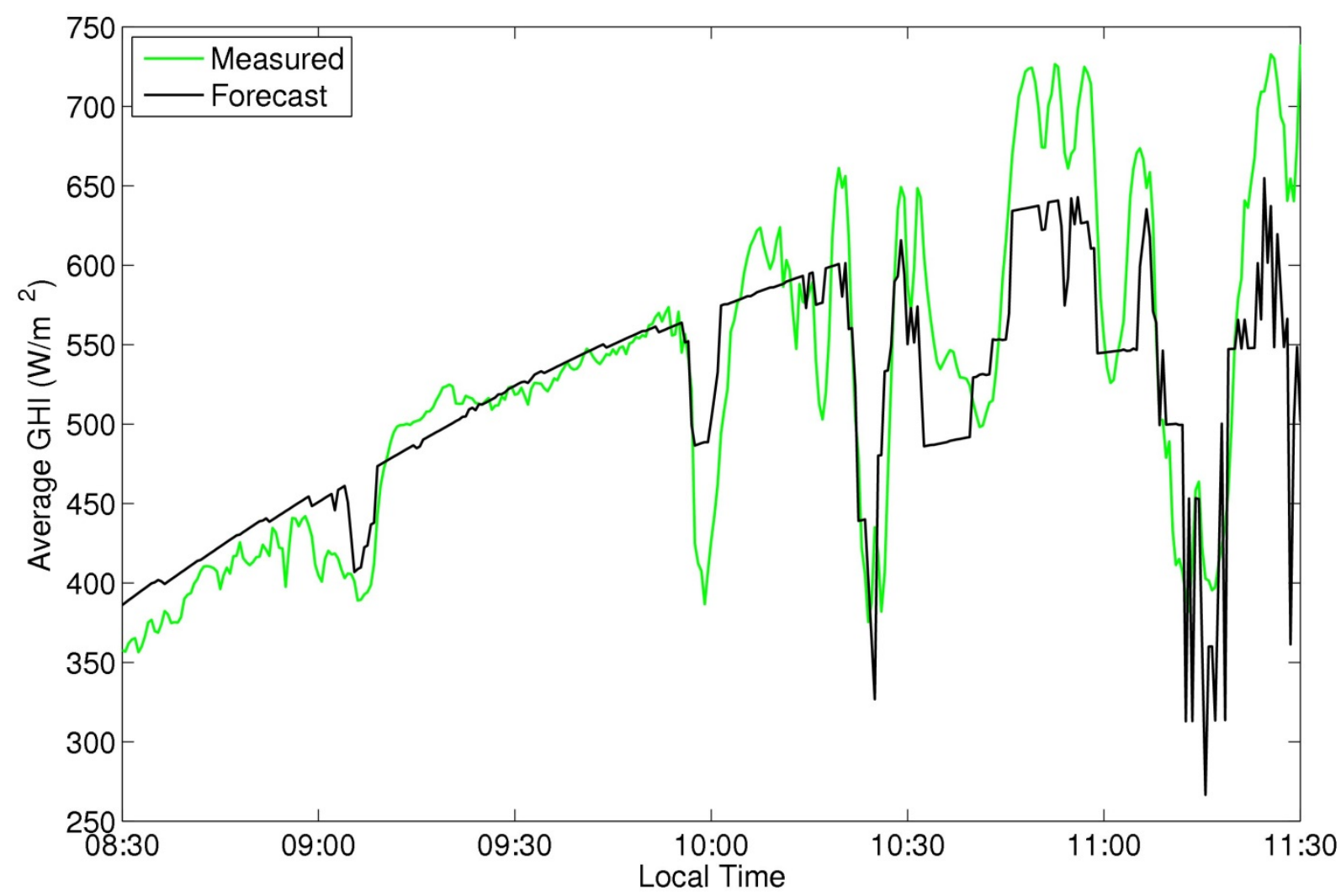

Figure 7-4. Example sky imager-based 5-minute-ahead irradiance forecasts. Location: Universtity of California at San Diego, November 14, 2012. Image from University of California at San Diego Center for Energy Research

\subsubsection{Satellite-Based Forecasts}

Forecasts of several hours ahead require observations of cloud fields in large areas. For example, assuming a maximum cloud velocity of $160 \mathrm{~km} / \mathrm{h}$, a region of approximately $2,000 \mathrm{~km}$ by 2,000 $\mathrm{km}$ has to be covered to track arriving clouds 6 hours ahead. Satellite data with their broad coverage (see Section 4.4) are an appropriate source for these horizons.

Cloud and irradiance information from satellite images can be derived by a variety of methods, as presented in Chapter 4. In principle, all of them can be applied to satellite-based irradiance forecasting with cloud motion vectors. There are also many approaches to derive atmospheric cloud motion vectors, which are commonly used in operational weather forecasting to describe wind fields at upper levels in the atmosphere.

Satellite-based nowcasting schemes have been developed in recent years based on cloud motion vectors or sectoral cloud tracking (Hammer et al. 2003, Schroedter-Homscheidt et al. 2011). The satellite-based forecasting scheme from the University of Oldenburg in Germany (Lorenz, Heinemann, and Hammer 2004, Kühnert, Lorenz, and Heinemann 2013), described exemplarily here, uses images of the geostationary MSG satellites (See Chapter 4). The semiempirical HELIOSAT method (Hammer et al. 2003; see Chapter 4) is applied to obtain information about clouds and irradiance. A characteristic feature of the method is the dimensionless cloud index, which gives information about the cloud transmissivity. 
Cloud motion vectors are derived by identifying corresponding cloud patterns in two consecutive images (see Figure 7-5). Rectangular areas, the "target areas," are defined with a size of approximately $90 \mathrm{~km}$ by $90 \mathrm{~km}$ to be large enough to contain information about temporally stable cloud structures and small enough that cloud motion for the this area can be described by a single vector. Mean square pixel differences between target areas in consecutive images $\left(\mathrm{n}_{0}\right.$ and $\mathrm{n}_{-1}$ ) are calculated for displacements in all directions (Figure 7-5a-c). The maximum possible displacement ("search area") is determined by maximum wind speeds at typical cloud heights. The displacement that yields the minimum mean square pixel difference for a given target area is assigned as motion vector (Figure 7-5d). The derived motion vectors are applied to the cloud index image $\mathrm{n}_{0}$ to predict future cloud conditions. A smoothing filter is applied to the predicted cloud index image to eliminate randomly varying small-scale structures that are hardly predictable. Finally, irradiance is derived from the predicted cloud index images using the HELIOSAT method.

The SolarAnywhere short-term forecasting scheme (Perez et al. 2009, Perez and Hoff 2013) for the United States based on GOES satellite images (see Section 4.4.1) follows a similar approach to detect cloud motion and is also based on a semiempirical cloud index method (see Chapter 4). Another method presented in Schroedter-Homscheidt et al. (2011) discriminates the tracking of optically thin cirrus clouds from the tracking of optically thick cumulus or stratus clouds with respect to increased accuracy needs in direct irradiance nowcasting for concentrating technologies.

Müller and Remund (2013) propose a method that combines cloud index values retrieved from MSG satellites with wind fields from a NWP model. The wind fields are predicted with the WRF model (Skamarock et al. 2008) in hourly resolutions and applied to forward propagation of the observed cloud patterns. Information about the height of the monitored clouds is necessary to determine the corresponding NWP model level. Müller and Remund (2013) assume fixed cloud heights for this purpose. An advantage of the application of NWP wind fields compared to satellite-derived cloud motion vectors is the potential to describe changes in the direction and speed of cloud movement during the extrapolation process. 
image $n_{-1}$,

$\mathrm{t}-15 \mathrm{~min}$

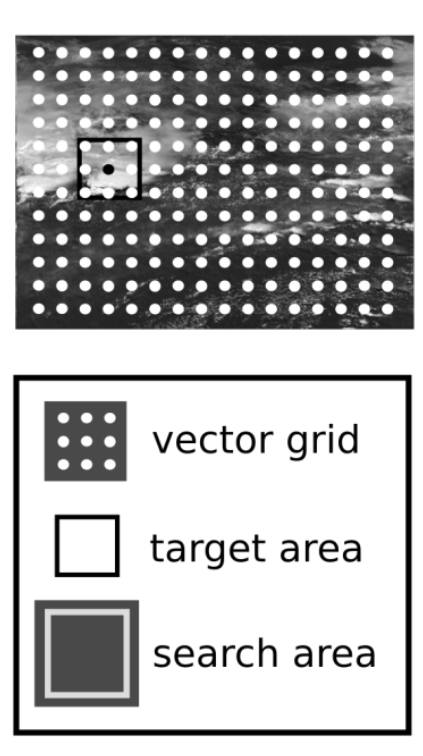

image $\mathrm{n}_{0}$,

time $\mathrm{t}$

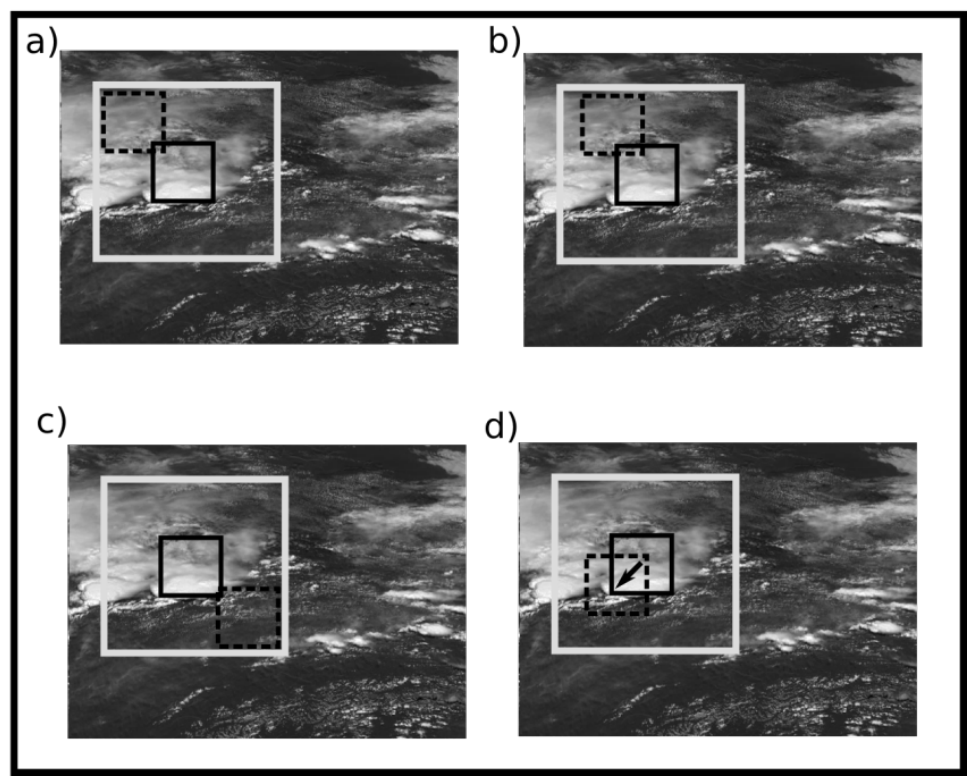

Figure 7-5. Schematic overview for detection of cloud motion in satellite images. Images reproduced from Kuehnert et al. 2013

A method for satellite-based short-term forecasting based on a physical cloud and irradiance retrieval scheme (see Chapter 4) is introduced by Miller et al. (2013). They process GOES satellite observations with the NOAA Pathfinder Atmospheres Extended (PATMOS-x) (Heidinger et al. 2014) retrieval package, a stand-alone radiative transfer code, and combine them with wind field data from the Global Forecast System (GFS) model. Cloud properties are retrieved with PATMOS-x in a first step. Next, the cloud fields are advected using GFS winds at the vertical level matching the cloud-top height as retrieved from PATMOS-x. Finally, solar irradiance at the surface is calculated with radiative transfer calculations using predicted cloud properties and additional atmospheric parameters.

\subsubsection{Irradiance Forecasting with NWPs}

NWP models are routinely operated by weather services to forecast the state of the atmosphere for several days ahead. Starting from initial conditions that are derived from worldwide observations, the temporal development of the atmospheric conditions is modeled by solving the basic equations describing the physical laws governing the atmosphere. This physical modeling is essential for any forecast more than several hours ahead. A comprehensive overview of NWP modeling is given in Kalnay (2003).

Global NWP models are applied to calculate the future state of the atmosphere for the complete Earth. To determine the initial state of a forecast, data assimilation tools (Jones and Fletcher 2013) are applied to make efficient use of worldwide meteorological observations, including measurement data from weather stations and buoys as well as satellite observations. The prognostic equations, describing the dynamics and physics of the atmosphere, are solved using numerical methods. These involve spatial and temporal discretization, in which the grid 
resolution is determined by the computational costs. Many physical processes occur on spatial scales much smaller than the grid size, including, for example, condensation, convection, turbulence, and scattering and absorption of radiation. The statistical effect of these unresolved processes on the mean flow is modeled with parameterizations of atmospheric physics. Today, the spatial resolution of global NWP models, run by approximately 15 weather services, is in the range of $16 \mathrm{~km}$ to $50 \mathrm{~km}$; the temporal resolution of the model output is 1 hour, 3 hour, or 6 hour, limited by storage requirements.

Mesoscale or regional models covering only part of the Earth and taking initial and lateral boundary conditions from a global NWP model allow for downscaling to a finer grid. Weather services typically operate mesoscale models with a spatial resolution in the range of $3 \mathrm{~km}$ to 20 $\mathrm{km}$ and provide hourly forecasts, but also higher resolutions are feasible. The higher spatial resolution allows for an explicit modeling of small-scale atmospheric phenomena.

For irradiance forecasting, the parameterizations of radiation transfer and cloud properties are of special importance. Larson (2013) compares the respective model configurations with respect to GHI for four operational NWP models, including the integrated forecast system (IFS) of the European Center for Medium-Range Weather Forecasts and GFS run by NOAA. In particular, he discusses deep and shallow cumulus parameterizations, turbulent transport, stratiform microphysics and prognosed hydrometers, cloud fraction and overlap assumptions, the description of aerosols, and the shortwave radiative transfer schemes. But he also emphasizes that "because of the strong feedback and interactions of physical processes in the atmosphere," other processes may have a significant impact on irradiance forecasting.

Today, most NWP models offer GHI as direct model output, and some also provide forecasts of direct and diffuse irradiances. Although in principle direct model output can be used for solar energy application, in practice mostly an additional post-processing is applied to improve forecast accuracy (see Section 7.3.4).

\subsubsection{Examples of Operational NWP Models}

As part of the IEA SHC's Task 36 and Task 46, irradiance forecasts of different NWP models have been investigated and evaluated. The following lists these with their spatial resolutions and output time intervals, exemplarily highlighting cloud fraction parameterizations and radiation schemes for some of the models. Additionally, references with respect to the application and evaluation of irradiance forecasts of these models in the context of solar energy forecasting are provided. A comparison of GHI forecasts based on these models for the United States, Canada, and Europe is given in Perez et al. (2013). It shall be emphasized that the sample of operational models and applications given here is not at all a complete list, but it reflects the work completed in the IEA SHC's Task 36 and Task 46.

The IFS of the European Center for Medium Range Weather Forecasting is a global model operated with a horizontal grid spacing of $16 \mathrm{~km}$ and 137 vertical levels for the high resolution deterministic forecasts ("Atmospheric Physics" 2014). Operational output is available with a temporal resolution of 3 hours up to 6 days ahead, with a higher resolution of 1 hour accessible in the framework of research projects. An overview on the current IFS physical parameterizations is given in the European Center for Medium Range Weather Forecasting. In particular, "the radiation code is based on the Rapid Radiation Transfer Model (Mlawer et al. 
1997, Iacono et al. 2008). Cloud-radiation interactions are taken into account in detail by using the values of cloud fraction and liquid, ice and snow water contents from the cloud scheme using the McICA method (Monte Carlo Independent Column Approximation; Pincus, Barker, and Morcrette 2003) and McRad method (Morcrette et al. 2008)". The prognostic scheme for clouds and large-scale precipitation is based on Tiedtke (1993). In Lorenz et al. (2009), European Center for Medium Range Weather Forecasting irradiance forecasts are analyzed with respect to different properties relevant for their application for PV power prediction. Lorenz et al. (2011) propose and evaluate an approach for regional PV power prediction for improved grid integration based on European Center for Medium Range Weather Forecasting forecasts.

NOAA's GFS ("Dataset I-i Atmospheric Fields-High-Resolution Forecast” 2014) is currently operated at a spatial resolution of $50 \mathrm{~km}$ with 47 levels, and output is provided at three-hourly intervals up to 8 days ahead. Model physics related to clouds and radiation are summarized in Larson (2013); here, it shall only be mentioned that cloud fraction is a diagnostic variable in the GFS model in contrast to the IFS model. Mathiesen and Kleissl (2011) give an evaluation of intraday GHI forecasts of the GFS compared to IFS forecasts from the European Center for Medium Range Weather Forecasting and the North American Model.

Environment Canada's Canadian Meteorological Centre operates the Global Environmental Multiscale. The model is run in different configurations, including a regional deterministic configuration (Mailhot et al. 2006) generating forecasts up to 48 hours ahead at a 7.5-minute time step and with a spatial resolution of approximately $15 \mathrm{~km}$ at the grid center, in Canada. Pelland, Gallanis, and Kallos (2011) have investigated solar irradiance and PV power forecasting with post-processing applied to the high-resolution global environmental multiscale forecasts.

The mesoscale WRF (Skamarock et al. 2008) model has been developed as a series of opensource models in a collaborative effort of several institutes led by the National Center for Atmospheric Research in the United States. The WRF model is non-hydrostatic with multiple nesting capabilities offering various parameterizations for the different physical processes. This allows its configuration for specific conditions for a certain application or a region of interest. The radiation scheme proposed in Dudhia et al. (1998) is applied primarily to solar energy applications. No cloud fraction parameterization is currently implemented for WRF, and the grid boxes are described as either fully cloudy or clear. Because clouds can be highly variable in space, this can be a disadvantage (Larson 2013), especially if the model is not run with a high spatial resolution.

The application of the WRF, initialized with GFS, for solar irradiance forecasting - and energy meteorology applications in general - is a research focus at the University of Jaen in Spain. Ruiz-Arias et al. (2008) have investigated the relative performance of two different planetary boundary layer parameterizations for Mesoscale Model of $5^{\text {th }}$ generation (MM5), the preceding model of WRF, for different sky conditions. A topographic downscaling procedure for the solar irradiance in mountainous regions and its application to the WRF is proposed in Ruiz-Arias et al. (2010). Lara-Fanego et al. (2011) have evaluated 3-day-ahead hourly and 10-minute WRF forecasts of GHI and DNI in Andalusia (Southern Spain). In cooperation with the National Center for Atmospheric Research (Ruiz-Arias et al. 2013) have performed surface clear-sky shortwave radiative closure intercomparisons in the WRF model and proposed a simple 
parameterization of the shortwave aerosol optical properties for surface direct and diffuse irradiances assessment (Ruiz-Arias, Dudhia, and Gueymard 2014).

WRF for solar irradiance forecasting initialized with GFS is also operated at Meteotest in Switzerland (Remund et al. 2008), at GL-Garrad Hassan (Kleissl 2013), as part of the operational air quality forecasting program at the Atmospheric Sciences Research Center of the University of Albany (2010) and at AWS Truepower in the United States.

The High Resolution Limited Area Model is a hydrostatic NWP model operated by several national meteorological services in Europe-for example, the Spanish Agencia Estatal de Meteorología and the Danish Meteorological Institute. ${ }^{48}$ The Danish Meteorological Institute runs its highest resolution High Resolution Limited Area Model model "SKA" for an area covering Northwestern Europe with a grid size of 0.03 degrees $(3 \mathrm{~km})$ and 65 vertical levels. The High Resolution Limited Area Model uses the clear-sky irradiance scheme of Savijärvi (1990) and the cloud scheme of Wyser, Rontu, and Savijärvi (1999).

The regional weather forecasting system SKIRON (Kallos 1997) is operated for solar energy applications at Spain's National Renewable Energy Center (Gaston et al. 2009).

\subsubsection{Statistical Methods and Post-Processing}

Statistical learning models are widely applied for solar irradiance and power forecasting. The functional dependence between input variables (predictors) and forecast values (predictands) is established in a training phase by learning from historic data, assuming that patterns in the historical data sets are repeated in the future and thus may be exploited for forecasting. Statistical methods include classical regression methods, such as autoregressive and autoregressive integrated moving average models, and artificial intelligence techniques, such as artificial neural networks, $\mathrm{k}$ nearest neighbors, or support vector regression. An overview of different statistical approaches used for solar irradiance forecasting is given in Coimbra and Pedro (2013) and Diagne et al. (2012). Here, we reference selected examples for the majority of cases investigated in the IEA SHC's Task 36 and Task 46.

Statistical models can be used to derive irradiance forecasts solely based on measurements without involving any physical modeling (time series models with no exogenous input). They also play an important role in enhancing the output of NWP models and can be applied to cloud motion forecasts (see Figure 7-6). Different terminology is used for this combination of statistical and physical forecasting methods, depending on the perspective of the researchers. The community of statistical modeling and artificial intelligence refers to these models as "statistical models with exogenous input." Meteorologists commonly use the terms statistical post processing or, more specifically, model output statistics in the context of NWP, which is the terminology adopted here.

Post-processing methods are applied to forecasts of physical models to

- Reduce systematic forecast errors,

\footnotetext{
${ }^{48}$ See http://www.hirlam.org/.
} 
- Account for local effects (e.g., topography, surface)

- Account for the influence of selected variables in more detail (e.g., aerosols)

- Derive parameters that are not provided as model output (For example, today direct irradiance is not a standard output parameter of NWP models.)

- Combine the output of different models.

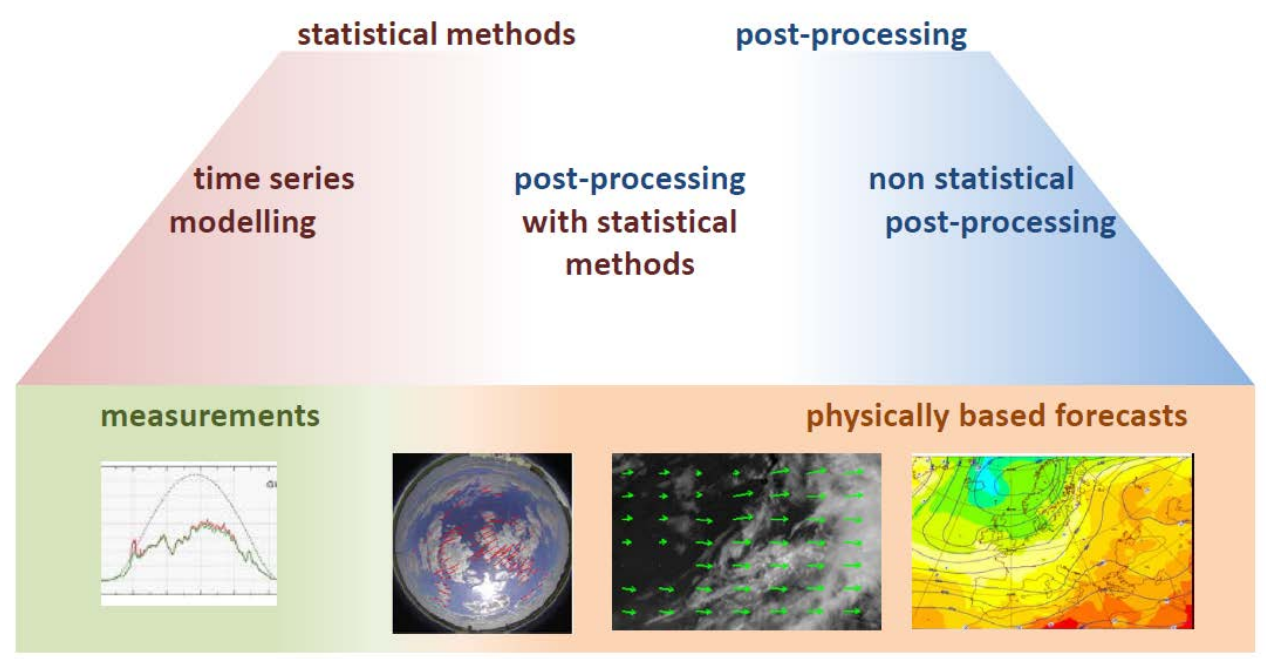

Figure 7-6. Overview of the application of statistical methods and post-processing

In addition to statistical post-processing techniques, there are also nonstatistical methods for post-processing (see Figure 7-6). A traditional method to obtain improved local forecasts from NWP model output is to involve forecast experts' human knowledge. Forecasts of direct or diffuse irradiance can be derived using empirical or physical models from GHI forecasts. Finally, the application of smoothing filters or spatial averaging reduces fluctuations of forecasts in variable situations, which can be beneficial if the correlation between measurements and forecasts is low (see section 7.3.6).

In the following paragraphs, we give a short overview of different statistical and post-processing methods.

\subsubsection{Time-Series Models}

Intra-hour or hours-ahead solar irradiance or PV power forecasting with time-series models uses recent measurements of irradiance or PV power as a basic input, possibly complemented by measurements of other variables. Examples are the application of a coupled autoregressive and dynamical system model for forecasting solar radiation on an hourly time scale described in Huang et al. (2013), the comparison of artificial neural networks and classical time series models in Reikard (2009), and the short-term PV power prediction approach of Bacher, Madsen, and Nielsen 2009.

For any statistical model, the selection and availability of appropriate input variables as well as an optimized preprocessing of these data is of critical importance for good forecast performance. 
Also, the choice of the model configuration - for example, the artificial neural network architecture or the assignment of support vector regression model parameters determining the degree of generalization is essential. Finally, setup of the training sample - for example, the number of days and sites used for training - has an influence on forecast accuracies. Coimbra and Pedro (2013) show the benefit of the application of a genetic algorithm to identify the most suitable artificial neural network architecture, preprocessing scheme, and training data.

In the following, we shortly discuss advantages and limits of purely statistical approaches. Timeseries models take the advantage of high autocorrelation for short time lags in time series of solar irradiance and cloud cover. Any physical forecasting model has an inherent uncertainty regardless of the forecast horizon, caused by limits in spatial and temporal resolutions, uncertainty in input parameters, and simplifying assumptions within the model. Therefore, for the very short-term scale of typically up to 1 hour or 2 hours ahead, forecasts based on accurate on-site measurements are advantageous; however, the high autocorrelation of irradiance time series is decreasing rapidly with increasing time lags, limiting the effectiveness of the time-series approach for horizons beyond several hours ahead. For these horizons, it is essential to account for dynamic phenomena, such as motion and formation or dissolution of clouds, which are influenced not only by local irradiance but by regional weather conditions, with the extension of the influencing area increasing with increasing forecast horizons. Physical models can describe these phenomena and model complex atmospheric interactions throughout large areas. Bacher, Madsen, and Nielsen. (2009) perform a comparison of an autoregressive model for hourly solar power forecasting with and without exogenous input. They found that measurement data are the most important input to the model up to 2 hours ahead and NWP forecast parameters are the adequate input for next-day horizons.

\subsubsection{Model Output Statistics}

Model output statistics are widely used to refine the output of NWP models, primarily to account for local variations in weather and surface conditions (Glahn and Lowery 1979) using measurements and/or climatology for specific locations as a basis to adapt the forecasts. For example, MOS techniques constitute a powerful tool to adapt the results from NWP or satellitebased models to site-specific conditions (Gueymard et al. 2012). For solar irradiance forecasting, satellite-derived values may be used instead of ground measurements. The set of predictors consists of NWP output and may be extended by including any relevant information-for example, prior observations and climatological values.

Traditionally, the term model output statistic is associated with the use of regression equations. A wider concept of model output statistics includes any statistical approach that relates NWP output to observed variables; some examples are given in the following. Lorenz et al. (2009) apply a bias correction in dependence on the solar elevation and the clear-sky index to European Center for Medium Range Weather Forecasting irradiance forecasts (see also Section 7.3.7). An approach using Kalman-filtering to improve irradiance forecasts of the Canadian Global Environmental Multiscale model is introduced in Pelland et al. (2011). Marquez and Coimbra (2011) investigate the application of artificial neural networks to predicted variables from the NWS forecasting database, and Gaston et al. (2009) use a machine-learning algorithm to enhance SKIRON solar irradiance forecasts. The statistical forecast tool BLUE FORECAST (Natschlaeger et al. 2008) is based on the GFS model and integrates different methods of data 
mining such as ridge regression, automatic quadratic models, or neural networks to process the forecasts.

\subsubsection{Combinations of Different Models}

Combining the output of different models can increase the forecast accuracy considerably when compared to single-model forecasts. First, simple averaging is beneficial for models with similar accuracy, exploiting the fact that forecast errors of different models are usually not perfectly correlated (Perez et al. 2013).

Combination methods using statistical tools may additionally account for strengths and weaknesses of the different models for certain situations - for example, by adapting the contribution of each model depending on the weather situation. In particular, they may also be applied to establish an irradiance forecasting tool covering horizons from several minutes to several days ahead by integrating measurements, climate monitoring, and NWP forecasts with an optimized weighting depending on the forecast horizon (Lorenz et al. 2012).

\subsubsection{Post-Processing With Empirical Models}

Empirical models allow for deriving parameters not provided as direct output of NWP models (direct model output) from predictions of related variables. Perez et al. (2007) propose a solar radiation forecast model relating sky-cover predictions from the National Digital Forecast Database to the to the clear-sky index of global irradiance.

The PV power forecasting approaches presented in Lorenz et al. (2011) and Pelland et al. (2012) involve empirical models to derive the POA irradiance as input for PV simulation models. Transposition of the predicted global horizontal to POA irradiance first requires decomposition to its components horizontal beam and diffuse irradiance. For that purpose, a number of empirical diffuse or direct fraction models are available, originally developed for application to measurements or satellite data, such as Skartveith, Olseth, and Tuft (1998) and Perez et al. (1992). Next, the two components are converted to the array plane. The transposition of the direct irradiance is straightforward and subject to geometric considerations only. The transposition of the diffuse irradiance requires a model for the directional distribution of radiance over the sky, describing anisotropic effects such as horizon brightening and circumsolar irradiance (Perez et al. 1987, Gueymard 1987, Hay 1979). A validation and comparison of different separation models is provided in a number of publications, including Boland, Huang, and Ridley (2013); De Miguel et al. (2001); Dervishi and Mahdavi (2012); Gueymard (2011); Gueymard and Ruiz-Arias (2014); Ruiz-Arias et al. (2010); and Tapakis, Michaelides, and Charalambides (2015). Similar validation studies but for transposition models are provided by Behr (1997); David, Lauret, and Boland (2013); Gueymard (2009); Ineichen (2011); and Kambezidis, Psiloglou, and Gueymard (1994). Finally, the validation of combined separation and transposition models has been undertaken by Gueymard (2009); Orehounig, Dervishi, and Mahdavi (2014); and Lave et al. (2015).

\subsubsection{Physical Post-Processing Approaches}

Physical post-processing approaches can be used to derive parameters that are not provided as direct model output. In particular, they are employed for direct irradiance forecasting and for a better consideration of aerosols. A direct and diffuse irradiance forecasting approach for solar 
energy applications based on chemical transport and NWP is presented in Breitkreuz et al. (2009). Forecasts of the AOD are inferred from particle forecasts of a chemical transport model. These aerosol forecasts together with other remote sensing data (ground albedo, ozone) and NWP parameters (water vapor, clouds) are used as input to radiation transfer calculations to derive the irradiance forecasts. Another physical post-processing procedure for direct irradiance forecasting based on WRF model output and satellite retrievals is proposed and evaluated in Lara Fanego et al. (2011).

\subsubsection{Meteorologists' Interpretation of NWP Output}

Many forecast providers involve forecast experts' human knowledge for deriving their forecast products. Traumueller and Steinmaurer (2010) describe the combination of a clear-sky model with cloud cover forecasts, derived from meteorologists by analyzing and comparing the output of different global and local NWP models. Especially in situations difficult to forecast, such as fog, this method offers potential for improvement. Also, the setup of the U.S. National Digital Forecast Database involves human input, as described in Perez et al. (2010).

\subsection{Evaluation of Irradiance Forecasts}

Forecast evaluation provides users with necessary information about forecast accuracy and helps them choose between different forecasting products or assess the risk when using a forecast as a basis for decisions. In research, detailed evaluations are an indispensable basis for model testing and further model development.

Many different aspects can contribute to forecast evaluation. An extensive overview of forecast verification methods is given in Joliffe and Stephenson (2003). Depending on the target group and the aim of the accuracy assessment, different methods to assess prediction quality will be selected. Forecast users are often interested in the forecast "end use accuracy," specified with a basic set of accuracy measures most important for the intended application; however, with the increasing importance of forecast information, their interest in detailed evaluation methods is growing.

The quality of forecasts is evaluated by assessing their similarity to reference data with visual analysis and statistical error measures. Most often, irradiance measurements are used as reference data - commonly referred as ground truth data; nevertheless, it is subject to uncertainties (see Section 3.3). Also, satellite-retrieved irradiance values or the output of a detailed physical model may serve as a reference.

A coherent set of basic evaluation measures and procedures as well as reference data sets were compiled in a collaborative effort of the IEA SHC's Task 36 and the European MESoR project (Beyer et al. 2009) to allow for a transparent and comparable evaluation of different solar radiation forecast products. This work is continued and extended in the IEA SHC's Task 46.

Here, we give an outline of these evaluation methods, including statistical error measures, comparison to persistence as a trivial reference model (skill scores), and the analysis of probability density functions as well as the analysis of forecast performance in dependence on various parameters characterizing irradiance and cloud conditions. An example evaluation illustrates these concepts of accuracy assessment and the benefit of baseline post-processing approaches investigated within the IEA SHC's Task 36. 


\subsubsection{Measurement and Forecast Data}

Different GHI forecasting approaches are evaluated against hourly pyranometer measurements of 18 weather stations of the German Weather Service in Germany (Figure 7-7) for the period from March 2013 to February 2014.

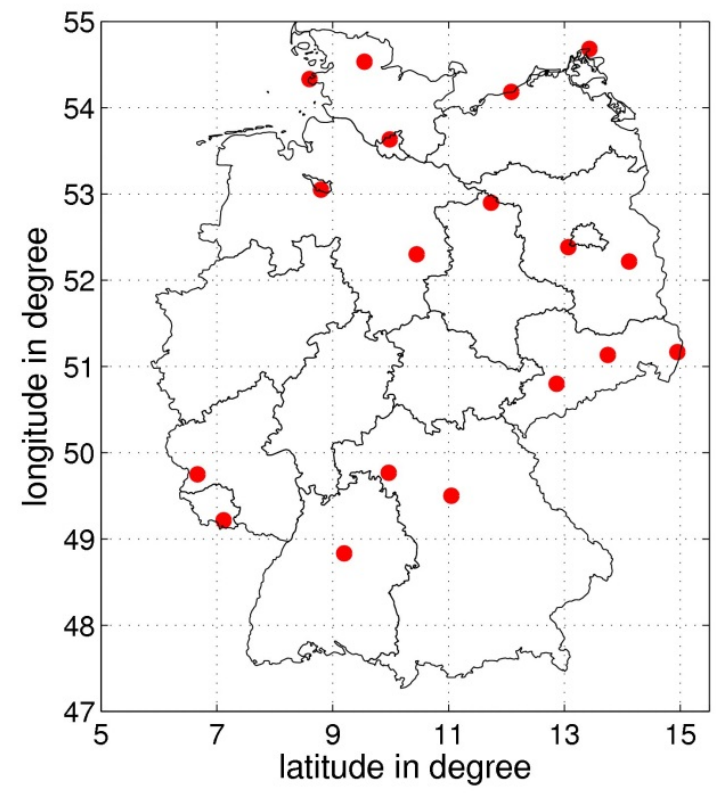

Figure 7-7. Evaluation sites in Germany

These data are a subset of the forecasts and measurements currently assembled in the context of a benchmarking study of NWP based forecasts performed in the IEA SHC's Task 46.

Here, forecasts from a global and a regional NWP model are compared:

- Irradiance forecasts of the high-resolution deterministic global IFS model, operated at the European Center for Medium Range Weather Forecasting

○ Grid size: $0.125^{\circ}$

- Output time interval: 3 hours

- Forecast base time used here: 0:00 UTC

○ Forecast horizons: 1 hour to 24 hours ahead.

- Irradiance forecasts of the Danish Meteorological Institute's highest resolution regional High Resolution Limited Area Model model SKA:

- Area: Northwestern Europe, initialized with IFS data

○ Grid size: 0.03 degree $(3 \mathrm{~km})$

- Output time interval: 1 hour

○ Forecast base times: 0:00 UTC, 06:00 UTC, 12:00 UTC, 18:00 UTC 
- Forecast horizons: 4 hours to 9 hours ahead.

Different base-line post-processing approaches were applied to both data sets:

- Spatial averaging

- IFS forecasts are averaged over 1 degree by 1 degree.

- The SKA model forecasts are derived for two averaging versions: from the nearest neighbor (denoted as "SKA" in the following) and averaged over 20-by20 grid boxes corresponding to approximately $60 \mathrm{~km}$ by $60 \mathrm{~km}$ ("SKA $20 \times 20$ ").

- Temporal interpolation: Hourly irradiance values are derived from 3-hour IFS forecasts averaged over 1 degree by 1 degree by linear interpolation of the clear-sky index (Lorenz et al. 2009), denoted as "IFS" in the following

- Temporal averaging: For "SKA $20 \times 20$ " forecasts, temporal averages over 5 hours of the clear-sky index is analyzed: $G H I_{\text {pred }}(t)=G H I_{\text {clear }}(t) \sum_{i=-2}^{i=2} k t^{*}(t+i)$ ("SKA av").

- Statistical post-processing

○ Linear regression for GHI and the clear-sky index kt*

- Bias correction in dependence on the solar elevation and kt* (Lorenz et al. 2009).

Training is performed with a sliding window technique using measurements of the previous 30 days of all German Weather Service sites together. The number of training data pairs is a critical issue, and it was found that using a network of stations for training is favorable compared to using data from individual stations only for the bias correction.

\subsubsection{Statistical Error Measures}

Statistical error measures are applied for quantitative forecast evaluation. Here, a number of error measures based on first-order statistics are presented.

The error of a single measurement is given as:

$$
\varepsilon_{i}=I_{\text {pred }, i}-I_{\text {meas }, i}
$$

where $I_{\text {pred, } i}$ denotes a predicted irradiance value (GHI or DNI), and $I_{\text {meas }, i}$ is the corresponding measured value.

For evaluating solar and wind power predictions, it is common practice to use the RMSE as a main score for assessing forecast accuracy:

$$
\text { rmse }=\frac{1}{\sqrt{N}} \sqrt{\sum_{i=1}^{N} \varepsilon_{i}^{2}}
$$

with $\mathrm{N}$ the number of data pairs. Only daytime values are considered for the evaluation. Relative errors for irradiance forecast are generally derived by normalization with respect to mean measured irradiance. In contrast, relative errors of PV power forecasts for utility applications are often given in relation to the installed power rather than to average measurements (e.g., Lorenz et al. 2011). 
The RMSE can be split into different parts, related to systematic and stochastic components of forecast errors. The bias

$$
\text { bias }=\bar{\varepsilon}=\frac{1}{N} \sum_{i=1}^{N} \varepsilon_{i}
$$

describes the difference between the mean values of predicted and measured values (systematic error). For a positive bias, the predicted values exceed the measurements in average.

The standard deviation of the errors stderr:

$$
\text { stderr }=\sigma(\varepsilon)=\frac{1}{\sqrt{N}} \sqrt{\sum_{i=1}^{N}\left(\varepsilon_{i}-\bar{\varepsilon}\right)^{2}}
$$

gives information on the fluctuation of the errors around their mean value. It may be further decomposed into one part related to the amplitude error $\left(\sigma\left(I_{\text {pred }}\right)-\sigma\left(I_{\text {meas }}\right)\right)$ and another part related to the correlation coefficient $r$ of the time series, defined as:

$$
r=\frac{\sum_{i=1}^{n}\left(I_{\text {pred }, i}-\overline{I_{\text {pred }}}\right) \cdot\left(I_{\text {meas }, i}-\overline{I_{\text {meas }}}\right)}{\sigma\left(I_{\text {pred }}\right) \sigma\left(I_{\text {meas }}\right)}
$$

With the complete decomposition of the RMSE:

$$
\operatorname{rmse}^{2}=\operatorname{bias}^{2}+\left(\sigma\left(\mathrm{I}_{\text {pred }}\right)-\sigma\left(\mathrm{I}_{\text {meas }}\right)\right)^{2}+2 \sigma\left(\mathrm{I}_{\text {meas }}\right) \sigma\left(\mathrm{I}_{\text {pred }}\right)(1-\mathrm{r})
$$

The parts of the errors that can be easily corrected with a simple post-processing based on linear transformations can be identified.

A linear transformation of a time series with the coefficients $\alpha$ and $\beta$ is given by:

$$
I_{\text {pred }, L R}=\alpha I_{\text {pred }}+\beta
$$

To achieve a minimum root mean square erro, the mean value and the standard deviation $\sigma\left(l_{\text {pred }}\right)$ of the predicted time series are adjusted in a way that the bias is removed and the stderr is minimized (see also Beyer et al. 2009). The minimum RMSE achievable with linear transformations is determined by the correlation $r$ between measurements and predictions and the amplitude of the measurements:

$$
r m s e_{L R}=\sigma\left(I_{\text {meas }}\right) \sqrt{1-r}
$$

with the amplitude of the predictions scaled to

$$
\sigma\left(I_{\text {pred,LR }}\right)=\sigma\left(I_{\text {meas }}\right) r
$$

The same variance of predicted and measured time series results in the smallest possible RMSE only for a perfect correlation. For correlation coefficients smaller than one, a reduction of the 
variance of the predicted time series according to Equation 7-8 improves forecast accuracy in terms of RMSE.

This is illustrated in Figure 7-8, showing a considerably higher RMSE for the nearest neighbor SKA forecasts than the IFS forecasts, but only a slightly smaller correlation coefficient. A clear reduction of the RMSE from $46 \%$ to $43 \%$ is achieved with linear regression for the SKA forecasts, with $\sigma\left(I_{\text {pred }}\right)$ reduced from $1.05 \sigma\left(I_{\text {meas }}\right)$ to $0.9 \sigma\left(I_{\text {meas }}\right)$. For the IFS forecasts, with the ratio $\sigma\left(I_{\text {pred }}\right) / \sigma\left(I_{\text {meas }}\right)$ close to the correlation coefficient $r$ already for the original forecasts and a small bias, the improvement with linear regression is within the limits of the given precision.

Another common measure to assess forecast accuracy is the mean absolute error:

$$
\text { mae }=\frac{1}{N} \sum_{i=1}^{N}\left|\varepsilon_{i}\right|
$$

recommended in Hoff et al. (2012) as a preferred measure, in particular for reporting relative errors.

From a user's point of view, ultimately the impact of forecast errors on their application will be decisive for the choice of the most suitable error measure. The mean absolute error is appropriate for applications with linear cost functions-i.e., when the costs caused by a wrong forecast are proportional to the forecast error. The RMSE is more sensitive to large forecast errors and hence suitable for applications when small errors are more tolerable and larger errors cause disproportionately high costs, which is the case for many applications in the energy market and for grid management issues. 

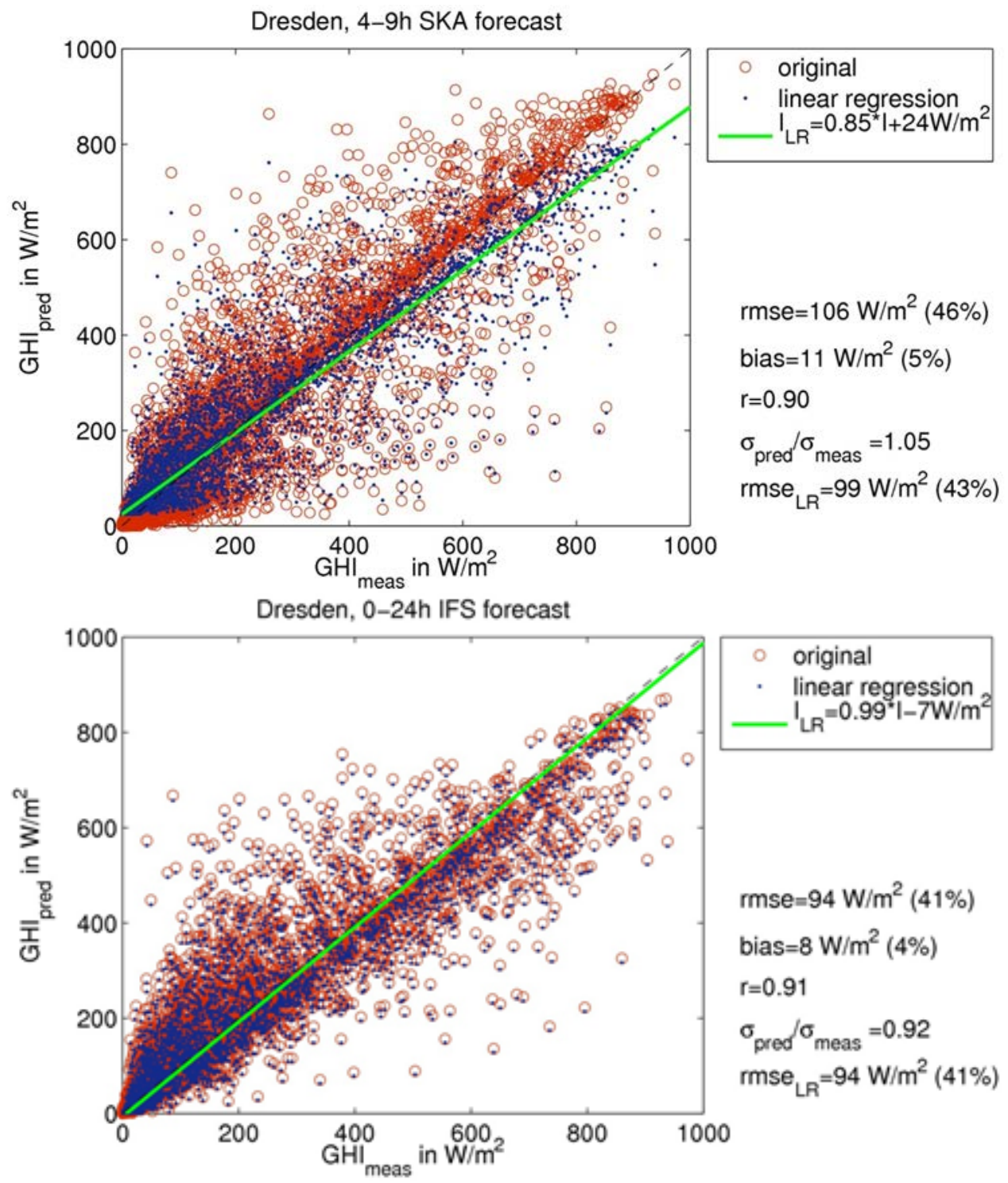

Figure 7-8. Scatterplots of predicted and measured GHI for (top) high-resolution SKA and (bottom) IFS forecasts. The original forecasts are shown in red, the forecast processed with the linear regression of $\mathrm{GHI}$ is shown in blue, and the regression equation is visualized in green. The data are from Dresden, Germany, March 1, 2013-February 28, 2014.

\subsubsection{Evaluation in Dependence of Solar Elevation and Analysis of the Clear-Sky Index}

A special feature of irradiance forecasts is their strong deterministic component because of the daily and seasonal course of the sun. Figure 7-9 shows the characteristic course of the RMSE throughout the day time for the $\mathrm{SKA}_{\mathrm{av}}$ and IFS forecasts. The solar elevation determines maximum possible irradiances and therefore also influences the magnitudes of forecast errors.

To investigate the non-deterministic part of the forecast errors, primarily caused by cloud forecast errors, the clear-sky index is analyzed.

$$
\mathrm{Kt}^{*}=\mathrm{GHI} / \mathrm{GHI}_{\text {clearsky }}
$$


Here, the clear-sky model of Dumortier (Fontoynont 1998, Dumortier 1998) with the METEONORM high-resolution database (Remund 2009) for the turbidity input is used to derive $\mathrm{GHI}_{\text {clearsky. }}$ As shown in Section 4.4, clear-sky irradiance can be estimated with good accuracy. Still, it should be kept in mind that the clear-sky index does not depend only on the analyzed forecasts, but that there is some influence of the used clear-sky model and atmospheric input parameters.

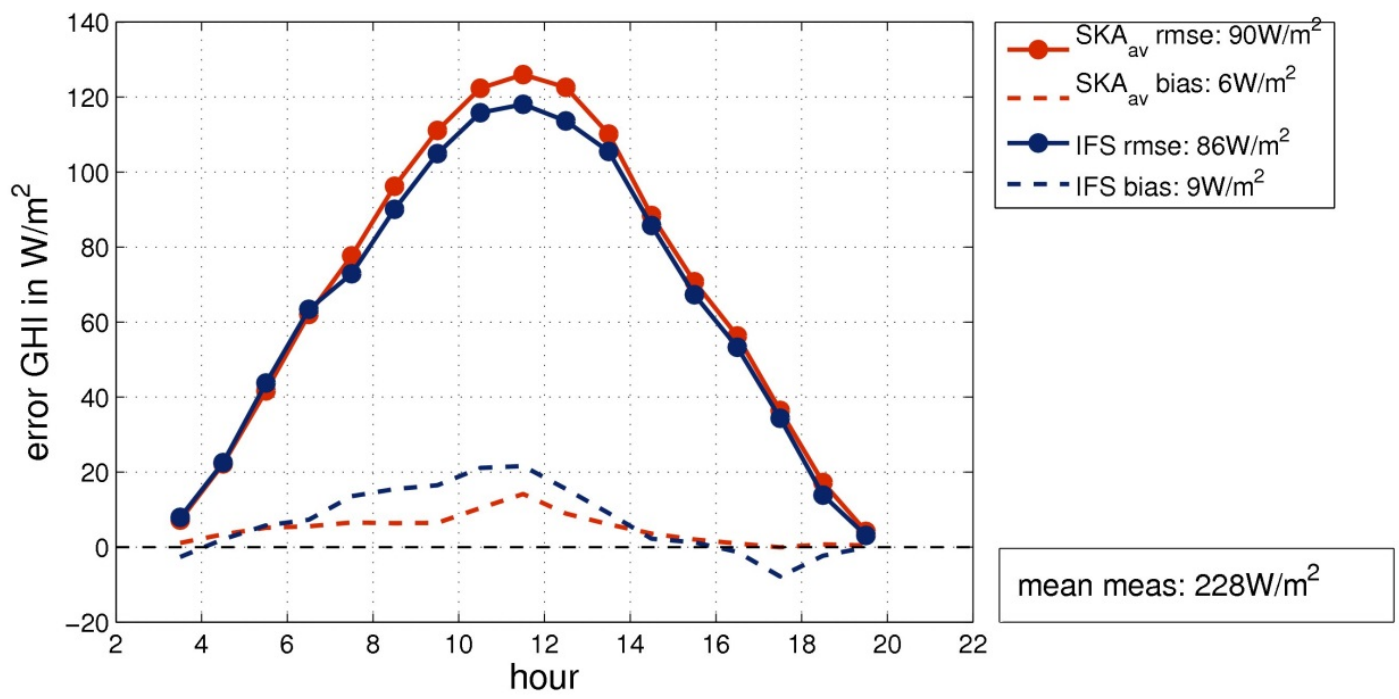

Figure 7-9. (Solid lines with dots) RMSE and (dashed lines) bias of (red) SKA av and (blue) IFS GHI forecasts through the time of the day. The data are from 18 German Meteorological Service sites, March 1, 2013-February 28, 2014.
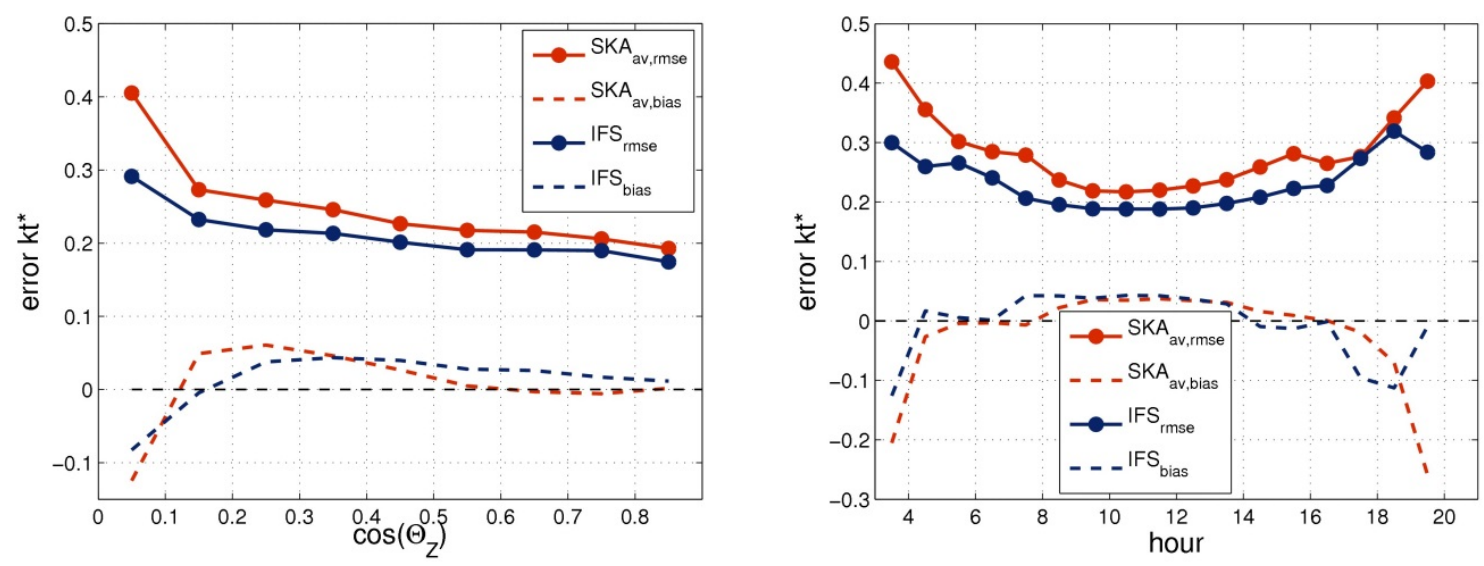

Figure 7-10. (Solid lines with dots) RMSE and (dashed lines) bias of (red) SKA av and (blue) IFS kt* forecasts through the (right) time of the day and (left) cosine of the SZA $\cos \Theta_{z}$. The data are from 18 German Meteorological Service sites, March 1, 2013-February 28, 2014.

Figure 7-10 shows that the RMSE of the clear-sky index kt* is increasing for morning and evening hours with low solar elevations, as also the magnitude of the bias. For low solar elevations, there is a high sensitivity to three-dimensional effects for broken clouds situations as well as high sensitivity to errors in the atmospheric turbidity (AOD; WV) for clear-sky modeling, both of which contribute to larger RMSE values; however, when considering overall 
error measures for GHI, the effect of the low accuracy of kt* for low solar elevations is mitigated by multiplication with small clear-sky irradiances.

Care has to be taken when applying statistical models to the clear-sky index kt* instead of GHI, which is potentially beneficial because the deterministic trend is reduced. A statistical adjustment for $\mathrm{kt}^{*}$ will give equal weight to errors in $\mathrm{kt}^{*}$ for all solar elevations. Therefore, it may adapt too much to the larger systematic errors of kt* for low solar elevations and not fully tap the potential for adjustments for higher solar elevations, which have a larger influence on irradiance forecast errors. A model to explicitly consider the dependence of the bias on the solar elevation is introduced in Section 7.3.7.
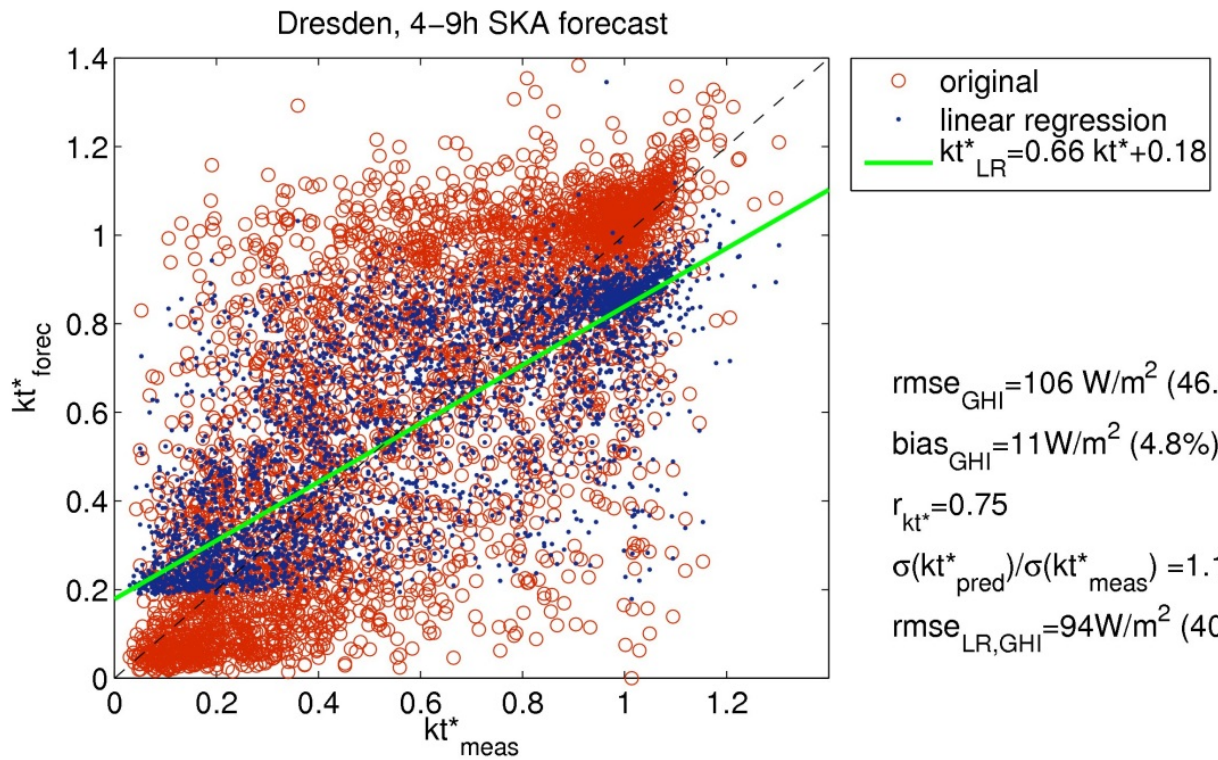

$$
\begin{aligned}
& \text { rmse }_{\mathrm{GHI}}=106 \mathrm{~W} / \mathrm{m}^{2}(46.0 \%) \\
& \text { bias }_{\mathrm{GHI}}=11 \mathrm{~W} / \mathrm{m}^{2}(4.8 \%) \\
& \mathrm{ktt}_{\mathrm{kt}^{\star}}=0.75 \\
& \sigma\left(\mathrm{kt}^{\star}{ }_{\text {pred }}\right) / \sigma\left(\mathrm{kt}^{\star}{ }_{\text {meas }}\right)=1.14 \\
& \text { rmse }_{\mathrm{LR}, \mathrm{GHI}}=94 \mathrm{~W} / \mathrm{m}^{2}(40.9 \%)
\end{aligned}
$$

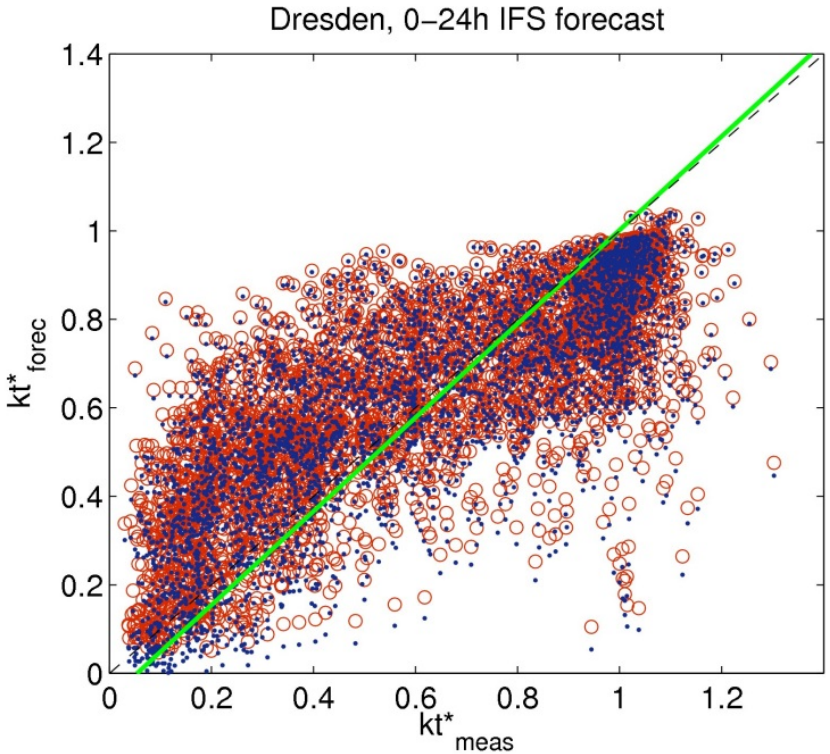

$$
\begin{aligned}
& \begin{array}{|ll|}
\hline \circ & \text { original } \\
\cdot & \text { linear regression } \\
& \mathrm{kt}^{*}{ }_{\mathrm{LR}}=1.06 \mathrm{kt}^{*}-0.06 \\
\hline
\end{array} \\
& \text { rmse }_{\mathrm{GHI}}=94 \mathrm{~W} / \mathrm{m}^{2}(41.0 \%) \\
& \text { bias }_{\mathrm{GHI}}=8 \mathrm{~W} / \mathrm{m}^{2}(3.5 \%) \\
& r_{k^{*}}=0.76 \\
& \sigma\left(\mathrm{kt}^{*}{ }_{\text {pred }}\right) / \sigma\left(\mathrm{kt}^{*}{ }_{\text {meas }}\right)=0.71 \\
& \mathrm{rmse}_{\mathrm{LR}, \mathrm{GHI}}=93 \mathrm{~W} / \mathrm{m}^{2} \text { (40.6\%) }
\end{aligned}
$$

Figure 7-11. Scatterplot of predicted over measured $\mathbf{k t}^{*}$ for (top) high-resolution SKA and (bottom) IFS forecasts. The original forecasts are shown in red, the forecast processed with the linear regression of $\mathbf{k t}^{*}$ is shown in blue, and the regression equation is visualized in green. The data are from Dresden, Germany, March 1, 2013-February 28, 2014; $\cos \left(\Theta_{\mathrm{z}}\right)>0.1$ for kt ${ }^{*}$ evaluation, $\cos \left(\Theta_{\mathrm{z}}\right)$ $>0.0$ for GHI RMSE and bias. 
Scatterplots and the effect of linear regression for the clear-sky index kt* of the SKA and IFS example forecasts are shown in Figure 7-11. Here, the analysis is limited to $\cos \left(\Theta_{Z}\right)>0.1$ to avoid overadaptation to large errors in low solar elevations. Correlation coefficients for $\mathrm{kt}^{*} \mathrm{with}_{\mathrm{kt}^{*}} \sim$ 0.75 are much smaller than for GHI with $\mathrm{r}_{\mathrm{GHI}} \sim 0.9$, because the deterministic trend caused by solar elevation is mostly removed and the amplitude of the predictions with linear regression of $\mathrm{kt}^{*}$ are adapted accordingly (Equation 7-8). Compared to the linear regression performed for GHI (Figure 7-8), a further reduction of the RMSE can be achieved for the SKA forecasts; whereas the impact for the IFS forecasts is still small.

\subsubsection{Persistence and Skill}

The comparison to a trivial reference model is a frequently applied check to assess the quality of a forecast. The most common reference model for short-term forecasts is persistence assuming that the current situation does not change and taking actual or recently measured values as forecast values.

For solar irradiance forecasting, the deterministic component of solar irradiance caused by the path of the sun should be considered as an additional constraint. For day-ahead forecasting, a simple approach reproducing the daily course of irradiance is persistence of the measured value of the previous day at the same time as a forecast value, which does not require any model for the daily irradiance pattern:

$$
\mathrm{GHI}_{\text {per }, 24 \mathrm{~h}}(\mathrm{t})=\mathrm{GHI}_{\text {meas }}(\mathrm{t}-24 \mathrm{~h})
$$

A more flexible option is using persistence of the clear-sky index $\mathrm{kt}^{*}$ meas in combination with a clear-sky model instead of persistence of irradiance values. For forecast horizons of several hours $(\Delta \mathrm{t})$ ahead, persistence $\mathrm{GHI}_{\mathrm{per}, \Delta \mathrm{t}}$ for the time $\mathrm{t}$ is then defined as:

$$
\mathrm{GHI}_{\text {per kt } * \Delta \mathrm{t}}(\mathrm{t})=\mathrm{GHI}_{\text {clear }}(\mathrm{t}) \mathrm{k}_{\text {meas }}^{*}(\mathrm{t}-\Delta \mathrm{t})
$$

Considering average values of the clear-sky index throughout a certain period instead of single value can additionally reduce the RMSE (for the effect of temporal averaging, see also Section 7.3.6). For day-ahead forecasting, persistence of the average clear-sky index of the previous day is a suitable reference model that yields considerably smaller errors than the simple approach $\mathrm{GHI}_{\text {per,24h }}$ described above (see Beyer et al. (2009).

Another common reference model is based on climatological mean values. An investigation for German weather stations (Beyer et al. 2009) showed a superior performance when using climatological mean values of kt* compared to persistence of the average daily clear-sky index from 2 days onward. This may be different for other climates; in any case, it is worthwhile to investigate the use of climatological mean values as a second reference model in addition to persistence.

The performance of the investigated forecast model compared to a reference model is evaluated with skill scores. They are defined as the difference of the scores for the model forecast and the reference forecast divided by the difference between the respective scores for a perfect forecast and the reference. For example, the RMSE skill score SS $_{\mathrm{RMSE}}$ is given as 


$$
\mathrm{SS}_{\mathrm{RMSE}}=\frac{\text { RMSE }_{\mathrm{ref}}-\mathrm{RMSE}_{\text {model }}}{\mathrm{RMSE}_{\mathrm{ref}}}
$$

where $\mathrm{RMSE}_{\text {ref }}$ refers to the reference model and $\mathrm{RMSE}_{\text {model }}$ to the investigated forecasting algorithm. Its value thus ranges between 1 (perfect forecast) and 0 (reference forecast). A negative value indicates performance worse than the reference. Skill scores may be applied only for comparisons to a simple reference model and also for intercomparisons to different forecasting approaches (improvement scores $[i s]$ ).

\subsubsection{Probability Density Function of the Clear-Sky Index}

Analyzing the ability of a forecast model to resemble observed probability density functions or frequency distributions of irradiance and clear-sky index can give useful information for model understanding.

Figure 7-12 shows the probability density function of the clear-sky index derived from measurements compared to the corresponding pdfs of SKA and IFS forecasts. The probability density function of the observations shows a clear peak for $\mathrm{kt}^{*}=1$, corresponding to clear-sky situations. This indicates that there is no systematic bias in the clear-sky model applied to derive $\mathrm{kt}^{*}$. The high-resolution SKA forecasts show too many very clear $\left(\mathrm{kt}^{*}>1\right)$ situations and those with very low clear-sky index because of too much cloud water in thick clouds; whereas intermediate cloud conditions are underrepresented. The opposite behavior is found for IFS forecasts. The 3-hour output time interval and averaging throughout 1 degree by 1 degree contribute to the larger share of intermediate $\mathrm{kt}^{*}$ values. The clear-sky "peak" near the kt* values of $0.9-0.95$ for the IFS forecasts indicates an underestimation of the irradiance for clear-sky conditions.

Although it gives insight to model performance, similarity of the distribution functions of measurements and forecasts is not a sufficient forecast, because it does not include any information about the correct timing of the modeled events, which is essential for forecasting. For example, persistence will result in a probability density function very similar to the observed probability density function (see also Perez et al. 2013), but with higher RMSE values than the investigated SKA and IFS forecasts. These yield reasonable accuracies despite the considerable deviations of the probability density function of the clear-sky index compared to the observed probability density function.

A quantitative evaluation of the agreement between the observed and forecasted distribution functions can be done using the Kolmogorov-Smirnoff integral (Espinar et al. 2008). It is usually applied to distribution functions of GHI rather than to kt* (Beyer et al. 2009, Perez et al. 2013). 


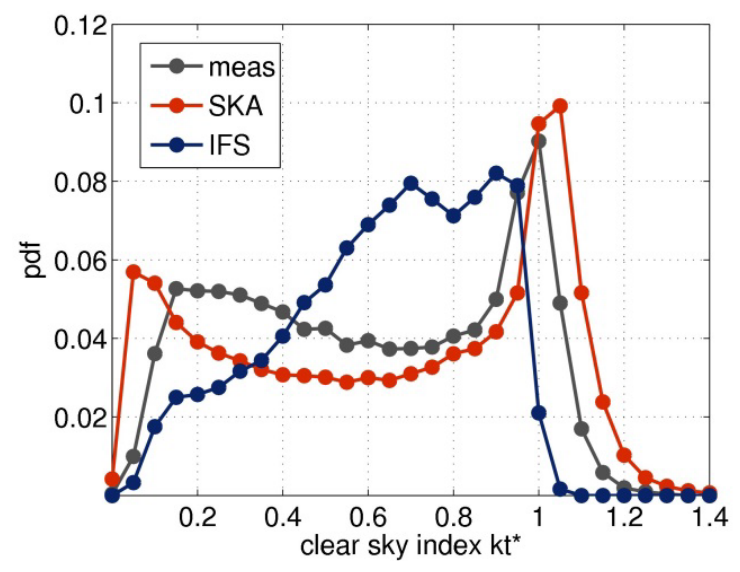

Figure 7-12. Probability density function of the clear-sky index derived from (gray) measurements, (red) high-resolution SKA forecasts, and (blue) IFS forecasts. The data are from 18 German Meteorological Service sites, March 1, 2013-February 28, 2014; $\cos \left(\Theta_{\mathrm{Z}}\right)>0.1$.

\subsubsection{Evaluation in Dependence on Cloud Variability and Spatial and Temporal Averaging}

Cloud variability has a strong impact on forecast accuracy. An evaluation of the SKA forecasts in dependence on the variability of the clear-sky index of the measurements, here represented by the standard deviation of kt* ${ }_{\text {meas }}$ throughout 5 hours, shows increasing RMSE values with increasing variability (Figure 7-14). This is also illustrated in Figure 7-13. The forecasts show a good agreement with measurements for the clear-sky day (Figure 7-13, left) with basically constant clear-sky index and considerable deviations from the measurements for the day with variable clouds (Figure 7-13, right).

The impact of linear regression in dependence on cloud variability is analyzed in Figure 7-14, left. The reduction of fluctuations with linear regression is beneficial in variable situations with low correlation, but it has a negative impact in situations with low variability. In particular, for clear-sky situations, the reduction of clear-sky index to values below 1 by linear regression (Figures 7-8 and 7-11) leads to an underestimation of the irradiances. 

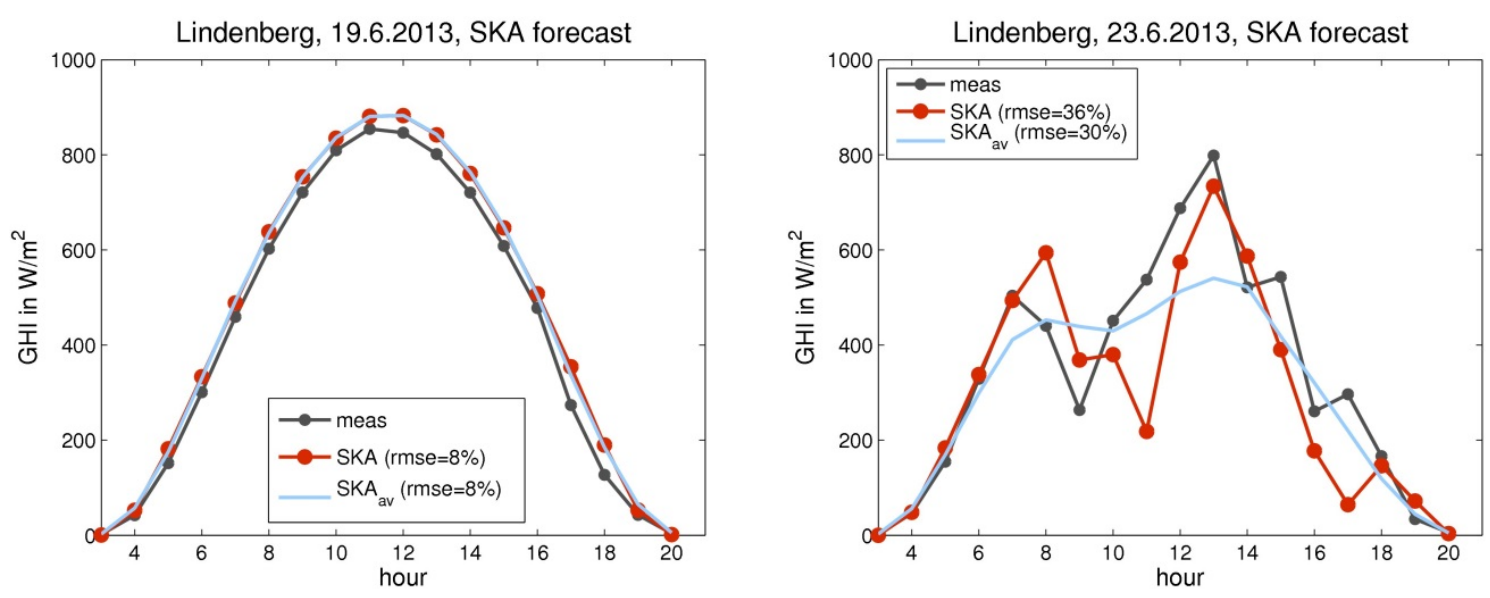

Figure 7-13. Example days comparing measurements to SKA forecasts with different spatial and temporal averaging-(red) SKA: nearest grid point with hourly resolution; (light blue) SKA av: 5hour moving average of clear-sky index of the average throughout 20-by-20 grid points. (Left) Clear-sky; data from Lindenberg, Germany, June 19, 2013; and (right) variable cloud conditions; data from Lindenberg, Germany, March 23, 2013.

The application of smoothing filters or spatial and temporal averaging is a possibility to reduce fluctuations in variable situations and to preserve the quality of the original forecasts in homogeneous clear-sky and overcast situations. An additional advantage of this approach is that no additional measurement data are required for the adaptation. Figure 7-13, right, and Figure 714 , right, show clearly smaller RMSE values of the averaged forecasts compared to the original forecast for variable situations and even a small improvement for low-variability situations. The optimum region size and time interval for averaging depends on the correlation between forecast to measurements. They have not been systematically optimized for the example shown here to illustrate the principle benefit of spatial as well as temporal averaging. Combining the first averaging and second linear regression can further reduce forecast errors (see Figure 7-14, right). 

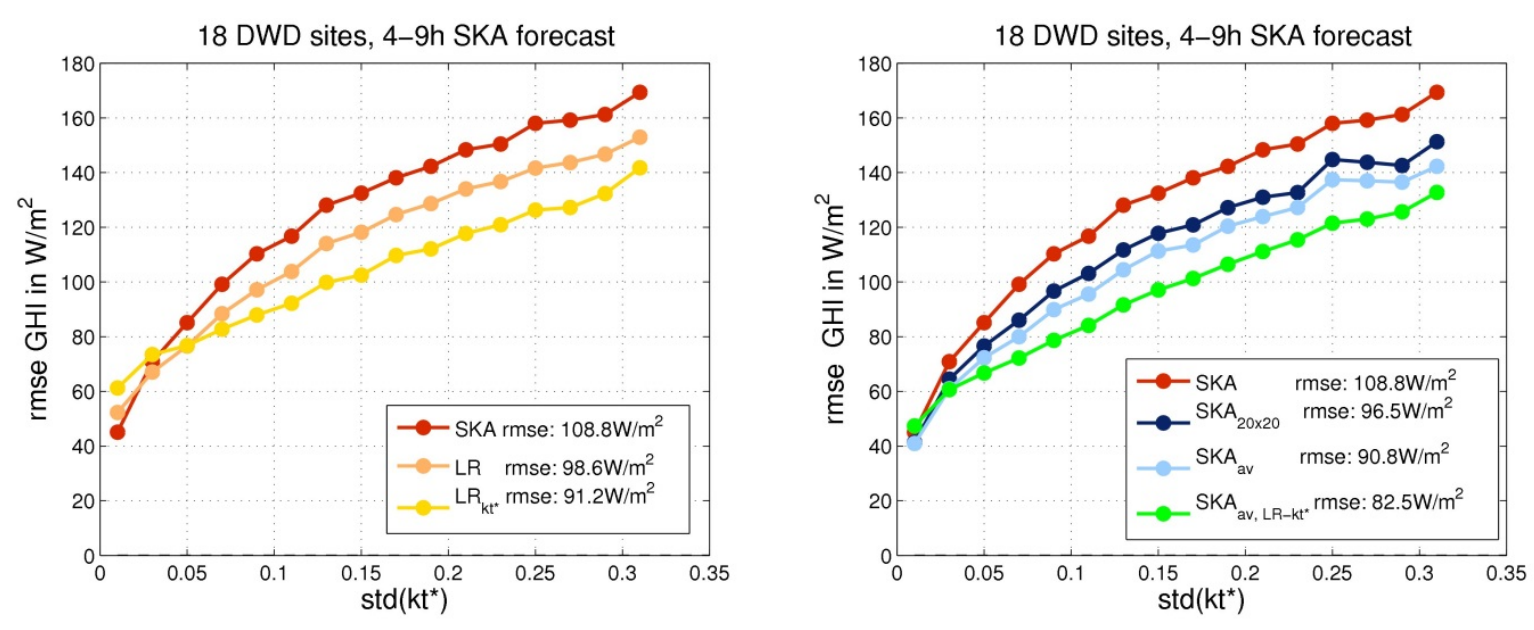

Figure 7-14. RMSE in dependence of the standard deviation of $\mathbf{k t}^{*}{ }_{\text {meas }}$ (throughout 5 hours) for (left) SKA forecasts with the application of linear regression ([red] SKA: nearest grid point, [orange] LR: linear regression for GHI, and [yellow] LR-kt* for linear regression for kt*) and (right) with different spatial and temporal averaging ([red] SKA: nearest grid point, [dark blue] SKA $\mathbf{A}_{20 \times 20}$ averaged throughout 20-by-20 grid points, [light blue] SKA ${ }_{a v}$ 5-hour gliding mean of clear-sky index of the average throughout 20-by-20 grid points, and [green] SKA ${ }_{\mathrm{av}}$, LR.kt: linear regression of $\mathrm{kt}^{\star}$ applied to $\mathrm{SKA}_{\mathrm{AV}}$ ). The data is from 18 German Meteorological Service sites, April 3, 2013February 28, 2014; training set: last 30 days, all sites.

However, Figure 7-13, right, also shows that potentially useful information on cloud variability is lost when looking at average values. The SKA forecasts give a reasonable representation of the hour-to-hour variability, which is not the case anymore for the average forecasts. Mathiesen et al. (2013) suggest providing forecasts of cloud variability as an additional parameter, complementing spatially and/or temporally averaged irradiance values yielding better RMSE scores.

Spatial averaging has the greatest impact in NWP models with high temporal and spatial resolutions. A detailed evaluation of irradiance forecasts from the Canadian GEM model resulted in a reduction of forecast errors by $10 \%$ to $15 \%$ when averaged throughout several hundred kilometers (Pelland et al. 2011). A similar improvement is achieved for WRF forecasts provided by Meteotest as average values of 10-by-10 model pixels, corresponding to an area of $50 \mathrm{~km}$ by $50 \mathrm{~km}$ (Mueller and Remund 2012). Mathiesen and Kleissl (2011) report $100 \mathrm{~km}$ by100 km as a suitable averaging area for irradiance forecasts of the GFS model and the NAM model, which is also WRF based. The benefit of horizon-dependent smoothing filters for CMV forecasts is shown in Lorenz et al. (2004) and Kuehnert et al. (2013).

\subsubsection{Evaluation and Bias Correction in Dependence on Sun Elevation and Clear- Sky Index}

Systematic deviations of NWP output variables often depend on the meteorological situation as well as on the solar elevation (see also Section 7.3.3). An analysis of the forecast bias of the two investigated models in dependence on the predicted clear-sky index $\left(\mathrm{k}^{*}\right.$ pred $)$ and the cosine of the SZA $\left(\cos \theta_{z}\right)$ is given in Figure 7-15. The (left) IFS forecasts show a clearly nonlinear dependence of the bias of the predicted cloud conditions with a considerable overestimation of the irradiance for intermediate cloud situations $\left(0.3<\mathrm{kt}^{*}{ }_{\text {pred }}<0.8\right)$. (Figure $7-11$, bottom, shows a 
similar result.) The averaged $\mathrm{SKA}_{\mathrm{av}}$ forecasts overestimate for situations predicted as clear-sky and underestimate in situations predicted as overcast. This is similar to the behavior found for the original SKA forecasts in the scatterplot shown in Figure 7-11 (top). Also the dependence of the bias on the solar elevation is clearly shown in Figure 7-15. The magnitude and the patters of the bias over the clear-sky index are changing with $\cos \left(\Theta_{\mathrm{Z}}\right)$.
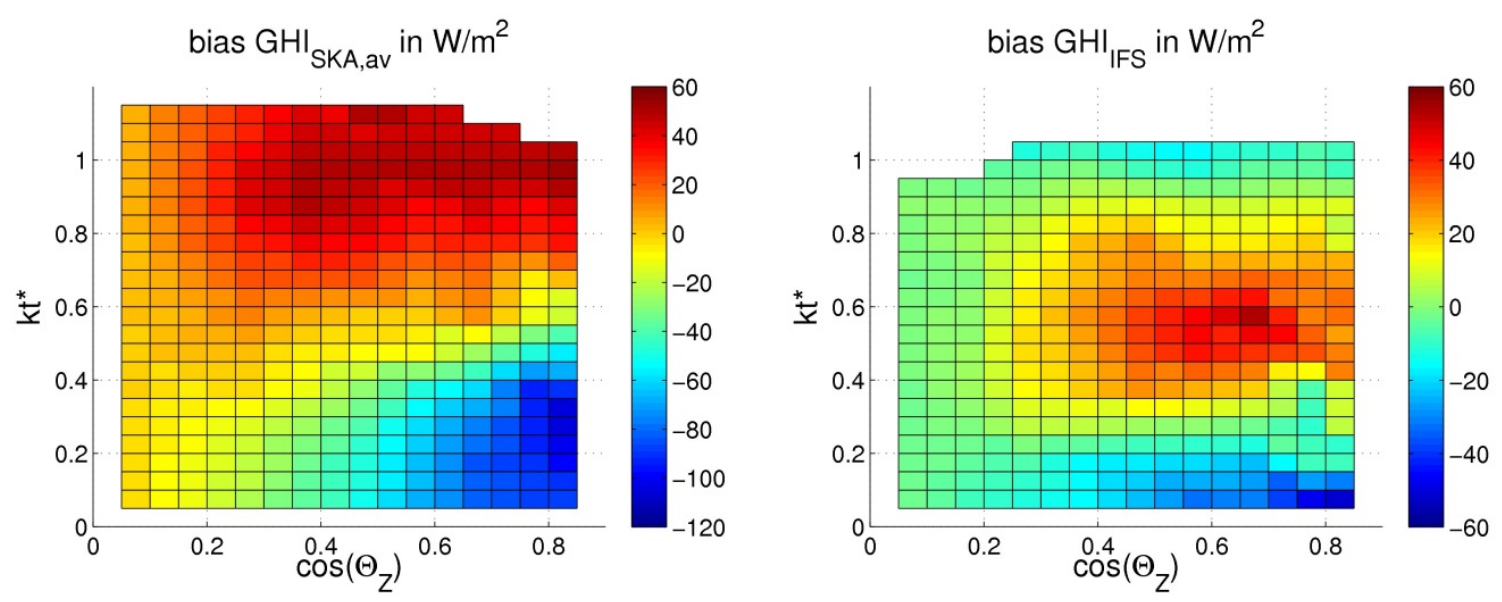

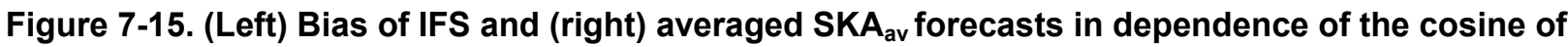
the SZA and the predicted clear-sky index kt*. The data are from 18 German Meteorological Service sites, March 3, 2013-February 28, 2014.

The given analysis can be used as a basis for a weather-dependent bias correction (Lorenz et al. 2009). To avoid the systematic deviations in dependence on the forecasted cloud conditions, first the bias is modeled as a $4^{\text {th }}$ degree polynomial function of the predicted clear-sky index kt* and the cosine of the SZA $\left(\theta_{z}\right)$. The corrected forecasts are then obtained by subtracting the modeled bias $\left(\mathrm{kt}^{*}, \theta_{\mathrm{z}}\right)$ from the original predicted values. Training of the fit functions is performed with a sliding window technique using measurements of the previous 30 days of weather stations in the region of interest.

This approach has been adapted and evaluated also for other NWP models and different climates. Mathiesen and Kleissl (2011) found improved accuracies when applying it to three different NWP models - GFS, North American Model, and IFS - for stations in the continental United States, as did Pelland et al. (2011) for their application to the Canadian GEM model, and Mueller and Remund (2011) for WRF forecasts for Switzerland.

Figure 7-16 shows the RMSE of the two forecast models in dependence on the two parameters $\mathrm{kt}^{*}{ }_{\text {pred }}$ and $\cos \theta \mathrm{z}$. For the IFS model, forecast errors are comparatively small for situations predicted as clear sky $\left(\mathrm{kt}^{*}{ }_{\text {pred }} \approx 1\right)$ or overcast $\left(\mathrm{kt}^{*}{ }_{\text {pred }}<0.2\right)$. The largest deviations among measurements and forecasts are found for situations with variable cloud cover, corresponding to intermediate values of the clear-sky index of the IFS forecasts. These patterns are similar for the averaged $\mathrm{SKA}_{\mathrm{av}}$ forecasts, but because of the higher variability in the forecasts, high RMSE values are found for a wider range of clear-sky index values. The knowledge of the situationspecific RMSE based on predicted conditions can provide a basis for the estimation of weatherspecific uncertainty information (see Lorenz et al. 2009). 

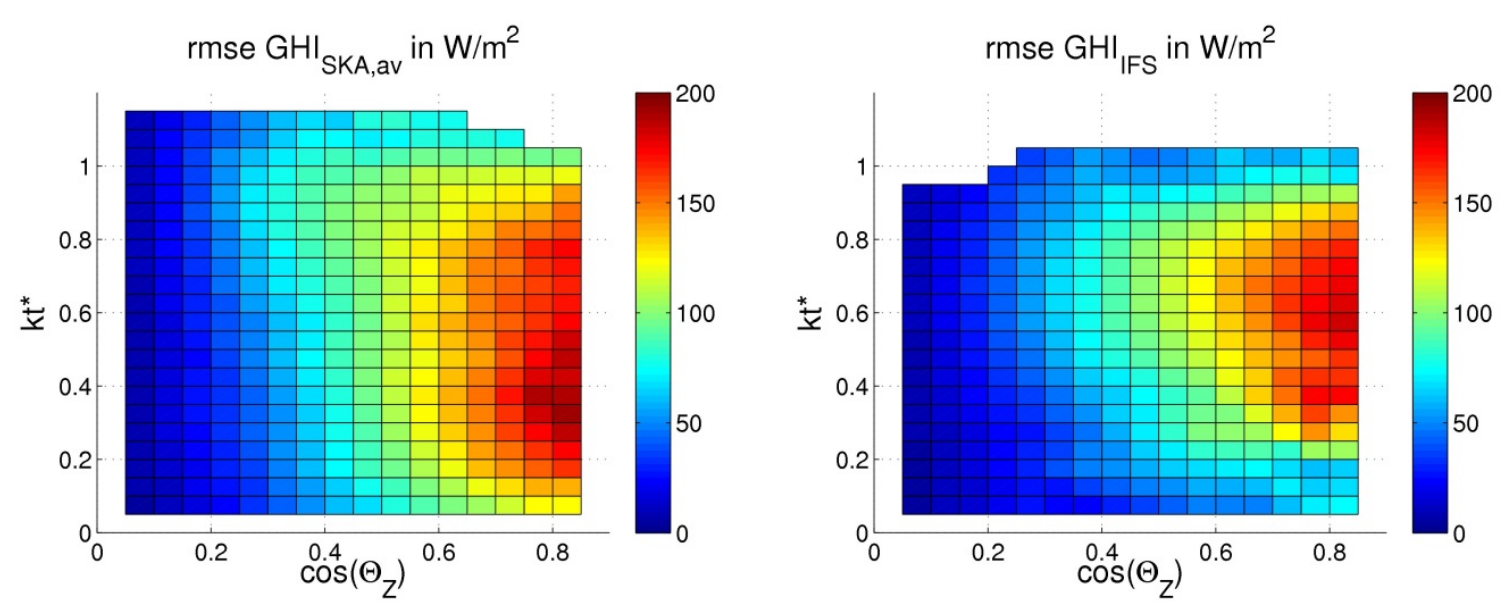

Figure 7-16. (Left) RMSE of IFS and (right) averaged SKA forecasts in dependence of the cosine of the SZA and the predicted clear-sky index kt*. The data are from 18 German Meteorological Service sites, March 3, 2013-February 28, 2014.

\subsubsection{Comparison of Different Post-Processing Approaches to Site-Specific and Regional Forecasting}

The impact of the different post-processing approaches presented in the previous sections is compared to site-specific and regional forecasts here. Regional forecasts, important for utility applications, are calculated as the average of the considered 18 German Meteorological Service sites.

The results of this evaluation, summarized in Figure 7-17, are shortly discussed in the following:

- RMSE values of the regional forecasts are approximately half the errors of single-site forecasts for the data set investigated here. Because of spatial averaging effects, the course of average irradiance forecasts and measurements is smoother than in single sites, which shows in particular a high variability for partly cloudy conditions (Figure 7-13, right). The deviations among forecasts and measurements are smaller for the regional average values, because forecast errors partly compensate each other. The reduction of errors when considering a set of stations instead of a single station is determined by the cross-correlation of forecast errors between the sites, depending on their distance from each other (Lorenz et al. 2009), as well as on the forecast model. An analysis of regional forecast errors with a larger number of sites for different region sizes and different forecast models is given in Lorenz et al. (2009), Kuehnert et al. (2013), and Lorenz et al. (2012).

- For single-site forecasts, there is a considerable improvement of $16.4 \%$ (is $\left.\mathrm{RMSE}=\left(\mathrm{RMSE}_{\mathrm{av}}-\mathrm{RMSE}_{\text {highres }}\right) / \mathrm{RMSE}_{\text {highres }}\right)$; see also Equation 7-13) with spatial and temporal averaging for the hourly, high-resolution SKA forecasts, as outlined in more detail before. As expected, averaging has less impact on forecast performance when considering regional forecasts (improvement: 5.4\%).

- Statistical post-processing with measurements applied to the average SKA forecasts further reduces RMSE values. Linear regression based on $\mathrm{kt}$ * performs better than linear regression directly applied to GHI, with a more pronounced difference for regional 
forecasts. Best results are achieved with the bias correction in dependence of the solar elevation and clear-sky index, although the improvement over linear regression with kt* is only small ( $1.8 \%$ for regional forecasts). Overall, with this approach the improvement compared to the average SKA forecasts amounts to $9.5 \%$ for single sites and of $21.4 \%$ for regional averages. For regional forecasts, there is a much larger impact of systematic deviations than for single-site forecasts with larger RMSE values.
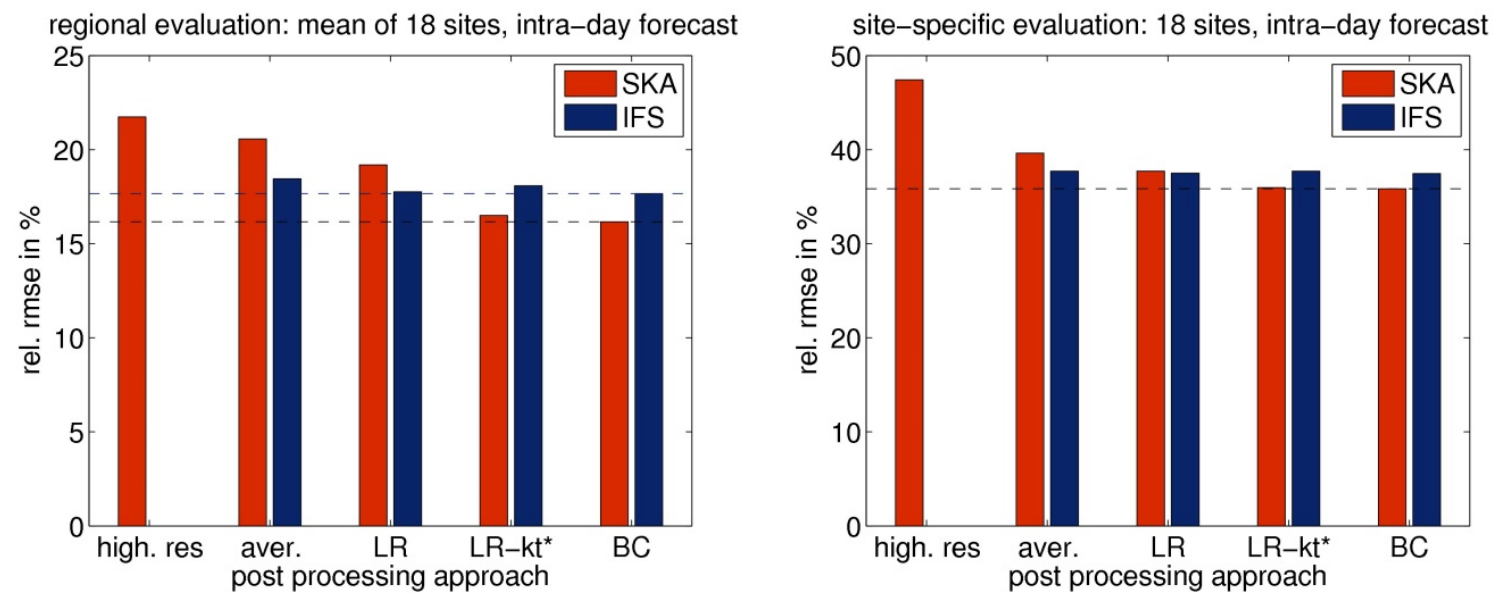

Figure 7-17. RMSE of IFS and SKA GHI forecasts with different post-processing approaches for (left) single site forecasts and (right) regional forecasts, derived as mean value of all sites. The data are from 18 German Meteorological Service sites, April 3, 2013-February 28, 2014; training set: last 30 days, all sites.

- The comparison of irradiance forecasts of the high-resolution regional SKA model to the IFS forecasts based on 3-hour output and averaged throughout $100 \mathrm{~km}$ by $100 \mathrm{~km}$ reveals smaller RMSE values of the IFS forecasts as long as no statistical post-processing is involved and when compared to the averaged $\mathrm{SKA}_{\mathrm{av}}$ forecasts. When applying the bias correction in dependence of the solar elevation and clear-sky index, SKA $\mathrm{BC}_{\mathrm{BC}}$ forecasts show smaller RMSE values than the IFS $\mathrm{B}_{\mathrm{BC}}$ forecast with the same post processing. For IFS forecasts, the impact on the investigated post-processing schemes is only small, with an improvement of $7.8 \%$ for regional forecasts using the bias correction. 


\section{Future Work}

Advancing renewable energy technologies will require improvements to our understanding of solar radiation resources. This chapter briefly describes the areas of research and development identified by NREL as emerging technology needs.

\subsection{Forecasting Solar Radiation}

Solar power forecasting will be an essential component of the future energy supply system using large amounts of fluctuating solar power. Already today, power forecasting systems contribute to the successful grid integration of considerable amounts of solar power. As a consequence of the need for precise and detailed forecast data in the energy sector, increasing research is underway on the forecasting of solar irradiance and solar electricity generation.

Current research in irradiance and solar power forecasting covers all the different approaches presented. Improvements in NWP-based irradiance forecasting may be expected as these models continue to develop with respect to resolution, data assimilation, and parameterizations of clouds and radiation. In particular, the development and application of rapid-update-cycle models has a high potential to improve intraday forecasting. Forecasting techniques based on cloud motion will benefit from enhancements in cloud detection approaches for both satellite-based and skyimager-based methods. With respect to statistical methods, apart from model development, the availability of high-quality and up-to-date measurement data of solar irradiance and solar power will be of critical importance. Finally, an optimized combination of different physical and statistical models will be of advantage for any solar power prediction system.

Specification of the expected uncertainty of solar irradiance or power predictions for different weather situations gives valuable additional information to forecast users and is more and more requested by them. Forecast uncertainty can be quantified with confidence intervals or in the form of fully probabilistic forecasts. Probabilistic forecasting may be based on the use of NWP ensemble prediction systems as well as on the statistical analysis of the distributions of historic predictions and measurements. As in deterministic forecasting, ultimately the combination of physical and statistical methods will lead to the best results. Nevertheless, a similar activity for direct irradiance forecast verification is still missing.

Finally, a proper accuracy assessment is essential for further model development as well as for users that rely on forecasts as a basis for decision making. The work performed in the IEA SHC's Task 36 to establish a transparent framework for forecast evaluation is currently continued and extended in the IEA SHC Task 46. This includes the investigation of new evaluation measures and concepts - for example, to assess variability information about different spatial and temporal scales as well as NWP and CMV model intercomparison studies for different climatic regions.

\subsection{High-Resolution Temporal Data}

Electrical generation by solar thermal electric power systems is directly proportional to the available DNI. Historical solar resource data are typically available for hourly time intervals. More subhourly time-series data (15 minutes or less) with dense spatial coverage (e.g., $5 \mathrm{~km}$ or less) are needed to address load-following simulations and related economic considerations. A first such database is now available as MACC-RAD and is described in Chapter 4 and Chapter 5. 
Currently available instrumentation and measurement equipment can acquire solar irradiance data in intervals as often as 1 second (Wilcox and Myers 2008). Research is underway to deploy solar resource measurement stations that will provide high-resolution data at single locations and within the collector fields.

\subsection{Site-Specific Resource Data}

Characterizing the spatial variability of solar irradiance throughout distances of $1 \mathrm{~km}$ or less is important for improving the siting, design, and performance monitoring of a solar energy conversion system. The 1991-2010 NSRDB update, along with the 1961-1990 update, provides historical solar irradiance data for specific locations from 1961 through 2010, with a resulting spatial scale of approximately $100 \mathrm{~km}$. Additionally, the satellite derived and physics-based NSRDB 2014 update provides subhourly solar irradiance data for 4-km grid cells from 2005 through 2012. Methods for increasing the spatial resolution of satellite-based models for estimating solar irradiance at the surface are under development.

Because the maintenance of meteorological ground stations is often a fundamental problem for collecting high-quality data, instruments that require less maintenance than those described in ISO 9060 are of interest. Such sensors must be standardized to increase their applicability to solar resource assessments.

Data from large solar energy plants and from the corresponding resource assessment that may have high-quality radiometric instrumentation need to be systematically investigated.

\subsection{Additional Measurands}

Although DNI, GHI, or POA are the most important meteorological input parameters for the prediction of the solar power plant yield, several additional parameters must also be provided for accurate yield analysis. This involves familiar parameters such as wind speed, ambient temperature, humidity, and pressure as well as solar energy-specific measurands. Examples of such parameters are soiling, the sunshape/circumsolar radiation, and the extinction of radiation between the mirror and the receivers. Often, no site-specific information on these properties is available, which results in remarkable impacts on the accuracy of the yield prediction. Hence, these parameters have to be studied in more detail in the future.

\subsection{Effects of Climate Change on Solar Resource Assessments}

It is not yet clear what impact changes in atmospheric aerosol loading from natural causes or industrial pollution, changing patterns of precipitation and cloudiness, temperature extremes, and other climatic variables will have on the more recent solar resource data when estimating the performance of a CSP plant throughout the system design life (e.g., 25 years). Research is needed to advance climate modeling capabilities and merge the output with advanced system performance models.

\subsection{Need for Cross-Disciplinary Analysis Projects}

The use of solar resource and meteorological data to address complex problems such as timedependent utility load estimations, cloud transient effects on grid stability, and solar generation dispatching requires close collaboration among analysts, utility planners, and the resource and meteorology communities. Shared knowledge will advance the identification of resource data needs and the development of methods for improved resource data and information to meet those needs. 


\section{References}

\section{Chapter 2: Overview of Solar Radiation Resource Concepts}

ASTM. (2006). E490, 2000a: Standard Solar Constant and Zero Air Mass Solar Spectral Irradiance Tables. West Conshohocken, PA: ASTM International.

ASTM. (2006). G173-03: Standard Tables for Reference Solar Spectral Irradiances-Direct Normal and Hemispherical on $37^{\circ}$ Tilted Surface. West Conshohocken, PA: ASTM

International; 21 pp.

Badescu, V., ed. (2008). Modeling Solar Radiation at the Earth's Surface. Berlin, Germany: Springer.

Barnett, T.P.; Ritchie, J.; Foat, J.; Stokes, G. (1998). "On the Space-Time Scales of the Surface Solar Radiation Field.” Journal of Climate (11); pp. 88-96.

BIPM; IEC; IFCC; ISO; IUPAP; OIML. (1995). Guide to the Expression of Uncertainty in Measurement. Geneva: ISO TAG4.

Blanc, P., Espinar, B., Geuder, N., Gueymard, C., Meyer, R., Pitz-Paal, R., Reinhardt, B., Renné, D., Sengupta, M., Wald, L., Wilbert, S. (2014). "Direct Normal Irradiance Related Definitions and Applications: The Circumsolar Issue." Solar Energy 110(0); pp. 561-577.

http://dx.doi.org/10.1016/j.solener.2014.10.001.

Buie, D.; Monger, A.G. (2001). "The Effect of Circumsolar Radiation on Solar Concentrating Systems." The International Solar Energy Society's Solar World Congress Proceedings; Adelaide, Australia.

De Toma, G.; White, O.R.; Chapman, G.A.; Walton, S.R. (2004). "Solar Irradiance Variability: Progress in Measurement and Empirical Analysis." Advances in Space Research (34); pp. 237242.

Deepak, A.; Adams, R.R. (1983). "Photography and Photographic-Photometry of the Solar Aureole." Applied Optics (22:11); pp. 1,646-1,654.

Erbs, D.G.; Klein, S.A.; Duffie, J.A. (1982). "Estimation of the Diffuse Radiation Fraction for Hourly, Daily, and Monthly Average Solar Radiation.” Solar Energy (28:4); pp. 293-304.

Evans, D.; Grether, D.; Hunt, A.; Wahlig, M. (1980). "Spectral Character of Solar and Circumsolar Radiation." Presented at the 25th Annual Meeting of the American Section of the International Solar Energy Society, Phoenix, Arizona.

Grether, D.; Nelson, J.; Wahlig, M. (1975). "Measurement of Circumsolar Radiation." Proceedings of the Society of Photo-Optical Instrumentation Engineers, vol. 68: Solar Energy Utilization. Bellingham, WA: Society of Photo-Optical Instrumentation Engineers.

Gueymard C.A.; Ruiz-Arias J.A. (2014). "Performance of Separation Models to Predict Direct Irradiance at High Frequency: Validation over Arid Areas." EuroSun 2014 ISES Conference 
Proceedings; Aix-les-Bans, France.Hay, J.E. (1979). Study of Shortwave Radiation on Nonhorizontal Surfaces: Report. 79-12. Downsview, Ontario: Atmospheric Environment Service.

Gueymard, C. (2008). "Prediction and Validation of Cloudless Shortwave Solar Spectra Incident on Horizontal, Tilted, or Tracking Surfaces." Solar Energy (82); pp. 260-271.

Gueymard, C.A. (1988). "An Anisotropic Solar Irradiance Model for Tilted Surfaces and Its Comparison with Selected Engineering Algorithms.” Solar Energy (40); pp. 175.

Gueymard, C.A. (2001). "Parameterized Transmittance Model for Direct Beam and Circumsolar Spectral Irradiance.” Solar Energy (71:5); pp. 325-346.

Iqbal, M. (1983). An Introduction to Solar Radiation. New York: Academic Press.

Kalapatapu, R.; Chiesa, M.; Armstrong, P.; Wilbert, S. (2012). "Measurement of Dni Angular Distribution with a Sunshape Profiling Irradiometer." SolarPACES Conference Proceedings; September 11-14, 2012, Marrakesh, Morocco.

Klucher, T.M. (1979). "Evaluation of Models to Predict Insolation on Tilted Surfaces." Solar Energy (23); pp. 111-114.

Liu, B.Y.H.; Jordan, R.C. (1960). "The Interrelationship and Characteristic Distribution of Direct Diffuse and Total Solar Radiation." Solar Energy (3); pp. 1-19.

Liu, B.Y.H.; Jordan, R.C. (1961). "Daily Insolation on Surfaces Tilted Towards Equator." ASHRAE Transactions (67); pp. 526-541.

Major, G. (1992). "Estimation of the Error Caused by the Circumsolar Radiation when Measuring Global Radiation as a Sum of Direct and Diffuse Radiation." Solar Energy (48:4); pp. 249-252.

Major, G. (1994). Circumsolar Correction for Pyrheliometers and Diffusometers: WMO-TD 635. Geneva, Switzerland: World Meteorological Organization.

Marion, W.; Riordan, C.; Renné, D. (1992). Shining On: A Primer on Solar Radiation Data. NREL/TP-463-4856. Golden, CO: National Renewable Energy Laboratory.

Maxwell, E. (1987). A Quasi-Physical Model for Converting Hourly Global Horizontal to Direct Normal Insolation. Golden, CO: Solar Energy Research Institute. Accessed November 10, 2014 : http://rredc.nrel.gov/solar/pubs/PDFs/TR-215-3087.pdf.

Mayer, B. (2009). "Radiative Transfer in the Cloudy Atmosphere." European Physics Journal Conferences; pp. 75-99.

Mayer, B.; Kylling, A. (2005). "Technical Note: The libRadtran Software Package for Radiative Transfer Calculations: Description and Examples of Use." Atmospheric Chemistry and Physics Discussions (5:2); pp. 1,855-1,877. 
Mie, G. (1908). "Beitrage zur Optiktrüber Medien Speziell Kolloidaler Metalosungen.” Annalen der Physik, Vierte Folge (25:3); pp. 377-445.

Mueller, G. (1897). Die Photometrie der Gestirne. Leipzig, Germany: Wilhelm Engelmann.

Myers, D.R. (2011). "Quantitative Analysis of Spectral Impacts on Silicon Photodiode Radiometers.” Solar 2011 Conference Proceedings; May 17-21, 2011, Raleigh, North Carolina. NREL/CP-5500-50936. Golden, CO: National Renewable Energy Laboratory.

Neumann, A.; Witzke, A.; Jones, S.A.; Schmitt, G. (2002). "Representative Terrestrial Solar Brightness Profiles.” Journal of Solar Energy Engineering (124:2); pp. 198-204.

Noring, J.E.; Grether, D.F.; Hunt, A.J. (1991). Circumsolar Radiation Data: The Lawrence Berkeley Laboratory Reduced Data Base. NREL/TP-262-4429. Golden, CO: National Renewable Energy Laboratory.

Orgill, J.F.; Hollands, K.G. (1977). "Correlation Equation for Hourly Diffuse Radiation on a Horizontal Surface.” Solar Energy (19:4); pp. 357-359.

Pastiels, R. (1959). Contribution à l'Étude du Problème des Méthodes Actinométriques. Brussels, Belgium : Institut Royal Météorologique de Belgique.

Perez, R.; Seals, R.; Ineichen, P.; Stewart, R.; Menicucci, D. (1987). “A New Simplified Version of the Perez Diffuse Irradiance Model for Tilted Surfaces.” Solar Energy (39:3); pp. 221-231.

Perez, R.; Seals, R.; Zelenka, A.; Ineichen, P. (1990). "Climatic Evaluation of Models That Predict Hourly Direct Irradiance from Hourly Global Irradiance: Prospects for Performance Improvements." Solar Energy (44:2); pp. 99-108.

Rabl, A.; Bendt, P. (1982). "Effect of Circumsolar Radiation on Performance of Focusing Collectors.” Journal of Solar Energy Engineering (104:3); pp. 237-250.

Rayleigh, L. (1871). "On the Light from the Sky, Its Polarization, and Colour.” Philosophical Magazine; pp. 107-120, 274-279.

Reindl, D.T., et al. (1990). "Evaluation of Hourly Tilted Surface Radiation Models.” Solar Energy (45); pp. 9-17.

Temps, R.C.; Coulson, K.L. (1977). "Solar Radiation Incident Up On Slopes of Different Orientations.” Solar Energy (19); pp. 179-184.

Watt, A. (1980). Circumsolar Radiation. SAND-80-7009. Cedaredge, CO: Watt Engineering Ltd.

Wilbert, S. (2014). Determination of Circumsolar Radiation and Its Effect on Concentrating Solar Power. Ph.D. Thesis. Aachen, Germany: Rheinisch-Westfälische Technische Hochschule Aachen University. urn:nbn:de:hbz:82-opus-51715. Accessed November 11, 2014: http://darwin.bth.rwth-aachen.de/opus3/volltexte/2014/5171/. 
Wilbert, S.; Pitz-Paal, R.; Jaus, J. (2012). "Circumsolar Radiation and Beam Irradiance Measurements for Focusing Collectors.” COST WIRE Workshop Proceedings; May 22-23, 2012, Riso, Denmark.

Wilbert, S.; Pitz-Paal, R.; Jaus, J. (2013). "Comparison of Measurement Techniques for the Determination of Circumsolar Irradiance." American Institute of Physics Conference Proceedings (1556:1); April 15-17, 2013, Miyazaki, Japan, pp. 162-67.

Wilcox, S.; Anderberg, M.; Beckman, W.; DeGaetano, A.; George, R.; Gueymard, C.; Lott, N.; Marion, W.; Myers, D.; Perez, R.; Renné, D.; Stackhouse, P.; Vignola, F.; Whitehurst, T. (2007). National Solar Radiation Database 1991-2005 Update: User's Manual. NREL/TP-581-41364, Golden, CO: National Renewable Energy Laboratory.

Wilcox, S.; Gueymard, C.A. (2010). "Spatial and Temporal Variability of the Solar Resource in the United States.” Solar 2010 Conference Proceedings; May 17-22, 2010, Phoenix, Arizona.

Willmott, C.J. (1982). "On the Climatic Optimization of the Tilt and Azimuth of Flat-Plate Solar Collectors.” Solar Energy (28); pp. 205-216.

WMO. (2008). WMO Guide to Meteorological Instruments and Methods of Observation: WMO No. 8. 7 th edition. Geneva, Switzerland. http://www.wmo.int/pages/prog/gcos/documents/gruanmanuals/CIMO/CIMO_Guide-

7th_Edition-2008.pdf.

Young, A.T. (1981). "On the Rayleigh-Scattering Optical Depth of the Atmosphere." Journal of Applied Meteorology and Climatology (20); pp. 328-330.

http://dx.doi.org/10.1175/1520-0450(1981)020<0328:OTRSOD>2.0.CO;2.

\section{Chapter 3: Measuring Solar Radiation}

Alados-Arboledas, L.; Batlles, F.J.; Olmo, F.J. (1995). "Solar Radiation Resource Assessment by Means of Silicon Cells." Solar Energy (54:3); pp. 183-191.

ASTM (2005). E816-05: Standard Test Method for Calibration of Pyrheliometers by Comparison to Reference Pyrheliometers. West Conshohocken, PA: ASTM International.

ASTM. (2005). E824-05: Transfer of Calibration from Reference to Field Radiometers. West Conshohocken, PA: ASTM International.

ASTM. (2005). G167-05: Standard Test Method for Calibration of a Pyranometer Using a Pyrheliometer. West Conshohocken, PA: ASTM International; 21 pp.

ASTM. (2005). G183-05: Standard Practice for Field Use of Pyranometers, Pyrheliometers and UV Radiometers. West Conshohocken, PA: ASTM International.

Augustyn, J.; Geer, T.; Stoffel, T.; Kessler, R.; Kern, E.; Little, R.; Vignola, F. (2002). "Improving the Accuracy of Low Cost Measurement of Direct Normal Solar Irradiance." Solar 2002 Conference Proceedings; June 15-20, 2002, Reno, Nevada. 
Badosa, J.; Wood, J.; Blanc, P.; Long, C.N.; Vuilleumier, L.; Demengel, D.; Haeffelin, M. (2014). "Solar Irradiances Measured Using Spn1 Radiometers: Uncertainties and Clues for Development." Atmospheric Measurement Techniques (7:8); pp. 8,149-8,191.

Bahm, R.J.; Nakos, J.C. (1979). The Calibration of Solar Radiation Measuring Instruments: Final Report. BER-1(79)DOE-684-1. Albuquerque, NM: University of New Mexico College of Engineering.

Bergholter, U.; Dehne, K. (1994). "SCAPP: A Compact Scanning Pyrheliometer/Pyranometer System for Direct, Diffuse, and Global Solar Radiation." WMO-IOM No. 57. Geneva, Switzerland: World Meteorogolical Organizaion; p. 245ff.

Dutton, E.G.; Michalsky, J.J.; Stoffel, T.; Forgan, B.W.; Hickey, J.; Nelson, D.W.; Alberta, T.J.; Reda, I. (2000). "Measurement of Broadband Diffuse Solar Irradiance Using Current Commercial Instrumentation with a Correction for Thermal Offset Errors." Journal of Atmospheric and Oceanic Technology (18:3); pp. 297-314.

Espinar, B.; Wald, L.; Blanc, P.; Hoyer-Klick, C.; Schroedter-Homscheidt, M.; Wanderer, T. (2011). Report on the Harmonization and Qualification of Meteorological Data: Project ENDORSE_Energy Downstream Service: Providing Energy Components for GMES_Grant Agreement No. 262892. Paris, France: Armines. Accessed November 11, 2014 : http://www.endorse-fp7.eu/public deliverables.

Finsterle, W. (2011). WMO International Pyrheliometer Comparison: IPC-XI 27 Sept.-15 Oct. 2010—WMO IOM No. 108. Davos, Switzerland: World Meteorological Organization.

Fröhlich, C. (1991). "History of Solar Radiometry and the World Radiation Reference." Metrologia (28); pp. 111-115.

Geuder, N.; Affolter, R.; Goebel, O.; Dahleh, B.; Al Khawaja, M.; Wilbert, S.; Pape, B.; Pulvermüller, B. (2010). "Validation of Direct Beam Irradiance Measurements from Rotating Shadowband Pyranometers in a Different Climate." SolarPACES Conference Proceedings; September 21-24, 2010, Perpignan, France.

Geuder, N.; Affolter, R.; Kraas, B. (2012). "Experiences on Radiation Measurements with Rotating Shadowband Irradiometers." COST WIRE Workshop Payerne Proceedings; September 21, 2012, Payerne, Switzerland.

Geuder, N.; Hanussek, M.; Haller, J.; Affolter, R.; Wilbert, S. (2011). “Comparsion of Corrections and Calibration Procedures for Rotating Shadowband Irradiance Sensors." SolarPACES Conference Proceedings; September 20-23, 2011, Granada, Spain.

Geuder, N.; Pulvermüller, B.; Vorbrügg, O. (2008). “Corrections for Rotating Shadowband Pyranometers for Solar Resource Assessment.” Solar Energy Applications Conference Proceedings; Part of SPIE Optics + Photonics, August 10-14, 2008, San Diego, California.

Geuder, N.; Pulvermüller, B.; Vorbrugg, O.; Butrón, J.T.; Kistner, R.; Behrens, K.; Grewe, R.-D. (2006). "Examination of Different Irradiation Sensors: Operation Experiences and Comparative 
Study." 4th International Conference on Experiences with Automatic Weather Stations Proceedings; May 24-26, 2006, Lisbon, Portugal.

Geuder, N.; Quaschning, V. (2006). "Soiling of Irradiation Sensors and Methods for Soiling Correction.” Solar Energy (80:11); pp. 1,402-1,409.

Geuder, N.; Wolfertstetter, F.; Wilbert, S.; Schueler, D.; Luepfert, R.A.E.; Espinar, B. (2014). "Screening and Flagging of Solar Irradiation and Ancillary Meteorological Data." SolarPACES Conference Proceedings; September 16-19, 2014, Beijing, China.

Gueymard, C. (2009). "Direct and Indirect Uncertainties in the Prediction of Tilted Irradiance for Solar Engineering Applications.” Solar Energy (83); pp. 432-444.

Gueymard, C.A.; Myers, D.R. (2009). "Evaluation of Conventional and High-Performance Routine Solar Radiation Measurements for Improved Solar Resource, Climatological Trends, and Radiative Modeling." Solar Energy (83); pp. 171-185.

Habte, A.; Wilcox, S.; Stoffel, T. (2014). Evaluation of Radiometers Deployed at the National Renewable Energy Laboratory's Solar Radiation Research Laboratory. NREL/TP-5D00-60896. Golden, CO: National Renewable Energy Laboratory.

Harrison, L.; Michalsky, J.; Berndt, J. (1994). “Automated Multi-Filter Rotating Shadowband Radiometer: An Instrument for Optical Depth and Radiation Measurements." Applied Optics (33:22), August; pp. 5,118-5,125.

Holben, B.; Eck, T., et al. (1998). "AERONET_A Federated Instrument Network and Data Archive for Aerosol Characterization.” Remote Sensing of Environment (66:1); pp. 1-16.

Ineichen, P.; Perez, R. (2002). “A New Airmass Independent Formulation for the Linke Turbidity Coefficient.” Solar Energy (73:3); pp. 151-157.

Iqbal, M. (1983). An Introduction to Solar Radiation. Academic Press, New York.

ISO. (1990). ISO 9060:1990: Specification and Classification of Instruments for Measuring Hemispherical Solar and Direct Solar Radiation. Geneva, Switzerland.

ISO. (1992). ISO 9847: 1992 (E): Solar Energy-Calibration of Field Pyranometers by Comparison to a Reference Pyranometer. Geneva, Switzerland; 20 pp.

ISO. (1993). ISO 9846: 1993(E): Solar Energy-Calibration of a Pyranometer Using a Pyrheliometer. Geneva, Switzerland; 24 pp.

ISO. (2008). Guide 100-2008: Evaluation of Measurement Data-Guide to the Expression of Uncertainty in Measurement. Comité International des Poids et Mesures. www.bipm.org/utils/common/documents/jcgm/JCGM_100_2008_E.pdf.

Journée, M.; Bertrand, C. (2011). "Quality Control of Solar Radiation Data within the RMIB Solar Measurements Network.” Solar Energy (85:1); pp. 72-86. 
Kalapatapu, R.; Chiesa, M.; Armstrong, P.; Wilbert, S. (2012). "Measurement of Dni Angular Distribution with a Sunshape Profiling Irradiometer." SolarPACES Conference Proceedings; September 11-14, 2012, Marrakesh, Morocco.

Kern, E.C. (2010). "Calibration Methods for Silicon Photodiode Pyranometers used in Rotating Shadowband Radiometers." SolarPACES Conference Proceedings; September 21-24, 2012, Perpignan, France.

King, D.L.; Myers, D.R. (1997). "Silicon Photodiode Pyranometers: Operational Characteristics, Historical Experiences, and New Calibration Procedures." 26th IEEE Photovoltaic Specialists Conference Proceedings; May 13-17, 1997, Washington, D.C.

LI-COR Biosciences. (2005). LI-COR Terrestrial Radiation Sensors-Instruction Manual. Lincoln, Nebraska: LI-COR, Inc.

Lindner, P. (1984). "A New Sunshine Duration Sensor." Instruments and Observing Methods: WMO-IOM No. 15-Technical Conference on Instruments and Cost-Effective Meteorological Observations Proceedings; September 24-28, 1984, Nordwijkerhout, The Netherlands.

Long, C.N.; Dutton, E.G. (2002). "Baseline Surface Radiation Network: Global Network Recommended QC Tests-V2.0." Accessed September 25, 2014: http://epic.awi.de/30083/1/BSRN_recommended_QC tests_V2.pdf.

Maxwell, E.; Wilcox, S.; Rymes, M. (1993). Users Manual for SERI QC Software: Assessing the Quality of Solar Radiation Data. NREL/TP-463-5608 DE93018210. Golden, CO: National Renewable Energy Laboratory. Accessed September 26, 2014: http://www.nrel.gov/docs/legosti/old/5608.pdf.

Maxwell, E.L; Wilcox, S.M.; Cornwall, C.; Marion, B.; Alawaji, S.H.; Mahfoodh, M.; ALAmoudi, A. (1999). Progress Report for Annex II-Assessment of Solar Radiation Resources in Saudi Arabia 1993-1997. NREL/TP-560-25374. Golden, CO: National Renewable Energy Laboratory.

Micek, L.V. (1981). Direct Beam Insolation Measurement Errors Caused by Pyrheliometer Misalignment. M.S. Thesis. San Antonio, TX: Trinity University.

Michalsky, J.J., et al. (2011). “An Extensive Comparison of Commercial Pyrheliometers Under a Wide Range of Routine Observing Conditions." Journal of Atmospheric and Oceanic Technology (28); pp. 752-766.

Morrison, R. (1998). Grounding and Shielding Techniques. 4th edition. New York: John Wiley and Sons.

Myers, D.; Wilcox, S. (2009). Relative Accuracy of 1-Minute and Daily Total Solar Radiation Data for 12 Global and 4 Direct Beam Solar Radiometers. NREL/CP-550-45734. Golden, CO: National Renewable Energy Laboratory. 
Myers, D.; Wilcox, S.; Marion, W.; Al-Abbadi, N.; Mahfoodh, M.; Al-Otaibi, Z. (2002). Final Report for Annex II-Assessment of Solar Radiation Resources in Saudi Arabia 1998-2000. NREL/TP-50-31546. Golden, CO: National Renewable Energy Laboratory.

Myers, D.R. (2011). "Quantitative Analysis of Spectral Impacts on Silicon Photodiode Radiometers.” American Solar Energy Society Solar Conference Proceedings; May 17-20, Raleigh, North Carolina. Boulder, CO: American Solar Energy Society.

Pape, B.; Battles, J.; Geuder, N.; Zurita, R.; Adan, F.; Pulvermüller, B. (2009). "Soiling Impact and Correction Formulas in Solar Measurements for CSP Projects." SolarPACES Conference Proceedings; September 15-18, 2009, Berlin, Germany.

Reda, I. (1996). Calibration of a Solar Absolute Cavity Radiometer with Traceability to the World Radiometric Reference. NREL/TP-463-20619. Golden, CO: National Renewable Energy Laboratory.

Reda, I. (2011). Method to Calculate Uncertainties in Measuring Shortwave Solar Irradiance Using Thermopile and Semiconductor Solar Radiometers. NREL/TP-3B10-52194. Golden, CO: National Renewable Energy Laboratory.

Reda, I.; Dooraghi, M.; Habte, A. (2013). NREL Pyrheliometer Comparison: September 16 to 27, 2013 (NPC-2013). NREL/TP-3B10-60749. Golden, CO: National Renewable Energy Laboratory.

Reda, I.; Myers, D.; Stoffel, T. (2008). "Uncertainty Estimate for the Outdoor Calibration of Solar Pyranometers: A Metrologist Perspective." Measure (3:4), Dec.; pp. 58-66; NREL/JA581-41370.

Reda, I.; Stoffel T.; Myers, D. (2003). “A Method to Calibrate a Solar Pyranometer for Measuring Reference Diffuse Irradiance.” Solar Energy (74); pp. 103-112.

Spencer, J.W. (1982). “A Comparison of Methods for Estimating Hourly Diffuse Solar Radiation from Global Solar Radiation.” Solar Energy (29:1); pp.19-32

Stoffel, T.L.; Reda, I.; Myers, D.R.; Renné, D.; Wilcox, S.; Treadwell, J. (2000). "Current Issues in Terrestrial Solar Radiation Instrumentation for Energy, Climate, and Space Applications." Metrologia) (37:5); pp. 399-401.

Taylor, B.N.; Kuyatt, C.E. (1987). Guidelines for Evaluation and Expressing the Uncertainty of NIST Measurement Results: NIST Technical Note 1297. Gaithersburg, MD: National Institute of Standards and Technology.

Vignola, F. (2006). "Removing Systematic Errors from Rotating Shadowband Pyranometer Data." Solar 2006 Conference Proceedings; July 7-13, 2006, Denver, Colorado.

Vignola, F.; Long, C.N.; Reda, I. (2009). “Testing a Model of IR Radiative Losses.” NREL/CP3B0-46411. Presented at the Society of Photo-Optical Instrumentation Engineers; August 2-6, 2009, San Diego, CA. 
Vignola, F.; Michalsky, J.; Stoffel, T. (2012). Solar and Infrared Radiation Measurements. Abbas Ghassemi, ed. New York: Energy and the Environment Series, CRC Press.

Vuilleumier, L.; Sommer, N.; Paglia, G.; Hauser, M.; Ruffieux, D.; Félix, C.; Calpini, B. (2013). "Performance Evaluation of Radiation Sensors for the Solar Energy Sector." 2nd International Conference on Energy \& Meteorology Proceedings; June 25-28, 2013, Toulouse, France.

Wilcox, S.; Cormack, P. (2011). "Implementing Best Practices for Data Quality Assessment of the National Renewable Energy Laboratory's Solar Resource and Meteorological Assessment Project." Solar 2011 Conference Proceedings; May 16-21, 2011, Raleigh, North Carolina.

Wilcox, S.; Myers, D. (2008). Evaluation of Radiometers in Full-Time Use at the National Renewable Energy Laboratory Solar Radiation Research Laboratory. NREL/TP-550-44627. Golden, CO: National Renewable Energy Laboratory.

WMO. (2008). WMO Guide to Meteorological Instruments and Methods of Observation: WMO No. 8. 7th edition. Geneva, Switzerland. http://www.wmo.int/pages/prog/gcos/documents/gruanmanuals/CIMO/CIMO Guide7th_Edition-2008.pdf.

\section{Chapter 4: Modeling Solar Radiation-Current Practices}

Badescu, V.; Gueymard C.A.; Cheval, S.; Oprea, C.; Baciu, M.; Dumitrescu, A.; Iacobescua, F.; Milso, I.; Rada, C. (2012). "Computing Global and Diffuse Solar Hourly Irradiation on ClearSky: Review and Testing of 54 Models." Renewable and Sustainable Energy Reviews (16); pp. 1636-1656.

Ben Djemaa, A.; Delorme, C. (1992). “A Comparison Between One Year of Daily Global Irradiation From Ground-Based Measurements Versus METEOSAT Images From Seven Locations in Tunisia.” Solar Energy (48:5); pp. 325-333.

Beyer, H.; Costanzo, C.; Heinemann, D. (1996). "Modifications of the HELIOSAT Procedure for Irradiance Estimates from Satellite Images.” Solar Energy (56:3); pp. 207-221.

Bird, R.E.; Hulstrom, R.L. (1981). A Simplified Clear-Sky Model for Direct and Diffuse Insolation on Horizontal Surfaces. SERI/TR-642-761. Golden, CO: Solar Energy Research Institute.

Cano, D.; Monget, J.M.; Albuisson, M.; Guillard, H.; Regas, N.; Wald, L. (1986). “A Method for the Determination of the Global Solar Radiation from Meteorological Satellite Data." Solar Energy (37:1); pp. 31-39.

Cebecauer, T.; Suri, M.; Perez, R. (2010). "High Performance MSG Satellite Model for Operational Solar Energy Applications.” ASES Annual Conference Proceedings; May 17-22, 2010, Phoenix, Arizona.

Chen, M.; Zhuang, Q.; He, Y. (2014). “An Efficient Method of Estimating Downward Solar Radiation Based on the MODIS Observations for the Use of Land Surface Modeling." Remote Sensing (6); pp. 7,136-7,157. 
Czeplak, G.; Noia, M.; Ratto, D.F. (1991). "An Assessment of a Statistical Method to Estimate Solar Irradiance at the Earth's Surface from Geostationary Satellite Data." Renewable Energy (1); pp. 737-743.

Darnell, W.L.; Staylor, W.F. (1992). "Seasonal Variation of Surface Radiation Budget Derived From International Satellite Cloud Climatology Project C1 Data.” Journal of Geophysical Research (97:15); pp. 741-760.

Darnell, W.L.; Staylor, W.F.; Gupta, S.K.; Denn, M. (1988). "Estimation of Surface Insolation Using Sun-Synchronous Satellite Data.” Journal of Climate (1); pp. 820-835.

Dedieu, G.; Deschamps, P.Y.; Kerr, Y.H. (1987). "Satellite Estimation of Solar Irradiance at the Surface of the Earth and of Surface Albedo Using a Physical Model Applied to Meteosat Data." Journal of Climate and Applied Meteorology (26); pp. 79-87.

Delorme, C.; Gallo, A.; Oliveiri, J. (1992). "Quick Use of WEFAX Images from METEOSAT To Determine Daily Solar Radiation in France.” Solar Energy (49:3); pp. 191-197.

Diabate, L.; Demarcq, H.; Michaud-Regas, N.; Wald, L. (1988). "Estimating Incident Solar Radiation at the Surface From Images of the Earth Transmitted by Geostationary Satellites: The HELIOSAT Project.” International Journal of Solar Energy (5); pp. 261-278.

Diak G.R.; Gautier, C. (1983). "Improvements to a Simple Physical Model for Estimating Insolation from GOES Data." Journal of Climate and Applied Meteorology (22); pp. 505-508.

Dumortier, D. (1998). “The Satellite Model of Turbidity Variations in Europe." Presented at the Sixth SATELLIGHT Meeting; September 1998, Freiburg, Germany.

Dürr, B.; Zelenka, A. (2009). "Deriving Surface Global Irradiance over the Alpine Region From METEOSAT Second Generation Data by Supplementing the HELIOSAT Method." International Journal of Remote Sensing (30); pp. 5,821-5,841.

Espinar, B.; Hoyer-Klick, C.; Lefèvre, M.; Schroedter-Homscheidt, M.; Wald, L. (2014). User's Guide to the MACC-RAD Services on Solar Energy Radiation Resources: Part C-MACC-II Project Report D_122.6, Version v3.0. Public report accessible via http://www.gmesatmosphere.eu.

Fatichi, S.; Ivanov, V.Y.; Caporali, E. (2011). "Simulation of Future Climate Scenarios with a Weather Generator.” Advances in Water Resources (34); pp. 448-467.

Fontoynont, M., et al. (1998). "Satellight: A WWW Server Which Provides High Quality Daylight and Solar Radiation Data for Western and Central Europe." 9th Conference on Satellite Meteorology and Oceanography Proceedings; Paris, France, pp. 434-437.

Frouin, R.; Gautier, C.; Katsaros, K.B.; Lind, R.J. (1988). “A Comparison of Satellite and Empirical Formula Techniques for Estimating Insolation over the Oceans." Journal of Applied Meteorology (27); pp. 1,016-1,023. 
Frulla, L.A.; Gagliardini, D.A.; Grossi Gallegos, H.; Lopardo, R. (1988). "Incident Solar Radiation on Argentina From the Geostationary Satellite GOES: Comparison with Ground Measurements." Solar Energy (41:1); pp. 61-69.

Gautier, C. (1988). "Surface Solar Irradiance in the Central Pacific During Tropic Heat: Comparisons Between in situ Measurements and Satellite Estimates." Journal of Climate (1); pp. 600-608.

Gautier, C.; Diak, G.; Masse, S. (1980). "A Simple Physical Model to Estimate Incident Solar Radiation at the Surface From GOES Satellite Data." Journal of Applied Meteorology (19); pp. $1,005-1,012$.

Gautier, C.; Frouin, R. (1984). "Satellite-Derived Ocean Surface Radiation Fluxes." Proceeding of the Workshop on Advances in Remote Sensing Retrieval Methods; Williamsburg, Virginia.

George, R.; Wilcox, S.; Anderberg, M.; Perez, R. (2007). "National Solar Radiation Database (NSRDB): 10 Km Gridded Hourly Solar Database." Solar 2007 Conference Proceedings; July 712, 2007, Cleveland, Ohio. NREL/CP-581-41599. Golden, CO: National Renewable Energy Laboratory.

Gueymard C.A. (1993). "Critical Analysis and Performance Assessment of Clear-Sky Irradiance Models Using Theoretical and Measured Data." Solar Energy (51); pp.121-138.

Gueymard C.A. (2003a). "Direct Solar Transmittance and Irradiance Predictions with Broadband Models: Part I—Detailed Theoretical Performance Assessment.” Solar Energy (74); pp. 355-379; Corrigendum (76); pp. 513 (2004).

Gueymard C.A. (2003b). "Direct Solar Transmittance and Irradiance Predictions with Broadband Models: Part II-Validation with High-Quality Measurements." Solar Energy (74); pp. 381-395; Corrigendum (76); pp. 515 (2004).

Gueymard C.A.; Myers D. (2008). "Validation and Ranking Methodologies for Solar Radiation Models." Badescu, V., ed. Modeling Solar Radiation at the Earth's Surface. Berlin, Germany: Springer.

Gueymard C.A.; Ruiz-Arias J.A. (2015). "Validation of Direct Normal Irradiance Predictions Under Arid Conditions: A Review of Radiative Models and Their Turbidity-Dependent Performance." Renewable and Sustainable Energy Reviews (45); pp. 379-396.

Gueymard, C.A. (2008). "REST2: High-Performance Solar Radiation Model for Cloudless-Sky Irradiance, Illuminance and Photosynthetically Active Radiation-Validation with a Benchmark Dataset." Solar Energy (82); pp. 272-285.

Gueymard, C.A. (2012). "Clear-Sky Irradiance Predictions for Solar Resource Mapping and Large-Scale Applications: Improved Validation Methodology and Detailed Performance Analysis of 18 Broadband Radiative Models." Solar Energy (86); pp. 2145-2169. 
Gueymard, C.A. (2014). "A Review of Validation Methodologies and Statistical Performance Indicators for Modeled Solar Radiation Data: Towards a Better Bankability of Solar Projects." Renewable and Sustainable Energy Reviews (39); pp. 1,024-1,034.

Hammer, A.; Heinemann, D.; Hoyer, C.; Kuhlemann, R.; Lorenz, E.; Muller, R.; Beyer, H.G. (2003). "Solar Energy Assessment Using Remote Sensing Technologies." Remote Sensing of Environment (8); pp. 423-432.

Hammer, A.; Lorenz, E.; Kemper, A.; Heinemann, D.; Beyer, H.G.; Schumann, K.; Schwandt, M. (2009) "Direct Normal Irradiance for CSP Based on Satellite Images of Meteosat Second Generation.” SolarPACES Conference Proceedings; September 15-18, 2009, Berlin, Germany.

Hay, J.E.; Hanson, K.; Hanson, J. (1978). “A Satellite-Based Methodology for Determining Solar Irradiance at the Ocean Surface during GATE." Bulletin of the American Meteorological Society (59); pp. 1,549.

Heidinger, A.K. (2003). "Rapid Daytime Estimation of Cloud Properties over a Large Area from Radiance Distributions." Journal of Atmospheric and Oceanic Technology (20); pp. 1,237-1,250.

Heidinger, A.K.; Foster, M.J.; Walther, A. Zhao, X. (2014). "The Pathfinder AtmospheresExtended (PATMOS-x) AVHRR Climate Data Set." Bulletin of the American Meteorological Society (95); pp. 909-922. http://dx.doi.org/10.1175/BAMS-D-12-00246.1.

Hu, Y.X.; Stamnes, K. (1993). "An Accurate Parameterization of the Radiative Properties of Water Clouds Suitable for Use in Climate Models.” Journal of Climate (6); pp. 728-742.

Ineichen, P. (2008). “A Broadband Simplified Version of the Solis Clear Sky Model.” Solar Energy (82); pp. 758-762.

Istvan, L. Personal communication.

Joseph, J.H.; Wiscombe, W.J.; Weinman, J.A. (1976). "The Delta-Eddington Approximation for Radiative Transfer.” Journal of Atmospheric Science (33); pp. 2,452-2,459.

Justus, C.; Paris, M.V.; Tarpley, J.D. (1986). "Satellite-Measured Insolation in the United States, Mexico and South America." Remote Sensing of Environment (20); pp. 57-83.

Kato, S.; Ackerman, T.P.; Mather, J.H.; Clothiaux, E.E. (1999). “The k-Distribution Method and Correlated-k Approximation for a Shortwave Radiative Transfer Model." Journal of Quantitative Spectroscopy \& Radiative Transfer (62); pp. 109-121.

Kerschegens, M.; Pilz, U.; Raschke, E. (1978). “A Modified Two-Stream Approximation for Computations of the Solar Radiation in a Cloudy Atmosphere.” Tellus (30); pp. 429-435.

Kinne, S., et al. (2005). "An AeroCom Initial Assessment-Optical Properties in Aerosol Component Modules of Global Models." Atmospheric Chemistry and Physics Discussions (5); pp. $8,285-8,330$. 
Klink, J.C.; Dollhopf, K.J. (1986). An Evaluation of Satellite-Based Insolation Estimates for Ohio. Journal of Climate and Applied Meteorology (25); pp. 1,741-1,751.

Kriebel, K.T.; Gesell, G.; Kästner, M.; Mannstein, H. (2003). "The Cloud Analysis Tool APOLLO: Improvements and Validation." International Journal of Remote Sensing (24); pp. 2,389-2,408.

Kriebel, K.T.; Saunders, R.W.; Gesell, G. (1989). "Optical Properties of Clouds Derived from Fully Cloudy AVHRR Pixels.” Beiträge zur Physik der Atmosphäre (62:3), August; pp. 165171.

Kylling, A.; Stamnes, K; Tsay, S.C. (1995). "A Reliable and Efficient Two-Stream Algorithm for Radiative Transfer: Documentation of Accuracy in Realistic Layered Media." Journal of Atmospheric Chemistry (21); pp. 115-150.

Laszlo, I.; Ciren, P.; Liu, H.; Kondragunta, S.; Tarpley, J.D.; Goldberg. M.D. (2008). "Remote Sensing of Aerosol and Radiation from Geostationary Satellites." Advances in Space Research (41); pp. 1,882-1,893.

Lefèvre, M.; Oumbe, A.; Blanc, P.; Espinar, B.; Gschwind, B.; Qu, Z.; Wald, L.; SchroedterHomscheidt, M.; Hoyer-Klick, C.; Arola, A.; Benedetti, A.; Kaiser, J.W.; Morcrette, J.-J. (2013). "McClear: A New Model Estimating Downwelling Solar Radiation at Ground Level in ClearSky Conditions." Atmospheric Measurement Techniques (6); pp. 2,403-2,418. http://www.atmos-meas-tech.net/6/2403/2013/amt-6-2403-2013.html.

Lefèvre, M.; Wald, L.; Diabate, L. (2007). "Using Reduced Datasets ISCCP-B2 From the Meteosat Satellites To Assess Surface Solar Irradiance.” Solar Energy (81); pp. 240-253.

Lohmann, S.; Schillings, C.; Mayer, B.; Meyer, R. (2006). “Long-Term Variability of Solar Direct and Global Irradiance Derived from ISCCP Data and Comparison with Re-Analysis Data." Solar Energy (80); pp. 1,390-1,401.

Lorenz, E. (2007). Improved Diffuse Radiation Model: MSG-Report for the EC-Project PVSAT2: Intelligent Performance Check of PV System Operation Based on Satellite Data. NNE5-20010571. Deliverable D4.2b. Oldenburg, Germany: University of Oldenburg.

Marion, W.; Wilcox, S. (1994). Solar Radiation Data Manual for Flat-Plate and Concentrating Collectors. NREL/TP-463-5607. Golden, CO: National Renewable Energy Laboratory.

Massey Jr., F.J. (1951). “The Kolmogorov-Smirnov Test for Goodness of Fit.” Journal of the American Statistical Association (56); pp. 68-78.

Mayer, B.; Kylling, A. (2005). "The libRadtran Software Package for Radiative Transfer Calculations: Description and Examples of Use." Atmospheric Chemistry and Physics Discussions (5:2); pp. 1,855-1,877.

Möser, W.; Raschke, E. (1983). "Mapping of Global Radiation and Cloudiness from METEOSAT Image Data.” Meteorologische Rundschau (36); pp. 33-41. 
Möser, W.; Raschke, E. (1984). "Incident Solar Radiation over Europe Estimated From METEOSAT Data." Journal of Climate and Applied Meteorology (23); pp. 166-170.

Moussu, G.; Diabaté, L.; Obrecht, D.; Wald, L. (1989). "A Method for the Mapping of the Apparent Ground Brightness Using Visible Images from Geostationary Satellites.” International Journal of Remote Sensing (10:7); pp. 1,207-1,225.

Mueller, R.W.; Dagestad, K.F.; Ineichen, P.; Schroedter, M.; Cros, S.; Dumortier, D.; Kuhlemann, R.; Olseth, J.A.; Piernavieja, G.; Reise, C.; Wald, L.; Heinnemann, D. (2004). "Rethinking Satellite Based Solar Irradiance Modelling: The SOLIS Clear Sky Module." Remote Sensing of Environment (90:2); pp. 160-174.

Myers, D.R. (2013). Solar Radiation: Practical Modeling for Renewable Energy Applications. Ghassemi, A., ed. New York: Energy and the Environment Series, CRC Press; 182 pp.

Nullett, D. (1987). "A Comparison of Two Methods of Estimating Insolation over the Tropical Pacific Ocean Using Cloudiness from Satellite Observations.” Solar Energy (39:3); pp. 197-201.

Nunez. M. (1990). "Solar Energy Statistics for Australian Capital Regions.” Solar Energy (44); pp. 343-354.

Oumbe, A.; Qu, Z.; Blanc, P.; Lefevre, M.; Wald, L. (2014). "Decoupling the Effects of Clear Atmosphere and Clouds to Simplify Calculations of the Broadband Solar Irradiance at Ground Level." Geoscientific Model Development (7); pp. 1,661-1,169.

Pavolonis, M.; Heidinger, A.K.; Uttal, T. (2005). "Daytime Global Cloud Typing From AVHRR and VIIRS: Algorithm Description, Validation, and Comparisons." Journal of Applied Meteorology (44); pp. 804-826.

Pereira, E.B.; Abreu, S.L.; Stuhlmann, R.; Rieland, M.; Colle, S. (1996). "Survey of the Incident Solar Radiation Data in Brazil by Use of METEOSAT Satellite Data." Solar Energy (57:2); pp. $125-132$.

Perez, R.; Ineichen, P. (2002). “A New Operational Model for Satellite-Derived Irradiances: Description and Validation.” Solar Energy (73); pp. 307-317.

Perez, R.; Ineichen, P.; Maxwell, E.; Seals, R.; Zelenka, A. (1992). “Dynamic Global-to-Direct Irradiance Conversion Models.” ASHRAE Transactions-Research Series (98:1); pp. 354-369.

Perez, R.; Ineichen, P.; Moore, K.; Kmiecik, M.; Chain, C.; George, R.; Vignola, F. (2002). “A NewOperational Satellite-to-Irradiance Model.”Solar Energy (73:5); pp. 307-317.

Perez, R.; Seals, R.; Ineichen, P.; Stewart, P.; Menicucci, D. (1987). “A New Simplified Version of the Perez Diffuse Irradiance Model for Tilted Surfaces: Description Performance Validation.” Solar Energy (39); pp. 221-232.

Pinker, R.T.; Ewing, J.A. (1985). "Modeling Surface Solar Radiation: Model Formulation and Validation.” Journal of Climate and Applied Meteorology (24); pp. 389-401. 
Pinker, R.T.; Frouin, R.; Li, Z. (1995). “A Review of Satellite Methods to Derive Surface Shortwave Irradiance.” Remote Sensing of Environment (51); pp. 108-124.

Pinker, R.T.; Laszlo, I. (1992). "Modeling of Surface Solar Irradiance for Satellite Applications on a Global Scale.” Journal of Applied Meteorology and Climatology (31); pp. 194-211.

Pinker, R.T.; Laszlo, I. (1992). "Modeling Surface Solar Irradiance for Satellite Applications on a Global Scale.” Journal of Applied Meteorology (31); pp. 194-211.

Raschke, E.; Stuhlmann, R.; Palz, W.; Steemers, T.C. (1991). Solar Radiation Atlas of Africa. Rotterdam, Amsterdam: A.A. Balakema Publishers, for the Commission of European Communities; p. 155.

Remund, J. (2009). Aerosol Optical Depth and Linke Turbidity Climatology: Description for Final Report of IEA SHC Task 36. Bern, Switzerland: Meteotest.

Renné, D.S.; Perez, R.; Zelenka, A.; Whitlock, C.; DiPasquale, R. (1999). Advances in Solar Energy: An Annual Review of Research and Development. Vol. 13. Goswami, D. Y., and Boer, K.W., eds. Boulder, CO: American Solar Energy Society.

Rigollier, C.; Lefèvre, M.; Wald L. (2004). "The Method HELIOSAT-2 for Deriving Shortwave Solar Radiation Data From Satellite Images.” Solar Energy (77); pp. 159-169.

Ruiz-Arias, J.A.; Cebecauer, T.; Tovar-Pescador; Š́ri , M. (2010). "Spatial Disaggregation of Satellite-Derived Irradiance Using a High-Resolution Digital Elevation Model." Solar Energy (84:9); pp. 1,644-1,657.

Sadler, J.C.; Oda, L.; Kilonsky, B.J. (1976). Pacific Ocean Cloudiness from Satellite Observations. Honolulu, HI: University of Hawaii Department of Meteorology.

Schiffer, R.A.; Rossow, W.B. (1983). "The International Satellite Cloud Climatology Project (ISCCP): The First Project of the World Climate Research Programme." Bulletin of the American Meteorological Society (64); pp. 779-784.

Schillings, C.; Mannstein, H.; Meyer, R. (2003a). "Operational Method for Deriving High Resolution Direct Normal Irradiance from Satellite Data.” Solar Energy (76:4); pp. 475-484.

Schmetz, J. (1989). "Towards a Surface Radiation Climatology: Retrieval of Downward Irradiances from Satellites." Atmospheric Research (23); pp. 287-321.

Schmetz, J.; Pili, P.; Tjemkes, S.; Just, D.; Kerkmann, J.; Rota, S.; Ratier, A. (2002). “An Introduction to Meteosat Second Generation (MSG)." Bulletin of the American Meteorological Society (83); pp. 977-992.

Schubnell, M. (1992). "Sunshape and Its Influence on the Flux Distribution in Imaging Solar Concentrators.” Journal of Solar Energy Engineering (114:4); pp. 260. 
Sengupta, M.; Gotseff, P. (2013). Evaluation of Clear-Sky Models for Satellite-Based Irradiance Estimates. NREL/TP-5D00-60735. Golden, CO: National Renewable Energy Laboratory. http://www.nrel.gov/docs/fy14osti/60735.pdf.

Sengupta, M.; Habte, A.; Gotseff, P.; Weekley, A.; Lopez, A.; Anderberg, M.; Molling, C.; Heidinger, A. (2014a) "A Physics-Based GOES Satellite for Use in NREL's National Solar Radiation Database.” Preprint. Prepared for the European Photovoltaic Solar Energy Conference and Exhibition, September 22-26, 2014, Amsterdam, Netherlands. NREL/CP-5D00-62776. Golden, CO: National Renewable Energy Laboratory.

Sengupta, M.; Habte, A.; Gotseff, P.; Weekley, A.; Lopez, A.; Molling, C.; Heidinger, A. (2014b) “A Physics-Based GOES Satellite Product for Use in NREL's National Solar Radiation Database." Solar 2014 Conference Proceedings; July 6-10, 2014, San Francisco, California. NREL/CP-5D00-62237. Golden, CO: National Renewable Energy Laboratory.

Shaltout, M.A.M.; Hassen, A.H. (1990). "Solar Energy Distribution over Egypt Using METEOSAT Photos.” Solar Energy (45:6); pp. 345-351.

Stowe, L.L.; Davis, P.A.; McClain, E.P. (1999). "Scientific Basis and Initial Evaluation of the CLAVR-1 Global Clear Cloud Classification Algorithm for the Advanced Very High Resolution Radiometer." Journal of Atmospheric and Oceanic Technology (16); pp. 656-681.

Stuhlmann, R.; Rieland, M.; Raschke, E. (1990). “An Improvement of the IGMK Model To Derive Total and Diffuse Solar Radiation at the Surface From Satellite Data." Journal of Applied Meteorology (29); pp. 586-603.

Tarpley, J.D. (1979). "Estimating Incident Solar Radiation at the Surface from Geostationary Satellite Data." Journal of Applied Meteorology (18); pp. 1,172-1,181.

Vindel, J.M.; Polo, J.; Antonanzas-Torres, F. (2013). "Improving Daily Output of Global to Direct Solar Irradiance Models with Ground Measurements." Journal of Renewable and Sustainable Energy (5); 063123.

Whitlock, C.H.; Charlock, T.P.; Staylor, W.F.; Pinker, R.T.; Laszlo, I.; Ohmura, A.; Gilgen, H.; Konzelman, T.; DiPasquale, R.C.; Moats, C.D.; LeCroy, S.R.; Ritchey, N.A. (1995). "First Global WRCP Shortwave Surface Radiation Budget Dataset." Bulletin of the American Meteorological Society (76); pp. 905-922.

Wilbert, S.; Reinhardt, B.; DeVore, J.; Röger, M.; Pitz-Paal, R.; Gueymard, C.; Buras, R. (2013). "Measurement of Solar Radiance Profiles with the Sun and Aureole Measurement System." Journal of Solar Energy Engineering (135:4); pp. 041002-041002. doi: 10.1115/1.4024244.

Yang, P.; Liou, K.N.; Wyser, K.; Mitchell, D. (2000). "Parameterization of the Scattering and Absorption Properties of Individual Ice Crystals." Journal of Geophysical Research (105); pp. 4,699-4,718.

Zelenka, A.; Perez, R.; Seals R.; Renné, D. (1999). "Effective Accuracy of Satellite-Derived Irradiance." Theoretical and Applied Climatology (62); pp. 199-207. 
Zhang, Y.C.; Rossow, W.B.; Lacis, A.A.; Oinas, V.; Mishchenko, M.I. (2004). "Calculation of Radiative Fluxes from the Surface to Top of Atmosphere Based on ISCCP and Other Global Datasets: Refinements of the Radiative Transfer Model and the Input Data." Journal of Geophysical Research (109).

\section{Chapter 5: Historical Solar Resource Data}

Arguez, A.; Vose, R.S. (2011). "The Definition of the Standard WMO Climate Normal: The Key to Deriving Alternative Climate Normals." Bulletin of the American Meteorological Society (92); pp. 699-704.

Dumortier, D. (1998). "Daylight Availability in Freiburg and Nantes: Two Sites Close in Latitude.” Presented at the Sixth SATELLIGHT Meeting; September 1998, Freiburg, Germany, September.

Gueymard, C.A.; Myers, D.R. (2009). "Evaluation of Conventional and High-Performance Routine Solar Radiation Measurements for Improved Solar Resource, Climatological Trends, and Radiative Modeling." Solar Energy (83); pp. 171-185.

Habte, A.; Lopez, A.; Sengupta, M.; Wilcox, S. (2014). Temporal and Spatial Comparison of Gridded TMY, TDY, and TGY Data Sets. NREL/TP-5D00-60886. Golden, CO: National Renewable Energy Laboratory; 30 pp.

Hall, I.; Prairie, R.; Anderson, H.; Boes, E. (1978). Generation of Typical Meteorological Years for 26 SOLMET Stations. SAND78-1601. Albuquerque, NM: Sandia National Laboratories.

Hegner, H.; Müller, G.; Nespor, V.; Ohmura, A.; Steigrad, R.; Gilgen, H. (1998). Technical Plan for BSRN Data Management (WRMC Technical Report No. 2): WMO-TD No. 882. Geneva, Switzerland: World Meteorgological Organization.

Hulstrom, R.L., ed. (1989). Solar Resources. Cambridge, MA: MIT Press.

Ineichen, P. (2013). Long-Term Satellite Hourly, Daily and Monthly Global, Beam and Diffuse Irradiance Validation: Interannual Variability Analysis. Geneva, Switzerland: University of Geneva. http://www.cuepe.ch/archives/annexes-iae/ineichen-2014 long-term-saf-validation.pdf.

Ineichen, P. (2014). "Long Term Satellite Global, Beam, and Diffuse Irradiance Validation." Energy Procedia (48); pp. 1,586-1596.

Kalnay, E.; Kanamitsu, M.; Kistler, R.; Collins, W.; Deaven, D.; Gandin, L.; Iredell, M.; Saha, S.; White, G.; Woollen, J.; Zhu, Y.; Chelliah, M.; Ebisuzaki, W.; Higgins, W.; Janowiak, J.; Mo, K.C.; Ropelewski, C.; Wang, J.; Leetmaa, A.; Reynolds, R.; Jenne, R.; Joseph, D. (1996). "The NCEP/NCAR 40-Year Reanalysis Project.” Bulletin of the American Meteorological Society (77); pp. 437-471.

Kistler, R.; Kalnay, E.; Collins, W.; Saha, S.; White, G.; Woollen, J.; Chelliah, M.; Ebisuzaki, W.; Kanamitsu, M.; Kousky, V.; van den Dool, H.; Jenne, R.; Fiorino, M. (2001). "The NCEP-NCAR 50-Year Reanalysis: Monthly Means CD-ROM and Documentation." Bulletin of the American Meteorological Society (82); pp. 247-267. 
Lohmann, S.; Schillings, C.; Mayer, B.; Meyer, R. (2006). "Long-Term Variability of Solar Direct and Global Irradiance Derived From ISCCP Data and Comparison with Re-Analysis Data." Solar Energy (80); pp. 1,390-1,401.

Marion, W. (1994). Summary Information and Data Sets for the HBCU Solar Measurements Network. NREL/TP-463-7090. Golden, CO: National Renewable Energy Laboratory.

Marion, W.; Myers, D. (1992). A Comparison of Data from SOLMET/ERSATZ and the National Solar Radiation Database. NREL/TP-463-5118. Golden, CO: National Renewable Energy Laboratory.

Marion, W.; Urban, K. (1995). User's Manual for TMY2s-Typical Meteorological Years Derived from the 1961-1990 National Solar Radiation Database. NREL/TP-463-7668. Golden, CO: National Renewable Energy Laboratory.

Maxwell, E.; Wilcox, S.; Rymes, M. (1993). Users Manual for SERI QC Software: Assessing the Quality of Solar Radiation Data. NREL/TP-463-5608 DE93018210. Golden, CO: National Renewable Energy Laboratory. Accessed September 26, 2014: http://www.nrel.gov/docs/legosti/old/5608.pdf.

Maxwell, E.L. (1998). "METSTAT-The Solar Radiation Model Used in the Production of the National Solar Radiation Database (NSRDB).” Solar Energy (62); pp. 263-279.

Myers, D.R.; Stoffel, T.L.; Reda, I.; Wilcox, S.M.; Andreas. A. (2002). "Recent Progress in Reducing the Uncertainty in and Improving Pyranometer Calibrations." Transactions of the ASME (124); pp. 44-50.

NASA. (2008). "Surface Meteorology and Solar Energy: Accuracy." https://eosweb.larc.nasa.gov/cgi-bin/sse/sse.cgi?skip@larc.nasa.gov+s06\#s06.

NCDC. (1978). User's Manual-Hourly Solar Radiation-Surface Meteorological Observations. TD-9724. Asheville, NC: National Climatic Data Center.

NCDC. (1979a). Final Report-Hourly Solar Radiation-Surface Meteorological Observations. TD-9724. Asheville, NC.

NCDC. (1979b). SOLDAY User's Manual (TD9739) Daily Solar Radiation-Surface Meteorological Data. Asheville, NC: Environmental Data and Information Service.

NREL. (1993). User's Manual for SERI_QC Software-Assessing the Quality of Solar Radiation Data. NREL/TP-463-5608. Golden, CO: National Renewable Energy Laboratory.

NSRDB. (1992). User's Manual-National Solar Radiation Database (1961-1990). Version 1.0. Golden, CO: National Renewable Energy Laboratory; Asheville, NC: National Climatic Data Center.

NSRDB. (1995). Final Technical Report: National Solar Radiation Database (1961-1990)_ Volume 2. NREL/TP-463-5784. Golden, CO: National Renewable Energy Laboratory. 
Olseth J.A.; Skartveit A. (1998). "High Latitude Global and Diffuse Radiation Estimated From METEOSAT Data." Presented at the European Conference on Applied Climatology, ECAC 98; Vienna, Austria, October 19-23, 1998.

Page J. (1996). "Technical Report for the Second SATELLIGHT Meeting”; June 1996; Bergen, Norway.

Perez, R.; Ineichen, P.; Moore, K.; Kmiecik, M.; Chain, C.; George, R.; Vignola, F. (2002). “A New Operational Satellite-to-Irradiance Model.” Solar Energy (73:5); pp. 307-317.

Pinker, R.T.; Laszlo, I. (1992). "Modeling Surface Solar Irradiance for Satellite Applications on a Global Scale.” Journal of Applied Meteorology (31); pp. 194-211.

Remund, J.; Lefèvre, W.L.; Ranchin, M.; Page, T. (2003). Worldwide Linke Turbidity Information, ISES Solar World Congress. Göteborg, Sweden: Solar Energy for a Sustainable Future.

Rigollier, C.; Bauer, O.; Wald, L. (2000). "On the Clear Sky Model of the 4th European Solar Radiation Atlas with Respect to the HELIOSAT Method." Solar Energy (68:1); pp. 33-48. See also Geiger, M.; Diabaté ,L.; Ménard L.; Wald, L. (2002). "A Web Service for Controlling the Quality of Global Solar Irradiation.” Solar Energy (73:6); pp. 475-480.

Sengupta, M.; Habte, A.; Gotseff, P.; Weekley, A.; Lopez, A.; Anderberg, M.; Molling, C.; Heidinger, A. (2014). "Physics-Based GOES Product for Use in NREL's National Solar Radiation Database." Preprint. Prepared for the European Photovoltaic Solar Energy Conference and Exhibition, Sept. 22-26, 2014. NREL/CP-5D00-62776. Golden, CO: National Renewable Energy Laboratory.

Thornton, P.E.; Hasenauer, H.; White. M.A. (2000). "Simultaneous Estimation of Daily Solar Radiation and Humidity from Observed Temperature and Precipitation: An Application Over Complex Terrain in Austria." Agricultural and Forest Meteorology (104); pp. 255-271.

Thornton, P.E.; Running, S.W. (1999). “An Improved Algorithm for Estimating Incident Daily Solar Radiation from Measurements of Temperature, Humidity, and Precipitation." Agriculture and Forest Meteorology (93); pp. 211-228.

Wilcox, S. (2007). National Solar Radiation Database 1991-2005 Update: User's Manual. NREL/TP-581-41364. Golden, CO: National Renewable Energy Laboratory.

Wilcox, S.; Marion, W. (2008). "Development of an Updated Typical Meteorological Year Data Set for the United States.” Solar 2008 Conference Proceedings; May 3-8, 2008, San Diego, California.

Wilcox, S.; Marion, W. (2008). Users Manual for TMY3 Data Sets. NREL/TP-581-43156. Revised May 2008. Golden, CO: National Renewable Energy Laboratory.

Wilcox, S.; Myers. D. (2008). Evaluation of Radiometers in Full-Time Use as the National Renewable Energy Laboratory Solar Radiation Research Laboratory. NREL/TP-550-44627. 
Golden, CO: National Renewable Energy Laboratory.

http://www.nrel.gov/docs/fy09osti/44627.pdf.

Wilcox, S.M. (2012). National Solar Radiation Database 1991-2010 Update: User's Manual. NREL/TP-5500-54824. Golden, CO: National Renewable Energy Laboratory; 479 pp.

Zelenka, A.; Perez, R.; Seals, R.; Renné, D. (1999). "Effective Accuracy of Satellite-Derived Irradiance." Theoretical and Applied Climatology (62); pp. 199-207.

\section{Chapter 6: Applying Solar Resource Data to Concentrating Solar Power Projects}

Ballestrín, J.; Marzo, A. (2011). "Solar Radiation Attenuation in Solar Tower Plants." Solar Energy (86:1); pp. 388-392.

Belhomme, B.; Pitz-Paal, R.; Schwarzbözl, P.; Ulmer, S. (2009). “A New Fast Ray Tracing Tool for High-Precision Simulation of Heliostat Fields.” Journal of Solar Energy Engineering $(131: 3)$.

Bender, G.; Davidson, F.; Eichelberger, F.; Gueymard, C.A. (2011). "The Road to Bankability: Improving Assessments for More Accurate Financial Planning." Solar 2011 Conference Proceedings; May 16-21, 2011, Raleigh, North Carolina.

Bendt, P.; Rabl, A. (1981). "Optical Analysis of Point Focus Parabolic Radiation Concentrators." Applied Optics (20:4); pp. 674-683.

Bendt, P.; Rabl, A.; Gaul, H.W.; Reed, K.A. (1979). Optical Analysis and Optimization of Line Focus Solar Collectors. SERI/TR-34-092. Golden, CO: National Renewable Energy Laboratory. Accessed May 12, 2014:

http://citeseerx.ist.psu.edu/viewdoc/download?doi=10.1.1.129.5191\&rep=rep1\&type=pdf.

Beyer, H.G.; Martinez, J.P.; Suri, M.; Torres, J.L.; Lorenz, E.; Müller, S.C.; Hoyer-Klick, C.; Ineichen, P. (2009). Management and Exploitation of Solar Resource Knowledge: Ca-Contract No. 038665-D 1.1.3. Report on Benchmarking of Radiation Products. http://www.mesor.org/docs/MESoR Benchmarking of radiation products.pdf.

Blair, N.; Dobos, A.; Freeman, J.; Neises, T.; Wagner, M.; Ferguson T.; Gilman P.; Janzou, S. (2014). System Advisor Model, SAM-2014.1.14: General Description. NREL/TP-6A20-61019. Golden, CO: National Renewable Energy Laboratory.

Buck, R. (2010). Solar Power Raytracing Tool SPRAY User Manual. Cologne, Germany: German Aerospace Center.

Carow, J.R. (2008). Anpassung langjähriger Satelliten-Strahlungszeitreihen an Bodenmesswerte eines Jahres. Diploma Thesis. Nordhausen, Germany: University of Applied Science. 
Cebecauer, T.; Suri, M. (2010). “Accuracy Improvements of Satellite-Derived Solar Resource Based on GEMS Re-Analysis Aerosols." SolarPACES Conference Proceedings; September 2124, 2010, Perpignan, France.

Chhatbar, K.; Meyer, R. (2011). "The Influence of Meteorological Parameters on the Energy Yield of Solar Thermal Power Plants." SolarPACES Conference Proceedings; September 20-23, 2011, Granada, Spain.

Dersch, J.; Schwarzbözl, P.; Richert, T. (2011). "Annual Yield Analysis of Solar Tower Power Plants with Greenius.” Journal of Solar Energy Engineering (133:3); 9 pp.

doi:10.1115/1.4004355.

Dobos, A.P. (2014) PVWatts Version 5 Manual. NREL/TP-6A20-62641. Golden, CO: National Renewable Energy Laboratory.

Ellis, A.; Behnke, M.; Barker, C. (2011). "PV System Modelling for Grid Planning Studies." 37th IEEE Photovoltaic Specialists Conference Proceedings; June 19-24, 2011, Seattle, Washington.

Freeman, J.; Whitmore, J.; Blair, N.; Dobos, A. (2014). Validation of Multiple Tools for Flat Plate Photovoltaic Modeling Against Measured Data. NREL/TP-6A20-61497. Golden, CO: National Renewable Energy Laboratory.

Freeman, J.; Whitmore, J.; Kaffine, L.; Blair, N.; Dobos, A. (2013). System Advisor Model: Flat Plate Photovoltaic Performance Modeling Validation Report. NREL/TP-6A20-60204. Golden, CO: National Renewable Energy Laboratory.

Gueymard C.A. (2013). "Aerosol Turbidity Derivation from Broadband Irradiance Measurements: Methodological Advances and Uncertainty Analysis." Solar 2013 Conference Proceedings; April 16-20, 2013, Baltimore, Maryland.

Gueymard, C.A.; George, R. (2005). "Gridded Aerosol Optical Depth Climatological Datasets over Continents for Solar Radiation Modeling." International Solar Energy Society Solar World Congress Proceedings; Orlando, Florida.

Gueymard, C.A.; Gustafson, W.T.; Bender, G.; Etringer, A.; Storck, P. (2012). "Evaluation of Procedures to Improve Solar Resource Assessments: Optimum Use of Short-Term Data from a Local Weather Station to Correct Bias in Long-Term Satellite-Derived Solar Radiation Time Series. World Renewable Energy Forum Conference Proceedings; May 13-17, 2012. Denver, $\mathrm{CO}$.

Gueymard, C.A.; Wilcox, S.M. (2009). "Spatial and Temporal Variability in the Solar Resource: Assessing the Value of Short-Term Measurements at Potential Solar Power Plant Sites." Solar 2009 Conference Proceedings; May 11-16, 2009, Buffalo, New York.

Gueymard, C.A.; Wilcox, S.M. (2011). "Assessment of Spatial and Temporal Variability in the U.S. Solar Resource from Radiometric Measurements and Predictions from Models Using Ground-Based or Satellite Data." Solar Energy (85); pp. 1,068-1,084. 
Habte, A.; Lopez, A.; Sengupta, M.; Wilcox, S. (2014). Temporal and Spatial Comparison of Gridded TMY, TDY, and TGY Data Sets. NREL/TP-5D00-60886. Golden, CO: National Renewable Energy Laboratory; 30 pp.

Hanrieder, N.; Wehringer, F.; Wilbert, S.; Wolfertstetter, F.; Pitz-Paal, R.; Campos, A.; Quasching, V. (2012). "Determination of Beam Attenuation in Tower Plants." SolarPACES Conference Proceedings; September 11-14, 2012, Marrakesh, Morocco.

Harmsen, E.W.; Tosado-Cruz, P.; Mecikalski, J.R. (2014). "Calibration of Selected Pyranometers and Satellite-Derived Solar Radiation in Puerto Rico.” International Journal of Renewable Energy Technology (5); pp. 43-54.

International Electrotechnical Commission. (1998). International Standard IEC 61724:

Photovoltaic System Performance Monitoring E Guidelines for Measurements, Data Exchange and Analysis. Geneva, Switzerland.

Janotte, N. (2012). Requirements for Representative Acceptance Tests for the Prediction of the Annual Yield of Parabolic Trough Solar Fields. PhD Thesis. Aachen, Germany: RheinischWestfälische Technische Hochschule Aachen University.

Kearney, D. (2011). Utility-Scale Parabolic Trough Solar Systems-Performance Acceptance Test Guidelines. NREL/SR-5500-48895. Golden, CO: National Renewable Energy Laboratory. http:/www.nrel.gov/docs/fy11osti/48895.pdf.

Kearney, D. (2013). Utility-Scale Power Tower Solar Systems: Performance Acceptance Test Guidelines. NREL/SR-5500-57272. Mehos, M. NREL Technical Monitor. Golden, CO: National Renewable Energy Laboratory.

Killius, N.; Schroedter-Homscheidt, M. (2012). Data Fusion of Satellite and Ground Measurement Time Series. EU-Project Report ENDORSE D3.5-Part B, September 2012.

Kistler, B.L. (1986). A User's Manual for Delsol3: A Computer Code for Calculating the Optical Performance and Optimal System Design for Solar Thermal Central Receiver Plants. Livermore, CA: Sandia National Laboratories.

Kurtz, S.; Krishnani, P.; Freeman, J.; Flottemesch, R.; Riley, E.; Dierauf, T.; Newmiller, J.; Ngan, L.; Jordan, D.; Kimber, A. (2014). "PV System Energy Test." 40th IEEE Photovoltaic Specialists Conference Proceedings; June 8-13, 2014, Denver, Colorado.

Leary, P.L.; Hankins, J.D. (1979). User's Guide for MIRVAL: A Computer Code for Comparing Designs of Heliostat-Receiver Optics for Central Receiver Solar Power Plants. SAND-77-8280. Livermore, CA: Sandia National Laboratories.

Mehos, M.; Perez, R. (2005). “Mining for Solar Resources: U.S. Southwest Provides Vast Potential.” Imaging Notes (20:2); pp. 12-15; NREL/JA-550-37799. Golden, CO: National Renewable Energy Laboratory. 
Meyer, R.; Torres Butron, J.; Marquardt, G; Schwandt, M.; Geuder, N.; Hoyer-Klick, C.; Lorenz, E.; Hammer, A.; Beyer, H.G. (2008). "Combining Solar Irradiance Measurements and Various Satellite- Derived Products to a Site-Specific Best Estimate.” SolarPACES Conference Proceedings; March 4-7, 2008, Las Vegas, Nevada.

Mieslinger, T.; Ament, F.; Chhatbar, K.; Meyer, R. (2014). “A New Method for Fusion of Measured and Model-Derived Solar Radiation Time Series." International Conference on Solar Heating and Cooling for Buildings and Industry Proceedings; September 23-25, Freiburg, Germany; Energy Procedia (48); pp. 1,617-1,626.

Pitman, C.L.; Vant-Hull, L.L. (1982). “Atmospheric Transmission Model for a Solar Beam Propagating between a Heliostat and a Receiver." Presented at the Annual Meeting of the American Solar Energy Society; June 1-5, 1982, Houston, Texas.

Quaschning, V.; Ortmanns, W.; Kistner, R.; Geyer, M. (2001). “Greenius: A New Simulation Environment for Technical and Economical Analysis of Renewable Independent Power Projects." Solar Forum 2001-Solar Energy: The Power to Choose Proceedings; April 21-25, 2001, Washington, DC.

Ruiz-Arias, J.A.; Quesada-Ruiz, S.; Fernández, E.F.; Gueymard, C.A. (2015). “Optimal Combination of Gridded and Ground-Observed Solar Radiation Data for Regional Solar Resource Assessment." Solar Energy (112); pp. 411-424.

Sarver, T.; Al-Qaraghuli, A.; Kazmerski, L.L. (2013). "A Comprehensive Review of the Impact of Dust on the Use of Solar Energy: History, Investigations, Results, Literature, and Mitigation Approaches." Renewable and Sustainable Energy Reviews (22:0); pp. 698-733.

Schroedter-Homscheidt, M.; Oumbe, A. (2013). "Validation of an Hourly Resolved Global Aerosol Model in Answer to Solar Electricity Generation Information Needs." Atmospheric Chemistry and Physics (13:7); pp. 3,777-3,791.

Schumann, K.; Beyer, H.G.; Chhatbar, K.; Meyer, R. (2011). "Improving Satellite-Derived Solar Resource Analysis with Parallel Ground-Based Measurements." International Solard Energy Society Solar World Congress Proceedings; August 28-September 2, 2011, Kassel, Germany.

Schwarzbözl, P. (2009). The User's Guide to HFLCAL: A Software Program for Heliostat Field Layout Calculation. Software release Visual HFLCAL VH12. Cologne, Germany: German Aerospace Center.

Sengupta, M.; Wagner, M.J. (2011). "Impact of Aerosols on Atmospheric Attenuation Loss in Central Receiver Systems.” SolarPACES Conference Proceedings; September 20-23, 2011, Granada, Spain.

Thøgersen, M.LL.; Motta, M.; Sørensen, T.; Nielsen, P. (2007). "Measure-Correlate-Predict Methods: Case Studies and Software Implementation.” European Wind Energy Conference Proceedings; May 7-10, 2007, Milan, Italy. 
Thuman, C.; Schnitzer, M.; Johnson, P. (2012). "Quantifying the Accuracy of the use of Measure-Correlate-Predict Methodology for Long-Term Solar Resource Estimates.” Solar 2012 Conference Proceedings; May 13-17, 2012, Denver, Colorado.

Tomson, T.; Russak, T.V.; Kallis, V.A. (2008). "Dynamic Behavior of Solar Radiation.” Badescu, V., ed. Modeling Solar Radiation at the Earth's Surface. Berlin, Germany: Springer.

van Sark, W.G.J.H.M.; Reich, N.H.; Müller, B.; Armbruster, A.; Kiefer, K.; Reise, C. (2012). "Review of Performance Ratio Development." World Renewable Energy Forum Proceedings; May 13-17, 2012, Denver, Colorado.

Vittitoe, C.; Biggs, F. (1981). User's Guide to HELIOS: A Computer Program for Modeling the Optical Behavior of Reflecting Solar Concentrators: Part 1. SAND 91-1180. Albuquerque, NM: Sandia National Laboratories.

Wendelin, T. (2003). "SolTRACE: A New Optical Modeling Tool for Concentrating Solar Optics." International Solar Energy Conference Proceedings; March 15-18, 2003, Kohala Coast, Hawaiit; pp. 253-260.

Wilbert, S. (2014). Determination of Circumsolar Radiation and Its Effect on Concentrating Solar Power. PhD Thesis. Aachen, Germany: Rheinisch-Westfälische Technische Hochschule Aachen University. http://darwin.bth.rwth-aachen.de/opus3/volltexte/2014/5171/.

Wolfertstetter, F.; Pottler, K.; Geuder, N.; Affolter, R.; Merrouni, A.A.; Mezrhab, A.; Pitz-Paal, R. (2014). "Monitoring of Mirror and Sensor Soiling with Tracs for Improved Quality of Ground Based Irradiance Measurements." Energy Procedia (49); pp. 2,422-2,432.

\section{Chapter 7: Forecasting Solar Radiation}

"Air Quality Forecasting at the Atmospheric Sciences Research Center." (2010). Atmospheric Sciences Research Center, University at Albany, State University of New York. http://asrc.albany.edu/research/aqf/.

“Atmospheric Physics." (2014). European Center for Medium Range Weather Forecasting. http://www.ecmwf.int/en/research/modelling-and-prediction/atmospheric-physics.

"Dataset I-i Atmospheric Fields-High-Resolution Forecast." (2014). "Global Forecast System." (2014). National Weather Service, Environmental Modeling Center. http://www.emc.ncep.noaa.gov/index.php?branch=GFS.

"Modeling and Prediction." (2014). European Center for Medium Range Weather Forecasting. http://www.ecmwf.int/en/research/modelling-and-prediction.

Bacher, P.; Madsen, H.; Nielsen, H.A. (2009). "Online Short-Term Solar Power Forecasting." Solar Energy (83); pp. 1,772-1,783.

Behr, H.D. (1997). "Solar Radiation on Tilted South Oriented Surfaces: Validation of Transfer Models." Solar Energy (61); pp. 399-413. 
Beyer, H.G.; Martinez, J.P.; Suri, M.; Torres, J.L.; Lorenz, E.; Müller, S.C.; Hoyer-Klick, C.; Ineichen, P. (2009). Management and Exploitation of Solar Resource Knowledge: Ca-Contract No. 038665-D 1.1.3. Report on Benchmarking of Radiation Products. http://www.mesor.org/docs/MESoR_Benchmarking_of_radiation_products.pdf

Boland, J.; Huang, J.; Ridley, B. (2013). "Decomposing Global Solar Radiation Into its Direct and Diffuse Components." Renewable and Sustainable Energy Reviews (28); pp. 749-756.

Breitkreuz, H. ; Schroedter-Homscheidt, M. ; Holzer-Popp, T. ; Dech, S. (2009). "Short-Range Direct and Diffuse Irradiance Forecasts for Solar Energy Applications Based on Aerosol Chemical Transport and Numerical Weather Modeling." Journal of Applied Meteorology and Climatology (48) ; pp. 1766.

Chow, C.W. ; Urquhart, B.; Lave, M., Domniguez, A. ; Kleissl, J. ; Shields, J. ; Washom, B. (2011). "Intra-Hour Forecasting with a Total Sky Imager at the UC3 San Diego Solar Energy Testbed." Solar Energy (85:11); pp. 2,881-2893. doi:10.1016/j.solener.2011.08.025.

Coimbra, F.M.; Pedro, H.T.C. (2013). "Stochastic Learning Methods." Kleissl, J., ed. Solar Energy Forecasting and Resource Assessment. Elsevier.

David, M.; Lauret, P.; Boland, J. (2013). "Evaluating Tilted Plane Models for Solar Radiation Using Comprehensive Testing Procedures at a Southern Hemisphere Location." Renewable Energy (51); pp. 124-131.

De Miguel, A.; Bilbao, J.; Aguar, R.; Kambezidis, K.; Negro, E. (2001). "Diffuse Solar Irradiation Model Evaluation in the North Mediterranean Belt Area." Solar Energy (70); 143153.

Dervishi, S.; Mahdavi, A. (2012). "Computing Diffuse Fraction of Global Horizontal Solar Radiation: A Model Comparison.”Solar Energy (86); pp. 1,796-1,802.

Diagne, M.; David, M.; Lauret, P.; Boland, J.; Schmutz, N. (2013). "Review of Solar Irradiance Forecasting Methods and a Proposition for Small-Scale Insular Grids." Renewable and Sustainable Energy Reviews (27), November; pp. 65-76.

Dudhia, J. (1989). "Numerical Study of Convection Observed During the Winter Monsoon Experiment Using a Mesoscale Two-Dimensional Model." Journal of the AtmosphericSciences (46); pp. 3,077-3,107. doi:10.1175/1520-0469(1989)0462.0.CO;2.

Dumortier, D. (1998). "The Satellite Model of Turbidity Variations in Europe." Presented at the Sixth SATELLIGHT Meeting; September 1998, Freiburg, Germany.

Dumortier, D. (2009). Management and Exploitation of Solar Resource Knowledge: Contract No. 038665-D 1.2.1 \& D1.2.2 Description of Solar Resource Products: Summary of Benchmarking Results and Examples of Use. http://www.mesor.org/docs/MESoR_Handbook_on_best_practices.pdf. 
Espinar, B.; Ramirez, L.; Drews, A.; Beyer, H.G.; Zarzalejo, L.F.; Polo, J.; Martin, L. (2008). "Analysis of Different Error Parameters Applied to Solar Radiation Data from Satellite and German Radiometric Stations.” Solar Energy (83:1).

European Center for Medium Range Weather Forecasting. http://www.ecmwf.int/en/forecasts/datasets/dataset-i-i-atmospheric-fields-high-resolutionforecast.

Fontoynont, M, et al. (1998). Satellight: A WWW Server Which Provides High-Quality Daylight and Solar Radiation Data for Western and Central Europe." 9th Conference on Satellite Meteorology and Oceanography Proceedings; May 1998, Paris; pp. 434-437.

Gastón, M.; Lorenz, E.; Lozano, S.; Heinemann, D.; Blanco, M.; Ramírez, L. (2009). "Comparison of Global Irradiance Forecasting Approaches." SolarPACES Conference Proceedings; September 15-18, 2009, Berlin, Germany.

Gauchet, C.; Blanc, P.; Espinar, B.; Charbonnier, B.; Demengel, D. (2012). "Surface Solar Irradiance Estimation with Low-Cost Fish-Eye Camera." Workshop on Remote Sensing Measurements for Renewable Energy Proceedings; May 22-23, 2012, Risoe, Denmark.

Ghonima, M.S.; Urquhart, B.; Chow, C.W.; Shields, J.E.; Cazorla, A.; Kleissl, J. (2012). "A Method for Cloud Detection and Opacity Classification Based on Ground-Based Sky Imagery." Atmospheric Measurement Techniques (5); pp. 2,881-2,892. doi:10.5194/amt-5-2881-2012, 2012.

Glahn, H.R.; Lowry, D.A. (1972). "The Use of Model Output Statistics (MOS) in Objective Weather Forecasting." Journal of Applied Meteorology and Climatology (11); pp. 1,203-1,211.

Gueymard C.A.; Ruiz-Arias J.A. (2014). "Performance of Separation Models to Predict Direct Irradiance at High Frequency: Validation over Arid Areas.” EuroSun 2014 ISES Conference Proceedings; Aix-les-Bans, France.

Gueymard, C.A. (2009). "Direct and Indirect Uncertainties in the Prediction of Tilted Irradiance for Solar Engineering Applications.” Solar Energy (83); pp. 432-444.

Gueymard, C.A.; Gustafson, W.T.; Bender, G.; Etringer, A.; Storck P. (2012). "Evaluation of Procedures to Improve Solar Resource Assessments: Optimum Use of Short-Term Data from a Local Weather Station to Correct Bias in Long-Term Satellite-Derived Solar Radiation Time Series." World Renewable Energy Forum Conference Proceedings; May 13-17, 2012. Denver, $\mathrm{CO}$.

Hammer, A.; Heinemann, D.; Hoyer, C.; Kuhlemann, R.; Lorenz, E.; Müller, R.; Beyer, H.G. (2003). "Solar Energy Assessment Using Remote Sensing Technologies." Remote Sensing of Environment (86); pp. 423-432.

Heinle, A.; Macke, A.; Srivastav, A. (2010). "Automatic Cloud Classification of Whole Sky Images.” Atmospheric Measurement Techniques (3); pp. 557-567. doi:10.5194/amt-3-557-2010, 2010. 
Hirsch, T.; Martin, N.; Gonzalez, L.; Biencinto, M.; Wilbert, S.; Schroedter-Homscheidt, M.; Chenlo, F.; Feldhoff, J.F. (2014). Technical Report on the Functional Requirements for the Nowcasting Method. DLR, CIEMAT.

Hoff, T.E.; Perez, R.; Kleissl, J.; Renne, D.; Stein, J. (2012). "Reporting of Irradiance Modeling Relative Prediction Errors." Progress in Photovoltaics: Research and Applications (21); pp. $1,514-1,519$.

Huang, J.; Korolkiewicz, M.; Agrawal, M.; Boland, J. (2013). "Forecasting Solar Radiation on an Hourly Time Scale Using a Coupled AutoRegressive and Dynamical System (CARDS) Model." Solar Energy (87), January; pp. 136-149. ISSN 0038-092X. http://dx.doi.org/10.1016/j.solener.2012.10.012.

Iacono, M.; Delamere, J.; Mlawer, E.; Shephard, M.; Clough, S.; Collins, W. (2008). "Radiative Forcing by Long-Lived Greenhouse Gases: Calculations with the AER Radiative Transfer Models." Journal of Geophysical Research (113D); 13103.

Ineichen, P. (2011). Global Irradiance on Tilted and Oriented Planes: Model Validations. Geneva, Switzerland: University of Geneva. http://archive-ouverte.unige.ch/unige:23519.

Inman, R.H.; Pedro, H.T.C.; Coimbra, C.F.M. (2013). "Solar Forecasting Methods for Renewable Energy Integration." Progress in Energy and Combustion Science (39); pp. 535-576.

Jolliffe, I.T.; Stephenson, D.B. (2003). Forecast Verification: A Practitioner's Guide in Atmospheric Science. New York: Wiley.

Jones, A.S.; Fletcher, S.J. (2013). "Data Assimilation in Numerical Weather Prediction and Sample Applications.” Kleissl, J., ed. Solar Energy Forecasting and Resource Assessment. Elsevier.

Kallos, G. (1997). “The Regional Weather Forecasting System SKIRON.” Symposium on Regional Weather Prediction on ParallelComputer Environments Proceedings; October 15-17, 1997, Athens, Greece.

Kalnay, E. (2003). Atmospheric Modeling, Data Assimilation and Predictability. Cambridge, UK: Cambridge University Press.

Kambezidis, H.D.; Psiloglou, B.E.; Gueymard, C.A. (1994). "Measurements and Models for Total Solar Irradiance on Inclined Surface in Athens, Greece.” Solar Energy (53); pp. 177-185.

Kleissl, J., ed. (2013). Solar Energy Forecasting and Resource Assessment. Elsevier.

Klucher, T.M. (1979). "Evaluation of Models to Predict Insolation on Tilted Surfaces." Solar Energy (23); pp. 111-114.

Kraas, B.; Schroedter-Homscheidt, M.; Madlener, R. (2013). "Economic Merits of a State-ofthe-Art Concentrating Solar Power Forecasting System for Participation in the Spanish Electricity Market.” Solar Energy (93); pp. 244-255. 
Kühnert, J.; Lorenz, E.; Heinemann, D. (2013). "Satellite-Based Irradiance and Power Forecasting for the German Energy Market.” Kleissl, J., ed. Solar Energy Forecasting and Resource Assessment. Elsevier.

Lara-Fanego, V.; Ruiz-Arias, J.A.; Pozo-Vázquez, D.; Santos-Alamillos, F.J.; Tovar-Pescador, J. (2011). "Evaluation of the WRF Model Solar Irradiance Forecasts in Andalusia (southern Spain)." Solar Energy (86:8), August; pp. 2,200-2,217.

Larson, V.E. (2013). "Forecasting Solar Irradiance with Numerical Weather Prediction Models." Kleissl, J., ed. Solar Energy Forecasting and Resource Assessment. Elsevier.

Lave, M.; Hayes, W.; Pohl, A.; Hansen, C.W. (2015). "Evaluation of Global Horizontal Irradiance to Plane of Array Irradiance Models at Locations across the United States." IEEE Journal of Photovoltaics (99); pp. 1-10.

Long, C.N.; DeLuisi, J.J. (1998). "Development of an Automated Hemispheric Sky Imager for Cloud Fraction Retrievals." 10th Symposium on Meteorological Observations and Instrumentation Proceedings; January 11-16, 1998, Phoenix, Arizona.

Lorenz, E.; Heinemann, D. (2012). "Prediction of Solar Irradiance and Photovoltaic Power." Comprehensive Renewable Energy (1); pp. 239-292. doi: 10.1002/pip.1224.

Lorenz, E.; Heinemann, D.; Hammer, A. (2004). "Short-Term Forecasting of Solar Radiation Based on Satellite Data." EuroSun 2004 Proceedings; June 20-23, Freiburg, Germany; pp. 841848.

Lorenz, E.; Scheidsteger, T.; Hurka, J.; Heinemann, D.; Kurz, C. (2011). "Regional PV Power Prediction for Improved Grid Integration." Progress in Photovoltaics: Research and Applications (19); pp. 757-771. doi: 10.1002/pip.1033

Lorenz, E; Hurka, J; Heinemann, D, Beyer H.G. (2009). "Irradiance Forecasting for the Power Prediction of Grid-Connected Photovoltaic Systems.” IEEE Journal of Special Topics in Earth Observations and Remote Sensing (2); pp. 2-10.

Lucas, B.; Kanade, T. (1981). "An Iterative Image Registration Technique with an Application to Stereovision.” Imaging Understanding Workshop Proceedings; April, Pittsburgh, Pennsylvania, pp. 121-130.

Macke, A.; HOPE-Team. (2014). "The HD(CP)² Observational Prototype Experiment HOPE: An Overview." ACP Special Issue: $H D(C P)^{2}$ Observational Prototype Experiment.

Madhavan, B.L.; Kalisch, J.; Macke, A. (2014). "Shortwave Surface Radiation Budgeting Network for Observing Small-Scale Cloud Inhomogeneity Fields." ACP Special Issue: HD $(C P)^{2}$ Observational Prototype Experiment.

Mailhot, J.; Belair, S.; Lefaivre, L.; Bilodeau, B.; Desgagne, M.; Girard, C.; Glazer, A.; Leduc, A.M.; Methot, A.; Patoine, A.; Plante, A.; Rahill, A.; Robinson, T.; Talbot, D.; Tremblay, A.; 
Vaillancourt, P.A.; Zadra, A. (2006). "The 15-km Version of the Canadian Regional Forecast System." Atmosphere-Ocean (44); pp. 133-149.

Marquez, R.; Coimbra, C.F.M. (2011). "Forecasting of Global and Direct Solar Irradiance Using Stochastic Learning Methods, Ground Experiments and the NWS Database." Solar Energy (85); pp. 746-756.

Mathiesen, P.; Kleissl, J. (2011). "Evaluation of Numerical Weatherprediction for Intra-Day Solar Forecasting in the Continental United States." Solar Energy (85); pp. 967-977.

Miller, S.; Rogers, M.; Heidinger, A.; Laszlo, I.; Sengupta, M. (2013). "Cloud Advection Schemes for Short-Term Satellite-Based Insolation Forecasts." Solar 2013 Conference Proceedings; April 16-20, 2013, Baltimore, Maryland.

Mlawer, E.J.; Taubman, S.J.; Brown, P.D.; Iacono, M.;J.; Clough, S.A. (1997). "Radiative Transfer for Inhomogeneous Atmospheres: RRTM, a Validated Correlated-k Model for the Longwave." Journal of Geophysical Research (102D); pp. 16,663-16,682.

Morcrette, J.J.; Barker, H.W.; Cole, J.N.S.; Iacono, M.J.; Pincus, R. (2008). "Impact of a New Radiation Package, McRad, in the ECMWF Integrated Forecasting System." Monthly Weather Review (136:12); pp. 4,773-4,798. doi:10.1175/2008MWR2363.1.

Mueller, S.C.; Remund, J. (2011). IEA/SHC Task 36: Radiation Forecast Switzerland: Final Report on Activity C3 IEA SHC Task 36. Cedar, MI: Solar Heating and Cooling Program, International Energy Agency.

Natschläger, T.; Traunmüller, W.; Reingruber, K.; Exner, H. (2008). "Lokal Optimierte Wetterprognosen zur Regelung Stark Umweltbeeinflusster Systeme.” SCCH, Blue Sky. Tagungsband Industrielles Symposium Mechatronik Automatisierung; pp. 281-284. Clusterland Oberösterreich $\mathrm{GmbH} / \mathrm{Mechatronik-Cluster.}$

Orehounig, K.; Dervishi, S.; Mahdavi, A. (2014). "Computational Derivation of Irradiance on Building Surfaces: An Empirically-Based Model Comparison.” Renewable Energy (71); pp. 185-192.

Pelland, S.; Gallanis, G.; Kallos, G. (2011). "Solar and Photovoltaic Forecasting through Postprocessing of the Global Environmental Multiscale Numerical Weather Prediction Model." Progress in Photovoltaics: Research and Applications (21:3), November; pp. 284-296.

Perez, R.; Hoff, T. (2013). “Solar Anywhere Forecasting.” Kleiss1, J., ed. Solar Energy Forecasting and Resource Assessment. Elsevier.

Perez, R.; Ineichen, P.; Maxwell, E.; Seals, R.; Zelenka, A. (1992). "Dynamic Global to Direct Irradiance Conversion Models.” ASHRAE Transactions-Research Series; pp. 354-369.

Perez, R.; Kivalov, S.; Schlemmer, J.; Hemker, K., Jr. (2009). "Validation of Short and Medium Term Operational Solar Radiation Forecasts in the U.S.” Solar Energy (84:12); pp. 2,161-2,172. http://dx.doi.org/10.1016/j.solener.2010.08.014. 
Perez, R.; Kivalov, S.; Schlemmer, J.; Hemker, K.; Renne, D.; Hoff, T.E. (2010. "Validation of Short- and Medium-Term Operational Solar Radiation Forecasts in the U.S." Solar Energy (84:5); pp. 2161e72.

Perez, R.; Kmiecik, M.; Schlemmer, J.; Root, L.; Moore, K.; Stackhouse, P. (2007). "Evaluation of PV Generation Capacity Credit Forecast on Day-Ahead Utility Markets." Solar 2007

Proceedings; July 8-12, 2007, Cleveland, Ohio.

http://www.asrc.cestm.albany.edu/perez/directory/LoadMatch.html.

Perez, R.; Lorenz, E.; Pelland, S.; Beauharnois, M.; van Knowe, G.; Hemker, K., Jr.;

Heinemann, D.; Remund, J.; Müller, S.C.; Traunmüller, W.; Steinmauer, G.; Pozo, D.; Ruiz-

Arias, J.; Lara-Fanego, V.; Ramirez-Satigosa, L.; Gaston-Romero, M.; Pomares, L.M. (2013).

"Comparison of Numerical Weather Prediction Solar Irradiance Forecasts in the US, Canada and

Europe." Solar Energy (94); pp. 305-326.

Perez, R.; Moore, K.; Stackhouse, P. (2007). "Forecasting Solar Radiation-Preliminary

Evaluation of an Approach Based Upon the National Forecast Database." Solar Energy (81:6); pp. 809-812.

Perez, R.; Seals, R.; Ineichen, P.; Stewart, R.; Menicucci, D. (1987). “A New Simplified Version of the Perez Diffuse Irradiance Model for Tilted Surfaces.” Solar Energy (39); pp. 221-231.

Pincus, R.; Barker, H.W.; Morcrette, J.J. (2003). “A Fast, Flexible, Approximate Technique for Computing Radiative Transfer in Inhomogeneous Cloud Fields.” Journal of Geophysical Research (108). (D13): 4376, doi:10.1029/2002JD003322.

Prahl, C.; Wilbert, S.; Kilius, N.; Schroedter-Homscheidt, M.; Hanrieder, N.; Müller, S. (2014).

"Kurzfristige Vorhersagen der solaren Direktstrahlung für optimierten Betrieb von

Solarkraftwerken.” Presented at the Sonnenkolloquium; Cologne, Germany.

Reikard, G. (2009). "Predicting Solar Radiation at High Resolutions: A Comparison of Time Series Forecasts.” Solar Energy (83:3); pp. 342-349. doi:10.1016/j.solener.2008.08.007.

Remund, J. (2009). Aerosol Optical Depth and Linke Turbidity Climatology: Description for Final Report of IEA SHC Task 36. Bern, Switzerland: Meteotest.

Remund, J.; Perez, R.; Lorenz, E. (2008). "Comparison of Solar Radiation Forecasts for the USA." 23rd European Photovoltaic SolarEnergy Conference Proceedings; Valencia, Spain.

Remund, J.; Perez, R.; Lorenz, E. (2008). "Comparison of Solar Radiation Forecasts for the USA." 23rd European Photovoltaic Solar Energy Conference Proceedings; Valencia, Spain.

Rikos, E.; Tselepis, S.; Hoyer-Klick, C.; Schroedter-Homscheidt, M. (2008). "Stability and Power Quality Issues in Microgrids under Weather Disturbances: An Implementation to the Kythnos Island Power System." IEEE Journal of Special Topics in Earth Observations and Remote Sensing (1); pp. 170-179. doi:10.1109/JSTARS.2008.2010557. 
Ruiz-Arias, J.; Pozo-Vázquez, D.; Sánchez-Sánchez, N.; Montávez, J.P.; Hayas-Barrú, A.; Tovar-Pescador, A. (2008). "Evaluation of Two MM5-PBL Parameterizations for Solar Radiation and Temperature Estimation in the South-Eastern Area of the Iberian Peninsula." Nuovo Cimento (31-C:5-6); pp. 825-842. http://matras.ujaen.es/pdf/ruizarias2009evaluation.pdf.

Ruiz-Arias, J.A.; Cebecauer, T.; Tovar-Pescador; Š́ri, M. (2010). "Spatial Disaggregation of Satellite-Derived Irradiance Using a High-Resolution Digital Elevation Model." Solar Energy (84:9); pp. 1,644-1,657.

Ruiz-Arias, J.A.; Dudhia, J.; Gueymard, C.A. (2014). "A Simple Parameterization of the ShortWave Aerosol Optical Properties for Surface Direct and Diffuse Irradiances Assessment in a Numerical Weather Model.” Geoscientific Model Development (7); pp. 1,159-1,174. doi:10.5194/gmd-7-1159-2014.

Ruiz-Arias, J.A.; Dudhia, J.; Santos-Alamillos, F.J.; Pozo-Vázquez, D. (2013). "Surface ClearSky Shortwave Radiative Closure Intercomparisons in the Weather Research and Forecasting Model." Journal of Geophysical Research: Atmospheres (118); pp. 9,901-9,913. doi:10.1002/jgrd.50778.

Savijärvi, H. (1990). "Fast Radiation Scheme for Mesoscale and Short-Range Forecast Models." Journal of Applied Meteorology and Climatology (29); pp. 437-447.

Shields, J.E.; Johnson, R.W.; Koehler, T.L. (1993). "Automated Whole Sky Imaging Systems for Cloud Field Assessment." 4th Symposium on Global Change Studies Proceedings; American Meteorological Society; pp. 228-231.

Skamarock, W.C.; Klemp, J.B.; Dudhia, J.; Gill, D.O.; Barker, D.M.; Duda, M.G.; Huang, X.Y.; Wang, W.; Powers, J.G. (2008). A Description of the Advanced Research WRF Version 3. NCAR/TN-475+STR. Boulder, CO: Mesoscale and Microscale Meteorology Division, National Center for Atmospheric Research.

Skartveit, A.; Olseth, J.A.; Tuft, M.E. (1998). "An Hourly Diffuse Fraction Model with Correction for Variability and Surface Albedo." Solar Energy (63); pp. 173-183.

Steinmaurer, G. (2008). "The Development of the Simulation Environment for the Energy Management of the Solar Assisted District Heating Grid in Wels." 1st International Conference on Solar Heating, Cooling and Buildings (EuroSun 2008) Proceedings; October 7-10, Lisbon, Portugal.

Tapakis, R.; Michaelides, S.; Charalambides, A.G. (2015). "Computations of Diffuse Fraction of Global Irradiance: Part 1-Analytical Modeling." Solar Energy (forthcoming).

Tiedtke, M. (1993). "Presentation of Clouds in Large-Scale Models." Monthly Weather Review (121:11); pp. 3,040-3,061. doi:10.1175/1520-0493(1993)1212.0.CO;2.

Traunmüller, W.; Steinmaurer, G. (2010). "Solar Irradiance Forecasting, Benchmarking of Different Techniques and Applications to Energy Meteorology." 3rd International Conference 
on Solar Heating, Cooling and Buildings (EuroSun 2010) Proceedings; September 28-October 1, 2010, Graz, Austria.

Urquhart, B., et al. (2013). "Sky Imaging Systems for Short-Term Forecasting.” Kleissl, J., ed. Solar Energy Forecasting and Resource Assessment. Elsevier.

Urquhart, B.; Kurtz, B.; Dahlin, E.; Ghonima, M.; Shields, J.E.; Kleissl, J. (2014). "Development of a Sky Imaging System for Short-Term Solar Power Forecasting." Atmospheric Measurement Techniques Discussions (7:5); 4,859-4,907.

Wittmann, M.; Breitkreuz, H.; Schroedter-Homscheidt, M.; Eck, M. (2008). "Case Studies on the Use of Solar Irradiance Forecast for Optimized Operation Strategies of Solar Thermal Power Plants." IEEE Journal of Special Topics in Earth Observations and Remote Sensing (1); pp. 18 27.

Wood-Bradley, P.; Zapata, J.; Pye, J. (2012). Cloud Tracking with Optical Flow for Short-Term Solar Forecasting. Canberra, Australia: Solar Thermal Group, Australian National University.

Wyser, K.; Rontu, L.; Savijärvi, H. (1999). "Introducing the Effective Radius into a Fast Radiation Scheme of a Mesoscale Model." Contributions to Atmospheric Physics (72:3); pp. 205-218.

\section{Chapter 8: Future Work}

Wilcox, S.M.; Myers, D.R. (2008). Evaluation of Radiometers in Full-Time Use at the National Renewable Energy Laboratory Solar Radiation Research Laboratory. NREL/TP-550-44627. Golden, CO: National Renewable Energy Laboratory. 


\section{Appendix A: Radiometer Manufacturers and Distributors}

This list is provided for information only.

Analytical Spectral Devices, Inc.

5335 Sterling Drive, Suite A

Boulder, CO 80301 USA

Telephone: 303-444-6522

Fax: 303-444-6852

http://www.asdi.com/

Spectral irradiance measurements

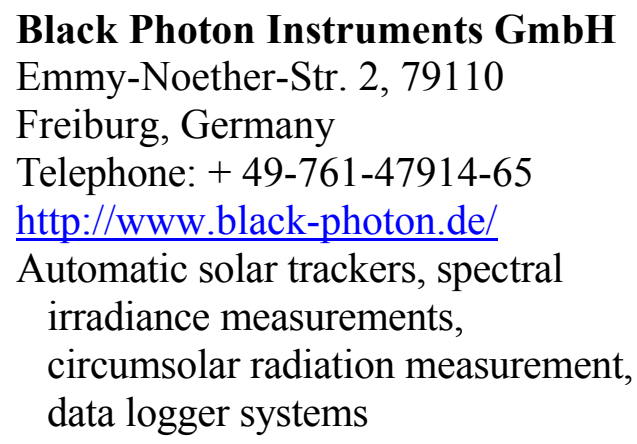

\section{Brusag}

Chapfwiesenstrasse 14

CH-8712 Stäfa

Switzerland

Telephone: +41-1-926-74-74

Fax: +41-1-926-73-34

http://www.brusag.ch/

Automatic solar trackers

Campbell Scientific, Inc.

815 West 1800 North

Logan, UT 84321 USA

Telephone: 435-753.2342 (Info.)

Telephone: 435-750.9681 (Orders)

Fax: 435-750.9540

E-mail: info@campbellsci.com

http:/www.campbellsci.com/

Data logger systems, weather stations

\section{Casella London Limited}

Regent House

Britannia Walk

London N1 7ND
Telephone: 01-253-8581

Telex: 26-16-41

Radiometers

\section{CSP-Services GmbH}

Friedrich-Ebert-Ufer 30

51143 Cologne, Germany

Telephone: +49-2203-959003-0

http://www.cspservices.de/

Rotating shadowband irradiometer

Davis Instruments Corporation

3465 Diablo Avenue

Hayward, CA 94545 USA

Telephone: 510-732-9229

Fax: 510-670-0589

http://www.davisnet.com/

Weather stations

\section{DAYSTAR}

3250 Majestic Ridge

Las Cruces, NM 88011 USA

Telephone: 505-522-4943

www.raydec.com/daystar

Radiometers

\section{Delta-T Devices, Ltd.}

130 Low Road, Burwell

Cambridge, CB25 0EJ

UK

U.S. Distributor:

Gary L. Woods, Sales Manager

garywoods@dynamax.com

http://www.dynamax.com/

Telephone: 800-896-7108 (Toll free)

Telephone: 281-564-5100

Fax: 281-564-5200

Radiometers, weather stations, data loggers 
EKO Instruments Trading Co.,

Ltd.

21-8

Hatagaya 1-chome

Shibuyaku, Tokyo 151

Japan

Telephone: 81-3-3469-4511

Fax: 81-3-3469-4593

Telex: J25364 EKOTRA

www.eko.co.jp/ 1

U.S. Distributor:

SC-International, Inc.

346 W. Pine Valley Drive

Phoenix, AZ 85023 USA

Telephone: 602-993-7877

Fax: 602-789-6616

Radiometers, trackers, data loggers

The Eppley Laboratory, Inc.

12 Sheffield Avenue

Newport, RI 02840 USA

Telephone: 401-847-1020

Fax: 401-847-1031

http://www.eppleylab.com/

Radiometers, trackers, data loggers

Hukseflux Thermal Sensors B.V.

Elektronicaweg 25

2628 XG Delft

The Netherlands

Telephone: +31-15-2142669

Fax: +31-152574949

http://www.hukseflux.com/

Radiometers

Hukseflux U.S. Sales Representative

Robert Dolce

HuksefluxUSA

P.O. Box 850

Manorville, NY 11949 USA

Telephone: 631-251-6963

E-mail: rdolce@HuksefluxUSA.com

Irradiance, Inc.

41 Laurel Drive

Lincoln, MA 01773 USA

Phone/Fax: 781-259-1134 http://www.irradiance.com/

Rotating shadowband radiometer

Kipp \& Zonen, Delft BV

Delftechpark 36

2628 XH Delft

The Netherlands

Telephone: 31-15-2755-210

info@kippzonen.com

http://www.kippzonen.com/

U.S. Sales Representative

Victor Cassella

Kipp \& Zonen

125 Wilbur Place

Bohemia, NY 11716 USA

Telephone: 631-589-2065 ext. 22

Fax: 631-589-2068

Email:vc@kippzonen.com

Radiometers, trackers, data loggers, weather stations

LI-COR, Inc.

4421 Superior Street

Lincoln, NE 68504 USA

Telephone: 800-447-3576 (Toll free)

Telephone: 402-467-3576

Fax: 402-467-2819

http://licor.com/

Radiometers, data loggers, weather stations

Matrix, Inc.

537 S. 31st Street

Mesa, AZ 85204 USA

Telephone: 480-832-1380

Radiometers

\section{Medtherm Corporation}

P.O. Box 412

Huntsville, AL 35804 USA

Telephone: 256-837-2000

Fax: 256-837-2001

http://medtherm.com/

Cavity radiometers 
MicroStrain, Inc.

459 Hurricane Lane, Suite 102

Williston, VT 05495

Telephone: 800-449-3878

http://www.microstrain.com/

Wireless sensors, data loggers

\section{Middleton Solar}

Factory 20, 155 Hyde Street

Yarraville, Victoria 3013 Australia

Telephone: +61-3-9396-1890

Fax: +61-3-9689-2384

http://www.middletonsolar.com/

Radiometers

Ocean Optics, Inc.

830 Douglas Ave.

Dunedin, FL 34698 USA

Telephone: 727-733-2447

Fax: 727-733-3962

http://www.oceanoptics.com/

European Sales Office: Geograaf 24

6921 EW DUIVEN The Netherlands

Telephone: +31-(0)-26-319-0500

Fax: +31-(0)-26-319-05-05

\section{Onset}

470 MacArthur Boulevard

Bourne, MA 02532 USA

Telephone: 508-743-3210

http://www.onsetcomp.com/

HOBO loggers, radiometers, met

sensors
PH. Schenk GmbH \& Co KG

Jedleseer Strasse 59

A-1210 Wien, Austria

Telephone: +43/1-271-51-31-0

E-mail: office@schenk.co.at

http://www.schenk.co.at/schenk

Radiometers

\section{Solar Light Company}

721 Oak Lane

Philadelphia, PA 19126 USA

Telephone: 215-927-4206

http://solarlight.com/

Radiometers

\section{Suntrace GmbH}

Brandstwiete 46

20457 Hamburg

Germany

Telephone: +49-40-767-96-38-0

http://www.suntrace.de/home.html

Rotating shadowband irradiometer

Yankee Environmental Systems,

Inc.

Montaque Industrial Park

101 Industrial Road

P.O. Box 746

Turners Falls, MA 01376 USA

Telephone: 413-863-0200

http://www.yesinc.com/

Radiometers, data systems, sky imagers 


\section{Appendix B: International Energy Agency Solar Heating and Cooling Programme}

The Solar Heating and Cooling Programme (SHC) was founded in 1977 as one of the first multilateral technology initiatives ("Implementing Agreements") of the International Energy Agency (IEA). Its mission is "to enhance collective knowledge and application of solar heating and cooling through international collaboration to reach the goal set in the vision of solar thermal energy meeting $50 \%$ of low temperature heating and cooling demand by $2050 . "$

The member countries collaborate on projects (referred to as "Tasks") in the fields of research, development, and demonstration and test methods for solar thermal energy and solar buildings.

A total of 53 such projects have been initiated to date, 39 of which have been completed.

Research topics include

- Solar Space Heating and Water Heating (Tasks 14, 19, 26, 44)

- Solar Cooling (Tasks 25, 38, 48, 53)

- Solar Heat or Industrial or Agricultural Processes (Tasks 29, 33, 49)

- Solar District Heating (Tasks 7, 45)

- Solar Buildings/Architecture/Urban Planning (Tasks 8, 11, 12, 13, 20, 22, 23, 28, 37, 40, $41,47,51,52)$

- Solar Thermal and PV (Tasks 16, 35)

- Daylighting/Lighting (Tasks 21, 31, 50)

- Materials/Components for Solar Heating and Cooling (Tasks 2, 3, 6, 10, 18, 27, 39)

- Standards, Certification, and Test Methods (Tasks 14, 24, 34, 43)

- Resource Assessment (Tasks 1, 4, 5, 9, 17, 36, 46)

- Storage of Solar Heat (Tasks 7, 32, 42).

In addition to the project work, special activities include

- SHC International Conference on Solar Heating and Cooling for Buildings and Industry

- Solar Heat Worldwide - annual statistics publication

- Memorandums of understanding with solar thermal trade organizations

- Workshops and conferences. 


\section{Members}

- Australia

- Austria

- Belgium

- Canada

- China

- Denmark

- European

Commission
- Finland

- France

- Germany

- Italy

- Mexico

- Netherlands

- Norway

- Portugal
- Singapore

- South Africa

- Spain

- Sweden

- Switzerland

- Turkey

- United Kingdom

- United States

\section{Sponsors}

- ECREEE - Economic Community of West African States Centre for Renewable Energy and Energy Efficiency

- European Copper Institute

- Gulf Organization for Research and Development

- RCREEE_-Regional Center for Renewable Energy and Energy Efficiency

\section{Further Information}

For up-to-date information about the IEA SHC, including many free publications, please visit www.iea-shc.org. 